The copyright of this thesis vests in the author. No quotation from it or information derived from it is to be published without full acknowledgement of the source. The thesis is to be used for private study or noncommercial research purposes only.

Published by the University of Cape Town (UCT) in terms of the non-exclusive license granted to UCT by the author. 


\title{
UNIVERSITY OF CAPE TOWN
}

Department of Civil Engineering

Water Research Group

\section{DESIGN AND PERFORMANCE OF BNR ACTIVATED SLUDGE SYSTEMS WITH FLAT SHEET MEMBRANES FOR SOLID-LIQUID SEPARATION}

\author{
By \\ Geoff J.G. du Toit \\ $B S c$, Engineering (Hons.) - Civil \\ University of Cape Town
}

Thesis submitted in partial fulfilment of the requirements for the degree

Master of science in Engineering at the University of Cape Town

December, 2006 


\section{DECLARATION BY CANDIDATE}

\section{GEOFFREY JOHN GUINNESS DU TOIT}

Hereby declare that I know the meaning of plagiarism and state that all the work in this document, save for that which is properly acknowledged, is my own. This work has not been submitted for a degree at another University.

Signed by candidate

December 2006 


\section{SYNOPSIS}

\section{BACKGROUND}

Wastewater treatment technologies have developed out of the need to protect receiving water bodies from the increasingly concentrated municipal and industrial waste streams generated through human activity. Of the methods available to clean wastewaters, biological nutrient removal (BNR) activated sludge (AS) is applied throughout South Africa and internationally as it has many advantages, notably it is cheaper to operate, does not introduce salinity into the water and is a simple and robust process. One of the key steps in the BNR AS process is the separation of biomass from the water. This is traditionally achieved by means of secondary settling tanks (SSTs), however recently the use of membranes for solid-liquid separation has gained popularity for the following reasons:

- Membranes are able to retain all solids and thus are insensitive to the settling characteristics of sludges,

- they can be run at high concentrations and hence smaller reactor volumes are required,

- membranes can produce a guaranteed high quality effluent free of pathogens and in some cases viruses too.

- Additionally smaller reactor volumes and the obviation of SSTs allow a substantial wastewater treatment plant footprint reduction.

Hence the combination of membranes in BNR AS is being increasingly applied. Membrane applications are becoming common in Europe, North America and Asia where much research has been conducted on the performance of membranes. The majority of the research has focused on the physical membrane performance, investigating the mechanisms of fouling, or on the membrane biological reactor (MBR) performance in removing organic compounds or nitrogen compounds from wastewater. There are however few case studies investigating BNR using membranes despite speculation that the inclusion of membranes may indeed affect the nature of the activated sludge biomass (Witzig et al., 2002).

In this investigation 6 case studies are reported from the literature in which various BNR configurations using membranes were proposed and investigated:

- Monti et al. (2006) compared two AS systems in a UCT configuration, one a MBR and the other using a conventional SST, with the same system design and operational parameters. Both systems had the same sludge mass and hence the influence of sludge concentration on BNR performance was not assessed.

- Lesjean et al. $(2003,2005)$ conducted a 4-year study on pre-denitrification and post-denitrification configurations of MBR wastewater treatment systems at bench and pilot scale. The systems were run at varying sludge ages and high solids concentrations. Excellent nutrient removal was observed in both configurations without additional carbon dosing in the post-denitrification system. However the systems were found to be generally under loaded, and precipitation of $\mathrm{P}$ due to calcium and ferric ions was observed which compromised observations of biological $\mathrm{P}$ removal. 
- Ahn et al. (2003) operated two lab scale MBR systems as a sequencing anaerobic/anoxic MBR (SAM) and the other a MLE system. P removal was observed in the SAM system, but at the cost of poor $N$ removal. BNR removal was observed but not optimised.

- Mouthon-Bello and Zhou (2005) conducted a study on a submerged MBR in an anoxic-anaerobic-aerobic configuration at 20 and 50 day sludge ages. Alum was dosed to the aerobic reactor to aid $P$ removal. This configuration made the anaerobic reactor redundant and biological $P$ removal could not be observed.

- Fleisher et al. (2005) investigated the BNR performance of an MBR system in a 5-stage configuration in order to ascertain whether biological and chemical $P$ removal could be achieved concurrently. They successfully demonstrated that BEPR could be achieved, in addition using chemical precipitation in the MBR reactor to completely remove all remaining $P$. The 5 -stage configuration was also successful in reducing $\mathrm{TN}$ to $<3 \mathrm{mgN} / \ell$. Fleischer et al. (2005) also modelled the observed system performance and suggested that current simulations (IWA ASM2d) adequately predicted the BNR performance of the system. Lastly they investigated the solids produced from the membrane system in order to determine if they differed from conventional solids and observed that a higher density cake could be produced from the MBR sludge than from conventional sludge.

- Ramphao et al. (2004) investigated the BNR performance of (wo systems in UCT configurations. In contrast to the study by Monti et al. (2006) the systems were run at their design solids concentrations, i.e. aerobic solids concentrations were $4500 \mathrm{mgTSS} / \ell$ in the conventional system compared to $18000 \mathrm{mgTSS} / \ell$ in the MBR system. The MBR system produced an effluent that was consistently equal to, or better than, the conventional effluent. It was found that the current BNR simulations could adequately predict system performance, but solids production in the MBR system was substantially higher than expected.

The research has shown that the inclusion of membranes in the system does not adversely affect the BNR performance, and also that at high concentration sludges, as are characteristic of MBR systems, the BNR performance remains consistent. However these studies have only indicated that MBRs are feasible and have not investigated in depth how the performance is affected - notably how to optimise BNR in MBR systems. The studies have demonstrated the inability to compare systems without the kinetic constants for modelling being established, as each investigation is on different wastewaters and serves different BNR objectives. Additionally information important to design such as the oxygen transfer efficiency in high solids concentration sludges remains much debated in the literature (Wagner and Pöpel, 1998, Günder and Krauth, 1999).

\section{RESEARCH OBJECTIVES}

This investigation followed on directly from the work reported in Ramphao et al. (2004) in which the feasibility of a MBR for BNR treatment was investigated. From the conclusions drawn in Ramphao et al. (2004) it was recommended that further investigations into the kinetics of the MBR system be undertaken, to better understand the influence of the membranes and how the concentration of the sludge impacts on the biological activity and behaviour of the micro-organisms in the system. 
Additionally design specific information on the oxygen transfer of the high concentration sludge required further investigation. Thus the research objectives for this investigation were:

- To verify the results obtained in the initial investigation (Ramphao et al., 2004) with particular emphasis on explaining the phenomena of increased sludge production;

- To gain a better understanding of the operating conditions and considerations of MBR BNR systems including oxygen transfer in high concentration sludges;

- To provide a parent system from which further testing into the kinetics of a MBR BNR system could be performed (Parco et al. 2006).

\section{RESEARCH APPROACH}

In order to address these objectives two parallel lab-scale membrane (MBR) and conventional (CAS) activated sludge systems (Figs. 1 and 2) were operated under laboratory conditions allowing their behaviour to be monitored and their performance compared. In order to verify the previous results of Ramphao et al. (2004) the same original experimental apparatus and operational conditions were adopted and testing continued.

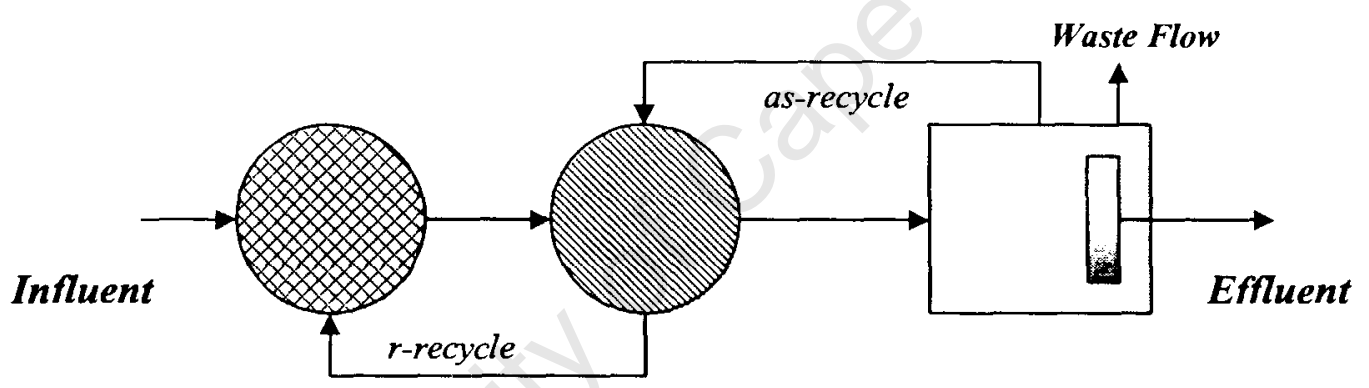

Anaerobic Anoxic Aerobic

Figure 1: $\quad$ Schematic layout of the MBR UCT system

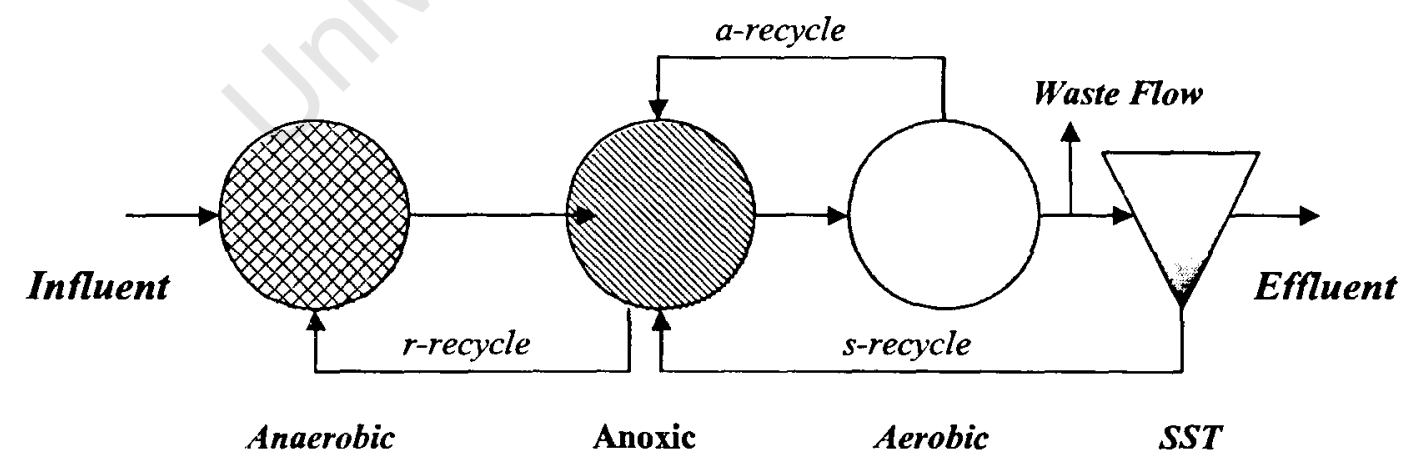

Figure 2: $\quad$ Schematic layout of the conventional UCT system

- Both systems were run in a UCT configuration, which was chosen because it allows denitrification and $\mathrm{P}$ removal to act independently of each other; the anaerobic reactor is protected from recycled nitrate from the anoxic reactor provided the recycles do not overload the anoxic reactor with nitrate. As far as possible system design and operational parameters such as zone mass fractions 
and inter-reactor recycles were kept the same in both systems. A summary of the system design and operating parameters is presented in Table 1.

Table 1: $\quad M B R$ and conventional UCT systems' design and operating parameters.

\begin{tabular}{|l|c|c|}
\hline SYSTEM PARAMETERS & MBR UCT & CAS UCT \\
\hline Sludge Age (d) & 20 & 20 \\
\hline Anaerobic (R1) Volume $(\ell)$ & 19 & 5.6 \\
\hline Anoxic (R2) Volume $(\ell$ ) & 21 & 6.2 \\
\hline Aerobic (R3) Volume $(\ell$ ) & $34^{1}$ & 13.2 \\
\hline Anaerobic (R1) Mass Fraction (\%) & $12.6^{2}$ & $12.6^{2}$ \\
\hline Anoxic (R2) Mass Fraction (\%) & $27.9^{2}$ & $27.9^{2}$ \\
\hline Aerobic (R3) Mass Fraction (\%) & $59.5^{2}$ & $59.5^{2}$ \\
\hline s-sludge Return Recycle (SST to R2) & - & $1: 1$ \\
\hline a-recycle Return Recycle (R3 to R2) & $3: 1$ & $2: 1$ \\
\hline r-recycle Return Recycle (R2 to R1) & $1: 1$ & $1: 1$ \\
\hline Hydraulic retention time (d) & 0.53 & 1.67 \\
\hline Aerobic MLVSS conc. (mgVSS/ $\ell$ ) & 12500 & 3600 \\
\hline Aerobic MLSS conc. (mgTSS/ $\ell$ ) & 18000 & 5000 \\
\hline Influent flow ( $\ell / d)$ & 140 & 15 \\
\hline Feed COD concentration (mg/ $\ell$ ) & 1000 & 1000 \\
\hline Waste flow from Aerobic Reactor $(\ell / d)$ & 2.85 & 1.1 \\
\hline OUR (mgO/ $\ell$ ) & 135 & 37 \\
\hline Membrane Flux (m $\left.{ }^{3} / \mathrm{m}^{2} / \mathrm{d}\right)$ & 0.239 & - \\
\hline
\end{tabular}

${ }^{1}$ Effective aerobic volume. MBR UCT Aerobic reactor is in fact $32 \ell$, with a side OUR -aeration reactor for OUR measurement of $2 \ell$.

${ }^{2}$ For the given $\mathrm{a}, \mathrm{s}$ and $\mathrm{r}$ recycle values

There were however some differences between the two MBR and CAS UCT systems:

- The major difference between the two systems was the influent flow to the MBR system, which was fed a little over three times (3.1) the organic load of the CAS system in order to generate the solids concentration required for effective cross-flow scour (greater that $12000 \mathrm{mgTSS} / \ell$ versus 4000 $\operatorname{mgTSS}(\ell)$. As the same feed was used for both systems, the higher influent flow rate to the MBR system resulted in a decreased hydraulic retention time (13 hours versus 40 hours).

- Sludge distribution and zone mass fractions in the MBR UCT system differed slightly from that in the conventional UCT system, in that they are linked to the a- and r-recycles due to the solids liquid separation step occurring in the aerobic reactor creating a concentration effect (Ramphao et al., 2004). Additionally in the MBR UCT system the measured recycles were found to differ from the design recycles resulting in system mass fractions that differed slightly from the design mass fractions: Average mass fractions for the MBR UCT system were anaerobic:anoxic:aerobic $=0.139: 0.277: 0.584$ versus the original design values of $0.126: 0.279: 0.595$. In the conventional UCT system only the r-recycle influenced the system mass fractions and was found to 
remain close to the design value, hence the system mass fractions were assumed to be the same as those determined in design, 0.126:0.279: 0.595. This resulted in slight differences in the system mass fractions of the two systems.

The two UCT systems were fed screened ( $1 \mathrm{~mm}$ mesh) raw (unsettled) sewage diluted to $800 \mathrm{mgCOD} / \ell$, increased to $1000 \mathrm{mgCOD} / \ell$ by adding $200 \mathrm{mgCOD} / \ell$ sodium acetate to accentuate BEPR. Ammonia was added to maintain an influent TKN/COD ratio of 0.10 and phosphorus was added to ensure no $\mathrm{P}$ limitation. Additionally sodium bicarbonate was added to the feed to maintain $\mathrm{pH}>7$ in both systems. The sewage was collected in $2 \mathrm{~m}^{3}$ batches from the Mitchells Plain Wastewater Treatment Plant (Cape Town), stored at $4^{\circ} \mathrm{C}$ and served as feed for about two weeks.

Both systems were monitored daily via the standard engineering parameters presented in Table 2. Additionally recycle flow rates and trans-membrane pressure (TMP, constant flux $0.24 \mathrm{~m}^{3} / \mathrm{m}^{2} / \mathrm{d}$ ) were monitored daily. Mixed liquor samples were analysed microscopically for filaments and floc structure monthly. Sewage readily biodegradable COD (RBCOD) was measured daily according to Ekama et al., (1986) and as a check by flocculation-filtration (Mbewe et al., 1995). Daily sludge was wasted from the systems in accordance with the required sludge age of 20 days. OUR was measured in a side stream reactor to the aerobic reactor. This was necessitated by the requirement for constant aeration in the aerobic reactor in order to achieve effective scour across the membranes.

Table 2: $\quad$ Sampling position and parameter measurement

\begin{tabular}{|c|c|c|c|c|c|c|c|c|c|c|c|}
\hline TEST & $\mathrm{COD}$ & $\mathrm{TKN}$ & $\mathrm{FSA}$ & $\mathrm{NO}_{3}$ & $\mathrm{NO}_{2}$ & $\mathrm{~T}-\mathrm{P}$ & $\mathrm{TSS}$ & VSS & OUR & DSVI & $\mathrm{pH}$ \\
\hline & 1 & 2 & 3 & 4 & 5 & 6 & 7 & 8 & 9 & 10 & 11 \\
\hline Influent & $\mathrm{U}$ & $\mathrm{F} ; \mathrm{U}$ & $\mathrm{F}$ & & & $\mathrm{U}$ & & & & & \\
\hline Anaerobic & & & & $\mathrm{F}$ & $\mathrm{F}$ & $\mathrm{F}$ & $\mathrm{U}$ & $\mathrm{U}$ & & & \\
\hline Anoxic & & & & $\mathrm{F}$ & $\mathrm{F}$ & $\mathrm{F}$ & $\mathrm{U}$ & $\mathrm{U}$ & & & \\
\hline $\begin{array}{c}\text { Aerobic } \\
\text { Final }\end{array}$ & $\mathrm{U}$ & $\mathrm{U}$ & & $\mathrm{F}$ & $\mathrm{F}$ & $\mathrm{F}$ & $\mathrm{U}$ & $\mathrm{U}$ & $*$ & $*$ & $*$ \\
\hline
\end{tabular}

F = Filtered through Schleicher \& Schull ME 25/21 $0.45 \mu \mathrm{m}$ membrane filters.

$\mathrm{U} \quad=$ Unfiltered samples

* $\quad=$ Direct measurement taken (filtering not applicable)

The numbers on the test methods below refer to Standard Methods (1985), though some have been adapted to suit the requirements of the UCT wastewater research laboratory (WRL).

1. COD Chemical Oxygen Demand, open reflux method; 5220 (B)

2. TKN Total Kjeldahl Nitrogen, micro-kjeldahl method; 4500 - Norg (C)

3. FSA Free and Saline ammonia, titrimetric method; $4500-\mathrm{NH}_{3}$ (B), (E)

4. $\mathrm{NO}_{3} \quad$ Hydrazine reduction (Technicon Auto-Analyzer); $4500-\mathrm{NO}_{3}(\mathrm{H})$

5. $\mathrm{NO}_{2} \quad$ Hydrazine reduction (Technicon Auto-Analyzer); $4500-\mathrm{NO}_{2}(\mathrm{H})$ 
6. T-P Total Phosphorus; Sulphuric acid/Persulphate digestion at $100^{\circ} \mathrm{C}$ followed by molybdate-vanadate colour development for orthophosphate (Standard Methods, 1985 - Method 424C III)

7. TSS Total suspended solids dried at $103-105^{\circ} \mathrm{C} ; 2540$ (D)

8. VSS Volatile suspended solids ignited at $600^{\circ} \mathrm{C} ; 2450$ (E)

9. DSVI Dilute Sludge Volume Index; (Ekama and Marais, 1984b), 271 (D)

10. OUR Oxygen Utilization Rate; automated (Randall, et al., 1991), 271 (B)

11. $\mathrm{pH} \quad \mathrm{pH}$ meter, Hanna Instruments model $\mathrm{HI} 9023 ; 4500-\mathrm{H}^{+}(\mathrm{B})$

In order to investigate the oxygen transfer efficiencies of the system unsteady state aeration testing and steady state aeration testing were conducted in the aerobic reactor with tap water and mixed liquor at various concentrations respectively. Due to the disruptive nature of these tests this testing was conducted once the main investigation had been completed

\section{EXPERIMENTAL SYSTEM RESULTS}

\subsection{Steady-State Periods}

The steady-state investigation was conducted for 449 days with a total of 29 sewage batch periods. Each scwage batch was accepted as a steady-state period. For every sewage batch data outside the range mean $\pm 1.96 \times$ sample standard deviation $(95 \%$ confidence interval), were rejected. All remaining data were considered valid and averaged to represent the "average" response of the system for that sewage batch (steady-state) period. These steady-state averages were used to calculate average ratios of process characteristics.

\subsection{Mass Balances}

Nitrogen and COD mass balances were performed for each sewage batch period in order to verify the accuracy and reliability of the analytical data, and to provide an early warning sign if the data was poor. Good $\mathrm{N}$ and COD mass balances were achieved for the MBR system of $96 \%$ and $103 \%$ respectively. However consistently low mass balances were achieved for the conventional system of $80 \%$ and $83 \%$ respectively. The poor mass balances in the conventional system were investigated extensively and the low balances attributed to unaccounted for sludge losses which were largely as a consequence of spillages.

\subsection{Mixed Liquor Solids}

For all the MBR and conventional system reactors the mixed liquor solids parameters, MLSS, MLVSS, COD and TKN were monitored regularly from the beginning of the investigation. The information on the variation of mixed liquor concentrations with time was necessary in order to interpret the BNR performance of the systems.

\subsubsection{Sludge Age (Rs)}

As far as possible the sludge age of the system was consistently maintained at 20 days by wasting the appropriate mixed liquor volume from the aerobic reactor. In the event of an unintentional mixed liquor loss from the system due to i) a spill from the 
reactors, ii) a burst interconnecting pipe, or iii) foam removal, knowledge of the total solids content of the system allowed the mass of mixed liquor lost to be determined and approximate mixed liquor mass to be wasted reduced accordingly over the subsequent days.

\subsubsection{MLSS and MLVSS concentrations}

Throughout the investigation there were minor and major unintended mixed liquor losses, typically through spillages or while cleaning the system. Where ever possible sludge was retained, filtered through a $2 \mathrm{~mm}$ mesh to break up sludge "clots" and to prevent the accidental addition of foreign objects into the system, and returned to the system. When sludge was lost the total mixed liquor lost was calculated from the difference in solids concentration from the day prior to the spill, and the loss compensated for by not wasting for the equivalent number of days following the spill.

The MLSS concentration in the aerobic reactor remained within the range 16000 to $19000 \mathrm{mgTSS} / \ell$ and the anoxic and anaerobic concentrations were within the ranges of $12000-14000 \mathrm{mgTSS} / \ell$ and $6000-8000 \mathrm{gTSS} / \ell$ respectively for the MBR system. This is less than the design solids concentration, but is attributed to a lower average $S_{t i}$ and higher $f_{S, u p}$ than were used for design.

Similarly the conventional system showed lower MLSS concentrations than expected with aerobic mixed liquor solids concentrations consistently within the range $2500-$ $3500 \mathrm{mgTSS} / \ell$ compared to the expected range $4500-5500 \mathrm{mgTSS} / \ell$.

\subsubsection{Mixed Liquor Characteristics}

In order to quantify the mixed liquor in both systems the VSS, TSS, COD and TKN concentrations of the mixed liquor were measured. Investigation average ratios between these parameters are listed in Table 4.

Table 3: $\quad$ Mixed liquor parameters

\begin{tabular}{|l|l|l|l|}
\hline Parameter & Unit & MBR System & Conv. System \\
\hline VSS/TSS & $\mathrm{mgVSS} / \mathrm{mgTSS}$ & 0.809 & 0.814 \\
\hline COD/NSS & $\mathrm{mgCOD} / \mathrm{mgVSS}$ & 1.402 & 1.496 \\
\hline TKN/NSS & $\mathrm{mgN} / \mathrm{mgVSS}$ & 0.085 & 0.094 \\
\hline
\end{tabular}

- Both systems exhibited high VSS/TSS ratios which are not characteristic of BEPR systems. In BEPR systems the development of a PAO population is encouraged. PAO's have internally a low VSS/TSS ratio due to the additional inorganic polyphosphate in their cell mass.

- Although the COD/VSS ratios differ substantially from each other in the two systems, they both fall very close to the expected and theoretical $f_{\mathrm{CV}}$ values of 1.48 and 1.42 respectively (WRC., 1984).

- The COD/VSS ratio indicates that the COD incorporated into the mixed liquor was lower in the MBR system than in the conventional system. However the comparison of mass balances showed a greater proportion of COD was removed from the MBR system via the mixed liquor wasted than in the conventional system. In order for this to occur proportionally more mixed liquor would need to be wasted from the MBR system than the conventional 
system in order to achieve higher COD removals through wasting, particularly with lower COD incorporated in the mixed liquor. This is only possible, at the same sludge age, if the sludge production in the MBR system was substantially greater than that in the conventional system.

\subsubsection{Sludge Production}

Ramphao et al. (2004) reported that sludge production in the two UCT systems differed significantly. A number of explanations were suggested, however more data was required in order to validate the observations. Hence one of the objectives for this investigation was to validate the observed discrepancy in sludge production in the two systems. However the poor COD balances in the conventional system make the sludge production data incomparable.

- With the exception of sewage batch 4 the sludge production in the MBR system was consistently higher than that of the conventional UCT system by on average $50 \%$.

- Average sludge productions for the two systems were 0.311 and 0.205 (mgVSS/d)/(mgCOD/d) for the MBR and conventional UCT systems respectively. Ramphao et al. (2004) reported similar results, 0.32 and 0.22 (mgVSS/d)/(mgCOD $/ \mathrm{d}$ ) respectively.

- The higher sludge production in the MBR UCT system can be accommodated in the steady state design model by increasing the unbiodegradable particulate COD fraction $\left(f_{S \text {.up }}\right)$. This was demonstrated by the high $f_{S \text {,up }}$ values observed in the MBR system of 0.224 in the investigation of Ramphao et al. (2004) and 0.200 in this investigation.

As was noted by Ramphao et al., (2004) a number of factors contribute to the higher sludge production in the MBR system:

- The retention of solids by membranes in the MBR system resulted in approximately $17.2 \mathrm{mgTSS} / \ell$ accumulating in the MBR system that would have been lost through the SST in the conventional UCT system. This would have "increased" sludge production by $0.018(\mathrm{mgVSS} / \mathrm{d}) /(\mathrm{mgCOD} / \mathrm{d})$.

- In the MBR UCT system, organics that would be considered as soluble in the conventional system are retained. This is demonstrated by the difference in the $0.45 \mu \mathrm{m}$ filtered effluent COD system averages from the MBR and conventional UCT systems of $8 \mathrm{mgCOD} / \ell$. This would account for approximately 0.008 in the difference in the $\mathrm{f}_{\mathrm{S} \text {,up }}$ values above.

Additionally Ramphao et al., (2004) proposed two other explanations for the difference in sludge production in the two systems.

- Higher P removal in the MBR UCT system suggests a greater PAO population which would produce more sludge per unit influent COD than OHOs due to their lower endogenous respiration rates (Wentzel et al., 1990).

- Particulate organics that are biodegradable in the conventional UCT system are no longer biodegradable in the MBR system due to factors such as high MLSS concentrations, or different floc morphology. 
In the literature previous studies comparing conventional and MBR BNR systems run under the same operating conditions have indicated that the sludge production of the two systems were very similar (Masse et al., 2006, Monti et al., 2006), however in both investigations the systems were run at the same COD loading rate per unit reactor volume. Masse et al., (2006) included the sludge lost through the SST in sludge wasting calculations in order to compare sludge productions in the two systems.

Additionally sludge production in nitrification-denitrification (ND) and ND biological excess phosphorus removal (BEPR) systems operated using the same wastewater source, for sludge ages in the region of 20 days, have produced sludge in comparable magnitudes to those observed in the conventional system ranging from 0.18 to 0.31 $(\mathrm{mgVSS} / \mathrm{d}) /(\mathrm{mgCOD} / \mathrm{d})$. Thus it would appear that there is an increase in sludge production in the MBR system linked to the increased MLSS concentration in the MBR system and the retention by membranes of all solids.

\subsection{SYSTEM REMOVALS AND EFFLUENT QUALITY}

The average removals of both the MBR and conventional UCT systems for this investigation are summarised in Table 4.

Table 4: $\quad$ Summary of the influent and effluent qualities, and the resultant removals, of both UCT systems.

\begin{tabular}{|c|c|c|c|c|c|c|}
\hline \multirow{2}{*}{\multicolumn{2}{|c|}{ Parameter }} & \multirow{3}{*}{$\begin{array}{l}\text { Influent } \\
951.2 \\
\end{array}$} & \multicolumn{2}{|c|}{ MBR UCT } & \multicolumn{2}{|l|}{ Conv. UCT } \\
\hline & & & Effluent & Efficiency & Effluent & Efficiency \\
\hline $\mathrm{COD}$ & $\mathrm{mgCOD} / \mathrm{l}$ & & 42.0 & $95.6 \%$ & $74.6^{1}\left(50^{2}\right)$ & $92.2 \%$ \\
\hline TKN & $\mathrm{mgN} / \mathrm{l}$ & 106.5 & 1.7 & $98.4 \%$ & $3.7^{1}\left(2.0^{2}\right)$ & $96.5 \%$ \\
\hline FSA & $\mathrm{mgN} / \mathrm{I}$ & 81.7 & 0.7 & $99.1 \%$ & 1.3 & $98.4 \%$ \\
\hline $\mathrm{NO} 3$ & $\mathrm{mgN}-\mathrm{NO} 3 / \mathrm{l}$ & 0 & 18.0 & - & 18.1 & - \\
\hline TN & $\mathrm{mgN} / \mathrm{l}$ & 106.5 & 19.7 & $81.5 \%$ & $21 .{ }^{*}$ & $79.5 \%$ \\
\hline TP & $\mathrm{mgP} / \mathrm{I}$ & 30.3 & 9.0 & $21.3 \mathrm{mgP} / \mathrm{linf}$ & 13.6 & $16.7 \mathrm{mgP} / \mathrm{linf}$ \\
\hline TSS & mgTSS/l & N/A & 0.0 & - & 21.5 & - \\
\hline e. coli & $\mathrm{CFU} / 100 \mathrm{ml}$ & N/A & $<10$ & - & 2250 & - \\
\hline
\end{tabular}

${ }^{1}$ unfiltered sample; ${ }^{2} 0.45$ filtered sample;

$\mathrm{N} / \mathrm{A}=$ value not available

\subsubsection{COD Removals}

The COD removal efficiency of the MBR system (96\%) was superior to that of the conventional system (92\% unfiltered, $95 \% 0.45 \mu \mathrm{m}$ filtered). These results were comparable to those observed by Ramphao et al. (2004) of 96\% COD removal in the MBR UCT system and 93\% unfiltered and 94\% $0.45 \mu \mathrm{m}$ filtered in the conventional UCT system.

The difference in filtered COD removals from both systems is attributed to the smaller pore size of the membranes which retain organics that would otherwise be considered soluble in a conventional system. However membrane specifications state that the nominal pore size of the Kubota $\mathbb{B}$ membranes used in this study were $0.4 \mu \mathrm{m}$, while the membranes used to filter the conventional system effluent are only marginally larger $(0.45 \mu \mathrm{m})$, thus the improved filterability of the membrane system is 
attributed rather to the development of a dynamic gel layer which reduces the effective pore size of the membranes.

The MBR unfiltered "effluent" COD values (measured from the supernatant of the DSVI test on MBR aerobic sludge) were consistently higher than those in the conventional system which confirmed that the MBR system retains and accumulates unsettleable material which would flow out with the effluent in a conventional system. This was observed in the DSVI test in which the supernatant of the conventional system mixed liquor would become clear in time, whereas the MBR supernatant remained cloudy.

The difference between the filtered $(50 \mathrm{mgCOD} / \ell)$ and unfiltered $(75 \mathrm{mgCOD} / \ell)$ effluent COD measured in the conventional UCT system is attributed to the loss of non-settleable solids through the SST. Approximately $21.5 \mathrm{mgTSS} / \ell$ were lost as COD in the effluent.

After Ramphao et al. (2004), differences in the MBR UCT effluent COD and the conventional UCT effluent are accommodated in the steady state design models as differences in the soluble COD fractions $\left(f_{\mathrm{S}, \text { us }}\right)$ which were 0.044 and 0.068 respectively.

\subsubsection{N Removals}

The TKN removal efficiency of the MBR system (98\%) was marginally better than that of the conventional system (97\% unfiltered, $98 \% 0.45 \mu \mathrm{m}$ filtered). This is again attributed to the retention of solids by the membranes that would have been lost in the effluent of the conventional UCT system. FSA removal was also very similar for both systems $99 \%$ in the MBR system and $98 \%$ in the conventional system. Thus near complete nitrification was achieved in both systems.

Effluent nitrate concentrations were virtually the same for both systems (18.0 and $18.1 \mathrm{mgN}-\mathrm{NO}_{3} / \ell$ in the MBR and conventional UCT systems respectively) resulting in similar total nitrogen (TN) removals, $(81.5 \%$ for the MBR system versus $79.5 \%$ for the conventional system).

Nitrogen is removed from BNR systems either by incorporation of nitrogen in mixed liquor and its subsequent removal through wasting, or through nitrification/ denitrification.

- The influent $\mathrm{N}$ incorporated in the mixed liquor was lower in the MBR UCT system than in the conventional UCT system. This corresponds to the observation above of lower COD incorporated in the MLVSS.

- Regardless, $\mathrm{N}$ removal through sludge wasting was higher in the MBR UCT system than the conventional UCT system, this is largely due to the higher sludge production in the MBR system, and consequent increased sludge mass wasted per unit influent $\mathrm{N}$.

- The MBR UCT system additionally displayed higher $\mathrm{N}$ removals through denitrification. This was achieved despite similar mass fractions and that the conventional UCT system anoxic reactor was frequently fully loaded. 


\subsubsection{P Removals}

In both systems TP was dosed in excess of the amount the system could remove in order to demonstrate BEPR. Thus $\mathrm{P}$ removal performance is represented by $\mathrm{P}$ removals. System average $P$ removals of $21.3 \mathrm{mgP} / \ell$ and $16.7 \mathrm{mgP} / \ell$ were achieved. Clearly however, the $\mathrm{P}$ removal performance of the conventional UCT system was inferior to that of the MBR system. This was because:

i) The anoxic P uptake was more prevalent in the conventional system with $22.1 \%$ of $\mathrm{P}$ uptake taking place in the anoxic reactor, in contrast to only $8.5 \%$ anoxic $\mathrm{P}$ uptake in the MBR system. Additionally the conventional anoxic reactor was regularly overloaded with $\mathrm{NO}_{3}$ as evidenced by consistent anoxic $\mathrm{NO}_{3}$ concentrations $>1 \mathrm{mgNO}_{3} / \ell$. Ekama and Wentzel (1999) and $\mathrm{Hu}$ et al. (2002) report a reduction in BEPR with increasing anoxic $P$ uptake BEPR.

ii) The above observations indicate that the conventional system was not operated optimally (anoxic reactor overloaded). Low MLSS concentrations, as reported earlier reduced the denitrification potential of the anoxic reactor, thus allowing $\mathrm{NO}_{3}$ to be recycled to the anaerobic reactor. Hence, as the conventional system was not performing optimally no comparison could be drawn between the two systems on whether the presence of membranes changed the $P$ removal efficiency of the MBR system.

\subsubsection{Microbial Removals}

Periodic effluent samples were tested from both systems for the indicator microorganism e-coli using the membrane filtration method. Results indicated pathogen counts were not detectable in the MBR UCT system whereas in the conventional UCT system pathogen counts ranged from 580 to $5600 \mathrm{CFU} / 100 \mathrm{ml}$.

Clearly from the removals described above the MBR UCT system produced an effluent that was equal, if not superior in quality to the conventional UCT system. Due to complete retention of solids, and pathogens, the membrane effluent has a higher quality for reuse purposes.

\subsection{OXYGEN TRANSFER RATE TESTING}

Presently, one of the most important considerations for the design of MBR plants is the feasibility of running the systems at high solids concentrations. In previous literature it has been noted that the oxygen transfer efficiency (OTE) of systems decreases substantially at high solids concentrations (Cornel et al., 2003, Krampe and Krauth, 2003).

Following the intensive study on BNR performance in the MBR system oxygen transfer efficiencies of the activated sludge at high MLSS concentrations were determined by performing tests on the sludge in the aerobic reactor. Oxygen mass transfer co-efficient $\left(\mathrm{K}_{\mathrm{LA}}\right)$ values were determined for a number of air flow rates in the operating range of the aeration system. Steady state tests were then carried out on the activated sludge once it had reached endogenous conditions (to reduce the interference by variations in feed characteristics). For a single MLSS concentration a 
number of readings were taken at different airflow rates and the alpha values observed were averaged. Alpha values of $0.17-0.28$ (21000mgTSS $/ \ell), \quad 0.38-0.68$ (17000mgTSS $/ \ell)$ and $0.53-0.80(11000 \mathrm{mgTSS} / \ell)$ were observed.

The wide range of Alpha values observed is a concern. It was concluded that the predominant reason for the variations in observed Alpha values was the sensitivity of the steady state equation to variations in the OUR and $C_{L}$ values, and fluctuations in the airflow readings. As the system was run at a long sludge age under endogenous conditions, limited OUR activity resulted in OUR readings that were typically very low and conversely $C_{L}$ readings were very high, hence small differences have large effects. In order to compensate for high $C_{L}$ values only low airflows could be run, at which the air flow rotameter was less sensitive and variable. It was assumed that by averaging a number of Alpha readings at the same MLSS concentration a more accurate Alpha value could be determined for that MLSS concentration. In conclusion it is recommended that future tests are run on sludge with a far shorter solids retention time, thus providing a greater active fraction in the activated sludge, and hence an increased OUR at endogenous respiration. Additionally sufficiently sensitive air flow rate instrumentation is required to accurately monitor the airflow into the aerobic reactor. It was noted that a number of factors affect the oxygen transfer into the sludge. At a lab scale the geometry of the system and the nature of the aeration (coarse bubble aeration) have a substantial influence on the Alpha value. Thus only the OTE of the lab scale MBR can be calculated and must be interpreted with care on full scale. Hence further studies in conditions resembling full scale are required to accurately calculate Alpha and OTE.

Previous studies on high concentration sludge had indicated a close correlation between viscosity and oxygen mass transfer. Samples from the OTR testing were analysed for rheology. A linear relationship between Alpha and viscosity was observed suggesting further investigations into the viscosity of high concentration sludges should be undertaken to better understand and predict Alpha values.

\section{MEMBRANE PERFORMANCE}

Although the focus of this investigation was the BNR performance of the MBR system, the performance of the membranes was also monitored. Throughout the investigation, the TMP of the membranes was measured in order to ascertain how the membrane filterability deteriorated over time. A constant increase in TMP of approximately $0.29 \mathrm{~mm} / \mathrm{d}$ was observed. However the presence of unbiodegradable colloidal material in the mixed liquor adversely influenced the TMP, which agreed with observations of Fleischer et al. (2005). On completion of the investigation a full chemical clean was performed on the membranes using a 1.0\% Hypochlorite solution. Applying a negative pressure on the effluent line of the membranes encouraged the hypochlorite to flow through the membranes. Within 24 hours of the chemical clean the membrane TMP had returned to its original TMP at the start of the investigation. This indicated that the increase of TMP is reversible with effective cleaning of the membranes. 


\section{CONCLUSIONS}

From this investigation, the following conclusions could be drawn:

- Membranes in a BNR system are a feasible nutrient removal solution with excellent organic and nutrient removal performance. The presence of membranes and consequently operating the system at high sludge concentrations did not adversely affect BNR performance, but produced an effluent of equal or superior quality to that produced by a conventional system using SSTs. In addition pathogen counts indicated that all pathogens were retained by the membranes. Thus the membrane effluent is safer and more viable for reuse purposes.

- Higher sludge productions of 0.311 and $0.320(\mathrm{mgVSS} / \mathrm{d}) /(\mathrm{mgCOD} / \mathrm{d})$ were observed in the MBR system in both this investigation and by Ramphao et al. (2004). This higher sludge production is accommodated in steady state design theory by increasing the unbiodegradable particulate COD fraction ( $\mathrm{f}_{\mathrm{S} \text {.up }}$ ) to 0.200 in this investigation and 0.224 for Ramphao et al. (2004). The increased sludge production in the MBR is justified in part by the retention of all solids by the membranes. Similarly the unbiodegradable soluble COD fraction ( $\left.f_{S, \text { us }}\right)$ must be decreased to account for the additional retention of "soluble" COD which is attributed to the finer membrane pore size.

- A theoretical evaluation of the BNR performance of the MBR system indicated that the current steady state BNR theory was able to closely predict the system performance for COD removal and nitrification. However for denitrification the $\mathrm{D}_{\mathrm{PP}}$ was under predicted requiring $\mathrm{K}_{2}{ }^{\top} \mathrm{T}$ to be adjusted from 0.145 to $0.216 \mathrm{mgN} / \mathrm{mgVSS} / \mathrm{d}$ at $20^{\circ} \mathrm{C}$ in order to match observed and predicted values. The BEPR predictions for aerobic $P$ uptake BEPR were close to those observed when the system PAO population reached a steady state (sewage batches $18-25)$. $\mathrm{f}_{\mathrm{XBGP}}$ observed in this period $(0.376 \mathrm{mgP} / \mathrm{mgVSS})$

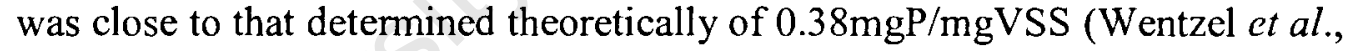
1990).

- Aeration testing was performed on the system, in order to determine alpha values for the high concentration sludge. Alpha values of $0.5-0.6$ for $\sim 15$ $000 \mathrm{mg}$ TSS $/ \ell$ and $0.2-0.3$ for $\sim 20000 \mathrm{mgTSS} / \ell$ were determined, which are higher than other values reported in the literature. These values are however specific to the laboratory system run in which factors such as reactor geometry and high aeration turbulence would have affected oxygen transfer in the system. Additionally the low sensitivity of the measuring apparatus resulted in substantial variance of results.

- The filterability of the membranes can be influenced by fine colloidal material, however observations indicated that the filterability would return to previous levels once colloids are removed from solution by assimilation into the mixed liquor.

\section{RECOMMENDATIONS FOR FURTHER STUDY}

Following from this investigation two recommendations are proposed:

- Accurate knowledge of the oxygen transferability in high concentration sludge is an important design consideration. However in this investigation the difficulty in measuring this parameter at a lab scale was realised. It is 
recommended that this parameter needs to be quantified on a full scale and be determined at a fully operational BNR MBR WWTP.

- The relationship between Alpha and viscosity of activated sludge needs to be investigated further in order to better understand the influence of high concentration sludges on oxygen mass transfer. 


\section{ACKNOWLEDGEMENTS}

I wish to express my gratitude to the following people for the contribution they made whether academically or personally to the research reported here:

- Prof. George Ekama and Assoc. Prof Mark Wentzel-My supervisors for their active guidance, enthusiasm and untiring support throughout this project.

- Mr Taliep Lakay and Mr Hector Mafungwa - Lab manager and lab assistant respectively who's vast experience and willingness to help made the onerous task of running three systems that much more bearable.

- The staff of the Civil Engineering workshop Messrs Eike von Guerard, Charles Nicholas and Theo Monyana for their construction and maintenance of the laboratory equipment, and swift assistance when repairs were required.

- Mr Peter Tapscot for filamentous organism identification and Mrs Ingrid Thompson of Scientific Services branch of the Cape Metropolitan Council for pathogen counts.

- Prof. Paul Slabbert and Dr. Veruscha Fester for conducting rheometric studies on the MBR mixed liquor.

- Mpho Ramphao who conducted the first phase of this investigation and set a high standard from which to develop this report.

- My lab mates, mentors and friends Ashley Muller, Valentina Parco, Johann Poinapen, Pierre van $Z y l$ and Michael Mebrahtu for their support and encouragement.

- My family for helping maintain perspective and humour throughout, Karen Vickers for her boundless energy and inspiration while here and Ryan Miller for being my anchor whenever I faltered.

I also wish to acknowledge the support of the following organisations that funded and supported the project in cash and kind:

- Water Research Commission (WRC)

- National Research Foundation (NRF)

- University of Cape Town (UCT) 


\section{TABLE OF CONTENTS}

DECLARATION

(i)

SYNOPSIS

(ii)

ACKNOWLEDGEMENTS

(xvi)

TABLE OF CONTENTS

(xvii)

LIST OF SYMBOLS

CHAPTER 1 INTRODUCTION

1.1 BACKGROUND

1.2 IMPACT OF MEMBRANES ON BNRAS DESIGN AND PERFORMANCE

1.3 PREVIOUS WORK ON MBR BNR

1.4 OBJECTIVES OF RESEARCH

1.5 RESEARCH APPROACH

CHAPTER 2 LITERATURE REVIEW

2.1 INTRODUCTION

2.2 CURRENT MEMBRANE TECHNOLOGY

2.2.1 Membrane Configurations

2.2.2 Commercial Membrane Application

2.3 MEMBRANE DESIGN CONSIDERATIONS

2.3.1 System Design

2.3.2 Modelling of MBR Systems

2.4 OPERATIONAL ISSUES OF MBR SYSTEMS

2.4.1 Operational Experience of MBR Systems

2.4.2 Sludge Production

2.4.3 Aeration

2.4.4 Membrane Flux and Fouling

2.4.5 Membrane Foaming

2.4.6 Energy Consumption

2.5 MICROBIOLOGY OF MBR SYSTEMS

2.5.1 Microbiology

2.5.2 Effluent Quality

(2.10)

2.6 ORGANIC AND NUTRIENT REMOVAL PERFORMANCE

2.6.1 COD Removal

2.6.2 Nitrogen Removal

2.6.3 Phosphorus Removal

2.7 PREVIOUS STUDIES ON BNR IN MBR SYSTEMS

2.7.1 Ruhleben WWTP, Berlin, Germany

2.7.2 Korea Institure for Science and Technology

2.7.3 University of British Columbia

2.7.4 University of Guelph 
xviii
2.7.5 Loudoun County Sanitation Authority
2.7.6 UCT Water Research Group
2.7.7 Summary
2.8 CONCLUSIONS

CHAPTER 3 METHODOLOGY

3.1 INTRODUCTION

3.2 RESEARCH APPROACH

3.3 ACTIVATED SLUDGE SYSTEMS DESCRIPTION

3.3.1 Activated Sludge Systems Operated

3.3.2 UCT systems design and operating parameters

3.3.3 MBR UCT System Description

3.3.4 Conventional UCT System Description

3.3.5 Square Wave Feed Activated Sludge System

3.3.6 Operating Procedures and Conditions

3.3.7 System Seeding

3.4 INFLUENT FEED

3.4.1 Wastewater Collection and Storage

3.4.2 Feed Preparation

3.5.1 UCT Activated Sludge Systems

3.5.2 Square Wave Feed Activated Sludge System

3.6.1 Initial Flux Testing

3.6.2 Oxygen Transfer Rates

3.6.3 Residence Time Distribution

3.7 OXYGEN TRANSFER TESTING

3.7.1 Oxygen Transfer Theory

3.7.2 Unsteady State (USS) Test

3.7.3 Steady State Testing

3.7.4 Beta Testing $(\beta)$

\subsection{CLOSURE}

3.8.1 MBR System Operation

3.8.2 Conventional UCT Operation

3.8.3 Operation of MBR system at longer (40d) Rs

CHAPTER 4 SQW and MBR SYSTEMS EXPERIMENTAL RESULTS AND ANALYSIS

4.1 INTRODUCTION

4.2 SYSTEM CONDITIONS AND STEADY STATE PERIODS (4.2)

4.2.1 System Operating Conditions

4.2.2 Steady State Periods

4.3 SQW ACTIVATED SLUDGE SYSTEM

4.4 MBR SYSTEM ANALYSIS AND RESULTS

4.4.1 Recycles and Mass Fractions

4.4.2 Membrane and Bioreactor Testing

4.4.2.1 Initial Membrane and Bioreactor testing 
4.5 MEMBRANE PERFORMANCE

4.6 MIXED LIQUOR SOLIDS

4.6.1 Sludge Age (Rs)

4.6.2 MLSS and MLVSS Concentrations

4.6.3 COD/VSS Ratios

4.6.4 System VSS/TSS Ratios

4.6.5 System TKN/VSS Ratios

4.6.6 Sludge Production

4.6.7 System Mixed Liquor Settleability and Microbial Analysis

4.7 MASS BALANCES

4.7.1 Nitrogen Mass Balance

4.7.2 COD Mass Balance

4.8 INFLUENT UNBIODEGRADABLE COD FRACTION (4.29)

4.8.1 Unbiodegradable Soluble COD Fraction $\left(\mathrm{f}_{\mathrm{S}, \mathrm{us}}\right)$

4.8.2 Unbiodegradable Particulate COD Fraction ( $f_{S \text {.up }}$ )

4.9 SYSTEM PERFORMANCE

4.9.1 COD Removal

4.9.2 Nitrogen Removal

4.9.3 BEPR

4.10 THEORETICAL EVALUATION OF ND AND BEPR PERFORMANCE IN THE MBR SYSTEM

4.10.1 Nitrification

4.10.2 Denitrifiation

4.10 .3 a-Recycle Ratio

4.10.4 Effluent Nitrate Concentration

4.10.5 Theoretical BEPR Performance

4.11 CLOSURE

CHAPTER 5 CONVENTIONAL UCT SYSTEM EXPERIMENTAL RESULTS

5.1 INTRODUCTION

5.2 SYSTEM CONDITIONS AND STEADY STATE PERIODS (5.1)

5.3 MIXED LIQUOR SOLIDS

5.3.1 MLSS and MLVSS CONCENTRATIONS

5.3.2 System VSS/TSS Ratios

5.3.3 COD to VSS Ratio ( $\left.f_{C V}\right)$

5.3.4 TKN/VSS Ratio $\left(f_{N}\right)$

5.3.5 Re-evaluation of VSS Values

5.3.6 Sludge Production

5.3.7 Diluted Sludge Volume Index

5.4 MASS BALANCES

5.4.1 Nitrogen Mass Balance

5.4.2 COD Mass Balance

5.5 INFLUENT UNBIODEGRADABLE COD FRACTIONS

5.5.1 Unbiodegradable Soluble Cod Fraction ( $f_{\mathrm{S}, \mathrm{us}}$ )

5.5.2 Unbiodegradable Particulate COD Fraction ( $\left.f_{S . u p}\right)$

5.5.3 Revision of Sludge Production

5.5.4 Discussion 
5.6 SYSTEM PERFORMANCE

5.6.1 COD Removal Performance

5.6.2 Nitrogen Removal

5.6.3 Biological Excess Phosphorus Removal (BEPR)

5.7 CLOSURE

CHAPTER 6 COMPARISON OF SYSTEM PERFORMANCES

$\begin{array}{llc}6.1 & \text { INTRODUCTION } & (6.1) \\ 6.2 & \text { SYSTEM OPERATIONAL PARAMETERS } & (6.1) \\ 6.3 & \text { SYSTEM REMOVALS AND EFFLUENT QUALITY } & (6.2) \\ & 6.3 .1 \quad \text { COD Removals } & (6.2) \\ & 6.3 .2 \quad \text { N removals } & (6.3) \\ & 6.3 .3 \quad \text { P removals } & (6.4) \\ & 6.3 .4 \quad \text { Microbial Removals } & (6.4) \\ 6.4 & \text { MASS BALANCES } & (6.4) \\ 6.5 & \text { SLUDGE PRODUCTION } & (6.6) \\ 6.6 & \text { MIXED LIQUOR CHARACTERISTICS } & (6.8) \\ 6.7 & \text { NITROGEN REMOVAL } & (6.9) \\ 6.8 & \text { BIOLOGICAL EXCESS PHOSPHORUS REMOVAL (BEPR) } \\ & \text { BEIIAVIOUR } & (6.9) \\ 6.9 & \text { CLOSURE } & (6.10)\end{array}$

CHAPTER 7 CONCLUSIONS AND RECOMMENDATIONS

7.1 INTRODUCTION

7.2 CONCLUSIONS

7.3 RECOMMENDATIONS FOR FURTHER STUDY

APPENDICES

APPENDIX A

APPENDIX B

APPENDIX C

APPENDIX D

APPENDIX E

APPENDIX F

APPENDIX G
MBR AND SQW DAILY MEASUREMENTS

CONVENTIONAL UCT DAILY MEASUREMENTS

NITROGEN AND COD MASS BALANCES

CONSTRUCTION AND INTERPRETATION OD STATISTICAL PLOTS

CAPE METROPOLITAN SCIENTIFIC SERVICES PATHOGEN COUNTS

AERATION AND OXYGEN TRANSFER TESTING IN THE MBR SYSTEM

40 DAY LONG SLUDGE AGE MBR DAILY MEASUREMENTS 


\section{LIST OF SYMBOLS}

\begin{tabular}{|c|c|}
\hline AA & Anoxic-Aerobic (filaments) \\
\hline$A_{C}$ & Acetate, sodium acetate $\left(\mathrm{CH}_{3} \mathrm{COONa}\right)$ \\
\hline ADWF & Average Dry Weather Flow \\
\hline $\mathrm{AE}$ & Aerobic \\
\hline $\mathrm{AOB}$ & Ammonia-Oxidising Bacteria \\
\hline AS & Activated Sludge \\
\hline ASM & Activated Sludge Model \\
\hline AVSS & Active volatile suspended solids \\
\hline $\mathrm{AX}$ & Anoxic \\
\hline BEPR & Biological Excess Phosphorus Removal \\
\hline B & Beta-effect of impurities on $\mathrm{C}_{\mathrm{S}}$ \\
\hline$b_{\mathrm{HT}}$ & Specific endogenous mass loss rate of $\mathrm{OHOs}$ at temperature $T(1 / \mathrm{d})$ \\
\hline$b_{\mathrm{GT}}$ & Specific endogenous mass loss rate of PAOs at temperature $T(1 / d)$ \\
\hline BNR & Biological Nutrient Removal \\
\hline BSP & Bench Scale Plant \\
\hline CAS & Conventional Activated Sludge \\
\hline cfu & Colony forming units \\
\hline $\mathrm{CO}_{2}$ & Carbon dioxide \\
\hline COD & Chemical Oxygen Demand \\
\hline $\mathrm{C}_{\mathrm{S}}$ & Saturated DO concentration under site conditions $(\mathrm{mgO} / \ell)$ \\
\hline $\mathrm{C}_{\mathrm{L}}$ & $\mathrm{DO}$ concentration in reactor $(\mathrm{mgO} / \ell)$ \\
\hline CLSM & Confocal Laser Scanning Microscopy \\
\hline d & Day \\
\hline${ }^{\circ} \mathrm{C}$ & Degrees Celcius \\
\hline$\Delta$ & Indicates a change in parameter value \\
\hline$\triangle \mathrm{OUR}$ & Change in OUR $(\mathrm{mgO} / \ell / \mathrm{h})$ \\
\hline DO & Dissolved Oxygen (mgO) \\
\hline DPAO & Denitrifying Phosphate Accumulating Organism \\
\hline $\mathrm{D}_{\mathrm{PP}}$ & Total system denitrification potential (mgN/ $\ell$ ) \\
\hline DSVI & Diluted Sludge Volume Index (ml/gTSS) \\
\hline EPS & Extracellular Polymeric Substances \\
\hline EBPR & Excess Biological Phosphorus Removal \\
\hline f & Fraction of (unbiodegradable) endogenous residue. \\
\hline $\mathrm{F} / \mathrm{M}$ & Food to Micro-organism ratio \\
\hline $\mathrm{f}_{\mathrm{CV}}$ & COD to VSS ratio of the mixed liquor (COD/VSS) \\
\hline$f_{E H}$ & Endogenous residue fraction of OHOs \\
\hline$f_{\mathrm{EG}}$ & Endogenous residue fraction of PAOs \\
\hline$f_{i}$ & VSS to TSS ratio of the mixed liquor ( $\mathrm{mgN} / \mathrm{mgCOD}$ ) \\
\hline FISH & Fluorescence In Situ Hybridization \\
\hline fm & Reactor zone mass fraction \\
\hline$f_{n}$ & Nitrogen to COD ratio of the mixed liquor ( $\mathrm{mgN} / \mathrm{mgCOD}$ ) \\
\hline$f_{N}$ & Fractional TKN content of VSS (mgN/mgVSS) \\
\hline FSA & Free and Saline Ammonia \\
\hline $\mathrm{f}_{\mathrm{ts}}$ & RBCOD fraction with respect to total COD in wastewater \\
\hline$f_{\text {up }}$ & $\begin{array}{l}\text { Fraction of unbiodegradable particulate (with respect to total)COD in } \\
\text { the influent wastewater }\end{array}$ \\
\hline
\end{tabular}


$\mathrm{f}_{\mathrm{us}} \quad$ Fraction of unbiodegradable soluble (with respect to total) COD in the influent wastewater

$f_{X B P G} \quad$ Fractional polyp content of PAOs

g gram

GAO Glycogen Accumulating Organism

h Hour

$\mathrm{HgCl} \quad$ Mercury chloride

HPLC High Pressure Liquid Chromatograph

HRT Hydraulic Retention Time

IWA International Water Association (formerly IAWQ, IAWPRC)

IAWPRC International Association for Water Pollution Research and Control

IAQW International Association on Water Quality (formerly IAWPRC)

ISS Inorganic Settleable Solids (mgISS/ $\ell$ )

$\mathrm{K}_{1 \mathrm{~T}} \quad$ Specific denitrification rate with $\mathrm{RBCOD}$ as substrate

(mgN/mgAVSS/h), at temperature $\mathrm{T}$

$\mathrm{K}_{2 \mathrm{~T}} \quad$ Specific denitrification rate with SBCOD as substrate (mgN/mgAVSS/h), at temperature $\mathrm{T}$

$\mathrm{K}_{3 \mathrm{~T}} \quad$ Specific denitrification rate with endogenous generated SBCOD as substrate $(\mathrm{mgN} / \mathrm{mgAVSS} / \mathrm{h})$, at temperature $\mathrm{T}$

$\mathrm{K}_{\mathrm{LA}} \quad$ Oxygen mass transfer co-efficient $(1 / \mathrm{h})$

Kpa Kilo-Pascal

kWh Kilo-Watt hour

$\ell \quad$ Litres

m Meters

MBR Membrane Biological Reactor

mg Milligram

$\min \quad$ Minute

ML Mixed Liquor

MLE Modified Lutzack-Ettinger (activated sludge system)

MLSS Mixed Liquor Suspended Solids

mm Millimeters

MPWWTP Mitchells Plain Wastewater Treatment Plant

$\mathrm{M}_{\mathrm{Sti}} \quad$ Mass of total COD in the influent wastewater (mgCOD)

MUCT Modified UCT system

$\mathrm{M}_{\mathrm{Xa}} \quad$ Mass of active biomass (mgAVSS)

$\mathrm{M}_{\mathrm{Xv}} \quad$ Mass of volatile suspended solids (mgVSS)

$\mathrm{N} \quad$ Elemental Nitrogen

$\mathrm{N}_{2} \quad$ Molecular dinitrogen

$\mathrm{NaCl}$ Sodium Chloride

$\mathrm{N}_{\mathrm{ae}} \quad$ Effluent ammonia concentration (mgN/ $\ell$ )

$\mathrm{N}_{\mathrm{C}} \quad$ Nitrification Capacity

ND Nitrification-Denitrification

NDBEPR Nitrification-Denitrification Biological Excess Phosphorus Removal

$\mathrm{NH}_{4} \quad$ Ammonium (mgN/ $\ell$ )

$\mathrm{N}_{\text {nd }} \quad$ NOx concentration denitrified $(\mathrm{mgN} / \ell)$

$\mathrm{N}_{\mathrm{ne}} \quad$ Effluent NOx $(\mathrm{mgN} / \ell)$

$\mathrm{N}_{\mathrm{nL}} \quad$ Nitrate Load

$\mathrm{NO}_{2} \quad$ Nitrite $(\mathrm{mgN} / \ell)$

$\mathrm{NO}_{3} \quad$ Nitrate $(\mathrm{mgN} / \ell)$

$\mathrm{NO}_{\mathrm{x}} \quad$ Nitrite and Nitrate $(\mathrm{mgN} / \ell)$ 


\begin{tabular}{|c|c|}
\hline NOB & Nitrite-Oxidising Bacteria \\
\hline $\mathrm{N}_{\text {ousi }}$ & Organic unbiodegradable soluble influent Nitrogen $(\mathrm{mgN} / \ell)$ \\
\hline $\mathrm{O}$ & Elemental Oxygen \\
\hline $\mathrm{O}_{2}$ & Molecular Oxygen \\
\hline $\mathrm{OHO}$ & Ordinary Heterotrophic Organism \\
\hline OSR & Oxygen Supply Rate \\
\hline OTE & Oxygen Transfer efficiency \\
\hline OTR & Oxygen Transfer Rate \\
\hline OUR & Oxygen Utilization Rate $(\mathrm{mgO} / \ell / \mathrm{h})$ \\
\hline $\mathrm{P}$ & Elemental Phosphorus \\
\hline $\mathrm{P}$ & Atmospheric Pressure \\
\hline $\mathrm{p}$ & Saturated vapour pressure \\
\hline PAO & Polyphosphate Accumulating Organism \\
\hline PHA & Polyhydroxyalkanoate \\
\hline PolyP & Polyphosphate \\
\hline $\mathrm{PP}$ & Pilot Plant \\
\hline PWWF & Peak Wet Weather Flow \\
\hline$Q_{i}$ & Influent wastewater flowrate $(\ell / d)$ \\
\hline $\mathrm{Q}_{w}$ & Sludge waste flow $(\ell / \mathrm{d})$ \\
\hline RBCOD & Readily Biodegradable COD (mgCOD $/ \ell$ ) \\
\hline $\mathrm{R}_{\mathrm{s}}$ & Sludge Age (d) \\
\hline $\mathrm{R}_{\mathrm{sm}}$ & Minimum Sludge Age for nitrification to occur (d) \\
\hline RTD & Residence Time Distribution \\
\hline s & Second \\
\hline SAFR & Specific Air Flow Rate \\
\hline $\mathrm{SB}$ & Sewage Batch \\
\hline SBCOD & Slowly Biodegradable COD $(\mathrm{mgCOD} / \ell)$ \\
\hline $\mathrm{S}_{\mathrm{bi}}$ & Biodegradable COD in the influent wastewater $(\mathrm{mgCOD} / \ell)$ \\
\hline $\mathrm{S}_{\mathrm{bs}}$ & Biodegradable soluble COD (mgCOD $/ \ell)$ \\
\hline SCFA & Short-Chain Fatty Acids \\
\hline SMBR & Submerged Membrane Biological Reactor \\
\hline SQW & Square-wave (fed activated sludge system) \\
\hline SRT & Solids Retention Time \\
\hline SSD & Sample Standard Deviation \\
\hline SST & Secondary Settling Tank \\
\hline $\mathrm{S}_{\mathrm{ti}}$ & Total influent wastewater COD concentration $(\mathrm{mgCOD} / \ell)$ \\
\hline $\mathrm{S}_{\mathrm{te}}$ & Total effluent COD \\
\hline $\mathrm{S}_{\mathrm{us}}$ & Unbiodegradable COD in influent \\
\hline $\mathrm{T}$ & Temperature $\left({ }^{\circ} \mathrm{C}\right)$ \\
\hline TKN & Total Kjeldhal Nitrogen concentration (mgN/ $\ell$ ) \\
\hline TMP & Trans-Membrane Pressure $(\mathrm{mm})$ \\
\hline TP & Total Phosphorus \\
\hline TSS & Total suspended solids (mgTSS/ $\ell$ ) \\
\hline$\mu \mathrm{m}$ & Micro-meter (micron) \\
\hline UCT & University of Cape Town \\
\hline UF & Ultra-Filtration \\
\hline V & Volume \\
\hline VFA & Volatile Fatty Acids \\
\hline$V_{p}$ & Total system (process) volume \\
\hline VSS & Volatile Suspended Solids (mgVSS/ $\ell$ ) \\
\hline
\end{tabular}


xxiv

$\begin{array}{ll}\text { WW } & \text { Wastewater } \\ \text { WRC } & \text { Water Research Commission } \\ \text { WRG } & \text { Water Research Group } \\ \text { WRL } & \text { Water Research Laboratory } \\ X_{\mathrm{a}} & \text { Active biomass concentration (mgAVSS/ } \ell \text { ) } \\ \mathrm{X}_{\mathrm{V}} & \text { Volatile suspended solids concentration }(\mathrm{mgVSS} / \ell) \\ \mathrm{Y}_{\mathrm{H}} & \text { Ordinary heterotrophic cell yield coefficient } \\ \mathrm{Y}_{\mathrm{H}, \mathrm{AE}} & \text { Ordinary heterotrophic cell yield coefficient under aerobic conditions }\end{array}$ 


\section{CHAPTER 1}

\section{INTRODUCTION}

\subsection{BACKGROUND}

Throughout the world development and population growth are placing an increasing strain on water resources as demand for water for agriculture, industry and municipal use increase. Growing out of this increased demand is a awareness by individuals and governments that water bodies need to be safeguarded from pollutants, both in order to protect the environment and maintain the quality of life of the people who use and are in contact with these water bodies.

In South Africa, which is a water scarce country, the protection of water resources has long been (since the 1960's) a national priority. With a rapidly growing population, particularly in urban areas, the importance of protecting receiving water bodies from pollution is vitally important. Of the methods available to clean wastewaters, biological nutrient removal (BNR) is applied throughout South Africa as it has many advantages, notably it is cheaper to operate, does not introduce salinity into the water and is a simple and robust process to operate.

An essential step of the BNR process is the separation of solids from liquids in activated sludge systems. Traditionally this has been achieved using secondary settling tanks (SSTs), however more recently the use of membrane filters for solidliquid separation has gathered momentum internationally as membranes offer certain significant advantages over conventional SST separation techniques. These are:

1) The membranes retain all reactor solids, preventing solids overflow with the effluent. This ensures a high quality effluent.

2) In addition to retaining all biological solids membranes, due to pore sizes of $\sim 0.1 \mu \mathrm{m}$, are also effective in filtering pathogens and in some cases viruses from the effluent allowing the upgrading of the water to reuse or even potable standards easier to achieve.

3) Systems can be run at high reactor sludge concentrations, 15 to $20 \mathrm{~g} / \ell$, which require smaller reactor volumes. This not only means that the plant footprint is reduced, but also that plant capacity can be greatly increased with the existing plant infrastructure.

4) The plant footprint is further reduced as no SSTs are required.

Thus, coupled with the conventional BNR activated sludge technology, membranes for solid liquid separation are a very viable option for present and future wastewater applications. Many research groups internationally have focused their attention on investigating the performance of membranes, as certain factors, namely cost, fouling, and uncertainty on the lifespan of the membranes have limited their widespread application. Additionally a number of groups have investigated the biological performance of activated sludge systems using membranes, confirming that the high 
concentrations induced by the membranes do not adversely affect the biological performance of COD removal and nitrification.

Stricter water quality guidelines internationally have made nutrient removal from wastewaters a far greater priority (Lesjean et al., 2003, Howell, 2004, Gunder and Krauth, 1999). These guidelines require a guaranteed quality effluent which can be achieved far easier with a secure solids-liquid separation medium, such as is provided by membranes. In some cases where water reclamation is required the water can be treated up to potable standards with substantially less infrastructure than would have been required with conventional water treatment techniques. Hence there is a need to understand the impact of membranes on biological nutrient removal (BNR) activated sludge processes. However only a limited number of studies have been conducted on BNR system performance with membranes.

\subsection{IMPACT OF MEMBRANES ON BNR ACTIVATED SLUDGE DESIGN AND PERFORMANCE}

The design of BNR systems and the approach to wastewater treatment plant design is significantly influenced by the introduction of membranes for solid-liquid separation (Ramphao et al., 2005). As membranes retain all solids, and are more economically run at high solids concentrations they impose different selection criteria on the organisms present and can alter the bioceonosis of the system mixed liquor (Kraume et al., 2005).

Ramphao et al. (2005) provided an elegant discussion on the influence of membranes on design. They concluded that, with the zone mass fractions determined, the aerobic reactor is sized on the volume required to house the membranes and aeration devices, with the membrane surface area determined by the peak wet weather flow (PWWF). The system sludge age is determined by the desired solids concentration in the reactors. This design contrasts to conventional designs in that the reactor volumes are based on the membrane surface area required to meet the peak wet weather flow rather than the system organic load and required reactor solids concentration.

\subsection{PREVIOUS WORK ON MBR BNR}

Previous studies have been conducted on BNR in MBR systems. Monti et al. (2006) compared two AS systems in a UCT configuration, one a MBR and the other using a conventional SST, with the same system design and operational parameters. Both systems had the same sludge mass and hence the influence of sludge concentration on BNR performance was not assessed.

Lesjean et al. $(2003,2005)$ conducted a 4-year study on pre-denitrification and postdenitrification configurations of MBR wastewater treatment systems at bench and pilot scale. The systems were run at varying sludge ages and high solids concentrations. Excellent nutrient removal was observed in both configurations without additional carbon dosing in the post-denitrification system. However the systems were found to be generally underloaded, and precipitation of $P$ due to calcium and ferric ions was observed which compromised observations of biological $\mathrm{P}$ removal. 
Ahn et al. (2003) operated two lab scale MBR systems as a sequencing anaerobic/anoxic MBR (SAM) and the other a MLE system. P-removal was observed in the SAM system, but at the cost of poor $N$ removal. BNR removal was observed but not optimised.

Fleisher et al. (2005) investigated the BNR performance of an MBR system in a 5stage configuration in order to ascertain whether biological and chemical $\mathrm{P}$ removal could be achieved concurrently. They successfully demonstrated that BEPR could be achieved, using chemical precipitation in the MBR reactor to completely remove all remaining $P$. The 5-stage configuration was also successful in reducing $T N$ to $<3 \mathrm{mgN} / \ell$. In addition Fleischer et al. (2005) modelled the observed system performance and suggested that current simulations (IWA ASM2d) adequately predicted the BNR performance of the system. Lastly they investigated the solids produced from the membrane system in order to determine if they differed from conventional solids and observed that a higher density cake could be produced from the MBR sludge than from conventional sludge.

Ramphao et al. (2004) investigated the BNR performance of two systems in UCT configurations. In contrast to the study by Monti et al. (2006) the systems were run at their design solids concentrations, ie aerobic solids concentrations were $4500 \mathrm{mgTSS} / \ell$ in the conventional system compared to $18000 \mathrm{mgTSS} / \ell$ in the MBR system. The MBR system produced an effluent that was consistently equal to, or better than, the conventional effluent. It was found that the current BNR simulations could adequately predict system performance, but solids production in the MBR system was substantially higher than expected.

The research has shown that the inclusion of membranes in the system does not adversely affect the BNR performance, and also that at high concentration sludges, as are characteristic of MBR systems, the BNR performance remains consistent. However these studies have only indicated that MBRs are feasible and have not investigated in depth how the performance is affected - notably how to optimise BNR in MBR systems. Additionally information important to design such as the oxygen transfer efficiency in high solids concentration sludges remains much debated in the literature (Wagner and Popel, 1998, Gunder and Krauth, 1999).

\subsection{OBJECTIVES OF RESEARCH}

This investigation followed on directly from the work reported in Ramphao et al. (2004) in which the feasibility of a MBR for BNR treatment was investigated. From the conclusions drawn in Ramphao et al. (2004) it was recommended that further investigations into the kinetics of the MBR system be undertaken, to better understand the influence of the membranes and how the concentration of the sludge impacts on the biological activity and behaviour of the micro-organisms in the system. Specific areas requiring further research included:

- Verifying the results obtained in the initial investigation (Ramphao et al., 2004) with particular emphasis on explaining the phenomena of increased sludge production;

- Gaining a better understanding of the operating conditions and considerations of MBR BNR systems; 
- Providing a parent system from which further testing into the kinetics of a MBR BNR system could be performed (Parco et al. 2006).

\subsection{RESEARCH APPROACH}

In order to address these objectives two parallel lab-scale conventional and membrane activated sludge systems were operated under laboratory conditions allowing their behaviour to be monitored and their performance compared. In order to verify the previous results the same original experimental apparatus and operational conditions were adopted from Ramphao et al. (2004) and testing continued. Samples drawn from these parent systems would enable kinetic testing of the membrane sludge to be performed. The kinetic studies on the MBR system are reported in Parco et al. (2006). Additionally the MBR system was, subsequent to the completion of the parallel investigation, run at a longer sludge age by Mahimba et al. (2006) in order to investigate sludge production in the MBR system further.

Chapter 2 of this report is a literature review of current knowledge in MBR systems for BNR applications. The methodology of the research approach is presented in Chapter 3, followed by the results of the MBR and conventional systems presented in Chapters 4 and 5 respectively. A comparative discussion is presented in Chapter 6 while conclusions and recommendations for further study are presented in Chapter 7. 


\section{CHAPTER 2}

\section{LITERATURE REVIEW}

\section{$2.1 \quad$ INTRODUCTION}

The application of membranes to solid liquid separation in biological reactors was first suggested in 1969 (Smith 1969, cited in Brindle et al., 1996). Since then membranes have evolved significantly, particularly in the last decade through research and application. Churchouse et al. (1999) note that up until the mid 90s the advancement of MBR technology was hindered by two primary disadvantages, namely that it was expensive and there was concern over the membrane failure rate. "MBR's were untested, complex and generally small scale" (Churchouse et al., 1999). However in the last 10 years there has been a dramatic increase in their use for wastewater treatment with typical applications to industrial wastewaters, domestic wastewaters and specific municipal wastewaters where a smaller plant footprint, water reuse or stringent discharge standards were required (Lesjean et al., 2004; Yang et al., 2005).

Advantages reported for MBR systems are that:

1) All biological solids and high molecular weight solutes (Sutton et al., 1994, cited in Brindle and Stephenson, 1996) are retained in the bioreactor producing an effluent that is of consistently completely solids free and of a high quality. This makes membrane systems very suitable for reuse applications (Howell, 2003).

2) As all solids are retained in the bioreactor the solids concentration can be increased substantially, thus reducing the required volume of the bioreactors.

3) The process can be run at long sludge ages ( $>50$ days) which favours slower growing micro-organisms making the system more robust to load variations and toxic shocks (Lesjean et al., 2004, Brindle et al., 1996).

4) The microscopic pore size prevents pathogens and micro-organisms passing into the effluent making tertiary treatment, and reuse of the wastewater easier.

Applications for MBRs range from small to large scale aerobic municipal water treatment plants, and aerobic and anaerobic industrial water treatment plants. Anaerobic MBRs have been shown to be very efficient (Brindle et al., 1996). Various membrane sizes based on pore sizes are available from micro-filtration $(0.1-0.3 \mu \mathrm{m})$ which will retain all bio-solids and some viruses, ultra-filtration $(5 \mathrm{~nm}-0.1 \mu \mathrm{m})$ retaining all solids and viruses, and nano-filtration $(2 \mathrm{~nm})$ which serves specific niche applications. Due to their excellent performance, low trans-membrane pressure (TMP) requirements and cost, micro-filtration units are used for most applications (Fane and Chang, 2002). 


\subsection{CURRENT MEMBRANE TECHNOLOGY}

\subsubsection{Membrane Configurations}

Currently three membrane configurations, hollow fibre submerged membranes, plateand-frame submerged membranes and external tubular side stream modules are available on the marketplace (Lesjean et al., 2004).

Up until the mid-90's mainly external side-stream configurations were used whereby sludge from the reactor was pumped from the bioreactor and through an external membrane. High power requirements with resulting high costs were thus the primary limiting factor to the widespread application of MBR's up to this point. In the late 80 's it was proposed to include membranes in the bioreactor (Yamamoto et al., 1989) and by the mid 90's the submerged membrane bioreactor (SMBR) had become more common place. To scour the membranes cross-flow velocities were applied to the surface of the membranes. Initially cross flow velocities were achieved by hydraulic means, for instance using paddles. However the use of aeration to provide cross flow velocity instead greatly improved the viability of MBRs by reducing power requirements (solid liquid separation occurred in the bioreactor) and the biological oxygen requirements and membrane scouring could be achieved with one process (Brindle et al., 1996).

Lesjean et al. (2004) drew the following conclusions from comparing the three configurations:

1) Submerged membrane configurations required less capital costs and consumed substantially less energy.

2) Although hollow fibre modules were more cost-competitive than flat-sheet modules, they required more equipment.

3) A broader range of materials is available for flat sheet and tubular side-stream membranes making them more versatile for various applications where properties like resistance to chemicals and heat are important. Hence flat sheet and tubular side-stream membranes are more suitable to difficult industrial applications.

4) Due to higher MLSS concentrations external side-stream MBR systems are generally more compact and require a smaller surface area.

5) Both submerged configurations require the biological reactor to be large enough to accommodate the membranes. Flat sheet membranes require a greater volume to surface area than hollow fibre membranes. Thus in applications where the volume required for the solid liquid separation step is limited, hollow fibre, or external tubular configurations would be preferential.

The majority of municipal wastewater MBR applications use an SBMR configuration whereas industrial applications commonly use the external configuration.

\subsubsection{Commercial Membrane Application}

Up until the early 90's commercial membrane technology was limited to small flow applications where connections to larger treatment works were not feasible. As noted above this limitation was due to the high power costs of external MBRs and was overcome with the advent of SMBR systems (Yang et al., 2005). 
In North America four main manufacturers provide membrane bioreactor systems. They are Zenon Environmental Inc. (Canada), USFilter Memcor (USA), Kubota (Japan) and Mitsubishi-Rayon (Japan) (Yang et al., 2005). Of these Kuboto supplies flat sheet panel membranes, whereas the other three all supply hollow fibre membranes. Other major membrane producers include Puron AG for hollow fibre membrance; Toray Industries Inc., Hans Huber AG and A3 GmbH for plate and frame configurations; Anglagentechnik $\mathrm{GmbH}$, Techsep and Wehrle who provide external side-stream membranes (Lesjean et al., 2004).

According to Yang et al. (2005), in 2005 there were 2259 MBR wastewater plants worldwide, of which 1527 were municipal plants and 732 industrial. Most MBR applications are still on a medium to small scale in terms of capacity: In North America of the 219 municipal wastewater treatment plants using MBR technology only 17 are larger than $10000 \mathrm{~m}^{3} / \mathrm{d}$, with the largest capacity plant in operation a 29 $600 \mathrm{~m}^{3} / \mathrm{d}$. In Japan small scale SMBRs are used extensively for water recycling (Judd, 2002).

It is interesting to note the distribution of membrane configurations internationally. Hollow fibre membranes dominate the North American market, while in contrast flatpanel membranes dominate the international markct. This is attributed to the presence of Zenon in North America and Kuboto in South East Asia where MBR systems are enjoying strong support (Yang et al., 2005).

Lesjean et al., (2004), argue that for medium to large scale municipal wastewater applications hollow fibre configurations are most competitive, whereas in small to medium scale municipal wastewater applications plate and frame technologies would have an advantage. Judd (2002) compared hollow fibre and plate-and-frame configurations from two full scale plants. He noted that the hollow fibre membranes were cheaper, but more complex, requiring backwashing equipment and regular cleaning. In contrast plate-in-frame systems were more expensive, but provided greater hydrodynamic control, were easier to maintain and ran at a lower flux. Both systems produced no significant odours, but foaming did occur in the aeration basin due to the high aeration rates.

For industrial applications external side-stream MBRs have enjoyed preference due to their perceived suitability to high temperature, high organic strength, extreme $\mathrm{pH}$, high toxicity and low filterability (Yang et al., 2005). However Yang et al (2005) reports that there are no apparent reasons submerged membranes could not treat such industrial wastewaters as well. Lesjean et al. (2004) are in agreement and argue that all three MBR configurations are competitive for industrial wastewater treatment.

\subsection{MEMBRANE DESIGN CONSIDERATIONS}

\subsubsection{System Design}

Ramphao et al. (2004a) conducted a study on BNR AS system design with a SMBR in place of a conventional SST. They concluded that the use of membranes for solidliquid separation in BNR AS systems makes a significant difference to the design of BNR systems and the approach to wastewater treatment plant design. In particular 
anaerobic, anoxic and aerobic zone mass fractions can be varied (within a range) by adjusting the inter-reactor recycles allowing the optimization of biological $\mathrm{N}$ and $\mathrm{P}$ removal corresponding to the influent wastewater characteristics. Essentially the simplified design procedure is as follows: With the zone mass fractions and recycle ratios determined for biological nutrient removal the PWWF determines the surface area of membranes required based on their specified operational flux. The membranes have an aerobic zone volume and aeration requirement. If the aeration requirement for the membranes can meet the biological aeration demand then the membrane one requirement will size the aerobic reactor. If the membrane aeration requirement cannot meet the biological aeration requirement then the aerobic reactor must be sized to include the membranes and additional aeration devices. Thus accurate aeration information is essential to the design. The sludge age of the system is then determined by the required MLSS concentration in the aerobic reactor. For a detailed design procedure examining the impact of submerged membranes on the design of BNR AS systems see Ramphao et al. (2004a).

Bratby (2002) presented considerations for the choice of membranes for a WWTP upgrade, where space constrictions and high water reuse quality necessitated the use of membranes. Both hollow fibre and flat sheet membranes were considered with principle differences in their design being the operational MLSS concentration (10 $000 \mathrm{mgTSS} / \ell$ and $12500 \mathrm{mgTSS} / \ell$ respectively), the aerobic zone volume of the membranes and the biological and membrane aeration requirements. The flat sheet membranes, in a single storey configuration, had a lower surface area to reactor volume ratio $\left(8.5 \mathrm{~m}^{2} / \mathrm{m}^{3}\right)$, compared to the hollow fibre membranes $\left(27.6 \mathrm{~m}^{2} / \mathrm{m}^{3}\right)$, resulting in difficulties accommodating both the membranes and adequate aeration devices. However double storey plate and frame configurations (as recommended by Kubota for such an application) were not considered. Bratby (2002) indicated concern regarding several uncertainties in the operation of MBR full scale plants including operation and aeration performance at high MLSS concentrations.

\subsubsection{Modelling of MBR Systems}

In order to design activated sludge systems using membranes a theoretical model is required. Bratby (2002) reported doubt in design that the simulation package BioWin could adequately predict the system performance due to high MLSS and retention of all solids, and recommended that simulations be calibrated to full scale plants to resolve this. Cornel et al. (2003) state that operational experience shows the MBR's can be modelled by the activated sludge models ASM1 and ASM3 by the International Water Association (IWA). Lee et al., (2002b) developed a model combining ASM 1 with a membrane fouling model in order to predict the performance of the membranes. Guadix et al. (2004) developed a model for the prediction of membrane performance and life-cost.

\subsection{OPERATIONAL ISSUES OF MBR SYSTEMS}

\subsubsection{Operational Experience of MBR Systems}

Yang et al. (2005), conducted a survey of MBR plant operators in North America to assess the performance of full scale MBR systems. Benefits of MBR's cited by operators in Yang et al. (2005) include: 
1) High quality effluent

2) Space savings enabling plant upgrading without land expansion

3) Shorter start up times compared to conventional plants

4) Low operating and maintenance manpower requirements

Disadvantages cited in Yang et al. (2005) included:

1) Bioreactor foaming

2) Low Oxygen transfer efficiencies

3) Membrane fouling

4) Requirement for rigorous membrane cleaning

5) Lower than anticipated membrane permeability

Of these disadvantages membrane fouling remains the primary concern amongst operators.

\subsubsection{Sludge Production}

COD is removed from biological systems by the utilization of carbon compounds with oxygen for synthesis of new biomass, production of $\mathrm{CO}_{2}$ and energy. However Pirt (1965, cited in Witzig, 2002)) noted that micro-organisms use available COD mainly for maintenance at low growth rates, thus reducing sludge production. As sludge production and its subsequent need for disposal is a major cost of wastewater treatment plants, Kraume et al. (2005) argue that "wastewater treatment plants should be designed and operated such that pollutants are diverted from assimilation via biosynthesis to energy requiring functions associated with non-growth activities". In theory this is achievable by keeping biomass as close to a food limited environment as possible thereby uncoupling metabolism so that catabolism of substrate continues unaffected while anabolism is constrained (Stephenson, cited in Gander et al., 2000), thus achieving a reduction in biomass yield. The above can be achieved in long sludge ages (Low F/M) activated sludge systems but the COD mass balance measures that influent COD not harvested as sludge production is COD directed to oxygen demand.

Maintaining low $\mathrm{F} / \mathrm{M}$ ratios result in minimum sludge production (Brindle et al., 1996). Low sludge productions of $0.26 \mathrm{kgTSS} / \mathrm{kgBOD}$ for an aerobic MBR with sludge age of 57 days (Davies et al., 1998) and $0.2 \mathrm{kgTSS} / \mathrm{kgCOD}$ for a MLE MBR system with 50 day sludge age (Buisson et al., 1998) have been reported. Rosenberger et al. (2002) ran a submerged membrane bioreactor with anoxic and aerobic zones for 534 days with minimal sludge wasting and concluded that for very low feed to microorganism (F/M) ratios virtually no excess sludge was produced apart from the accumulation of inorganic particulates. Rosenberger et al., (2002) suggested that bacteria in the highly concentrated sludge of an MBR are limited by organic carbon and the physiological state of most cells was not characteristic for growing cells. Masse et al (2006) reported a decrease of sludge production from 0.31 to 0.13 $\mathrm{mgVSS} / \mathrm{mgCOD}$ as SRT increased from 9 to 110 days in a fully aerobic SMBR system. In terms of COD balance based activated sludge models, the low sludge production at long sludge ages means high oxygen demand because the endogenous process has continued for a long time equivalent to enhanced aeration in which aeration sludge digestion as well as wastewater treatment takes place in the activated sludge reactor. 
The minimal sludge production in the MBR at long sludge ages was also investigated by Witzig et al (2002). They noted that net biomass production is limited by the amount of energy provided, the maintenance energy demand, and cell decay and lysis which led to two hypotheses for zero net biomass generation:

1) Growth-death concept: Whereby limited substrate results in the growth rate decreasing until it equals and death rate. Hence a net zero sludge production. This hypothesis however assumes that all dead material is reused, and makes no account of inorganic material accumulating in the reactor as is suggested by Marais and Ekama (1976).

2) Maintenance energy concept: Proposed by Pirt (1965, cited in Witzig, 2002) energy for maintenance is used in preference to growth. Therefore bacterial growth will only occur if energy is supplied in excess of the maintenance energy requirements of the sludge. This hypothesis does not take into account death of organisms.

Long SRTs do have two specific drawbacks: decreased biomass viability, and the retention and accumulation of non-reactive compounds which can lead to microbial inhibition and toxicity, fouling and limited sludge disposal alternatives (Brindle et al, 1996). In industrial anaerobic MBRs with long sludge ages the accumulation of sulphur compounds is of concern (Brindle et al, 1996).

Sludge production is expected to be very similar for MBR and conventional activated sludge (CAS) systems receiving the same waste water for the same SRT, with a small increase in sludge production in the MBR system due to the retention of all solids by the membrane versus the loss of some solids through the SST (Urbain et al (1994), Masse et al., 2006). Masse et al. (2006) operated two parallel aerobic AS systems, one with membrane solid liquid separation, the other with a SST. The solids lost in the effluent of the SST were measured and incorporated into the sludge wasted daily. At a SRT of 10 days the sludge production for both systems was very close demonstrating that sludge production in MBR and CAS systems is the same at the same SRT and same wastewater source (unbiodegradable particulate COD fraction, f.up)

\subsubsection{Aeration}

Aeration system design required knowledge of the capacity of the aeration device to transfer oxygen to the wastewater (Baker et al., 1975). Factors impact on this capacity relate both to physical variables (reactor geometry, the mixing intensity, temperature, viscosity, reactor depth etc.) and process-related variables (SRT, nutrient and organic loading, process configuration) in the system (Baker et al., (1975), Wagner and Pöpel (1998), Günder and Krauth (1999), Cornel et al., (2003))

Baker et al., (1975) proposed that viscosity, not MLSS concentration is a fundamental factor affecting oxygen transfer. Viscosity has a more direct influence on oxygen transfer as viscosity decreases the rate of oxygen movement from the gaseous to the liquid phase (Baker et al., 1975, Rosenberger et al. 2002b and Gunder 2001 cited in Krampe and Krauth, 2003). The increased concentration of soluble organic matter associated with the mixed liquor increases with the increase in solids concentration. 
From experimentation over a large range of solids concentrations (up to 50mgTSS/ $\ell$ ) Baker et al., 1975 concluded that oxygen transfer rate $\left(K_{L A}\right)$ decreases logarithmically with an increase in viscosity.

Krampe and Krauth (2003) investigated the oxygen transfer efficiencies (OTE) of various aeration devices and found fine bubble aerators to be the most efficient at solids concentrations up to $18 \mathrm{mgTSS} / \ell$. Thereafter the device efficiencies converged. Günder and Krauth (1999) ran three membrane bioreactors with submerged plate and frame, hollow fibre and external tubular membranes respectively. All three systems were fed the same wastewater and the sludges generated showed no significant differences. Alpha (the ratio of oxygen transfer rates in mixed liquor and pure water) was monitored as the sludge concentration in the systems increased. They noticed a significant decrease in alpha with increased MLSS concentration, from 0.5 at $8 \mathrm{gTSS} / \ell$ to 0.15 at $25 \mathrm{gTSS} / \ell$. Judd (2002) noted that "Clearly the effectiveness of aeration is a key aspect of MBR technologies". The drive for low sludge production MBRs clearly has cost implications on the oxygen demand and hence transfer rate costs of the system.

\subsubsection{Membrane Flux and Fouling}

Membranes retain all solids larger than their pore size in the mixed liquor solution. Thus membrane failure does not compromise the effluent, instead the membranes foul and the effluent flux $\left(\mathrm{m}^{3}\right.$ effluent $/ \mathrm{m}^{2}$ surface area/ $\mathrm{d}$ ) decreases (Trussel et al., 2006). Factors that affect membrane performance (after Rosenberger et al., 2006) can be listed as:

1) Membrane material and construction

2) Hydrodynamic conditions (flux, trans-membrane pressure (TMP), cross-flow velocity)

3) Operational conditions (temperature, SRT, inflow, dissolved oxygen concentration)

4) Activated sludge characteristics (MLSS, extra-cellular polymeric substances (EPS), viscosity)

Initially the solids fraction of the activated sludge was believed to have the greatest impact on membrane fouling (Brindle and Stephenson, 1996). However more recently the non-settleable fraction of activated sludge, containing colloids and solutes, is attributed to membrane fouling (Rosenberger et al., 2006, Trussel et al., 2006, Fleischer et al., 2005). Rosenberger et al. (2006) concluded that the concentration of polysaccharides and other non-settleable organic compounds (eg. proteins and organic colloids), and hydrophilic properties impacted on fouling. The analysed organic substances, extra-cellular polymeric substances (EPS), are of microbial origin and are produced and degraded in the activated sludge, making the fouling of membranes by EPS a dynamic process, and easily disturbed. Factors that influence EPS include dissolved organic carbon concentrations, SRT (Trussel et al., 2006), substrate type, COD:N:P ratio (Mahendraker et al., 2005), feed concentration, organic loading rate, reactor type, aeration rates ( $\mathrm{Ji}$ and $\mathrm{Zhou}, 2006)$ and temperature.

Ishiguro (1994, cited in Brindle and Stephenson, 1996) noted the phenomenon of concentration polarization whereby solutes would accumulate on the membrane 
surface forming a gel layer. This gel layer acts as a secondary membrane which reduces the flux, but retains smaller particles improving the membrane filtration. Chiemchaisri (1993, cited in Brindle and Stephenson, 1996) showed that due to the gel effect membranes with differing manufactured pore sizes $(0.03 \mu \mathrm{m}$ and $0.1 \mu \mathrm{m})$ produced permeate with virus concentrations in the same order, i.e. both membranes had effectively the same pore size. Rosenberger et al., (2006) confirmed the presence of a dynamic gel layer by demonstrating that the retention of polysaccharides improved from $70 \%$ to $100 \%$ in new and used membranes respectively while operating at similar fluxes.

Howell (1995, cited in Gander et al., 2000) hypothesised that there is a critical flux below which membrane fouling is minimal. Thus if membranes were run at a "subcritical" flux the fouling is greatly reduced. Ognier et al., $(2002,2004)$ and Cho and Fane and Chang (2002) both cited in Rosenberger et al., (2006) explain membrane failure by assuming a gradual decrease of membrane pore size over time due to macro-molecular adsorption, increasing permeate velocity in pores. As the permeate velocity increases the critical flux is approached ultimately causing failure.

Thus membrane deterioration will occur over time requiring an increasing TMP to maintain the system flux (Fane and Chang, 2002). This can be achieved either by allowing an increase in the reactor head, or applying a suction pressure on the permeate line. Membrane fouling is typically controlled by surface shear often applied as a crossflow velocity, or back-flushing, or a combination of the two (Fane and Chang, 2002).

Most manufacturers recommend periodic chemical cleaning to remove biofouling or chemical precipitation (scaling) using a $0.5-2 \%$ hypochlorite solution and $1-2 \%$ citric acid solution respectively (Darton, 1997, Kennedy, pers com). SMBR membranes typically require cleaning every 6 months, but systems that are run at low fluxes have been run for 18 months without cleaning and maintained operational flux, while external tubular membranes, run at high fluxes with rapid cross flow velocities reportedly require cleaning on a weekly basis (Gander et al., 2000).

\subsubsection{MBR Foaming}

Foaming in the aerobic or anoxic reactors of MBR systems has been reported (Monti et al., 2006, Trussel et al., 2006) and is attributed to the high solids concentration in the reactors, and a lack of flow though in reactor design. Trussel et al. (2006) who observed foaming in an aerobic system controlled the foaming by placing the outlet from the aerobic reactor at the surface level of the mixed liquor recycling the foam and preventing its accumulation over time.

\subsubsection{Energy Consumption}

Cornel et al. (2003) reported high power consumption in MBR plants, 2 to 3 times higher than in conventional activated sludge plants. At MLSS concentrations of $15 \mathrm{mgTSS} / \ell$ power requirements for filtration and aeration only of 1.0 to $2.0 \mathrm{kWh} / \mathrm{m}^{3}$ and 2.5 to $3.5 \mathrm{kWh} / \mathrm{m}^{3}$ were reported for submerged MBR systems and external sidestream systems respectively (Günder and Krauth, 1999; Cornel, 2003). Of the submerged membrane power requirement $2 / 3$ of the energy was required for 
generating sufficient cross-flow to control fouling. For all configurations low alpha values (section 2.4.3) due to the higher MLSS concentrations increased the power requirement for aeration. Günder and Krauth (2003) note that for the plate-and-frame and hollow fibre configurations, running the system at MLSS concentrations of $25 \mathrm{gTSS} / \ell$ resulted in aeration power requirements closer to $3.0 \mathrm{kWh} / \mathrm{m}^{3}$.

\subsection{MICROBIOLOGY OF MBR SYSTEMS}

\subsubsection{Microbiology}

Many advantages of membranes have already been listed above, however their impact on the biology of MBRs is also significant. Witzig et al. (2002) note the following modifications to the activated sludge biology in MBRs:

i) a higher, often substantially so, MLSS concentration;

ii) The retention, and hence selective cultivation of slow growing, nonsettleable bacteria which would otherwise be washed out in CAS plants (Liebig et al ,2001);

iii) The complete retention of cysts of parasites, eggs of worms and virtually all bacteria thus providing an effluent of very high quality.

Witzig et al. (2002) conducted a study into the microbial community structure and physiological state of high concentration biomass in an MLE type MBR nitrogen removal system. The system was run with minimal excess sludge removal and observations were made at sludge ages from $20 \mathrm{~d}$ to $60 \mathrm{~d}$. They concluded that the bacterial population was able to mineralize the substrates (COD and N) at high and stable rates despite high biomass concentrations. Comparisons with CAS systems treating the same wastewater suggested that the MBR sludge population was more substrate limited than CAS systems. Grazing organisms were absent in the MBR reactor which are an important mechanism for cell elimination and ecological selection in CAS systems. Masse et al. (2006) operated two parallel aerobic AS systems at the same loading rates and SRT's, one a MBR and the other a CAS system, and conducted investigations on the sludge morphology. They observed a higher number of non-flocculating bacteria in the MBR, but the same mean floc size in both systems. As the SRT increased the floc size in the MBR system decreased and more dispersed micro-organisms were observed in the MBR. Conversely, in the CAS system, as SRT increased more filamentous organisms were observed.

Manser et al. (2005) undertook an investigation specifically into the performance of nitrifiers in MBR reactors. In their study two pilot scale plants in identical MLE configurations, one a MBR, the other a CAS system, were run over an 8 month period using the same influent wastewater. Systems were run at a $20 \mathrm{~d}$ sludge age with the same operation parameter, i.e. the same wastewater, average MLSS concentrations (3680 $\mathrm{mgTSS} / \ell$ and $3650 \mathrm{mgTSS} / \mathrm{l}$ respectively) and the anoxic and aerobic mass fractions were kept the same $\left(f_{\operatorname{manx}}=f_{\text {maer }}=0.50\right)$. The only difference was the scale at which the systems were run. The CAS system had a total reactor volume of $15 \mathrm{~m}^{3}$, while the MBR system had a total reactor volume of $0.35 \mathrm{~m}^{3}$.

Manser et al. (2005) noted that both systems exhibited similar maximum nitrification rates. Biofilm development on the membranes was found to have negligible 
contribution to overall nitrification, suggesting that cross-flow velocities and aeration prevent development of stable biofilm on the membranes.

Manser et al. (2005) concluded, based on observations on CAS and MBR systems run at the same sludge concentrations, that the presence of membranes does not directly influence the nitrifying community, rather the nitrifying community is influenced by operating conditions (sludge age and MLSS concentration) and wastewater characteristics. Manser et al. (2005) hypothesised that this is also true of other microbiological populations. The membrane does not enhance nitrification performance or protect the system from overloading as these are biological processes. However Manser et al. (2005) noted that smaller MBR floc sizes due to vigorous aeration result in less mass transfer effects. Manser et al. (2005) suggested that conventional models are adequate for nitrification, however further research would be required at the operating conditions suggested for MBR systems (ie high sludge concentrations).

\subsubsection{Effluent Quality}

Membrane separation provides an effluent of high quality. Membranes are able to disinfect waters resulting in the retention of all pathogenic micro-organisms in the sludge mass (Gander et al., 2000). Günder and Krauth (1999) report that in three membrane systems that they investigated salmonella was absent from the system effluents, as were all indicator bacteria.

\subsection{ORGANIC AND NUTRIENT REMOVAL PERFORMANCE}

\subsubsection{COD Removal}

An abundance of literature has been published on the COD removal performance of MBR systems in either submerged (Cote et al. 1997, Trussel et al. 2006, Gander et al., 2000) or external configurations (Günder and Krauth, 1999). COD removal is performed by the utilization of COD for metabolism with oxygen supplied to the system, or the incorporation of COD in the activated sludge which is removed through regular sludge wasting. Due to the inherent ability of membranes to retain all solids within the system COD removal is reliable. Consistently impressive COD removals ranging from $90-98 \%$ are reported at sludge ages from 2 to $>50$ days (Trussel et al., 2006, Buisson et al., 1998, Masse et al. 2006).

Günder and Krauth (1999) ran three membrane systems using submerged hollow fibre membranes, submerged plate in frame membranes and external tubular membranes with pore sizes of $0.1 \mu \mathrm{m}, 0.4 \mu \mathrm{m}$ and $0.1 \mu \mathrm{m}$ respectively. However the COD removals for all three systems were virtually the same due to the formation of the dynamic gel layer described in Section 2.4.4.

Masse et al., (2006) reported excellent retention of EPS in the supernatant of the sludge, which contribute significantly to the improved COD removal of membrane biological reactors. Ramphao et al. (2004) showed the effluent COD of their nitrification-denitrification (ND) biological excess phosphorus removal (BEPR) submerged flat plate MBR system was substantially lower $(35 \mathrm{mgCOD} / \ell)$ than the 
$0.45 \mu \mathrm{m}$ membrane filtered COD $(57 \mathrm{mgCOD} / \ell)$ of a control system fed the same influent COD.

\subsubsection{Nitrogen Removal}

The mechanism for nitrogen removal in MBR systems is the same as in conventional activated sludge systems (Kraume et al., 2005). Ammonia, from the influent and released into solution from the utilization of organics in the wastewater and sludge, is converted first to $\mathrm{NO}_{2}$ then to $\mathrm{NO}_{3}$ in the aerobic reactor by autotrophic nitrifier organisms. $\mathrm{NO}_{3}$ in the anoxic reactor is used as a terminal electron acceptor by facultative heterotrophic organisms for the utilization of COD and is converted to nitrogen gas and released into the atmosphere. Additionally nitrogen is incorporated into the sludge mass through synthesis and removed through sludge wasting.

\section{Nitrification}

Nitrogen removal has been shown to be equal or better in MBRs than in conventional activated sludge (CAS) systems due to longer SRT and smaller floc sizes which allow better transport of nutrients and dissolved oxygen to the flocs (Hakani et al., 1990, cited in Gander et al., 2000). Kraume et al. (2005) report that most MBR plants achieve total nitrification $\left(<1 \mathrm{mgN}-\mathrm{NH}_{4} / \ell\right.$ ). However the high MLSS concentrations, which increase the sludge viscosity and affect its rheology, result in poor mixing and the formation of anoxic micro-zones, which can induce simultaneous nitrification denitrification (Rosenberger et al., 2002) in the aerobic MBR. This can be beneficial but is at the cost of membrane fouling and poor aeration performance induced by high MLSS concentrations.

As with CAS systems nitrification in MBR systems is sensitive to feed characteristics and operational parameters: SRT (Trussel et al., 2006), DO concentration (Hakani et al. 1990, cited in Gander et al., 2000), temperature (Kisino et al., 1996), organic loading, $\mathrm{pH}$ and levels of key nutrients in the feed.

As discussed in Section 2.5.1 the ability of the membranes to retain all microorganisms can affect the microbial population. Kraume et al. (2005) suggest that this will change the specific nitrification rate due to the shift in biocoenosis. Li et al. (2005) compared the nitrification performance of a SMBR to a CAS system. Both systems were run at long sludge ages and fed a synthetic ammonia containing inorganic wastewater. The nitrification performance of the systems was monitored as the hydraulic retention time (HRT) of the systems was decreased. In the CAS system, as the flow through the reactor increased, washout of micro-organisms with the effluent occurred. However Li et al. (2005) reported that this was not selective as the microbial community structure remained unchanged from before washout occurring, as non-settleable solids would wash out regardless of the HRT. In the SMBR system, as all microbial organisms were retained, the microbial community diversity only increased with the extension of the operating period. In both systems nitrification efficiency of $98 \%$ was achieved for hydraulic retention times of $\geq 10 \mathrm{~h}$ and $\geq 20 \mathrm{~h}$ for the SMBR and CAS systems respectively, illustrating the insensitivity of the SMBR system to high flow through rates. Fleischer et al. (2005) noted that the nitrifiers in an 
MBR system could recover from nitrification inhibition far faster than nitrifiers in conventional systems indicating that nitrification in MBR systems is far more robust.

Parco et al, (2006) showed that the specific growth rate of nitrifiers $\left(\mu_{\mathrm{nm}}\right)$ decreases in the MBR system compared with a conventional system. It is hypothesised that the decrease in $\mu_{\mathrm{nm}}$ is due to the retention of non-settleable, slower growing nitrifiers in the MBR system. Parco et al. (2006) however concluded that an increase in the minimum sludge age for nitrification $\left(R_{s m}\right)$ has little influence on the performance of MBR systems as they are typically run at sludge ages substantially longer than $R_{\mathrm{sm}}$.

\section{Denitrification}

Two configurations for denitrification were reviewed in the literature: Predenitrification and post-denitrification.

Pre-denitrification utilizes a primary anoxic reactor, placed upstream of the aerobic reactor and receives the influent flow. A recycle from the aerobic reactor loads $\mathrm{NO}_{3}$ and sludge mass to the anoxic reactor where the $\mathrm{NO}_{3}$ can be utilized as electron acceptor for synthesis of biomass and the $\mathrm{N}$ released as nitrogen gas. Advantages of the pre-denitrification configuration cited in Kraume et al. (2005) are:

1) Substantially higher denitrification rates are possible due to the presence of influent COD: the readily available carbon source stimulates faster denitrification rates compared to endogenous denitrification rates.

2) $\mathrm{As}_{\mathrm{NO}_{3}}$ is used as electron acceptor the oxygen demand in the aerobic reactor is reduced.

3) Half the alkalinity removed in the nitrification step is recovered by denitrification, stabilizing the $\mathrm{pH}$.

With a pre-denitrification reactor, nitrate removal is limited by the rate of the recycle, and so complete $\mathrm{N}$ removal is practically not possible.

Post-denitrification utilizes the secondary anoxic reactor downstream of the aerobic reactor, ensuring all $\mathrm{NO}_{3}$ generated in the aerobic reactor passes through the anoxic reactor and so theoretically can be denitrified. However, as most of the influent COD is degraded in the upstream aerobic reactor denitrification in the post-anoxic reactor is carbon limited, typically with slow endogenous denitrification rates. COD (methanol) dosing to the secondary anoxic reactor may be required to increase denitrification rates and to enable complete denitrification. Gnirss et al. (2003) states the following advantages for post-denitrification with carbon dosing over pre-denitrification in MBR systems:

1) The saving in biological oxygen demand by using influent organics in preference to carbon dosing is considered insignificant due to the high energy requirements in the aerobic MBR for aeration and cross flow velocity scour of the membranes.

2) MBR systems tend to result in poor primary anoxic denitrification rates $\left(K_{1}\right)$ making denitrification in primary and secondary anoxic reactors comparable. Reasons for decreased $\mathrm{K}_{1}$ values in MBR systems cited by Vocks et al., 2005 were the increased MLSS concentration and high oxygen carry over from the 
aerobic reactors. Vocks et al. (2005) also reported increased secondary anoxic denitrification $\left(\mathrm{K}_{3}\right)$ rates linked to an increased glycogen accumulating organism (GAO) population that could utilise stored glycogen as carbon source in the post-anoxic reactor. The occurance of GAO bacteria in full scale plants fed municipal wastewater is rare and so this observation should not be accepted as a general conclusion for all secondary anoxic reactors.

3) Less pumping and recycling equipment is required to obtain $\mathrm{N}$ removal and complete $\mathrm{N}$ removal is possible.

Kraume et al. (2005) proposes post-denitrification with carbon dosing, on the basis of (1) above, as a promising configuration in MBR technology.

\subsubsection{Phosphorus Removal}

Two methods of $P$ removal are typically employed. The first is chemical precipitation whereby phosphate is transformed to a precipitating iron, aluminium or calcium salt which accumulates in the sludge and is removed in the waste stream (Kraume et al., 2005). Disadvantages of this method, listed in Lesjean et al. (2003), are an increase in sludge production (up to $25 \%$ ), additional chemical consumption, increase in the salinity in the effluent, adverse impacts on biological nitrification and reduced sludge reuse in agricultural and other applications.

The second method to remove $\mathrm{P}$ is for it to be incorporated into the biological sludge mass. Although this occurs normally to a small degree $(0.03 \mathrm{mgP} / \mathrm{mgVSS})$ it can be augmented by biological excess phosphorus removal (BEPR). With BEPR the growth of specialised organisms in the sludge, phosphorus accumulating organisms (PAOs) that store substantial intracellular polyphosphates $(0.38 \mathrm{mgP} / \mathrm{mgVSS}$, Wentzel 1990$)$, are encouraged through the implementation of an anaerobic reactor at the head of the activated sludge reactor. The presence of PAOs in significant numbers, which take up volatile fatty acids (VFAs) produced from the influent readily biodegradable COD in the anaerobic reactor, increase the overall $P$ content of the sludge, and excess $P$ removal is achieved through normal sludge wasting. Wentzel and Ekama (1997) noted that increasing sludge age above 5 days decreases $P$ removal per unit influent COD. This is due to the reduced PAO active fraction of the sludge as only active PAOs retain internal polyP which, upon death the $\mathrm{P}$ is returned to solution through lysis. Thus the sludge age of the system cannot be increased without impacting significantly on $\mathrm{P}$ removal efficiency. Hence for MBRs with longer sludge ages, $\mathrm{P}$ removal has been ensured with additional chemical precipitation (Kraume et al., 2005).

Because MBRs consistently retain all solids in the bioreactor, the significant contribution that the loss of solids in the effluent makes in CAS systems to the effluent $\mathrm{P}$ concentration is prevented. Additionally the aerobic separation step in MBRs "fixes" the phosphorus in the sludge when the effluent is separated from the sludge (Gnirss et al., 2003). Therefore MBRs can produce better consistent $P$ removal. 


\subsection{PREVIOUS STUDIES ON BNR IN MBR SYSTEMS}

\subsubsection{Ruhleben WWTP, Berlin, Germany.}

In order to investigate NDBEPR in MBR systems under pre-denitrification and postdenitrification conditions Lesjean et al. $(2003,2005)$ ran a bench scale plant (BSP) and two pilot plants (PP1 and PP2) in parallel with the conventional Ruhleben WWTP in Berlin. The BSP plant was run first in the pre-denitrification MUCT configuration (BSP1), and subsequently in the post-denitrification configuration (BSP2), in both cases with an upstream anaerobic reactor. PP1 was a UCT configuration, and PP2 a post-denitrifying system with upstream anaerobic reactor. All systems were fed wastewater from the same source and monitored over a 2-year period. System configurations and parameters are listed in Table 2.1.

Table 2.1: $\quad$ System parameters for the Ruhleben $M B R$ investigation

\begin{tabular}{|c|c|c|c|c|c|}
\hline & Unit & \multicolumn{3}{|c|}{ MBR } & Conv. \\
\hline System & & $\mathrm{BSP} 1$ & $\mathrm{PP} 1$ & $\mathrm{PP} 2$ & Ruhleben \\
\hline Influent Flow & $\mathrm{m}^{3} / \mathrm{d}$ & 0.24 & 2.6 & 2.9 & 240000 \\
\hline Influent COD & $\mathrm{mgCOD} / \ell$ & 998 & 740 & 740 & 740 \\
\hline Influent TN & $\mathrm{mgN} / \ell$ & 70 & 61 & 61 & 61 \\
\hline Influent TP & $\mathrm{mgP} / \ell$ & 10.5 & 9.1 & 9.1 & 9.1 \\
\hline Reactor Vol. & $\mathrm{m}^{3}$ & 0.21 & 2.0 & 2.2 & 198500 \\
\hline Sludge Age & $\mathrm{d}$ & 15 & 26 & 26 & $15-18$ \\
\hline Aerobic TSS & $\mathrm{gTSS} / \ell$ & 6.2 & 10 & 10 & $3-5$ \\
\hline Approx $\mathrm{f}_{\text {Mana }}$ & $\%$ & 0.09 & 0.10 & 0.10 & not available \\
\hline Approx $\mathrm{f}_{\text {Manx }}$ & $\%$ & 0.40 & 0.45 & 0.50 & not available \\
\hline Approx $\mathrm{f}_{\text {Maer }}$ & $\%$ & 0.51 & 0.45 & 0.40 & not available \\
\hline
\end{tabular}

In all MBR systems the COD and nutrient removal was comparable or better than the conventional system indicating that the membranes did not adversely affect nutrient removal, but in fact improved effluent quality. The MBR systems produced effluent COD removals of $96 \%$ (36mgCOD/ $\ell$ effluent) in comparison to the $95 \%$ of the conventional WWTP.

Initially the BSP system was run to assess the feasibility of BEPR in a MBR system and run in a MUCT configuration (Adam et al., 2002). This was followed by retrofitting the BSP to investigate the feasibility of a post-denitification MBR configuration. Following the initial BSP investigations the two pilot scale systems, PP1 and PP2, were run for sludge ages of 26, 22, 8 and 15 days respectively over successive 6 month periods at $12-15^{\circ} \mathrm{C}$. Both pilot systems exhibited similar COD, $\mathrm{N}-\mathrm{NH}_{4}$ and TP removals. Effluent $\mathrm{P}$ concentrations in all systems were consistently low $(0.5 \mathrm{mgP} / \ell)$, however, due to the presence of calcium and ferric ions in the wastewater, precipitation of $\mathrm{P}$ was observed which left doubt as to the extent of biological P-removal performance. Thus in order to assess the biological $\mathrm{P}$ removal performance the BSP system was spiked with excess $P$ such that the system would not be $\mathrm{P}$-limited. Taking into account the $\mathrm{P}$ precipitation the biological $\mathrm{P}$ removal was estimated as $14-19 \mathrm{mg} \mathrm{P} / \ell$ in BSP1 and $24-26 \mathrm{mgP} / \ell$ in BSP2. The improved $\mathrm{P}$ removal performance in BSP2 can be attributed to the immediate transition of anaerobic to aerobic zones hence preventing anoxic $\mathrm{P}$ uptake which often decreases $\mathrm{P}$ removal. However only details of the BSP2 system mass fractions were reported, hence it is difficult to fully assess the $P$ removal performance. It was noted that the $P$ 
content in the sludge was typically $2-3 \% \mathrm{P} / \mathrm{TS}$ in PP1 and PP2, however in the BSP system, where excess $\mathrm{P}$ was supplied, 6.4 and $7 \% \mathrm{P} / \mathrm{TS}$ were observed in BSP1 and BSP2 respectively.

As was expected total $\mathrm{N}$ removal was better in PP2 with almost complete $\mathrm{N}$ removal, $94 \%(3.6 \mathrm{mgN} / \ell)$, compared to $82 \%(11.0 \mathrm{mgN} / \ell) \mathrm{N}$ removal in PP1 due to the recycle limitation. This is an advantage of the post-denitrification system. However at short sludge ages the denitrification of the PP2 system became unstable and could not produce consistent $\mathrm{N}$ removal, while PP1 could. Vocks et al. (2005) investigated denitrification rates in the systems by running batch tests on anoxic sludge. They reported $K_{1}$ rates (presumably on influent RBCOD) of up to $3.2 \mathrm{mgN} / \mathrm{mgVSS}$.h in the primary anoxic reactor, and noted $\mathrm{K}_{3}$ rates (presumably on endogenous organics) of 0.2-0.6 mgN/mgVSS.h in the secondary anoxic reactor of PP2 which are faster than those expected for endogenous respiration which is assumed to provide the carbon source in post-denitrification systems. The improved post-denitrification rates were hypothesised by Vocks et al. (2005) to be due to stored glycogen in denitrifying organisms.

A major concern of post-denitrification is that slower denitrification rates require a substantially larger anoxic mass fraction than pre-denitrification configurations. Hence there is a need to dose COD to the secondary anoxic reactor in order to decrease the $f_{\text {Manx }}$ requirements. It is interesting to note that both systems had effectively denitrified mid-way through the flow through anoxic reactors suggesting that they were underloaded and hence the systems were not optimized in so far as the mass fractions of the different zones were concerned. Thus, from this investigation, it is difficult to gauge the impact of post-denitrification over pre-denitrification in terms of system anoxic mass fractions.

\subsubsection{Korea Institute of Science and Technology}

Ahn et al. (2003) operated two lab scale MBRs, one as a sequencing anaerobic/anoxic membrane bioreactor (SAM) and the other a MLE MBR configuration. System mass fractions were $\mathrm{f}_{\mathrm{Man} / \mathrm{ax}}=40 \%$ and $\mathrm{f}_{\text {Maer }}=60 \%$ respectively. The systems were monitored for 35 days after achieving a sludge age of 70 days, and a MLSS concentration of $10100-11100 \mathrm{mgTSS} / \ell$. The feed composition ratio was $61 / 9.5 / 1$ (mgCOD/TN/TP). P removal was substantially better in the SAM system $(93 \%$ $0.26 \mathrm{mgP} / \ell)$ than in the MLE system $(45 \% 2.0 \mathrm{mgP} / \ell)$, due to the presence of the anaerobic zone in the SAM. However the BEPR potential of the system was not demonstrated as excess $P$ was not dosed with the influent. TN removal in the SAM system was poor, $60 \%$, due to the reduced anoxic zone mass fraction and hence reduced denitrification. The MLE system achieved $67 \% \mathrm{TN}$ removal. The authors noted that better TN removal could have been obtained with a larger anoxic mass fraction. The experiments demonstrate that the performance of parallel MLE and BEPR systems cannot be compared because each has a different objective and favours the removal of one nutrient or the other.

\subsubsection{University of British Columbia}

Monti et al. (2006) compared and evaluated the enhanced biological phosphorus removal (EBPR) performance of two $2500 \ell$ pilot scale BNR systems using 
membranes and SST's respectively for solid liquid separation. A commendable effort was made in the study to keep the operational and design parameters of both systems identical. The only differences were the method of solid liquid separation and the presence of the SST which effectively increased the system volume of the conventional (C)EBPR system.

Both systems were in UCT configurations with system volume fractions of $0.11,0.28$ and 0.61 for the anaerobic, anoxic and aerobic reactors. Solid liquid separation was effected in the aerobic reactor for both (in the CEBPR system sludge from the SST was recycled back to the aerobic reactor). The systems were operated at sludge ages of 12 days, and HRT of $10 \mathrm{~h}$ and $7 \mathrm{~h}$ for different periods of the study. The systems were loaded equally with feed, and wasted according to the total sludge in the system including that in the SST of the conventional system, to achieve a SRT of 12 days. The operational and system parameters are summarised in Table 2.2.

Table 2.2: $\quad$ System and operational parameters for UBC MBR and Conv. comparison study

\begin{tabular}{|c|c|c|c|}
\hline System & Units & MBR & Conv \\
\hline Reactor vol. & $\ell$ & 2228 & $2228(+900)$ \\
\hline Sludge age & $\mathrm{d}$ & 12 & $12(9.5)$ \\
\hline Aerobic TSS & $\mathrm{mgTSS} / \ell$ & $3500^{\top}$ & $2800^{\top}$ \\
\hline $\mathrm{f}_{\text {Mana }}$ & - & 0.04 & $0.04(0.03)$ \\
\hline $\mathrm{f}_{\text {Manx }}$ & - & 0.21 & $0.21(0.37)$ \\
\hline $\mathrm{f}_{\text {Maer }}$ & - & 0.75 & $0.75(0.60)$ \\
\hline
\end{tabular}

= approximate values;

Values in parentheses take into account the influence of the clarifier on the conventional system.

Due to the presence of two large clarifiers ( $450 \ell$ each), which contained approximately $20 \%$ of the CEBPR system sludge, the MLSS concentrations in the reactors of the CEBPR system were consistently lower, at $80 \%$ of those in the membrane (M)EBPR system, 3500 and $2800 \mathrm{mgTSS} / \ell$ in the MEBPR and CEBPR systems respectively. Hence although the CEBPR and MEBPR systems had proportionally the same mass fractions, the SST in the CEBPR system caused a lower effective aerobic mass fraction and aerobic sludge age (9.5 d vs $12 \mathrm{~d}$ in the MEBPR system).

The COD removal of both systems was good with 92 and $90 \%$ COD removal in the MEBPR and CEBPR systems respectively. Monti et al. (2006) noted that the COD removed via utilization with oxygen in the MEBPR system was substantially higher ( $27 \%$ of COD removal vs $18 \%$ in the CEBPR system). This observation was attributed to the higher effective aerobic sludge age and supported by the decreased sludge production in the MEBPR. The CEBPR system produced $15 \%$ more sludge than the MEBPR system which is also attributed to a longer aerobic sludge age and the additional degradation capacity of the system. Monti et al. (2006) noted that previous literature argue both greater and lesser sludge production for MBR systems, and suggest that sludge production is rather a function of system configuration and operating conditions than merely retention of more solids by membranes. $\mathrm{N}$ removal by both systems was good, with superior denitrification in the CEBPR system attributed to denitrification in the SST. 
The EBPR performance varied in both systems through the study. Frequent Bio-P failure was attributed to

i) VFA limitation due to changes in temperature, dilution of COD in the influent feed and reduced denitrification capacity of the MEBPR system which resulted in nitrate being recycled to the anaerobic reactor. This limited the VFA's available for sequestration by the PAOs in the anaerobic reactor.

ii) The shorter aerobic sludge age in the CEBPR system resulted in greater sludge production and hence increased P-removal capacity.

The lower sludge yield (due to increased sludge age) and lower denitrification capacity of the MEBPR (due to increased anoxic volume in the CEBPR system) affected the MEBPR system's P removal performance. However Monti et al. (2006) suggested that this could be compensated for by carbon addition to ensure P-release is not carbon-limited and by including post-denitrification in the system design to increase denitrification capacity. On average both systems produced comparably low soluble $\mathrm{P}$ in the effluent illustrating that EBPR is feasible with membranes. Additionally the MEBPR system retained all solids in the aerobic reactor and hence no $P$ escaped as sludge with the effluent producing a reduced TP concentration compared to the CEBPR system. However the extent of biological $\mathrm{P}$ removal could not be determined as the system was P-limited. It must be noted that both $f_{\text {iviaua }}$ and $\mathrm{f}_{\text {Manx }}$ were very small thus limiting PAO and denitrification activity respectively.

Prior to concluding the study the CEBPR system was converted to a MEBPR system by the insertion of a membrane module in the aerobic reactor. The system was monitored for four months and the system adapted quickly. This indicated that a smooth upgrade of a MEBPR system is possible.

Monti et al. (2006) concluded that under carbon-limited conditions the CEBPR system performed better due to its increased sludge production and additional denitrification capability, though this was at the cost of the additional volume requirements for the SST. The MEBPR system proved to be more robust as it recovered far faster than the CEBPR system after bio-P failure due to its ability to retain all solids.

\subsubsection{University of Guelph}

Mouthon-Bello and Zhou (2005) conducted a study on a pilot submerged membrane bioreactor with BNR. The pilot scale plant was operated at an average of $18.5^{\circ} \mathrm{C}$ in an anoxic-anaerobic-aerobic configuration with mass fractions $(0.17: 0.33: 0.50)$ over two periods for sludge ages of 20 days and 50 days respectively. An average influent feed ratio of 100:17:3 mgCOD:mgN:mgP was fed to the system. Influent unbiodegradable COD fractions of $0.09 \mathrm{mgCOD} / \ell$ and $0.20 \mathrm{mgCOD} / \ell$ for $\mathrm{f}_{\mathrm{S} \text {,us }}$ and $\mathrm{f}_{\mathrm{S} \text {.up }}$ respectively were assumed. At a 20 day sludge age removals of $89 \%(35 \mathrm{mgCOD} / \ell), 82 \%$ $(11 \mathrm{mgTN} / \ell))$ and $94 \%(0.05 \mathrm{mgP} / \ell)$ were reported, compared to removals of $92 \%$ $(31 \mathrm{mgCOD} / \ell), 78 \%(14 \mathrm{mgTN} / \ell)$ and $90 \%(0.1 \mathrm{mgP} / \ell)$ at a $50 \mathrm{~d}$ sludge age.

In order to maintain system mass fractions the volumes of the reactors and the sludge recycle were adjusted for the two sludge ages. Consequently the changed sludge recycle affected the nitrate load $\left(\mathrm{N}_{\mathrm{nL}}\right)$ to the anoxic reactor and the denitrification 
potential $\left(D_{P P}\right)$ was influenced by increased reactor volume and solids concentration without any change to influent COD. Thus there were differences in the $\mathrm{N}$ removal performance at the two sludge ages that are system specific. Ferrous chloride was dosed to the influent feed thus providing high $\mathrm{P}$ removal through precipitation but no indication of biological P-removal performance. Due to the reactor configuration of having the anoxic reactor upstream of the anaerobic reactor most readily biodegradable COD would have been utilized for denitrification resulting in limited COD for acquisition by POAs and hence limited biological P removal potential. Thus without $C O D$ dosing to the anaerobic reactor, which was not reported, little biological $P$ removal would have been possible thus making the anaerobic zone mass fraction redundant.

A significant difference in the aerobic and effluent $\mathrm{NO}_{3}$ concentrations was reported ( $8.7 \mathrm{mgN}-\mathrm{NO}_{3} / \ell$ vs $17.5 \mathrm{mgN}-\mathrm{NO} 3 / \ell$ at 50 day sludge age) which could not be readily accounted for despite efforts to minimize biological activity while handling the aerobic sample. Sludge production at a 20 day sludge age was reported as $0.39(\mathrm{mgTSS} / \mathrm{d}) /(\mathrm{mgCOD} / \mathrm{d})$.

\subsubsection{Loudoun County Sanitation Authority}

Fleisher et al. (2005) conducted a pilot scale sludy over an 8 month period using MBR technology coupled with BNR activated sludge and chemical treatment systems to achieve very strict effluent nutrient discharge requirements of $<3 \mathrm{mgN} / \ell$ and $<0.1 \mathrm{mgP} / \ell$. Three MBR BNR configurations were investigated, namely:

- a 4-stage process with a de-aeration stage between the MBR reactor and the primary anoxic reactor,

- a 4 stage system, and

- a 5-stage system including an anaerobic reactor at the head of the system. 

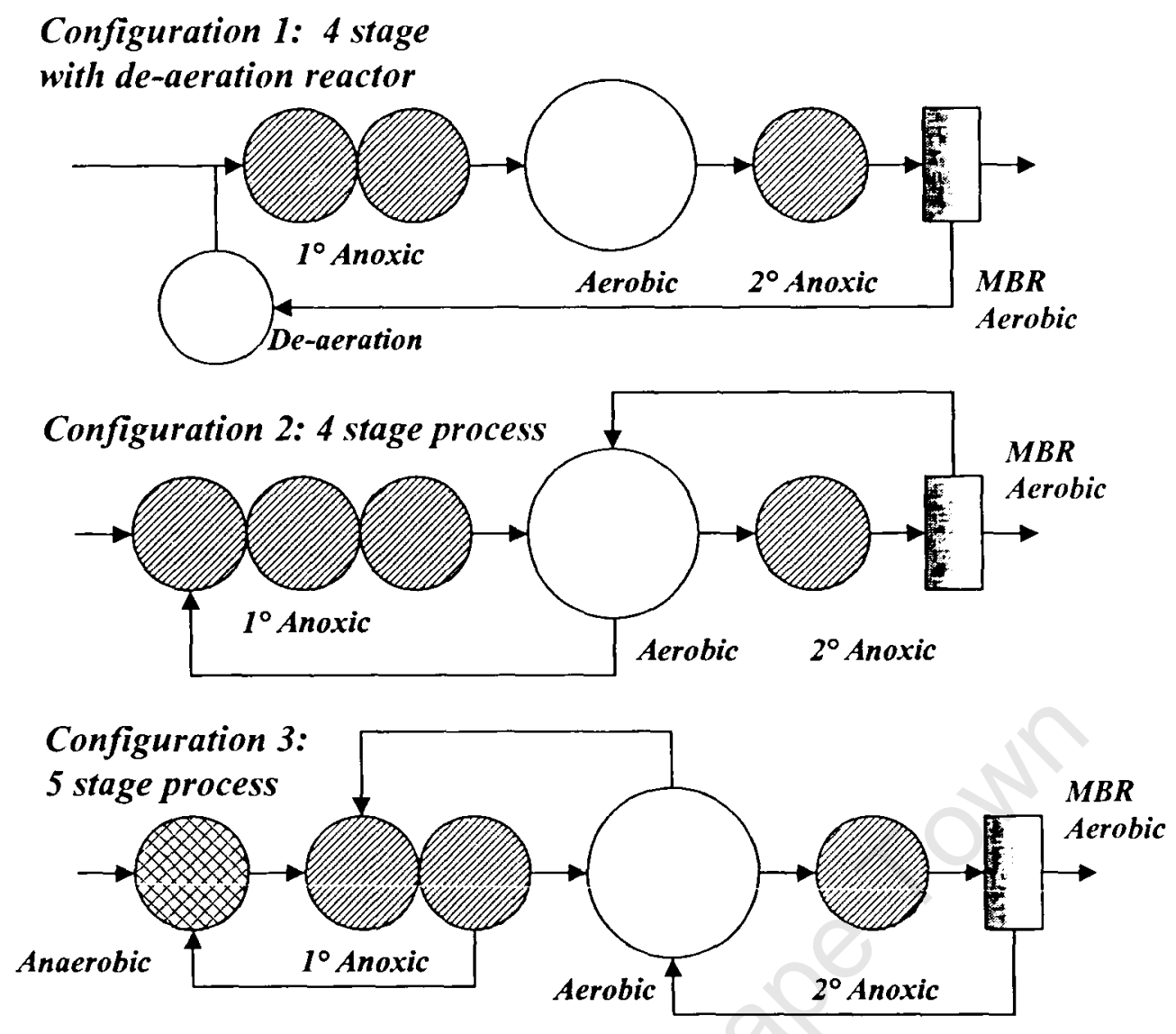

Figure 2.1: $\quad$ Three BNR configurations were investigated for the Loudoun County MBR study: a 4-stage system with de-aeration reactor, a 4-stage process and a 5 stage process including an upstream anaerobic reactor.

A schematic of the systems is presented in Fig 2.1. The first two configurations were run in order to ascertain the influence of recycled oxygen from the heavily aerated MBR reactor to the anoxic zones of the system on the BNR performance of the system. Following these investigations the system was run in the third configuration with varied concentrations of methanol dosed to the $2^{\circ}$ anoxic reactor and alum dosed to the MBR aerobic reactor in order to observe their effects on $\mathrm{N}$ removal and $\mathrm{P}$ removal respectively.

Influent wastewater nutrient ratios of 100:13:2 $\mathrm{mgCOD} / \mathrm{mgN} / \mathrm{mgP}$ were reported. The systems were run at sludge ages of between 19-23 days and no indication of the system recycles and mass fractions was given. This omission is unfortunate as these parameters are necessary to assess system $\mathrm{N}$ and $\mathrm{P}$ removal performance.

System average MLSS concentrations varied through the investigation from 4000 to $9000 \mathrm{mgTSS} / \ell$ with a system average VSS/TSS ratio of 0.80 for BNR performance alone and 0.70 with the additional influence of chemical precipitation due to alum dosing. The alum was dosed in the MBR aerobic reactor in order that as much BEPR as was possible would have taken place prior to chemical precipitation. However de Haas et al. (2001) showed that with simultaneous precipitation chemical dosing can progressively displace BEPR, particularly when low P-concentrations $(<1.0 \mathrm{mgP} / \ell)$ are required. 
The membrane filterability was found to be strongly influenced by the filtration characteristics of the sludge. Fouling was assumed to be a result of colloidal solids not naturally incorporated into the activated sludge flocs. The addition of alum however caused these colloids to be included in the activated sludge flocs and hence improved sludge filterability. The system solids were found to be floc forming despite the turbulent conditions induced by the MBR. Pathogen counts were negligible with ecoli counts of $<2 \mathrm{cfu} / 100 \mathrm{ml}$ and $5-\log$ virus removal.

Expected nitrification performance, determined from model predictions, was observed with a total $\mathrm{N}$ concentration of $3 \mathrm{mgN} / \ell$ obtained with methanol dosing to the $2^{\circ}$ anoxic reactor to aid denitrification in the third configuration. When nitrification inhibition occurred the system would recover quickly demonstrating that the MBR system retained a robust nitrifying population. Prior to alum dosing BEPR was observed with $\mathrm{P}$ concentrations dropping from $6 \mathrm{mgP} / \ell$ influent to $3 \mathrm{mgP} / \ell$ effluent. With the addition of alum almost complete $P$ removal was possible $(<0.1 \mathrm{mgP} / \ell)$. Both $\mathrm{N}$ and $\mathrm{P}$ removal performances were closely predicted using IWA ASM $2 \mathrm{~d}$ indicating that ASM $2 \mathrm{~d}$ could adequately model BNR performance.

Sludge production varied according to the organic load on the system and the chemical dusing of methanol and alum. Prior to chemical dosing an average system sludge production of $0.25(\mathrm{mgVSS} / \mathrm{d}) /(\mathrm{mgCOD} / \mathrm{d})$ was observed. The sludge was additionally examined for dewatering and sludge handling purposes. In comparison to conventional sludges treated from the same influent wastewaters a higher solids cake could be produced from the MBR sludge (14.7\% vs $12.7 \%)$. It was concluded that MBR MLSS characteristics were within those expected for a long SRT BNR process.

\subsubsection{UCT Water Research Group}

Ramphao et al (2004) conducted an in depth study of the BNR performance of a MBR system. Two UCT configured systems were run in parallel: the first a MBR BNR system with membranes located in the aeration reactor, and the second a conventional activated sludge system with a secondary settling tank. Both systems were run at their design solids concentration, i.e. the conventional system at low solids $(\sim 3500 \mathrm{mgTSS} / \ell)$ and the MBR system at high solids $(\sim 18000 \mathrm{mg} / \mathrm{TSS} / \ell)$ for effective cross-flow scour. They were fed from the same feed, and design parameters such as the sludge age, zone mass fractions and recycles were kept the same. In order to assess the BNR performance of the systems RBCOD was dosed into the feed in order to accentuate BEPR performance, thus allowing $\mathrm{P}$ removal to occur without being P limited. The UCT WRG investigation (Ramphao et al., 2004) forms the basis of this investigation and is referred to as the Phase 1 investigation. The objectives and methodology of this investigation stem directly from the initial work of Ramphao et al. (2004) and are described in detail in Chapter 3.

\subsubsection{Summary}

In summary six investigations were reviewed in which membranes had been used in conjunction with BNR processes, and where nutrient removal had been observed and quantified. Table 2.3 summarises the investigations, their design configurations and operational parameters, and the removals they reported. 
Table 2.3: Summary of MBR BNR investigations reviewed

\begin{tabular}{|c|c|c|c|c|c|c|c|c|c|}
\hline \multirow[t]{2}{*}{ Study } & \multirow[t]{2}{*}{$\begin{array}{l}\text { System } \\
\text { configuration }\end{array}$} & \multicolumn{3}{|c|}{ Zone mass fractions } & \multirow{2}{*}{\begin{tabular}{|l|}
$\begin{array}{l}\text { Aerobic } \\
\text { MLSS }\end{array}$ \\
gTSSII \\
\end{tabular}} & \multirow{2}{*}{ Rs } & \multirow{2}{*}{$\frac{\text { N-removal }}{\%}$} & \multirow{2}{*}{\begin{tabular}{|c|} 
P-removal \\
$\%$ \\
\end{tabular}} & \multirow{2}{*}{$\frac{\text { modelling }}{-}$} \\
\hline & & fmana & $\operatorname{fman} x$ & fmaer & & & & & \\
\hline \multirow{2}{*}{$\begin{array}{l}\text { Ruhleben, Germany } \\
\text { (Lesjean et al. 2005) }\end{array}$} & Pre-denitrification & 0.1 & 0.45 & 0.45 & 10 & 26 & $\begin{array}{c}82 \\
(11.0 \mathrm{mgN} / \mathrm{l}) \\
\end{array}$ & $\begin{array}{c}99(\mathrm{ppt}) \\
(0.06 \mathrm{mgP} /))\end{array}$ & no \\
\hline & Post denitrification & 0.1 & 0.5 & 0.4 & 10 & 26 & $\begin{array}{c}94 \\
(3.6 \mathrm{mgN} / \mathrm{l}) \\
\end{array}$ & $\begin{array}{c}99\left(\mathrm{ppt}^{1}\right) \\
(0.07 \mathrm{mgP} / 1) \\
\end{array}$ & no \\
\hline \multirow{2}{*}{$\begin{array}{l}\text { Korea IST, S Korea (Ahn } \\
\text { et al . 2004) }\end{array}$} & SAM & \multicolumn{2}{|c|}{0.4} & 0.6 & 10.5 & 70 & 60 & $\begin{array}{c}93 \\
(0.26 \mathrm{mgP} /) \\
\end{array}$ & no \\
\hline & MLE & 0 & 0.4 & 0.6 & 10.5 & 70 & 67 & $45(2.0 \mathrm{mgP} / 1)$ & no \\
\hline $\begin{array}{l}\text { UBC, Canada (Monti et } \\
\text { al. 2006) }\end{array}$ & UCT & 0.04 & 0.21 & 0.75 & 3.5 & 12 & 63.8 & $\begin{array}{c}97(\mathrm{bio}) \\
(0.2 \mathrm{mgP} / i) \\
\end{array}$ & no \\
\hline \multirow{2}{*}{$\begin{array}{l}\text { Guelph, Canada } \\
\text { (Mouthon \& Zhou, 2005) }\end{array}$} & \multirow{2}{*}{$\begin{array}{l}\text { anaerobic-anoxic- } \\
\text { aerobic }\end{array}$} & 0.33 & 0.17 & 0.5 & 15 & 20 & $\begin{array}{c}82 \\
(11.0 \mathrm{mgN} / 1)\end{array}$ & $\begin{array}{c}94(\mathrm{ppt}) \\
(0.05 \mathrm{mgP} / 1)\end{array}$ & no \\
\hline & & 0.33 & 0.17 & 0.5 & 16 & 50 & $\begin{array}{c}78 \\
(14 \mathrm{mgN} / 1)\end{array}$ & $\begin{array}{c}90\left(\mathrm{ppt}^{1}\right) \\
(0.1 \mathrm{mgP} / \mathrm{l}) \\
\end{array}$ & no \\
\hline Loudoun County, USA & 5-stage bardenpho & $n / a$ & $n / a$ & n/a & 9 & 21 & $\begin{array}{l}92(3 \mathrm{mgN} / 1- \\
\text { COD dosed })\end{array}$ & $\begin{array}{c}99 \text { (bio + ppt') } \\
(<0.1 \mathrm{mgP} / \mathrm{l})\end{array}$ & yes ASM 2d \\
\hline $\begin{array}{l}\text { UCT WRG, RSA } \\
\text { (Ramphao et al. 2004) }\end{array}$ & UCT & 0.13 & 0.28 & 0.59 & 18 & 20 & $\begin{array}{c}74 \\
(26.4 \mathrm{mgN} / 1)\end{array}$ & $\begin{array}{c}\text { BEPR: } 26 \\
\text { mgP// removal }\end{array}$ & yes UCTPHO \\
\hline
\end{tabular}

$=$ P precipitated

(COD removal not reported as $>90 \%$ in all cases regardless of system)

\subsection{CONCLUSIONS}

Significant amounts of research have been conducted and publishcd on the influence and performance of membranes for solid liquid separation in AS sludge systems. Much of the research has been focused on the membrane performance, to understand the mechanisms of fouling and minimize them thus improving the lifespan of membranes and their cost, or on the membrane performance in removing organic compounds or nitrogen compounds.

With increasingly stringent water quality standards internationally for discharge and reuse (Lesjean et al., 2003, Howell, 2004) there is a need for wastewater treatment plants to provide effluents of a reliable and excellent standard. This can be achieved by combining membrane technology with BNR processes. Despite numerous studies on COD removal and nitrification, little research has been published on the impact of membranes on BNR performance, particularly on biological excess P-removal (BEPR), and on the applicability of current BNR simulation packages to membrane applications.

It is however difficult to compare the performances of the various systems as different configurations will give different results and system performances are specific to operating conditions and influent wastewater characteristics. Systems run in parallel can give an indication of comparative system performance but differences in recycles and mass fractions will occur due to the nature of membranes and SSTs, thus to base performance comparisons on effluent measurements alone is faulted. By running batch tests on the systems and reporting kinetic rates only systems run at the same sludge age and fed the same wastewater can be compared with each other. Thus in order to make general comparisons between different investigations the kinetic constants used for modelling, such as unbiodegradable fractions and specific kinetic rates need to be established. These are generally unavailable thus hindering useful comparisons of different investigations. Such kinetic constants are reported for this investigation in Parco et al (2007). 


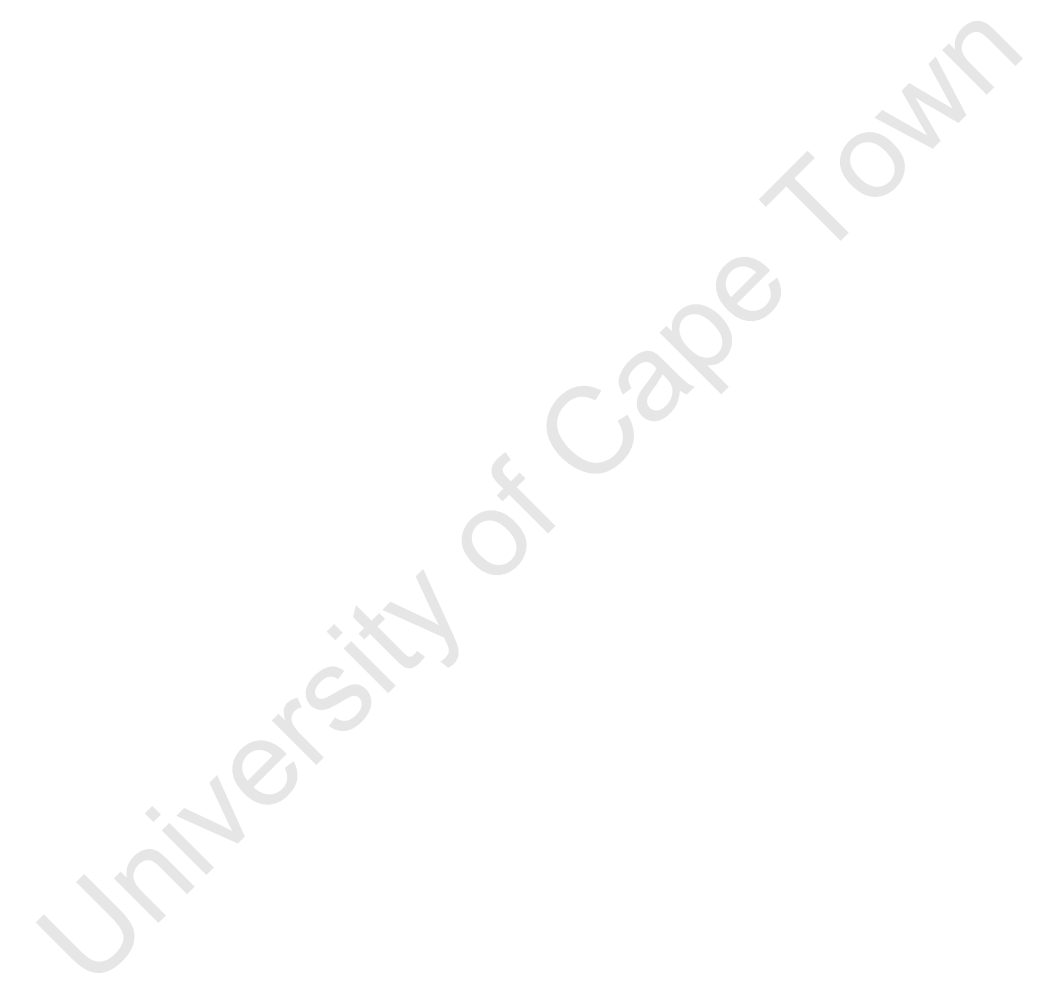




\section{CHAPTER 3}

\section{METHODOLOGY}

\section{$3.1 \quad$ INTRODUCTION}

As described in Chapters 1 and 2 membranes for solid liquid separation in activated sludge systems offer many advantages over conventional activated sludge (CAS) systems. Membranes can produce a reliable, high quality, solids free effluent which is largely disinfected and very suitable for reuse. Membranes obviate the need for secondary settling tanks (SST's). In addition membranes allow activated sludge plants to run at high mixed liquor concentrations providing a substantial reduction in plant footprint. These advantages make membranes for solid liquid separation an attractive option in cases requiring a guaranteed high quality effluent (e.g. recycling and reuse of wastewater, or discharge to sensitive water bodies), or where space is a priority (e.g. Plant upgrades in dense city areas or areas where land is very valuable). Moreover, membrane activated sludge plants can be run at long sludge ages which together with the high mixed liquor solids concentrations produce a stable, concentrated sludge which reduces sludge thickening and further stabilization processes.

However, as noted in Ramphao et al. (2004) the biocoenosis of mixed liquor in membrane systems differs from CAS systems due to unique selection properties; membranes retain all micro-organisms in the system, whereas CAS systems only retain those micro-organisms that flocculate and settle in the SST's; membrane systems run at considerably higher mixed liquor solids concentrations (12000 $18000 \mathrm{mgTSS} / \ell)$ versus $(3000-5000 \mathrm{mgTSS} / \ell)$ in CAS systems. The effects of these differences on design and performance of fully aerobic biologically mediated COD removal and nitrification are well established (Brindle and Stephenson, 1996), and have been investigated for denitrification and P-removal (Kaume et al., 2005, Yang et al., 2006). However the optimization of membrane biological reactor (MBR) biological nutrient (nitrogen and/or phosphorus) removal (BNR) systems, the applicability of steady state design models to MBR BNR systems and the impacts of membranes on BEPR remain to be established. The main aim of this research is to address these deficiencies.

In Chapter 1, the first phase (Phase 1) of this investigation was described. Phase 1 aimed to quantitatively establish the impact of membranes on the design, operation and performance of BNR activated sludge systems (Ramphao et al., 2004), and a substantial initial investigation was carried out into the impacts of membranes on design, and steady state and kinetic performance of MBR BNR systems (Ramphao et al., 2004). Hence the objectives of this thesis served to:

- Verify the results obtained in the initial investigation, with particular emphasis on explaining the phenomena of excessive sludge production,

- Gain a better understanding of the operating conditions and considerations of MBR BNR systems,

- Provide a parent system from which further investigations into the kinetics of a MBR BNR system could be performed. 
In order to address these objectives two parallel lab-scale conventional (CAS) and membrane (MBR) activated sludge systems were operated under laboratory conditions allowing their behaviour to be monitored and their performance compared. In order to verify the previous results the same original experimental apparatus and operational conditions were adopted and testing continued. This chapter describes the set-up, operation, and monitoring of these systems; behaviour and performances are described in Chapter 4 and 5 for the MBR and CAS BNR systems respectively, and Chapter 6 compares the system performances with each other and previous studies on similar systems.

\subsection{RESEARCH APPROACH}

With the research approach for Phase 2 adopted from Phase 1, two parallel BNR activated sludge systems were run, at lab scale, under controlled conditions, one a MBR system and the other a CAS system with a SST. The two systems had identical design and operating parameters, such as anaerobic, anoxic and aerobic mass fractions, recycles and sludge ages. The major difference between the two systems was the amount of feed fed to the MBR system, which was fed a little over three times (3.1) the organic load of the CAS system in order to generate the solids concentration required for effective cross-flow scour (greater that $12000 \mathrm{mgTSS} / \ell$ versus 4000 $\operatorname{mgTSS} / \ell$ ). As the same feed was used for both systems, the higher influent flow rate to the MBR system resulted in a decreased hydraulic retention time (13 hours versus 40 hours). The two systems were chosen to run in a UCT configuration with three internal recycles, called:

- a-aerobic mixed liquor to anoxic rector,

- $\mathrm{r}$ - anoxic mixed liquor to anaerobic reactor,

- $\mathrm{s}$ - return sludge from SST to anoxic reactor.

The MBR system had only two internal recycles as membranes replaced the SST and were located in the aerobic reactor (see Fig. 3.1). Thus the "s" recycle was incorporated into the "a" recycle represented henceforth as the "as" recycle and which was increased accordingly. The UCT configuration was chosen because it allows denitrification and phosphorus removal to act independently of each other; the anaerobic reactor is protected from recycled nitrate from the anoxic reactor-provided the recycles do not overload the anoxic reactor with nitrate.

\subsection{ACTIVATED SLUDGE SYSTEMS DESCRIPTION}

\subsubsection{Activated Sludge Systems Operated}

From Section 3.2 above, the steady state activated sludge laboratory-scale systems run for this research were:

- Long sludge age (20d) MBR-UCT system - this configuration was an adaption of the UCT configuration with membranes in the aerobic reactor replacing the function of an SST. Kubota ${ }^{\circledR}$ panel membranes were used (Fig. $3.1)$. 
- Long sludge age (20d) UCT system with SST - this was operated as a control against which to compare the impact of the membranes on the BNR performance of the MBR system (Fig. 3.2).

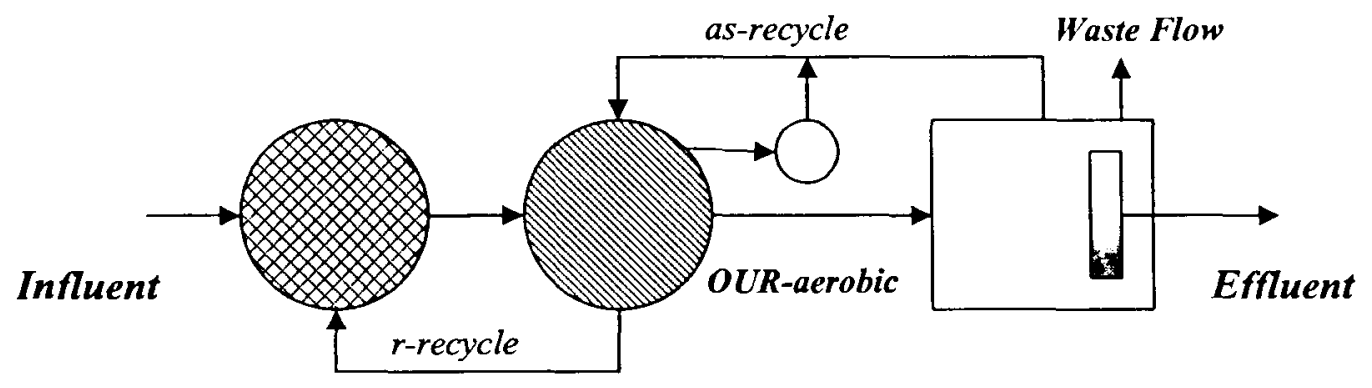

Anaerobic

Anoxic

Aerobic

Figure 3.1: Schematic layout of the MBR UCT system

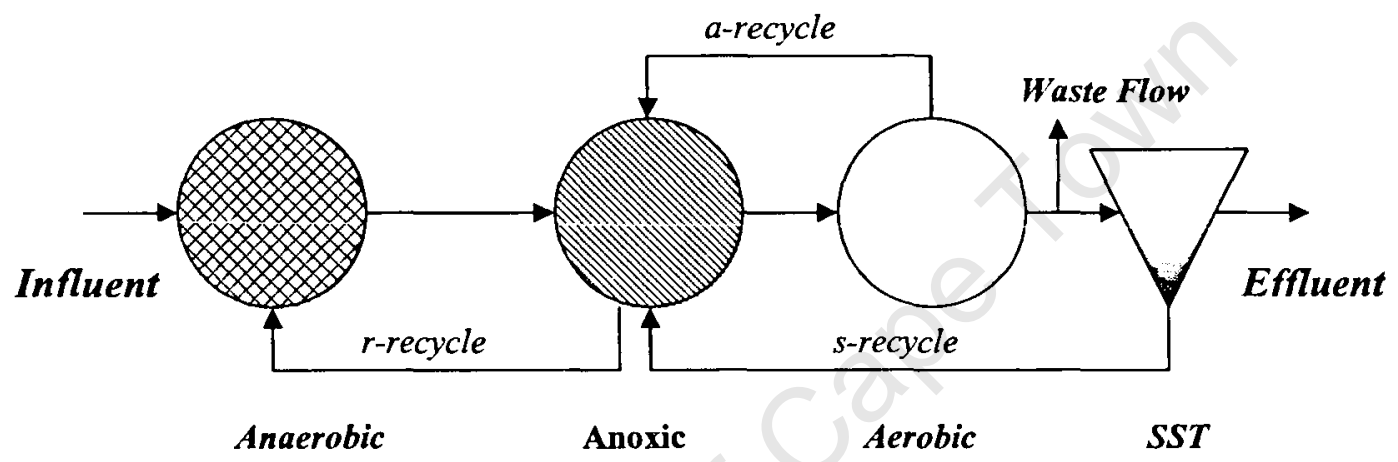

Figure 3.2: Schematic layout of the conventional UCT system

Readily biodegradable (RB)COD has a significant impact on nutrient removal in BNR activated sludge, regardless of the solid-liquid separation mechanism (Wentzel et al., 1990). To quantify the RBCOD the technique developed by Ekama et al.(1986) was used requiring the following activated sludge system to be operated:

- Short sludge age (2 days) square wave (SQW) fed activated sludge system (Fig. 3.3).

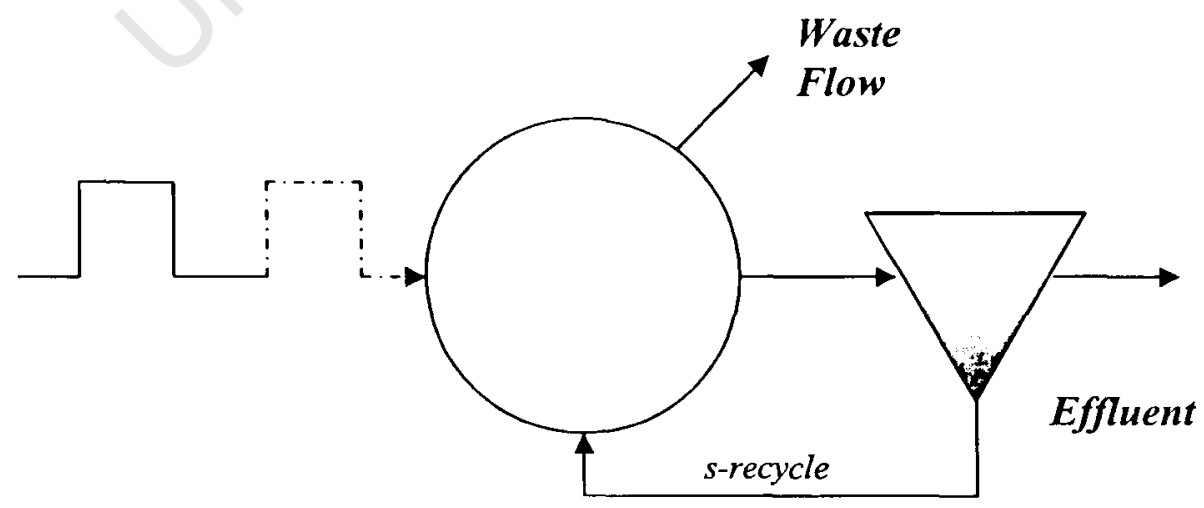

Aerobic

Reactor

Figure 3.3: $\quad$ Schematic of the SQW activated sludge system. 


\subsubsection{UCT systems design and operating parameters}

The two activated sludge systems have, as far as practically possible, identical design parameters, such as anaerobic, anoxic and aerobic mass fractions, recycles and sludge ages. The main difference was to be the influent flow rate (Section 3.2). The volume requirements for the panel membranes used in the research set the size of the MBR aerobic reactor and hence (for the selected anaerobic and anoxic mass fractions) the total system volume. Operating the conventional UCT system with the same volume was not possible due to the large volumes of influent wastewater required. Accordingly the total reactor volume of the MBR system was about 2.96 times larger than that of the conventional system (not including the SST volume).

The influent feed COD concentration was set at $1000 \mathrm{mgCOD} / \ell$. The influent flow rates of $140 \ell / \mathrm{d}$ and $15 \ell / \mathrm{d}$ for the MBR and conventional UCT systems respectively were calculated to achieve the design reactor mixed liquor concentrations of $18000 \mathrm{mgTSS} / \ell$ and $5000 \mathrm{mgTSS} / \ell$ respectively using the BNR kinetic model UCTPHO (Wentzel et al., 1992). Model inputs used were: a 20 day sludge age, where the sludge production and oxygen demand in the systems per unit COD were the same for both systems: $5.19 \mathrm{mgVSS} / \mathrm{mgCOD}$ load per day, $6.92 \mathrm{mgTSS} / \mathrm{mgCOD}$ load per day and $0.706 \mathrm{mgO} / \mathrm{d}$ per mgCOD load per day respectively. The South African raw wastewater unbiodegradable particulate $\left(f_{S, \text { up }}\right)$ and soluble $\left(f_{S, u s}\right)$ COD fractions of 0.13 and 0.05 respectively and $20 \%$ readily biodegradable COD (RBCOD) with respect to the total influent COD $\left(\mathrm{S}_{\mathrm{ti}}\right)$ and sludge VSS/TSS ratio of 0.75 were used. It was anticipated that the membrane mixed liquor concentrations would be higher still than the design value as the membranes would retain all solids, some of which would naturally flow through the SST of the control system.

The initial MBR and conventional system design operating parameters are listed in Table 3.1. 
Table 3.1: $\quad$ Initial MBR and conventional UCT systems' design and operating parameters.

\begin{tabular}{|l|c|c|}
\hline SYSTEM PARAMETERS & MBR UCT & CAS UCT \\
\hline Sludge Age (d) & 20 & 20 \\
\hline Anaerobic (R1) Volume $(\ell)$ & 19 & 5.6 \\
\hline Anoxic (R2) Volume $(\ell)$ & 21 & 6.2 \\
\hline Aerobic (R3) Volume ( $\ell$ ) & $34^{1}$ & 13.2 \\
\hline Anaerobic (R1) Mass Fraction (\%) & $12.6^{2}$ & $12.6^{2}$ \\
\hline Anoxic (R2) Mass Fraction (\%) & $27.9^{2}$ & $27.9^{2}$ \\
\hline Aerobic (R3) Mass Fraction (\%) & $59.5^{2}$ & $59.5^{2}$ \\
\hline s-sludge Return Recycle (SST to R2) & - & $1: 1$ \\
\hline a-recycle Return Recycle (R3 to R2) & $3: 1$ & $2: 1$ \\
\hline r-recycle Return Recycle (R2 to R1) & $1: 1$ & $1: 1$ \\
\hline Hydraulic retention time (d) & 0.53 & 1.67 \\
\hline Aerobic MLVSS conc. (mgVSS/ $\ell$ ) & 12500 & 3600 \\
\hline Aerobic MLSS conc. (mgTSS/ $\ell$ ) & 18000 & 5000 \\
\hline Influent flow ( $\ell / d$ ) & 140 & 15 \\
\hline Feed COD concentration (mg/ $\ell$ ) & 1000 & 1000 \\
\hline Waste flow from Aerobic Reactor $(\ell / d)$ & 2.85 & 1.1 \\
\hline OuR (mgO/ $\ell$ ) & 135 & 37 \\
\hline Membrane Flux (m ${ }^{3} / \mathrm{m}^{2} / \mathrm{d}$ ) & 0.239 & - \\
\hline
\end{tabular}

'effective aerobic volume. MBR UCT Aerobic reactor is in fact $32 \ell$, with a side OUR -aeration reactor for OUR measurement of $2 \ell$.

${ }^{2}$ For the given $\mathrm{a}, \mathrm{s}$ and $\mathrm{r}$ recycle values

\subsubsection{MBR UCT System Description}

The MBR UCT system reactors were constructed of clear Perspex with cylindrical anaerobic and anoxic reactors with volumes of $28 \ell$ each. Though the anaerobic and anoxic reactors were to hold $19 \ell$ and $21 \ell$ respectively the additional head would aid in unblocking the flow through pipes should they block which would commonly occur with small pipes and sludge of high concentration (Ramphao et al., 2004). Together the design volumes gave a total unaerated mass fraction of approximately $40.5 \%$ at the design recycles (as and $r$ ).

The aerobic membrane bioreactor used for this study was a rectangular aeration tank with an effective maximum capacity of $40 \ell$, dimensions $185 \times 260 \times 1720 \mathrm{~mm}$. Two additional Perspex walls rising about two-thirds of the liquid height from the base were inserted into the bioreactor, joining between the shorter walls, approximately $100 \mathrm{~mm}$ in from and parallel to the longer walls; these two walls had $100 \times 150 \mathrm{~mm}$ slots at the base to effectively create an inner riser zone and an outer descender zone.

In the riser zone five panel membrane modules (Kubota®) were submerged. The membrane modules were secured in slots in the shorter walls of the riser zone, and anchored with a stainless steel pin (The membranes were Kubota $\mathbb{B}$ polyethylene, pore size $0.4 \mu \mathrm{m}, 0.225 \times 0.31$ rectangular panels $6 \mathrm{~mm}$ wide with effective filtration area 
$0.202 \mathrm{~m} \times 0.29 \mathrm{~m} \times 2=0.117 \mathrm{~m}^{2}$ each). Coarse bubble aeration was provided directly below the membrane modules, by a rigid PVC loop with small holes drilled on the upper surface connected to a low-pressure air supply $(100 \mathrm{kPa})$. The air served to supply oxygen to, and mix the activated sludge, and induce a cross flow velocity over the membrane surface to prevent fouling. The air rates were controlled by a Rotameter ${ }^{\circledR} 2000$ (GEC - Elliott Process Instrument); DO concentrations were regularly monitored (YSI Model 5739) and never fell below $2 \mathrm{mgO} / \ell$.

The effluent flow through the membranes was collected via flexible silicone tubing connected to the membrane outlets, into a common rigid PVC pipe that exited through the reactor sidewall. This outflow was connected via flexible silicone piping to an adjustable overflow piece. Membrane flux is directly proportional to the pressure head (trans-membrane pressure, TMP) on the membranes, which is the difference between the level inside the reactor and the level of the overflow pipe. The TMP was measured by comparing the level of a standpipe connected to the bottom of the reactor against the level of the outflow. The level of the reactor needed to remain constant and could thus be controlled by adjusting the level of the overflow. This was important so that sudden changes in influent flow due to a blockage or spillage, or gradual changes in TMP could be compensated for while keeping the reactor volume constant at $32 \ell$.

Since the aerobic reactor containing the membranes required constant aeration, the oxygen utilization rate (OUR) could not be measured in this reactor. Accordingly a small cylindrical Perspex reactor $(3 \ell)$ was included in the system configuration in which the OUR could be constantly monitored using an automated technique (Randall et al. 1991). Initially flow for the reactor was taken directly from the aerobic reactor and recycled back to the aerobic reactor. However this setup yielded lower OUR values than expected and was revised with the reactor fed instead from the anoxic reactor and run at the same hydraulic retention time as the aerobic reactor before being fed back into the as-recycle (Section 3.8.1). This change gave OUR values far closer to those expected from simulations. The $3 \ell$ OUR reactor had a solids concentration equal to that of the anoxic reactor and so the measured OUR had to be increased by the ratio of the aerobic to anoxic solids concentrations. As the $3 \ell$ aerobic reactor had a lower solids concentration it is considered to be equivalent to a $2 \ell$ reactor with the aerobic reactor solids concentration, and is hence-forth referred to as such.

The volume of the aerobic reactors was set at $34 \ell(32 \ell+2 \ell)$, yielding a process volume $\left(\mathrm{V}_{\mathrm{p}}\right)$ of $74 \ell$. The system was operated at a sludge age of 20 days (see Table 3.1 ), by maintaining a wasting rate of $2.85 \ell / \mathrm{d}$. Waste sludge was removed once per day at the end of the daily feed cycle, after sampling had been completed, taking into account any mixed liquor removed through sampling, cleaning or lost through spillage. The influent flow rate $\left(Q_{i}\right)$ of $140 \ell / d$ with a target COD concentration $\left(S_{t i}\right)$ of $1000 \mathrm{mgCOD} / \ell$, to give a target mass loading rate of $140000 \mathrm{mgCOD} / \mathrm{d}$. Pumping of influent and recycle flows (Table 3.1) was by means of a simple peristaltic pump with flow rate controlled by an inverter. Recycle flows were set relative to the influent flow by using the appropriate number of pump channels as multiples of the influent number of channels. Reactors were connected by soft silicone tubing ( $\sim 5 \mathrm{~mm}$ to $15 \mathrm{~mm}$ in diameter). Recycle flow rates were frequently checked with stop watch and measuring cylinder because these flows affect the mass fractions in the MBR system. 


\subsubsection{Conventional UCT System Description}

As with the MBR system, all reactors used in the conventional UCT system were constructed of clear Perspex tube $(\sim 5 \mathrm{~mm}$ wall $)$, with approximate dimensions (height $\mathrm{x}$ diameter): anaerobic, $410 \times 190 \mathrm{~mm}$; anoxic, $410 \times 190 \mathrm{~mm}$; aerobic, $470 \mathrm{x}$ $210 \mathrm{~mm}$; and SST $450 \times 60 \mathrm{~mm}$. The tubes were closed by Perspex disks for base plates and lids. The contents of the reactors were completely mixed by means of a motor driven paddle mixer $(\sim 100 \mathrm{rpm})$, mounted centrally on the lid of the reactors. To prevent hydraulic short circuiting each reactor was equipped with a pair of vertical side baffles situated opposite each other and extending about $2 \mathrm{~cm}$ into the bulk solution. The reactors had two paddles fitted to the mixing shaft, one situated at the bottom and one about $120 \mathrm{~mm}$ above it. These were positioned to ensure complete suspension of the reactor contents while avoiding turbulence at the liquid surface to minimize air entrapment into the bulk solution. The lid of each of the reactors had a circular opening ( $\sim 50 \mathrm{~mm}$ diameter) to allow sampling and for the placement of the DO probe in the case of the aerobic reactor. Each reactor had a single inlet and outlet situated about $100 \mathrm{~mm}$ apart on its base plate. The process volume was maintained in each reactor by an overflow tube at the desired overflow level on an adjustable vertical slide attached outside of the wall of the reactor. Aeration in the aerobic reactor was provided by low pressure compressed air entering the reactor through a small borc Pcrspex tube that terminated in a small fish tank bubble diffuser at the bottom of the reactor. The airflow was controlled manually by adjusting a hose clamp on the airline entering the reactor. The dissolved oxygen (DO) in the aerobic reactor was maintained between 2.0 and $5.0 \mathrm{mgO} / \ell$ and was controlled with an YSI Model 5739 DO probe and Hi-Tech Micro-system OUR meter (Randall et al. 1991).

The SST was a Perspex tube inclined at $60^{\circ}$ to the horizontal, fitted with a motor driven $(\sim 0.5 \mathrm{rpm})$ wiper blade rotating a half turn every minute to reduce attached growth on the inside wall and release any nitrogen gas generated from possible denitrification in the settled sludge. Mixed liquor from the aerobic reactor entered the SST at the upper end of the tilted bottom disc to facilitate settling, while clarified effluent overflowed at the top and was collected in an effluent bucket. The s-recycle was drawn from the bottom end of the bottom disc and pumped to the anoxic reactor. Like the MBR system all pumping was by means of a peristaltic pump with the recycles achieved by assigning a proportional number of channels to each recycle relative to the number assigned to feed, only with the conventional UCT system the pump also ran on a timer due to the low flows required. Reactor and SST connections were by means of soft silicone connection piping $(-5 \mathrm{~mm}-15 \mathrm{~mm}$ in diameter).

The total reactor volume $\left(\mathrm{V}_{\mathrm{p}}\right)$ for the conventional UCT system totalled $25 \ell$, and was divided into $5.6 \ell$ anaerobic, $6.2 \ell$ anoxic and $13.2 \ell$ aerobic to give an unaerated mass fraction of about 40.5 percent (ie $12.6 \%$ anaerobic and $27.9 \%$ anoxic). The system was operated at a sludge age of 20 days by maintaining a wasting rate $\left(Q_{w}\right)$ of $1.1 \ell / \mathrm{d}$. Waste sludge was removed once per day from the aerobic reactor at the end of the daily feed cycle taking into account any mixed liquor removed for sampling, cleaning or lost through spillages. The influent COD load to the system was set at a flow rate $\left(\mathrm{Q}_{\mathrm{i}}\right)$ of $15 \ell / \mathrm{d}$ with target $\mathrm{COD}$ concentration $\left(\mathrm{S}_{\mathrm{ti}}\right)$ of $1000 \mathrm{mgCOD} / \ell$, to give a target mass loading rate of $15000 \mathrm{mgCOD} / \mathrm{d}$ (Table 3.1 ). 


\subsubsection{Square Wave Feed Activated Sludge System}

In evaluating BNR it is essential to determine the RBCOD concentration in the influent. The sodium acetate added to the influent (see Section 3.4 below) is all RBCOD, and its contribution to the total RBCOD could be calculated theoretically. However the RBCOD of the sewage component needed to be measured. This was achieved using the short sludge age square wave fed activated sludge system (SQW) method developed by Ekama and Marais (1978) and described by Ekama et al. (1986).

A schematic layout of the SQW system is shown in Fig. 3.3. The SQW system comprised a single aerobic reactor and SST constructed and equipped in the same way as for the conventional UCT system above. The reactor dimensions were approximately (height $\times$ diameter) $400 \times 180 \mathrm{~mm}$, with a total system process volume $\mathrm{V}_{\mathrm{p}}=6.7 \ell$. A short sludge age of $\sim 2$ days was maintained by wasting $3.2 \ell / \mathrm{d}$ from the reactor at the end of each day's feed cycle. (In the original test a sludge age of $2 \frac{1 / 2}{2}$ days was used. This was shortened to 2 days to ensure no interference by nitrification).

Unlike the UCT systems the SQW system was operated in a $12 \mathrm{~h}-\mathrm{ON} / 12 \mathrm{~h}-$ OFF feed pattern, hence the square-wave name, with an influent flow rate $\left(Q_{i}\right)$ of $18 \ell / 0.5 \mathrm{~d}$ $=36 \ell / \mathrm{d}$ over a 12 hour period. The initial target COD concentration was $500 \mathrm{mgCOD} / \ell$ with a target mass loading of $9000 \mathrm{mgCOD} / \mathrm{d}$ at $18000 \mathrm{mgCOD} / \mathrm{d}$ instantaneous loading. However later this was changed to the unaugmented sewage feed of $800 \mathrm{mgCOD} / \ell$, with a mass loading of $\sim 16000 \mathrm{mgCOD} / \mathrm{d}$ at $\sim 32000$ $\mathrm{mgCOD} / \mathrm{d}$ instantaneous loading as this could be taken from the feed to the UCT systems prior to augmenting the feed with sodium acetate. The system was operated with an s-recycle ratio of $1: 1$ with respect to the influent flow, which was terminated when the feed pumping stopped at the end of the 12 hour feed period.

\subsubsection{Operating Procedures and Conditions}

All activated systems were operated in the Water Research Laboratory at UCT with the temperature controlled at $20^{\circ} \mathrm{C}\left( \pm 1{ }^{\circ} \mathrm{C}\right)$.

The $\mathrm{pH}$ in the aerobic reactors of the systems was regularly monitored. Sodium bicarbonate was dosed with the feed to maintain a stable $\mathrm{pH}$ between 7.2 and 7.9.

Operating procedures detailed by Burke et al (1984) and Clayton et al (1989) were followed including inter alia regularly brushing reactor walls and intermittent cleaning of reactors and tubing.

\subsubsection{System Seeding}

In Phase 1 both systems were seeded from SST underflow mixed liquor $(\sim 9000$ mgTSS/l) from the Potsdam WWTP (Milnerton, South Africa), which operates in the UCT configuration for BNR. This mixed liquor was diluted $1: 1$ prior to addition to the conventional UCT system, but allowed to settle further to $\sim 12000 \mathrm{mgTSS} / \ell$ prior to addition to the MBR UCT system. The writer continued to use the sludge that had developed in the reactors during Phase 1 for the Phase 2 investigation. The SQW 
system was started up with wasted sludge from a laboratory ND system because BEPR sludge cannot be used for influent RBCOD measurement.

\subsection{INFLUENT FEED}

\subsubsection{Wastewater Collection and Storage}

The influent wastewater used in the study was raw (unsettled) sewage from the Mitchells Plain Wastewater Treatment Plant (MPWWTP) in Cape Town, South Africa. This wastewater is mainly domestic with a small (less than 10 percent) industrial component. The wastewater was collected in approximately $2 \mathrm{~m}^{3}$ batches from the main outlet channel at the head of the works, just upstream of the influent screw lift pumps and before screening (coarse and fine) and grit removal. The collected wastewater was brought to the laboratory by tanker-truck and, while being agitated with high pressure air, was dispensed by gravity through an in-line macerator into individual $400 \ell$ stainless steel tanks in the laboratory's $4{ }^{\circ} \mathrm{C}$ cold room. The sewage was stored in this way for about 12 to 14 days after which it was discarded and a new sewage batch collected. Experience has shown that the storage of sewage in the cold room tanks longer than three weeks leads to septicity (hydrogen sulphide accumulation) and non-representative changes in sewage characteristics. Immediately after storage in the cold room, a COD test was done on the ncw sewage to determine the necessary dilution for feed preparation for experimental systems operated in the laboratory. Typically, undiluted raw sewage COD from the MPWWTP ranges between 1000 and $1600 \mathrm{mgCOD} / \ell$, though during periods of water restrictions in Cape Town the concentrations closer to $2000 \mathrm{mgCOD} / \ell$ were observed.

\subsubsection{Feed Preparation}

The target total influent COD's for both the conventional and MBR UCT systems were the same at $1000 \mathrm{mgCOD} / \ell$ of which $800 \mathrm{mgCOD} / \ell$ was the raw sewage and $200 \mathrm{mgCOD} / \ell$ was acetate. From the raw wastewater batch the daily feed batches were prepared by diluting the raw sewage with tap water to the targeted influent sewage COD concentration to $800 \mathrm{mgCOD} / \ell$. Since the total daily volumes for the MBR, conventional UCT and SQW systems were $140 \ell, 15 \ell$ and $20 \ell$ respectively, and all three systems were fed the same mixture of sewage to water, prior to dosing, a combined feed of $175 \ell$ was prepared. The raw sewage in stainless steel tanks was thoroughly mixed, then withdrawn via a valve outlet pipe at the bottom of each tank through a $1 \mathrm{~mm}$ stainless steel mesh (to minimize the blockages in the lab systems). The appropriate volume of screened sewage was then poured into a $200 \ell$ plastic drum where the volume was made up with the required amount of tap water. The SQW feed would be removed leaving $155 \ell$. The remaining wastewater, for the MBR and conventional UCT systems was further supplemented with artificial organic (sodium acetate) and inorganic (ammonium chloride and di-hydrogen potassium orthophosphate) compounds (composition developed earlier in BNR research) to increase the COD, TKN and phosphorus to $1000 \mathrm{mgCOD} / 1,100 \mathrm{mg}-\mathrm{N} / \ell$ and $40 \mathrm{mgP} / \ell$. Sodium acetate was selected to augment the sewage organics by $200 \mathrm{mgCOD} / \ell$ in order to enhance the biological $\mathrm{P}$ removal processes (Wentzel et al., 1988), thereby to accentuate potential effects of the membranes on these processes. The additional di-hydrogen potassium orthophosphate was added to the influent to ensure the system was not P-limited, allowing BEPR to be observed. 


\subsection{SAMPLING AND TESTING}

\subsubsection{UCT Activated Sludge Systems}

To evaluate the process performance of the MBR and conventional UCT systems, the following routine samples were taken (shown schematically in Fig. 3.4).

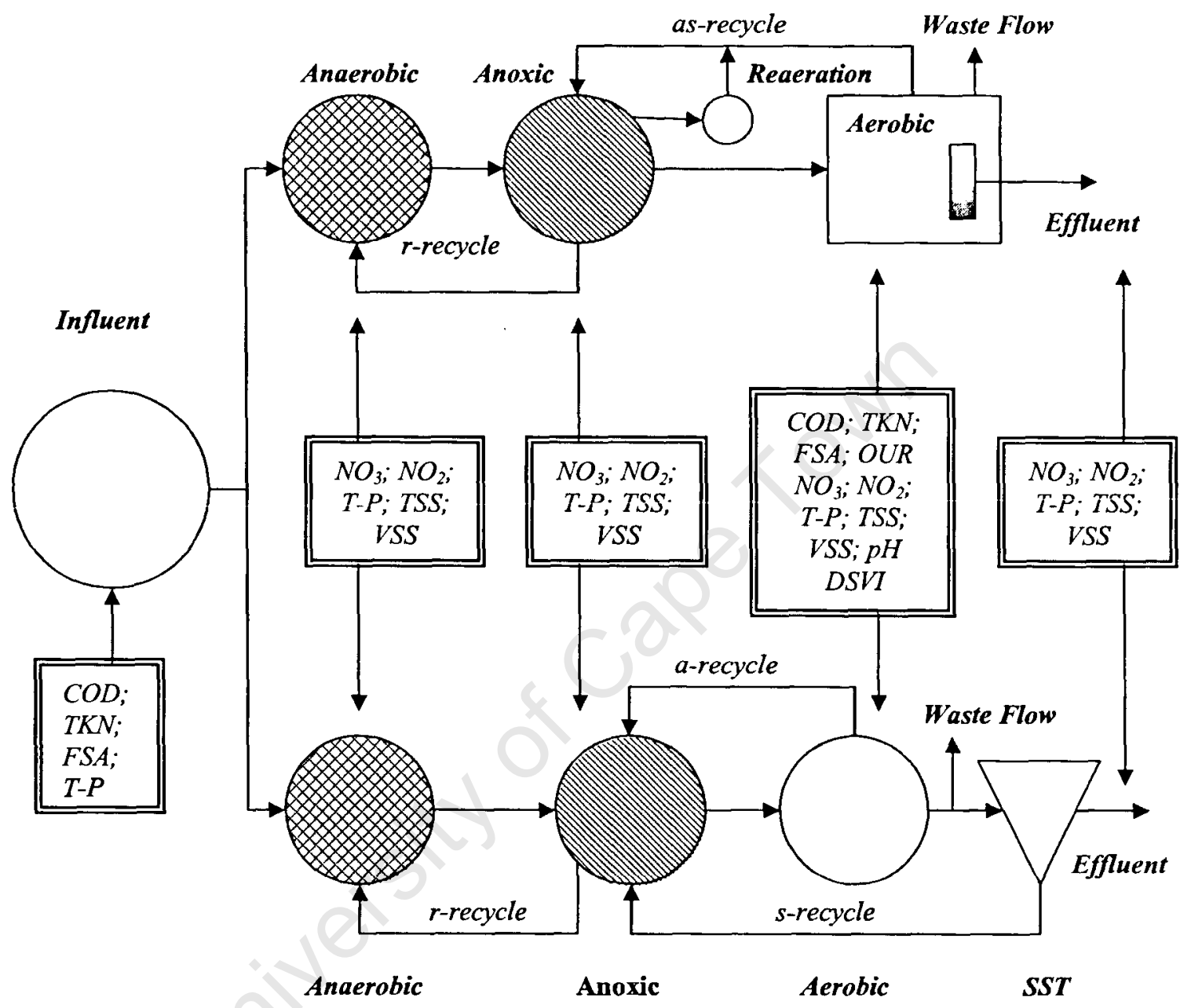

Fig 3.4: $\quad$ Schematic representation of routine sampling for the $M B R$ and conventional UCT systems.

\section{Influent Feed Sample}

Following the feed preparation of the diluted raw wastewater and augmentations, about $200 \mathrm{ml}$ was drawn out before feeding the systems and immediately preserved with one drop of mercuric chloride solution $\left(8.6 \mathrm{~g} / \ell \mathrm{HgCl}_{2}\right)$, and then placed in the $4^{\circ} \mathrm{C}$ cold room for later analysis, see Table 3.2.

\section{Reactor Mixed-Liquor Samples}

Near the end of each day's feed cycle, $100 \mathrm{ml}$ samples were drawn from the anaerobic, anoxic and aerobic reactors respectively of each system, and an additional $50 \mathrm{ml}$ sample was also drawn out of the OUR-aeration reactor of the MBR UCT system. As with the influent samples one drop of mercuric chloride solution $\left(8.6 \mathrm{~g} / \ell \mathrm{HgCl}_{2}\right)$ was 
added to the samples immediately after sampling to prevent any further biological activity. The samples were then placed in $50 \mathrm{ml}$ centrifuge tubes and centrifuged at $3500 \mathrm{rpm}$ or 10 minutes. A drop of polyelectrolyte flocculent $(1 \mathrm{~g} / \ell)$ was also added to the MBR UCT samples to make subsequent filtering easier (the high MLSS concentrations proved difficult to filter at times; tests in Phase 1 showed that polyelectrolyte had negligible effect on subsequent analysis). The centrifuged supernatants were filtered through $0.45 \mu \mathrm{m}$ membrane filters and stored for later total phosphorus, nitrate and nitrite analysis. The centrifuged solids were flushed into measuring crucibles with distilled water and tested for total settleable solids (TSS) and volatile settleable solids (VSS). During the analysis period it was noted that rapid denitrification in the MBR reactor samples compromised the nitrate measurements if samples were allowed to sit for any length of time. Hence samples were taken, poisoned and centrifuged as quickly as possible to prevent the occurrence of denitrification in the samples. The phenomenon of rapid denitrification was attributed to the high solids concentrations in the MBR system and is discussed further in Chapter 4, Section 4.7.

Additional $50 \mathrm{ml}$ and $25 \mathrm{ml}$ samples were drawn from the aerobic compartments of the conventional and MBR UCT systems respectively, then placed in $500 \mathrm{ml}$ and $1000 \mathrm{ml}$ volumetric flasks respectively, and then made up to volume with distilled water, to give respective dilutions 10 times (50 in $500 \mathrm{ml})$ and 40 times $(25 \mathrm{in} 1000 \mathrm{ml})$ for the conventional and MBR systems respectively. The diluted mixture was thoroughly mixed manually and then analysed for unfiltered COD and TKN (see Table 3.2).

Additionally $50 \mathrm{ml}$ and $300 \mathrm{ml}$ samples were drawn from the respective aerobic compartments of the MBR and conventional UCT systems, and diluted with secondary effluent from the respective systems in separate $1 \ell$ graduated cylinders to measure the diluted sludge volume index (DSVI, Ekama and Marais 1984b): The contents of the cylinder were shaken up and allowed to settle for 30 minutes, after which the settled volume was recorded. This value $\left(\mathrm{DSV}_{30}\right)$ was divided by the TSS concentration of the mixed liquor in the measuring cylinder to give the system DSVI in $\mathrm{ml} / \mathrm{gTSS}$; i.e. an estimation of the volume occupied by 1 gTSS of sludge in a SST (See Table 3.2).

\section{Effluent Samples}

At the end of each days feed cycle, a sample of the secondary effluent was drawn from the effluent bucket of each system. Conventional UCT samples were filtered through a $0.45 \mu \mathrm{m}$ membrane filter. There was no need to filter effluent samples from the MBR as the membrane pore sizes were smaller than those of the $0.45 \mu \mathrm{m}$ filter paper. These samples were filtered for COD, TKN, FSA, $\mathrm{NO}_{2}, \mathrm{NO}_{3}$, and TP (see Table 3.2). Effluent unfiltered samples for both systems were taken from the supernatant of the DSVI test described above. These samples were analysed for COD and TKN (See Table 3.2).

\section{Microbiological and Batch Test Samples}

Periodic sampling was performed on both the conventional and MBR systems for microbial analysis. All sampling was taken into consideration in calculating the daily sludge wasted and samples were prepared appropriately for their respective tests. 
Once monthly and fortnightly from March 2006 samples were analysed by a microbiologist for filament identification and floc morphology. Additionally from August 2006 samples were sent fortnightly to the Durban Institute of Technology for FISH analysis. Results are presented in Maharaj et al. (2006). All samples for microbial analyses were prepared appropriately with autoclaved equipment.

\section{In-situ Measurements}

The oxygen utilization rates (OUR) of the MBR and conventional UCT, and the SQW systems were measured continually and automatically using the technique detailed by Randall et al. (1991): A dissolved oxygen (DO) probe (YSI Model 5739) was placed in the aerobic activated sludge mixed liquor and connected to an automated DO meter/OUR data logger (HiTech Microsystems), which controlled reactor aeration between high and low DO set points via a solenoid valve on the air line. When the DO concentration in the mixed liquor reached the low set point $(\leq 2 \mathrm{mgO} / 1)$, the solenoid valve was opened and the reactor contents aerated until the DO concentration reached the high set point $(\geq 5 \mathrm{mgO} / \mathrm{l})$ and the air was switched off automatically. The decrease in DO with time was monitored until the DO reached the low setpoint again, when aeration recommenced and the cycle repeated. During each air off period in the cycle the slope of the DO-time data was automatically calculated by linear regression to give the OUR at that time, which together with the correlation coefficient, temperature and time, was stored by the meter. The OUR results for each day's feed cycle were downloaded from the DO meter to the PC the following day. The data was imported into a spreadsheet program where it was plotted and the average OUR for the day calculated. The DO meter and probe were routinely calibrated.

Daily the $\mathrm{pH}$ in the anoxic and aerobic reactor was measured by means of a $\mathrm{pH}$ probe connected to a $\mathrm{pH}$ meter.

In the MBR UCT system, the hydraulic head required to achieve the specific flux was measured daily, as the difference between the levels of the reactor contents (monitored by the standpipe) and the effluent (permeate) overflow weir, see Section 3.3.4.

\section{Summary}

Table 3.2 summarizes analyses routinely performed on the various system samples. Although all analyses refer to Standard Methods (1985), some have been adapted to suit the requirements in the UCT Wastewater Research Laboratory (WRL). 
Table 3.2: $\quad$ Sampling position and parameter measurement

\begin{tabular}{|c|c|c|c|c|c|c|c|c|c|c|c|}
\hline TEST & $\mathrm{COD}$ & $\mathrm{TKN}$ & $\mathrm{FSA}$ & $\mathrm{NO}_{3}$ & $\mathrm{NO}_{2}$ & $\mathrm{~T}-\mathrm{P}$ & $\mathrm{TSS}$ & $\mathrm{VSS}$ & OUR & DSVI \\
1 & 2 & 3 & 4 & 5 & 6 & 7 & 8 & 9 & 10 & 11 \\
\hline Influent & $\mathrm{U}$ & $\mathrm{F} ; \mathrm{U}$ & $\mathrm{F}$ & & & $\mathrm{U}$ & & & & & \\
\hline Anaerobic & & & & $\mathrm{F}$ & $\mathrm{F}$ & $\mathrm{F}$ & $\mathrm{U}$ & $\mathrm{U}$ & & & \\
\hline Anoxic & & & & $\mathrm{F}$ & $\mathrm{F}$ & $\mathrm{F}$ & $\mathrm{U}$ & $\mathrm{U}$ & & & \\
\hline Aerobic & $\mathrm{U}$ & $\mathrm{U}$ & & $\mathrm{F}$ & $\mathrm{F}$ & $\mathrm{F}$ & $\mathrm{U}$ & $\mathrm{U}$ & $*$ & $*$ & $*$ \\
\hline $\begin{array}{c}\text { Final } \\
\text { Effluent }\end{array}$ & $\mathrm{F} ; \mathrm{U}$ & $\mathrm{F} ; \mathrm{U}$ & $\mathrm{F}$ & $\mathrm{F}$ & $\mathrm{F}$ & $\mathrm{F}$ & & & & & \\
\hline
\end{tabular}

$\mathrm{F} \quad=$ Filtered through Schleicher \& Schull ME 25/21 $0.45 \mu \mathrm{m}$ membrane filters.

$\mathrm{U} \quad=$ Unfiltered samples

* $\quad=$ Direct measurement taken (filtering not applicable)

The numbers on the test methods below refer to Standard Methods (1985), though some have been adapted to suit the requirements of the UCT wastewater research laboratory (WRL).

1. COD Chemical Oxygen Demand, open reflux method; 5220 (B)

2. TKN Total Kjeldahl Nitrogen, micro-kjeldahl method; 4500 - Norg (C)

3. FSA Free and Saline ammonia, titrimetric method; $4500-\mathrm{NH}_{3}(\mathrm{~B})$, (E)

4. $\mathrm{NO}_{3} \quad$ Hydrazine reduction (Technicon Auto-Analyzer); $4500-\mathrm{NO}_{3}(\mathrm{H})$

5. $\mathrm{NO}_{2} \quad$ Hydrazine reduction (Technicon Auto-Analyzer); $4500-\mathrm{NO}_{2}(\mathrm{H})$

6. T-P Total Phosphorus; Sulphuric acid/Persulphate digestion at $100^{\circ} \mathrm{C}$ followed by molybdate-vanadate colour development for orthophosphate (Standard Methods, 1985 - Method 424C III)

7. TSS Total suspended solids dried at $103-105^{\circ} \mathrm{C} ; 2540$ (D)

8. VSS Volatile suspended solids ignited at $600{ }^{\circ} \mathrm{C} ; 2450$ (E)

9. DSVI Dilute Sludge Volume Index; (Ekama and Marais, 1984b), 271 (D)

10. OUR Oxygen Utilization Rate; automated (Randall, et al., 1991), 271 (B)

11. $\mathrm{pH}$ pH meter, Hanna Instruments model HI9023; $4500-\mathrm{H}^{+}$(B)

\subsubsection{Square Wave Feed Activated Sludge System}

The unfiltered influent sample for the SQW system was analysed for total COD, as described above. Also, OUR was monitored continually in-situ and reactor $\mathrm{pH}$ measured regularly, as described above.

\subsection{INITIAL TESTING}

\subsubsection{Initial Flux Testing}

In April 2005 new membranes were acquired for the investigation and the potential flux of the new membranes was evaluated to test whether the membranes could achieve the flux required for the MBR setup, and whether the physical design of the aerobic membrane bioreactor could accommodate the required head (TMP). Both the "old" and the "new" membranes were tested to allow a comparison of their performance.

The tests required timing the effluent flow from the aerobic reactor. The effluent would flow directly into an Imhoff cone and the TMP at the start and end of the test was measured with the mean TMP calculated for the test. Thus the flux through the 
membranes for a set TMP could be calculated and a relationship between flux and TMP developed. The tests were run using both distilled water and activated sludge mixed liquor.

\section{Continued Flux testing}

It was expected that over time the TMP of the membranes would increase, initially due to the establishment of a dynamic gel layer and over time due to progressive fouling. In order to track changes in the TMP over time the TMP was measured daily. Initially this was simply done by measuring the difference between the level in the reactor and the level of the effluent overflow weir. However, over time the results varied significantly due to fluctuations in the flow through the aerobic reactor. These fluctuations were due to a number of factors - primarily the pump speed which would vary throughout the day within its set range due to the liquid level of the feed tanks, and minor blockages in the feed lines. Additionally blockages, not obvious to the operator in the recycle piping would cause a build up of mixed liquor in one reactor and upon clearing surgeto the next reactor. In order to compensate for these fluctuations flux tests were adjusted whereby the TMP would be taken at the beginning and the end of the flux test and averaged. The TMP would then be adjusted by the quotient of the average daily flux divided by the measured flux. This method gave more consistent results, with the result that TMP could be monitored carefully in times of dynamic change - for instance during and after power failures when aeration had been off causing fouling to occur.

\subsubsection{Oxygen Transfer Rates}

To prevent fouling, scour across the membrane surface was provided by coarse bubble aeration installed directly below the membrane assembly. This aeration unit also had to provide sufficient oxygen to satisfy the biological oxygen demand (carbonaceous and nitrification), estimated at $\sim 135 \mathrm{mgO} / \ell / \mathrm{hr}$. Accordingly the oxygen transfer rate of the aeration assembly installed in the MBR UCT system aerobic reactor was experimentally determined in Phase 1 of the project using the unsteady state aeration of deoxygenated water. The results showed that the aeration supplied to the system was adequate and this was confirmed in Phase 2 by a DO concentration in the aerobic reactor consistently between $2-5 \mathrm{mgO} / \ell$.

\subsubsection{Residence Time Distribution}

To determine that the aerobic reactor containing the membranes was completely mixed in the MBR UCT system, despite the presence of the riser and descender zones, a residence-time distribution (RTD) test was carried out in Phase 1 by salt addition and conductivity measurements. The rate of appearance of conductivity in the effluent following a $\mathrm{NaCl}$ dose to the influent gives an indication of how well the reactor is mixed. The results of the tests in Phase 1 one indicated excellent mixing in the aerobic reactor (Ramphao et al., 2004). 


\subsection{OXYGEN TRANSFER TESTING}

\subsubsection{Oxygen Transfer Theory}

Aeration is one of the most important considerations in designing wastewater treatment plants and together with sludge handling is one of the primary costs in running a plant. Hence having a good understanding of oxygen transfer and providing an efficient aeration system will not only ensure that the plant works effectively but will reduce operation costs.

There are a number of factors which influence Oxygen Transfer Efficiency (OTE), which is the measure of how much oxygen introduced to the system is in fact transferred to the solution (Equation 3.1). These factors include temperature, pressure, geometry of the reactor and sludge concentration. The ability of the MBR to concentrate sludge allowed a study of the effect of concentration on the rate of oxygen transfer (OTR) and OTE to be conducted.

Typically in aeration tests concentration is taken into account through the Alpha $(\alpha)$ value. Alpha is derived as the quotient of the mass transfer coefficient $\mathrm{K}_{\mathrm{LA}}$ ' through the sludge divided by the $\mathrm{K}_{\mathrm{LA}}$ though tap water, Equation (3.1). Values vary considerably but are generally inversely proportional to the sludge concentration.

$$
\alpha=\frac{K_{L} a^{\prime}}{K_{L} a}
$$

In order to calculate Alpha two sets of tests need to be carried out. The first test is an Unsteady State (USS) test which would give the relationship between $\mathrm{K}_{\mathrm{LA}}$ (water) and the air flow rate as measured by a calibrated rotameter. The second test is a Steady State (SS) test conducted on the system while in operation. This would give $\mathrm{K}_{\mathrm{LA}}$ ' (mixed liquor) values in relation to a set airflow rate and a MLSS concentration. Thus Alpha values could be determined for a range of MLSS values by interpolating the $\mathrm{K}_{\mathrm{LA}}$ vs airflow relationship so that the $\mathrm{K}_{\mathrm{LA}}$ and $\mathrm{K}_{\mathrm{LA}}$ ' could be found for a particular MLSS value. The basis of both of these tests is Equation (3.2).

$$
\frac{d C}{d l}=K_{L} a\left(C s-C_{L}\right)-O U R
$$

Where:

$$
\begin{array}{ll}
\frac{d C}{d t} & =\text { change in DO concentration with time } \\
C s & =\text { saturated concentration of DO under site conditions }(\mathrm{mgO} / \mathrm{l}) \\
C_{L} & =\text { DO concentration in the reactor } \\
O U R & =\text { oxygen utilization rate in the reactor }
\end{array}
$$

With the $\mathrm{K}_{\mathrm{LA}}$ of the solution known the OTR and subsequently the OTE of the system can be calculated using Equations (3.3) - (3.5).

$$
\begin{aligned}
& \text { OTR }=K_{L} a \cdot C S . V \\
& O T E=O T R / O S R \cdot 100
\end{aligned}
$$


Where:

$$
\begin{aligned}
\mathrm{V} & =\text { Volume of the system aeration reactor } \\
\text { OSR } & =\text { Oxygen supply rate } \\
& =\text { Cor.Qair } \\
C_{O} & =\text { Oxygen content of air }\left(\mathrm{kgO} / \mathrm{m}^{3}\right) \\
\mathrm{Q}_{\text {air }} & =\text { Air flow rate }\left(\mathrm{m}^{3} / \mathrm{h}\right)
\end{aligned}
$$

\subsubsection{Unsteady State (USS) Test}

This test was conducted in the aerobic reactor which was drained of activated sludge, dismantled and thoroughly cleaned to remove all impurities which may impact the oxygen transfer in the reactor. Once clean the reactor was reassembled with "dummy" membranes in order to protect the system membranes from potentially aggressive salts used in the USS testing. The reactor was filled with distilled water to the operational volume of $32 \ell$. The system is then aerated for an hour in order to saturate the water completely with oxygen. A DO probe, calibrated the same morning was suspended upside down in the aerobic reactor to prevent bubbles collecting on the probes' membrane which would affect the DO readings. The expected saturated DO concentration $\left(\mathrm{C}_{\mathrm{S}}\right)$ from Equation (3.6) was checked with the saturated DO value to confirm correct calibration of the probe.

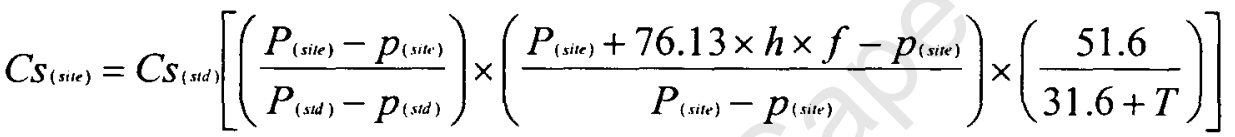

$$
\begin{aligned}
& P_{(s d)} \quad=\text { Pressure at } 1 \mathrm{~atm}(760 \mathrm{mmHg}) \\
& p_{\text {(sit) }}=\text { Saturated vapour pressure at site }(\mathrm{mmHg}) \\
& p_{(\text {sd })}=\text { Saturated vapour pressure at standard temperature }(\mathrm{mmHg}) \\
& 76.13 \times h=\text { pressure of } h \mathrm{~m} \text { of water }(\mathrm{mmHg}) \\
& f \quad=\text { fraction of submerged depth (from suface) at which pressure } \\
& \text { corresponds to the average concentration. Accepted 0.325. } \\
& T=\text { Temperature in }{ }^{\circ} \mathrm{C} \text {. }
\end{aligned}
$$

The temperature of the water in the reactor was checked before and after each iteration of the USS test, in order to take its influence into account. As the test was run in the Water Research Laboratory, in Cape Town at sea level the site pressure was assumed to be equal to the standard pressure. No correction was applied to $\mathrm{C}_{\mathrm{S}}$ to account for the effect of concentration of dissolved salts in the mixed liquor (Baker et al., 1975).

Each iteration of the test proceeded as follows: The air flow rotameter would be set at a value and the system aerated to saturation. A solution of Sodium Sulphate $( \pm 150$ $\left.\mathrm{mgNa}_{2} \mathrm{SO}_{3} / \ell\right)$ with Cobalt Chloride $\left(0.05 \mathrm{mgCo}^{2+} / \ell\right)$ as a catalyst was added to the aeration reactor in order to deoxygenate the reactor completely. Once all the sulphate has been utilised the DO will be retained in the water again. The DO was monitored with time as it increased from $0 \mathrm{mgO} / \ell$ to saturation. This process is repeated for a number of airflow rates. 
From Equation (3.2), and assuming that due to the lack of any impurities in the water the OUR is 0 , the $\mathrm{K}_{\mathrm{LA}}$ of a airflow rate is calculated by plotting the DO deficit, $\mathrm{C}_{\mathrm{S}}$ $C_{L}$, on a log scale versus time, the slope of which is $K_{L A}$ (Baker et al., 1975). Similarly $K_{L A}$ was calculated in this study from the plot of $\log \left(C_{S}-C_{L}\right)$ as in Equation (3.7). $\mathrm{K}_{\mathrm{LA}}$ is considered to increase with an increase in temperature due to lower viscosity and increased diffusivity (Baker et al., 1975). In order to adjust $\mathrm{K}_{\mathrm{LA}}(\mathrm{T})$ to standard conditions Equation (3.5) was applied

$$
\begin{aligned}
& K_{L a_{(T)}}=\left[\text { gradient } \log \left(C s-C_{L}\right) / 2.303\right] \\
& K_{L} a_{(20)}=K_{L} a_{(20)} / \theta^{(\mathrm{T}-20)}
\end{aligned}
$$

Where:

$$
\theta \quad=1.024
$$

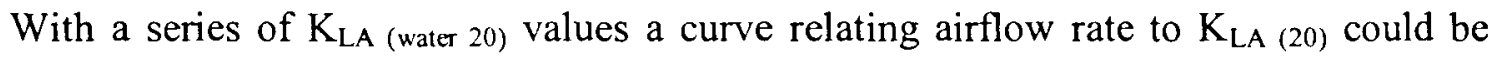
plotted.

\subsubsection{Steady State Testing}

In wastewater systems the DO concentrations in the activatcd mixed liquor is continually being depleted by microbial metabolism (Baker et al., 1975). Hence under steady state conditions an equilibrium is reached between the oxygen supplied and the oxygen used in the mixed liquor and the DO concentration in the reactor remains generally unchanged. Thus Equation (3.2) can be modified to Equation (3.9) and the $\mathrm{K}_{\mathrm{LA}}$ '(T) of the activated sludge can be calculated by measuring the $\mathrm{DO}$ concentration in the reactor and the OUR of the activated sludge. $\mathrm{K}_{\mathrm{LA}}{ }^{\prime}{ }_{(20)}$ is calculated using equation (3.8).

$$
K_{L} a_{(T)}=\frac{O U R}{\left(\beta \times C_{S}-C_{L}\right)}
$$

Where:

$$
\beta=\text { effect of impurities on } \mathrm{Cs}
$$

In order to run the test at constant OUR conditions the sludge was aerated without feed for 24 hours prior to testing to achieve endogenous conditions. This also served to concentrate the activated sludge. At the start of a test a portion of the activated sludge (2-3 $\ell)$ was removed for OUR testing in a separated batch reactor leaving the operating volume of approximately $32 \ell$ in the aerobic reactor. A number of average DO concentrations were measured at different air flow rates. MLSS samples were taken from both the aerobic reactor and the OUR batch reactor to determine the MLSS concentration during testing. Temperature was measured throughout. Once sufficient readings had been taken, all sludge was returned to the aerobic reactor and diluted. The excess activated sludge would be stored and the testing repeated at the new MLSS concentration.

Additionally mixed liquor samples at various concentrations were exported for analysis by the Flow Process Research Centre (FPRC) at the Cape Peninsula University of Technology for rheological analysis to determine the viscosity of the mixed liquor. 
Previous studies had indicated that there is a liner relationship between viscosity and Alpha values.

\subsubsection{Beta Testing $(\beta)$}

Beta represents the effect of impurities in the sludge on $\mathrm{C}_{\mathrm{S}}$ and is calculated using Equation (3.10). In order to assess the effect of impurities in the sludge $\beta$ was calculated by poisoning a $(3 \ell)$ sample of activated sludge with $100 \mathrm{ml}$ Mercuric Chloride $(\mathrm{HgCl})$ and measuring the saturated DO concentration achieved in the mixed liquor.

$$
\beta=C s_{(\text {sisuder })} / C s_{\text {(woter) }}
$$

\subsection{SYSTEM AND OPERATIONAL MODIFICATIONS}

\subsubsection{MBR System Operation}

The main system operational problems experienced in the MBR system in Phase 2 are summarised in Table 3.3.

Table 3.3: $\quad$ Membrane system operational problems and actions taken.

\begin{tabular}{|c|c|}
\hline $\begin{array}{l}\text { Sewage } \\
\text { Batch \# }\end{array}$ & Event (day) action taken; \\
\hline 2 & Foaming (26) brushed down foaming; Spills $(31,35) M^{\prime}$ filtered and returned \\
\hline 3 & \\
\hline 4 & $\begin{array}{l}\text { Foaming (throughout) Foam wasted (56); Spills }(66-69 ; 74) \mathrm{ML}^{1} \text { filtered and returned - } \\
\text { reduced wasting on subsequent days to recover lost } \mathrm{ML}\end{array}$ \\
\hline 5 & $\begin{array}{l}\text { anaerobic motor seized (92) motor repaired; small spill (94) } \mathrm{ML}^{1} \text { lost incorporated in } \\
\text { waste sludge }\end{array}$ \\
\hline 6 & Backlogs in Feed $(100 ; 102)$ \\
\hline 7 & Excessive foaming (111) in anoxic reactor - installation of wire foam mixer \\
\hline 8 & New OUR setup installed (d 133) \\
\hline 9 & Minor spill (140) $\mathrm{ML}^{\prime}$ lost incorporated in waste sludge \\
\hline 10 & Minor Spills $(149,153,162) \mathrm{ML}^{\prime}$ lost incorporated in waste sludge \\
\hline 11 & Power failure (176) washed membranes; \\
\hline 12 & $\begin{array}{l}\text { Pipes ruptured on feed pump - Spills }(191,198,200,203) \text { Changed feed piping } \\
\text { regularly }\end{array}$ \\
\hline 13 & 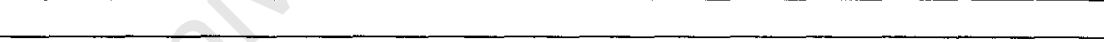 \\
\hline 14 & $\begin{array}{l}\text { Cold room off (d 219) sewage batch monitored for deterioration of waste water; Spill } \\
(224)\end{array}$ \\
\hline 15 & Spills $(237,241) M L^{1}$ filtered and returned \\
\hline 16 & Spill (254) ML' filtered and returned \\
\hline 17 & System feeding irregularly $(d 260)$ feed pump serviced and piping checked \\
\hline 18 & $\begin{array}{l}\text { Spill ( } 275) \mathrm{ML}^{1} \text { filtered and returned; Anoxic reactor outflow blocked draining aerobic } \\
\text { reactor exposing membranes }(271) \text { washed membranes; } \\
\text { re-aeration reactor mixer broken (274) repaired }\end{array}$ \\
\hline 19 & Spills $(294,298,305) \mathrm{ML}^{\top}$ filtered and returned \\
\hline 20 & Writer on leave \\
\hline 21 & Writer on leave \\
\hline 22 & Spills $(344-346 ; 348) \mathrm{ML}^{1}$ filtered and returned \\
\hline 23 & Spills $(359-360 ; 365) \mathrm{ML}^{1}$ filtered and returned; a-recycle blocked $(366)$ recycle cleared \\
\hline $24-$ & $\begin{array}{l}\text { Power failures }(374,377-8 ; 383) \text { washed membranes; } \\
\text { anaerobic and anoxic motors seized }(374) \text { replaced motors }\end{array}$ \\
\hline 25 & Power failure/Spill (386) washed membranes, ML filtered and returned \\
\hline 26 & Spills $(394,405)$ ML'filtered and returned \\
\hline 27 & Spills $(409,411) \mathrm{ML}^{9}$ filtered and returned \\
\hline 28 & Rapid deterioration in membrane permeability (from 407) Ran chemical clean (431) \\
\hline 29 & \\
\hline
\end{tabular}

' Mixed Liquor (ML) 


\section{Foaming}

Excessive foaming by $\mathrm{N}_{2}$ gas generation in the anoxic reactor was noticed early in the investigation (from day 26). Initially foam was brushed down into the mixed liquor on a daily basis, however this proved ineffective resulting in the development of a substantial foam layer which was physically removed from the surface of the anoxic reactor on day 56. The foam constituted a significant mass of sludge and its removal was accommodated as best as possible into the mass of sludge wasted. However a foam layer developed again shortly thereafter and floating balls were inserted into the anoxic reactor to prevent the development of foam with little success. Finally the foaming was remedied by inserting a wire stirrer slightly above the surface $(10 \mathrm{~mm})$ of the anoxic reactor (day 111). The stirrer disturbed the development of any further foam layer by incorporating foam back into the mixed liquor solution before it could consolidate. In order to prevent the aeration of the anoxic mixed liquor by the stirrer the anoxic reactor was lightly sealed by capping the sampling port, such that nitrogen gas could escape, but oxygen would not enter the reactor headspace. The reasons proposed for excessive foaming in the MBR anoxic reactor are discussed in Chapter 4, Section 4.6.2.

\section{OUR Measurement}

Due to the requirement of the membranes for constant aeration for cross-flow velocity scour, OUR in the aerobic reactor could not be measured by the method developed by Randall et al. (1991). Instead in Phase 1 a separate $3 \ell$ aerobic reactor (OUR-aeration reactor) was employed to measure OUR (Fig. 3.4). However in Phase 2 the OUR readings from this setup were found to be very low and attributed to the longer aerobic retention imposed on the re-aeration mixed liquor due to it being recycled from and returned to the aerobic reactor. Instead it was proposed to run the re-aeration reactor in a parallel configuration to the aerobic reactor, fed from the anoxic reactor and recycling back to the as-recycle with the same aerobic hydraulic residence time as in the aerobic reactor, Fig. 3.5 .

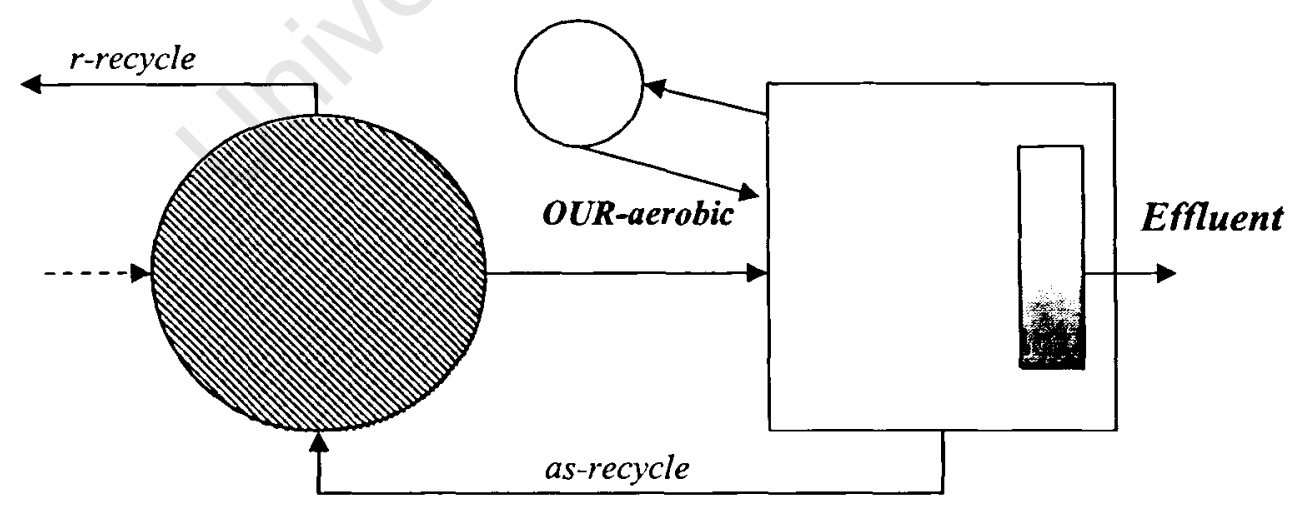

Anoxic

Aerobic

Figure 3.4: The original OUR setup, with re-aeration recycling mixed liquor directly from the aerobic reactor. 


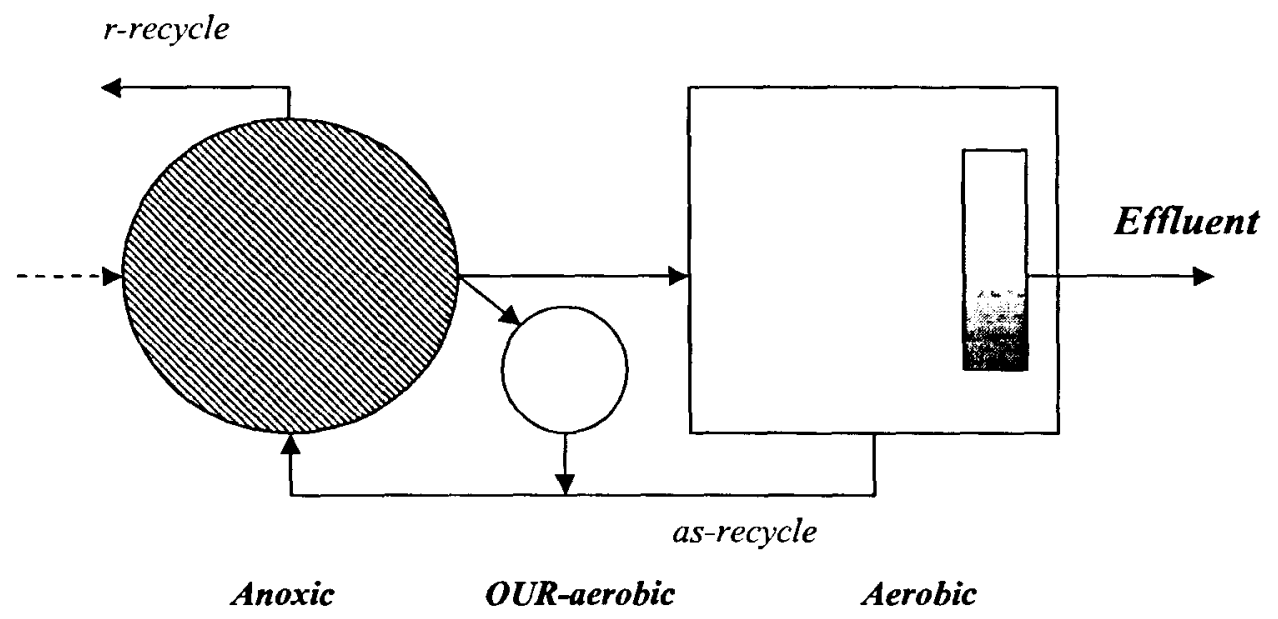

Figure 3.5: The modified OUR setup implemented on day 133 whereby the reaeration reactor ran in parallel to the anoxic reactor drawing mixed liquor from the anoxic reactor and feeding mixed liquor directly back into the as-recycle.

Due to the absence of the membrane concentration effect in the OUR-aeration reactor the mixed liquor solids concentration was effectively that of the anoxic reactor and needed to be adjusted to represent the OUR in the aerobic reactor. The OUR was simply increased proportionally with the MLSS concentration difference of the two reactors as in Equation 3.11. which gave OUR readings close to those calculated for the UCT system using UCTPHO.

$$
O U R_{a e r}=O U R_{r e} a e r \cdot \frac{M L S S_{a e r}}{M L S S_{r e} \text { aer }}
$$

\section{Power Failures}

On day 176 and from days 374 to 386 the Water Research Laboratory experienced several electrical power failures due to city wide power outages. The power failures resulted in membrane fouling, and backlogs in the feed volume due to no aeration, no reactor mixing and no feeding or recycling flows between reactors. Once power had been restored the TMP of the membranes would be checked, as in Section 3.6. If the TMP of the system had increased, indicating fouling on the membranes, the membranes would be removed and washed down with water only to remove mixed liquor caking on their surface. Additionally the sludge from the aerobic reactor would be drained and filtered through a $2 \mathrm{~mm}$ mesh screen to prevent potential pipe blockages from mixed liquor "clots". When a backlog in the feed had occurred the feed pump would be sped up appropriately and testing would only resume once a normal steady state flow was re-established. On day 374 power failures caused the anaerobic and anoxic reactor mixers to seize resulting in them having to be replaced with similar motors. 


\section{Mixed Liquor Blockages and Spills}

Regular blockages occurred in the system due to the high concentration of the MBR mixed liquor. Blockages would typically occur in the piping connecting reactors. These could be reduced by massaging the piping on a daily basis to remove any minor blockages and discourage wall growths. However sporadic major blockages resulting in spills from the system reactors occurred. Mixed liquor lost through spillages could usually be recovered from drip trays inserted underneath the MBR system and was filtered through a $2 \mathrm{~mm}$ mesh to ensure removal of large foreign particles that could damage the membranes and large mixed liquor clots that could block piping before returning mixed liquor to the system.

Silicon piping, used for the peristaltic pump feeding the MBR system and the recycles, was replaced on a weekly basis. However from sewage batches 12 to 19 the silicon piping would occasionally rupture prematurely. Buckets were placed under the pump in order to catch any spillages resulting from ruptured piping and the mixed liquor returned directly to the MBR system.

\section{Reactor Motor Failures}

Due to the increased MLSS concentration and continuous operation the mixing motors overheated and seized on days 92,174 and 274 requiring them to be replaced. In these cases if possible the reactors would be mixed manually using a brush periodically until the motor was repaired.

\subsubsection{Conventional UCT Operation}

The conventional UCT system remained essentially unmodified throughout the investigation. Occasional failures of the SST resulted in sludge overflows to the effluent collector bucket in which mixed liquor would settle and could be returned to the system. In the event that sludge was lost, either through spillages, or while cleaning the system, the lost sludge would be estimated and incorporated into the sludge wasted on that day, and if necessary on subsequent days.

\subsubsection{Operation of MBR system at a longer sludge age (40d)}

Following the parallel investigation the MBR UCT system was run by Mahimba et al. (2006) at a longer sludge age of 40 days in order to properly investigate the phenomenon of increased sludge production and high $f_{\mathrm{S} \text {, up }}$ in the system. In order to facilitate this transition certain design and operating parameters were changed, namely the feed volume was decreased from 140 to $80 \ell / d$ and the volume wasted was similarly decreased to $1.45 \ell / \mathrm{d}$ from $2.85 \ell$. The operation changes were determined in order to achieve an aerobic MLSS concentration of $18000 \mathrm{mgTSS} / \ell$, the same as in Phase 1 and 2 of the investigation. The system was run for two full sludge ages prior to the commencement of testing in order for the sludge to reach a steady state condition. No other significant changes were made to the system.

All the testing procedures from Phase 2 were retained in order to monitor the BNR performance of the 40 day slid 


\subsection{CLOSURE}

In this chapter the experimental methodology for the SQW, MBR UCT and conventional UCT systems run in this investigation is set out. The results from the experimental Phase 2 of the investigation are presented in Chapters 4 (SQW and MBR UCT system) and 5 (conventional UCT system). 


\section{CHAPTER 4}

\section{SQW and MBR SYSTEMS EXPERIMENTAL RESULTS AND ANALYSIS}

\subsection{INTRODUCTION}

In Chapter 3, the methodology of the project to investigate the impact of membranes for solid liquid separation on BNR behaviour and performance was described. This methodology was adopted and modified where necessary from Phase 1 of the project reported in Ramphao et al. (2004) in order to confirm and verify the results observed in Phase 1 and to expand on these. This required that operation of the three laboratory scale systems from Phase 1 be continued:

1. A square wave (SQW) activated sludge system in order to determine the RBCOD component of the influent waste water. This is an important parameter for evaluation of the BNR performance of activated sludge systems.

2. A MBR UCT system. This system, run in a UCT configuration, used membranes in the aerobic reactor for solid liquid separation. It allowed the BNR performance of a MBR system to be observed and studied.

3. A conventional UCT system with a SST. This system, also in a UCT configuration, acted as a control against which to compare the performance of the MBR system.

In this chapter, the experimental results from the SQW and MBR systems will be presented and analysed in detail.

\subsection{SYSTEM CONDITIONS AND STEADY STATE PERIODS}

\subsubsection{System Operating Conditions}

All three systems listed above were run continuously in the controlled conditions of the water research laboratory (WRL), which was kept at approximately $20{ }^{\circ} \mathrm{C}( \pm 1$ ${ }^{\circ} \mathrm{C}$ ). The two UCT systems were run continuously with close monitoring and control of the engineering and operational parameters. At least every two days samples were drawn from the systems and analysed in order to quantify and elucidate the BNR performance of the systems, see Chapter 3.

As described in Chapter 3 wastewater was collected in batches from the Mitchells Plain WasteWater Treatment Plant (MPWWTP, Cape Town, South Africa) and stored in an in-house cold room $\left(4^{\circ} \mathrm{C}\right)$. Each sewage batch served as influent feed for up to a maximum of three weeks, before being discarded and replaced with a new batch of sewage. The sewage batches were accepted to represent steady state periods, and thus the system results are divided into sewage batch periods, see Table 4.1. The investigation extended over 449 days, which included a total of 29 sewage batch periods. 
Table 4.1: $\quad$ Sewage batch periods and dates of operation of the three activated sludge systems.

\begin{tabular}{|c|c|c|c|c|}
\hline \multirow{2}{*}{$\begin{array}{l}\text { Sewage } \\
\text { Batch No }\end{array}$} & \multicolumn{2}{|c|}{ Date } & \multicolumn{2}{|c|}{ Day number } \\
\hline & From & To & From & To \\
\hline 2 & 5-Mar-05 & 18-Mar-05 & 21 & 36 \\
\hline 3 & 19-Mar-05 & 4-Apr-05 & 37 & 53 \\
\hline 4 & 5-Apr-05 & 26-Apr-05 & 54 & 75 \\
\hline 5 & 27-Apr-05 & 15-May-05 & 76 & 94 \\
\hline 6 & 16-May-05 & 31-May-05 & 95 & 110 \\
\hline 7 & 1-Jun-05 & 14-Jun-05 & 111 & 124 \\
\hline 8 & 15-Jun-05 & 23-Jun-05 & 125 & 132 \\
\hline 9 & 24-Jun-05 & 7-Jul-05 & 133 & 147 \\
\hline 10 & 8-Jul-05 & $27-J u l-05$ & 148 & 167 \\
\hline 11 & 28-Jul-05 & 17-Aug-05 & 168 & 188 \\
\hline 12 & 18-Aug-05 & 2-Sep-05 & 189 & 204 \\
\hline 13 & 3-Sep-05 & 16-Sep-05 & 205 & 218 \\
\hline 14 & 17-Sep-05 & 3-Oct-05 & 219 & 335 \\
\hline 15 & 4-Oct-05 & 12-Oct-05 & 236 & 244 \\
\hline 16 & 13-Oct-05 & 27-Oct-05 & 245 & 259 \\
\hline 17 & 28-Oct-05 & 10-Nov-05 & 260 & 273 \\
\hline 18 & 11-Nov-05 & 28-Nov-05 & 274 & 291 \\
\hline 19 & 29-Nov-05 & 19-Dec-05 & 292 & 312 \\
\hline 20 & 20-Dec-05 & 2-Jan-06 & 313 & 326 \\
\hline 21 & 3-Jan-06 & 17-Jan-06 & 327 & 341 \\
\hline 22 & 18-Jan-06 & 30-Jan-06 & 342 & 354 \\
\hline 23 & 31-Jan-06 & 14-Feb-06 & 355 & 369 \\
\hline 24 & 15-Feb-06 & 28-Feb-06 & 370 & 383 \\
\hline 25 & 1-Mar-06 & 8-Mar-06 & 384 & 391 \\
\hline 26 & 9-Mar-06 & 23-Mar-06 & 392 & 406 \\
\hline 27 & 24-Mar-06 & 7-Apr-06 & 407 & 421 \\
\hline 28 & 8-Apr-06 & 22-Apr-06 & 422 & 436 \\
\hline 29 & 23-Apr-06 & 5-May-06 & 437 & 449 \\
\hline
\end{tabular}

Since this research followed on from a previous investigation, all three systems had been operated from May 2003 (Ramphao et al., 2004). The writer assumed responsibility for operation and monitoring of the MBR and SQW systems in March 2005 and operated and monitored these two systems over the entire Phase 2 investigation. However, until sewage batch 15 the conventional UCT system was fed and monitored by laboratory staff. After this period, the writer took over control of operation, maintenance and monitoring of all three systems. During periods of extended absence by the writer (sewage batches 20 and 21) the systems were maintained by fellow post-graduate students and laboratory staff; however, no testing was conducted during these periods. Also, during sewage batch 1 analyses were incomplete because the writer was still learning and mastering the testing procedures.

\subsubsection{Steady State Periods}

Daily results for the MBR and conventional UCT systems are presented in Appendices $A$ and $B$ respectively and results for the SQW system included in Appendix A-1. The routine sampling and measurements taken from the two UCT 
systems were quantified and averaged as follows: Sewage batches were taken as steady state periods based on the assumption that no significant accumulation $( \pm$ ) would occur within the sewage batch and observations largely confirmed this.

Within a sewage batch, the first few days were allowed for the transition between steady state conditions and hence no testing was done on these days. Results recorded during a sewage batch period were analysed for consistency using a $95 \%$ confidence interval, whereby any results that fell outside this range (mean $\pm 1.96 \times$ sample standard deviation) were considered non-representative of the steady state and excluded (for a more detailed description of the statistical analysis see Appendix D). The remaining values were considered valid and averaged to generate an average response for the sewage batch. The average responses for each sewage batch (steady state period) were used for subsequent analysis of the performance of the systems. These averages are reported in Appendices A and B.

\subsection{SQW ACTIVATED SLUDGE SYSTEM}

The short sludge age square-wave (SQW) fed system used in this investigation was based on the conventional "standard" method to measure the RBCOD developed by Ekama and Marais (1978) and Ekama et al. (1986). As described in Chapter 3, in this investigation the system was operated at a short sludge age of 2 days. The OUR was measured continually and automatically using the technique detailed by Randall et al. (1991).

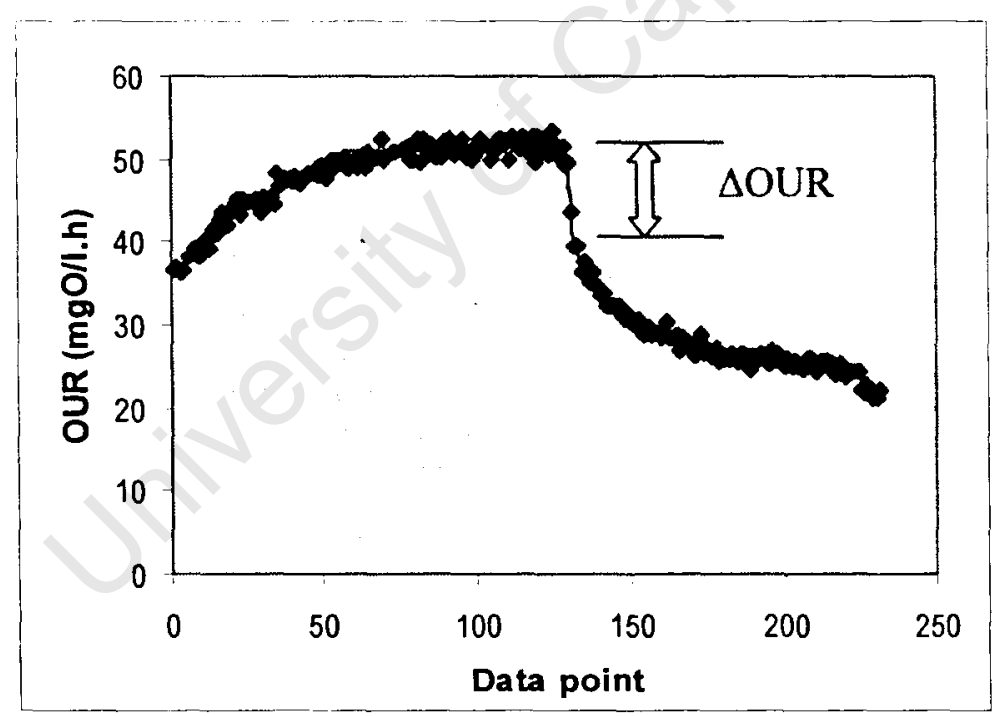

Figure 4.1: A typical OUR profile in the short sludge age SQW fed activated sludge system run in this investigation.

A typical OUR profile is shown in Fig 4.1. To calculate the RBCOD from the observed "drop" in OUR after feed termination ( $\triangle \mathrm{OUR}$ ) Equation (4.1) was used (Ekama et al., 1986):

$$
S_{b s i}=\frac{\left\{1 /\left(1-f_{C V} Y_{H}\right)\right\} \cdot \Delta O U R \cdot V_{P} \cdot 24}{Q}
$$

Where: $S_{b s i}=$ the RBCOD concentration of the wastewater $(\mathrm{mgCOD} / \ell)$

$f_{C V}=$ the COD to VSS ratio of the sludge (mgCOD $/ \mathrm{mgVSS}$ ) 


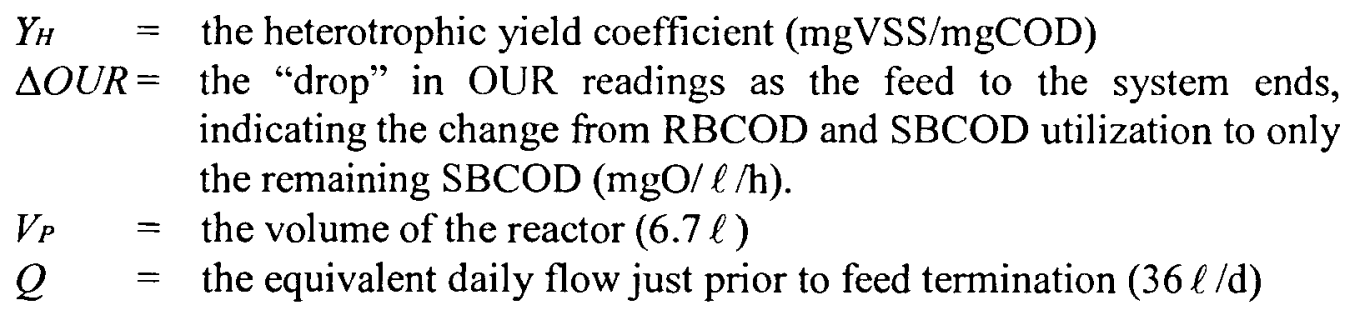

The influent wastewater RBCOD fraction with respect to the total COD $\left(f_{t s . S}\right)$ is given by Equation 4.2:

$$
f_{t s, S}=S_{b s i} / S_{t i}
$$

Using Equation (4.1) the daily RBCOD fractions for the sewage were calculated from the measured $\triangle$ OUR and $S_{t i}$ values, using the commonly accepted constants $Y_{H}=$ 0.45 and $\mathrm{f}_{\mathrm{cv}}=1.48$ (WRC, 1984, Ekama et al., 1986). The daily RBCOD fractions $\left(f_{t s, S}\right)$ from the SQW system are plotted in a linearized probability graph (see Appendix D) to check for normality, Fig. 4.2.

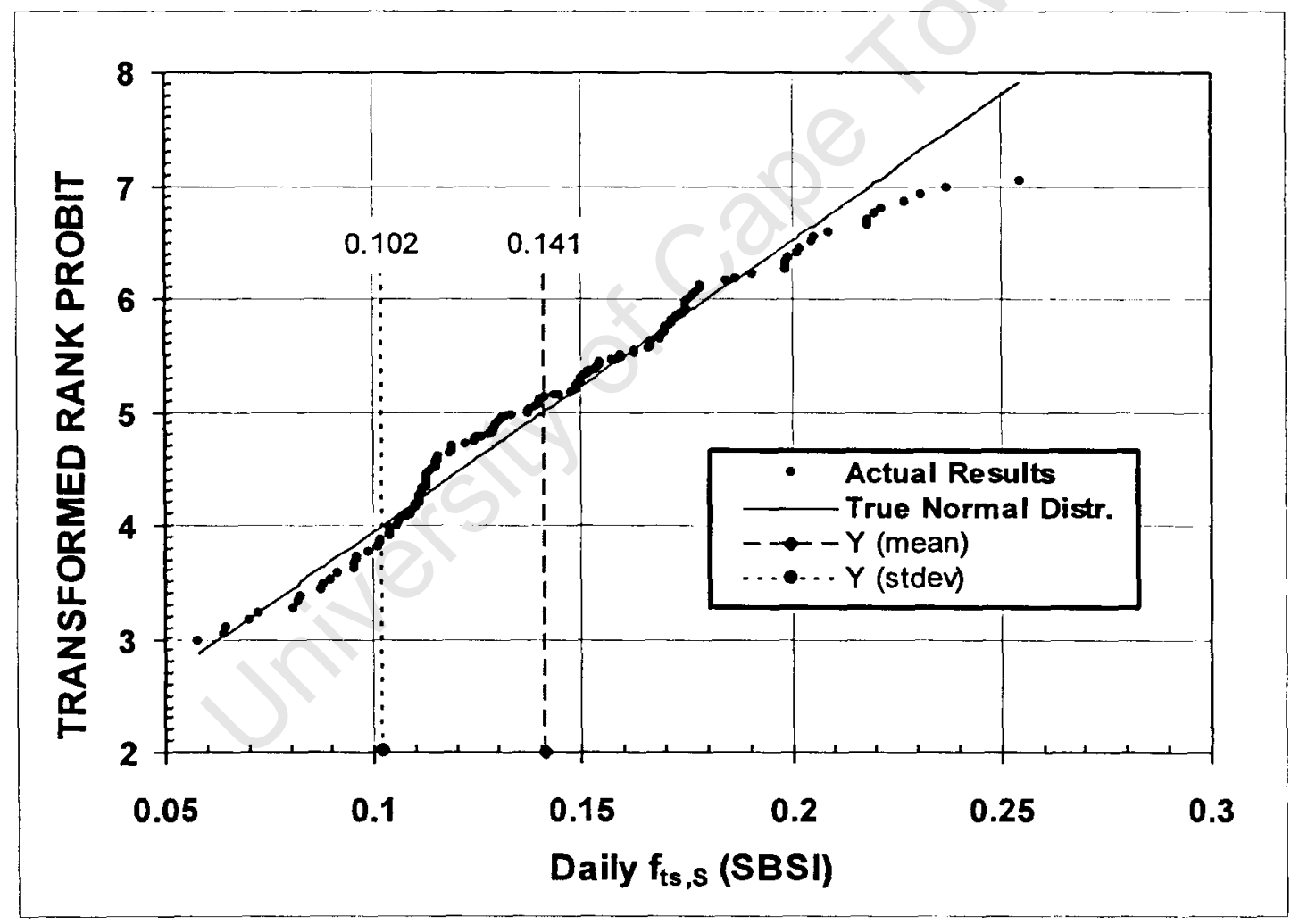

Figure 4.2: $\quad$ Statistical plot of the daily RBCOD as a fraction of the total influent COD $\left(f_{t s, S}\right)$ feed in the SQW influent feed.

The daily data, except for the few largest values, lie close to the normal distribution line, indicating a normal distribution. The mean $\mathrm{f}_{\mathrm{ts}, \mathrm{S}}$ of 0.141 is very close to that observed in the Phase 1 investigation of 0.145 (Ramphao et al., 2004), and falls within the range of previous $\mathrm{f}_{\mathrm{ts}, \mathrm{S}}$ values observed on sewage from the same source: 0.14 observed by Muller et al. (2003) and 0.16 by Ekama and Marais (1978). The sample standard deviation was 0.039 compared with 0.020 in Phase 1 (Rampaho et al., 2004). 
With the investigation average mean $\mathrm{f}_{\mathrm{ts}, \mathrm{S}}$ value of the sewage determined above and knowing that the sewage was augmented with $200 \mathrm{mgCOD} / \ell$ sodium acetate which is readily biodegradable, the total RBCOD content of the influent sewage feed $\left(f_{t s, T}\right)$ could be calculated using Equation (4.3):

$$
f_{i, T}=\frac{\left\{f_{t s .} .\left(S_{t i, T}-200\right)+200\right\}}{S_{t i, T}}
$$

Where $\mathrm{S}_{\mathrm{ti}, \mathrm{T}}=$ the total measured influent $\mathrm{COD}$ of the feed $(\mathrm{mgCOD} / \ell)$

Hence, the influent readily biodegradable component of the biodegradable $\operatorname{COD}\left(f_{b s, T}\right)$ could be calculated:

$$
f_{b s, T}=\frac{f_{t s, T}}{\left(1-f_{S, u s}-f_{S, u p}\right)}
$$

Where: $f_{S, u s}=$ unbiodegradable soluble fraction of the total influent COD

$f_{S, u p}=$ unbiodegradable particulate fraction of the total influent COD

Following the procedures above the sewage batch average $f_{t s, T}$ and $f_{b s, T}$ values were calculated using the unbiodegradable particulatc and soluble components of the influent ( $f_{S \text {, up }}$ and $f_{S \text {,us }}$ respectively) calculated in sections 4.8 .1 and 4.8.2 respectively. Table 4.2 lists the average $\mathrm{f}_{\mathrm{ts}, \mathrm{T}}$ and $\mathrm{f}_{\mathrm{bs}, \mathrm{T}}$ values for the various sewage batches.

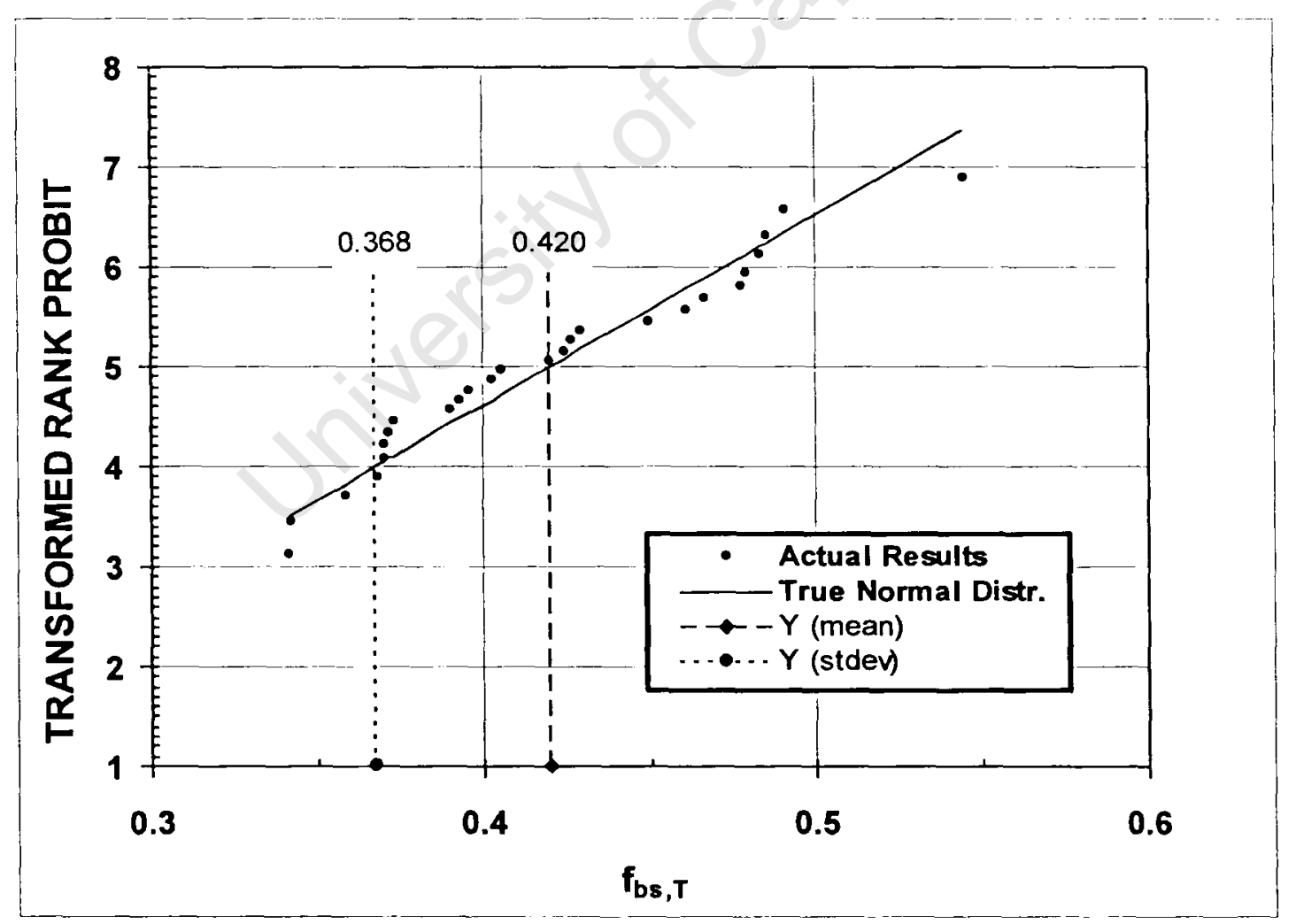

Figure 4.3: $\quad$ Statistical plot of $f_{b s, T}$ for sewage batch periods using $f_{S \text {,up }}$ and $f_{S \text {, us }}$ values generated with the MBR data. 
A statistical plot of the sewage batch $\mathrm{f}_{\mathrm{bs}, \mathrm{T}}$ data (Fig 4.3) indicates only minor deviation from the normal line. The mean is 0.420 , with a sample standard deviation of 0.052 , which is very close to that observed in Phase 1 of the project, mean $=0.431$ (Ramphao et al., 2004). The very high mean $\mathrm{f}_{\mathrm{bs}, \mathrm{T}}$ value is due to the addition of sodium acetate to the feed to augment BEPR.

Table 4.2: $\quad$ Sewage Batch $R B C O D$ as a fraction of total and biodegradable influent $C O D\left(f_{s t, T}\right.$ and $\left.f_{s b . T}\right)$

\begin{tabular}{|c|c|c|}
\hline $\begin{array}{c}\text { Batch } \\
\text { Number }\end{array}$ & $f_{\text {ts.I }}$ & $f_{\text {bs.I }}$ \\
\hline 2 & & \\
\hline 3 & 0.317 & 0.360 \\
\hline 4 & 0.297 & 0.341 \\
\hline 5 & 0.352 & 0.431 \\
\hline 6 & 0.323 & 0.461 \\
\hline 7 & 0.336 & 0.483 \\
\hline 8 & 0.368 & 0.569 \\
\hline 9 & 0.328 & 0.424 \\
\hline 10 & 0.322 & 0.404 \\
\hline 11 & 0.302 & 0.390 \\
\hline 12 & 0.290 & 0.393 \\
\hline 13 & 0.358 & 0.479 \\
\hline 14 & 0.285 & 0.376 \\
\hline 15 & 0.258 & 0.351 \\
\hline 16 & 0.285 & 0.371 \\
\hline 17 & 0.237 & 0.320 \\
\hline 18 & 0.325 & 0.482 \\
\hline 19 & 0.286 & 0.378 \\
\hline 20 & & \\
\hline 21 & & \\
\hline 22 & 0.291 & 0.356 \\
\hline 23 & 0.303 & 0.372 \\
\hline 24 & 0.336 & 0.562 \\
\hline 25 & 0.375 & 0.485 \\
\hline 26 & 0.339 & 0.435 \\
\hline 27 & 0.319 & 0.403 \\
\hline 28 & 0.258 & 0.367 \\
\hline 29 & 0.333 & 0.450 \\
\hline$A v e r a g e$ & 0.313 & 0.420 \\
\hline & & \\
\hline
\end{tabular}

\subsection{MBR SYSTEM ANALYSIS AND RESULTS}

In this section the experimental results and analysis for the MBR system are presented. As far as possible all system operation, sampling and testing were performed by the writer, in order to maintain consistency and a full understanding of the system and factors that may affect it.

\subsubsection{System Recycles and Mass Fractions}

From sewage batch 6 system recycle flow rates were measured on a weekly basis and found to vary considerably. Two possible causes for this variation are: 
i) the level of the feed tanks at the time of measurement affected the differential head pressure, particularly for the feed line, which would affect the recycle ratio, and

ii) undetected blockages in pipes (which were not always obvious) were suspected of affecting the flows through the recycle pipes. Hence, recycle values were checked by calculating the recycle ratios from total solids mass balances around the reactors, using Equations ( $4.5 \mathrm{a} \& \mathrm{~b}$ ) developed for the MBR system by Ramphao et al. (2004):

$$
r=\frac{X_{\text {Vana }}}{\left(X_{\text {Vanx }}-X_{\text {Vana }}\right)} \quad \text { and } \quad \text { as }=\frac{X_{\text {Vanx }}}{\left(X_{\text {Vaer }}-X_{\text {Vanx }}\right)}
$$

Where: $X_{\text {Vana, anx, aer }}=$ the VSS concentration of the anaerobic, anoxic an aerobic reactors respectively.

For most sewage batches the calculated and measured recycle ratio values corresponded closely, but as in Phase 1 these differed significantly from the expected (design) values (Ramphao et al., 2004). Thus, for analysis of system behaviour, the recycles used for sewage batch periods were the average of the measured and calculated values, see Table 4.3. For the early sewage batches (2-5) where recycle flows were not measured, the as-recycle had been incorrectly configured in a $4: 1$ ratio, hence a recycle of $4: 1$ is assumed as the measured value for these sewage batches.

The mass fractions ( $f_{\text {Mana, }} f_{\text {Manx }}, f_{\text {Maer, }}$ for anaerobic, anoxic and aerobic reactors respectively) were calculated for each reactor from the average sewage batch recycles above using Equation (4.6) and corresponded closely with those calculated from the measured reactor mixed liquor concentrations and volumes (Table 4.3).

$$
f_{\text {Mana }}=\frac{V_{a n a . X_{a n a}}}{\left(V_{a n a . X a n a}+V_{a n x .} X_{a n x}+V_{a e r .} X_{a e r}\right)}
$$

Where: $V \quad=$ the measured reactor volume

$$
\begin{aligned}
X_{a n a} & =X_{a n x .} r /(1+r) \\
X_{a n x} & =1 \\
X_{a e r} & =(1+a) / X_{\text {anx. }} a
\end{aligned}
$$

( $f_{\text {Manx }}$ and $f_{\text {Maer }}$ can similarly be calculated by substituting the values on the numerator of Equation (4.6) respectively) 
Table 4.3: $\quad$ Recycles and mass fractions for each sewage batch period

\begin{tabular}{|c|c|c|c|c|c|c|c|c|c|c|c|}
\hline \multirow[b]{2}{*}{ SB no. } & \multicolumn{4}{|c|}{ r-recycle } & \multicolumn{4}{|c|}{ a-recycle } & \multicolumn{3}{|c|}{ mass fractions } \\
\hline & design & measured & calculated & average & design & measured & calculated & average & $\mathrm{fm}_{\mathrm{ang}}$ & $\mathrm{fm}_{\mathrm{anx}}$ & $\mathrm{fm}_{\text {aer }}$ \\
\hline 2 & 1 & 1.00 & 1.39 & 1.19 & 3.00 & 4.00 & 4.37 & 4.19 & 0.141 & 0.285 & 0.574 \\
\hline 3 & 1 & 1.00 & 1.46 & 1.23 & 3.00 & 4.00 & 4.93 & 4.47 & 0.143 & 0.287 & 0.570 \\
\hline 4 & 1 & 1.00 & 1.53 & 1.26 & 3.00 & 4.00 & 2.67 & 3.33 & 0.140 & 0.277 & 0.583 \\
\hline 5 & 1 & 1.00 & 1.20 & 1.10 & 3.00 & 4.00 & 1.99 & 3.00 & 0.130 & 0.275 & 0.594 \\
\hline 6 & 1 & 1.29 & 1.25 & 1.27 & 3.00 & 3.11 & 2.92 & 3.01 & 0.138 & 0.273 & 0.589 \\
\hline 7 & 1 & 1.14 & 1.06 & 1.10 & 3.00 & 2.96 & 4.05 & 3.50 & 0.133 & 0.281 & 0.585 \\
\hline 8 & 1 & 1.28 & 1.28 & 1.28 & 3.00 & 2.99 & 4.93 & 3.96 & 0.144 & 0.283 & 0.573 \\
\hline 9 & 1 & 1.22 & 1.19 & 1.20 & 3.00 & 3.08 & 2.85 & 2.97 & 0.135 & 0.273 & 0.592 \\
\hline 10 & 1 & 1.15 & 1.08 & 1.12 & 3.00 & 3.17 & 4.34 & 3.75 & 0.135 & 0.283 & 0.581 \\
\hline 11 & 1 & 1.43 & 1.08 & 1.25 & 3.00 & 3.40 & 4.20 & 3.80 & 0.142 & 0.282 & 0.576 \\
\hline 12 & 1 & 1.44 & 1.29 & 1.36 & 3.00 & 3.31 & 4.74 & 4.02 & 0.147 & 0.282 & 0.571 \\
\hline 13 & 1 & 1.27 & 1.18 & 1.22 & 3.00 & 3.32 & 4.04 & 3.68 & 0.137 & 0.279 & 0.583 \\
\hline 14 & 1 & 1.14 & 1.60 & 1.37 & 3.00 & 2.96 & 3.83 & 3.40 & 0.142 & 0.271 & 0.587 \\
\hline 15 & 1 & 1.20 & 1.46 & 1.33 & 3.00 & 3.06 & 3.43 & 3.24 & 0.140 & 0.272 & 0.589 \\
\hline 16 & 1 & 1.07 & 1.24 & 1.15 & 3.00 & 2.84 & 3.11 & 2.97 & 0.132 & 0.273 & 0.608 \\
\hline 17 & 1 & 1.00 & 1.23 & 1.12 & 3.00 & 3.00 & 3.02 & 3.01 & 0.131 & 0.276 & 0.606 \\
\hline 18 & 1 & 1.27 & 1.39 & 1.33 & 3.00 & 3.38 & 3.08 & 3.23 & 0.140 & 0.271 & 0.600 \\
\hline 19 & 1 & 1.13 & 0.99 & 1.06 & 3.00 & 3.23 & 3.31 & 3.27 & 0.129 & 0.278 & 0.605 \\
\hline 20 & & & & & & & & & & & \\
\hline 21 & & & & & & a & 2 & & & & \\
\hline 22 & 1 & 1.15 & 1.29 & 1.22 & 3.00 & 3.32 & 4.19 & 3.75 & 0.141 & 0.282 & 0.588 \\
\hline 23 & 1 & 1.18 & 1.08 & 1.13 & 3.00 & 3.57 & 3.16 & 3.37 & 0.136 & 0.273 & 0.602 \\
\hline 24 & 1 & 1.23 & 1.16 & 1.20 & 3.00 & 3.13 & 3.62 & 3.37 & 0.136 & 0.278 & 0.599 \\
\hline 25 & 1 & 1.17 & 1.29 & 1.23 & 3.00 & 3.45 & 3.59 & 3.52 & 0.139 & 0.274 & 0.599 \\
\hline 26 & 1 & 1.23 & 1.23 & 1.23 & 3.00 & 3.91 & 2.95 & 3.43 & 0.139 & 0.276 & 0.598 \\
\hline 27 & 1 & 1.12 & 1.24 & 1.18 & 3.00 & 3.52 & 3.19 & 3.36 & 0.135 & 0.276 & 0.603 \\
\hline 28 & 1 & 1.10 & 1.26 & 1.18 & 3.00 & 3.15 & 3.35 & 3.25 & 0.133 & 0.271 & 0.610 \\
\hline 29 & 1 & 1.20 & 1.18 & 1.19 & 3.00 & 3.21 & 2.73 & 2.97 & 0.130 & 0.275 & 0.610 \\
\hline Ave & 1 & 1.17 & 1.25 & 1.21 & 3.00 & 3.35 & 3.56 & 3.45 & 0.137 & 0.277 & 0.591 \\
\hline $\begin{array}{l}\text { Ramphao } \\
\text { of al }\{2004\} \\
\end{array}$ & 1 & 1.47 & 1.51 & 1.52 & 3.00 & 3.29 & 2.92 & 3.01 & 0.142 & 0.264 & 0.594 \\
\hline
\end{tabular}

\subsubsection{Membrane and Bioreactor Testing}

\subsubsection{Initial Membrane and Bioreactor Testing}

In Phase 1 the bioreactor was tested for adequate mixing with a residence time distribution (RTD) test and adequate aeration using aeration of deoxygenated tap water (Ramphao et al., 2004). The RTD and aeration tests were not repeated in Phase 2 , though the dissolved oxygen (DO) concentration in the aeration reactor was checked regularly and consistently found to be between 2 and $5 \mathrm{mgO} / \ell$, which was adequate for the biological demand of the system. Air flow into the aerobic reactor was consistently in the range of $2.5 \mathrm{~m}^{3} / \mathrm{h}$ with a Specific Air Transfer Rate of $78 \mathrm{~m}^{3} /\left(\mathrm{m}^{3} \cdot \mathrm{h}\right)$.

In April 2005 new membrane panels were received for installation into the membrane bioreactor. The old membranes had been in operation since the beginning of the investigation in April 2003 and had not been chemically cleaned. Flux tests were 
performed on the new and old membranes and are presented in Fig. 4.4. After Günder and Krauth (1999) the permeability of membranes is calculated by the quotient of the flux $\left(\mathrm{m}^{3} / \mathrm{m}^{2} . \mathrm{d}\right)$ divided by the TMP $(\mathrm{m})$. Thus according to the initial flux tests, the permeability of the new and old membranes was determined as 5.68 and 2.09 $\mathrm{m}^{3} / \mathrm{m}^{2}$.m.d, demonstrating that the new membranes were almost 3 times as permeable as the old membranes.

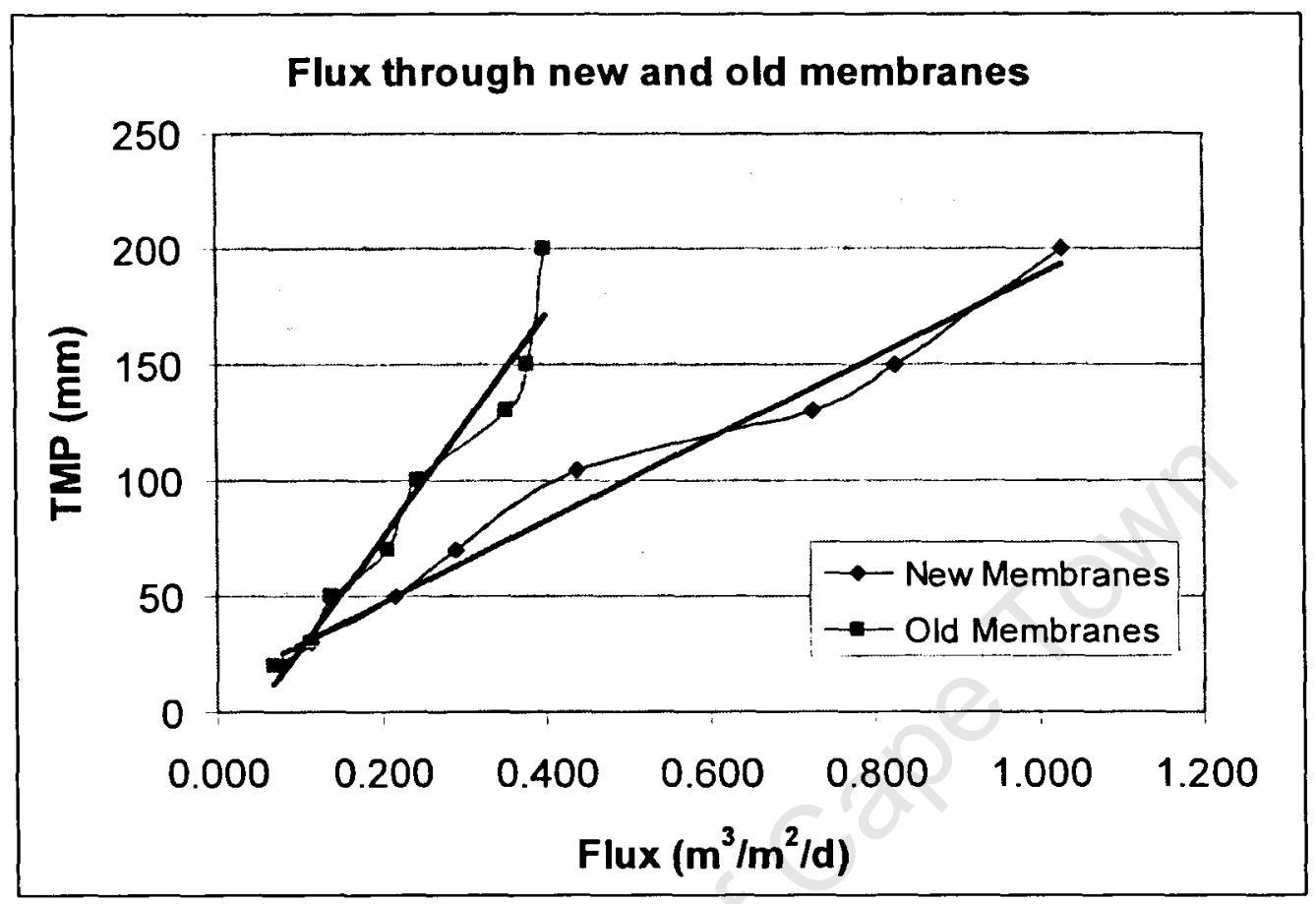

Figure 4.4: Results from flux tests on the old membranes and new membranes installed in April 2005.

\subsubsection{Oxygen Transfer Rate Testing}

Once the intensive study on BNR performance in the MBR system had been completed after sewage batch 29, (May 2006), the oxygen transfer rate (OTR) and oxygen transfer efficiencies (OTE) of the activated sludge at high MLSS concentrations were determined by performing tests on the sludge in the aerobic reactor. Details of the tests and methodology can be found in Chapter 3, Section 3.7.

\section{Unsteady State Testing}

Oxygen mass transfer co-efficient $\left(\mathrm{K}_{\mathrm{LA}}\right)$ values were determined for a number of air flow rates in the operating range of the aeration system (Appendix F-2). In Fig. 4.5 the curves representing the airflow rate-vs- $\mathrm{K}_{\mathrm{LA}}$ are presented for tests with membranes in the reactor, and tests with the membranes absent. The similarities of the curves suggest that the different geometry, and particularly the additional shear induced by the membranes had little impact on the oxygen transfer in the aerobic reactor. 


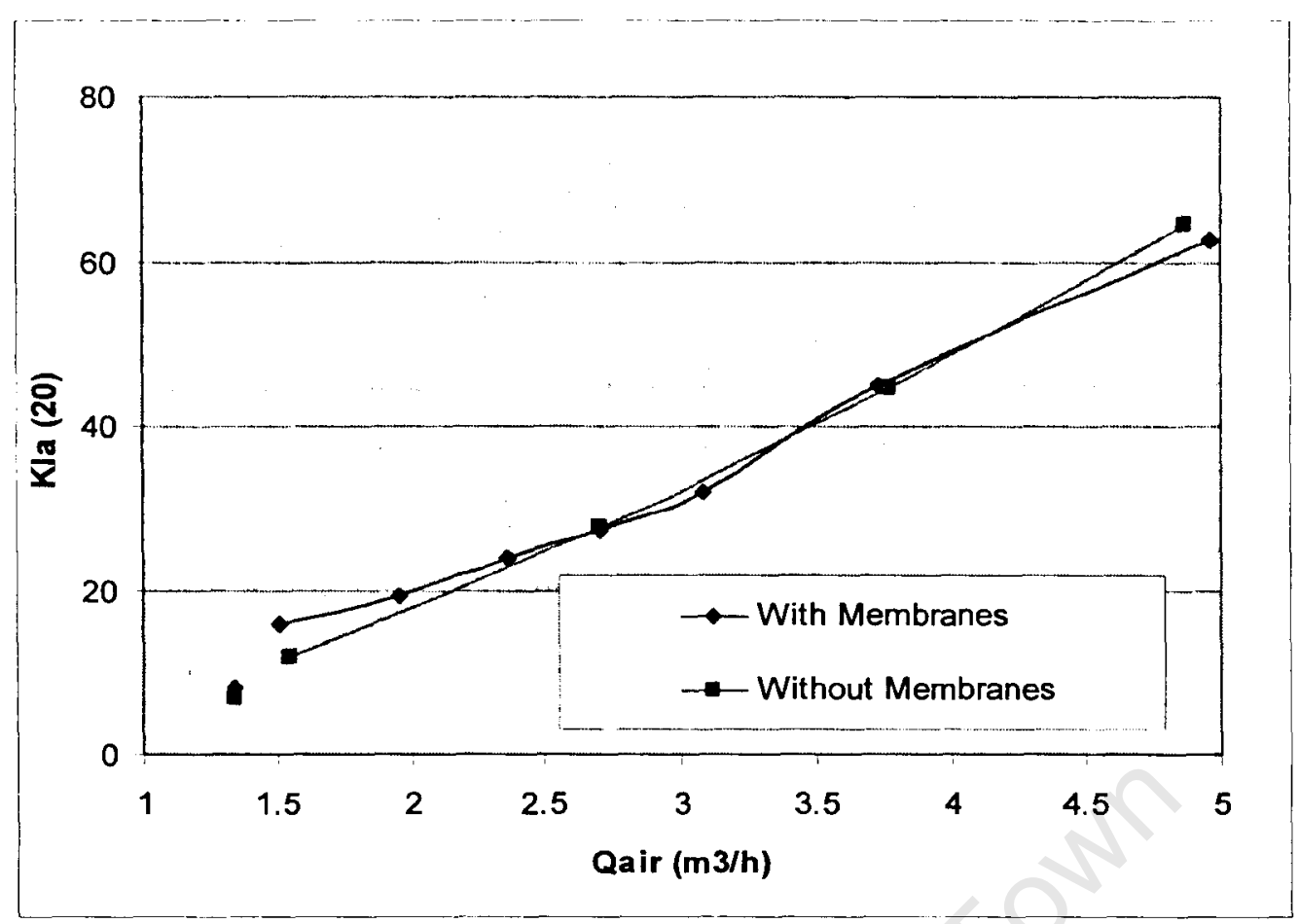

Figure 4.5: Reference curves for $\mathrm{K}_{\mathrm{LA}}(20)$ at various airflow rates ( $Q_{\text {air }}$ ) for testing with and without membranes installed in the reactor.

\section{Steady State Testing}

As described in Chapter 3, Section 3.7, steady state tests were carried out on the activated sludge once it had reached endogenous conditions to reduce the interference of differences caused by variations in feed (Appendix F-1). For a single MLSS concentration a number of readings were taken at different airflow rates (Fig. 4.6) and the Alpha values observed were averaged to give Fig. 4.7.

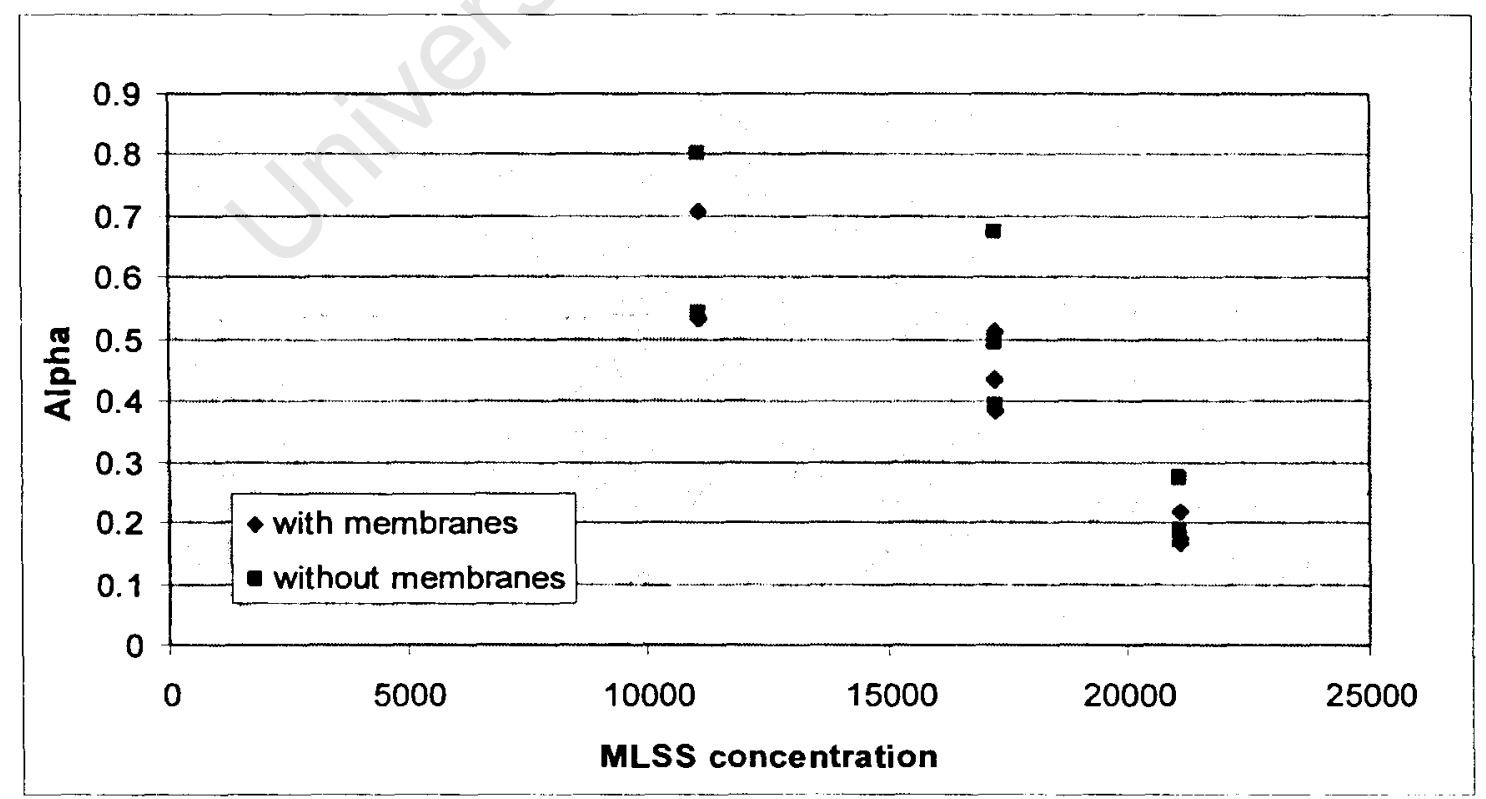

Figure 4.6.: The range of Alpha values observed at set MLSS values for the system with and without membranes respectively. 


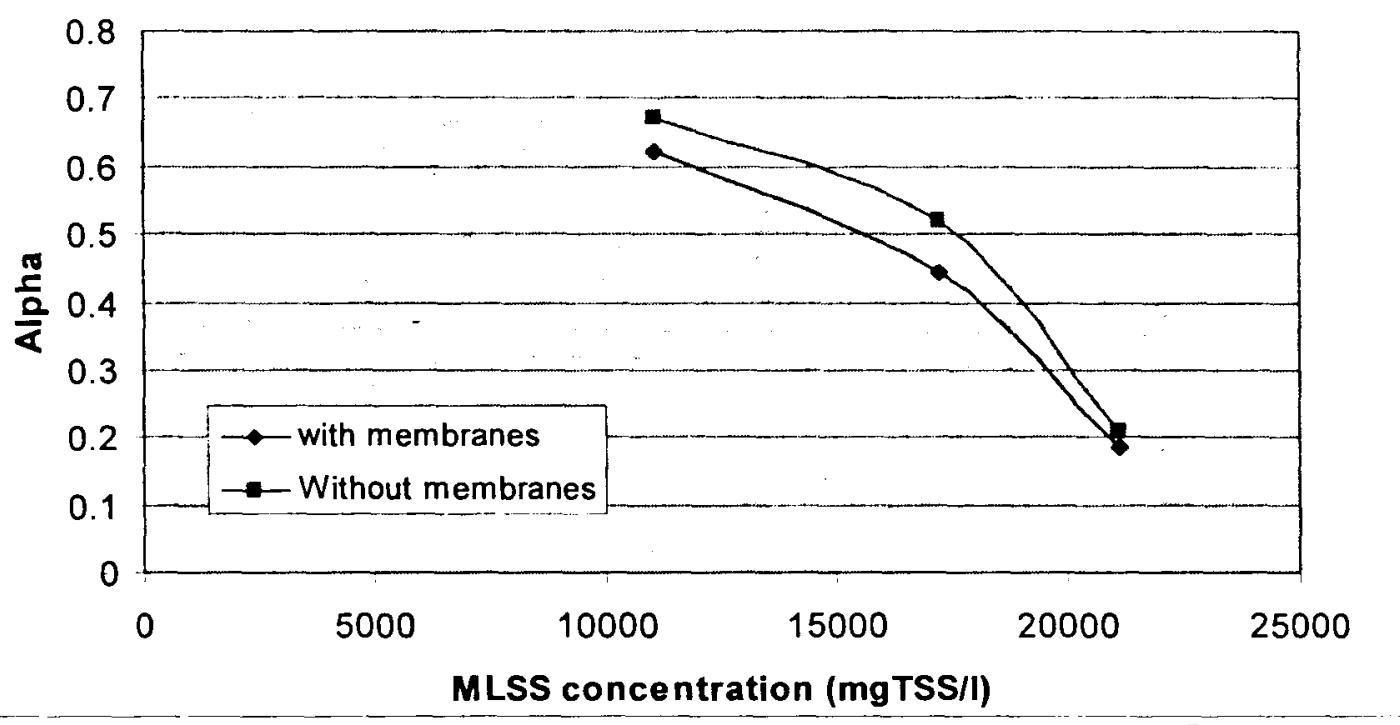

Figure 4.7: Averaged Alpha values at set MLSS concentrations for conditions with membranes and without membranes respectively.

The wide range of Alpha values observed is a concern. It was concluded that the predominant reason for the variations in observed Alpha values was the sensitivity of Equation (3.2) to variations in the measured OUR and $C_{L}$ values, and fluctuations in the airflow readings. As the system was run under endogenous conditions, limited OUR activity meant that OUR readings were typically very low and $C_{L}$ readings very high, hence minor differences in readings translated into major changes in calculated results. In order to compensate for high $\mathrm{C}_{\mathrm{L}}$ values only low airflows could be run, at which the rotameter was less accurate. It was hoped that by averaging a number of readings at the same MLSS concentration a more accurate Alpha value could be determined for that MLSS concentration. In conclusion it is recommended that future Alpha tests are run on sludge with a far shorter sludge age, thus providing a greater active fraction in the activated sludge, and hence an increased OUR at endogenous respiration. Additionally very sensitive airflow rate instrumentation is required to accurately monitor the airflow into the aerobic reactor.

\section{Measurement of OTR and OTE in the system}

Having established $\mathrm{K}_{\mathrm{LA}}$ and $\mathrm{K}_{\mathrm{LA}}$ ' values for clean water and mixed liquor respectively the OTR and OTE for the system were calculated using Equations (3.3) and (3.4). The same difficulties in measurement, noted above, also affected these calculations. Fig. 4.8a presents the OTR at various mixed liquor solids concentrations and in tap water against the specific air flow rate (SAFR) which is the volume of air per volume of liquid per hour. In this investigation the SAFR is very high in comparison to other studies (Cornel et al., 2003 investigated systems with SAFR in the range of $0-4$ $\mathrm{m}^{3} /\left(\mathrm{m}^{3} . \mathrm{h}\right)$ ) but this is attributed to the small volume of the aerobic reactor $(32 \ell)$ in this study and the requirement of substantial aeration for cross-flow velocity membrane scouring. The OTE values calculated for various mixed liquor solids concentrations are presented in Fig. 4.8b. The OTE values were very low but comparable to OTE values reported by Cornel et al. (2003) for coarse bubble aeration in mixed liquor at high solids concentrations. OTE values remained relatively constant at increasing SAFR rates, indicating that OTE is dependant on solids concentration, not flow rate. 


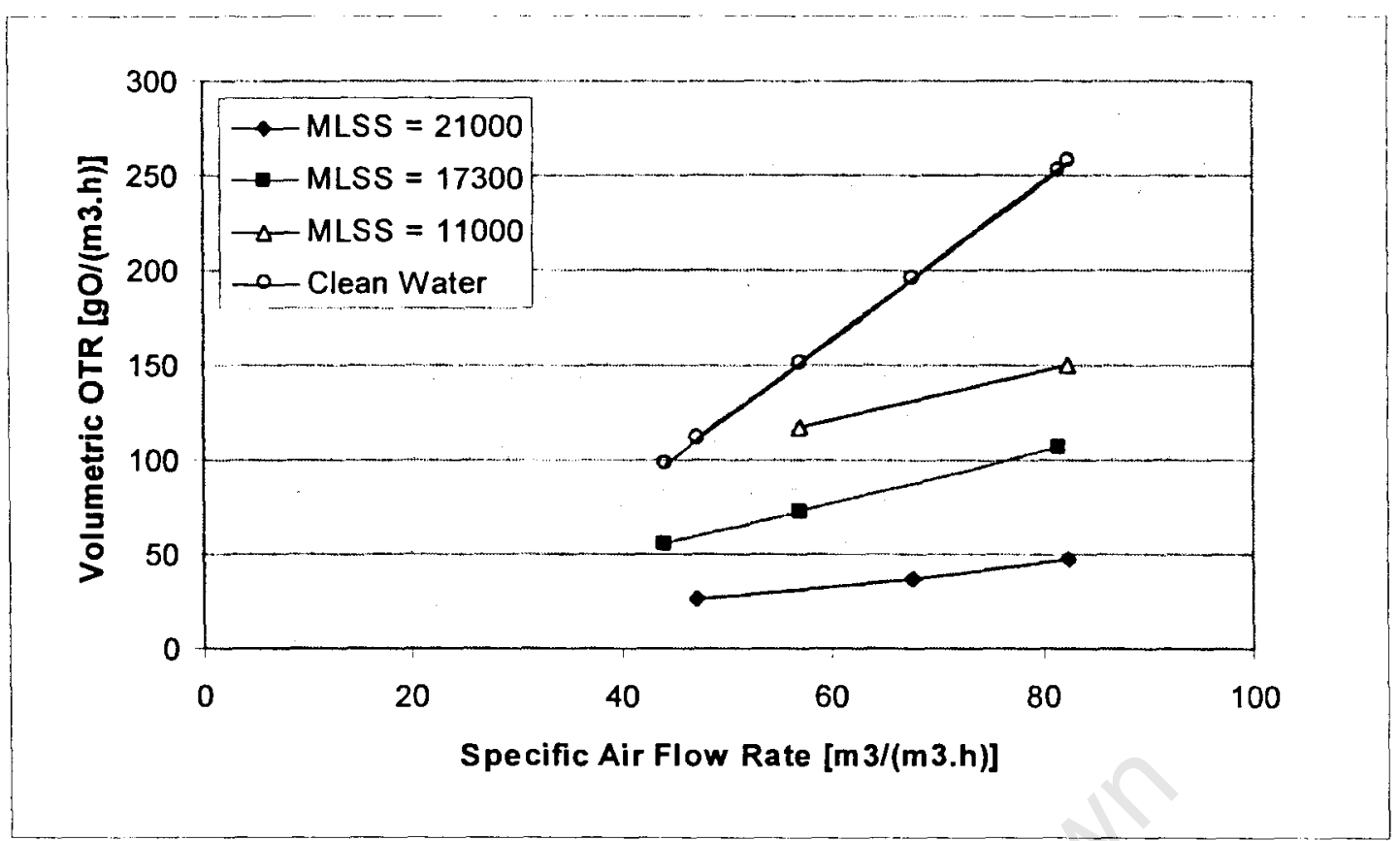

Figure 4.8a: Oxygen transfer rates (OTR) in clean water and various MLSS concentrations.

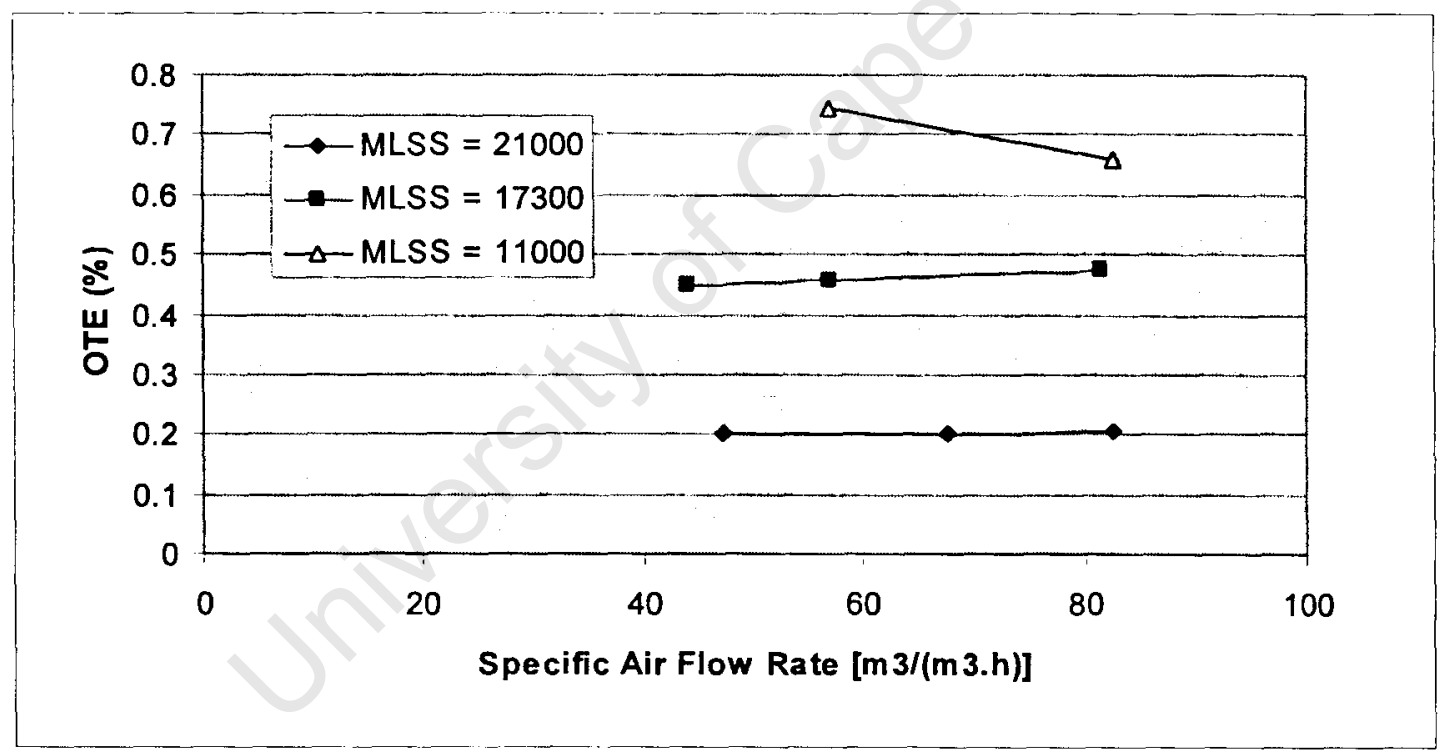

Figure 4.8b: Oxygen transfer efficiency (OTE) for various MLSS concentrations).

\section{Rheometric testing}

Baker et al. (1975) and Krampe J. and Krauth K. (2003), citing Rosenberger et al. $2002 \mathrm{~b}$ and Günder et al. (2001), indicated that there was a linear relationship between viscosity and Alpha, thus the relationship between the measured viscosity and Alpha values was investigated. Rheometric testing was conducted on the various mixed liquor concentrations to determine the viscosity of the mixed liquor at these concentrations (Appendix F-3). Figs. 4.9a and b present MLSS versus Alpha and viscosity, and the plot of Alpha versus the viscosity of the sludge at various shear rates of $58 / \mathrm{s}, 78 / \mathrm{s}$, $148 / \mathrm{s}$ and 280/s respectively. Although only three Alpha values were established there appears to be a linear regression suggesting that there is indeed a linear relationship 
between viscosity and Alpha. This relationship should be explored further in future investigations as it may allow a more accurate method of predicting Alpha in high concentration mixed liquor sludges. However such an investigation is dependant on the accurate measurement of Alpha in a high concentration sludge for which the writer experienced numerous difficulties as are described above.

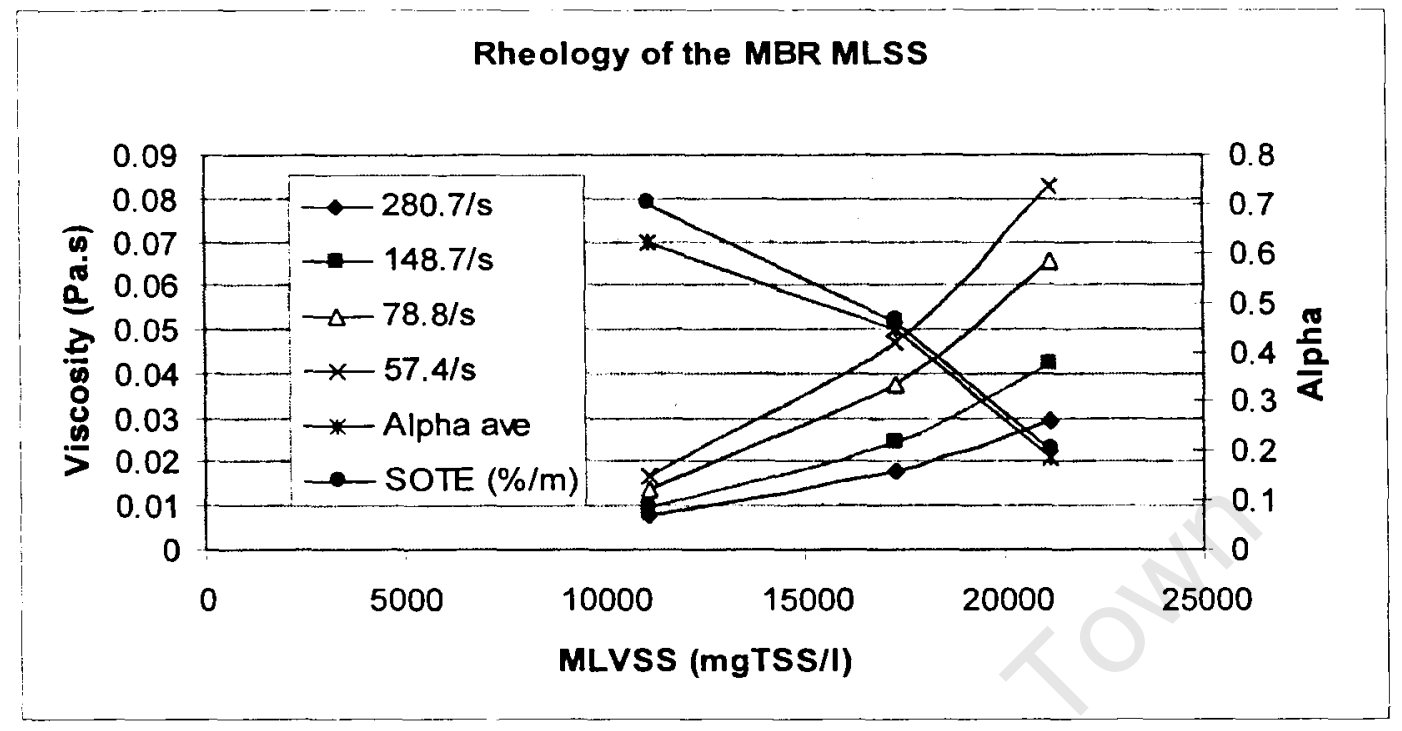

Figure 4.9a: Changes in viscosity and Alpha at various high concentration sludges.

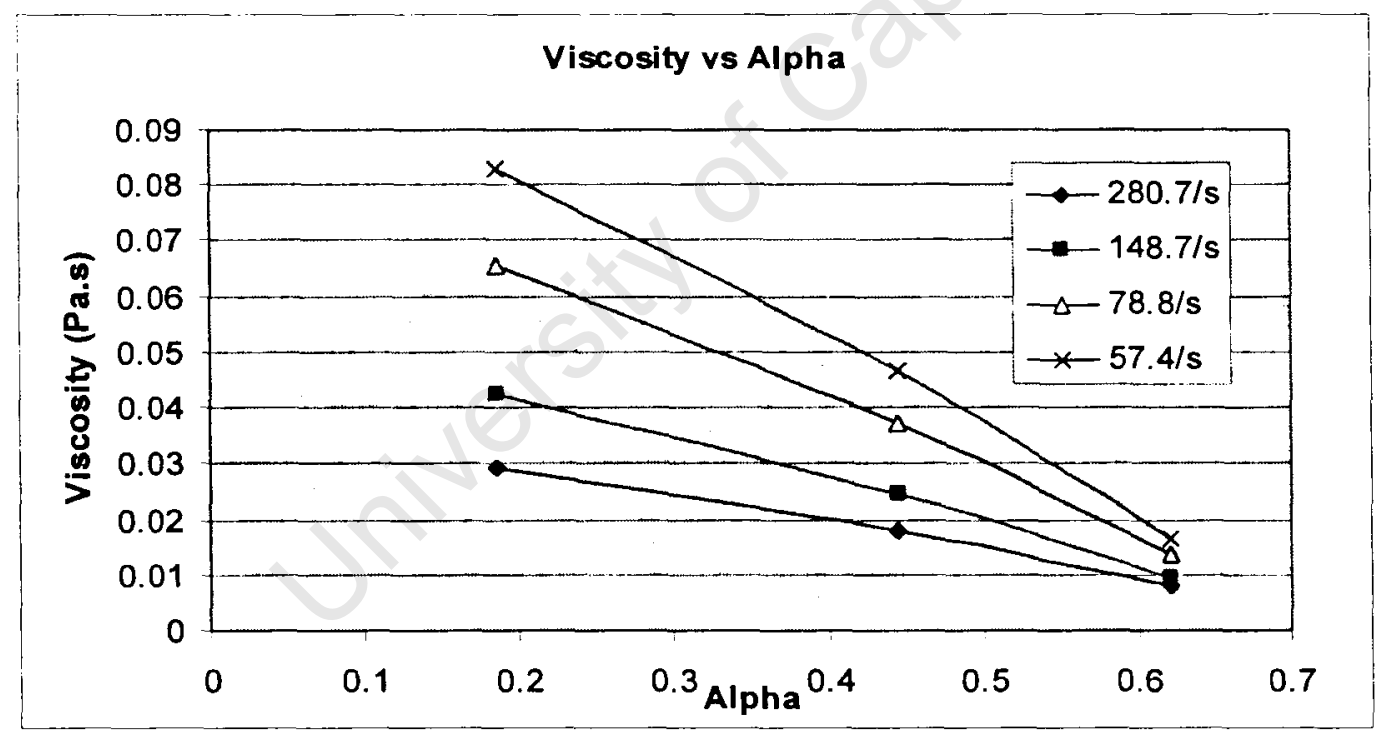

Figure 4.9b: A linear relationship between Alpha and viscosity was observed.

\section{Beta Testing}

Beta tests were conducted on the MBR sludge as described in Chapter 3, Section 3.7.4. It was found that the $\mathrm{C}_{\mathrm{S}}$ achieved in distilled water after aeration for an hour was the same as that observed in the sludge after substantial $\mathrm{HgCl}$ dosing had halted all biological activity. Thus a Beta value of 1.0 was assumed. 


\subsection{MEMBRANE PERFORMANCE}

The objective of this research was to investigate the BNR performance in a submerged MBR system. Hence, the performance of the membranes was not a research priority. However, membrane performance was monitored throughout the investigation by measuring trans-membrane pressure (TMP) and is reported in this section.

During the investigation, essentially a constant flux was selected (since the influent flowrate was constant) and the TMP varied to maintain this flux. Also the volume of the aerobic reactor needed to remain constant throughout the investigation. Accordingly the height of the effluent overflow pipe (Chapter 3, Section 3.6.2) was varied to maintain the constant flux and reactor volume. The TMP in mm water is the difference in height between the reactor liquid level and effluent overflow weir level. For the duration of Phase 1 TMP was monitored closely and a continual small increase of $0.12 \mathrm{~mm} / \mathrm{d}$ in the TMP of the membranes was noted. Similarly from the beginning of Phase 2 the TMP was continually monitored.

TMP is directly proportional to the flux passing through the membranes and hence to consistently monitor TMP required that the instantaneous flux be measured also. As the initial membrane tests indicated, the relationship between flux and TMP is linear. Thus, the measured TMP and instantaneous flux could be referenced back to the equivalent TMP at the expected steady state flux. This proved important late in the investigation as there were small but noticeable variations in the flux through the membranes induced by factors such as irregular pumping speeds and minor blockages in the piping connecting the reactors.

Until day 349 only the TMP was measured which, due to only minor fluctuations in the pump speeds and minor blockages, can be accepted as normally distributed and representative. However, from day 359 difficulties due to large mixed liquor spillages and intermittent power outages caused irregular pumping, and the effect of flux on TMP needed to be taken into account. Thus from day 371 , an adjusted $\mathrm{TMP}_{\text {adj }}$ is reported (Equation 4.7).

$$
T M P_{a d j}=\frac{T M P \cdot Q_{i}}{Q_{e}}
$$

Where: $Q_{i} \quad=$ the design influent flow of $140 \ell /$ day $/ 24 \mathrm{hrs}$

$Q_{e} \quad=$ the effluent flow measured during the TMP test $(\ell / \mathrm{hr})$ 


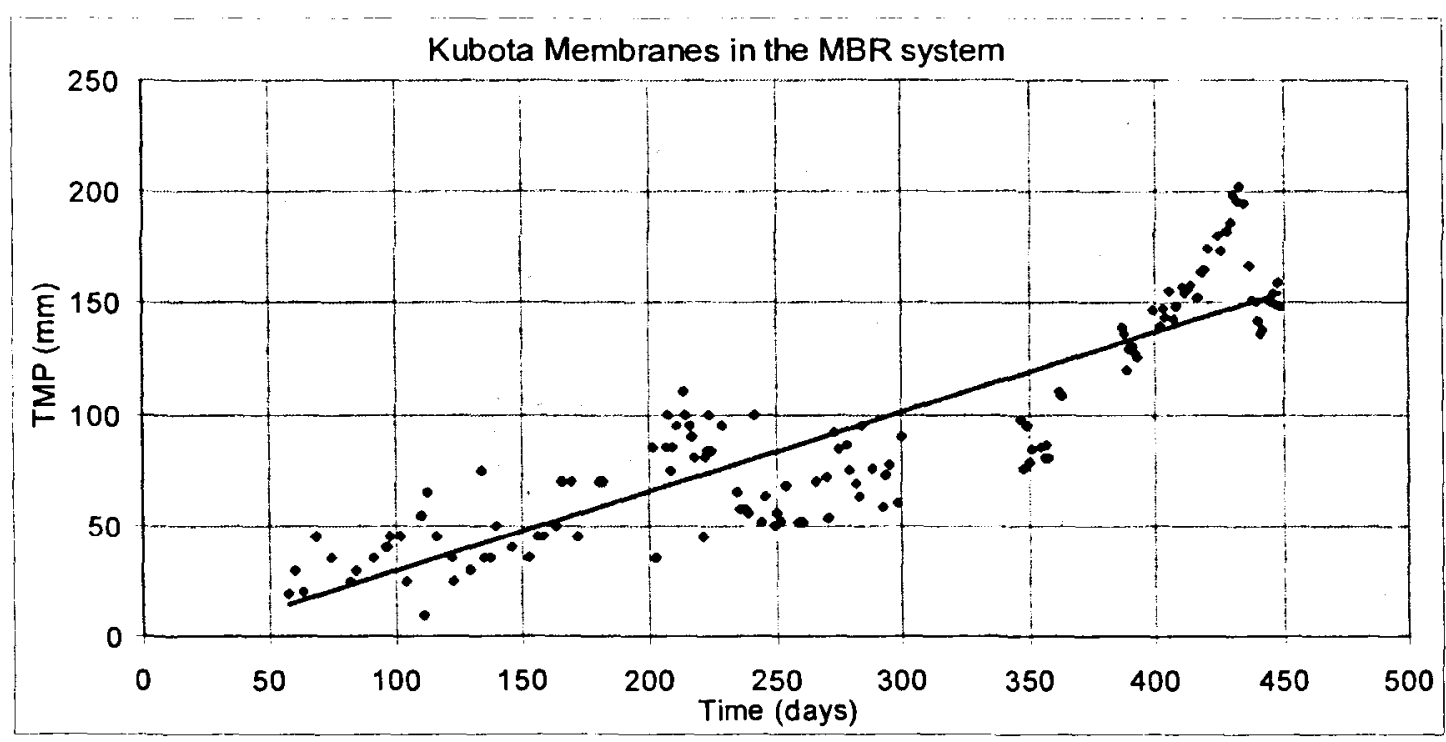

Figure 4.10: TMP changes in the MBR system were obsened for the duration of the project. A steady increase was observed with time though specific events like inorganic colloidal solids in the influent had a marked impact on the TMP.

The measured TMP with time over the investigation is shown in Fig. 4.10. Although the measured TMP shows considerable scatter due to variations at the time the measurements were taken, a consistent increase in the TMP over time was observed of about $0.29 \mathrm{~mm} / \mathrm{d}$. This is significantly larger than the increase noted in Phase 1 of $0.115 \mathrm{~mm} / \mathrm{d}$, and would have a significant influence on membrane life; over 20 years the increase in TMP would be $\sim 2.1 \mathrm{~m}$. Every two to three weeks the membranes were sprayed down with water, during regular system cleaning. However as with the Phase 1 investigation, no chemical cleans were performed on the membranes until the later stages of the investigation (sewage batch 28 ) in order to monitor the increase in TMP over time. No definitive explanation for this greater increase in TMP with time in Phase 2 compared to Phase 1 could be identified.

From day 374 to day 386 sporadic power failures were experienced in the lab, often at night, resulting in the membranes fouling due to discontinued or irregular aeration (no aeration results in no cross flow scouring). This resulted in an increased TMP. The fouling was in the form of a thin $(2 \mathrm{~mm})$, compact cake of sludge. In cases where the power failure had been brief, less than a half hour, the subsequent aeration of the membranes would remove much of the caking formed on the membranes. When the power failure had been longer than a half hour the caking that formed on the membranes was more resilient to the aeration and required manual cleaning by removal of the membranes from the system and water spraying. After water cleaning the TMP would return to levels shortly before the blockage. In full scale plants the potential of fouling due to power failures is circumvented by installing a stop valve on the permeate line which closes when power fails (Kennedy, pers. com.).

During sewage batches 27 and 28 (days 407 to 436) following the power failures, the TMP increased sharply from $142 \mathrm{~mm}$ (day 407) to $202 \mathrm{~mm}$ (day 433). Simultaneously, the filtration of the mixed liquor from the MBR reactors became increasingly difficult. An ISS test was performed on the sewage and a very high inorganic suspended solids (ISS) content, $0.162 \mathrm{mgISS} / \mathrm{mgCOD}$ was measured compared with 
averaged ISS concentrations of 0.041-0.050mgISS/mgCOD from 48 studies reported for sewage from the same wastewater source (Ekama and Wentzel, 2004). On investigation it was found that construction at the Mitchells Plain WWTP could have been the cause of additional fine ISS particles entering the sewage and could potentially have caused the increased TMP. Similar observations were reported by Fleischer et al. (2005) whereby the filterability of the sludge was significantly decreased by increased colloidal inorganics accumulating in the mixed liquor.

Up to this point in the study no chemical cleans had been performed on the membranes in order to allow the writer assess the deterioration of the membranes with time from biological factors. However the sudden increase in TMP necessitated that the membranes be thoroughly cleaned. Hence chemical and biological cleans using a $1.0 \%$ citric acid solution and $0.5 \%$ hypochlorite solution respectively as recommended by the manufacturer (Kennedy, pers. com.) were performed on the membranes on day 433. Prior to the chemical clean the system TMP was $202 \mathrm{~mm}$. Once the cleaning process had been performed the membranes were returned to the system and a TMP of $118 \mathrm{~mm}$ was measured in tap water. This improvement was substantially less than expected, which put in doubt the effectiveness of the chemical clean, and there was little improvement in TMP when the sludge was returned to the system, from $202 \mathrm{~mm}$ (day 433) to $194 \mathrm{~mm}$ (day 434). Only when the next sewage batch (29, day 437) was introduced, with ISS $=0.050$ mgISS/mgCOD, did the TMP drop rapidly to previous levels. Thus it appears that the TMP-flux relationship can be significantly influenced by fine colloidal ISS in the influent sewage. The rapid recovery suggested that the colloidal ISS is assimilated into the sludge over a period of only a few days. Thus this phenomenon should affect MBR systems with short and long sludge ages equally.

Kennedy (pers. com.) reported that on full scale membrane plants, chemical and biological cleans on membranes return the membrane TMP to original levels. Hence subsequent cleans were performed on the system membranes in October 2006. The membranes were thoroughly sprayed down with water and inserted into a $1 \%$ hypochlorite solution for an hour. However a slight suction was placed on the effluent outflow pipes of the membranes to circulate hypochlorite through the membranes. On removal the membranes which prior to cleaning had a light brown colouring had essentially been bleached and came out white. The initial TMP in the system was high, $460 \mathrm{~mm}$, but within 24 hours the TMP had dropped to $55 \mathrm{~mm}$, roughly the TMP observed when the new membranes had been installed in May 2005. The initial high TMP is explained by the progressive flushing of the soapy hypochlorite layer that had formed on the membranes. Additionally the failure of initial chemical cleans on day 433 is attributed to the old stock of hypochlorite used which may have degraded to the point of being ineffective.

\subsection{MIXED LIQUOR SOLIDS}

For all the MBR system reactors the mixed liquor solids parameters, MLSS, MLVSS, COD and TKN were monitored regularly from the beginning of the investigation. The information on the variation of mixed liquor concentrations with time was necessary in order to interpret the BNR performance of the system. 


\subsubsection{Sludge Age (Rs)}

As far as possible the sludge age of the system was maintained constant at 20 days by wasting $2.85 \ell / \mathrm{d}$ mixed liquor volume from the aerobic reactor (approximately $1 / 20^{\text {th }}$ of the system sludge mass). In the event of an unintentional mixed liquor loss from the system due to i) a spill from the reactors, ii) a burst interconnecting pipe, or iii) foam removal, knowledge of the total solids content of the system allowed the mass of mixed liquor lost to be determined and the approximate mixed liquor mass to be wasted was reduced accordingly over the following days.

\subsubsection{MLSS and MLVSS Concentrations}

After Phase 1 the mixed liquor in the systems was retained and the Phase 2 investigation commenced with the same sludge. Throughout the investigation there were minor unintended mixed liquor losses typically through spillages or while cleaning the system. Most of these were contained in drip trays below the reactors and the spilled mixed liquor was filtered through a $2 \mathrm{~mm}$ screen to avoid sludge "clots" forming which could cause blockages in the system, and to ensure that foreign objects weren't accidentally returned with the mixed liquor.

On a few occasions substantial amounts of mixed liquor were lost, Table 4.4. On each occasion the total mixed liquor lost was calculated as the difference in solids concentration from the day prior to the spill, and the loss was compensated by not wasting for the equivalent number of days following the spill.

Table 4.4: $\quad$ Sludge loss events in Phase 2

\begin{tabular}{|l|l|}
\hline Day & Event \\
\hline $48-56$ & Foaming: Steady solids accumulation in foam. \\
\hline $66,67,69$ and 74 & $\begin{array}{l}\text { Spillages: Extensive spillages resulting in substantial loss of solids and no } \\
\text { wasting until day 88. }\end{array}$ \\
\hline 359 and 360 & $\begin{array}{l}\text { Spillages: Recurrent spillages resulted in low solids and no wasting until } \\
\text { solids returned to 18 000mgTSS/ } \ell \text { on day 370. }\end{array}$ \\
\hline $\begin{array}{l}374-378,383 \\
\text { and } 386\end{array}$ & $\begin{array}{l}\text { Power failure: Regular power failures resulted in numerous minor spillages } \\
\text { as the membranes would foul overnight. }\end{array}$ \\
\hline
\end{tabular}

Foaming, exclusively in the anoxic reactor, was common and expected due to the high sludge concentration and thus the high volume of $\mathrm{N}_{2}$ gas released through denitrification. The foam generated was trapped on the reactor which did not allow for surface flow through. This was dealt with by brushing down the foam into the reactor liquid daily. However over one period (Days 48-56, Table 4.4) substantial foaming was observed, and when repeated brushing down did not help, it was physically removed from the system. In this case the mass of foam removed was taken into account when wasting sludge over the next few days. Between days 374 and 386 the laboratory experienced recurrent power failures which resulted in the systems not being fed and consequently mixed liquor was not wasted either. Hence longer sludge ages were measured for this batch period.

Apart from the periods noted above, the MLSS concentration in the aerobic reactor remained within the range of $16000-19000 \mathrm{mgTSS} / \ell$ and the anoxic and anaerobic concentrations were within the ranges of $12000-14000 \mathrm{mgTSS} / \ell$ and $6000-8000$ mgTSS/ $\ell$ respectively. These ranges are substantially less than those reported by 
Ramphao et al. (2004) in Phase 1. These differences are attributed to both to a lower $\mathrm{S}_{\mathrm{ti}}$ for Phase 2 (Section 4.9.1) and lower influent unbiodegradable particulate COD fraction ( $f_{S, u p}$, Section 4.8.2).

For assessment, the sewage batch average TSS and VSS concentrations in the three reactors are shown in Figs. 4.11. to 4.13. The TSS concentration of the $3 \ell$ re-aeration reactor is also shown in Fig. 4.12. Reactor concentrations were consistent over the investigation.

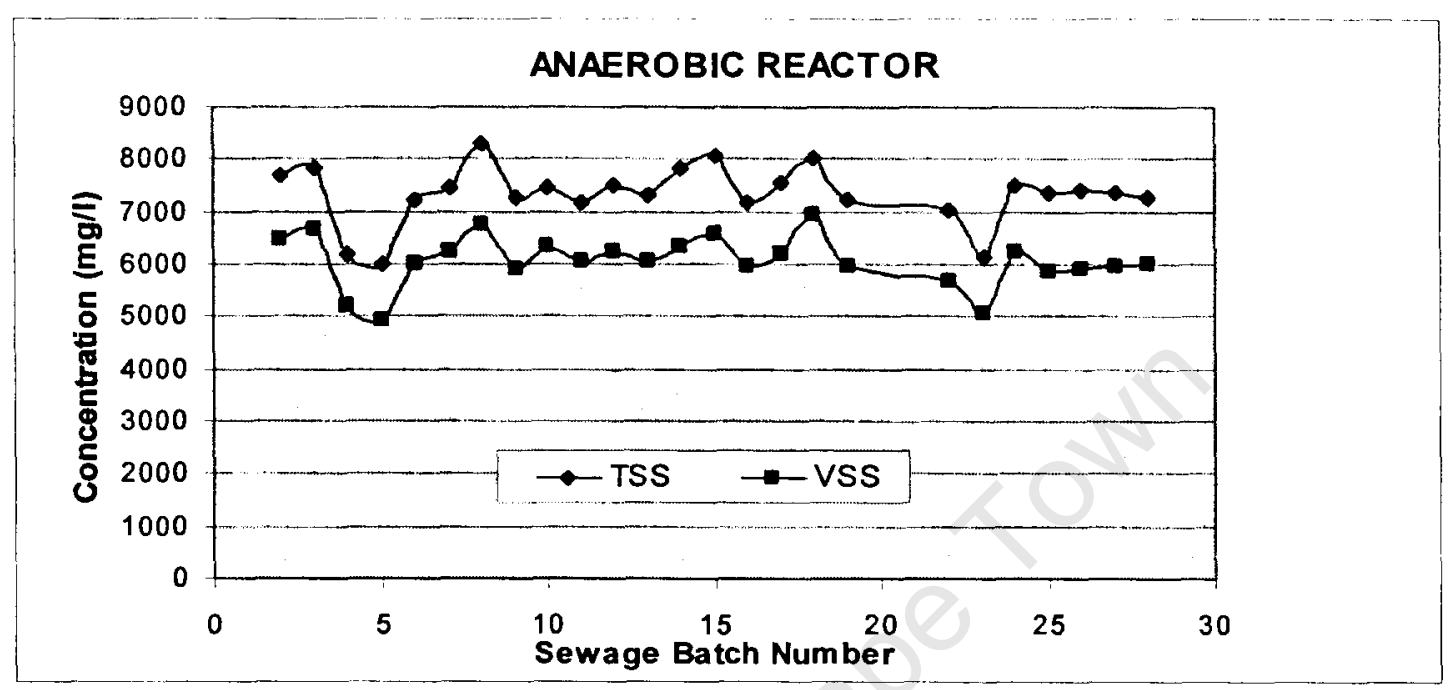

Figure 4.11.: Development of the mixed liquor total suspended solids (TSS) and the volatile suspended solids (VSS) concentrations in the anaerobic reactor.

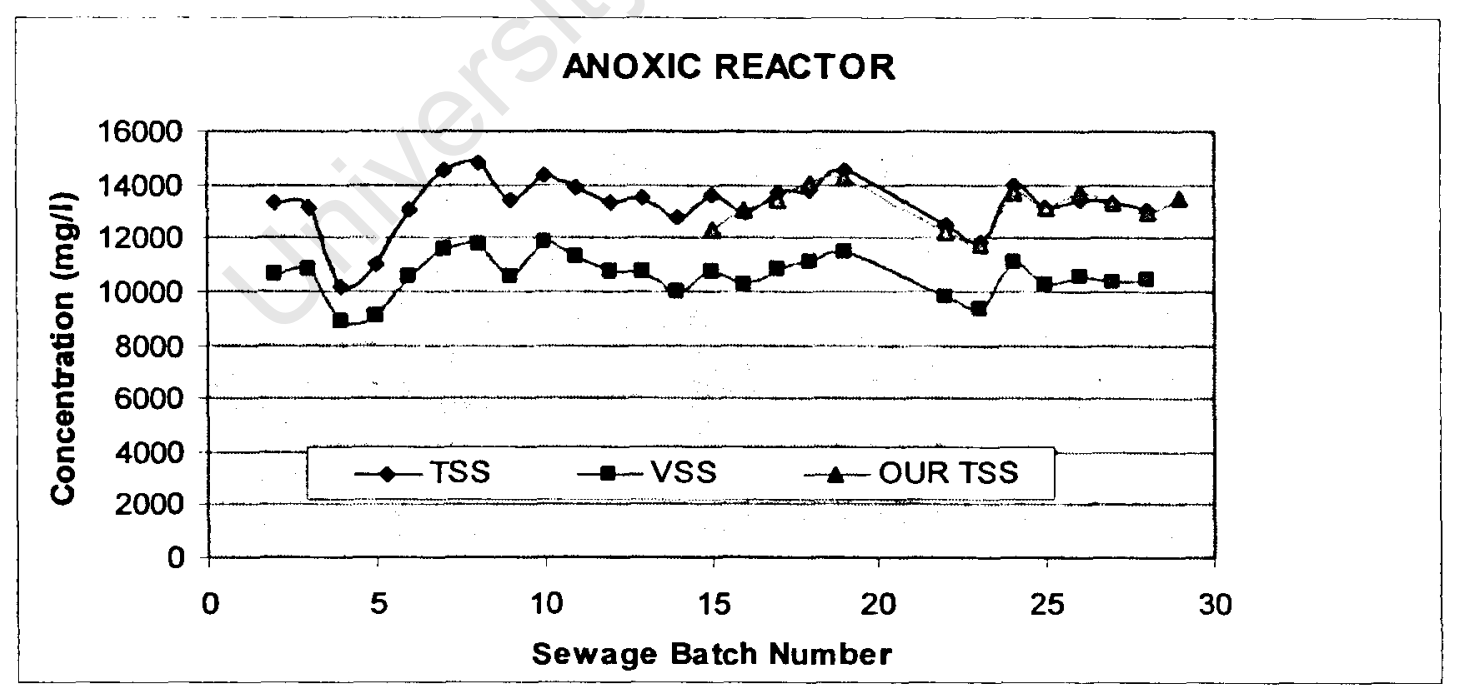

Figure 4.12.: Development of the mixed liquor total suspended solids (TSS) and the volatile suspended solids (VSS) concentrations in the anoxic and reaerobic reactors. 
AEROBIC REACTOR

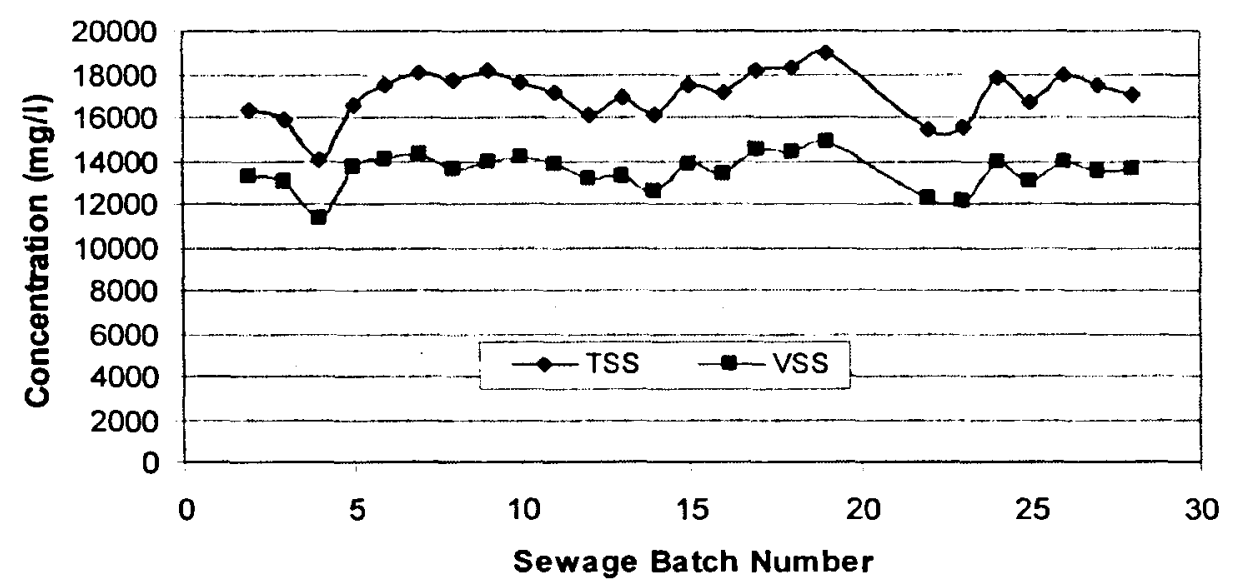

Figure 4.13.: Development of the mixed liquor total suspended solids (TSS) and the volatile suspended solids (VSS) concentrations in the aerobic reactor.

In an MBR system, mixed liquor distribution within the reactors is influenced directly by the recycles between reactors. In conventional systems the concentrations in the anoxic and aerobic reactors are very similar as the mixed liquor is concentrated after the aerobic reactor in the SST and then recycled to the anoxic reactor. In the MBR system, this concentration effect occurs within the biological reactor and thus there is a difference in concentration between the two reactors. This concentration difference is dependant on the a-recycle (Ramphao et al., 2004). The a-recycle measured in Phase 1 was marginally less than in Phase 2 (3.0 vs 3.5) and as a consequence the ratio between the TSS concentrations in the anaerobic and anoxic reactors was marginally less in Phase 2.

\subsubsection{COD/VSS Ratios}

The COD/VSS ratio $\left(f_{\mathrm{CV}}\right)$ was regularly measured for the aerobic reactor mixed liquor. The sewage batch average $f_{C V}$ values are plotted statistically in Fig. 4.14 giving a mean average of 1.40 (SSD $=0.059$. This $f_{C V}$ value is close to the theoretically estimated $f_{C V}$ of 1.42 (WRC, 1984) and is within the range of values $(1.3$ - 1.5) previously found in laboratory experiments in the Water Research Laboratory (WRL), (e.g. 1.37 Lee et al., 2002a; 1.42 Sneyders et al., 1998; 1.45 Mellin et al., 1998) for conventional systems. Further, the value is close to that measured in Phase 1, of 1.35 (Ramphao et al., 2004), and confirms that the membranes do not significantly influence this value. 


\section{Batch COD to VSS ratio (fcv) in MBR}

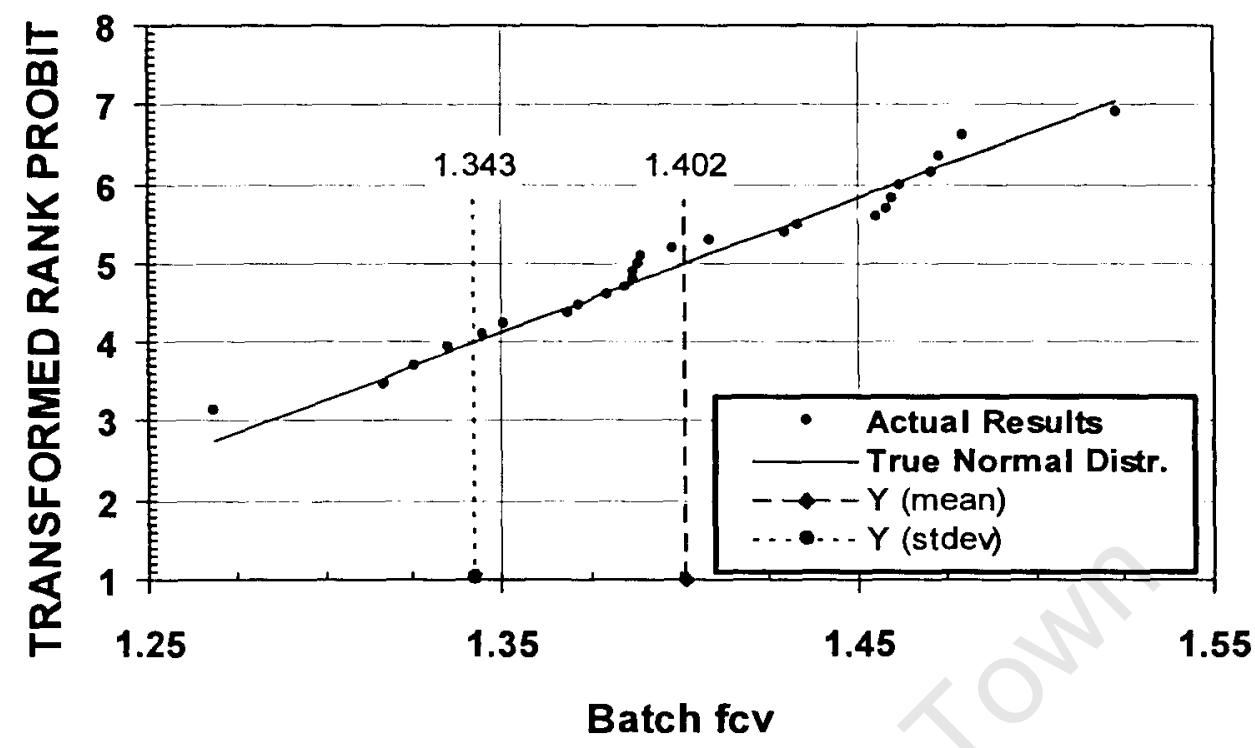

Figure 4.14.: Statistical plot of the sewage batch average COD to VSS ratio $\left(f_{C V}\right)$ in the MBR system

\subsubsection{System VSS/TSS Ratios}

The possibility that the membranes influence the VSS/TSS ratio $\left(f_{i}\right)$ of the mixed liquor through non-selective retention of all ISS was investigated. The ratio of VSS to TSS was measured in all three reactors giving investigation averages of 0.82 (anaerobic), 0.81 (anoxic) and 0.79 (aerobic). The difference in values for this ratio between reactors is expected and attributed to the release of inorganic phosphate and cations by PAO's in the anaerobic reactor, decreasing the inorganic content of the mixed liquor in that reactor (high $f_{i}$ ) and the uptake of phosphate and cations in the anoxic (marginal) and aerobic reactors which reincorporate inorganics in the mixed liquor (lower $\mathrm{f}_{\mathrm{i}}$ ). Throughout the investigation the VSS values in all three reactors tracked the TSS values consistently, Fig. 4.15. 


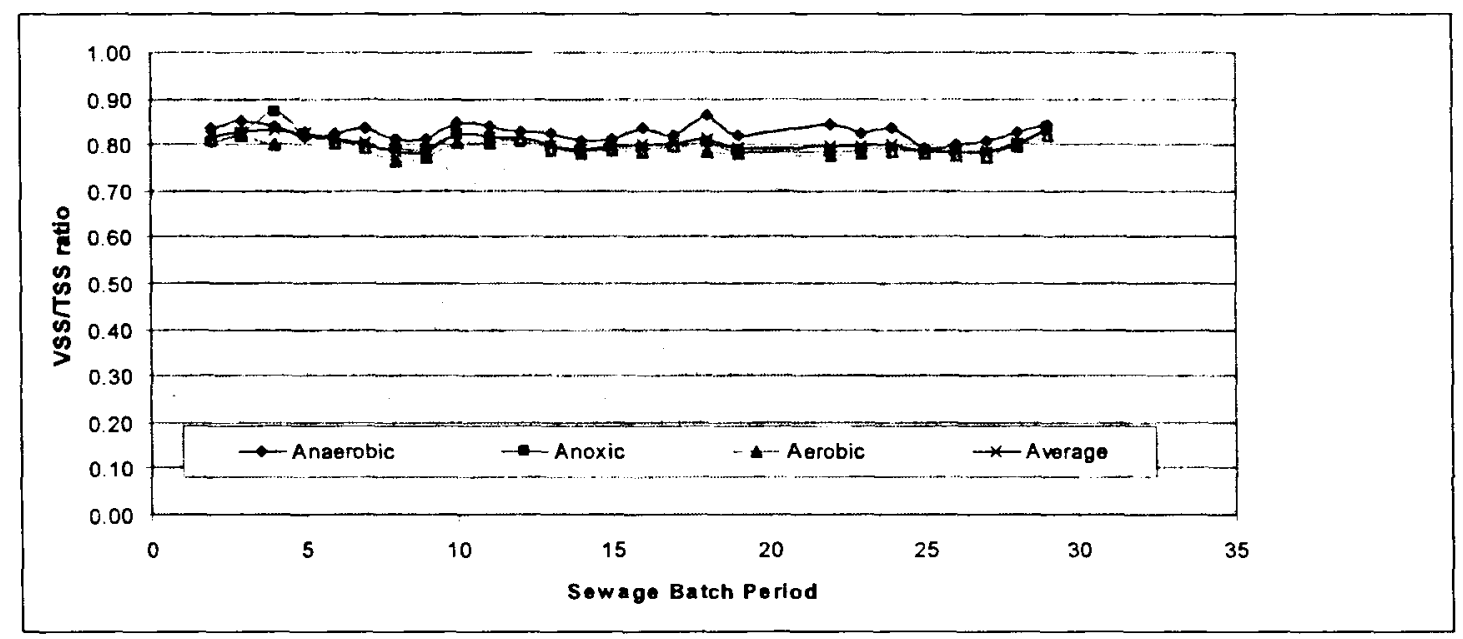

Figure 4.15: Comparison of the VSS/TSS ratios $\left(f_{i}\right)$ of the sludge in the anaerobic, anoxic and aerobic reactors.

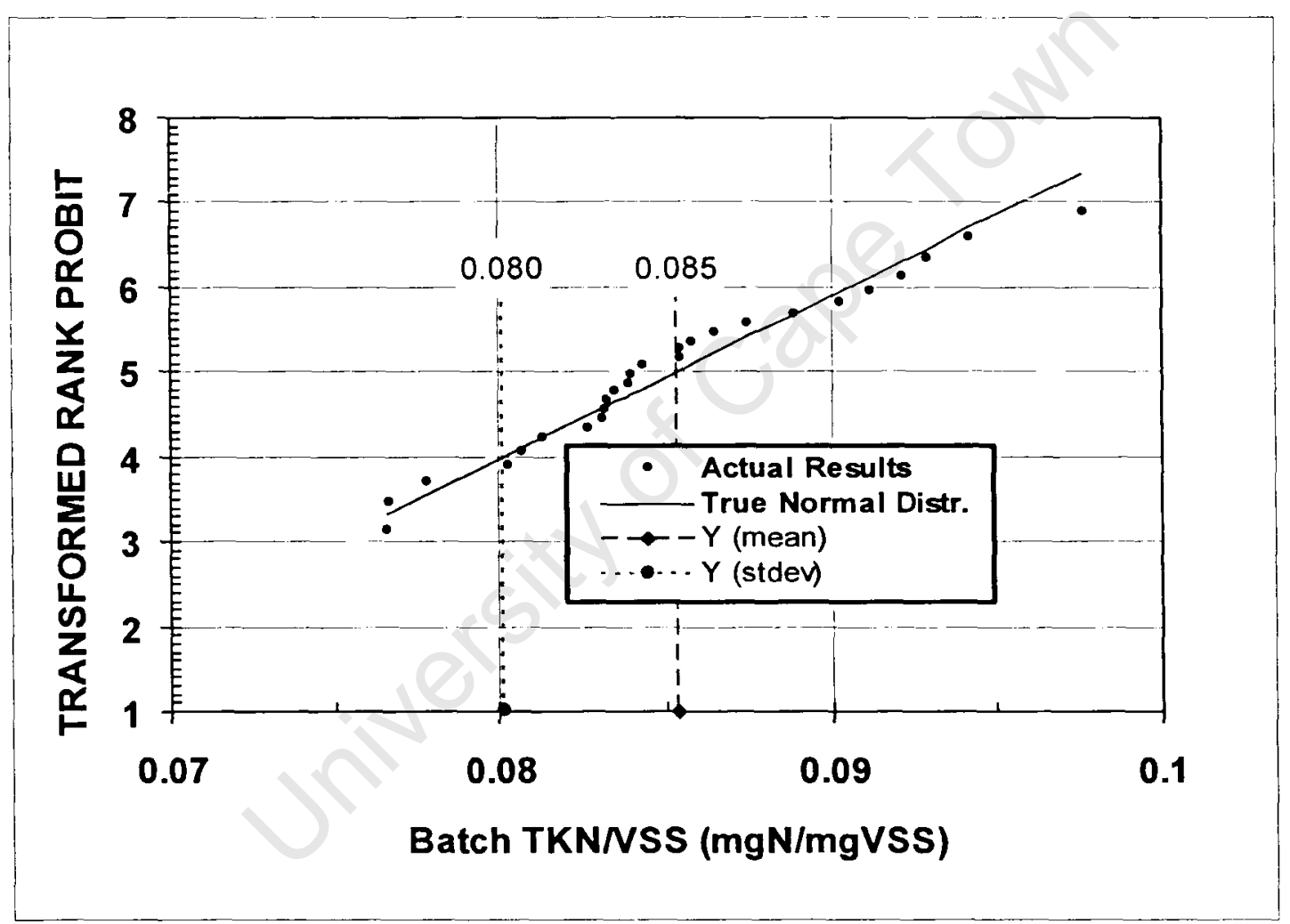

Figure 4.16: The statistical distribution of the daily VSS/TSS ratio (fi) in the MBR system.

The daily weighted average $f_{i}$ ratios of the three reactors are normally distributed (Fig. 4.16) with the mean $0.809(\mathrm{SSD}=0.028)$, which is close to the value determined in Phase 1 (mean $=0.791$ ). These values are higher than that expected $(\sim 0.75)$ for a BNR system accumulating significant biomass ISS through the intracellular storage of polyphosphate and associated counter ions. As noted in Phase 1 (Ramphao et al., 2004), this suggests that the membranes do not promote selective accumulation of ISS but rather may promote selective accumulation of organics as VSS. This will be examined later in the chapter. 


\subsubsection{System TKN/VSS Ratios}

The nitrogen content of the sludge was measured by the TKN/VSS ratio of the aerobic reactor mixed liquor. The results were fairly consistent throughout the test period and were normally distributed with a mean of $0.085(\mathrm{SSD}=0.005) \mathrm{mgN} / \mathrm{mgVSS}$, Fig. 4.17, which is close to the mean of 0.079 measured in Phase 1, (Ramphao et al., 2004). The mean does differ from the value of $0.10 \mathrm{mgTKN} / \mathrm{mgVSS} / \ell$ typically accepted for activated sludge mixed liquor (WRC,1984, Henze et al, 1987). However, as noted in Ramphao et al. (2004) the mean is very close to values of 0.086 .0 .086 and 0.083 obtained by Beeharry et al. (2001), Sneyders et al. (1998) and et al. (2002a) respectively for mixed liquor treating wastewaters from the same source (Mitchells Plain WWTP).

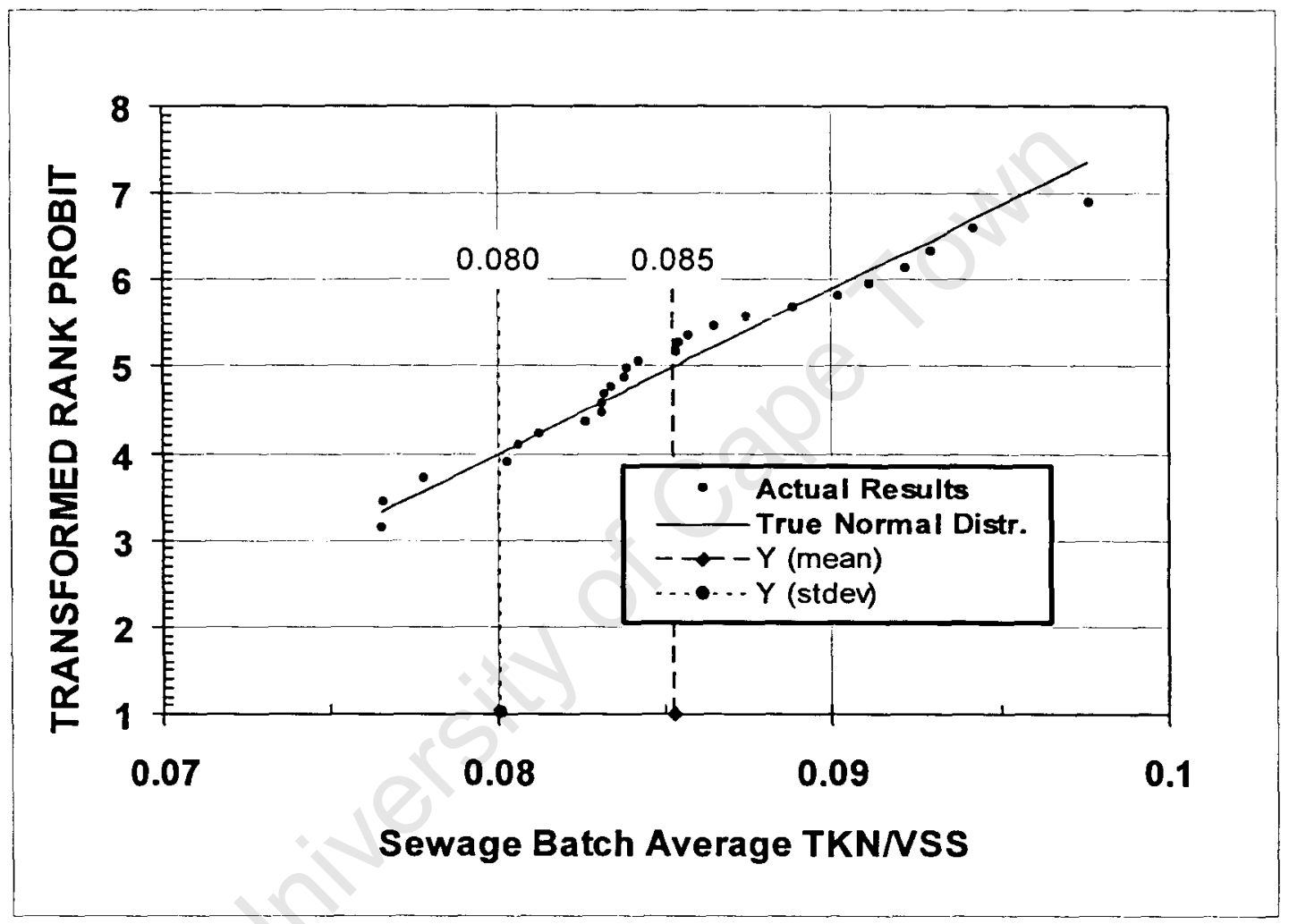

Figure 4.17: TKN/VSS ratio in the aerobic reactor.

The system mixed liquor solids were measured with three independent techniques, the VSS, COD and TKN. That the ratios between these measurements conform reasonably closely to theoretical and previously determined values provides substantive support that the mixed liquor solids have been accurately quantified.

\subsubsection{Sludge Production}

Sludge production is measured as the mass of MLVSS produced per unit influent COD load per day. Two methods were used to determine the sludge production, differing only in the method of calculating the sludge produced: the first calculated the sludge produced by the mass of sludge measured in the system divided by the sludge age, the second measured sludge produced by the mass of sludge wasted daily (Equations 4.8 and 4.9). The results from both methods were very close for the 
average of the sewage batches averages. The sludge production in the MBR UCT system varied over the study with a range of $0.24-0.39$ and a mean of $0.311 \mathrm{mgVSS} / \mathrm{mgCOD}$ (SSD $=0.035 \mathrm{mgVSS} / \mathrm{mgCOD}$ ) as shown in Fig. 4.18. This is very close to the sludge production observed in Phase 1 (Ramphao et al. 2004) of $0.32 \mathrm{mgVSS} / \mathrm{mgCOD}$, and indicates that the lower MLSS concentrations observed in Phase 2 were indeed due to lower influent COD in the system in Phase 2.

$$
\begin{aligned}
M L_{(\text {production })} & =\frac{M X_{v}}{R_{s .}\left(M S_{t i}-M S_{t e}\right)} \quad \text { or, } \\
M L_{(\text {production })} & =\frac{M X_{v(\text { waste })}}{\left(M S_{t i}-M S_{t e}\right)}
\end{aligned}
$$

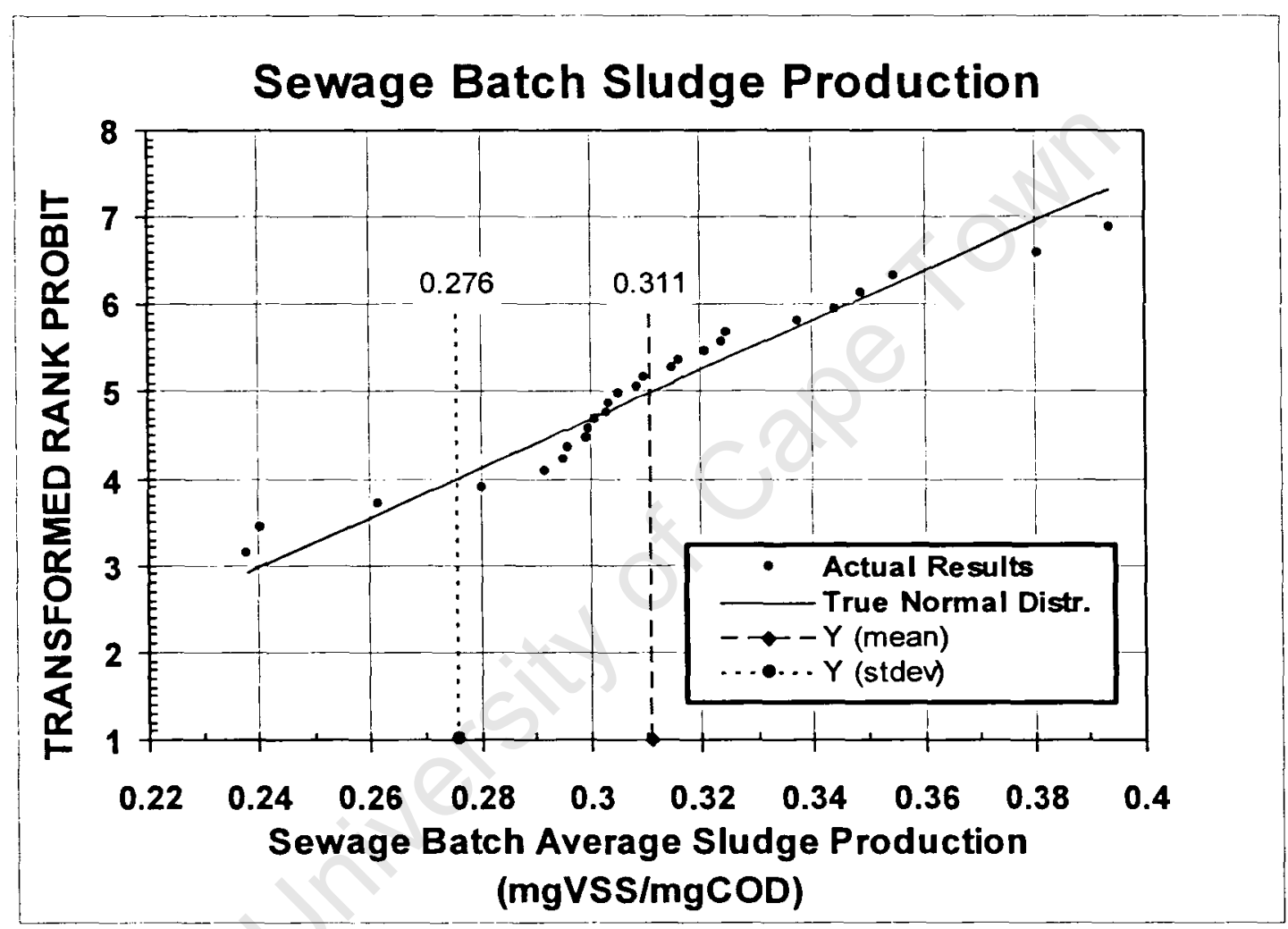

Figure 4.18: Statistical plot of the sewage batch average sludge production in the MBR system.

\subsubsection{System Mixed Liquor Settleability and Microbial Analysis}

MBR systems do not require mixed liquor to settle well due to the solid-liquid separation process occurring via membranes. However, in order to compare the settleability of the mixed liquor in the MBR system with the mixed liquor generated in the parallel conventional activated sludge system, diluted sludge volume index (DSVI) tests were conducted on the aerobic reactor mixed liquor in accordance to the method described in Chapter 3, Section 3.5.1.

Throughout the investigation the DSVI ranged consistently between 80 and 125 $\mathrm{ml} / \mathrm{gTSS}$ (Fig 4.19). The only exception to this trend was a higher DSVI of 
$142 \mathrm{ml} / \mathrm{gTSS}$ which was measured on day 429 . No event could be identified to account for this high value.

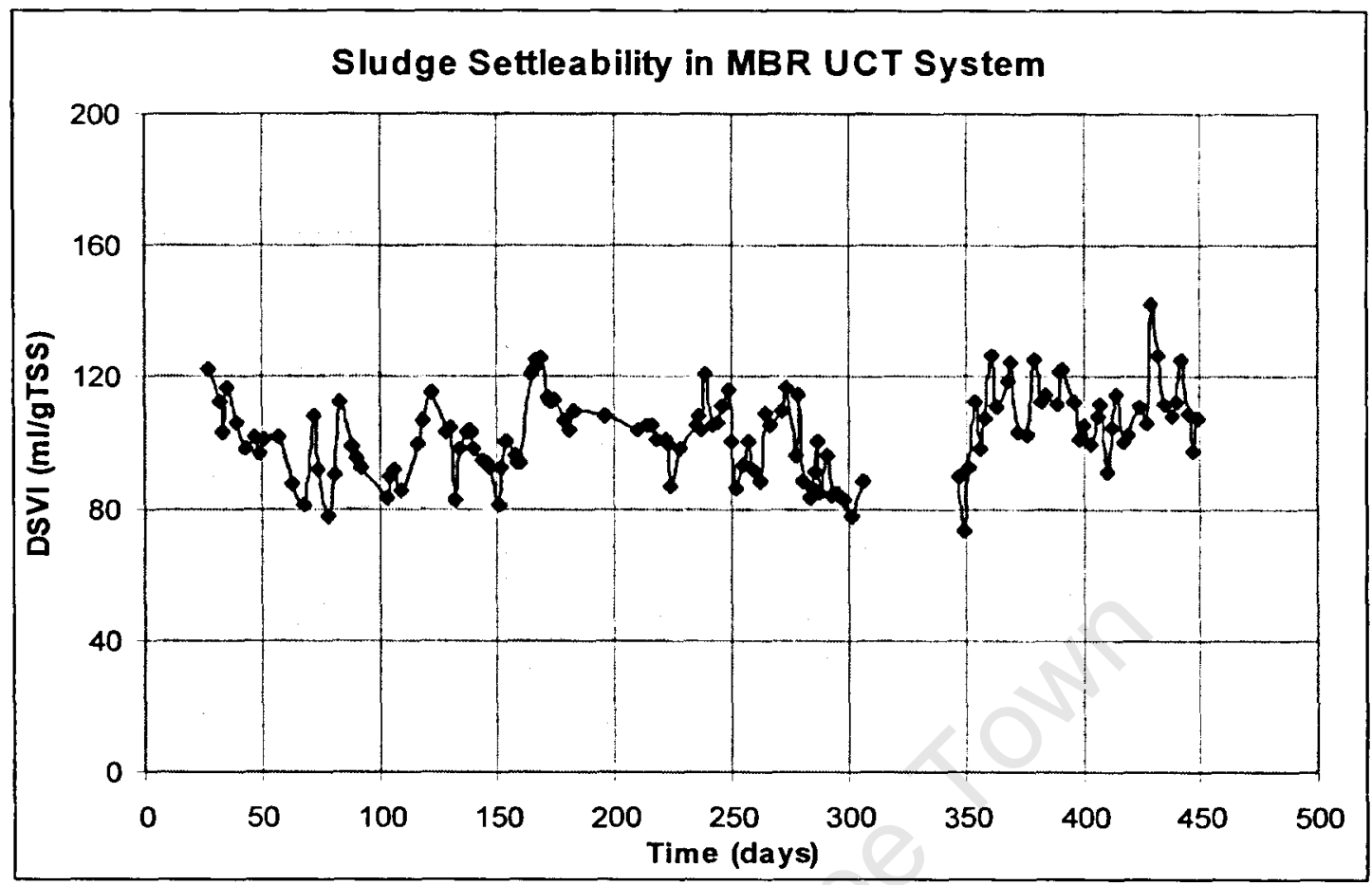

Figure: 4.19: General trend of diluted sludge volume index (DSVI for the MBR system)

During the investigation anoxic-aerobic (AA) filaments (M. Parvicella and Type 0092) were commonly found in the wastewater. As was noted in Phase 1 (Ramphao et al., 2004) these filaments relative abundance appeared to be linked to variations in the sludge settleability. A summary of the monthly, and from March 2006 fortnightly microbiological analyses for the MBR system is presented in Table 4.5. The microbial analysis was compared with the measured DSVI and although the DSVI remained relatively stable $(80-120 \mathrm{ml} / \mathrm{gTSS})$ there is an apparent trend for compact flocs to give lower DSVI $(<100 \mathrm{ml} / \mathrm{gTSS})$ and diffuse flocs a marginally higher DSVI $(>100 \mathrm{ml} / \mathrm{gTSS})$ as would be expected. It is important to note that in a lab scale MBR such as the one run in this investigation the aerobic reactor is very turbulent due to the membrane configuration and coarse bubble aeration, thus shear conditions are not conducive to large flocs forming as was observed in the microbiological analysis.

From August 2005 fortnightly samples were sent to the Microbiological laboratories at the Durban Institute of Technology for quantification of bacteria using Fluorescence In Situ Hybridization (FISH) and confocal laser scanning microscopy (CLSM). The microbial investigation, reported in Maharaj et al. (2006), concluded from the experiments that the membrane separation itself does not influence the nitrifying community composition. The composition and heterogeneity of the ammonia-oxidizing bacteria (AOB) population and its associated process stability appear to be dependent on other factors like wastewater composition and sludge age. Nitrite oxidizing bacteria (NOB) were in very low numbers, suggesting that they possibly had a higher affinity for metabolism rather than reproduction. It is hypothesized by Maharaj et al. (2006) that this function could also be assigned to other groups within the activated sludge population. 
Table 4.5: MBR system microbiological analyses recorded with system observations and DSVI.

\begin{tabular}{|c|c|c|c|c|c|c|c|c|c|}
\hline \multicolumn{10}{|c|}{ MBR system } \\
\hline \multirow[t]{2}{*}{ Date } & \multirow[t]{2}{*}{ Day $\#$} & \multirow[t]{2}{*}{$\begin{array}{c}\text { Sewaye } \\
\text { Batch }\end{array}$} & \multirow[t]{2}{*}{ Morfology floc } & \multirow[t]{2}{*}{$\begin{array}{c}\text { diameter } \\
\text { (umi) }\end{array}$} & \multicolumn{3}{|c|}{ Filamemous micing } & \multirow[t]{2}{*}{ DSVI } & \multirow[t]{2}{*}{ observations } \\
\hline & & & & & & Rank & Abundance & & \\
\hline $2005 / 03 / 24$ & 42 & 3 & round, compact & $<150$ & M.Panvicella & 1 & 2 & 122 & $\begin{array}{c}\text { foaming in AX } \\
\text { reactor }\end{array}$ \\
\hline $2005 / 14 / 21$ & 70 & 4 & $\begin{array}{l}\text { firm, round, } \\
\text { compact }\end{array}$ & $<150$ & Type 0092 & 1 & 4 & 90 & $\begin{array}{l}\text { foaming in } A X \text {, low } \\
\text { solids due to spills }\end{array}$ \\
\hline $2005 / 05 / 23$ & 102 & 6 & $\begin{array}{l}\text { firm, round, } \\
\text { compact }\end{array}$ & $150-500$ & Type 0092 & 1 & 3 & 84 & $n$ \\
\hline $2005: 06 / 22$ & 132 & 8 & $\begin{array}{l}\text { firm, round, } \\
\text { compact }\end{array}$ & $150-500$ & $\begin{array}{c}\text { M.Parvicella } \\
\text { Type } 0092\end{array}$ & $\begin{array}{l}2 \\
1\end{array}$ & $\begin{array}{l}2 \\
4\end{array}$ & 83 & $\mathrm{n}$ \\
\hline $2005 / 07 / 22$ & 162 & 10 & $\begin{array}{c}\text { firm, round, } \\
\text { compact }\end{array}$ & 150.500 & $\begin{array}{c}\text { M. Parvicella } \\
\text { Type } 0092\end{array}$ & $\begin{array}{l}2 \\
1 \\
\end{array}$ & $\begin{array}{l}3 \\
4 \\
\end{array}$ & 94 & $\begin{array}{l}\text { spills, low solids } \\
\text { conc. }\end{array}$ \\
\hline $2005 / 08 / 15$ & 186 & 11 & $\begin{array}{c}\text { weak, irregular. } \\
\text { diffuse }\end{array}$ & $<150$ & $\begin{array}{l}\text { M.Parvicella } \\
\text { Type } 0092\end{array}$ & $\begin{array}{l}2 \\
1 \\
\end{array}$ & $\begin{array}{l}2 \\
3\end{array}$ & 108 & $n$ \\
\hline $2005 / 09 / 19$ & 221 & 14 & $\begin{array}{l}\text { weak, irregular, } \\
\text { diffuse }\end{array}$ & $<150$ & $\begin{array}{l}\text { M.Panvicella } \\
\text { Type } 0092\end{array}$ & $\begin{array}{l}2 \\
1 \\
\end{array}$ & $\begin{array}{r}2 \\
2 \\
\end{array}$ & 100 & $n$ \\
\hline $2005 / 10 / 17$ & 249 & 16 & irregular, diffuse & $150-500$ & $\begin{array}{l}\text { M.Parvicella } \\
\text { Type } 0092 \\
\end{array}$ & $\begin{array}{l}1 \\
2 \\
\end{array}$ & $\begin{array}{l}4 \\
1 \\
\end{array}$ & 116 & $n$ \\
\hline $2005 / 11 / 29$ & 292 & 18 & $\begin{array}{c}\text { weak, irregular, } \\
\text { diffuse }\end{array}$ & $<150$ & $\begin{array}{l}\text { M.Panicella } \\
\text { Type } 0092\end{array}$ & $\begin{array}{l}2 \\
1 \\
\end{array}$ & $\begin{array}{l}2 \\
3 \\
\end{array}$ & 90 & $n$ \\
\hline $2006 / 01 / 24$ & 348 & 22 & $\begin{array}{l}\text { weak, irregular, } \\
\text { diffuse }\end{array}$ & $150-500$ & $\begin{array}{l}\text { M.Parvicella } \\
\text { Type } 0092\end{array}$ & $\begin{array}{l}1 \\
1 \\
\end{array}$ & $\begin{array}{l}2 \\
2 \\
\end{array}$ & 81 & $n$ \\
\hline $2006 / 0207$ & 362 & 23 & $\begin{array}{l}\text { weak, irregular. } \\
\text { diffuse }\end{array}$ & $<150$ & $\begin{array}{l}\text { M.Panicella } \\
\text { Type } 0092\end{array}$ & $\begin{array}{l}1 \\
2\end{array}$ & $\begin{array}{l}3 \\
3\end{array}$ & 198 & $\begin{array}{l}\text { major spills and } \\
\text { loss of solids }\end{array}$ \\
\hline $2006 / 02 / 28$ & 383 & 24 & $\begin{array}{l}\text { weak, irregular, } \\
\text { diffuse }\end{array}$ & $<150$ & $\begin{array}{l}\text { M.Parvicella } \\
\text { Type } 0092\end{array}$ & $\begin{array}{l}1 \\
1 \\
\end{array}$ & $\begin{array}{l}2 \\
2 \\
\end{array}$ & 113 & $\begin{array}{l}\text { fo aming in } A X \text {, } \\
\text { power failures }\end{array}$ \\
\hline $2006 / 03 / 15$ & 398 & 26 & round, compact & $<150$ & $\begin{array}{l}\text { M. Fanvicelia } \\
\text { Type } 0092\end{array}$ & $\begin{array}{l}2 \\
1 \\
\end{array}$ & $\begin{array}{l}2 \\
3 \\
\end{array}$ & ivi & it \\
\hline $2006 / 03 / 28$ & 411 & 27 & round, compact & $<150$ & $\begin{array}{l}\text { M.Parvicella } \\
\text { Type } 0092\end{array}$ & $\begin{array}{l}2 \\
1 \\
\end{array}$ & 0 & 97 & $\mathrm{n}$ \\
\hline $2006 / 04 / 11$ & 425 & 28 & irregular, diffuse & $<150$ & $\begin{array}{l}\text { M.Parvicella } \\
\text { Type } 0092\end{array}$ & $\begin{array}{l}1 \\
2\end{array}$ & $\begin{array}{l}3 \\
3\end{array}$ & 110 & $n$ \\
\hline $2006 / 04 / 25$ & 439 & 29 & $\begin{array}{c}\text { weak, irregular, } \\
\text { diffuse }\end{array}$ & $<150$ & $\begin{array}{l}\text { M. Panicella } \\
\text { Type } 0092\end{array}$ & $\begin{array}{l}1 \\
2 \\
\end{array}$ & $\begin{array}{l}2 \\
2 \\
\end{array}$ & 110 & $n$ \\
\hline
\end{tabular}

\subsection{MASS BALANCES}

Nitrogen and COD mass balances were performed for each sewage batch period in order to verify the accuracy and reliability of the analytical data, and to provide an early warning sign for data collection error. The mass balance procedure is described in Appendix $\mathrm{C}$ and attempts to account for all $\mathrm{N}$ or COD entering and exiting the system, based on the assumption that at steady state the measured $\mathrm{N}$ and COD entering the system in the influent should equal the measured $N$ and COD exiting the system through the effluent stream, sludge wasted, oxygen utilised and nitrogen denitrified. As above, sewage batches were accepted as steady state periods, and the sewage batch averages used to calculate the $N$ and COD mass balances. The COD and $\mathrm{N}$ mass balances obtained are listed in Table 4.6.

A mass balance should fall within the range of $90 \%-110 \%$ to indicate accurate measurements. Mass balances outside this range do not imply that all the data is poor, but simply indicate that one or more of the parameters measured is incorrect and needs to be interpreted with caution. In this study it was not possible to close the mass balances for the system for a number of sewage batches due to two main problems:

- Errors with the OUR measurement were detected early in the investigation but only solved during sewage batch 8 . Thus, the COD mass balance could not be performed for sewage batches 2 to 8 . The changed measurement procedure of OUR is described in detail in Chapter 3, Section 3.8.1. 
- Analytical nitrate $\left(\mathrm{NO}_{3}\right)$ and nitrite $\left(\mathrm{NO}_{2}\right)$ measurements appeared to be very low during the first sewage batches. It was suspected that a problem lay with the in-house Technicon auto-analyser used for these measurements. While this was being investigated and repaired, samples were processed on an external high pressure liquid chromatograph (HPLC), until accurate $\mathrm{NO}_{3}$ values were again obtained with the in-house Technicon auto-analyser. This meant that for sewage batches 2 to 8 inaccurate low $\mathrm{NO}_{3}$ values were probably the cause for low $\mathrm{N}$ mass balances. This would have implications for the COD balance, but as noted above COD mass balances could not be performed until sewage batch 8.

Table 4.6: $\quad$ Nitrogen and COD mass balances for the MBR system

\begin{tabular}{|c|c|c|}
\hline $\begin{array}{c}\text { Sewage } \\
\text { Batch Period }\end{array}$ & $\%$ Nitrogen Balance & $\%$ COD Balance \\
\hline 2 & $152.3 \%$ & No OUR Measurement \\
\hline 3 & $112.1 \%$ & No OUR Measurement \\
\hline 4 & $72.5 \%$ & No OUR Measurement \\
\hline 5 & $74.4 \%$ & No OUR Measurement \\
\hline 6 & $75.4 \%$ & No OUR Measurement \\
\hline 7 & $93.4 \%$ & No OUR Measurement \\
\hline 8 & $86.8 \%$ & No OUR Measurement \\
\hline 9 & $76.8 \%$ & $107.6 \%$ \\
\hline 10 & $101.4 \%$ & $98.3 \%$ \\
\hline 11 & $105.0 \%$ & $98.0 \%$ \\
\hline 12 & $96.4 \%$ & $107.4 \%$ \\
\hline 13 & $82.0 \%$ & $125.7 \%$ \\
\hline 14 & $96.6 \%$ & $113.4 \%$ \\
\hline 15 & $95.2 \%$ & $106.6 \%$ \\
\hline 16 & $91.7 \%$ & $108.4 \%$ \\
\hline 17 & $79.7 \%$ & $102.4 \%$ \\
\hline 18 & $88.3 \%$ & $112.4 \%$ \\
\hline 19 & $74.5 \%$ & $103.7 \%$ \\
\hline 20 & No testing & No testing \\
\hline 21 & No testing & No testing \\
\hline 22 & $102.1 \%$ & $93.0 \%$ \\
\hline 23 & $89.5 \%$ & $93.1 \%$ \\
\hline 24 & $118.5 \%$ & $123.8 \%$ \\
\hline 25 & $148.2 \%$ & $103.6 \%$ \\
\hline 26 & $96.1 \%$ & $93.5 \%$ \\
\hline 27 & $108.8 \%$ & $89.1 \%$ \\
\hline 28 & $113.0 \%$ & $84.9 \%$ \\
\hline 29 & $83.2 \%$ & $93.5 \%$ \\
\hline Average & $96.5 \%$ & $103.1 \%$ \\
\hline
\end{tabular}

Additionally it was observed that regularly the concentration of $\mathrm{NO}_{3}$ in the aerobic reactor was substantially lower than that of the effluent, whereas these should be the same. Kinetic studies indicated that the rate of denitrification in the concentrated mixed liquor was substantially faster than that in a conventional activated sludge system suggesting that denitrification was occurring between the sampling of the mixed liquor and its filtration. Hence, for the purposes of mass balances the effluent 
$\mathrm{NO}_{3}$ concentration has been used in place of the aerobic $\mathrm{NO}_{3}$ concentration measured. Mouthon-Bello and Zhou (2005) reported the same phenomena of significant differences in $\mathrm{NO}_{3}$ concentration between the aerobic supernatant and membrane permeate. They attributed this difference to the dynamic biological layer that forms on the membrane surface, but suggested that handling times prior to centrifuging could also be responsible for the differences.

\subsubsection{Nitrogen Mass Balance}

Nitrogen Mass balances for each sewage batch are listed in Table 4.6 and shown graphically in Fig. 4.17. Values ranged from 72.5 to $152 \%$ with an average of $96.5 \%$. Throughout the investigation the $\mathrm{N}$-mass balance varied with the majority $(\sim 60 \%)$ falling outside the acceptable $90-110 \%$ range. Early on in the study the large variance in $\mathrm{N}$-mass balances caused concern and subsequent efforts were made to identify reasons for the poor $\mathrm{N}$-mass balances. However despite continued efforts the variance continued. A number of reasons for the poor $\mathrm{N}$-mass balances are suggested in order of likelihood of taking place in this investigation:

- The N-balance is very sensitive to the a-recycle value. These recycles were measured on a weekly basis, but at different times to when samples were laken. The recycles could vary greatly throughout a day depending on the condition of piping, occurrence of minor blockages and the pressure head difference between the level of the feed tanks and the anaerobic reactor. For example changing the a-recycle by 0.5 from say $3: 1$ to $3.5: 1$ would increase the $\mathrm{N}$-balance by $6-10 \%$. Such a variation in the a-recycle is possible as is suggested by the differences between measured and calculated a-recycles in a number of batches (see Table 4.3).

- Sewage batches were shorter than the sludge age of the system, which could have caused brief periods where the system was not at steady state and may have influenced results.

- At high MLSS concentrations ( $>15000 \mathrm{mgTSS} / \ell$ ) other researchers have reported the incidence of anoxic micro-zones forming in the aerobic reactor (Kraume et al., 2005), whereby dissolved oxygen does not distribute to all flocs forming small anoxic zones where denitrification can occur. This would result in loss of nitrogen in the aerobic reactor and a drop in the N-balance.

The variation in the $\mathrm{N}$-balance results is very scattered on either side of $100 \%$ suggesting that the error in measurement was not a systematic one. The overall $\mathrm{N}$ mass balance is $96.5 \%$ and as a consequence, for overall analysis, the whole investigation average will be used.

Sewage batch periods in which specific conditions can explain poor mass balances are as follows: Sewage batch 2 is very high and is attributed to the familiarization of the writer with the testing regime. As noted above, the results in sewage batches 2 to 8 were subject to poor $\mathrm{NO}_{3}$ values and appear to bring down the mass of nitrogen leaving the system suggesting that problems in measuring $\mathrm{NO}_{3}$ remained unresolved throughout that testing period. Sewage batches 24 and 25 were characterised by intermittent power failures in the laboratory which interrupted aeration, and may have influenced nitrification resulting in a higher effluent TKN concentration than usual which would increase the mass balance. 
Of the $\mathrm{N}$ entering the system approximately $57.0 \%$ exited the system through denitrification, $24.6 \%$ through sludge wasting, $1.5 \%$ via effluent $\mathrm{TKN}$ and $17.2 \%$ through $\mathrm{NO}_{3}$ in the effluent (Fig. 4.20).

In Phase 1 Ramphao et al. (2004) reported mass balances consistently in the $90 \%$ to $110 \%$ range giving an average of $103.5 \%$ with a resulting breakdown of denitrification (51\%), effluent TKN (3.1\%), effluent nitrate/nitrite $(21.8 \%)$ and waste sludge $(24.4 \%)$. The difference between the two phases, particularly in denitrification and effluent nitrate/nitrite, is attributed directly to a different a-recycle (3.0 in Phase 1 versus 3.45 in Phase 2), thus more nitrate was loaded on the anoxic reactor for denitrification in Phase 2.

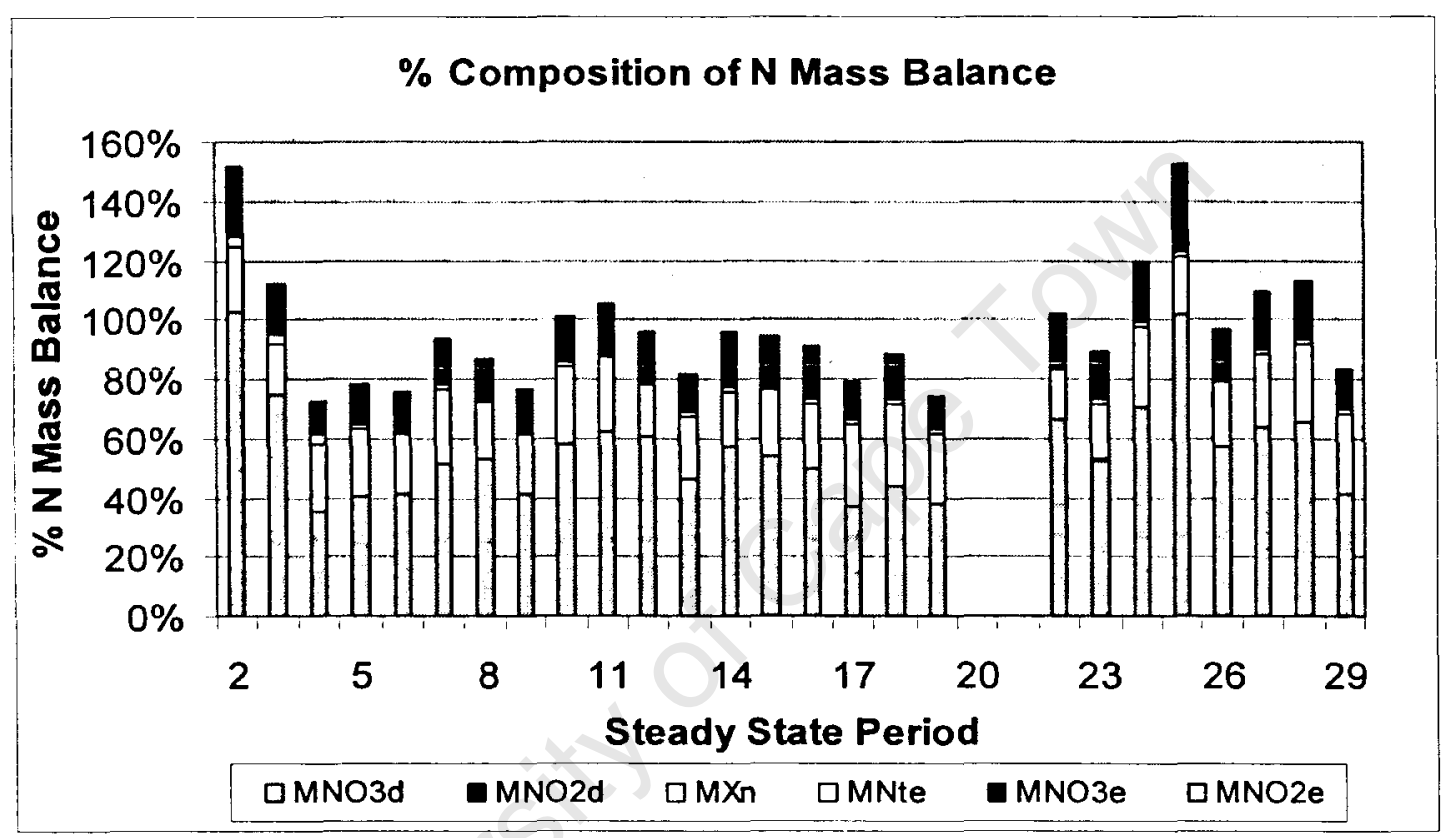

Figure 4.20: Percent composition of the Nitrogen Mass balance.

\subsubsection{COD Mass Balance}

As was noted earlier the COD mass balance could not be closed until sewage batch 8 due to difficulties in obtaining accurate readings for OUR in the aerobic reactor. COD mass balances for the duration of testing are listed in Table 4.6. Values from $85 \%$ to $126 \%$ were observed with 6 out of the 19 mass balances falling outside the $90 \%$ to $110 \%$ range. The average was $103.1 \%$.

Of these outliers all but sewage batches 13 and 24 fall close to the $90 \%$ to $110 \%$ range. Sewage batch 24 was affected by the power failures due to which spillages, and irregular feeding led to an unsteady state condition. The COD mass balance in sewage batch 13 is high due to a very high oxygen demand for Nitrogen linked to high $\mathrm{NO}_{3}$ values which also affect the $\mathrm{N}$-balance. No reasons for these high values can be put forward.

From Fig. 4.21, of the COD entering the system approximately $37.8 \%$ exited the system as oxygen consumed, $17.3 \%$ via denitrification, $40.4 \%$ was removed with the mixed liquor wasted and $4.5 \%$ exited the system via the effluent stream. In Phase 1 
Ramphao et al. (2004) achieved a lower COD mass balance with an investigation average of $90.5 \%$. In Phase 1, of the COD exiting the system approximately $33.6 \%$ was consumed with oxygen, $15.8 \%$ was consumed with denitrification, $46.8 \%$ was removed with mixed liquor wasted and $3.8 \%$ left the system via the effluent stream. The system COD-removal composition is comparable for both Phases 1 and 2 of the investigation.

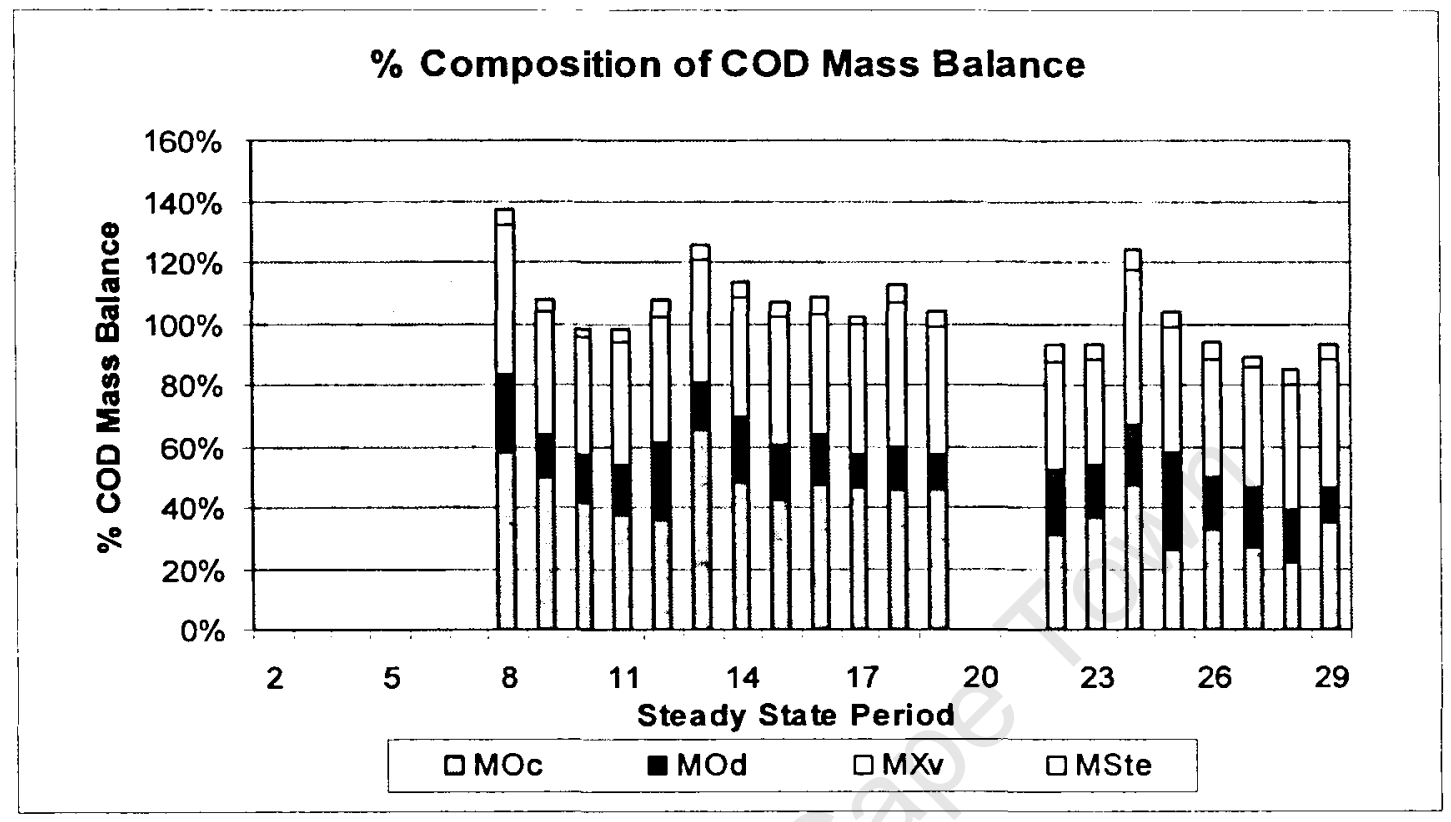

Figure 4.21: Percent composition of the COD- mass balance.

\subsection{INFLUENT UNBIODEGRADABLE COD FRACTIONS}

In Phase 1 , measured MLVSS concentrations, higher than predicted with UCTPHO simulations, prompted an investigation into the unbiodegradable soluble and particulate COD fractions of the influent feed. In order to simulate BNR systems the influent sewage needs to be characterised and values for the unbiodegradable soluble ( $\left.f_{\mathrm{S}, \text { us }}\right)$ and particulate $\left(\mathrm{f}_{\mathrm{S} \text {,up }}\right)$ COD fractions determined. In Phase 1 a much higher $\mathrm{f}_{\mathrm{S} \text {, up }}$ value was required to accurately simulate the system response. In order to substantiate the results from Phase 1, this exercise was repeated with the Phase 2 results.

\subsubsection{Unbiodegradable Soluble COD Fraction $\left(f_{\mathrm{S}, \mathrm{us}}\right)$}

It is assumed that all biodegradable soluble $\operatorname{COD}\left(\mathrm{S}_{\mathrm{bs}}\right)$ is utilised in the activated sludge system and thus any soluble COD that remains in the effluent $\left(\mathrm{S}_{\mathrm{te}}\right)$ must be unbiodegradable soluble $\left(\mathrm{S}_{\mathrm{us}}\right)$. Hence, Equation (4.8) can be used to determine $\mathrm{S}_{\mathrm{us}}$ as a fraction of the influent total $\operatorname{COD}\left(\mathrm{S}_{\mathrm{ti}}\right),\left(\mathrm{f}_{\mathrm{S}, \text { us }}\right)$ :

$$
\mathrm{f}_{\mathrm{S}, \mathrm{us}}=\mathrm{S}_{\mathrm{te}} / \mathrm{S}_{\mathrm{ti}}
$$

Batch averages of measured influent total $\operatorname{COD}\left(\mathrm{S}_{\mathrm{ti}}\right)$ and membrane filtered effluent $\operatorname{COD}\left(\mathrm{S}_{\mathrm{te}}\right)$ were used with Equation 4.8 to calculate $\mathrm{f}_{\mathrm{S} \text {,us. }}$. The sewage batch averages were plotted in a linearized probability graph (Fig. 4.22), giving an average $f_{S \text {, us }}$ of 0.044 , $(\mathrm{SSD}=0.009)$ (Table 4.6). This investigation average $\mathrm{f}_{\mathrm{S} \text {,us }}$ is slightly higher than that reported in Phase 1 (0.036), Ramphao et al. (2004). The influent feed 
(average $\mathrm{S}_{\mathrm{ti}}=951.8 \mathrm{mgCOD} / \ell$ ) was augmented with $200 \mathrm{mgCOD} / \ell$ sodium acetate, which is readily biodegradable. Thus, if the additional RBCOD is accounted for then the raw sewage from the Mitchells Plain WWTP would have a $f_{S, \text { us }}$ of 0.056 . This value lies in the lower limit of the range of typical values expected from South African wastewaters (WRC 1984). Also, it is well below other reported values from the same wastewater treatment plant cited in Ramphao et al. (2004): 0.085, Cronje et al (2000; 0.09, Ubisi et al (1997); 0.09, Mbewe et al (1995), and 0.096 Muller et al (2003).

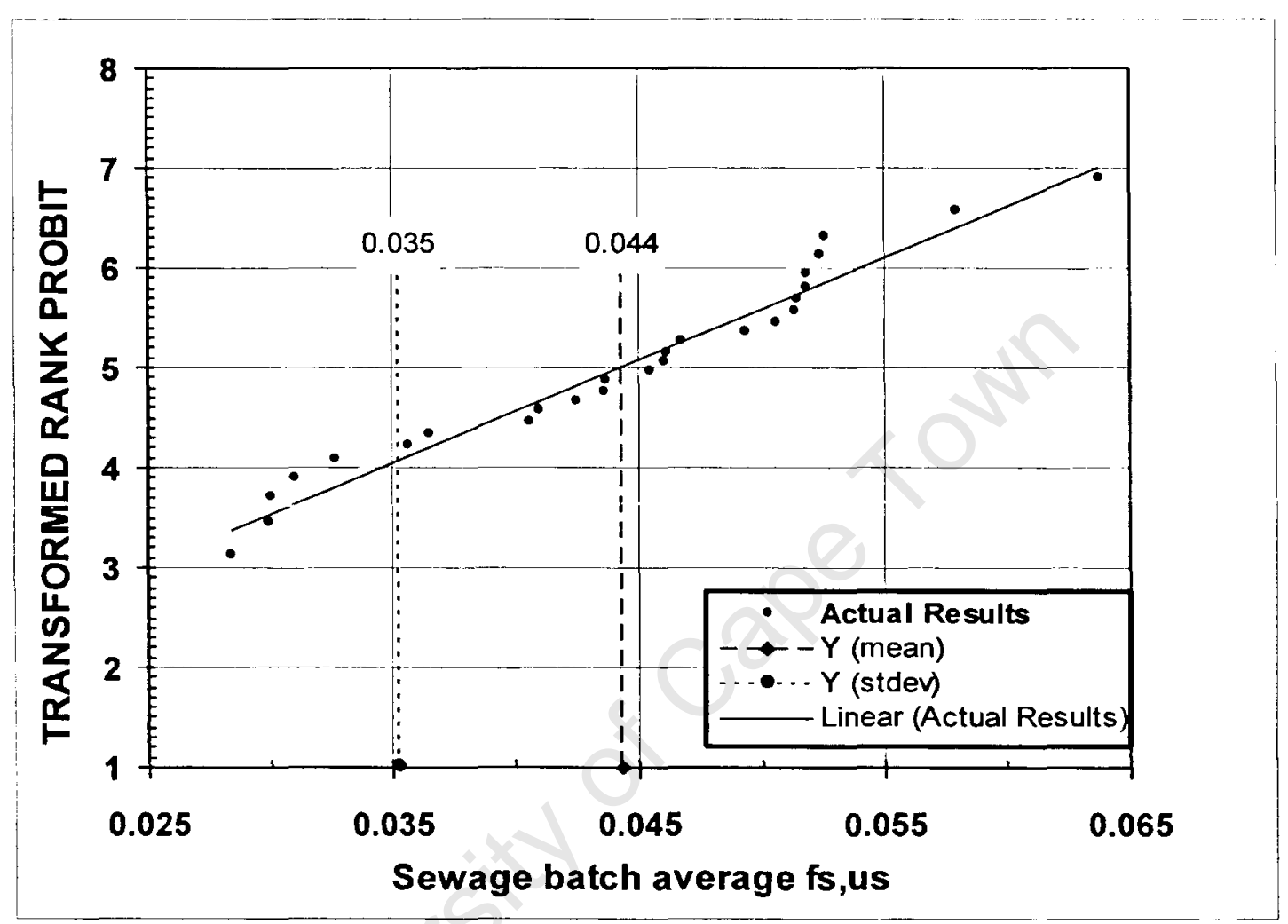

Figure 4.22: A statistical plot of the sewage batch average unbiodegradable $C O D$ fraction $\left(f_{S, u s}\right)$ of the influent feed. 
Table 4.7: $\quad$ Sewage batch unbiodegradable soluble $\left(f_{S, u s}\right)$ and particulate $\left(f_{S, u p}\right)$ fractions in the MBR UCT system.

\begin{tabular}{|c|c|c|}
\hline Batch Number & $\mathrm{f}_{\mathrm{s}, \text { us }}$ & $f_{s, \text { up }}$ \\
\hline 2 & 0.036 & 0.174 \\
\hline 3 & 0.029 & 0.087 \\
\hline 4 & 0.031 & 0.098 \\
\hline 5 & 0.039 & 0.144 \\
\hline 6 & 0.047 & 0.252 \\
\hline 7 & 0.037 & 0.269 \\
\hline 8 & 0.051 & 0.315 \\
\hline 9 & 0.042 & 0.184 \\
\hline 10 & 0.028 & 0.174 \\
\hline 11 & 0.041 & 0.185 \\
\hline 12 & 0.054 & 0.210 \\
\hline 13 & 0.046 & 0.208 \\
\hline 14 & 0.045 & 0.192 \\
\hline 15 & 0.042 & 0.218 \\
\hline 16 & 0.052 & 0.180 \\
\hline 17 & 0.030 & 0.222 \\
\hline 18 & 0.051 & 0.274 \\
\hline 19 & 0.051 & 0.195 \\
\hline 20 & & 0 \\
\hline 21 & & 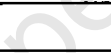 \\
\hline 22 & 0.058 & 0.127 \\
\hline 23 & 0.050 & 0.135 \\
\hline 24 & 0.064 & 0.340 \\
\hline 25 & 0.046 & 0.181 \\
\hline 26 & 0.049 & 0.168 \\
\hline 27 & 0.034 & 0.173 \\
\hline 28 & 0.046 & 0.236 \\
\hline 29 & 0.050 & 0.207 \\
\hline Average & 0.044 & 0.198 \\
\hline
\end{tabular}

The lower $f_{\mathrm{S} \text {,us }}$ determined here can be attributed to the finer pore size of the membranes which would retain colloidal and particulate COD that may otherwise have passed through the standard $0.45 \mu \mathrm{m}$ filter paper used to determine $S_{\text {us }}$ in conventional systems with SSTs. Hence, as noted in Phase 1 (Ramphao et al., 2004) the MBR system can be expected to attain a lower effluent COD concentration than the corresponding conventional system. Furthermore, in addition to retaining all suspended solids, the MBR system would retain organics considered "soluble" in a conventional system with SSTs, and these will probably reflect in the unbiodegradable particulate COD fraction $\left(f_{\mathrm{S}, \text { up }}\right)$; this aspect is examined in more detail below.

\subsubsection{Unbiodegradable Particulate COD Fraction $\left(f_{\mathrm{S}, \text { up }}\right)$}

The total mass of volatile (organic) suspended solids $\left(\mathrm{MX}_{\mathrm{v}}\right)$ in a BNR activated sludge system receiving a specific waste water is given by Equation (4.9) (Ramphao et al., 2004). Equation (4.9) is modified from that of the WRC (1984) and Ekama et al. (1986) models for ND systems and takes into account the phosphate accumulating 
organisms (PAO's) which contribute more to the MLVSS mass per COD mass utilized than the ordinary heterotrophs (OHOs) (Wentzel et al., 1990; Ramphao et al., 2004).

$$
\begin{aligned}
\frac{M X_{V}}{M S_{t i}}= & \left(1-f_{s, u s}-f_{S, u p}\right)\left[\left(1-\frac{\%}{100} f_{S b^{\prime} s}\right) \frac{Y_{H} R_{s}}{\left(1+b_{H T} R_{s}\right)}\left(1+f_{E H} b_{H T} R_{s}\right)\right. \\
& +\frac{\mathbf{\%}}{100} f_{S b^{\prime} s} \frac{Y_{G} R_{s}}{\left(1+b_{G T} R_{s}\right)}\left(1+f_{E H} b_{H T} R_{s}\right)+\frac{f_{S, u p}}{f_{C V}} R s
\end{aligned}
$$

Where:

$$
\begin{aligned}
& M X_{V} \quad=\text { VSS mass in biological reactor (kgVSS) } \\
& =V_{a n a} X_{V, a n a}+V_{a n x} X_{V, a n x}+V_{a e r} X_{V, a e r}+V_{\text {our }} X_{V}, \text { our } \\
& V_{\text {ana }} ; V_{\text {axx }} ; V_{\text {aer }} ; V_{\text {OUR }} \\
& =\text { Volume of anaerobic, anoxic, aerobic and OUR-aeration reactors }(\ell) \\
& X_{V, \text { ana }} ; X_{V, a n x} ; X_{V, \text { aer }} \text { and } X_{V, \text { ouR }} \\
& \text { = VSS concentrations in anaerobic, anoxic, aerobic and OUR-aeration } \\
& \text { reactors (mgVSS/ } \ell \text { ) } \\
& M S_{t i} \quad=\text { COD mass load on system }(\mathrm{kgCOD} / \ell) \\
& =\mathrm{Q}_{\mathrm{i}} \cdot \mathrm{S}_{\mathrm{ti}} \\
& \left.\mathrm{Q}_{\mathrm{i}} \quad=\text { influent feed flow (ADWF, } \ell / \mathrm{d}\right) \\
& \mathrm{f}_{\mathrm{S} \text {.up }} \quad=\text { unbiodegradable particulate COD fraction } \\
& \mathrm{f}_{\mathrm{S} \text {,us }} \quad=\text { unbiodegradable soluble COD fraction } \\
& \mathrm{f}_{\mathrm{Sb}} \text { 's } \quad=\text { influent readily biodegradable }(\mathrm{RB}) \mathrm{COD} \text { fraction with respect to } \\
& \text { biodegradable COD } \\
& \% \quad=\text { percentage influent RBCOD taken up by phosphate accumulating } \\
& \text { organisms (PAOs); } \\
& =0 \text { if system is nitrification-denitrification (ND) } \mathrm{N} \text { removal, i.e. no } \\
& \text { PAO's, }>0 \text { if system is ND biological excess phosphorus removal } \\
& \text { (NDBEPR), increasing with increasing BEPR (70-90\%). } \\
& \mathrm{Y}_{\mathrm{H}}, \mathrm{Y}_{\mathrm{G}}=\text { yield coefficient for ordinary heterotrophic organisms }(\mathrm{OHOs}) \text { and } \\
& \text { PAOs } \\
& =0.45 \mathrm{mgVSS} / \mathrm{mgCOD} \text { for both } \\
& \mathrm{b}_{\mathrm{HT}}, \mathrm{b}_{\mathrm{GT}} \quad=\text { endogenous respiration rate for OHOs and PAOs at } \mathrm{T}^{\circ} \mathrm{C} \\
& =0.24 / \mathrm{d} \text { and } 0.04 / \mathrm{d} \text { respectively at } 20^{\circ} \mathrm{C}\left(\theta_{\mathrm{b}}=1.029 \text { for both }\right) \\
& \theta_{\mathrm{b}} \quad=\text { temperature sensitivity coefficient for endogenous respiration } \\
& \text { Rs } \quad=\text { system sludge age (days) } \\
& \mathrm{f}_{\mathrm{EH}}, \mathrm{f}_{\mathrm{EG}} \quad=\text { endogenous residue fraction of the OHOs and PAOs } \\
& =0.20 \text { and } 0.25 \text { respectively } \\
& \mathrm{f}_{\mathrm{CV}} \quad=\mathrm{COD} / \mathrm{VSS} \text { ratio of organics }(\mathrm{mgCOD} / \mathrm{mgVSS}) \\
& \text { = sewage batch measured value }
\end{aligned}
$$

In Equation (4.9), values are available for the kinetic $\left(\mathrm{b}_{\mathrm{HT}}, \mathrm{b}_{\mathrm{GT}}, \theta_{\mathrm{b}}\right)$ and stoichiometric $\left(\mathrm{Y}_{\mathrm{H}}, \mathrm{Y}_{\mathrm{G}}, \mathrm{f}_{\mathrm{EH}}, \mathrm{f}_{\mathrm{EG}}\right.$ ) constants. Operational ( $\mathrm{Q}_{\mathrm{ADWF}}, \mathrm{V}_{\mathrm{ANA}}, \mathrm{V}_{\mathrm{ANX}}, \mathrm{V}_{\mathrm{AER}}$ and $\mathrm{R}_{\mathrm{S}}$ ) parameters varied slightly from sewage batch to sewage batch and accordingly were averaged for each sewage batch along with measured reactor VSS concentrations $\left(\mathrm{X}_{\mathrm{V}, \mathrm{ANA}}, \mathrm{X}_{\mathrm{V}, \mathrm{ANX}}\right.$ and $\left.\mathrm{X}_{\mathrm{V}, \mathrm{AER}}\right)$, wastewater characteristics $\left(\mathrm{S}_{\mathrm{ti}}, \mathrm{f}_{\mathrm{Sb}, \mathrm{s}}\right.$ and $\left.\mathrm{f}_{\mathrm{S}, \mathrm{us}}\right)$ and mixed liquor $\left(f_{C V}\right)$ characteristics. The percentage influent RBCOD taken up by polyphosphate accumulating organisms (\%) was assumed to be $90 \%$ based on literature (Wentzel et al., 1990). This leaves $\mathrm{f}_{\mathrm{S}, \text { up }}$ as the only unknown. Thus Equation 
(4.9) was solved for each sewage batch by successive substitution of $f_{S \text {, up }}$ until the calculated system VSS mass matches that measured. The resultant sewage batch $\mathrm{f}_{\mathrm{S} \text {.up }}$ are listed in Table 4.6 and plotted in Fig. 4.23. It must, however, be noted that as $\mathrm{f}_{\mathrm{S} \text {,up }}$ is the only unknown it becomes a "catch all" variable for any other values in the equation that may not be correct. Hence, the $f_{S \text {,up }}$ values calculated must be interpreted with care.

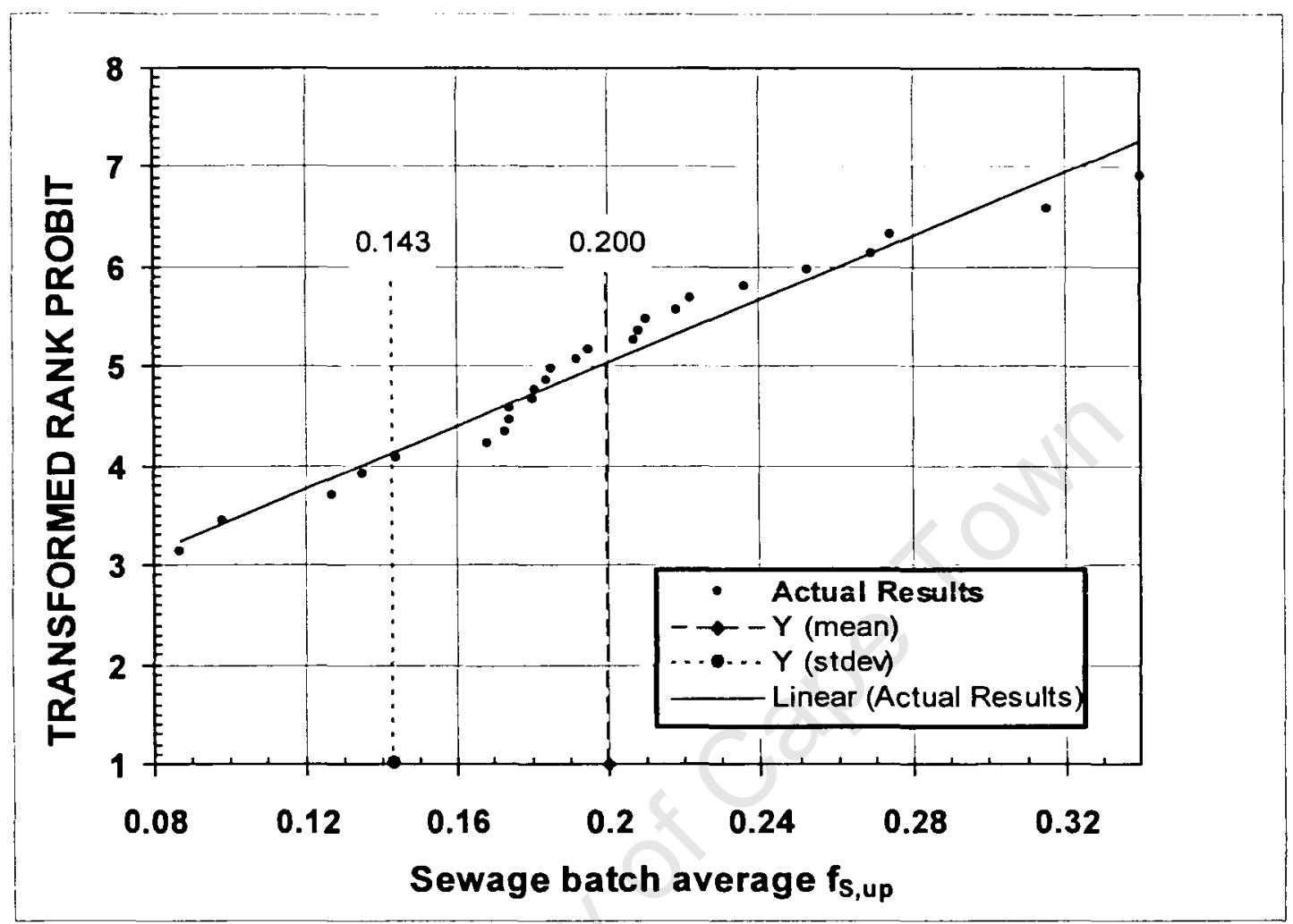

Figure 4.23: Statistical plot of batch average $f_{S, u p}$ for the MBR UCT system.

From Fig. 4.23, the $\mathrm{f}_{\mathrm{S} \text {,up }}$ values exhibit a normal distribution, with mean 0.200 (SSD $=$ $0.057)$. This mean is only slightly lower than that reported in Phase 1 , of 0.216 (Ramphao et al., 2004). Again, realising that $200 \mathrm{mgCOD} / \ell$ in the influent is Sodium Acetate and hence completely biodegradable, and that the overall average total is $952 \mathrm{mgCOD} / \ell$, the sewage $\mathrm{f}_{\mathrm{S} \text {, up }}=0.251$. This value is significantly higher than values reported in previous literature cited in Ramphao et al. (2004) for aerobic (0.108, Mbewe et al., 1995) and MLE (0.135, Warburton et al., 1991; 0.120, Ubisi et al., $1997 ; 0.160$, Beeharry et al., 2001;0.150, Lee et al 2002a) systems which used wastewater from the same source. However, the value does fall within the range measured for BEPR systems receiving wastewater from the same source $(0.06-0.32$, Ekama and Wentzel, 1999).

The total unbiodegradable COD fraction $\left(f_{S, u}\right)$ is calculated as the sum of the soluble $\left(f_{S, \text { us }}\right)$ and particulate $\left(f_{S, \text { up }}\right)$ unbiodegradable fractions. For Phase 1 and 2 of the investigation, the $f_{S, u}$ values are very similar, 0.252 and 0.242 respectively. 


\subsection{SYSTEM PERFORMANCE}

It was expected that the MBR system with its superior solids separation ability would provide a solids free effluent and hence improved effluent TKN and COD concentrations.

\subsubsection{COD Removal}

COD removal is one of the primary parameters on which wastewaters are monitored due to the detrimental effects of de-oxygenation within receiving water bodies. The COD conversion mechanism is primarily through synthesis where the carbonaceous material is either included in new cell mass and accumulates in the sludge, or is oxidised during the metabolism processes as $\mathrm{CO}_{2}$ with the electrons passed to oxygen to form water. Thus, COD is removed from the waste water through the wasted sludge or through oxygen utilization in the system.

The improved COD removal by the membranes could be seen in the very low $\mathrm{f}_{\mathrm{S} \text {,us }}$ fraction achieved which suggested that more COD was retained in the system than would have been expected in a conventional system.

In the investigation thrce categories of effluent COD were medsured (Chapter 3, Section 3.5.1): Membrane filtered effluent which was collected from the biological reactors' membrane outflow; $0.45 \mu \mathrm{m}$ filtered effluent which was the filtered supernatant from the centrifuged aerobic reactor mixed liquor sample; and an unfiltered "effluent" sample which was the supernatant from the DSVI settling test on the MBR sludge. The different effluent samples were intended to determine the difference in quality of the effluent in the absence of membranes.

Sewage batch average total influent and membrane filtered effluent COD concentrations are plotted in Fig. 4.24. Throughout the investigation reasonably consistent effluent values around $40 \mathrm{mgCOD} / \ell$ were obtained.

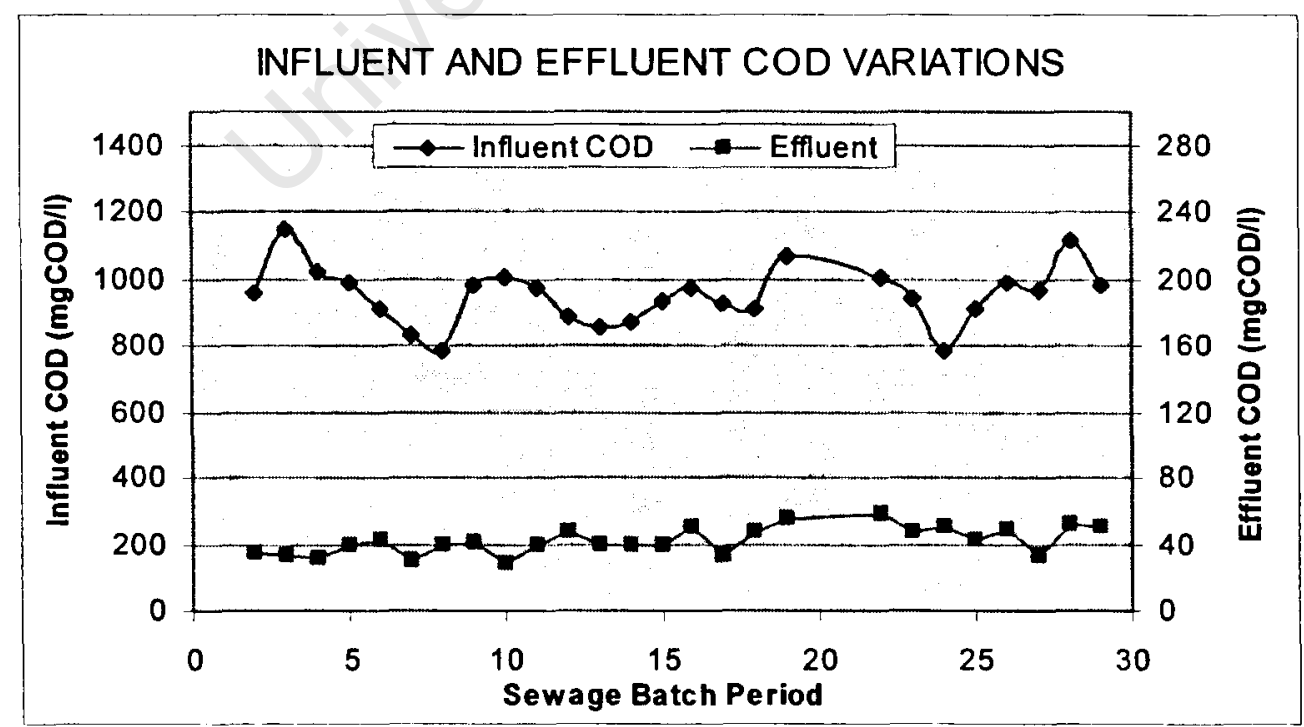

Figure 4.24: Influent and effluent COD variations for sewage batch averages. 

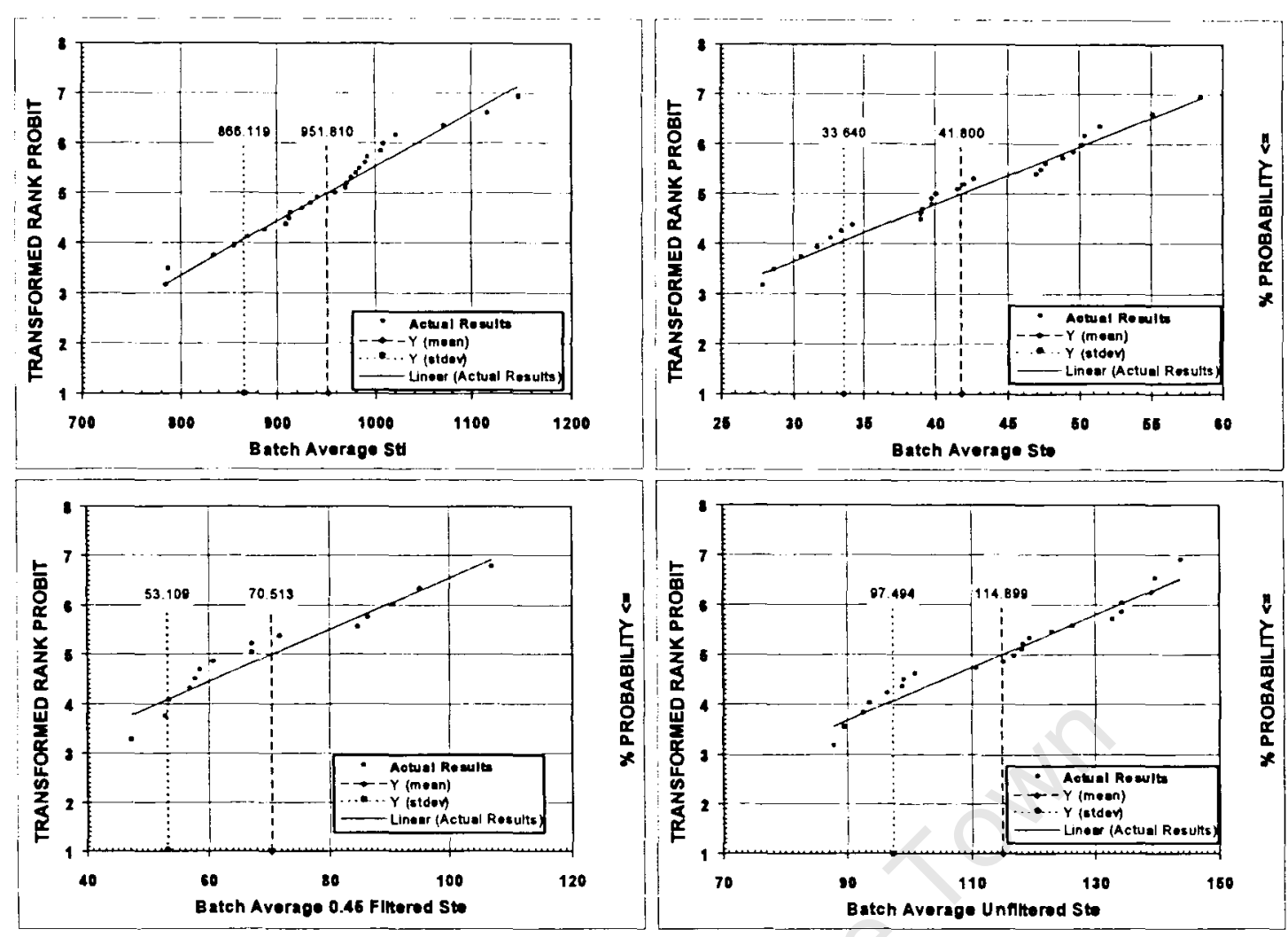

Figures 4.25a, b, $\boldsymbol{c}$ and d: Statistical distribution of the influent feed (Sti); membrane filtered effluent (Ste); Aerobic supernatant filtered through a $0.45 \mu \mathrm{m}$ filter; and "unfiltered" samples taken from the DSVI supernatant.

Fig. 4.25 shows statistical analyses of the influent and the three effluent COD concentrations, namely membrane effluent, $0.45 \mu \mathrm{m}$ filtered effluent and unfiltered effluent, illustrating that in all cases the data are normally distributed. The mean influent is $951.8 \mathrm{mgCOD} / \ell$ and the mean effluent $41.8 \mathrm{mgCOD} / \ell$ giving an effective COD removal of $95.7 \%$. Statistical analyses of the daily values recorded were not advisable due to one dominant factor that affected each batch, namely dilution of the batch with tap water (Chapter 3, Section 3.4.2). The investigation means for the 0.45 $\mu \mathrm{m}$ filtered and unfiltered effluent COD, presented in Table 4.8. clearly illustrate the efficient COD removal and solids/liquid separation by the system membranes, and that the membrane pore size is significantly less than $0.45 \mu \mathrm{m}$.

Table 4.8: $\quad$ Percent removals of the various effluent samples from the MBR system.

\begin{tabular}{|c|c|c|c|c|}
\hline & Influent & \multicolumn{3}{|c|}{ Effluent } \\
\cline { 3 - 5 } & COD & MBR & $\mathbf{0 . 4 5}$ & unfiltered \\
\hline mgCOD/l & 951.8 & 41.8 & 70.5 & 114.9 \\
\hline$\%$ removal & N/A & $95.7 \%$ & $92.7 \%$ & $88.1 \%$ \\
\hline
\end{tabular}

\subsubsection{Nitrogen Removal}

Nitrogen is removed from the waste water through nitrogen accumulation in the mixed liquor, removal with the waste flow and through sequential nitrification/ denitrification. Since the operational parameters sludge age and influent organic load were kept constant, the sludge production and wasting, and its associated $\mathrm{N}$ content, were relatively constant. Hence variations in the nitrogen removal were due to either 
variations in the TKN/COD load to the system, or to fluctuations in the nitrification/ denitrification performance.

Of the influent TKN the biodegradable organic nitrogen gets converted to ammonia through heterotrophic activity and adds to the influent ammonia. In nitrification this ammonia is converted sequentially to $\mathrm{NO}_{2}$ and then $\mathrm{NO}_{3}$ in the aerobic zone. The aerobic mass fraction achieved during the investigation was approximately $59.5 \%$, and the system was run at a long sludge age ( $~ 20$ days) which ensured complete nitrification. $\mathrm{pH}$ measured at regular intervals throughout the testing period indicated that the $\mathrm{pH}$ remained within the required range of $7-8.5$ so as not to inhibit autotrophic activity. Results of the effluent TKN and ammonia concentrations indicated that throughout the investigation complete nitrification was achieved.

In the UCT configuration, although much of the $\mathrm{NO}_{3}$ is recycled to the anoxic zone where denitrification occurs (the $\mathrm{NO}_{3}$ is consumed as electron acceptor in metabolism and nitrogen gas is released), a portion of the $\mathrm{NO}_{3}$ will always escape in the effluent from the aerobic reactor. Thus, the nitrogen removal must take into account the $\mathrm{NO}_{3}$ concentration in the effluent.

\section{Nitrification}
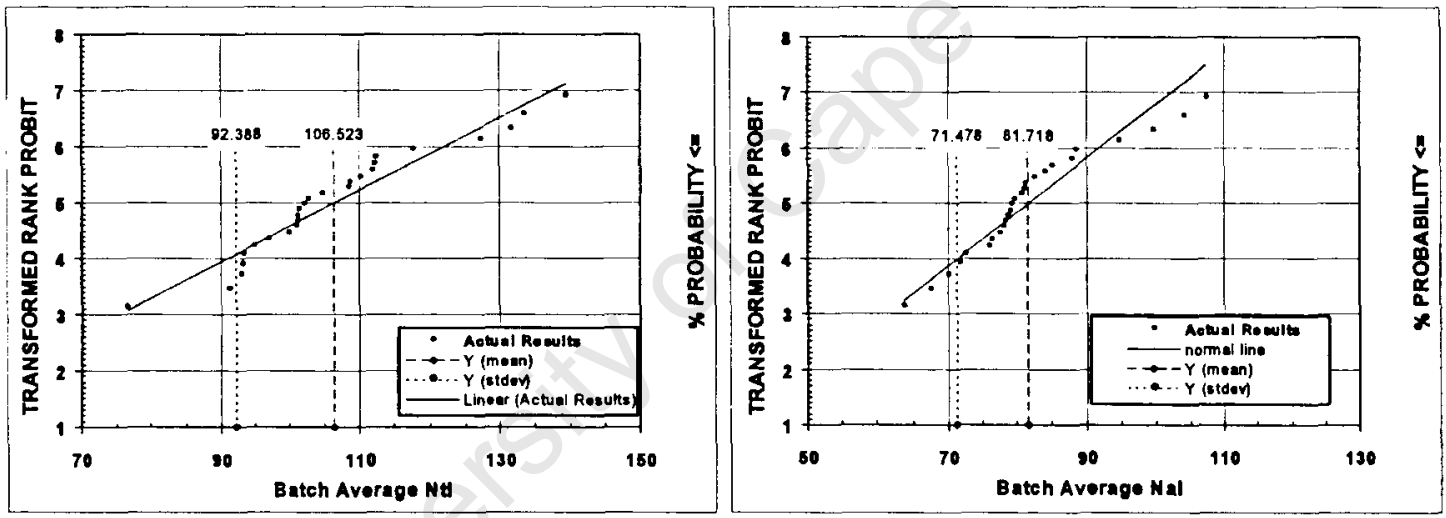

Figure 4.26 and $b$ : Statistical plots of the influent TKN $\left(N_{t i}\right)$ and FSA $\left(N_{a i}\right)$ concentrations

The sewage batch average influent TKN and FSA concentrations are plotted in Figs. $4.26 \mathrm{a}$ and $4.26 \mathrm{~b}$ respectively giving means for the investigation of $106.5( \pm 14.1)$ and $81.7( \pm 10.2) \mathrm{mgN} / \ell$ respectively. The data are not particularly well normally distributed, but this is to be expected as the concentration for each batch should vary since the COD was diluted to constant concentration and the TKN/COD ratios for each wastewater batch differed, see below. The effluent concentrations similarly are not normally distributed (Fig. $4.27 \mathrm{a}$ and $4.27 \mathrm{~b}$ ) due to the insensitivity of the TKN and FSA analytical tests at such low concentrations. Means for the batch average effluent TKN and FSA concentrations of $1.7( \pm 0.8)$ and $0.8( \pm 0.5) \mathrm{mgN} / \ell$ were obtained. 

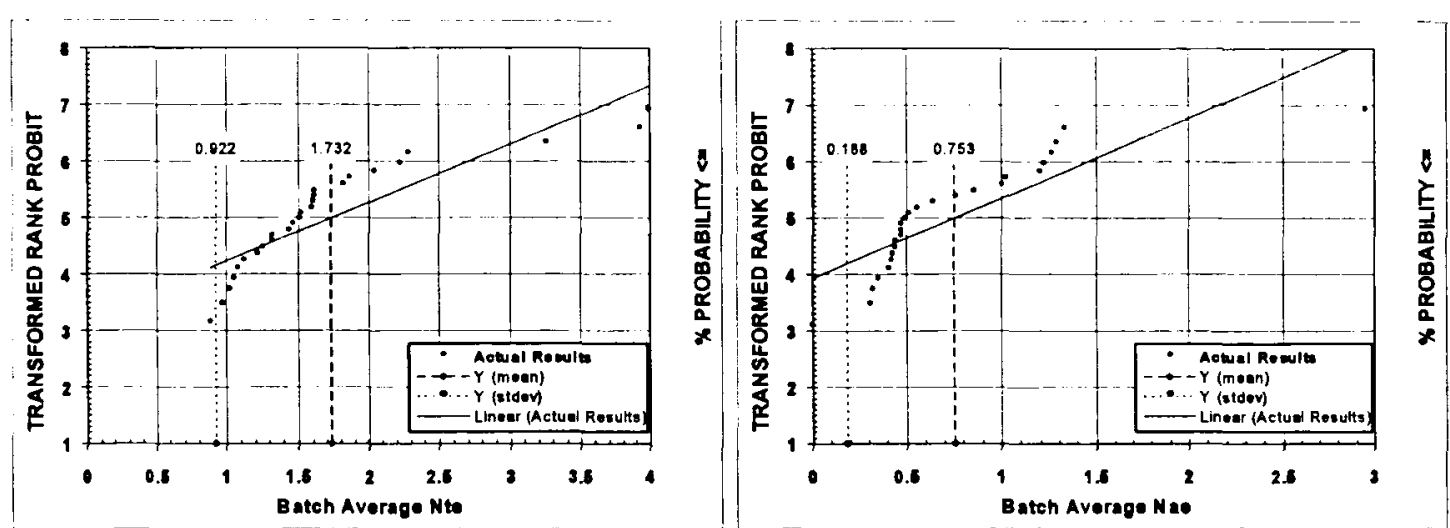

Figures 4.27 and b: Effluent TKN $\left(N_{t e}\right)$ and FSA $\left(N_{a e}\right)$ concentrations from the MBR system.

At the start of the investigation the influent TKN was dependant on the batch sewage TKN/COD ratio which varied considerably. This influent TKN was augmented with the addition of $\sim 20 \mathrm{mgN} / \ell$ ammonia $(200 \mathrm{mgCOD} / \ell$ sodium acetate was added to the feed in order to augment BEPR; however this caused a decrease in the TKN/COD ratio which thus needed to be corrected). Variations in the influent and effluent TKN and FSA concentrations are illustrated in Figs. 4.28 and 4.29. From sewage batch 13 it was decided to try maintain a constant influent TKN/COD ratio, of approximately 0.1 $\mathrm{mgN} / \mathrm{mgCOD}$ through addition of variable concentrations of ammonia. This strategy significantly reduced the variation in influent TKN/COD ratio, Fig.4.28.

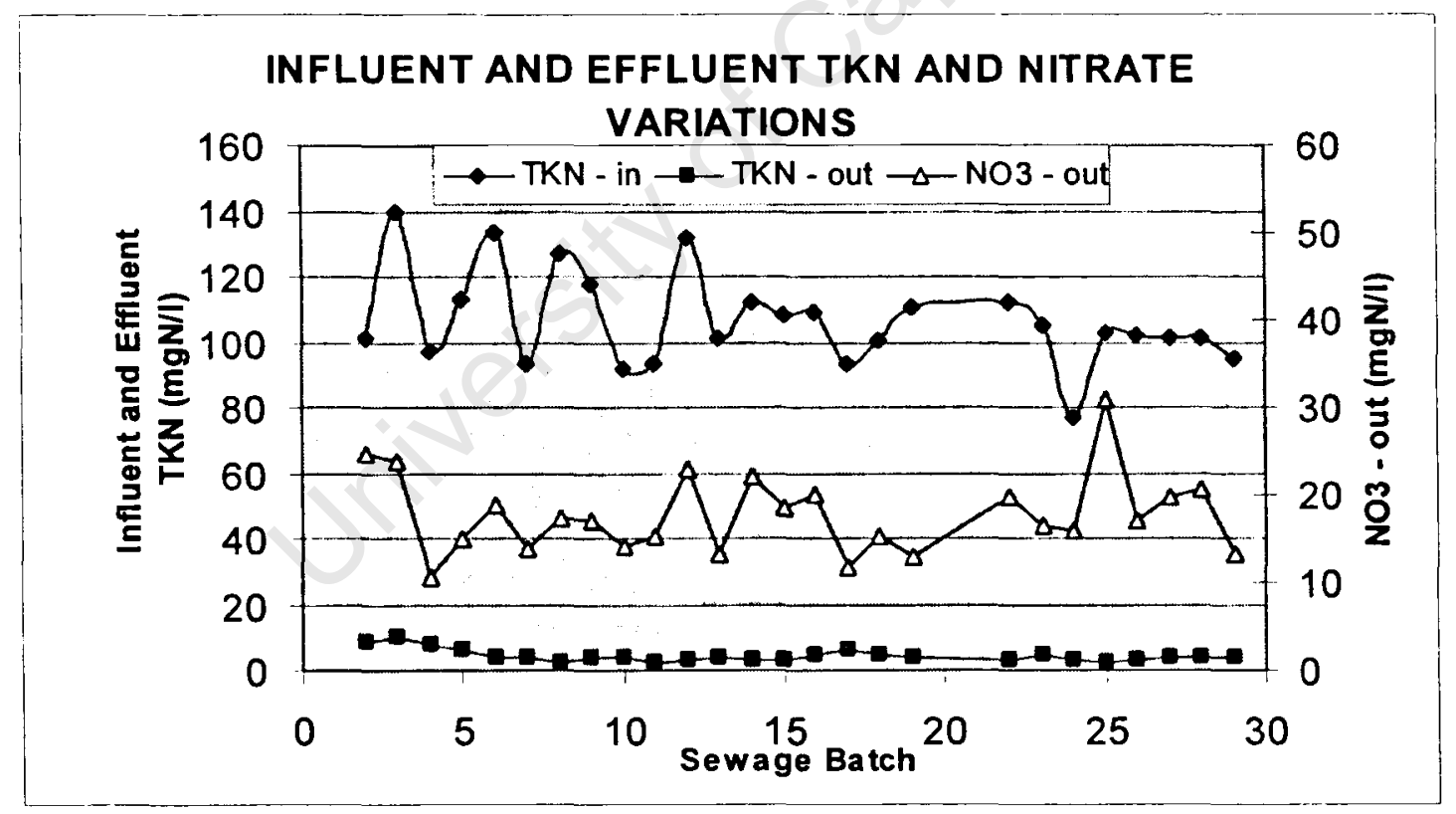

Figure 4.28: TKN influent and effluent variations with effluent $\mathrm{NO}_{3}$. 
INFLUENT AND EFFLUENT FSA AND NITRATE VARIATIONS

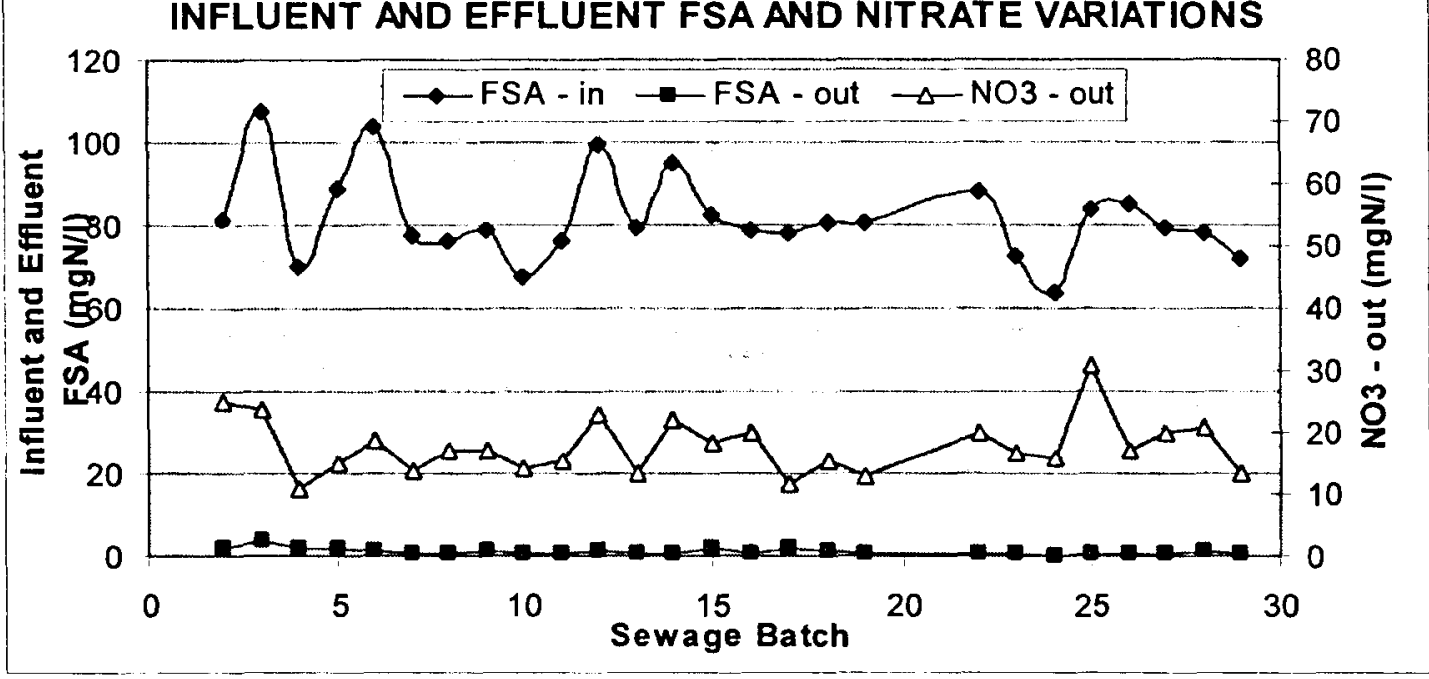

Figure 4.29: The FSA influent and effluent concentrations with the effluent $\mathrm{NO}_{3}$.

From the investigation means, the TKN and FSA removals were calculated. See Table 4.9. The TKN and FSA removals were high (98 and $99 \%$ respectively), confirming complete nitrification throughout the test period with the removals listed in the table below:

Table 4.9: $\quad$ TKN and FSA removals

\begin{tabular}{|c|c|c|c|}
\hline & Influent & Effluent & $\begin{array}{c}\% \\
\text { Removal }\end{array}$ \\
\hline & mgN/l & mgN/I & - \\
\hline TKN & 106.7 & 1.74 & $98.4 \%$ \\
\hline FSA & 82 & 0.75 & $99.1 \%$ \\
\hline
\end{tabular}

The difference between the effluent TKN $\left(\mathrm{N}_{\mathrm{te}}\right)$ and FSA $\left(\mathrm{N}_{\mathrm{ae}}\right)$ is assumed to be equal to the unbiodegradable soluble organic $\mathrm{N}$ concentration of the influent $\mathrm{TKN}\left(\mathrm{N}_{\text {ousi }}=\right.$ $1.74-0.75 \mathrm{mgN} / \ell=0.99 \mathrm{mgN} / \ell$ ). This gave $0.9 \%$ as a percent of the average influent TKN, which is close to the $1 \%$ value determined in Phase 1 (Ramphao et al., 2004). However, the influent feed was augmented with ammonia by $20 \%$ on average, which would increase the actual percent $\mathrm{N}_{\text {ousi }}$ of the raw sewage to $1.2 \%$. This is substantially lower than conventionally accepted values of 2.5 to $3.0 \%$ for typical South African waste waters (WRC, 1984). This may be attributed to the membranes retaining unbiodegradable soluble organics (with associated organic $\mathrm{N}$ ) that would otherwise escape with the effluent in a conventional system and be included in $\mathrm{N}_{\text {ouse }}$.

\section{Denitrification}

$\mathrm{NO}_{3}$ concentrations in the MBR system effluent $\left(\mathrm{N}_{\mathrm{ne}}\right)$ are shown in Figs.4.28 and 4.29. Variations in reactor $\mathrm{NO}_{3}$ concentrations are plotted with reactor phosphate concentrations in Figs. 4.27-4.30. For all reactors and the effluent, $\mathrm{NO}_{2}$ concentrations were less than $1 \mathrm{mgN}-\mathrm{NO}_{2} / \ell$ and could be considered negligible.

Denitrification occurs in the anoxic reactor. $\mathrm{NO}_{3}$ is returned with the "combined" asrecycle and is used by heterotrophic organisms as electron acceptor. The denitrification potential $\left(D_{p p}\right)$ is the maximum amount of $\mathrm{NO}_{3}$ that could be removed 
in the anoxic reactor, if sufficient $\mathrm{NO}_{3}$ was available. It is dependant on the anoxic mass fraction, the biodegradable COD that enters the anoxic reactor and the kinetics of denitrification. In NDBEPR systems the role of the influent RBCOD for denitrification is small due to it being taken up by the PAOs in the anaerobic reactor and their small contribution to denitrification (Hu et al., 2002). The $\mathrm{NO}_{3}$ load $\left(\mathrm{N}_{\mathrm{nL}}\right)$ is the mass of $\mathrm{NO}_{3}$ brought to the anoxic reactor via the recycles. It is dependant on the nitrification capacity, $\mathrm{N}_{\mathrm{C}},\left(\mathrm{mgN}-\mathrm{NO}_{3} / \ell\right.$ influent flow) which is the concentration of $\mathrm{NO}_{3}$ generated in the aerobic reactor via nitrification (hence the prerequisite of complete nitrification for designing and modelling denitrification), and the as-recycle ratio.

The actual mass of $\mathrm{NO}_{3}$ that is denitrified is dependant on the amount of $\mathrm{NO}_{3}$ loaded on the reactor, $N_{n L}$, relative to the $D_{P P}$. If $N_{n L} \geq D_{P P}$ then all the denitrification potential is used and denitrification is close to the maximum achievable. Denitrification is thus dependant on $D_{P P}$ and the residual nitrate that remains in the anoxic reactor is recycled to the anaerobic reactor and returned to the aerobic reactor. If $\mathrm{N}_{\mathrm{nL}}<\mathrm{D}_{\mathrm{PP}}$ then the anoxic reactor is underloaded and all $\mathrm{NO}_{3}$ to the anoxic reactor is denitrified giving an anoxic $\mathrm{NO}_{3}$ concentration of $0 \mathrm{mgN} / \ell$. In this case denitrification is effectively governed by $\mathrm{N}_{\mathrm{nL}}$. Hence, the anoxic reactor $\mathrm{NO}_{3}$ concentration is a good indicator of the state of denitrification (Fig. 4.32). The effluent with concentration $\mathrm{N}_{\text {ne }}$ is given by Equation (4.10):

$$
N_{n e}=\frac{N_{C}}{(a s+1)} \mathrm{mgN} / \ell
$$

and $\mathrm{N}_{\mathrm{nL}}$ and the nitrate concentration denitrified $\left(\mathrm{N}_{\mathrm{nd}}\right)$ are given by Equation (4.11):

$$
N_{n d} \& N_{n L}=N_{C}-N_{n e}=N_{C}-\frac{N_{C}}{a s+1}=\frac{a s \cdot N_{C}}{a s+1}
$$

As $\mathrm{N}_{\mathrm{nL}}$ is dependant on the influent TKN concentration and $\mathrm{D}_{\mathrm{PP}}$ is dependant on the influent COD concentration, the TKN/COD ratio of the influent should have a direct affect on the $\mathrm{N}_{\mathrm{ne}}$ concentration. As the TKN/COD ratio increases, $\mathrm{N}_{\mathrm{ne}}$ will increase, and visa versa, and this behaviour pattern is largely reflected in the experimental results, see Fig. 4.30. 


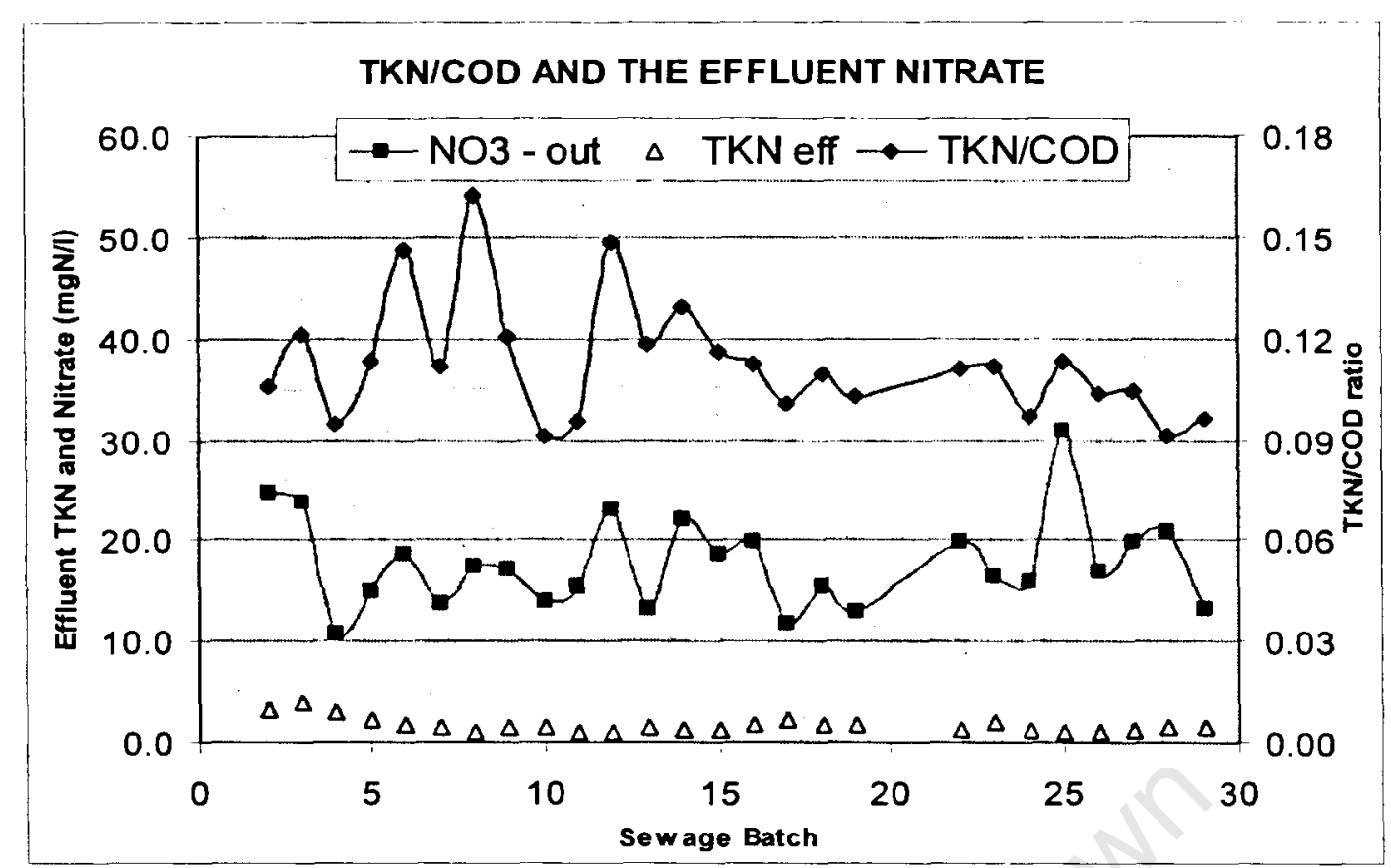

Figure 4.30: TKN/COD ratios of the influent feed and the effluent TKN and NO3 concentrations.

Anoxic $\mathrm{NO}_{3}$ concentrations were generally very low throughout the investigation: Apart from sewage batches 12 and 14 which had anoxic $\mathrm{NO}_{3}$ concentrations of 2.5 and $2.4 \mathrm{mgN} / \ell$ respectively, all other batches had values less than $1.3 \mathrm{mgN} / \ell$. The mean of the sewage batch averages over the investigation was $0.5 \mathrm{mgN} / \ell$ indicating that for the most part the $\mathrm{N}_{\mathrm{nL}}$ to the anoxic reactor equalled or slightly exceeded the $D_{P P}$. These observations indicated negligible $\mathrm{NO}_{3}$ recycled to the anaerobic reactor, and hence BEPR was operated independently of nitrate. Consistently overloaded anoxic reactors stimulate the growth of denitrifying PAOs and anoxic P uptake BEPR. This is observed from time to time in BEPR systems and causes a reduction in $P$ removal because the denitrifying PAOs use the influent RBCOD less efficiently than the aerobic PAOs. In the MBR system, $P$ uptake took place mostly in the aerobic reactor indicating negligible denitrification contribution by PAOs.

\section{System Nitrogen Removal}

The total system $\mathrm{N}$ removal is the difference between the influent TKN $\left(\mathrm{N}_{\mathrm{ti}}\right)$ and the sum of effluent $\mathrm{TKN}$ and $\mathrm{NO}_{3}$ (the $\mathrm{NO}_{2}$ is negligible). This value varied greatly from $13.7-32.0 \mathrm{mgTN} / \ell$. The batch average total nitrogen removals ranged from $68.9 \%$ to $86.1 \%$ with an average of $81.7 \%$. This is substantially higher than the 67 and $76 \%$ nitrogen removals observed in Phase 1, with a-recycle ratios $<2: 1$ and $3: 1$ respectively, and can be attributed in part to the higher a-recycle ratio in this Phase 2 investigation of $>3: 1$, which loaded the anoxic reactor with nitrate close to its denitrification potential.

\subsubsection{BEPR}

Biological Excess Phosphorus Removal (BEPR) is achieved in BNR systems by promoting the growth of phosphorus accumulating organisms (PAOs) which store a high concentration of intracellular polyphosphate (polyP), $0.38 \mathrm{mgP} / \mathrm{mgVSS}$ in 
comparison to $0.02 \mathrm{mgP} / \mathrm{mgVSS}$ in conventional heterotrophic organisms (Wentzel et al., 1990). This is achieved through incorporating an anaerobic/aerobic sequence with sewage fed to the anaerobic zone. In the anaerobic reactor PAOs store short chain fatty acids (SCFA) internally as polyhydroxyalkanoates (PHA). In order to convert the SCFAs to PHA, energy is required. This energy is sourced from complex polyP stored in the PAOs. In utilizing polyP, orthophosphate is released into the bulk liquid around the organism. Thus, in the anaerobic reactor SCFAs, which are generated from the influent RBCOD through acid fermentation by ordinary heterotrophic organisms (OHOs) in the mixed liquor, are sequestered and stored by the PAOs and as a result orthophosphates are released into solution. In the aerobic reactor the internally stored PHA is utilized as a carbon and energy source with oxygen as electron acceptor for growth and maintenance, and as an energy source for taking up orthophosphate from solution and forming polyP to replenish the polyP pool. The new PAO biomass that is generated in the growth process also takes up polyP, with the result that the $\mathrm{P}$ uptake is greater than the $\mathrm{P}$ release. The difference between the $\mathrm{P}$ uptake and $\mathrm{P}$ release is the $P$ removal.

Hence, under aerobic conditions there is a net increase in polyP bound up in PAO sludge mass and a net decrease of orthophosphates in solution. At steady state the production of new polyP and the reduction of orthophosphates in solution per day is equal to the polyP in the sludge wasted per day. In order to augment and hence accentuate the BEPR performance in the system the RBCOD in the influent was increased by dosing $200 \mathrm{mgCOD} / \ell$ influent sodium acetate. Additionally, to ensure that the system was not $\mathrm{P}$ limited, $20 \mathrm{mgP} / \ell$ influent orthophosphate was dosed in the daily feed.

To evaluate and observe the BEPR performance in the system, total $\mathrm{P}$ concentrations were measured on the influent, filtrate $(0.45 \mu \mathrm{m})$ of each reactor, and the effluent, and are illustrated in Figs. 4.31-4.34. However, as the P content of the mixed liquor was not measured a complete system $P$ mass balance could not be carried out on the system, as was the case with nitrogen and COD.

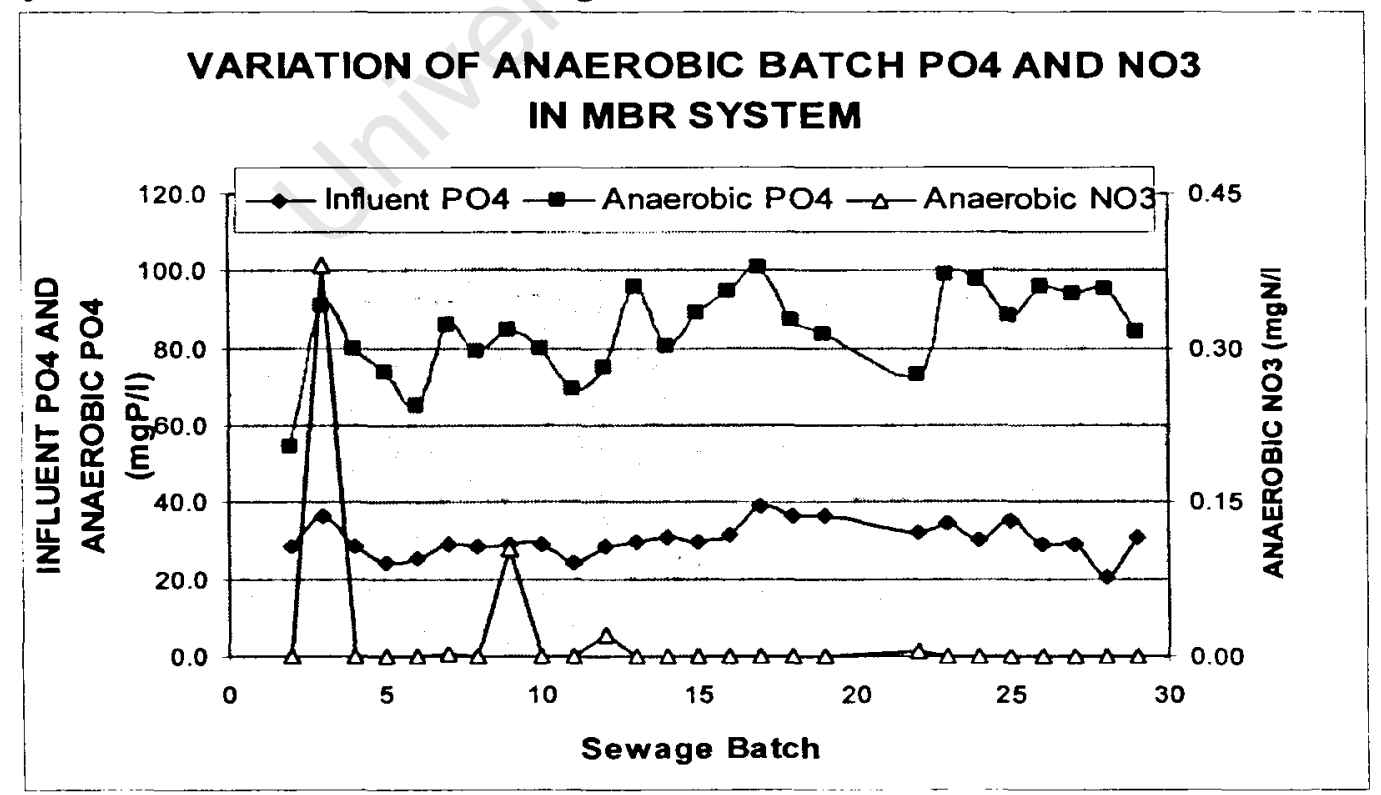

Figure 4.31: Time dependant variation in anoxic reactor total soluble phosphorus and anoxic nitrate concentrations. 


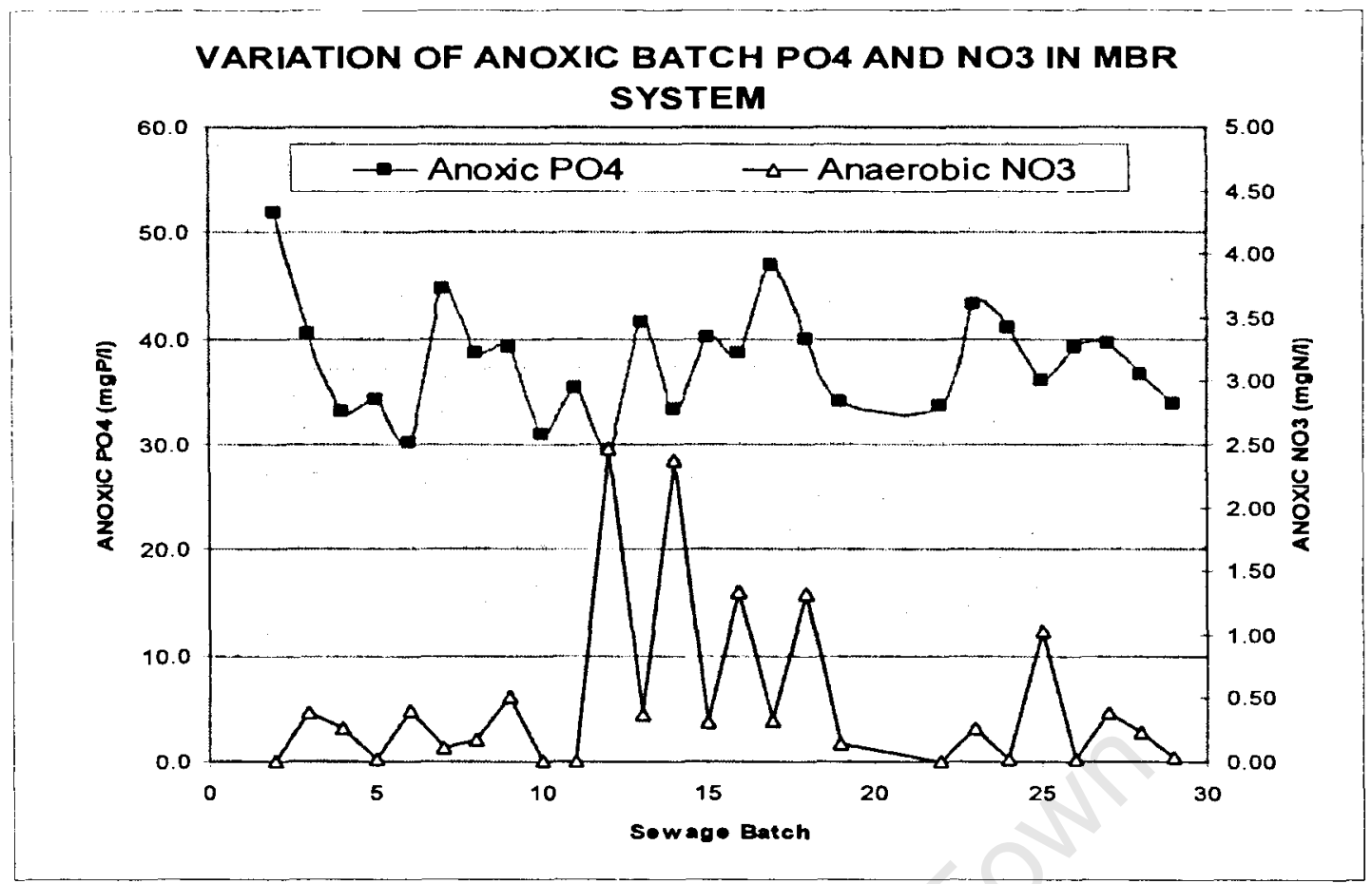

Figure 4.32: Time dependant variation in anoxic reactor total soluble phosphorus and anoxic nitrate concentrations

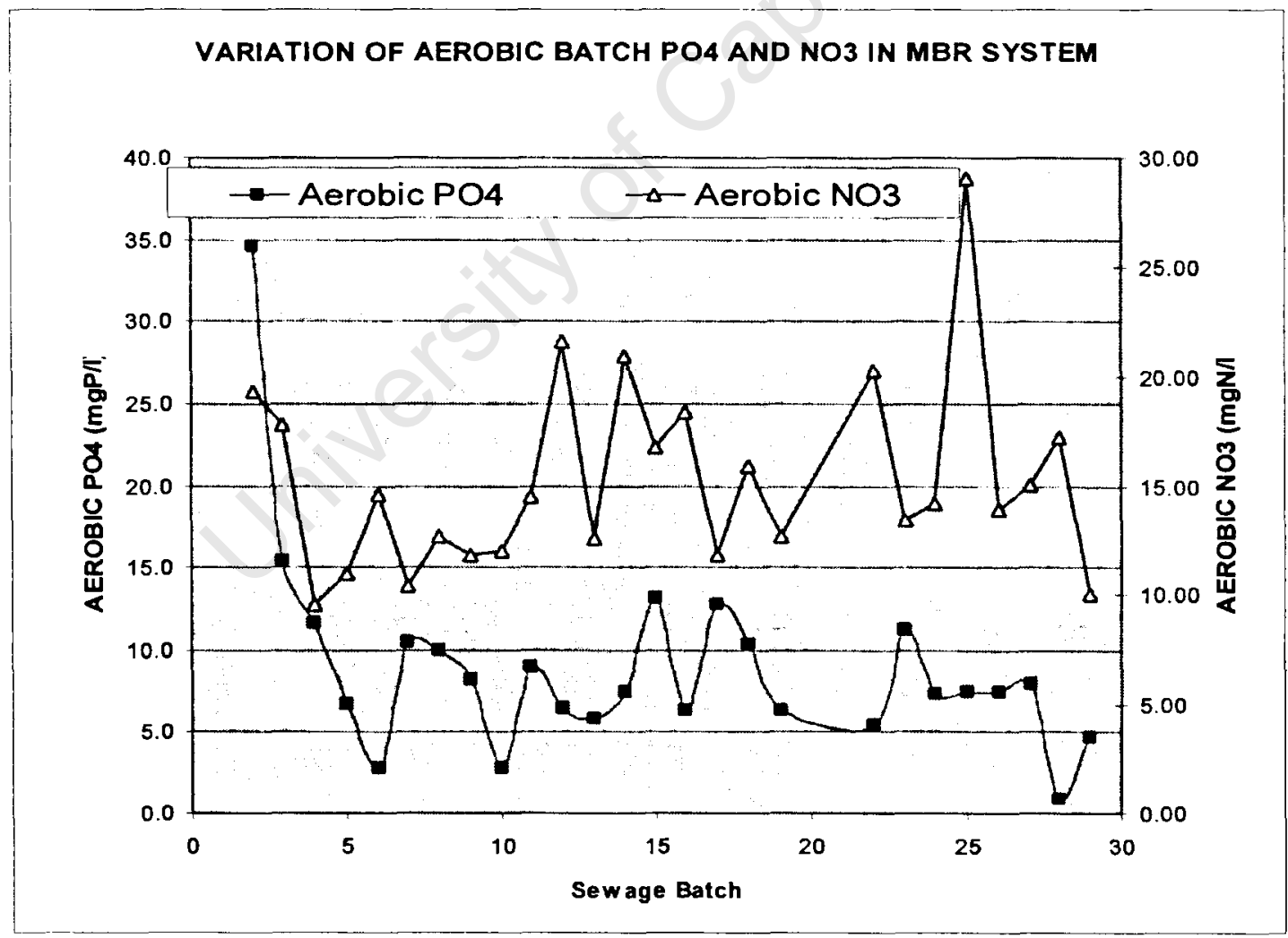

Figure 4.33: Time dependant variation in anoxic reactor total soluble phosphorus and anoxic nitrate concentrations 


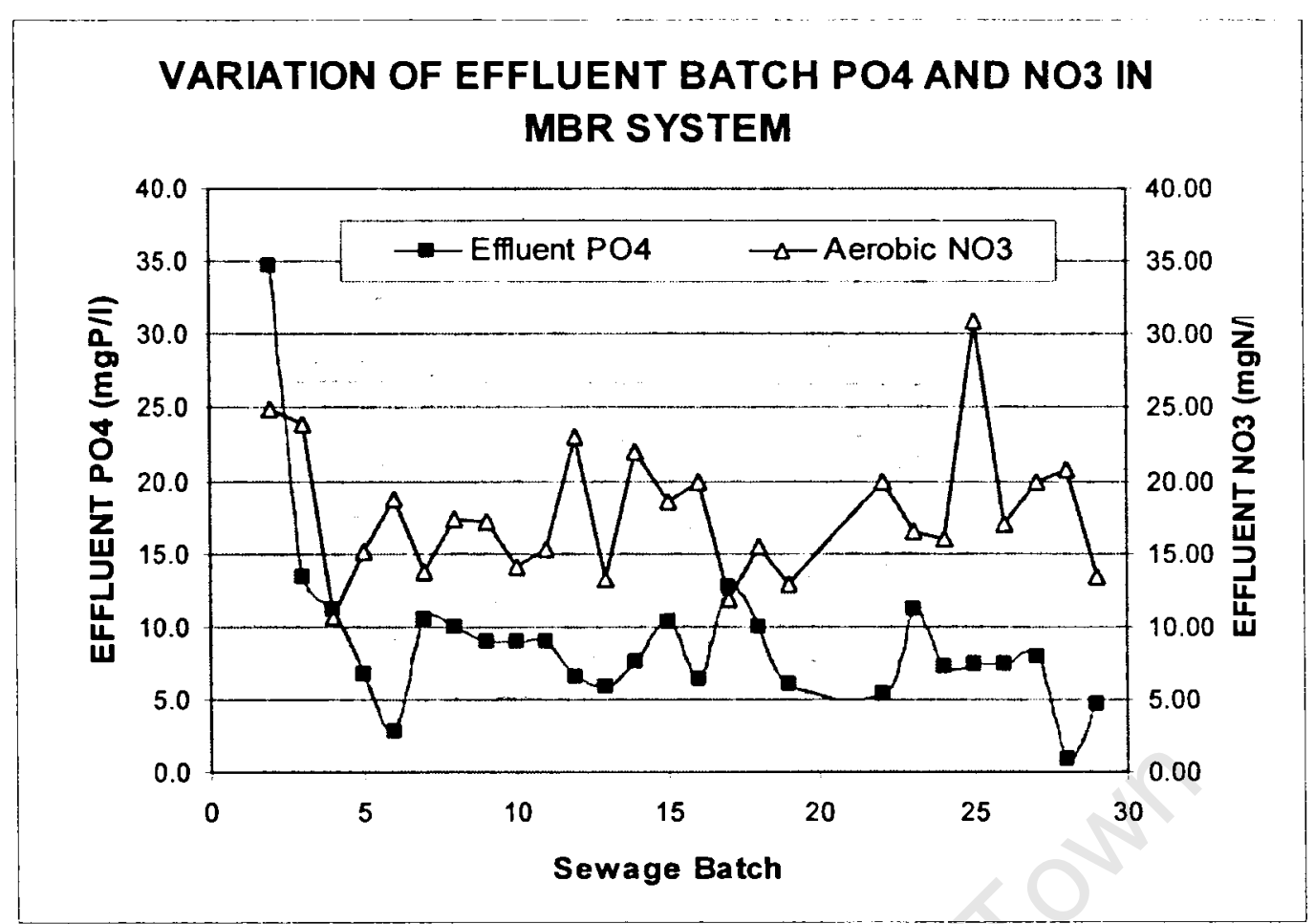

Figure 4.33: Time dependant variation in anoxic reactor total soluble phosphorus and anoxic nitrate concentrations

To evaluate the $\mathrm{P}$ removal performance across the reactors a $\mathrm{P}$ balance (Table 4.10) was set up, whereby the change in $P$ mass across each reactor was calculated via a reactor $P$ mass balance, i.e. the difference between $P$ mass entering the reactor and $P$ mass leaving the reactor. This difference, indicating $P$ uptake if positive and $P$ release if negative, divided by the influent flow gave the $\mathrm{P}$ uptake/release per litre influent flow, see Table 4.10. 
Table 4.10: Sewage batch average $P$ release (-ve) and $P$ uptake (+ve) in the reactors and across the membranes of the MBR system, and the total $P$ removal.

\begin{tabular}{|c|c|c|c|c|c|c|}
\hline $\begin{array}{c}\text { Batch } \\
\text { Number }\end{array}$ & Influent & Anaerobic & Anoxic & Aerobic & Membrane & $\begin{array}{l}\text { M (P.PO4) } \\
\text { removal }\end{array}$ \\
\hline & mgP/linf & mgP/linf & mgP/linf & mgP/linf & mgP/linf & mgP/linf \\
\hline 2 & 28.6 & -29.2 & -109.7 & 133.1 & -10.1 & -5.8 \\
\hline 3 & 36.2 & -109.1 & -50.8 & 182.6 & 0.6 & 22.8 \\
\hline 4 & 28.4 & -110.5 & 33.7 & 94.0 & 0.3 & 17.3 \\
\hline 5 & 24.0 & -93.2 & 5.6 & 104.8 & 1.7 & 17.2 \\
\hline 6 & 25.1 & -84.4 & -5.3 & 112.1 & -0.7 & 22.3 \\
\hline 7 & 29.1 & -101.6 & -33.0 & 153.3 & 0.3 & 18.7 \\
\hline 8 & 28.5 & -103.1 & -20.1 & 141.7 & 0.1 & 18.5 \\
\hline 9 & 29.3 & -108.9 & 8.4 & 120.8 & 0.5 & 20.3 \\
\hline 10 & 29.3 & -99.0 & -14.5 & 134.0 & -0.9 & 20.5 \\
\hline 11 & 24.0 & -87.5 & -25.3 & 127.9 & -0.3 & 15.0 \\
\hline 12 & 28.3 & -107.8 & 14.4 & 115.2 & 0.0 & 21.9 \\
\hline 13 & 29.6 & -132.8 & -10.8 & 167.3 & 0.1 & 23.8 \\
\hline 14 & 31.2 & -114.3 & 25.5 & 112.5 & 0.3 & 23.7 \\
\hline 15 & 29.8 & -124.0 & 22.0 & 121.4 & 1.4 & 19.4 \\
\hline 16 & 31.4 & -128.4 & 28.7 & 124.7 & 1.3 & 25.0 \\
\hline 17 & 39.1 & -121.4 & 8.8 & 139.0 & -0.5 & 26.4 \\
\hline 18 & 36.6 & -114.3 & 14.4 & 126.7 & -0.1 & 26.8 \\
\hline 19 & 36.5 & -100.1 & 16.8 & 113.8 & 1.0 & 30.4 \\
\hline 22 & 32.0 & -88.9 & -15.1 & 130.7 & 1.0 & 26.6 \\
\hline 23 & 34.7 & -127.0 & 9.2 & 141.3 & -0.3 & 23.5 \\
\hline 24 & 30.2 & -135.5 & 13.7 & 144.6 & 0.8 & 22.9 \\
\hline 25 & 35.2 & -117.2 & 17.3 & 127.7 & 0.5 & 27.8 \\
\hline 26 & 28.9 & -136.5 & 19.9 & 138.1 & 0.7 & 21.5 \\
\hline 27 & 29.2 & -128.7 & 31.2 & 118.6 & 5.5 & 21.2 \\
\hline 28 & 20.7 & -144.2 & 24.1 & 139.8 & 3.5 & 19.7 \\
\hline 29 & 31.0 & -112.8 & 21.3 & 117.9 & -0.6 & 26.3 \\
\hline Average & 30.3 & -113.3 & 5.6 & 130.0 & 0.7 & 22.4 \\
\hline $\begin{array}{l}\text { Ramphao } \\
\text { et al.,(2004) }\end{array}$ & 40.8 & -136.1 & 14.67 & 147.8 & 0.5 & 26.9 \\
\hline
\end{tabular}

The results for sewage batch 2 have been excluded from the investigation average as these were identified as being outliers and inconsistent with the rest of the data at the commencement of the investigation. The results of Ramphao et al. (2004) from Phase 1 are included in Table 4.10. The overall higher average results achieved in Phase 1 are attributed to a larger PAO population at steady state, and this is discussed later. Small differences in measured $P$ concentrations in the aerobic and effluent samples prompted the inclusion of a mass balance around the membranes in the aerobic reactor. However, apart from sewage batches 27 and 28 which followed on from the intermittent power failures, the changes in $\mathrm{P}$ across the membranes were very small. The anaerobic, anoxic and aerobic $\mathrm{P}$ release/uptake for the different sewage batches are shown in Fig. 4.34.

Both $\mathrm{P}$ uptake and release occurred in the anoxic reactor. $\mathrm{P}$ release occurred less often and typically early on in the investigation (sewage batches (2), 6-8, 10, 11, 13 and 
22), whereas $P$ uptake occurred more regularly and predominately in the latter half of the investigation. However, as a fraction of the total $\mathrm{P}$ uptake, anoxic $\mathrm{P}$ uptake was small $(8.5 \%)$.

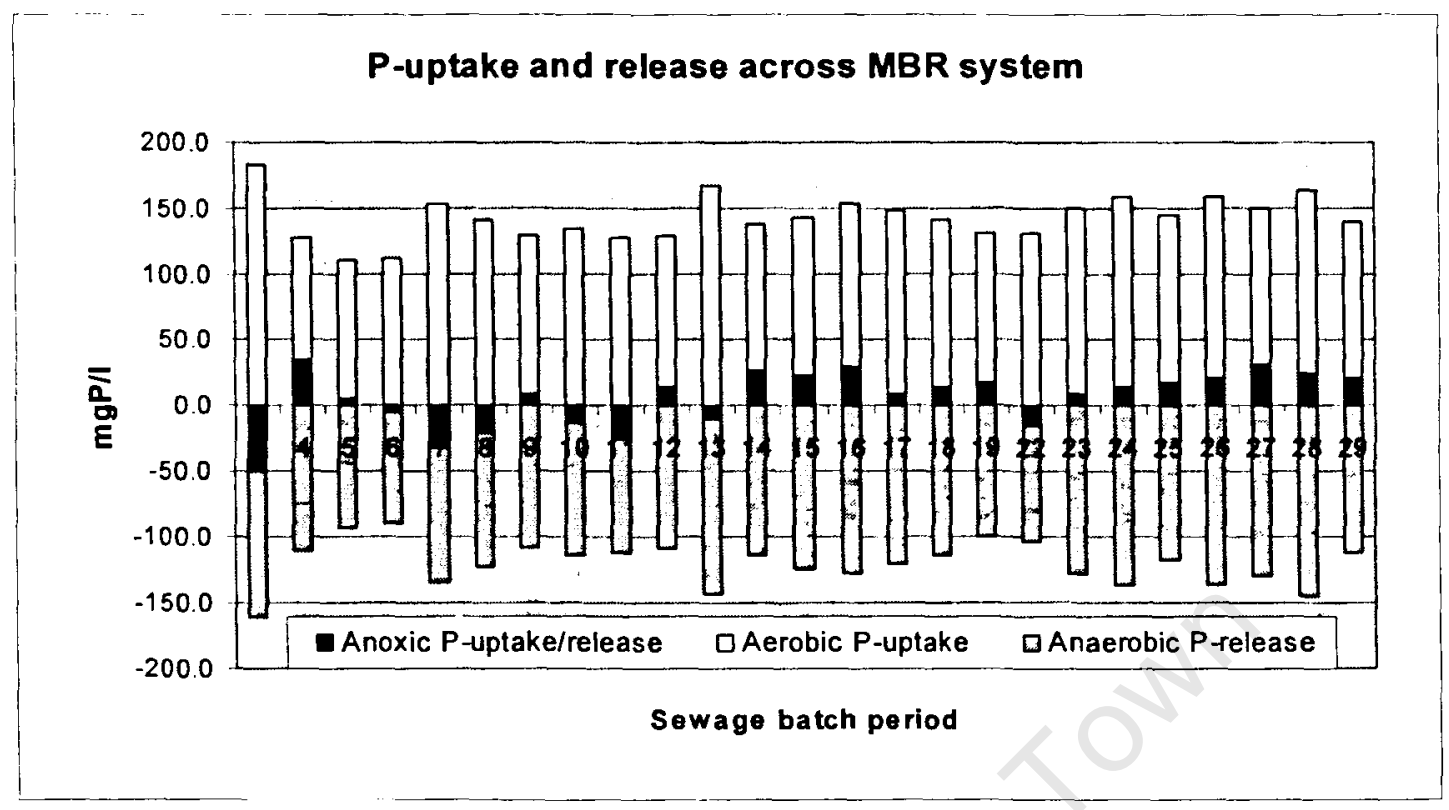

Figure 4.34: A stacked bar breakdown of Anaerobic $P$ release (-ve), anoxic $P$ release/uptake (-ve/+ve) and aerobic $P$ uptake $(+v e)$.

The magnitude of anoxic $P$ release corresponded quite closely with lower $P$ release in the anaerobic reactor (see Fig 4.35). If the anaerobic $P$ release was low, significant anoxic $\mathrm{P}$ release tended to occur. This is probably due to leakage of RBCOD through the anaerobic reactor to the anoxic reactor, possibly due to an initially low PAO population which increased with time in the investigation as reflected in the improved $\mathrm{P}$ removal. However, factors such as anoxic reactor $\mathrm{NO}_{3}$ concentration influence the anoxic $\mathrm{P}$ release/uptake (Hu et al., 2002) and hence no definitive conclusion can be drawn. 


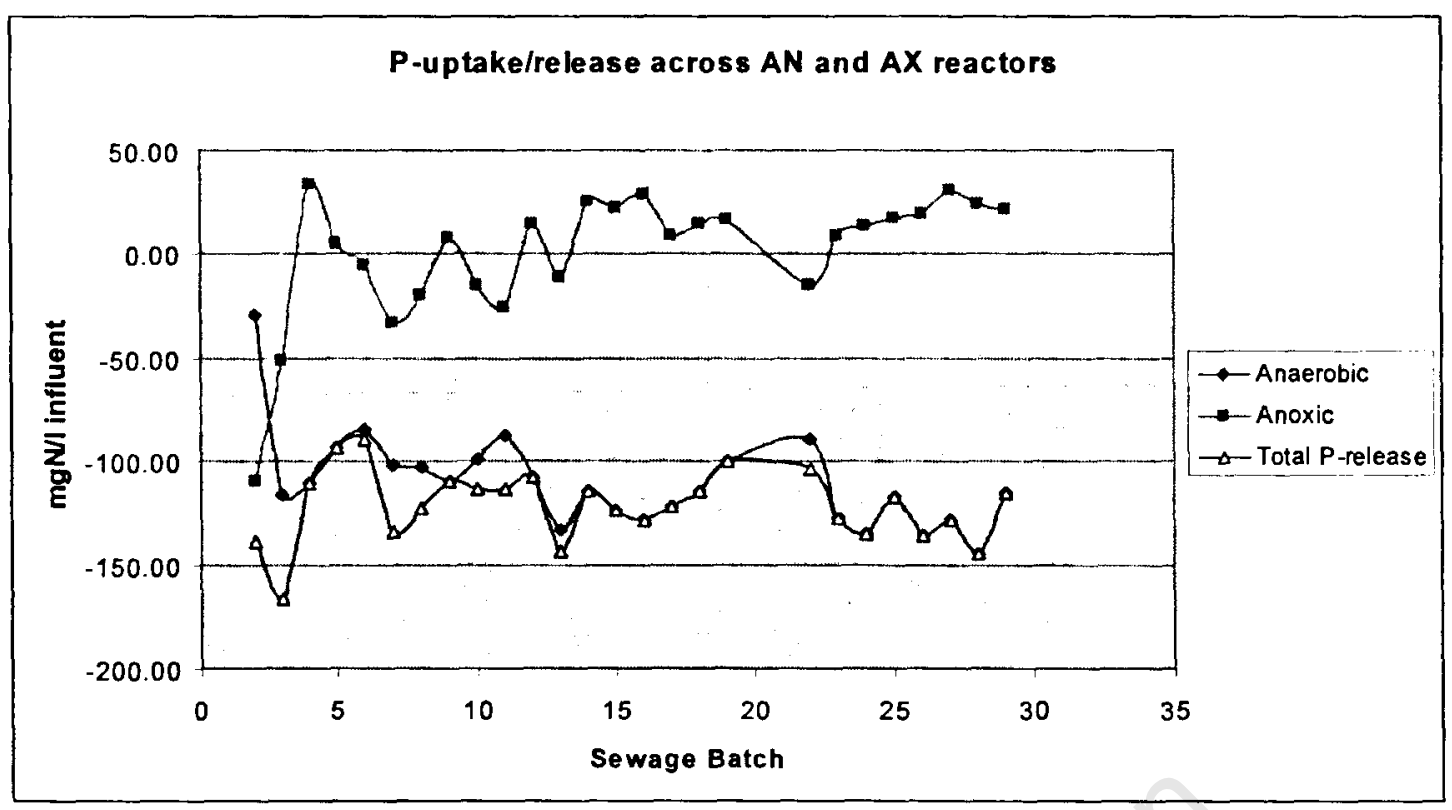

Figure 4.35: The average $P$ release and $P$ uptake across the $A N$ and $A X$ reactors appeared consistent.

System P removal (Fig. 4.36) showed a steady increase through the investigation, up until sewage batch 18 , after which it stabilized, suggesting that the slow growing PAO population only reached steady state around that time in the study. Sewage batch average $P$ removals are statistically plotted in Fig 4.37 , giving a mean $P$ removal for the investigation of $22.4 \mathrm{mgP} / \ell$, which is substantially lower than that observed in Phase 1 of $27.0 \mathrm{mgP} / \ell$ (Ramphao et al., 2004). However, if only the $P$ removals from sewage batch 18 onwards are considered, a mean removal of $24.7 \mathrm{mgP} / \ell$ is obtained which is substantially closer to the $P$ removal observed in Phase 1 above.

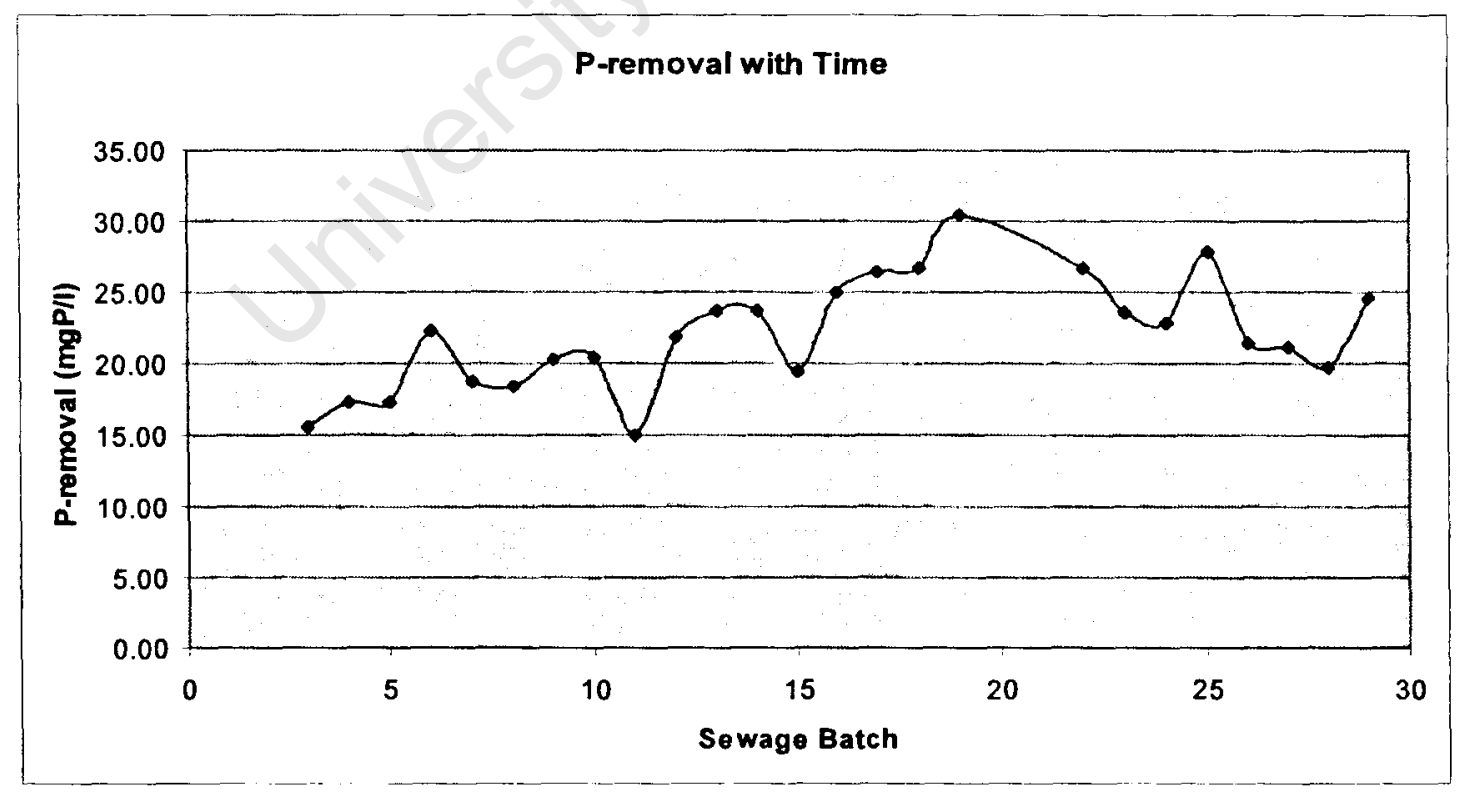

Figure 4.36: P-removal over time for the sewage batch periods. 


\section{P-removal in MBR system}

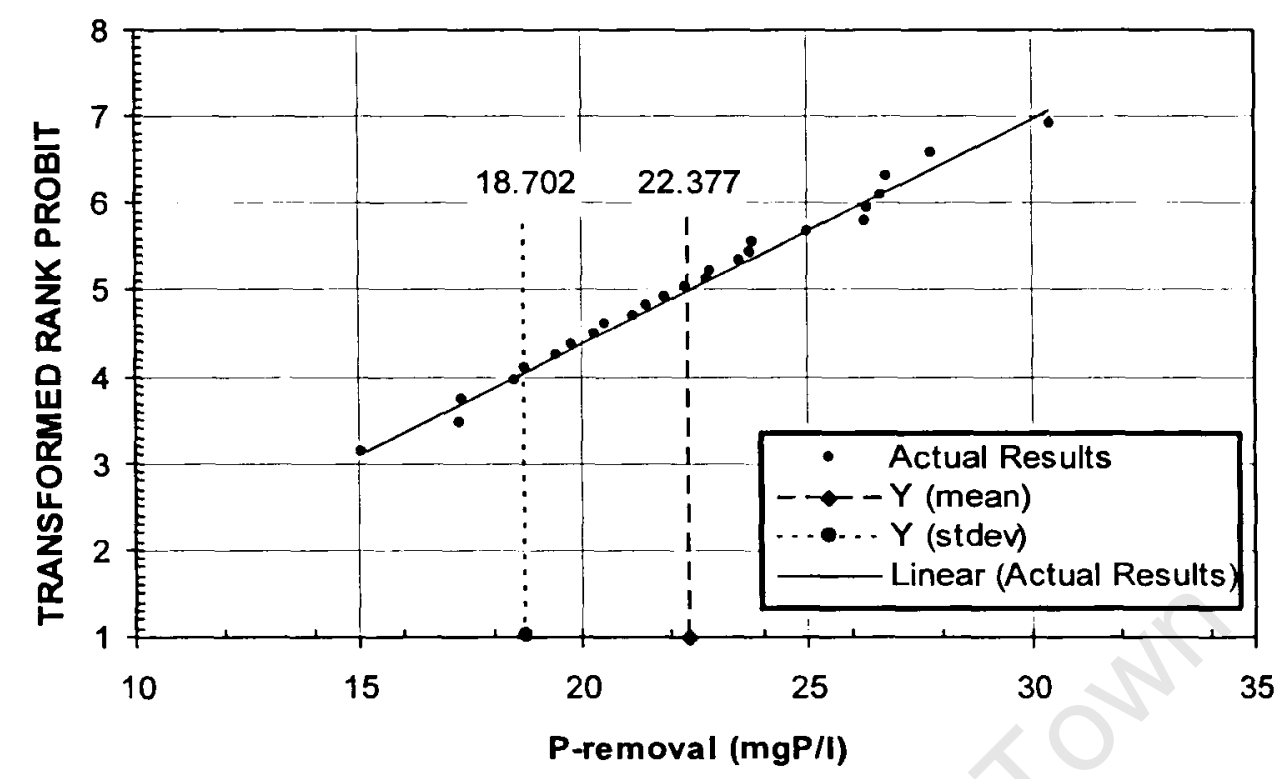

Figure 4.37: Statistical plot of the system $P$ removal for sewage batch periods.

The UCT configuration is designed to protect the anaerobic reactor from recycled $\mathrm{NO}_{3}$, whereby all $\mathrm{NO}_{3}$ entering the anoxic reactor is utilized for denitrification. If $\mathrm{NO}_{3}$ does enter the anaerobic reactor, it can be utilised with the available COD for denitrification by OHOs and thus less COD is available to the PAOs, which in turn reduces the $P$ release and subsequent $P$ uptake and hence $P$ removal. For every 1 $\mathrm{mgN}-\mathrm{NO}_{3} / \ell$ recycled to the anaerobic reactor $8.6 \mathrm{mgCOD} / \ell$ is no longer available to the PAOs, and the $\mathrm{P}$ removal reduces by approximately $0.85 \mathrm{mgP} / \ell$ (Wentzel et al., 1990).

Anoxic reactor $\mathrm{NO}_{3}$ concentrations for the different sewage batches are shown in Fig. 4.38 , together with the anaerobic $P$ release. On five occasions (sewage batches 12, 14, 16,18 and 25) the anoxic $\mathrm{NO}_{3}$ concentration exceeded $1 \mathrm{mgN}-\mathrm{NO}_{3} / \ell$ which would have been recycled to the anaerobic reactor. 


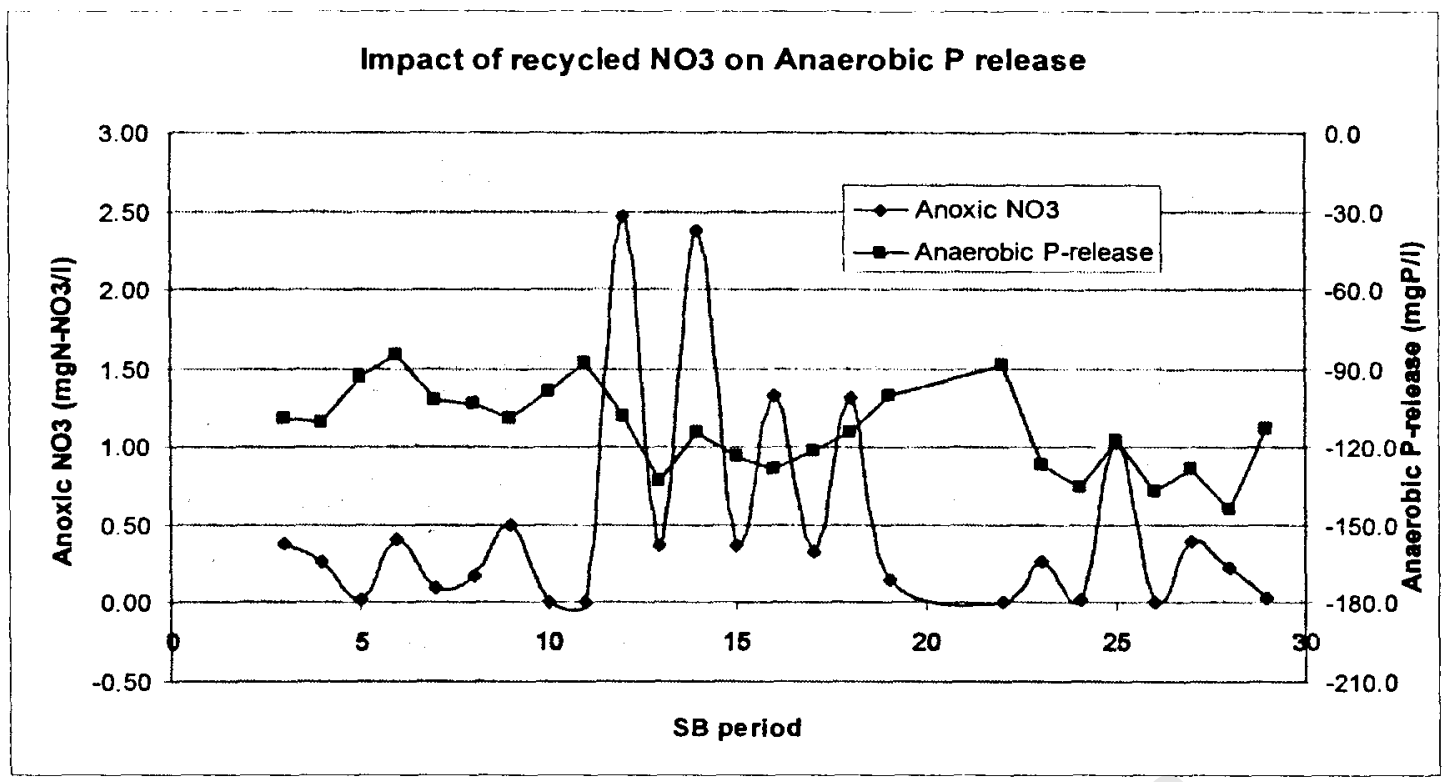

Figure 4.38: Anoxic $\mathrm{NO}_{3}$ concentration and its impact on anaerobic $P$ release.

The anaerobic $\mathrm{P}$ release is dependant inter alia on the influent RBCOD concentration. The more RBCOD available, the more PHA can be stored by the PAOs and hence the more $\mathrm{P}$ release will occur in the anaerobic reactor. In the calculations for characterizing the mixed liquor (Section 4.4.4) it was assumed that $90 \%$ of the influent RBCOD would be anaerobically converted through fermentation by OHOs to SCFAs and would thus be available to be utilized by PAOs. Additionally it was assumed that for every $1 \mathrm{mg} \mathrm{RBCOD}$ used $0.5 \mathrm{mgP}$ would be released in the anaerobic reactor (Wentzel et al., 1990). Thus plots of $90 \%$ RBCOD concentration for the sewage batches and the observed $P$ release/ 0.5 should show close correlation, see Fig. 4.39. The correlation is reasonably close, except for the first four sewage batches.

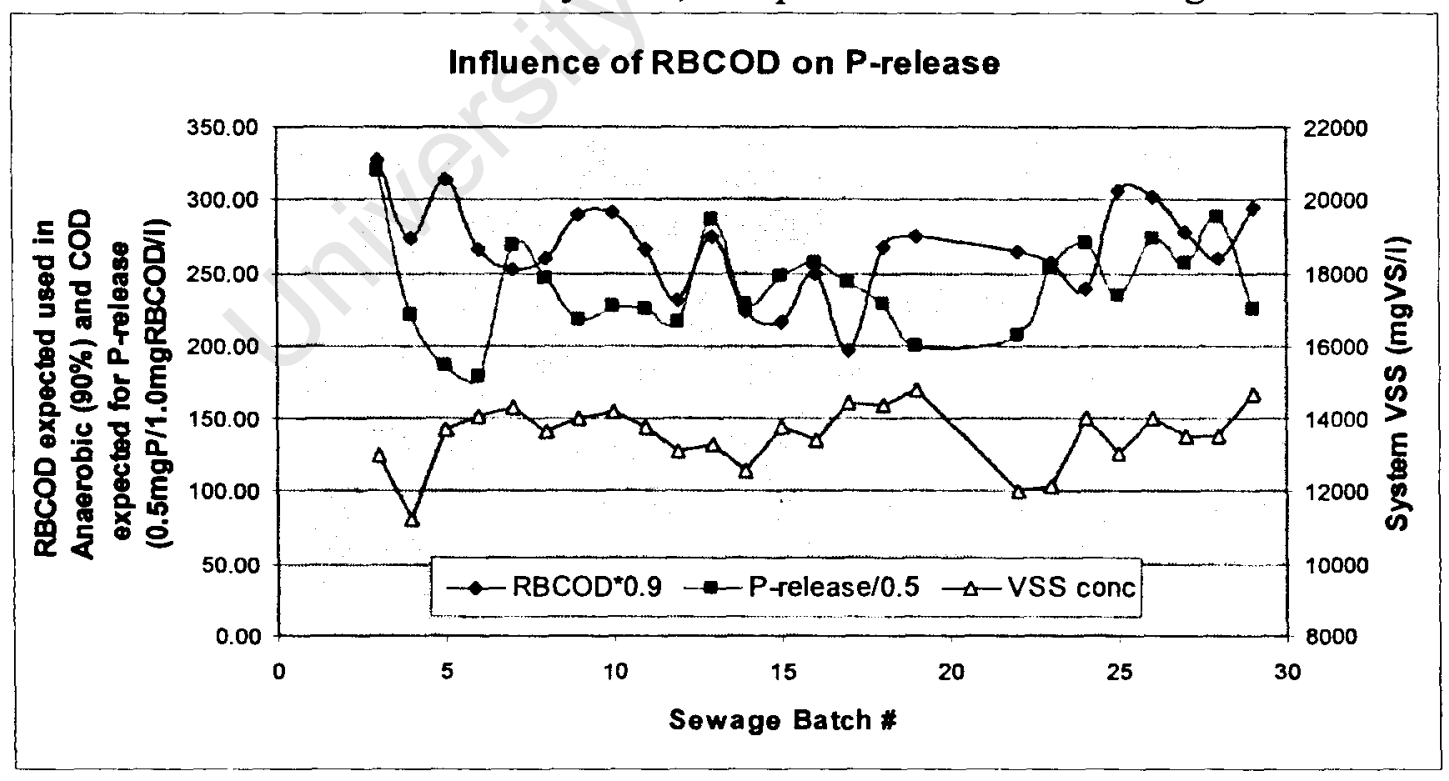

Figure 4.39: The relationship between $R B C O D P$ removal and VSS concentration. Apart from batches 2-4, 7-8, 19, 22 and 29 the values are very close supporting the assumptions made on the relationship between $R B C O D$ and $P$ release in the anaerobic reactor. It was assumed that the spills in batches 4 and 22 would have had an impact on the $P$ release as sludge loss would have meant a loss of PAO's which take time to develop. However the data does not seem to support this hypothesis. 
Additionally, from the understanding of BEPR, the $\mathrm{P}$ release and $\mathrm{P}$ removal, should track each other closely. This arises because the magnitude of $\mathrm{P}$ removal is relative to the new PAO cell mass, which is dependant on the amount of RBCOD stored in the anaerobic reactor with its consequent $P$ release. Sewage batch average anaerobic $P$ release and system $P$ removal are shown plotted in Fig 4.40. Up to sewage batch 16, both $\mathrm{P}$ release and $\mathrm{P}$ removal increase conforming to expectations. However, from sewage batch 19 the $P$ removal decreased, whereas the $P$ release increased contrary to expectation. No explanation for this latter behaviour is evident.

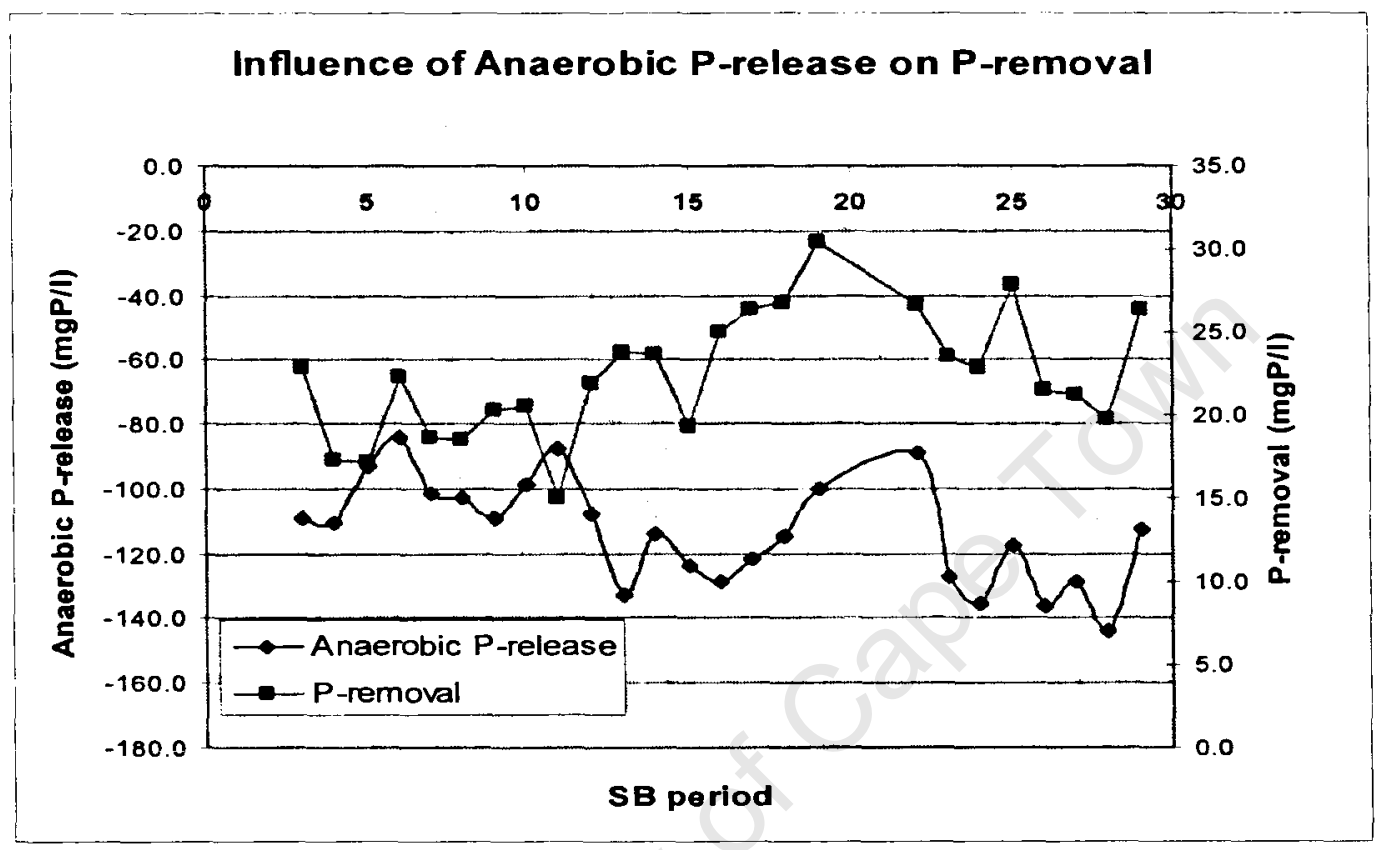

Figure 4.40: The relationship between anaerobic $P$ release and system $P$ removal is not clear.

\section{Anoxic P Uptake}

Early observations on BEPR systems indicated that $\mathrm{P}$ uptake took place only in the aerobic reactor, i.e. PAOs did not denitrify (Wentzel et al., 1990; Clayton et al., 1991). However more recent investigations indicate that some strains of PAOs can denitrify, termed denitrifying (D)PAOs (Kerry-Jasperson and Henze et al 1993; Baker and Dold, 1996; Kuba et al., 1993, 1997; Ekama and Wentzel, 1999; Hu et al., 2002) with associated anoxic $P$ uptake. With anoxic $P$ uptake by DPAOs, significantly reduced BEPR has been reported (Ekama and Wentzel, 1999; Hu et al., 2002), presumably due to less efficient utilization of the influent RBCOD (Hu et al., 2002). Conditions identified as contributing to anoxic BEPR include i) $\mathrm{NO}_{3}$ load to anoxic reactor exceeding the denitrification potential of the reactor, ii) low aerobic mass fractions, iii) anoxic-aerobic sequence of reactors, and iv) frequency of sludge alternation between anoxic and aerobic states (Hu et al., 2002).

The frequency of anoxic $\mathrm{P}$ uptake was evaluated in this investigation and in Figure 4.41. the occurrence of anoxic $P$ uptake is presented together with the concentration of $\mathrm{NO}_{3}$ in the anoxic reactor for the different sewage batches: 
- P release occurs in 8 sewage batches $(3,6-8,10-11,13,22)$, which apart from sewage batch 22 all occur in the first half of the study.

- Anoxic $P$ uptake as a percentage of the total $P$ uptake is very variable and ranged from $0-26.4 \%$ (sewage batch 4 ), with an average of $8.5 \%$.

- A correlation between anoxic $\mathrm{P}$ uptake and anoxic $\mathrm{NO}_{3}$ concentration was observed: Where anoxic P uptake was observed there was, in most cases, anoxic $\mathrm{NO}_{3}$ present (except sewage batches 5, 24, 26 and 29). The converse was less often true, that is where anoxic $\mathrm{NO}_{3}$ was present anoxic $\mathrm{P}$ uptake did not necessarily occur. This indicates that observable anoxic $\mathrm{NO}_{3}$ is a necessary, but not the only, requirement for anoxic P uptake.

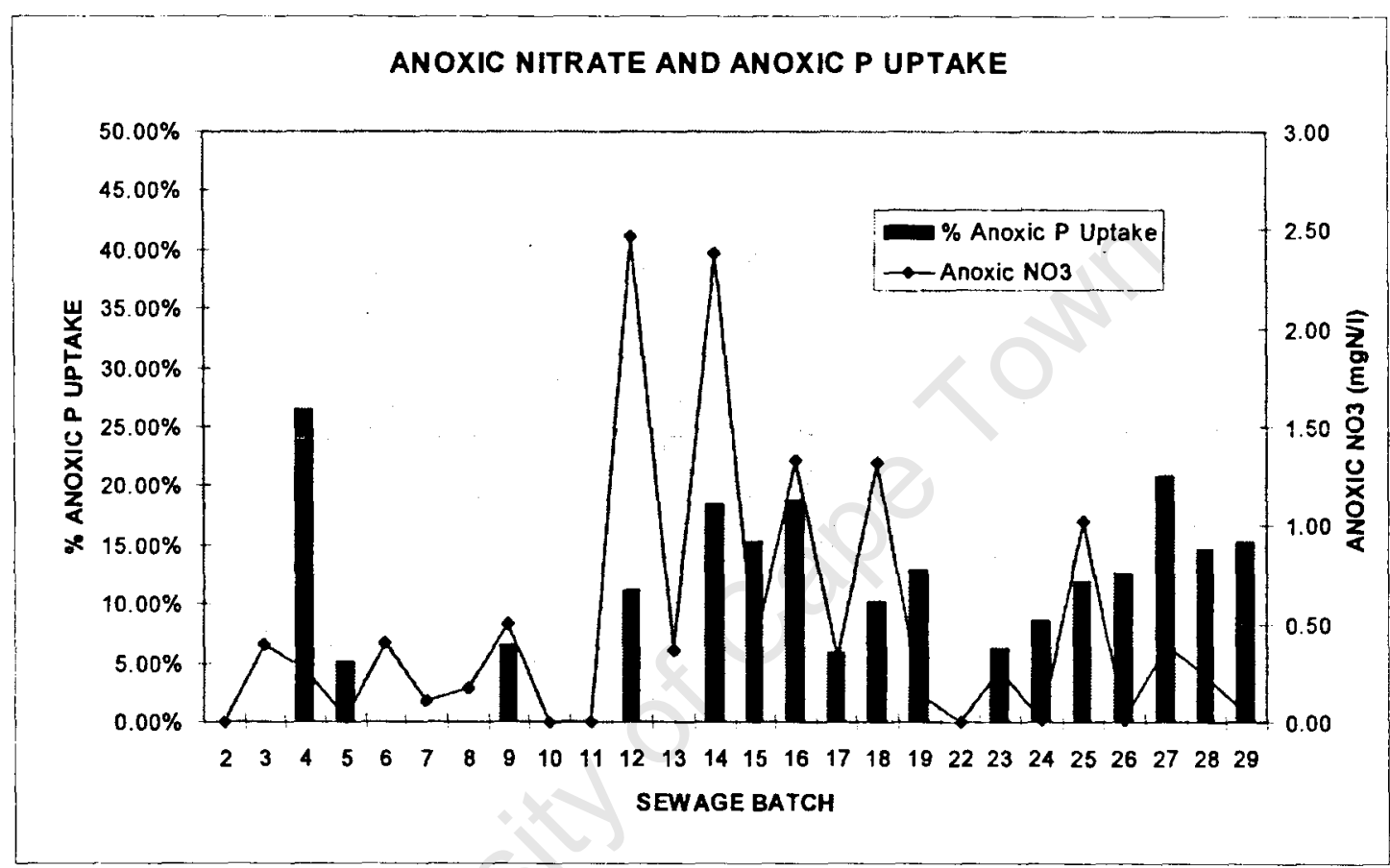

Figure 4.41: The frequency of anoxic P-uptake may be linked to the concentration of anoxic $\mathrm{NO}_{3}$.

\subsection{THEORETICAL EVALUATION OF ND AND BEPR PERFORMANCE IN THE MBR SYSTEM}

Theoretical evaluations of nitrification/denitrification (ND) and biological excess phosphorus removal (BEPR) were undertaken to assess the MBR UCT system performance.

\subsubsection{Nitrification}

In order to predict the nitrification and denitrification performance of the MBR UCT system, and hence the effluent $\mathrm{NO}_{3}$ concentrations, the steady state design model for BNR systems (WRC, 1984; WRC, in prep.) was used and predictions compared with measurements. 
For each steady state period the nitrification capacity of the system was predicted from (WRC, 1984):

$$
\mathrm{N}_{\mathrm{C}}=\mathrm{N}_{\mathrm{ti}}-\mathrm{N}_{\mathrm{S}}-\mathrm{N}_{\mathrm{te}}
$$

Where: $\mathrm{N}_{\mathrm{ii}} \quad=$ sewage batch average TKN in influent $(\mathrm{mgN} / \ell)$

$\mathrm{N}_{\mathrm{S}} \quad$ = nitrogen bound up in sludge production and wasted from the system (mgN/ $\ell$ influent)

$$
=\frac{f_{N} M X_{V}}{R_{S} Q_{i}}
$$

$\mathrm{f}_{\mathrm{N}}=$ fractional content of nitrogen in volatile suspended solids (VSS), (mgN/mgVSS)

$\mathrm{M}_{\mathrm{Xv}}$ = total mass of solids (VSS) in the system (mgVSS)

$\mathrm{R}_{\mathrm{S}} \quad=$ sludge age, taken as 20 (d)

$\mathrm{Q}_{\mathrm{i}} \quad=$ batch average influent flow, taken as $140(\ell)$

$\mathrm{N}_{\mathrm{te}} \quad=$ the effluent TKN concentration, calculated below $(\mathrm{mgN} / \ell)$ :

From nitrification theory (WRC, 1984) with a maximum specific growth rate of nitrifiers $\left(\mu_{\mathrm{nm}}\right)=0.45 / \mathrm{d}$ and half saturation constant $\mathrm{K}_{\mathrm{N}}=1.0 \mathrm{mgN} / \ell$, the effluent ammonia concentration $\left(\mathrm{N}_{\mathrm{ae}}\right)$ could be calculated. Thereafter, the effluent TKN concentration $\left(\mathrm{N}_{\mathrm{te}}\right)$ :

$\mathrm{N}_{\mathrm{te}}=\mathrm{N}_{\mathrm{ae}}+\mathrm{N}_{\text {ouse }}$

Where: $\mathrm{N}_{\text {ouse }}=$ effluent organic unbiodegradable soluble nitrogen, taken as the batch average difference between effluent TKN and FSA, see Section 4.4.7.2.

For comparison, the measured $\mathrm{N}_{\mathrm{te}}$ and $\mathrm{N}_{\mathrm{ae}}$ values were used. $\mathrm{N}_{\mathrm{C}}$ was measured as:

$\mathrm{N}_{\mathrm{C}}=\mathrm{N}_{\mathrm{ne}}+\mathrm{N}_{\mathrm{nd}}$

Where: $\mathrm{N}_{\mathrm{ne}} \quad=$ Effluent nitrate concentration

$\mathrm{N}_{\mathrm{nd}}=$ Nitrate denitrified in the anoxic (and anaerobic reactors), calculated from a nitrate mass balance around the anoxic and aerobic reactors.

From comparison of the predicted and measured values, Table 4.11:

- The measured $\mathrm{N}_{\mathrm{ae}}$ values were typically higher than those predicted, 0.63 $\mathrm{mgN} / \ell$ vs $0.24 \mathrm{mgN} / \ell$ respectively. This could be influenced by the sensitivity of the FSA test which, as discussed earlier, is not very sensitive at low concentrations. However, in the Phase 1 investigation Ramphao et al. (2004) found a substantial difference between the $\mathrm{N}_{\mathrm{ae}}$ measured and that predicted, $0.51 \mathrm{mgN} / \ell$ vs $2.16 \mathrm{mgN} / \ell$. This suggests that for modelling purposes a higher nitrifier half saturation constant $\left(\mathrm{K}_{\mathrm{N}}\right)$ value should be used. In this investigation using $\mathrm{K}_{\mathrm{N}}=2.9 \mathrm{mgN} / \ell$ as opposed to $1.0 \mathrm{mgN} / \ell$ gave similar measured and predicted $\mathrm{N}_{\mathrm{ae}}$ values $(0.70 \mathrm{mg} \mathrm{N} / \ell$ for both). 
Table 4.11: Theoretical evaluation of NDBEPR in the MBR system compared to measured values in Phase 2 and evaluation results from Phase 1 (Ramphao, et al., 2004)

\begin{tabular}{|c|c|c|c|c|c|c|c|c|c|c|c|c|c|c|c|c|c|c|c|c|c|c|c|c|c|c|c|c|c|c|c|}
\hline & & & & & & & & & & & & & & & & & & & & & & & & & & & & & & & \\
\hline \multirow{3}{*}{ 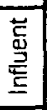 } & $5 \mathrm{ti}$ & (mgCODM) & 958 & 1148 & 1022 & 992 & 912 & 835 & 785 & 980 & 1006 & 976 & 888 & 856 & 870 & 934 & 971 & 926 & 914 & 1071 & & 1009 & 941 & 787 & 909 & 989 & 969 & 1116 & 983 & 151.5 & \\
\hline & $\mathrm{Nti}$ & $(\mathrm{mgN}$ & 101 & 39 & 97 & 113 & 34 & 93 & 27 & 118 & 91 & 93 & 132 & 101 & & 09 & 109 & 93 & 100 & 110 & & 112 & 105 & 77 & 103 & 102 & $\overline{01}$ & & 95 & & \\
\hline & $\mid$ Influent T & $\mathrm{mgN} / \mathrm{mgCO}$ & 0.11 & 0.12 & 0.09 & 0.11 & 15 & 0.11 & 0.16 & 0.12 & 0.09 & 0.10 & 0.15 & 0.12 & 0.13 & 0.12 & 0.11 & 0.10 & 0.11 & 0.10 & & 11 & 0.11 & 0.10 & 0.11 & 0.10 & 0.10 & 09 & 0.10 & 1 & \\
\hline & d & $\mathrm{mgN} /$ & 76.7 & 113.0 & 72.2 & 85.6 & 5.5 & 67.6 & 1014 & 3.2 & 65.3 & 68.5 & 108.5 & 78.2 & 897 & 83.2 & 82.8 & 66.1 & 71.1 & 827 & & 91.7 & 83.2 & 541 & 81.2 & 784 & 48 & 35 & $\overline{8.2}$ & & \\
\hline & & & 128.9 & 128.0 & 45.2 & 60.1 & 3.5 & 61.5 & 84.8 & & 9 & 73.6 & 13.3 & 60.0 & 85.9 & 76.9 & 73.9 & 46.2 & 59.5 & 54 & & 4.3 & 70.8 & 99 & 1353 & 75.3 & 47 & 87.0 & 2.6 & & \\
\hline & $N_{s}$ & $\mathrm{~N} / \mathrm{I}$ & 22.4 & 24.6 & 23.0 & 25.7 & 272 & 24.2 & 25.2 & 23.6 & 24.8 & 24.0 & 22.6 & 21.9 & 21.6 & 25.1 & 24.5 & 26.1 & 27.9 & 26 . & & 19.3 & 19.9 & 21.1 & 20.6 & 22.9 & 25.1 & 26.9 & 256 & & \\
\hline & Nte-p & $\mathrm{gNn}$ & 2.3 & 1.8 & 1.9 & 1.3 & 0.9 & & 0.7 & 1.0 & 1.4 & 0.8 & 0.7 & 1.3 & 1.1 & 0.3 & 1.6 & 1.2 & 1.1 & 1. & & 10 & 1.8 & 1.3 & 09 & 0.8 & 1.3 & 0.9 & 1.2 & & \\
\hline & & & 3 & 3.9 & 3.0 & 2.2 & & & 1 & & & 0.9 & & 1. & & 1.3 & 1.8 & 23 & 1. & & & & & 1.25 & & & & & & & \\
\hline & Nae-p & $\mathrm{NA}$ & 0.2 & 0.2 & 0.2 & 0 & & & 0. & 0 & 0.2 & 0.2 & 0.2 & 0.2 & 0.2 & 0.2 & 0.2 & 0.2 & 0.2 & 0 & & & 0.2 & & & 0.2 & 0.2 & & & & \\
\hline & Nae-n & & 1.2 & 2.4 & 1.3 & 1.2 & 1. & 0 & 0.5 & 0.7 & 04 & 0.3 & 0.6 & 0.4 & 0.4 & 1.3 & 0.4 & 1.3 & 1.0 & 0.5 & & 0.5 & 0.3 & 0.0 & 04 & 0.5 & 03 & 08 & & & \\
\hline & & & 49.0 & 55.1 & 46.6 & 45.3 & & 5.8 & 2.3 & 43.3 & 46.0 & & 39.4 & 40.0 & 38.9 & 39.5 & 42.1 & 40.1 & 35.2 & & & & 43.5 & 31.8 & 40.1 & 43.7 & 43.2 & 406 & 2.3 & & \\
\hline & Dpp. & $\mathrm{mgN} / \mathrm{mgCO}$ & 104.0 & 104.2 & 34.5 & 45.0 & 54.7 & 47.7 & 67.5 & 48.7 & 52.8 & 58.3 & 80.3 & 46.8 & 64.0 & 58.4 & 540 & 34.4 & 44.1 & 41. & & 74.5 & 54.3 & 53.9 & 04.4 & 58.3 & 64.9 & 663 & 393 & 58.12 & \\
\hline & $a-o p t-p$ & & 18 & 1.0 & 1.8 & 1.1 & 0.6 & 1.1 & 0.5 & 0.9 & 24 & 1.7 & 06 & \begin{tabular}{|l|}
1.0 \\
\end{tabular} & 08 & 0.9 & 10 & 1.6 & 1.0 & 1. & & 10 & \begin{tabular}{|l|}
1.1 \\
\end{tabular} & 1.4 & 1.0 & 1.3 & 1.4 & 1.2 & & & \\
\hline & a-sel & & 42 & & 3.3 & 3.0 & 3.0 & & 4.0 & 3.0 & & 3.8 & 40 & 3.7 & 3.4 & 32 & 3.0 & 3.0 & 3.2 & & & 38 & 3.4 & 3.4 & 3.5 & 3.4 & 34 & 3.3 & & 3.43 & \\
\hline & & & 48.8 & 55.0 & 46.6 & 5.2 & 37.4 & 35.9 & 32.4 & 43.3 & 45.9 & 43.4 & 394 & 39.8 & 38.7 & 39.6 & & 4012 & 35.2 & & & 6.1 & & & & & & & & & \\
\hline & & $\mathrm{mgN} /$ & 61.2 & 90.6 & 547 & 3.5 & 787 & 52.4 & 80.8 & 69.4 & \begin{tabular}{|l|}
51.4 \\
\end{tabular} & 54.2 & 86.6 & 61.3 & 69.2 & 62.8 & 61.8 & 48.8 & 537 & 63.2 & & 72.2 & \begin{tabular}{|l|}
641 \\
\end{tabular} & 19 & 63. & 606 & 6 & 558 & 08 & 9.95 & \\
\hline & & $\mathrm{mgNA}$ & 27.9 & 57.9 & 25.6 & & 68.1 & 31.7 & 69.0 & 49.8 & 19.4 & 25.1 & 69.1 & & 51.0 & 436 & 40.6 & 59 & 35.9 & 38.5 & & 45.6 & \begin{tabular}{|l|}
396 \\
\end{tabular} & 22.4 & 41.2 & 347 & 6 & 32.8 & 25.8 & & $\overline{4}$ \\
\hline & & & & & 107 & & 18.7 & 13.7 & 173 & 17.1 & 14.1 & 15 & 23.0 & & 219 & 18.5 & & & 15.4 & 12 & & 19 & & 6.0 & 10 & & & 20.7 & 3.3 & & \\
\hline & NIIE- d SEI & $\mathrm{mgNA}$ & 146 & 20.3 & 164 & 21.2 & 26.1 & 15.0 & 204 & 23.4 & 137 & 14.3 & 215 & 16.6 & 204 & 9.4 & 208 & 16.2 & 16.6 & 19.3 & & 19.2 & 190 & 12.4 & 179 & 177 & 17.2 & 17.2 & 17.1 & 1837 & 23. \\
\hline
\end{tabular}


- The differences in $\mathrm{N}_{\mathrm{ae}}$ are reflected in the $\mathrm{N}_{\mathrm{te}}$ values, and the correction to $\mathrm{K}_{\mathrm{N}}$ above brings the predicted and measured $\mathrm{N}_{\mathrm{te}}$ values very close (1.62 and 1.61 $\mathrm{mgN} / \ell$ respectively).

- Due to doubts about the reliability of the $\mathrm{NO}_{3}$ data up until sewage batch 9 only the sewage batch average values from sewage batch 9 onwards were taken into consideration in calculating the measured and predicted $\mathrm{N}_{\mathrm{C}}$ values. Their respective averages were very close, $76.16 \mathrm{mgN} / \ell$ versus $77.85 \mathrm{mgN} / \ell$, which correspond to the values reported in Ramphao et al. (2004), of 75 $\mathrm{mgN} / \ell$ for both measured and predicted values.

From the above observations, nitrification in the system can be closely predicted by the existing nitrification theory, and hence this theory can be applied directly to the design of MBR BNR systems. The unbiodegradable soluble organic nitrogen fraction determined in this investigation, taking into account the dosing of FSA into the influent, was 0.009 and should be used in design. Additionally, in this investigation the half saturation constant for the nitrifiers, $\mathrm{K}_{\mathrm{N}}$, was increased to $2.6 \mathrm{mgN} / \ell$ to match measured and calculated $N_{a e}$ (and $N_{t e}$ ) values. This adjustment in $K_{N}$ is however largely attributed to the insensitivity of TKN and FSA testing at very low concentration such as were measured in the MBR effluent. Thus a revision of $K_{N}$ is not recommended.

\subsubsection{Denitrification}

The theoretical denitrification potential of the primary anoxic rector $\left(D_{p p}\right)$ in the MBR UCT system was calculated according to the procedure set out in the new steady state design denitrification theory for NDBEPR systems, Equation (4.15) (WRC, in prep):

Where:

$$
D_{P P}=\frac{S_{b s N}(1+r)\left(1-f_{C V} Y_{H}\right)}{2.86}+\frac{f_{X 1} K^{\prime}{ }_{2 T}\left(S_{b i}-S_{s e q}\right) Y_{H} R s}{\left(1+b_{H T} R s\right)}
$$

$S_{b s N}=$ concentration of fermentable RBCOD leaving the anaerobic reactor, calculated according to Wentzel et al. (1990) (mgCOD/ $\ell$ )

$Y_{H}=$ the $\mathrm{OHO}$ and $\mathrm{PAO}$ biomass yield $=0.45 \mathrm{mgCOD} / \mathrm{mgCOD}$

$r \quad=$ recycle ratio, anoxic to anaerobic reactors

$2.86=$ the oxygen equivalence of nitrate (measured as $1 \mathrm{mgN}-\mathrm{NO}_{3}$ )

$f_{X 1} \quad=$ primary anoxic mass fraction (from Table 4.3)

$K^{\prime}{ }_{2 T}=\mathrm{OHO}$ specific denitrification rate in NDBEPR systems

$=0.145 \mathrm{mgN} / \mathrm{mgVSS} / \mathrm{d}$ at $20^{\circ} \mathrm{C}$

$S_{b i} \quad=$ influent biodegradable COD concentration $(\mathrm{mgCOD} / \ell)$

$=\mathrm{S}_{\mathrm{ti}}\left(1-\mathrm{f}_{\mathrm{S}, \text { us }}-\mathrm{f}_{\mathrm{S} \text {,up }}\right)$ with $\mathrm{f}_{\mathrm{S} \text {,us }}$ and $\mathrm{f}_{\mathrm{S}, \text { up }}$ available from Table 4.7 , Section 4.8 .

$S_{\text {seq }}=$ substrate sequestered by PAO's in the anaerobic reactor, calculated according to Wentzel et al. (1990) (mgCOD/ $\ell$ influent)

$b_{H T}=$ the rate of endogenous respiration for OHO's $(0.24 \mathrm{mgVSS} / \ell$ at $\left.20^{\circ} \mathrm{C}\right)$

Actual denitrification achieved was calculated from a mass balance around the anoxic and anaerobic reactors (as some, though very little, denitrification did occur in the 
anaerobic reactor as the r-recycle contained small amounts of measurable $\mathrm{NO}_{3}$ ). Comparing the predicted $\mathrm{D}_{\mathrm{PP}}$ with the actual denitrification (Table 4.11):

- Generally in all but three cases the DPP predicted was substantially less than that measured, $41.4 \mathrm{mgN} / \mathrm{mgCOD}$ versus $58.1 \mathrm{mgN} / \mathrm{mgCOD}$ despite the fact that virtually all nitrate entering the anoxic reactor was used $(\sim 0.5 \mathrm{mgN} / \ell$ anoxic nitrate) indicating that denitrification was limited by the nitrate load not the denitrification kinetics. Ramphao et al. (2004) observed similar deviations between measured and predicted values, $40.1 \mathrm{mgN} / \mathrm{mgCOD}$ and $54.1 \mathrm{mgN} / \mathrm{mgCOD}$ respectively.

- Reasons cited by Ramphao et al. (2004) for the difference were either i) incorrect $\mathrm{K}_{2 \mathrm{~T}}$ values for the OHOs in the Equation 4.15, or ii) anoxic P uptake that was observed in the system which implies denitrification by the PAOs, which is not included in the theoretical $D_{P P}$ equation, or both. The extent of denitrification with associated $\mathrm{P}$ uptake by the PAOs appears to be very variable (Ekama and Wentzel, 1999), from near zero anoxic P uptake (Clayton et al., 1989; 1991) to anoxic $\mathrm{P}$ uptake dominant over aerobic $\mathrm{P}$ uptake (Sorm et al., 1996). Experimental evidence tends to suggest that the magnitude of $P$ uptake is influenced by the anoxic mass fraction and the mass of $\mathrm{NO}_{3}$ loaded on the anoxic reactor relative to its denitrification potential (Hu et al., 2002) and this was supported by the observations here. However, as yet, it does not seem possible to make a definite statement as to exactly which conditions will induce the presence of denitrifying PAOs and associated anoxic P uptake in NDBEPR systems, or what the relative magnitude of these will be. Accordingly, it is difficult to incorporate such a process into predictive models. Clearly, further investigation is required in this area. In the meantime, denitrification design with existing theory will tend to be conservative, and performances exceeding predictions can be expected.

- Hence, in order to improve the denitrification predictions for this investigation the $\mathrm{K}_{2(20)}^{\prime}$ value was increased from $0.145 \mathrm{mgN} / \mathrm{mgCOD} / \mathrm{d}$ to $0.216 \mathrm{mgN} /$ $\mathrm{mgCOD} / \mathrm{d}$ at $20^{\circ} \mathrm{C}$ to match the average $\mathrm{D}_{\mathrm{PP}}$ predicted with that measured. This value falls within the range of values reported for $\mathrm{K}_{2 \mathrm{~T}}$ by $\mathrm{Hu}$ et al., (2002) for NDBEPR systems, $0.05-0.32 \mathrm{mgN} / \mathrm{mgVSS} / \mathrm{d}$.

\subsection{3 a-Recycle Ratio}

In evaluating the MBR system theory, the a-recycle noted below is the as-recycle, as described in Chapter 3. The optimum a-recycle ratio $\left(a_{o p t}\right)$, is the a-recycle that matches the nitrate load $\left(\mathrm{N}_{\mathrm{nL}}\right)$ to the primary anoxic reactor with its denitrification potential $\left(D_{P P}\right)$, i.e. at $a_{o p t} D_{P P}=N_{n L}$. The $N_{n L}$ is calculated as follows:

$$
N_{n L}=\frac{a s \cdot N_{C}}{(1+a s)}
$$

Where:

as $\quad=$ the as-recycle

$\mathrm{N}_{\mathrm{C}} \quad=$ the nitrification capacity, as calculated earlier in this chapter 
$\mathrm{a}_{0 \mathrm{pt}}$ values were determined for both the standard $\left(\mathrm{K}_{2(20)}^{\prime}=0.145 \mathrm{mgN} / \ell\right)$ and adjusted $\left(\mathrm{K}_{2(20)}^{\prime}=0.216 \mathrm{mgN} / \mathrm{mgCOD} / \mathrm{d}\right)$ theoretical $\mathrm{D}_{\mathrm{PP}}$ values and are presented in Table 4.11:

- a-opt values for $D_{P P}$ predicted (pred.) $=\mathrm{N}_{\mathrm{nL}}$ pred. were very low, 1.2 , in comparison to the measured a-recycle values in the system of 3.45:1. However this is clearly influenced by the $D_{\mathrm{PP}}$ pred. values which were substantially lower than the $\mathrm{D}_{\mathrm{PP}}$ values measured.

- Calculating $\mathrm{a}_{\text {opt }}$ with $\mathrm{D}_{\mathrm{PP}}$ pred. adjusted by increasing $\mathrm{K}_{2(20)}^{\prime}$ to $0.216 \mathrm{mgN} / \mathrm{mgCOD} / \mathrm{d}$ gives a higher $\mathrm{a}_{\mathrm{opt}}, 2.5: 1$, which is much closer to the measured a-recycle $\left(\mathrm{a}_{\mathrm{msrd}}\right)$.

- The average measured $D_{P P}$ and $N_{n L}$ in the anoxic reactor were very close, $58.1 \mathrm{mgN} / \ell$ and $58.6 \mathrm{mgN} / \ell$ respectively which corresponds closely with the measured anoxic $\mathrm{NO}_{3}$ concentration of $0.5 \mathrm{mgN} / \ell$ which would be the difference between the two. This indicates that on average the a-recycle was operating very close to, or just above the theoretical a-opt recycle.

\subsubsection{Effluent Nitrate Concentration}

At $\mathrm{N}_{\mathrm{nL}} \leq \mathrm{D}_{\mathrm{PP}}$ in the MBR UCT configuration the effluent $\mathrm{NO}_{3}$ concentration $\left(\mathrm{N}_{\mathrm{ne}}\right)$ is given by Equation 4.10. With Equation $4.10 \mathrm{~N}_{\text {ne }}$ is calculated for each sewage batch with the theoretical $\mathrm{a}_{\mathrm{opt}}$ and $\mathrm{a}_{\mathrm{msrd}}$. As noted above, measured anoxic reactor $\mathrm{NO}_{3}$ concentrations were negligible indicating that on average $N_{n L} \geq D_{P P}$, but only marginally so (anoxic $\mathrm{NO}_{3}=0.5 \mathrm{mgN} / \ell$ ). Measured and predicted $\mathrm{N}_{\mathrm{ne}}$ values are listed in Table 4.11.

- At $\mathrm{a}_{\text {opt }}=1.2$ a very high $\mathrm{N}_{\mathrm{ne}}$ is calculated of $40.0 \mathrm{mgN} / \ell$, which is reasonable since at this a-recycle far less $\mathrm{NO}_{3}$ is recycled to the anoxic reactor for denitrification. However, using the higher $\mathrm{a}_{\mathrm{opt}}=2.5$ calculated with the increased $K_{2}^{\prime}$ gives a lower $N_{n e}=23.1 \mathrm{mgN} / \ell$, which is far closer to the $\mathrm{N}_{\text {ne }}$ observed below.

- At the measured a-recycle ratio, the measured and predicted $\mathrm{N}_{\text {ne }}$ values are very close, $17.5 \mathrm{mgN} / \ell$ and $17.0 \mathrm{mgN} / \ell$ respectively. This is expected since the measured and predicted $N_{C}$ values are close and effectively the same arecycle value applies to both. This does however provide indirect confirmation of the value accepted for the a-recycle ratio. 


\subsubsection{Theoretical BEPR Performance}

In order to simulate the theoretical BEPR performance of the MBR system, the steady state design model of Wentzel et al. (1990) was used. The model required the readily biodegradable COD (RBCOD) concentration of the influent sewage to be known. This was measured in a separate system set up specifically for this purpose (Ekama et al., 1986) described in Section 4.3. Other waste water characteristics required as input to the model were obtained from the averages of the measured values for each sewage batch period $\left(\mathrm{S}_{\mathrm{ti}}\right)$ as well as from the estimated $\mathrm{f}_{\mathrm{S} \text {, us }}$ and $\mathrm{f}_{\mathrm{S} \text {, up }}$ values for these periods (Section 4.4.5). Operational parameters $\left(\mathrm{Q}_{i}, \mathrm{R}_{\mathrm{S}}\right.$, anaerobic mass fraction, r-recycle ratios) required in the model were also taken from batch average values, and the default kinetic and stoichiometric constants of Wentzel et al. (1990) were accepted. The design model equations have not been included in the text but are as in Wentzel et al. (1990).

In the steady state design model of Wentzel et al. (1990), PAO active mass has a higher $\mathrm{P}$ content to account for the polyphosphate stored by these organisms, expressed as a fractional $\mathrm{P}$ content, $\mathrm{f}_{\mathrm{XGBP}}$, in $\mathrm{mgP} / \mathrm{mgVSS}$. Wentzel et al. (1990) assigned a value of $f_{X G B P}=0.38 \mathrm{mgP} / \mathrm{mgVSS}$ to this constant. This was based on the $P$ removal response observed in BEPR systems with predominately aerobic $P$ uptake, and has been confimed in subsequent model applications to investigations with similarly dominant aerobic P uptake (Hu et al., 2002). However, in applications of the model to systems with anoxic $\mathrm{P}$ uptake, to account for the lower $\mathrm{P}$ removal observed in these systems, Ekama and Wentzel (1999) found that the value for $f_{X G B P}$ had to be reduced significantly, into the range of 0.1 to $0.26 \mathrm{mgP} / \mathrm{mgVSS}$ (Hu et al., 2002). Since anoxic $P$ uptake was observed in the MBR UCT system here, a similar reduction in $f_{X G B P}$ was expected. Accordingly, for each sewage batch the value for $f_{X G B P}$ was varied to match the model predicted $P$ removal to that measured, see Fig. 4.37 .

- The results varied considerably from sewage batch to sewage batch with a range of $0.153-0.425 \mathrm{mgP} / \mathrm{mgVSS}$ and an average of $0.303 \mathrm{mgP} / \mathrm{mgVSS}$ for the different sewage batches. Up to sewage batch 22 no explicit relationship between anoxic $P$ uptake and the $f_{X B G P}$ value could be discovered, probably due to the sewage batch periods being shorter than the sludge ages, and the increase in $\mathrm{P}$ removal towards steady state noted above. From sewage batch 22 , as the anoxic uptake increased, the $\mathrm{f}_{\mathrm{XBGP}}$ value decreased, and visa versa, in conformity with the observations of Ekama and Wentzel (1999).

- Using the average $f_{X B G P}=0.303 \mathrm{mgP} / \mathrm{mgVSS}$, the model was used to predict $P$ removal, Table 4.11 and Figs. 4.38 and 4.39. Overall the predictions are reasonably close to the measured values. 
Table 4.11: Comparison of measured and predicted $P$ release, $P$ uptake and $P$ removal, and the fraction $P$ content of $P A O s\left(f_{X B G P}\right)$ for the $M B R$ system, and the \% differences

\begin{tabular}{|c|c|c|c|c|c|c|c|c|c|c|}
\hline \multirow{2}{*}{$\begin{array}{l}\text { Sewage } \\
\text { Batch \# }\end{array}$} & \multicolumn{3}{|c|}{ P-release } & \multicolumn{3}{|c|}{ P-uptake } & \multicolumn{3}{|c|}{ P-removal } & \multirow[t]{2}{*}{ fxgbp } \\
\hline & meas & pred & $\%$ dif & meas & pred & $\%$ dif & meas & pred & \%dif & \\
\hline & $(\mathrm{mgP} / \mathrm{l})$ & $(\mathrm{mgP} / \mathrm{l})$ & & $(\mathrm{mgP} / \mathrm{l})$ & $(\mathrm{mgP} / \mathrm{l})$ & & $(\mathrm{mgP} / \mathrm{l})$ & $(\mathrm{mgP} / \mathrm{l})$ & & \\
\hline 2 & -138.9 & & & 133.1 & & & -5.8 & 6.9 & & \\
\hline 3 & -159.8 & -164.7 & $3 \%$ & 182.6 & 197.4 & $7 \%$ & 22.8 & 32.7 & $30 \%$ & 0.24 \\
\hline $4 x^{2}=$ & -110.5 & -136.5 & $19 \%$ & 127.8 & 164.1 & $22 \%$ & 17.3 & 27.6 & $38 \%$ & 0.205 \\
\hline 5 & -93.2 & -154.4 & $40 \%$ & 110.4 & 185.4 & $40 \%$ & 17.2 & 31.6 & $45 \%$ & 0.165 \\
\hline 6 & -89.7 & -126.6 & $29 \%$ & 112.1 & 153.8 & $27 \%$ & 22.3 & 27.2 & $18 \%$ & 0.287 \\
\hline 7325 & -134.6 & -119.4 & $-13 \%$ & 153.3 & 144.2 & $-6 \%$ & 18.7 & 24.8 & $25 \%$ & 0.25 \\
\hline 8 & -123.2 & -121.6 & $-1 \%$ & 141.7 & 125.6 & $-13 \%$ & 18.5 & 22.1 & $16 \%$ & 0.29 \\
\hline 9 & -108.9 & -140.2 & $22 \%$ & 129.2 & 169.7 & $24 \%$ & 20.3 & 29.5 & $31 \%$ & 0.225 \\
\hline 10 xistats & -113.5 & -144.3 & $21 \%$ & 134.0 & 175.2 & $23 \%$ & 20.5 & 30.2 & $32 \%$ & 0.223 \\
\hline 11 & -112.8 & -131.6 & $14 \%$ & 127.9 & 159.2 & $20 \%$ & 15.0 & 27.6 & $45 \%$ & 0.153 \\
\hline 12 & -107.8 & -101.5 & $-6 \%$ & 129.7 & 123.8 & $-5 \%$ & 21.9 & 22.3 & $2 \%$ & 0.37 \\
\hline 13 & -143.6 & -131.3 & $-9 \%$ & 167.3 & 157.7 & $-6 \%$ & 23.8 & 26.3 & $10 \%$ & 0.33 \\
\hline 14 & & -97.8 & $-17 \%$ & 138.0 & 126.3 & $-9 \%$ & 23.7 & 22.1 & $-7 \%$ & 0.42 \\
\hline 15 & -124.0 & -105.3 & $-18 \%$ & 143.4 & 136.1 & $-5 \%$ & 19.4 & 24.3 & $20 \%$ & 0.275 \\
\hline 16 & -128.4 & -116.8 & $-10 \%$ & 153.4 & 143.1 & $-7 \%$ & 25.0 & 25.5 & $2 \%$ & 0.37 \\
\hline 17 & -121.4 & -95.4 & $-27 \%$ & 147.7 & 135.6 & $-9 \%$ & 26.4 & 24.6 & $-7 \%$ & 0.42 \\
\hline 18 & -114.3 & -123.1 & $7 \%$ & 141.1 & 147.5 & $4 \%$ & 26.8 & 25.6 & $-5 \%$ & 0.405 \\
\hline 19 & -100.1 & -135.2 & $26 \%$ & 130.6 & 161.3 & $19 \%$ & 30.4 & 28.9 & $-5 \%$ & 0.405 \\
\hline \multicolumn{11}{|l|}{$\frac{74}{21>254}$} \\
\hline \multicolumn{11}{|l|}{21,5} \\
\hline & -104.0 & -132.4 & $21 \%$ & 130.7 & 152.9 & $15 \%$ & 26.6 & 26.0 & $-2 \%$ & 0.39 \\
\hline $23, t)$ & -127.0 & -125.9 & $-1 \%$ & 150.5 & 151.9 & $1 \%$ & 23.5 & 26.0 & $10 \%$ & 0.335 \\
\hline $248 x$ & -135.5 & -110.7 & $-22 \%$ & 158.3 & 128.3 & $-23 \%$ & 22.9 & 21.2 & $-8 \%$ & 0.425 \\
\hline 25 & -117.2 & -145.0 & $19 \%$ & 145.0 & 173.1 & $16 \%$ & 27.8 & 28.0 & $1 \%$ & 0.375 \\
\hline 26 & -136.5 & -149.1 & $8 \%$ & 158.0 & 184.9 & $15 \%$ & 21.5 & 30.3 & $29 \%$ & 0.24 \\
\hline 27 & -128.7 & -135.8 & $5 \%$ & 149.9 & 163.7 & $8 \%$ & 21.2 & 27.9 & $24 \%$ & 0.26 \\
\hline $36 \times 4$ & -144.2 & -127.4 & $-13 \%$ & 163.9 & 182.2 & $10 \%$ & 19.7 & 31.5 & $37 \%$ & 0.193 \\
\hline $29^{6}$ & -112.8 & -143.4 & $21 \%$ & 139.2 & 179.2 & $22 \%$ & 26.3 & 30.2 & $13 \%$ & 0.315 \\
\hline Average & -120.2 & -128.6 & $5 \%$ & 142.6 & 156.9 & $8 \%$ & 22.4 & 27.0 & $16 \%$ & 0.303 \\
\hline
\end{tabular}

- There was a steady increase in $\mathrm{P}$ removal from the beginning of the investigation to sewage batch 18 (Fig. 4.40). As noted in Section 4.9.3 this possibly indicates the development of the slow growing PAO population in the system, which would suggest that the system PAO population had not reached steady state until around sewage batch 18 . Thus, for this period the model overpredicted the measured values.

- From Fig. 4.40 it is evident that there is a period in the middle of the investigation, from sewage batches $12-25$, where the predictions and the measured values correspond very closely. The calculated $f_{X B G P}$ for this period alone is $0.376 \mathrm{mgP} / \mathrm{mgVSS}$, which is very close to the value used in the predictions of $0.38 \mathrm{mgP} / \mathrm{mgVSS}$.

- From sewage batch 25 to the end of the investigation predicted and measured $\mathrm{P}$ releases correspond closely (Fig. 4.38), but predicted $\mathrm{P}$ removals are 
significantly higher than those observed (Fig. 4.40). During this period, observed anoxic P-uptake increased (Fig 4.37) which would cause the reduction in P-removal.

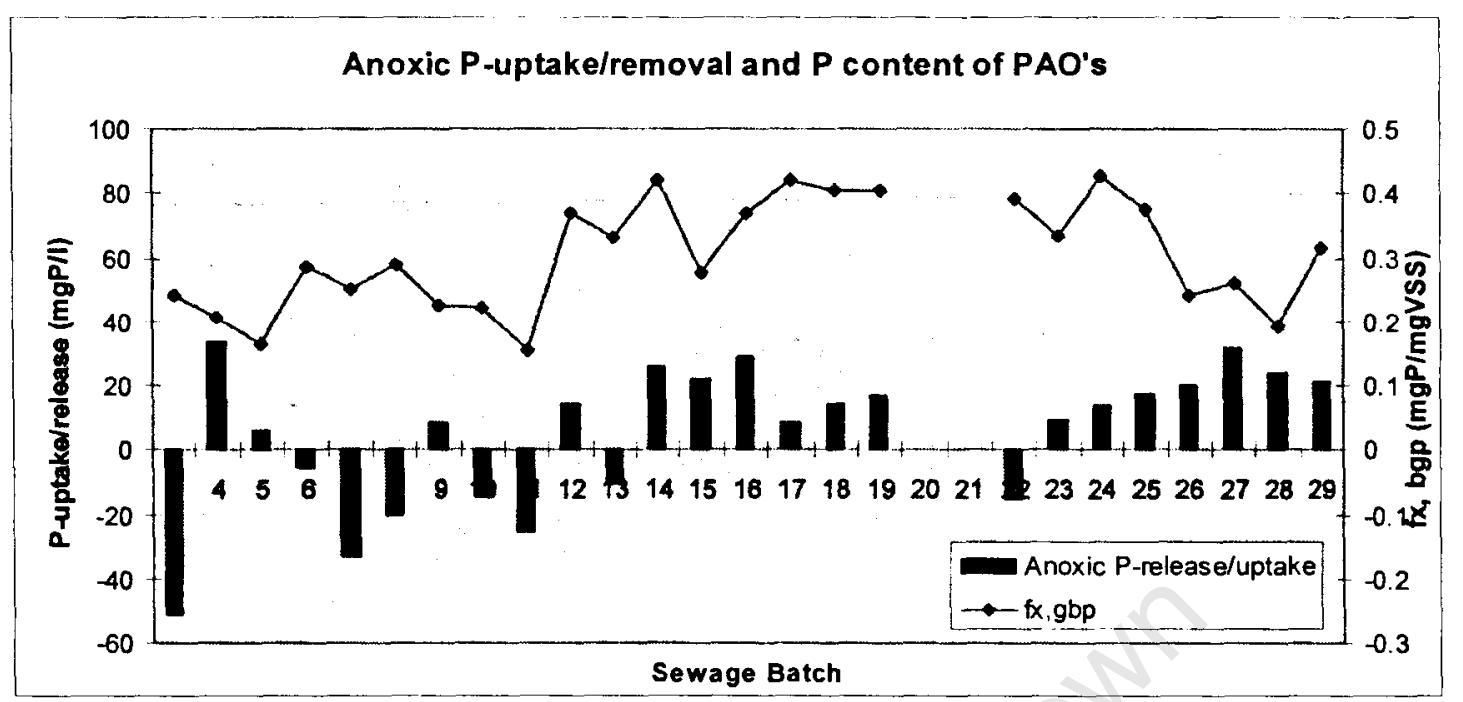

Figure 4.37: The P content of PAO's $\left(f_{X B G P}\right)$ and the P-update/release from the anoxic reactor.

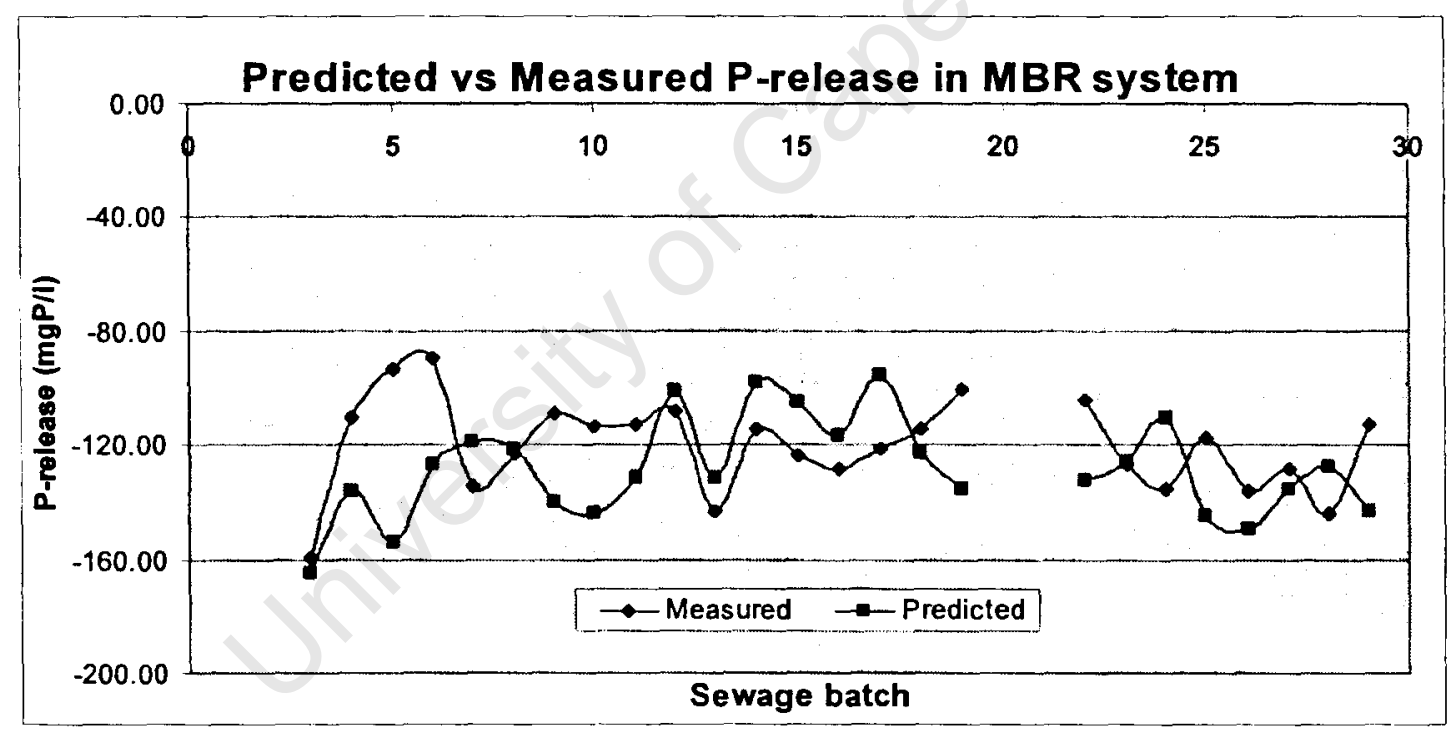

Figure 4.38: Comparison of predicted and measured $P$ release in the MBR system using $f_{X G B P}=0.38$. 


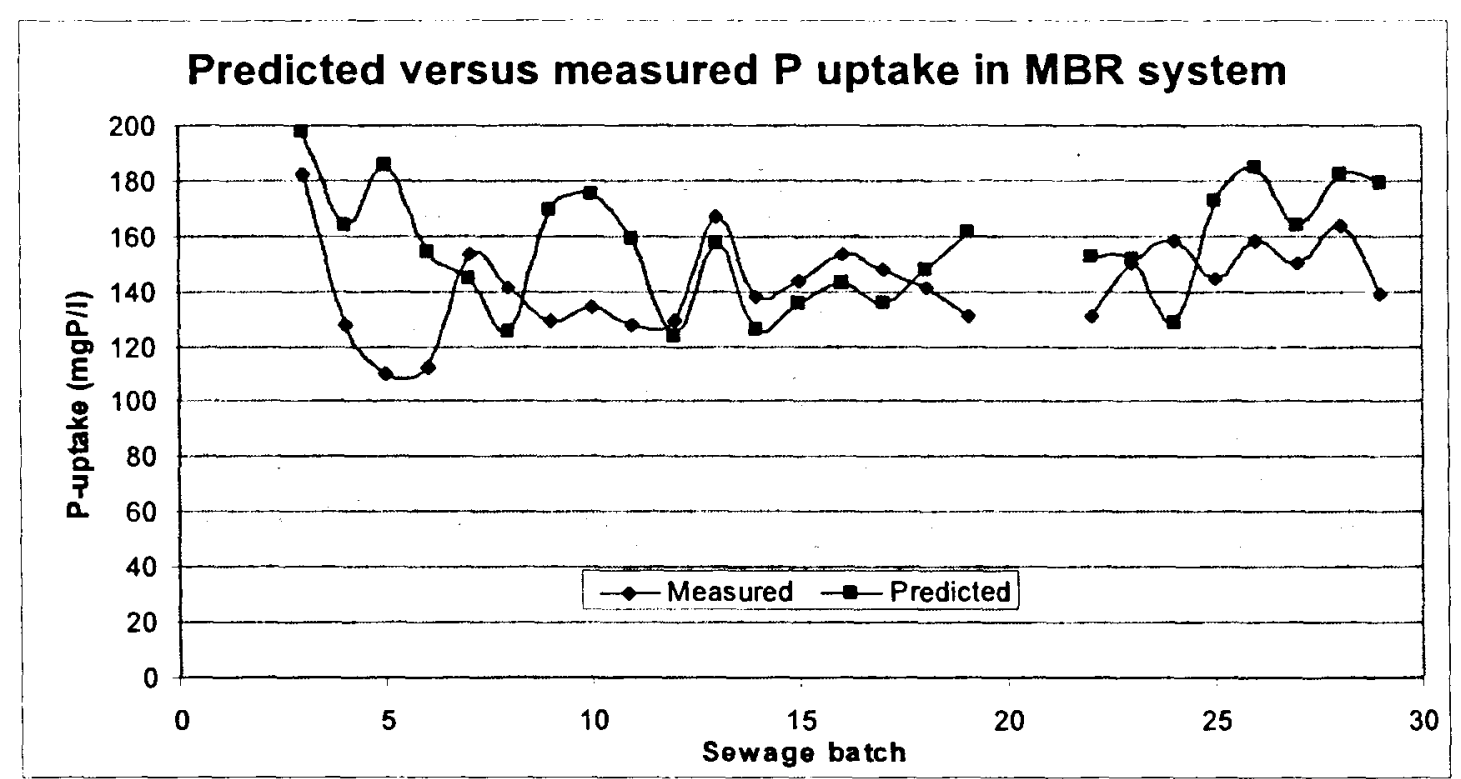

Figure 4.39: Comparison of predicted and measured $P$ uptake in the MBR system using $f_{X G B P}=0.38$

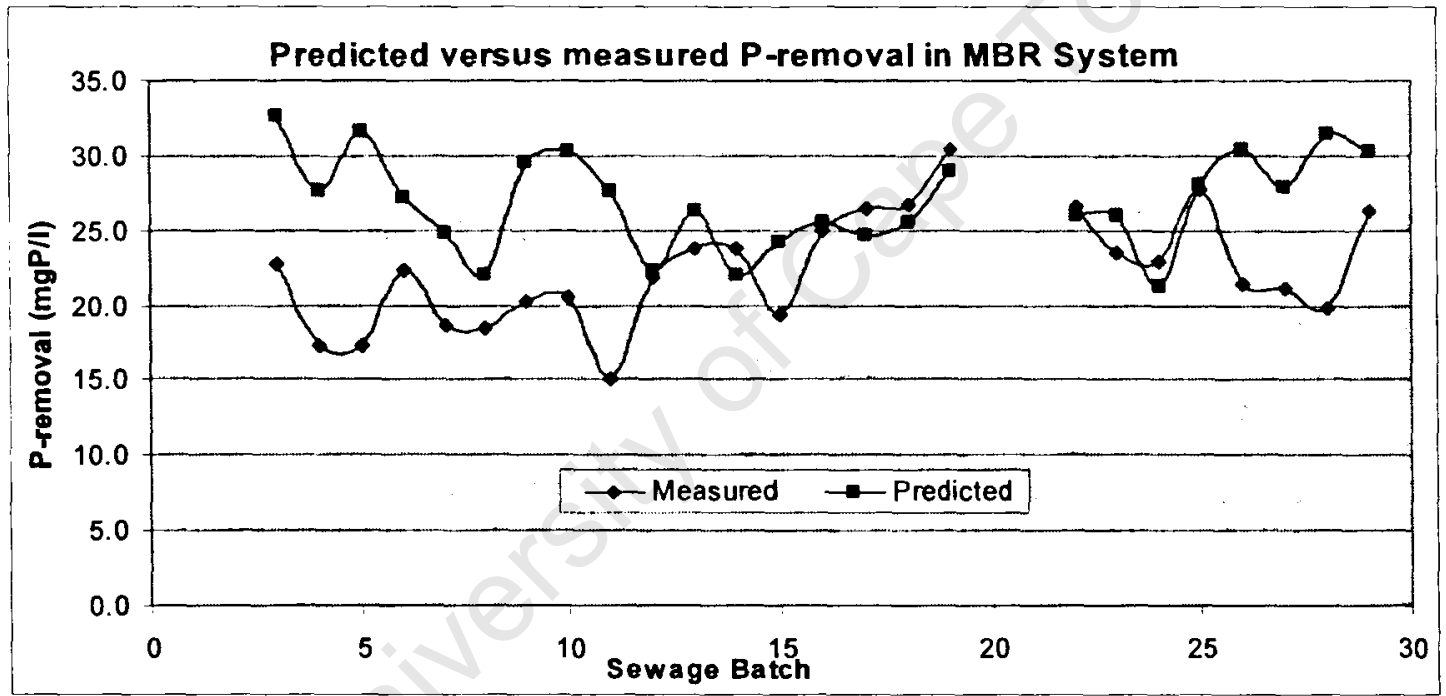

Figure 4.40: Comparison of predicted and measured $P$ removal in the MBR system keeping $f_{X B G P}=0.38$

\subsection{LONG SLUDGE AGE (40 DAY) INVESTIGATION}

\subsubsection{Introduction}

Following on from the Phase 2 investigation the sludge age of the MBR UCT system was increased to 40 days by adjusting the influent flow and sludge waste flows in order to maintain an MLSS concentration of approximately $18000 \mathrm{mgTSS} / \ell$. The system was operated and run by a BSc student, Muhimba et al. (2006) as partial fulfilment of his BSc (Eng) degree and results observed are reported here.

The observations of high sludge production and high calculated $f_{\mathrm{S} \text {,up }}$ values in Phase 2 of the investigation were of concern to the writer, hence it was proposed to run the system at a high sludge age in order to accent the sludge production in order that 
comparative observations could be made. In order to allow such a comparison all operational and design parameters were kept the same apart from the influent feed and sludge waste flows. Once steady state had been reached (after two sludge ages) the system was monitored for four sewage batch periods. The system data is presented in Appendix G.

\subsubsection{MLSS}

All sampling and testing was conducted as in Phase 2, (Chapter3, Section 3.5). On average a system sludge age of 39.5 days was achieved through controlled wasting from the aerobic reactor. Unexpected sludge loss did occur, however volumes lost were calculated and incorporated into the sludge wasting.

System MLSS concentrations varied considerably with ranges of 5000$7000 \mathrm{mgTSS} / \ell, 12000-17000 \mathrm{mgTSS} / \ell$ and $15000-20000 \mathrm{mgTSS} / \ell$ reported for the anaerobic, anoxic and aerobic reactors respectively. System VSS/TSS ratios were consistent within each reactor with $0.85 \mathrm{mgVSS} / \mathrm{mgTSS}, 0.82 \mathrm{mgVSS} / \mathrm{mgTSS}$ and $0.80 \mathrm{mgVSS} / \mathrm{mgTSS}$ reported for the anaerobic, anoxic and aerobic reactors respectively. These values are close to those reported in Phase 1 (Ramphao et al. 2004) and Phase 2. Initial $\mathrm{f}_{\mathrm{CV}}$ values were low (1.18 and 1.22 for sewage batches 1-2) however in sewage batches $3-4 \mathrm{f}_{\mathrm{CV}}$ values of 1.46 and 1.42 were obtained which are far closer to the expected $f_{C V}$ values. Similarly TKN/VSS values were particularly low for the first two sewage batches (0.077 and 0.072), but increased to values comparable to those in Phase 2 in the second two batch periods (0.095 and 0.090). These values indicated that MLSS concentrations were inconsistent in the first two sewage batches and only sewage batches 3-4 should be used for further analysis

A sludge production for the system was determined as $(18.8 \mathrm{mgVSS} / \mathrm{d}) /(\mathrm{mgCOD} / \mathrm{d})$. This value is substantially lower than sludge productions reported in Phase 1 and 2 of the project of 0.32 and 0.31 respectively. However a lower sludge production is expected for a longer sludge age as more sludge is degraded and COD is converted to $\mathrm{CO}_{2}$ through longer aeration.

\subsubsection{Mass Balances}

As a check for the accuracy of analytical measurements mass balances were performed as set out in Appendix C. Table 4.12. presents a summary of the system $\mathrm{N}$ and COD mass balances. Mass balances from sewage batches 1-2 were poor indicating that these sewage batches should be interpreted with caution. However sewage batches 3-4 fell within the range of acceptable confidence interval for mass balances of $90-110 \%$ and can be used for further analysis with confidence. Thus the previous observations of poor analytical results from sewage batches 1-2 are cofirmed.

Table 4.12: $\quad$ System $N$ and COD mass balances for the $M B R(R s=40 d)$ system.

\begin{tabular}{|c|c|c|}
\hline Sewage Batch \# & $\%$ Nitrogen Balance & $\%$ COD Balance \\
\hline 1 & $61 \%$ & $88 \%$ \\
\hline 2 & $89 \%$ & $92 \%$ \\
\hline 3 & $100 \%$ & $106 \%$ \\
\hline 4 & $99 \%$ & $99.23 \%$ \\
\hline
\end{tabular}




\subsubsection{System Removals}

System removals were typically consistent with the removals reported in Phases 1 and 2 of the investigation.

\section{COD removal}

Consistently low effluent COD concentrations were observed with an average COD concentration of $31.1 \mathrm{mgCOD} / \ell$ in the effluent. This value is lower than was observed in Phase $1(35.5 \mathrm{mgCOD} / \ell)$ and Phase $2(41.2 \mathrm{mgCOD} / \ell)$ and is attributed to the longer sludge age which may allow sufficient time to degrade COD otherwise considered unbiodegradable soluble.

\section{N removal}

High $\mathrm{N}$ removal was observed with investigation average effluent TKN and FSA concentrations of $1.9 \mathrm{mgN} / \ell$ and $1.0 \mathrm{mgN}-\mathrm{NH}_{4} / \ell$ respectively. Effluent nitrate concentrations of $20.7 \mathrm{mgN}-\mathrm{NO}_{3} / \ell$ and $0.9 \mathrm{mgN}-\mathrm{NO}_{2} / \ell$ were reported. All reported values were close to those observed in Phases 1 and 2 .

\section{P removal}

In order to assess $P$ removal performance a $P$ balance was set up across the system and is presented in Table 4.13. Only three sewage batch periods were evaluated due to difficulties with the spectrometer which measured $\mathrm{P}$ concentration. Clearly in this study anoxic $\mathrm{P}$ release was dominant over anaerobic $\mathrm{P}$ release which is unusual as both reactors were of similar size, RBCOD was present in the anaerobic reactor and $\mathrm{NO}_{3}$ was present in the anoxic reactor. However similar $\mathrm{P}$ removals to those achieved in Phase 1 and 2 were reported indicating that good $\mathrm{P}$ removal is possible even at very long sludge ages in an MBR system. As was done in Phase 1 and 2 the $f_{X B G P}$ required to match measured and predicted $P$ removals was calculated and are also reported in Table 4.13. These values varied considerably. No reasons are proposed for the unusual $\mathrm{P}$ removal performance of the system in this period of the study.

Table 4.13: $\quad$ Summary of $P$ release (-ve) and $P$ uptake (+ve) across the 40 day sludge age MBR system.

\begin{tabular}{|c|c|c|c|c|c|c|c|}
\hline $\begin{array}{l}\text { Sewage } \\
\text { Batch \# }\end{array}$ & Influent & Anaerobic & Anoxic & Aerobic & Membrane & $\begin{array}{c}\text { M (P-PO4) } \\
\text { removal }\end{array}$ & fxbgp \\
\hline & $\mathrm{mgP} / \mathrm{linf}$ & $\mathrm{mgP} / \mathrm{linf}$ & $\mathrm{mgP} / \mathrm{linf}$ & $\mathrm{mgP} / \mathrm{linf}$ & $\mathrm{mgP} / \mathrm{linf}$ & $\mathrm{mgP} / \mathrm{linf}$ & - \\
\hline 2 & 45.0 & -58.8 & -81.3 & 167.5 & 4.0 & 31.4 & 0.53 \\
\hline 3 & 37.2 & -41.6 & -81.7 & 130.3 & 11.9 & 18.8 & 0.22 \\
\hline 4 & 44.5 & -58.8 & -60.0 & 141.3 & 2.9 & 25.4 & 0.44 \\
\hline
\end{tabular}

\subsubsection{Influent unbiodegradable COD fractions and sludge production}

The primary reason for conducting this study at a longer sludge age was to further investigate the high sludge production and $f_{S \text {, up }}$ values observed in Phase 1 and 2 . Due to the lower effluent COD concentrations the $\mathrm{f}_{\mathrm{S} \text {, us }}$ value calculated for the 40 day sludge age investigation, of 0.029 , was lower than that calculated for Phase 1 and 2 of 
the investigation. Additionally the $\mathrm{f}_{\mathrm{S} \text {,up }}$ determined of 0.113 was consistently lower than that observed in Phase 1 and 2 of 0.224 and 0.200 respectively.

A number of reasons are proposed for these differences. Firstly this investigation was performed over a short period of time (6 weeks), the equivalent of one sludge age, which is not enough time to draw conclusions on the system performance. Additionally, though the consistent $\mathrm{f}_{\mathrm{S} \text {,up }}$ values calculated indicate that the system was at steady state, the system did experience frequent sludge losses due to spills and may have not actually achieved steady state resulting in low $f_{\mathrm{S}, \text { up }}$ values.

\subsection{CLOSURE}

In this section the MBR UCT system performance has been evaluated through detailed analysis of the data obtained from measurements on the system, and through application of steady state design theory to the system. It has been shown that the current steady state design models are indeed adequate for design of MBR BNR systems.

The results of the conventional system, which was used as a control against which to compare the impact of the membranes on the BNR performance of the MBR system are analyscd in Chapter 5. Chapter 6 compares the performances of the two systems in order to elucidate the relative performance of the MBR BNR system. 


\section{CHAPTER 5}

\section{CONVENTIONAL UCT SYSTEM EXPERIMENTAL RESULTS AND ANALYSIS}

\subsection{INTRODUCTION}

The conventional UCT system was operated as a control against which the BNR performance of the MBR UCT system could be evaluated. Both systems were run, as close as possible, to the same design and operating parameters, see Section 3.3.2, Chapter 3. The major differences between the two systems primarily comprised the increased organic loading on the MBR system (3.1 times) in order to achieve higher mixed liquor solids concentrations necessary for operation; and the reconfiguration of the $a$ - and $s$-recycles into the $a s$-recycle as the SST had been replaced by membranes in the MBR system.

The same parameters as in the MBR system were measured to assess the performance of the conventional UCT system (see Section 3.5, Chapter 3). All experimental data for the conventional system collected in the Phase 2 investigation is presented in Appendix B. In this chapter the performance of the conventional UCT system is evaluated.

Overall the conventional system data failed to show sufficient stability and mass balances consistently failed to close. Hence, some parameters could not be used to calibrate the results observed in the MBR system. Thus, in order to meet the project objective of calibrating the MBR performance, results from previous studies are used instead. However the performance of the system is discussed and where possible the data is compared.

\subsection{SYSTEM CONDITIONS AND STEADY STATE PERIODS}

Sewage batches and days of operation were as for the MBR UCT system, Table 4.1, Chapter 4. The same approach followed for the MBR UCT system: to identify sewage batches as steady state periods, identify outliers, average sewage batches behaviour and evaluate the system from sewage batch averages, was followed for the conventional UCT system. Detailed daily results are listed in Appendix B.

As was noted in Phase 1, the measured system recycles corresponded closely with the design recycle values and did not vary during the investigation (Ramphao et al., 2004). Accordingly, the expected recycle flow rates can be accepted for analysis. The RBCOD values calculated from the square wave (SQW) system described in Section 4.3, Chapter 4, are applied to this analysis also.

From the beginning of the investigation until sewage batch 15 the conventional system was operated by laboratory assistants while the writer focused his attention on the MBR system, and from day 236, sewage batch 15 , the writer ran both systems 
entirely. Unfortunately a thorough record and understanding of system operation was not available prior to sewage batch 15 .

\subsection{MIXED LIQUOR SOLIDS}

\subsubsection{MLSS and MLVSS Concentrations}

The Phase 2 investigation continued on from Phase 1 (Ramphao et al., 2004). In the interim period between Phase 1 and Phase 2 the conventional UCT system had continued being operated and monitored by lab assistants, with the same design operation conditions, but no testing and analysis. Thus at the beginning of the investigation the system was assumed to be at steady state and testing could commence with immediate effect.

Variations of solids concentrations with time are reported in Figs. 5.1 to 5.3. In sewage batches $4-11$ the solids concentrations varied sporadically, ranging from $3200-6700 \mathrm{mgTSS} / \ell$ before returning to a consistent range of $2500-3500 \mathrm{mgTSS} / \ell$ in the aerobic reactor. This variability was consistent in all three reactors suggesting that it is attributed to a systematic error in measurement over that period, which unfortunately was not noticed until sewage batch 11 . Doubts over the validity of measurements in the initial period resulted in them being excluded from further analysis of mixed liquor characteristics.

In sewage batches 2, 3, 12 - 29 the mixed liquor solids concentrations remained consistently within the range $2500-3500 \mathrm{mgTSS} / \ell$ which is substantially lower than the expected range $4500-5500 \mathrm{mgTSS} / \ell$ (see Table 3.1, Chapter 3). Ramphao et al., (2004) also reported lower solids concentrations in the system, $3500-5000$ $\operatorname{mgTSS} / \ell$.

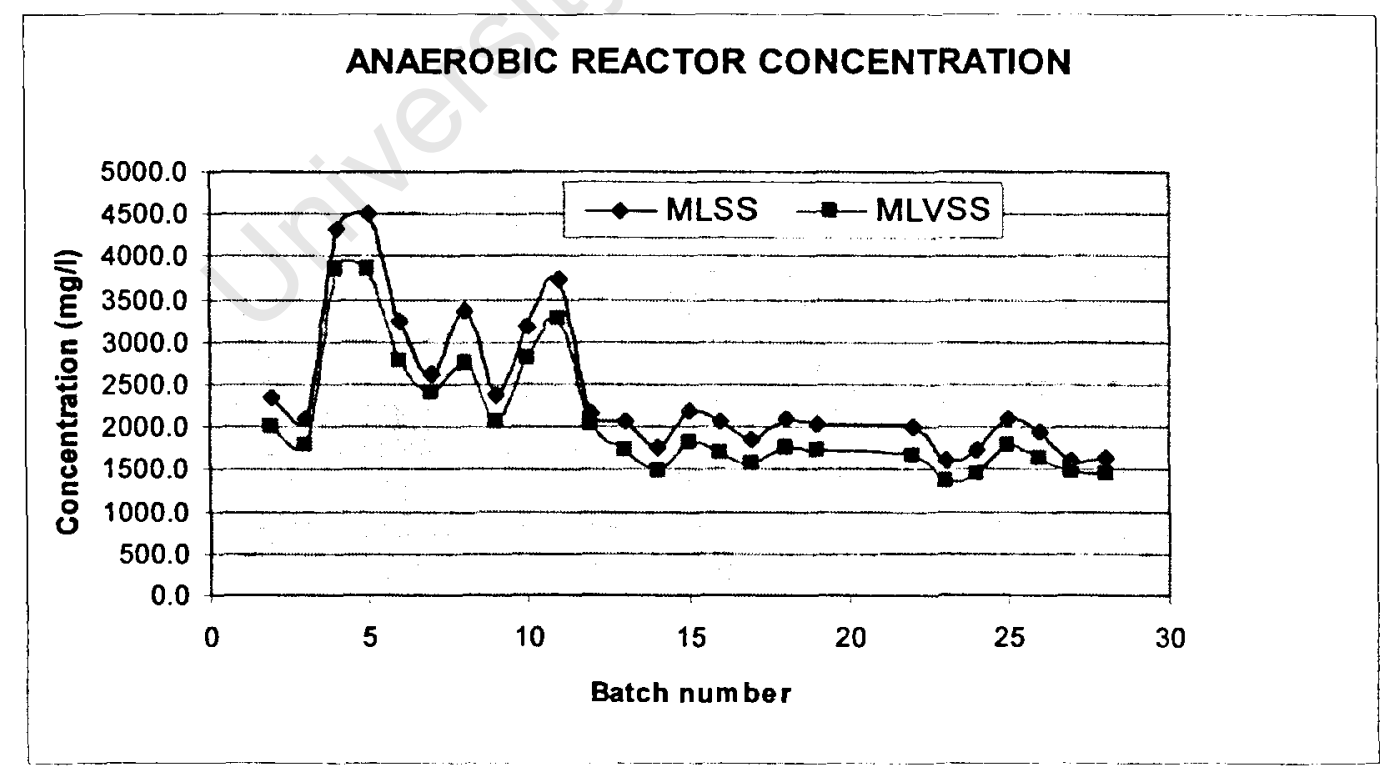

Figure 5.1: Anaerobic MLSS/MLVSS concentrations with time 


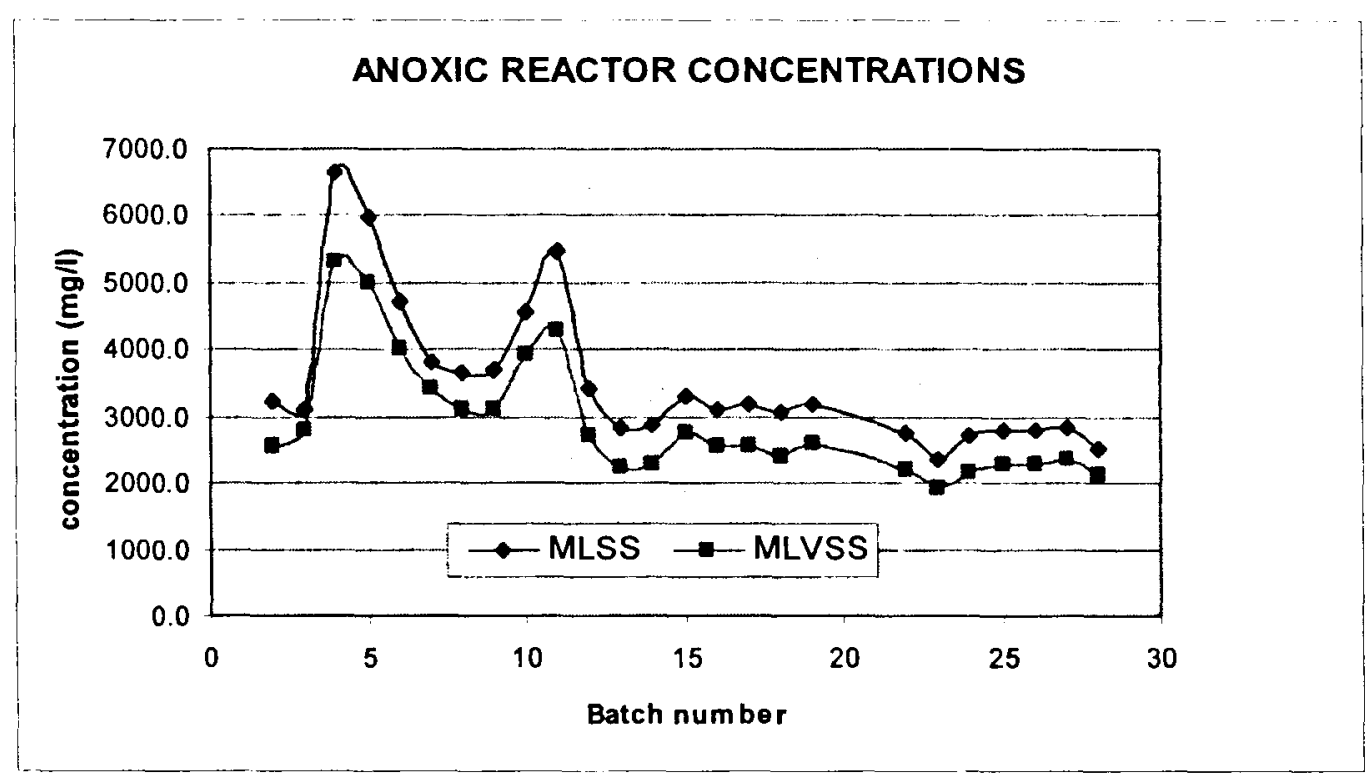

Figure 5.2: $\quad$ Anoxic MLSS/MLVSS concentrations with time

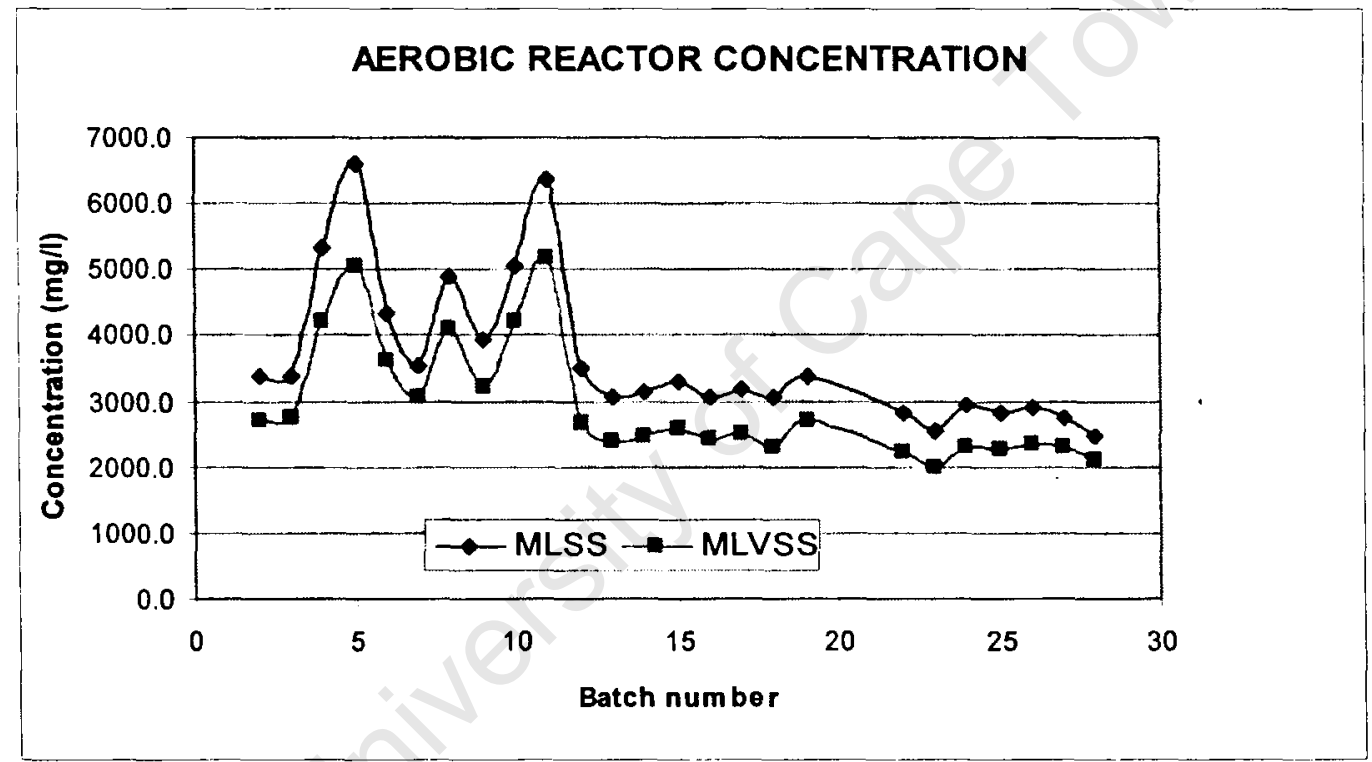

Figure 5.3: $\quad$ Aerobic MLSS/MLVSS concentrations with time

In an effort to improve solids concentrations in the conventional system $1.0 \ell$ sludge from the membrane system waste stream was added to the conventional system to boost the solids content of the sludge at the end of sewage batch 18 (day 291). A minor increase was noticed in the following sewage batch, however on returning from a period of leave, the writer observed that the system mixed liquor solids concentration had decreased to even lower concentrations.

\subsubsection{System VSS/TSS Ratios}

As can be seen from Figs 5.1 to 5.3 , the MLVSS tracked the MLSS values very closely. The VSS/TSS ratio of the sludge for each reactor is illustrated in Fig. 5.4. There was some variation in the VSS/TSS ratio for the first 11 sewage batches, however values generally remained consistent. VSS/TSS ratios of 0.85 $\mathrm{mgVSS} / \mathrm{mgTSS}$ (anaerobic), $0.82 \mathrm{mgVSS} / \mathrm{mgTSS}$ (anoxic) and $0.81 \mathrm{mgVSS} / \mathrm{mgTSS}$ 
(aerobic) were measured across the system with a mean weighted average of 0.814 ( $\mathrm{SSD}=0.027) \mathrm{mgVSS} / \mathrm{mgTSS}$, Fig. 5.5 , which is very close to that measured in Phase 1 with a mean $=0.817 \mathrm{mgVSS} / \mathrm{mgTSS}$ (Ramphao et al., 2004). Differences in VSS/TSS ratios between anaerobic, anoxic and aerobic solids are expected due to the $\mathrm{PAO}$ release and uptake behaviour.

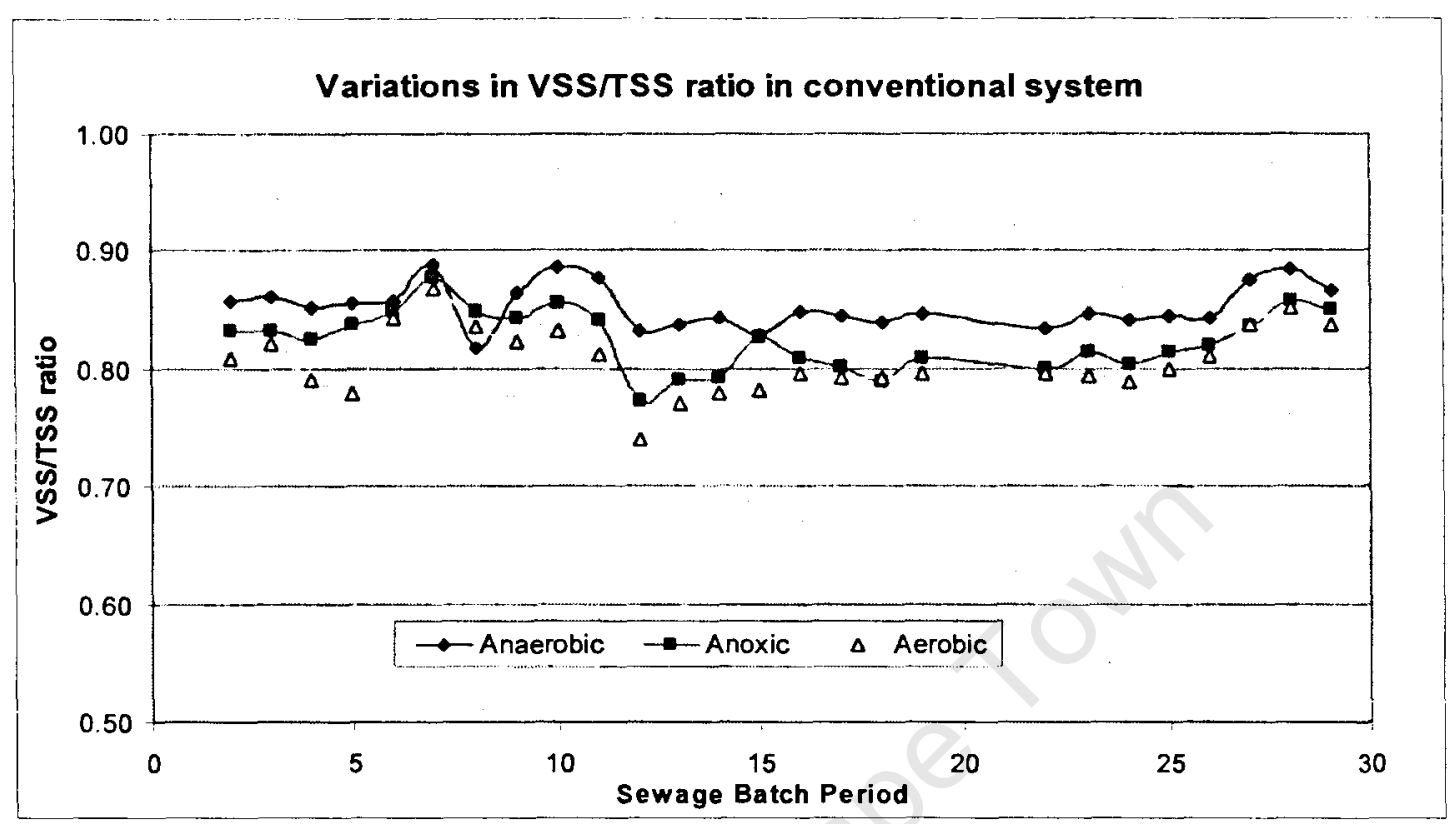

Figure 5.4: Variations of VSS/TSS ratios in the conventional UCT system over time.

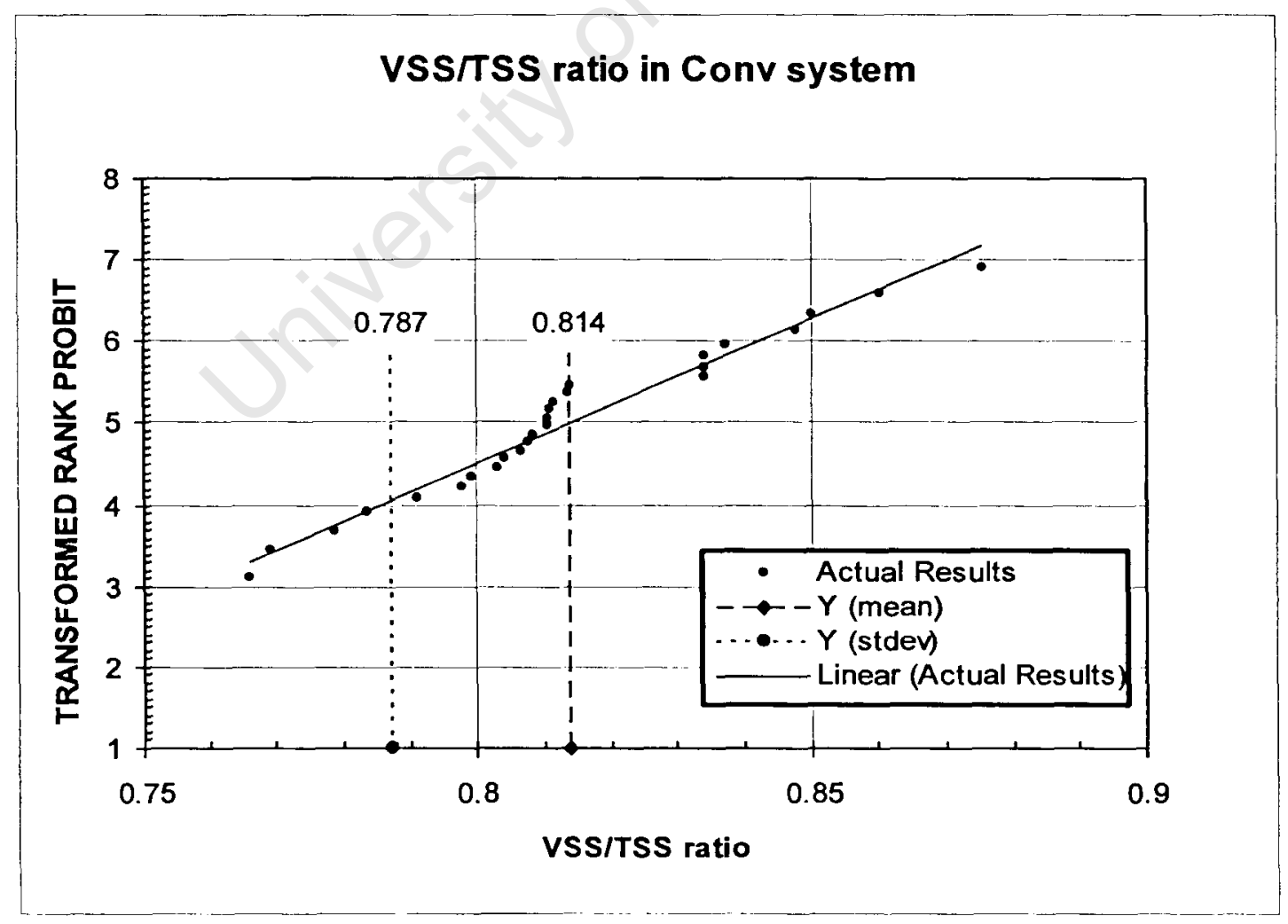

Figure 5.5: $\quad$ Statistical plot of the sewage batch average VSS/TSS ratios in the system. 


\subsubsection{COD to VSS Ratio $\left(f_{\mathrm{CV}}\right)$}

The variations in VSS concentration in sewage batches 4 - 11 adversely affected the COD/VSS $\left(\mathrm{f}_{\mathrm{cv}}\right)$ ratio providing evidence that the measured VSS values for this period were incorrect. Thus, to calculate the average $f_{c v}$ only the sewage batch averages from sewage batches 2,3 , and 12 to 29 were taken into account.

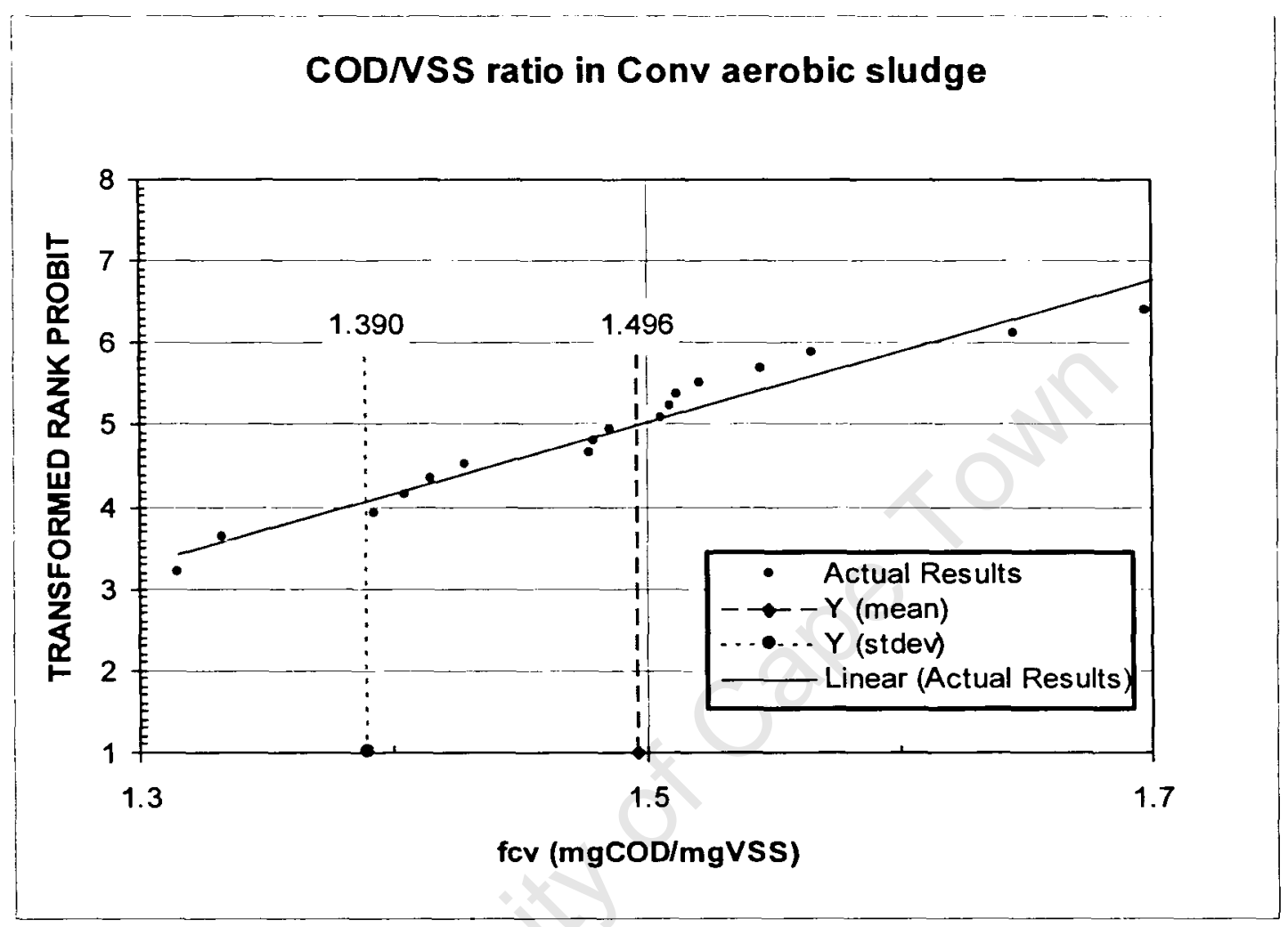

Figure 5.6: Statistical plot of the sewage batch average COD to VSS ratios $\left(f_{c v}\right)$ in the conventional UCT system.

The mean average $f_{c v}$ observed was $1.496(\mathrm{SSD}=0.106) \mathrm{mgCOD} / \mathrm{mgVSS}$, Fig. 5.6. This $f_{c v}$ value is close to the commonly accepted value of $f_{c v}$ of 1.48 (WRC, 1984) and is within the range of values $(1.3-1.5)$ previously found in laboratory experiments in the Water Research Laboratory (WRL), (see Chapter 4, Section 4.6.3 for comparisons with other values). Ramphao et al. (2004) reported an $\mathrm{f}_{\mathrm{cv}}$ of 1.36 in the conventional UCT system run in Phase 1 of the project.

\subsubsection{TKN/VSS Ratio}

The TKN/VSS ratios, like with the $\mathrm{f}_{\mathrm{cv}}$ values, showed substantial deviations over sewage batches 4 to 11 , again providing confirmation that errors lay with the VSS measurement in sewage batches 4 to 11. Taking the remaining data from batches $2,3,12-29$ a normal distribution was observed with a mean of $0.094(\mathrm{SSD}=0.009)$ $\mathrm{mgN} / \mathrm{mgVSS}$. This value is lower than that reported by Ramphao et al. (2004) of $0.113 \mathrm{mgN} / \mathrm{mgVSS}$ for the same system, but falls within the range of values noted in Chapter 4, Section 4.6 .4 , and is close to the value of $0.10 \mathrm{mgN} / \mathrm{mgVSS}$ commonly accepted for municipal waste-waters. 


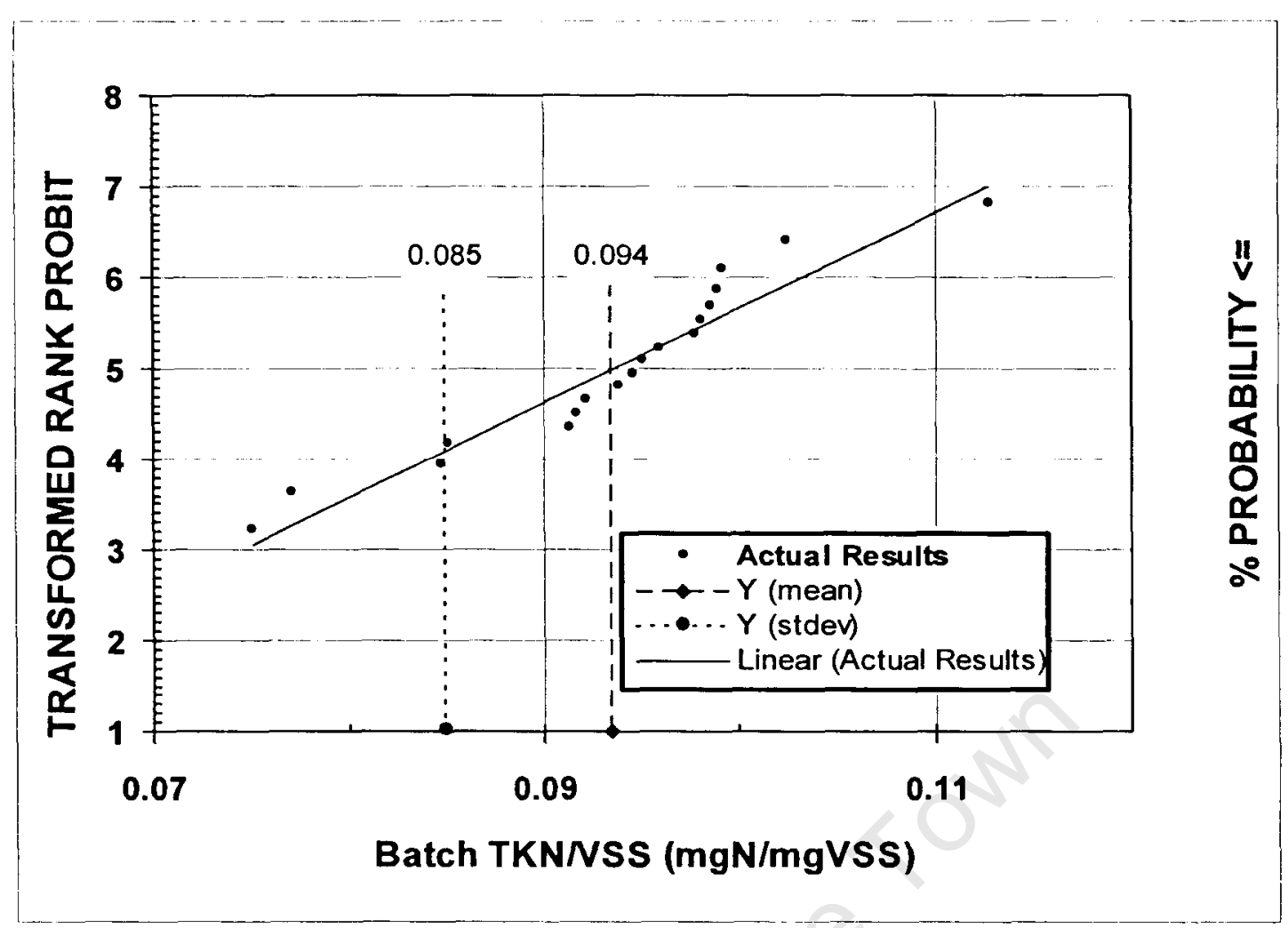

Figure 5.7: $\quad$ Statistical plot of the sewage batch average mixed liquor TKN/VSS ratios in the conventional UCT system.

\subsubsection{Re-evaluation of VSS Values}

As was noted earlier in this section there was substantial variation in the measured VSS results for sewage batches 4 to 11 . This resulted in the measured VSS values from sewage batches 4 to 11 being discarded for analysis. However aerobic sludge COD and TKN values, also taken during this period remained consistent. Thus, theoretically the suspect VSS concentrations could be recalculated using either the COD or TKN values and their respective measured fraction $f_{c v}$ or TKN/VSS, determined in Sections 5.3.3 and 5.3.4 above.

The VSS calculated from the sewage batch average aerobic COD concentrations and the calculated system average $\mathrm{f}_{\mathrm{cv}}$ are presented in Fig. 5.8. Similarly VSS calculated from the sewage batch average aerobic sludge TKN concentration and system average TKN/VSS results are presented in Fig 5.9. 


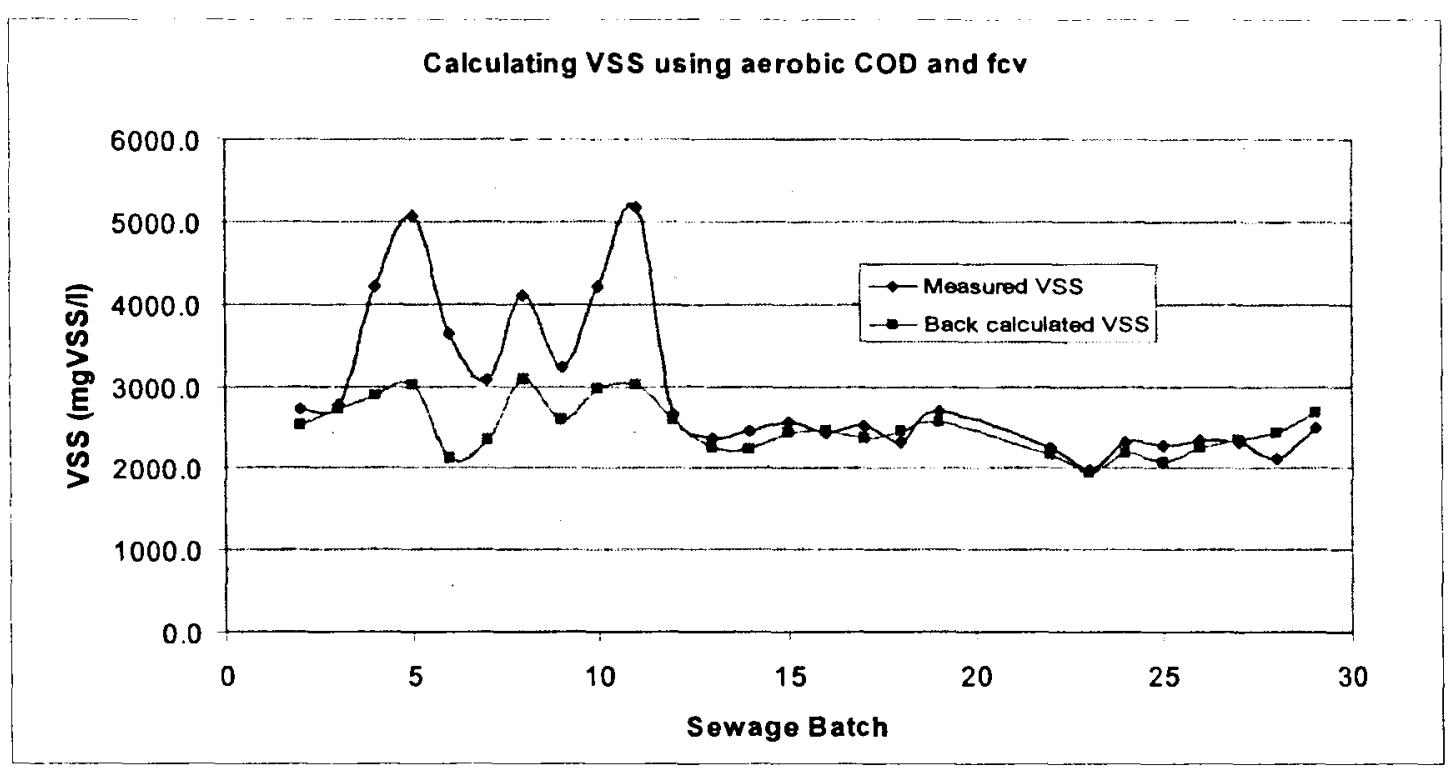

Figure 5.8: $\quad$ The measured and predicted VSS concentrations in the aerobic reactor using the aerobic COD concentration and average $f_{c} v$

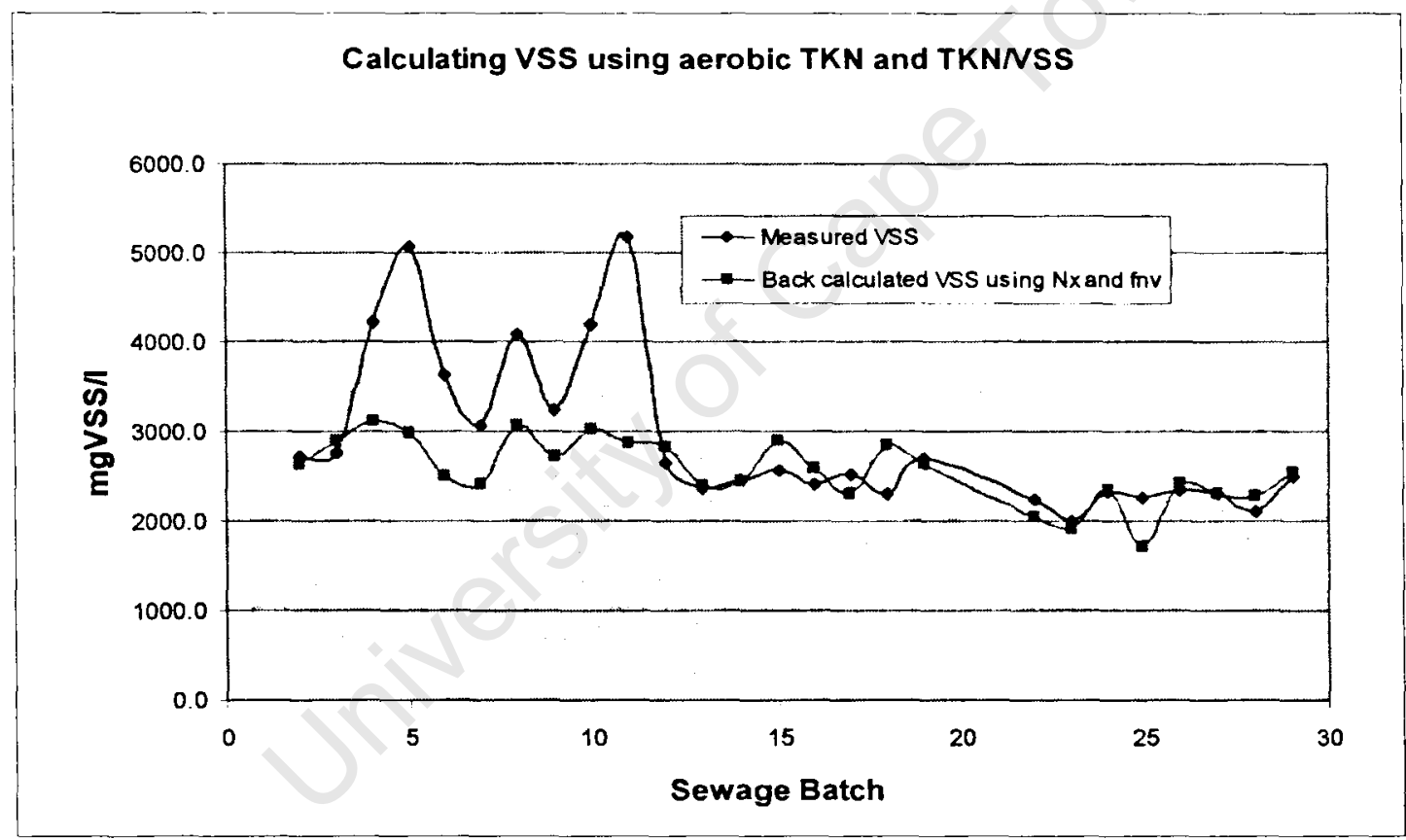

Figure 5.9: The measured and predicted VSS concentrations in the aerobic reactor using the aerobic TKN concentrations and average TKN/VSS.

- Both sets of results presented in Figs. 5.8 and 5.9 indicated that the solids concentrations should have remained consistently in the range of $2000-3000$ $\mathrm{mgVSS} / \ell$. The COD predictions seemed to track the measured values closer than the TKN predictions, thus demonstrating its applicability in predicting solids concentrations.

- Hence, for all further analysis MLVSS values are used calculated using mixed liquor COD concentrations instead of the direct MLVSS measurements. 


\subsubsection{Sludge Production}

In order to quantify the sludge production in the conventional UCT system Equations (4.8) and (4.9) (Chapter 4) were used. As discussed in Section 5.3.5 above, the measured VSS values were re-evaluated and all further analysis would use the MLVSS values calculated from the mixed liquor COD measurements as these predicted measured VSS in sewage batches $13-29$ more closely. Using the COD calculated MLVSS values the sludge production in the conventional UCT system ranged from $0.145-0.311 \mathrm{mgVSS} / \mathrm{mgCOD}$ with a mean sludge production of $0.205 \mathrm{mgVSS} / \mathrm{mgCOD}$ (SSD $=0.036$ ). This result differs significantly from that reported for the MBR system in Chapter 4 Section 4.3 .6 of $0.311 \mathrm{mgVSS} / \mathrm{mgCOD}$, but is close to the results reported for the conventional UCT system in Phase 1 (Ramphao et al., 2004) of $0.22 \mathrm{mgVSS} / \mathrm{mgCOD}$, and results of ND systems run at 20 day sludge ages using the same wastewater source: 0.20 (Ubisi et al., 1997) and 0.18 (Lee et al., 2002a). Note that these sludge ages are only valid for the 20 day sludge age of the MBR and conventional systems - difference sludge ages yield different sludge production rates.

\subsubsection{Diluted Sludge Volume Index}

The sludge settleability of the conventional system was monitored by means of the diluted sludge volume index (DSVI). Detailed results are presented in Appendix B, with the settleability trend during the investigation shown in Fig. 5.10. As was noted in Section 5.3.5 the VSS values used for the calculation of DSVI were calculated from the aerobic COD measurements.

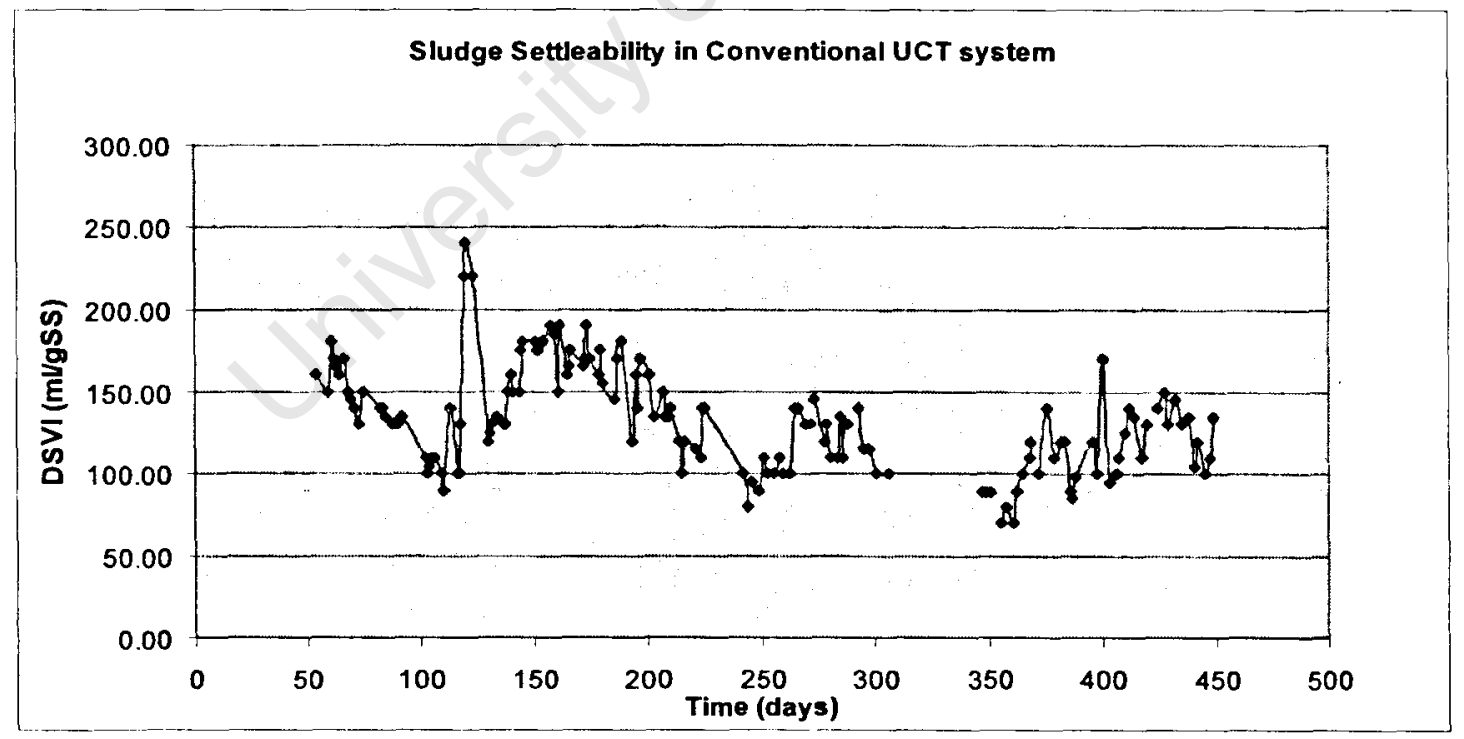

Figure 5.10.: General trend of diluted sludge volume index (DSVI).

Although DSVI records were kept from the beginning of the Phase 2 investigation, observations on the system performance were only recorded by the writer when he took over operation of the system on day 236 , sewage batch 15 .

From the start of the investigation the DSVI ranged between 100 and 180 . On day 119 aeration in the conventional system was blocked which may have resulted in a poor 
settling sludge developing over that period. From day 236 the DSVI increased from 100 to $150 \mathrm{ml} / \mathrm{gTSS}$. Mixed liquor spills were recorded on days 280 and 281 and on day 286 high ammonia concentrations were noted in the effluent indicating poor aeration which may have caused the increase in DSVI over this period. On day 291 $1.0 \ell$ sludge from the MBR waste stream was added to the conventional system to boost the solids concentration. The MBR sludge showed improved settleability (see Section 4.6.6) which may have influenced the conventional sludge as evidenced by a drop in DSVI from $150 \mathrm{ml} / \mathrm{gTSS}$ on day 291 to below $100 \mathrm{ml} / \mathrm{gTSS}$ on day 309 . Between days 374 and 383 the laboratory experienced regular power outages which affected system aeration and a similar increase in DSVI was noticed. On day 424 and the days immediately following the DSVI rose sharply to $220 \mathrm{ml} / \mathrm{gSS}$ due to no aeration in the sludge on that day, the level however returned to normal shortly thereafter.

In Phase 1 of the project a high DSVI ( $>150 \mathrm{ml} / \mathrm{mgSS})$ was noticed throughout the first 260 days, thereafter dropping to the range observed in this study.

Throughout the investigation filamentous organisms were present in the conventional sewage sludge, with a predominance of Microthrix Parvicella and Type 0092 organisms. A summary of the microbiological analyses performed on the conventional system is presented in Table 5.1 .

Table 5.1: Microbiological analyses for the conventional system.

\begin{tabular}{|c|c|c|c|c|c|c|c|c|c|}
\hline \multicolumn{10}{|c|}{ CONVENTIONAL SYSTEM } \\
\hline \multirow[t]{2}{*}{ Date } & \multirow[t]{2}{*}{ Day: } & \multirow[t]{2}{*}{$\begin{array}{c}\text { Sewage } \\
\text { Batch }\end{array}$} & \multirow[t]{2}{*}{$\begin{array}{c}\text { Morfology } \\
\text { floc }\end{array}$} & \multirow[t]{2}{*}{$\begin{array}{c}\text { Floc diameter } \\
\text { (um) }\end{array}$} & \multicolumn{3}{|c|}{ Filamentous microlganisms } & \multirow{2}{*}{ DSVI } & \multirow[t]{2}{*}{ nlservations } \\
\hline & & & & & & Rank & Abundance & & \\
\hline $2005-03-24$ & 42 & 3 & $\begin{array}{c}\text { weak, irregular, } \\
\text { diffuse }\end{array}$ & $150-500$ & $\begin{array}{l}\text { M.Parvicella } \\
\text { Type } 0092\end{array}$ & $\begin{array}{l}2 \\
1\end{array}$ & \begin{tabular}{|l|}
2 \\
4
\end{tabular} & $n$ & $n$ \\
\hline $2005-04-21$ & 70 & 4 & firm & $150-500$ & M.Panicella & 1 & 5 & 167 & $n$ \\
\hline $2005-05-23$ & 102 & 6 & $\begin{array}{l}\text { firm, irregular, } \\
\text { diffuse }\end{array}$ & $150-500$ & $\begin{array}{l}\text { M.Parvicella } \\
\text { Type } 0092 \\
\end{array}$ & $\begin{array}{l}2 \\
1 \\
\end{array}$ & $\begin{array}{l}2 \\
3 \\
\end{array}$ & 172 & $n$ \\
\hline $2005-06-22$ & 132 & 8 & $\begin{array}{c}\text { firm, irregular, } \\
\text { diffuse }\end{array}$ & $8^{150-500}$ & $\begin{array}{l}\text { M.Parvicelia } \\
\text { Type } 00092 \\
\end{array}$ & $\begin{array}{l}2 \\
1 \\
\end{array}$ & $\begin{array}{l}2 \\
3 \\
\end{array}$ & 128 & $\mathrm{n}$ \\
\hline $2005-07-22$ & 162 & 10 & $\begin{array}{l}\text { firm, round. } \\
\text { compact }\end{array}$ & $150-500$ & $\begin{array}{c}\text { M.Panicella } \\
\text { Type } 0092 \\
\text { Type } 1701\end{array}$ & $\begin{array}{l}2 \\
1 \\
3\end{array}$ & $\begin{array}{l}3 \\
4 \\
2\end{array}$ & 198 & $\mathrm{n}$ \\
\hline $2005-08-15$ & 186 & 11 & $\begin{array}{c}\text { weak, irregular, } \\
\text { diffuse }\end{array}$ & $150-500$ & $\begin{array}{c}\text { M.Parvicella } \\
\text { Type } 0092\end{array}$ & $\begin{array}{l}1 \\
2\end{array}$ & $\begin{array}{l}2 \\
2\end{array}$ & 191 & $n$ \\
\hline $2005-09-19$ & 221 & 14 & $\begin{array}{c}\text { weak, irregular, } \\
\text { diffuse }\end{array}$ & $150-500$ & $\begin{array}{l}\text { M.Parvicella } \\
\text { Type } 0092\end{array}$ & $\begin{array}{l}1 \\
2 \\
\end{array}$ & $\begin{array}{l}3 \\
1 \\
\end{array}$ & 115 & $\mathrm{n}$ \\
\hline $2005-10-17$ & 249 & 16 & \begin{tabular}{c|}
$\begin{array}{c}\text { firm, round. } \\
\text { compact }\end{array}$ \\
\end{tabular} & $150-500$ & $\begin{array}{l}\text { M.Parvicella } \\
\text { Type 0092 }\end{array}$ & $\begin{array}{l}2 \\
1\end{array}$ & $\begin{array}{l}1 \\
3 \\
\end{array}$ & 102 & irregular feeding \\
\hline $2005-11-29$ & 292 & 18 & $\begin{array}{c}\text { weak, round, } \\
\text { compact }\end{array}$ & $150-500$ & $\begin{array}{l}\text { M.Parvicella } \\
\text { Type } 0092\end{array}$ & $\begin{array}{l}2 \\
1 \\
\end{array}$ & $\begin{array}{l}2 \\
4 \\
\end{array}$ & 131 & high NH4 in effluent \\
\hline $2006-01-24$ & 348 & 22 & $\begin{array}{c}\text { weak, irregular. } \\
\text { diffuse }\end{array}$ & $150-500$ & $\begin{array}{l}\text { M.Parvicella } \\
\text { Type 0092 }\end{array}$ & $\begin{array}{l}2 \\
1\end{array}$ & $\begin{array}{l}2 \\
3\end{array}$ & 101 & $\mathrm{n}$ \\
\hline $2006-02-07$ & 362 & 23 & $\begin{array}{c}\text { firm, round, } \\
\text { campact }\end{array}$ & $150-500$ & $\begin{array}{l}\text { M.Parvicella } \\
\text { Type } 0092\end{array}$ & $\begin{array}{l}2 \\
1 \\
\end{array}$ & $\begin{array}{l}2 \\
3 \\
\end{array}$ & 111 & $n$ \\
\hline $2006-02-28$ & 383 & 24 & $\begin{array}{l}\text { firm, round, } \\
\text { compact }\end{array}$ & $150-500$ & \begin{tabular}{|l|} 
M.Parvicella \\
Type 0092 \\
\end{tabular} & 2 & $\begin{array}{l}3 \\
3 \\
\end{array}$ & 133 & $\begin{array}{l}\text { Sludge loss through } \\
\text { spills; power failures }\end{array}$ \\
\hline $2006-03-15$ & 398 & 26 & $\begin{array}{l}\text { firm, round, } \\
\text { compact }\end{array}$ & $150-500$ & $\begin{array}{l}\text { M.Parvicella } \\
\text { Type } 0092\end{array}$ & $\begin{array}{l}2 \\
1 \\
\end{array}$ & $\begin{array}{l}3 \\
4 \\
\end{array}$ & 122 & $n$ \\
\hline $2006-03-28$ & 411 & 27 & $\begin{array}{c}\text { firm, round, } \\
\text { compact } \\
\end{array}$ & $<150$ & $\begin{array}{c}\text { M.Parvicella } \\
\text { Type } 0092\end{array}$ & $\begin{array}{l}2 \\
2 \\
\end{array}$ & $\begin{array}{l}2 \\
2\end{array}$ & 162 & minor spill \\
\hline $2006-04-11$ & 425 & 28 & $\begin{array}{c}\text { weak, irregular, } \\
\text { diffuse }\end{array}$ & $<150$ & $\begin{array}{c}\text { M.Panicella } \\
\text { Type } 0092 \\
\end{array}$ & $\begin{array}{l}1 \\
2 \\
\end{array}$ & $\begin{array}{l}4 \\
3 \\
\end{array}$ & 220 & $\begin{array}{c}\text { low solids, poor } \\
\text { aeration }\end{array}$ \\
\hline $2006-04-25$ & 439 & 29 & $\begin{array}{c}\text { weak, irregular. } \\
\text { diffuse }\end{array}$ & $<150$ & $\begin{array}{c}\text { M.Panicella } \\
\text { Type } 0092\end{array}$ & $\begin{array}{l}1 \\
2\end{array}$ & $\begin{array}{l}2 \\
2\end{array}$ & 134 & $n$ \\
\hline
\end{tabular}




\subsection{MASS BALANCES}

Mass balances were performed on the conventional UCT system data, as was done for the MBR system, in order to evaluate the accuracy and reliability of the data. The range of $90-110 \%$ was considered to be acceptable for $\mathrm{N}$ and COD mass balances. The $\mathrm{N}$ and COD mass balances for the conventional system are shown in Table 5.2 and Figs. 5.11 and 5.12 respectively.

Table 5.2: $\quad$ Nitrogen and COD mass balances for the conventional UCT system.

\begin{tabular}{|c|c|c|}
\hline $\begin{array}{c}\text { Sewage Batch } \\
\text { number }\end{array}$ & $\begin{array}{c}\text { Percent N Mass } \\
\text { Balance }\end{array}$ & $\begin{array}{l}\text { Percent COD } \\
\text { Mass Balance }\end{array}$ \\
\hline & $\%$ & $\%$ \\
\hline 2 & $162.04 \%$ & $60.86 \%$ \\
\hline 3 & $87.59 \%$ & $87.48 \%$ \\
\hline 4 & $71.54 \%$ & $122.00 \%$ \\
\hline 5 & $105.68 \%$ & $82.38 \%$ \\
\hline 6 & $80.41 \%$ & $62.95 \%$ \\
\hline 7 & $108.87 \%$ & $123.92 \%$ \\
\hline 8 & $78.90 \%$ & $118.37 \%$ \\
\hline 9 & $77.75 \%$ & $97.92 \%$ \\
\hline 10 & $66.97 \%$ & $92.31 \%$ \\
\hline 11 & $76.68 \%$ & $99.71 \%$ \\
\hline 12 & $102.79 \%$ & $70.01 \%$ \\
\hline 13 & $62.90 \%$ & $85.67 \%$ \\
\hline 14 & $82.93 \%$ & $72.12 \%$ \\
\hline 15 & $71.47 \%$ & $75.09 \%$ \\
\hline 16 & $74.69 \%$ & $73.85 \%$ \\
\hline 17 & $57.34 \%$ & $87.05 \%$ \\
\hline 18 & $69.16 \%$ & $73.78 \%$ \\
\hline 19 & $55.93 \%$ & $75.16 \%$ \\
\hline 20 & no measurement & no measurement \\
\hline 21 & no measurement & no measurement \\
\hline 22 & $84.34 \%$ & $70.56 \%$ \\
\hline 23 & $76.14 \%$ & $77.89 \%$ \\
\hline 24 & $104.04 \%$ & $101.26 \%$ \\
\hline 25 & $88.26 \%$ & $84.47 \%$ \\
\hline 26 & $79.10 \%$ & $67.13 \%$ \\
\hline 27 & $78.25 \%$ & $78.87 \%$ \\
\hline 28 & $87.06 \%$ & $50.97 \%$ \\
\hline 29 & $61.66 \%$ & $68.52 \%$ \\
\hline Average & $79.61 \%$ & $78.20 \%$ \\
\hline
\end{tabular}

\subsubsection{Nitrogen Mass Balance}

As can be seen in Table 5.1 and Fig. 5.11, nitrogen mass balances ranging from $55.9 \%$ to $108.9 \%$ were obtained giving an average $\mathrm{N}$ mass balance of $79.6 \%$ (excluding sewage batch 2) for the entire investigation. Sewage batch 2 is an outlier and was not taken into account in assessing the $\mathrm{N}$ mass balances. This average is substantially lower than the averages obtained in Phase 1 of the investigation and 
provides substantial uncertainty on the data. Most values fell below $100 \%$ indicating that there was a systematic error in one or more of the measurements which accounted for the low mass balances.

The mass balance measurement is independent of the direct VSS measurement as aerobic $\mathrm{N}$ and COD results are used directly to calculate $\mathrm{N}$ and COD removal through sludge wasting. Thus the error lay in TKN and nitrite/nitrate measurements, or operational parameters: influent flow (Qi), waste flow $(\mathrm{Qw})$, or the a- and s-recycles.

Ramphao et al. (2004) reported a low, but adequate mass balance of $96 \%$ for Phase 1 noting that less nitrogen was accounted for as leaving than entering the system.

Of the nitrogen entering the system (comparisons with Ramphao et al., 2004 are in brackets):

- $39.3 \%$ left through the denitrification of nitrate/nitrite (37.9)

- $16.8 \%$ left as nitrogen in the waste sludge (37\%)

- $15.9 \%$ left as effluent nitrite/nitrate $(17.7 \%)$

- $7.5 \%$ left as effluent TKN $(2.8 \%)$

- $20.4 \%$ is unaccounted for $(4.6 \%)$

From the above comparison the denitrification and effluent $\mathrm{N}$ values are comparable, however the $\mathrm{N}$ in the sludge wasted is substantially less. This could indicate that an error in the amount of sludge wasted daily is responsible for the poor $\mathrm{N}$ mass balances, or sludge losses regularly occurred that the writer was not aware of. It is important to notice however that the TKN/VSS value for Phase 1 (0.113, Ramphao et al., 2004) was significantly higher than in Phase 2 (0.094) which would have greatly influenced the mass of $\mathrm{N}$ in the wasted sludge.

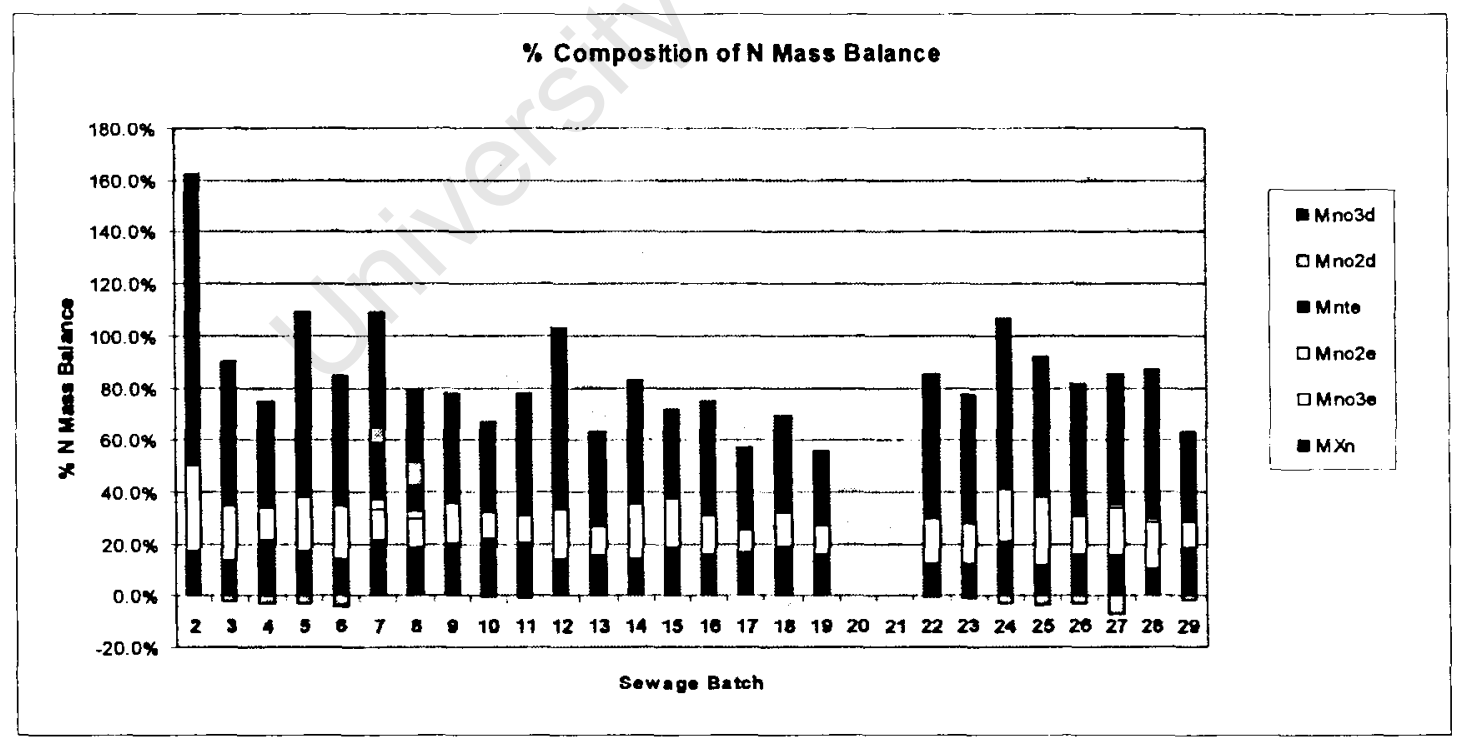

Figure 5.11: Graphical representation of the percentage nitrogen (N) mass balance for the sewage wastewater batches for the conventional UCT system. Percentages shown are for nitrate for denitrification (MNO3d), nitrite for denitrification (MNO2d), TKN nitrogen in the effluent stream (Nte), effluent nitrite and nitrate (MNO2e) and (MNO3e) respectively and nitrogen in the waste stream (MXn). 


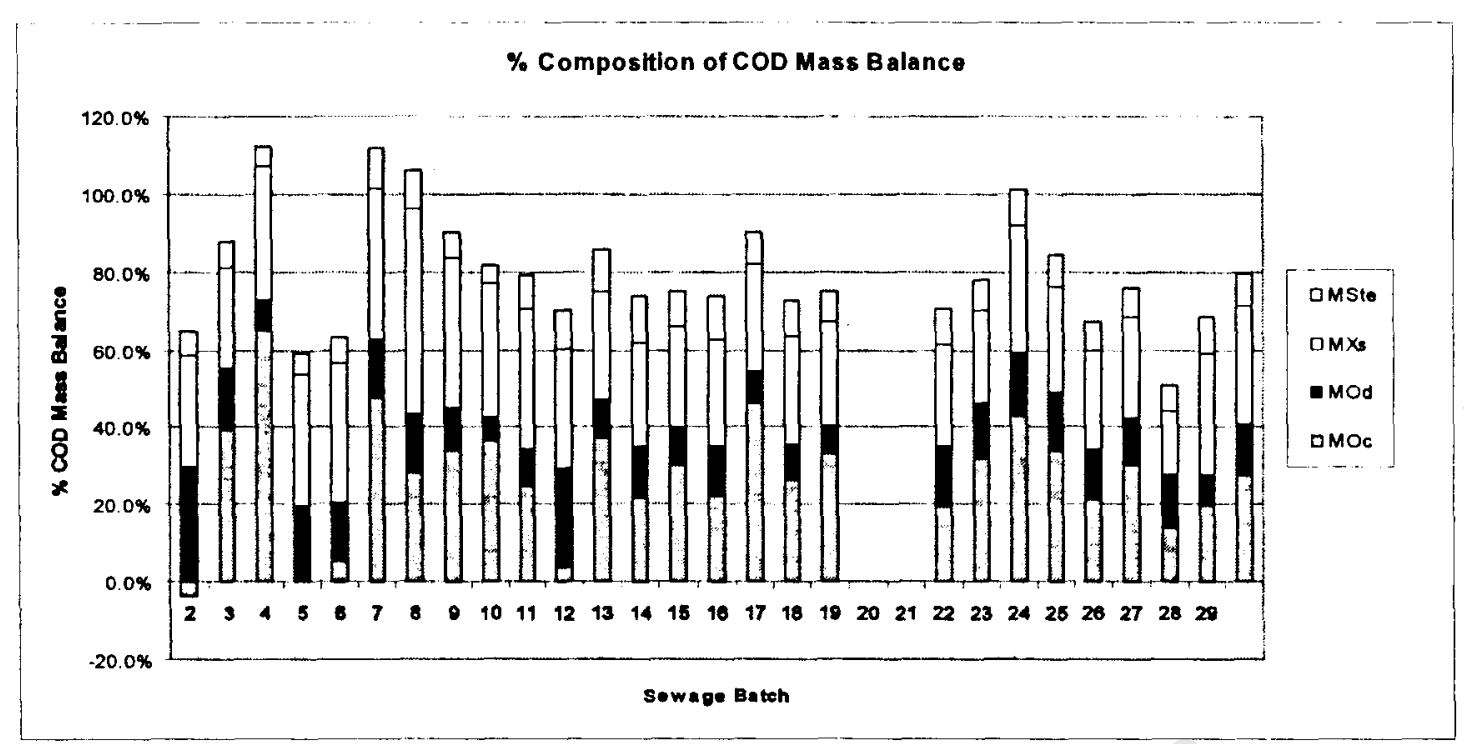

Figure 5.12: Graphical representation for the percentage COD mass balance for the sewage wastewater batches for the conventional UCT system. Percentages are also shown for the COD in the effluent stream (MSte), $C O D$ in the waste sludge (MXs), equivalent oxygen demand for denitrification (Mod) and the carbonaceous oxygen demand (MOc).

\subsubsection{COD Mass Balance}

As can be seen in Table 5.1 and Fig. 5.12, COD mass balances ranging from $51.0 \%$ to $123.9 \%$ were obtained, giving an average mass balance of $78.2 \%$ for the entire investigation. Again this result is substantially lower than that obtained in Phase 1, 87.1\% (Ramphao et al., 2004).

As with the nitrogen mass balances only a few sewage batch results fell above $100 \%$, with the majority of values falling consistently lower indicating a systematic error in the measurements. This too was noted in Phase 1 where COD leaving the system could not be accounted for.

A comparison of the COD leaving the system relative to the COD entering follows with the values of Ramphao et al. (2004) in brackets:

- $28.7 \%$ is removed as oxygen consumed $(45.2 \%)$

- $12.9 \%$ is removed through denitrification $(10.8 \%)$

- $30.3 \%$ is removed through sludge wasting $(26.4 \%)$

- $8.2 \%$ is removed in the effluent flow as COD (4.7\%)

- $20.6 \%$ is unaccounted for $(12.9 \%)$

From the above comparison the denitrification, sludge wasting and effluent COD give comparative removals, however OUR readings were substantially lower than those observed in Phase 1 (Ramphao et al., 2004). Thus it is suspected that the measured OUR readings were regularly less than they should have been. This is explained by the lower sludge mass in the conventional system reported earlier. Additionally as noted above for the $\mathrm{N}$-mass balance the unintentional loss of solids through spillages or inconsistent wasting may have resulted in the poor mass balances in the 
conventional system, as unaccounted for sludge loss would impact directly on mass balances.

\subsection{INFLUENT UNBIODEGRADABLE COD FRACTIONS}

The MLVSS concentrations observed in the system were substantially lower than those generated in the UCTPHO simulation (Dold et al., 1991) using unbiodegradable soluble and particulate COD fractions of $f_{S \text {,us }}=0.05$ and $f_{S \text {,up }}=0.13$. This had been observed in the conventional UCT system in Phase 1 and prompted an investigation into the influent unbiodegradable fractions. Similarly an attempt to understand the low MLVSS concentrations of the conventional UCT system is presented here.

\subsubsection{Unbiodegradable Soluble COD Fraction $\left(f_{\mathrm{S}, \mathrm{us}}\right)$}

The unbiodegradable soluble COD fraction $\left(\mathrm{f}_{\mathrm{S}, \mathrm{us}}\right)$ is calculated on the assumption that all biodegradable COD in the system is utilized, thus only the unbiodegradable soluble COD remains in the effluent. $f_{\mathrm{S} \text {, us }}$ with regard to the influent COD (sewage + acetate) was determined as in Chapter 4 , Section 4.8 .

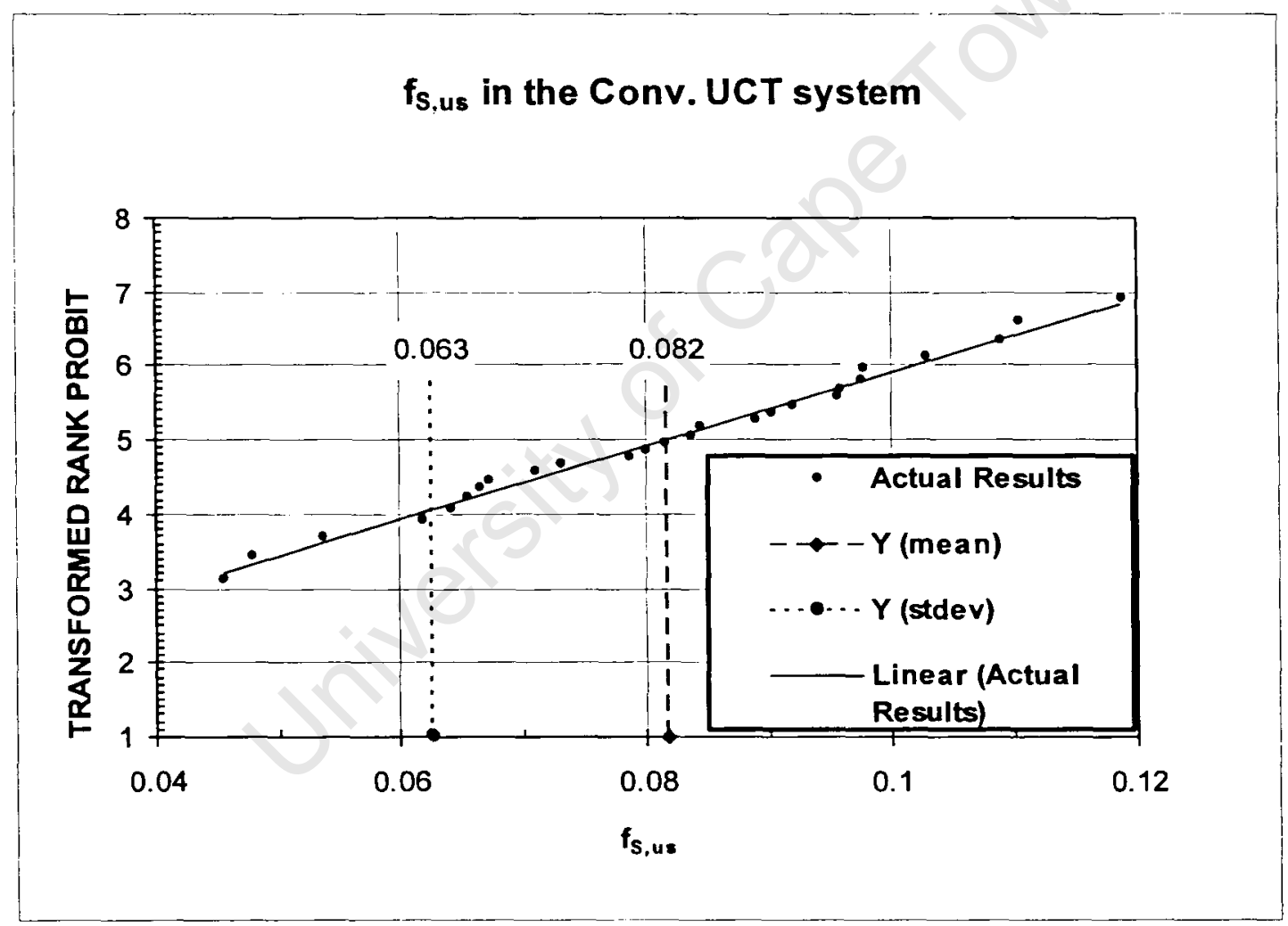

Figure 5.13: Statistical analysis of the unbiodegradable soluble component of the raw wastewater.

The average $\mathrm{f}_{\mathrm{S} \text {,us }}$ for each batch period was calculated from the corresponding batch influent and $0.45 \mu \mathrm{m}$ filtered effluent averages. The sewage batch average $\mathrm{f}_{\mathrm{S} \text {, us }}$ values are plotted on a linearized probability graph (Fig. 5.13) to check for normality. The data lie close to the true normal line indicating a normal distribution. The mean sewage batch average is $0.054(\mathrm{SSD}=0.012)$, very close to $\mathrm{f}_{\mathrm{S} \text {, us }}=0.058$ measured in Phase 1 (Ramphao et al., 2004). Noting that $200 \mathrm{mgCOD} / \ell$ in the influent was sodium acetate and hence completely biodegradable, and that the overall average total 
$\mathrm{COD}$ is $951.2 \mathrm{mgCOD} / \ell$, the raw wastewater $\mathrm{f}_{\mathrm{S} \text {.us }}=0.068$ before the addition of sodium acetate. This average falls within the range of typical values expected for raw domestic wastewaters in South Africa (0.04 to 0.10, WRC 1984) and compares favourably with values found by other researchers who used waste water from the same source: 0.085, Cronje et al. (2000); 0.09, Ubisi et al. (1997a,b); 0.075 Musvoto et al. (1992); 0.09, Mbewe et al. (1995) and 0.096, Muller et al. (2003).

\subsubsection{Unbiodegradable Particulate COD Fraction $\left(f_{S, \text { up }}\right)$}

The unbiodegradable particulate COD fraction $\left(\mathrm{f}_{\mathrm{S} \text {,up }}\right)$ was calculated using the same method outlined for the MBR UCT system (Chapter 4, Section 4.8.2). The calculation is dependant on the system VSS mass $\left(\mathrm{M}_{\mathrm{Xv}}\right)$ in the system, which as noted in Section 5.3 was calculated using the mixed liquor COD measurements. With the sewage batch average values for the conventional system applied directly into Equation (4.9) the $\mathrm{f}_{\mathrm{S}, \mathrm{up}}$ results plotted in Fig. 5.14 were obtained.

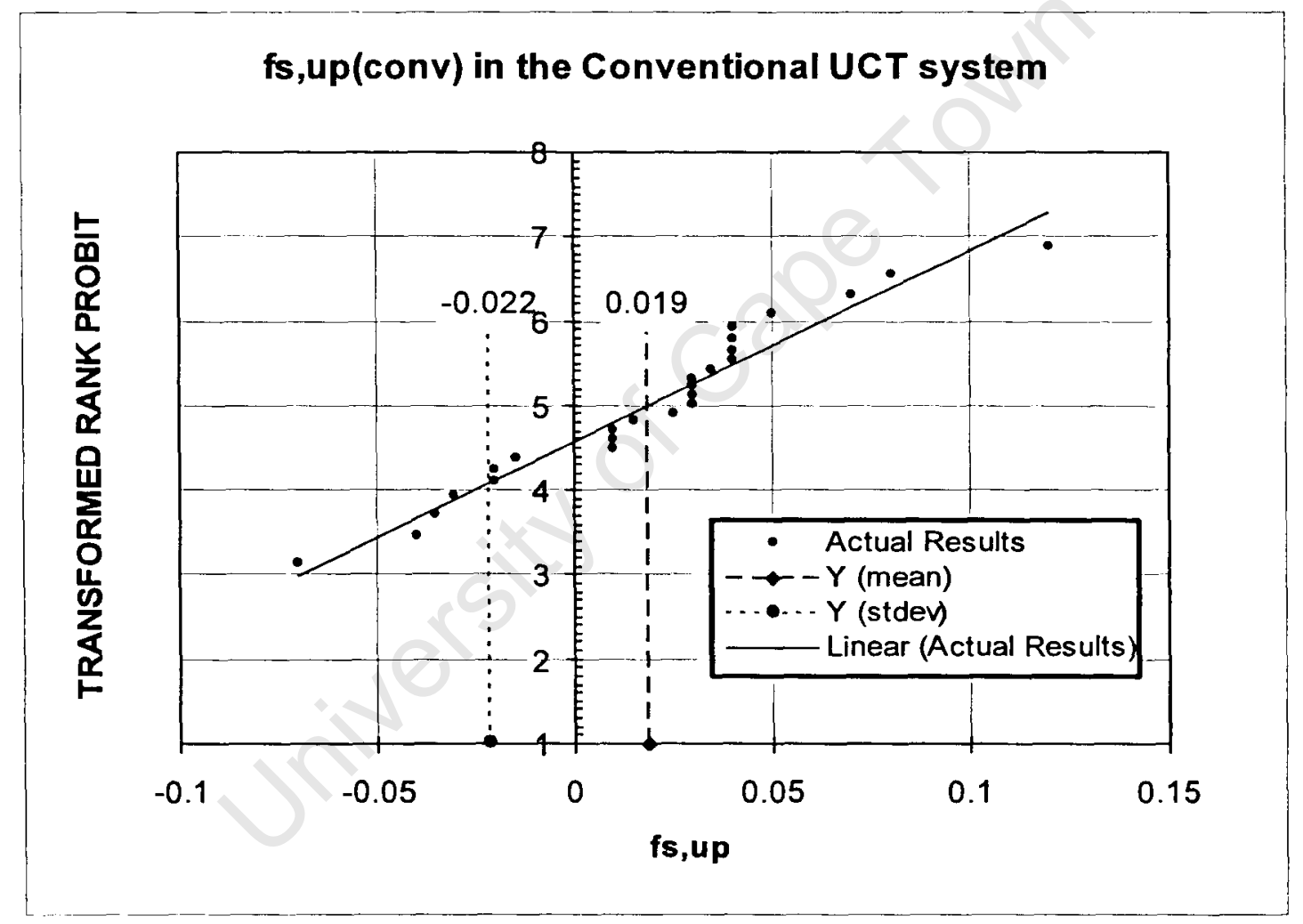

Figure 5.14: Statistical plot of the $f_{S, \text { up }}$ values determined in the conventional UCT system.

A mean value of $f_{\mathrm{S}, \text { up }}=0.019(\mathrm{SSD}=0.041)$ was observed. This result is significantly lower than that expected from raw domestic wastewaters in South Africa, $0.07-0.20$ (WRC, 1984) and corresponding values reported by other researchers using the same wastewater source: for aerobic (0.108, Mbewe et al., 1995), MLE (0.135, Warburton et al., 1991; 0.120, Ubisi et al., 1997; 0.155, Mellin et al., 1998; 0.160, Beeharry et al., 2001; 0.150, Lee et al 2002a), MUCT (0.15 Clayton et al., 1991; $0.29 \& 0.32$ Musvoto et al., 1992) and UCT (0.045 \& 0.062 Sneyders et al., 1998; 0.067 Ramphao et al., 2004) configurations. Such a low average $f_{\mathrm{S} \text {,up }}$ value (and in some sewage batches negative values) indicates that the mass of VSS in the system (as measured 
via the $C O D$ ) was comparatively much lower than for systems in which higher $\mathrm{f}_{\mathrm{S} \text {,up }}$ values were obtained.

An effort was made to try understand why the $\mathrm{f}_{\mathrm{S} \text {,up }}$ in the conventional system was so low, particularly in comparison to the MBR system which had a relatively high $\mathrm{f}_{\mathrm{S} \text {,up }}$ (0.20). Equation (4.9) is based on the assumption of a $100 \%$ COD mass balance, which as reported in Section 5.4.2 was not achieved in almost every sewage batch fed to the conventional system. The low COD mass balance could be taken into consideration in one of two ways:

- either it could be assumed that COD was lost from the system via the influent feed, in which case the reported influent COD was higher than the actual influent COD,

- or unaccounted for COD was lost from the system through increased uncontrolled sludge losses resulting in a shorter sludge age,

In the first instance, a reduced influent COD mass is assumed. Only the COD accounted for should be used as influent. This reduces the influent COD mass by $20 \%$ of the measured influent COD feed. This amounts to $3 \ell$ of the $15 \ell$ fed daily, which is a significant volume to lose without being able to account for it. Calculating the $\mathrm{f}_{\mathrm{S} \text {,up }}$ from influent COD mass gives an average $\mathrm{f}_{\mathrm{S} \text {,up }}$ of 0.125 (SSD $=0.074$ ). A statistical plot of the results (Fig. 5.15) shows a relatively normal distribution.

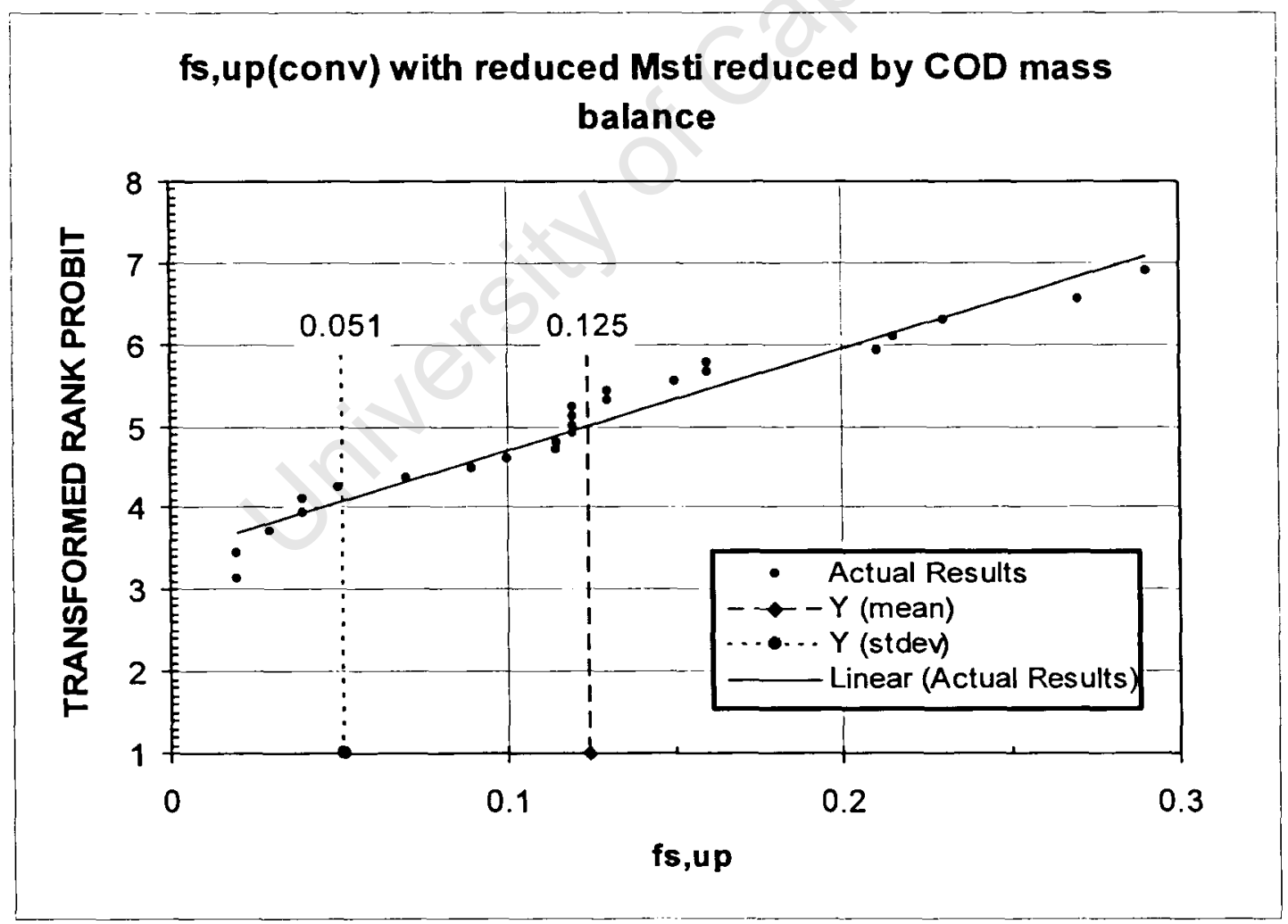

Figure 5.15: $f_{\text {S.up }}$ calculated taking the COD-mass balance into consideration

If it is accepted that the $\mathrm{MS}_{\mathrm{ti}}$ measurements were correct then the remaining variable in Equation 4.9 is the sludge age. In order to achieve an overall COD mass balance of $100 \%$ a sludge age of $12.2 \mathrm{~d}$, or an additional $0.9 \ell$ mixed liquor would need to have 
been wasted daily over and above the $1.1 \ell$ already wasted. This too seems unlikely, though frequent sludge spills may have occured without the knowledge of the writer. These were dealt with by the laboratory assistant.

\section{Alternative method of calculating $\mathrm{f}_{\mathrm{S} \text {,up }}$ for the conventional UCT system.}

An additional investigation into alternative methods of calculating $f_{\mathrm{S}, \text { up }}$ was conducted using the method described below:

The unbiodegradable fraction of influent $\operatorname{COD}\left(\mathrm{f}_{\mathrm{S}, \mathrm{u}}\right)$ can be calculated as in Equation (5.1):

$f_{s, u}=f_{s, u s}+f_{S, u p}$

If $f_{S, u}$ is accepted to be an influent wastewater characteristic and is constant for both the MBR system and the conventional UCT system, then $f_{S, \text { up }}$ for the conventional system could be calculated as the difference between $f_{\mathrm{S}, \mathrm{u}}$ measured in the MBR system and $f_{S, \text { us }}(0.45)$ measured in the conventional system, (Equation 5.2). (It is assumed that the difference between $f_{\mathrm{S}, \text { us }}\left(0.45\right.$ conv eff) and $f_{\mathrm{S}, \text { us (membrane efluent) is }}$ incorporated in the MBR $\mathrm{f}_{\mathrm{S} \text {, up }}$ fraction).

$$
f S_{, u p(\mathrm{conv})}=(f s, u p(\mathrm{MBR})+f s, u s(\mathrm{MBR} \text { eff }))-f s, u s(\mathrm{conv})
$$

Using sewage batch averages for $f_{S, u}(M B R)$ and $f_{S, u s}$ (conv) a new set of $f_{S, \text { up }}$ (conv) values were calculated, these are presented in a statistical plot, Fig. 5.16.

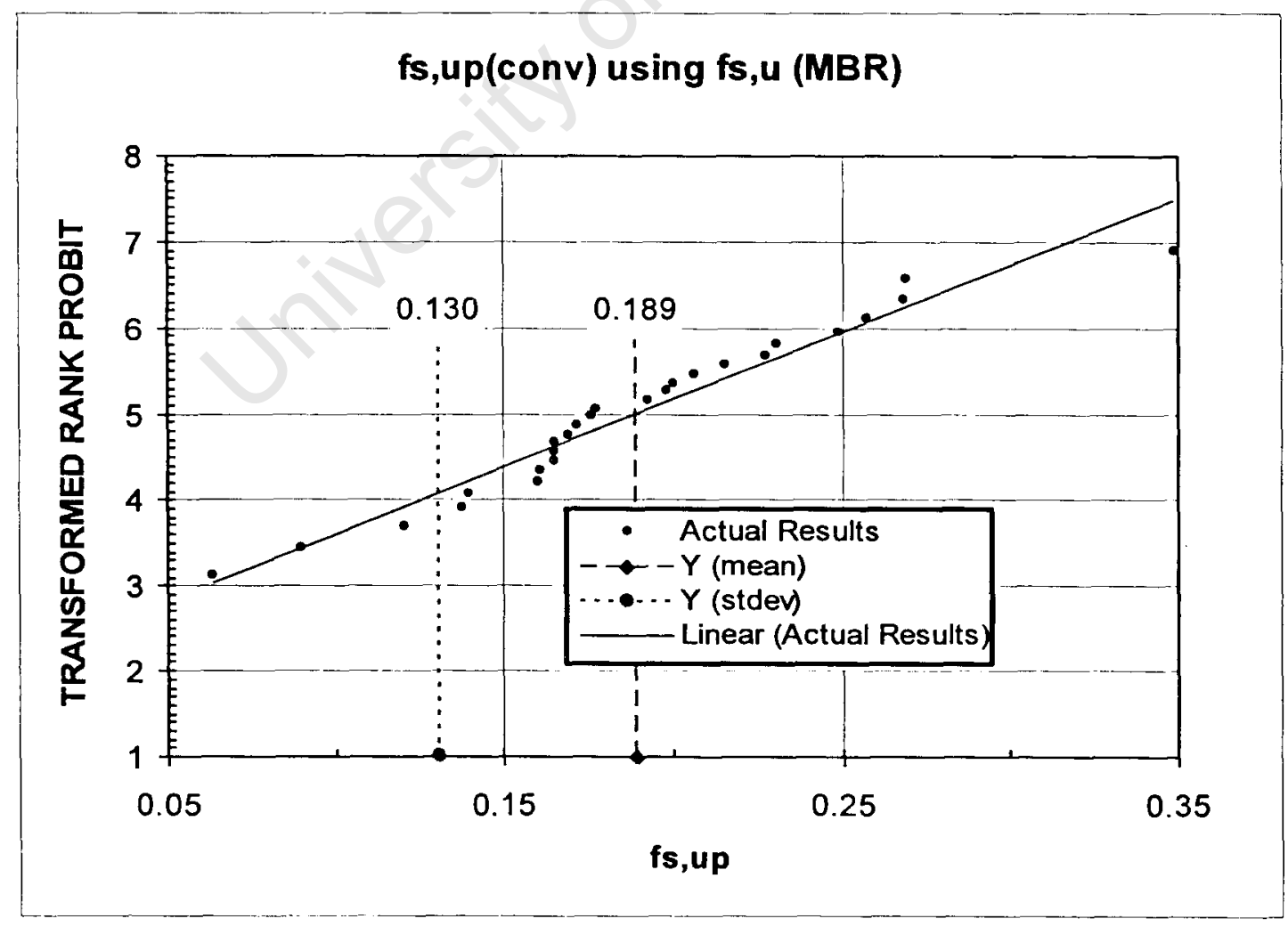

Figure 5.16: Statistical plot of recalculated $f_{\text {S.up }}$ values using the $f_{S . u}(M B R)$ values. 
If these values are applied back into the $\mathrm{M}_{\mathrm{Xv}} / \mathrm{M}_{\mathrm{Sti}}$ equation (Chapter 4 , Equation 4.9) and instead the sludge age $\left(R_{s}\right.$, and consequently the waste flow, $\left.Q_{w}\right)$ or influent flow $\left(Q_{i}\right)$ are used as variables then the equivalent variations in these parameters can be determined to suggest reasons for the poor COD balance in the conventional UCT system.

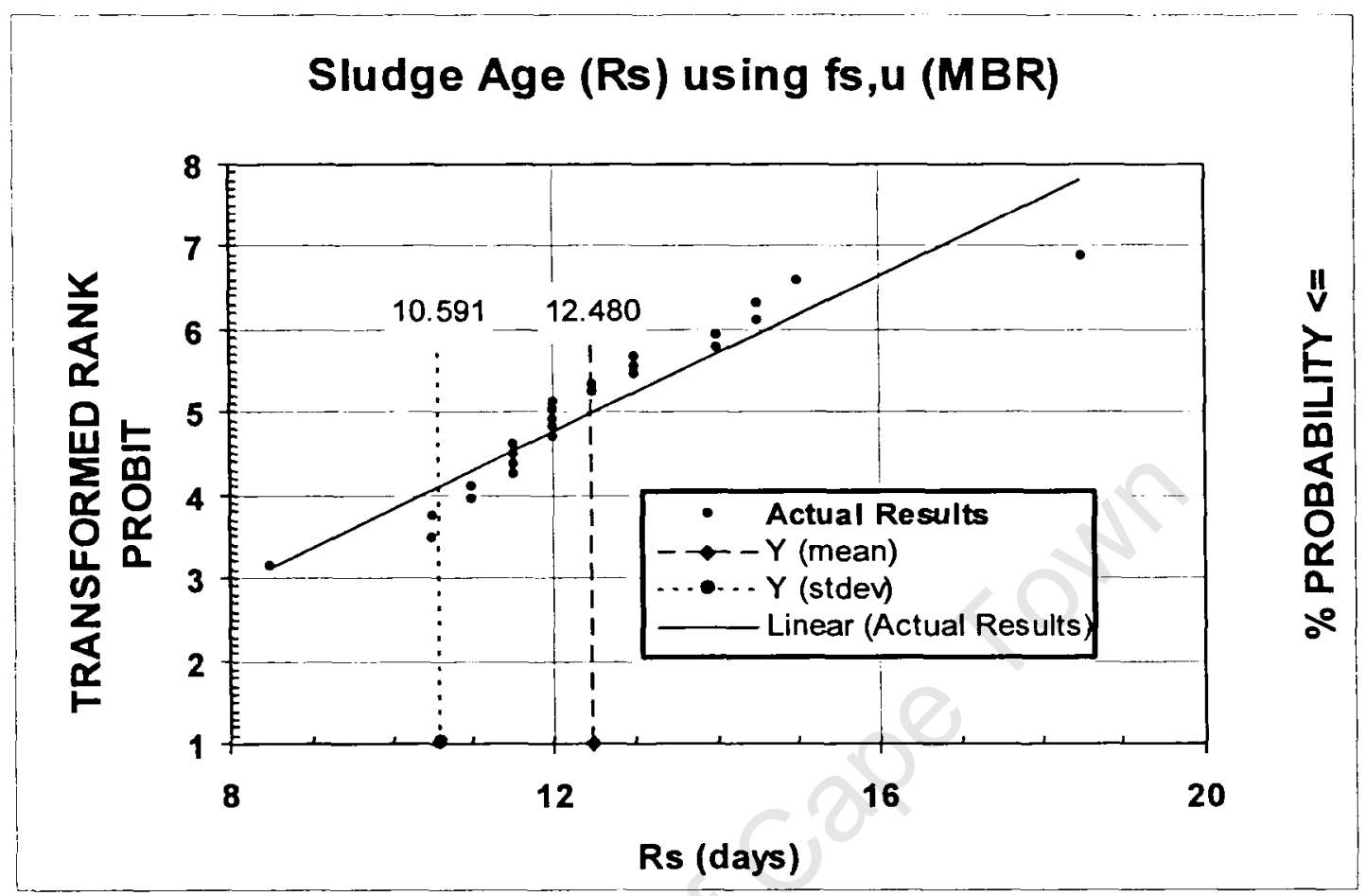

Figure 5.17: Variations in sludge age as determined using $f_{\text {S.up }}$ calculated using $M B R f_{S, u}$

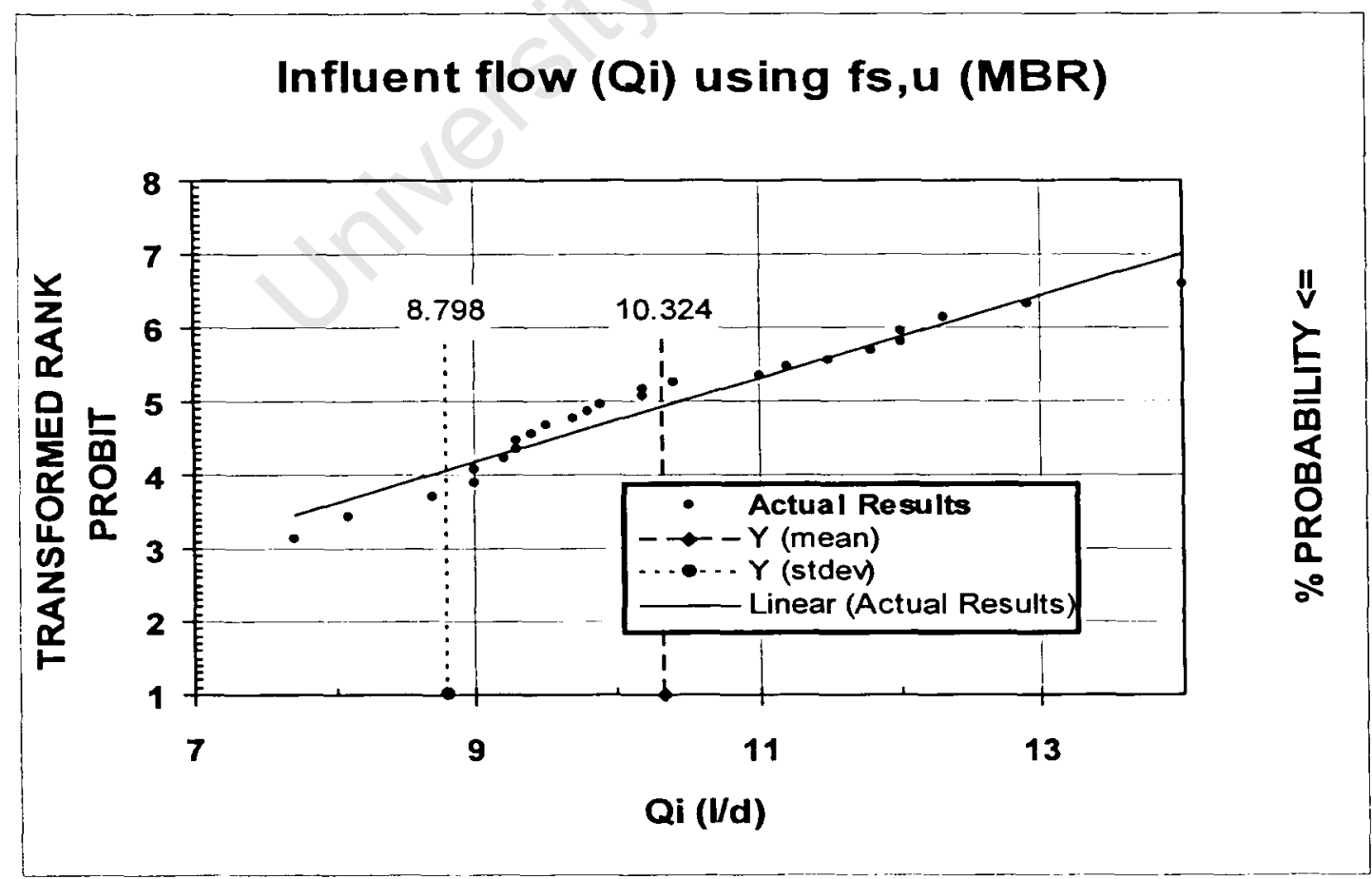

Figure 5.18: Variations in influent flow as determined $u$ sing $f_{\text {Sup }}$ calculated using $M B R f_{S, u}$ 
From the above investigation, in order for the $f_{S \text {, up }}$ in the conventional system to compare with the $f_{S, u}$ in the MBR investigation, the sludge age $\left(R_{s}\right)$ of the conventional system would have to decrease substantially from 20 days on average to 12.5 days, or similarly the influent flow rate $\left(Q_{i}\right)$ would have to decrease substantially from $15 \ell / \mathrm{d}$ to $10.3 \ell / \mathrm{d}$.

The results from Phase 1 (Ramphao et al., 2004) also indicated a significantly reduced $f_{\mathrm{S} \text {,up }}=0.067$ in the conventional UCT system, thus suggesting that the $\mathrm{f}_{\mathrm{S}, \mathrm{u}}$ value is not the same for the MBR and conventional UCT systems as is assumed above. In Phase 1 , the $\mathrm{f}_{\mathrm{S}, \mathrm{u}}$ for the MBR system was 0.252 but for the conventional UCT system 0.125 . Regardless it is concluded that unaccounted for sludge losses from the conventional UCT system resulted in poor mass balances and low $f_{S \text {,up }}$ values.

\subsubsection{Revision of Sludge Production}

The system sludge production is dependant on sludge age $\left(\mathrm{R}_{\mathrm{s}}\right), \mathrm{COD}\left(\mathrm{MS}_{\mathrm{ti}}\right)$ load on the system and influent COD characteristics. In Section 5.5.2 above, Rs and Qi values were proposed for the conventional system in accordance with revised COD mass balances. In order to determine the impact of these revisions on the system sludge production, the calculations of Section 4.6 .6 are repeated below:

- With the revised sewage batch average $R_{s}$ values above, the system sludge production ranged from $0.251-0.451 \mathrm{mgVSS} / \mathrm{mgCOD}$, with a mean of 0.351 $(\mathrm{SSD}=0.048)$. This value is higher than that observed in Phase 1 , but is in the range of sludge production values expected for a sludge age of 12.5 days.

- Retaining the original system sludge age of 20 days and instead using the revised influent flow rate $\mathrm{Q}_{i}$ as determined above the system sludge production ranged from $0.239-0.429 \mathrm{mgVSS} / \mathrm{mgCOD}$, with a mean of 0.317 $\mathrm{mgVSS} / \mathrm{mgCOD}(\mathrm{SSD}=0.049)$. This result is very close to that observed in the MBR UCT system of $0.311 \mathrm{mgVSS} / \mathrm{mgCOD}$.

\subsubsection{Discussion}

In this section difficulties with the calculation of the $\mathrm{f}_{\mathrm{S} \text {, up }}$ values for the conventional system have been presented. Reasons proposed for the unusually low $f_{S \text {, up }}$ values were a loss of COD from the influent, or an additional loss of sludge from the system. The writer was however unaware of either occurring. Due to the volumes lost in order to account for the decreased COD or sludge age it would appear that the loss of sludge was more likely, or a combination of the two occurred.

\subsection{SYSTEM PERFORMANCE}

\subsubsection{COD Removal}

Daily influent $\left(\mathrm{S}_{\mathrm{ti}}\right)$ and effluent $\left(\mathrm{S}_{\mathrm{te}}\right)$ COD concentrations are shown in Fig. 5.19. Both unfiltered and $0.45 \mu \mathrm{m}$ filtered $S_{\mathrm{te}}$ values are presented. 


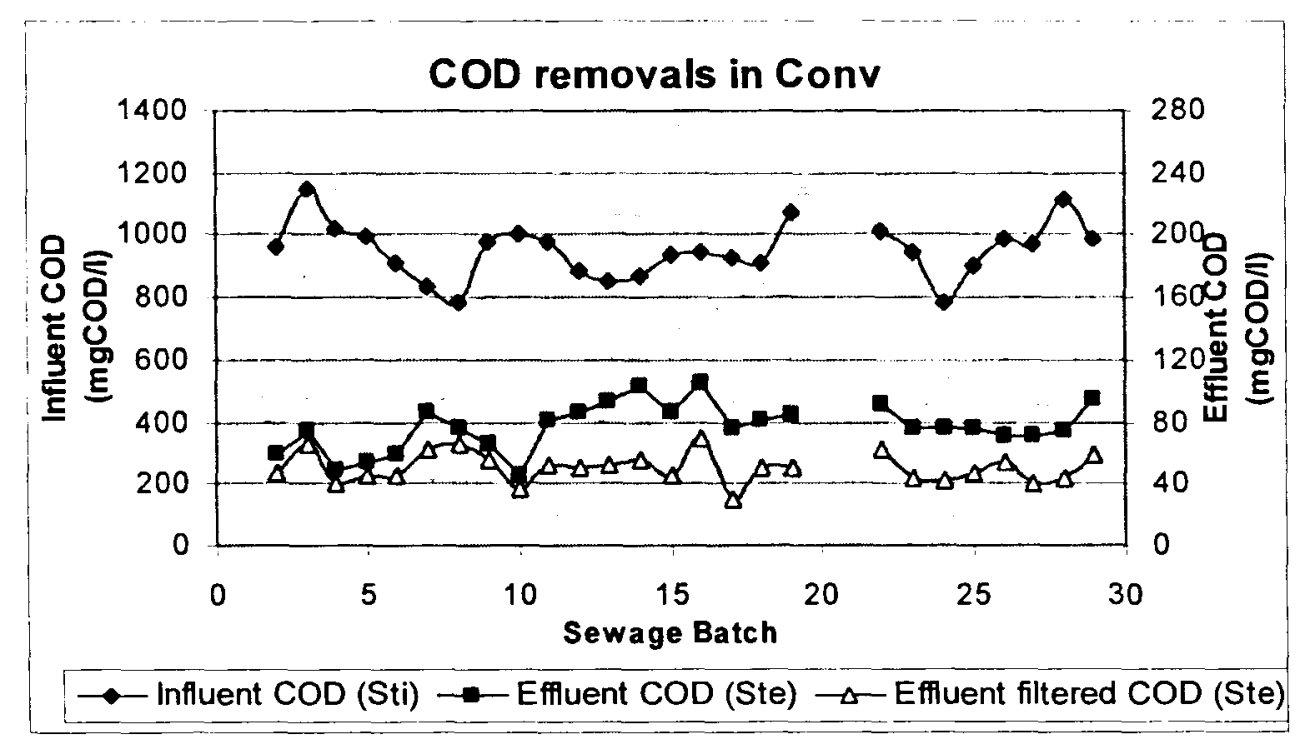

Figure 5.19: Comparison of influent $\left(S_{t i}\right)$, effluent unfiltered and effluent unfiltered $\left(S_{t e}\right)$ for each batch period.

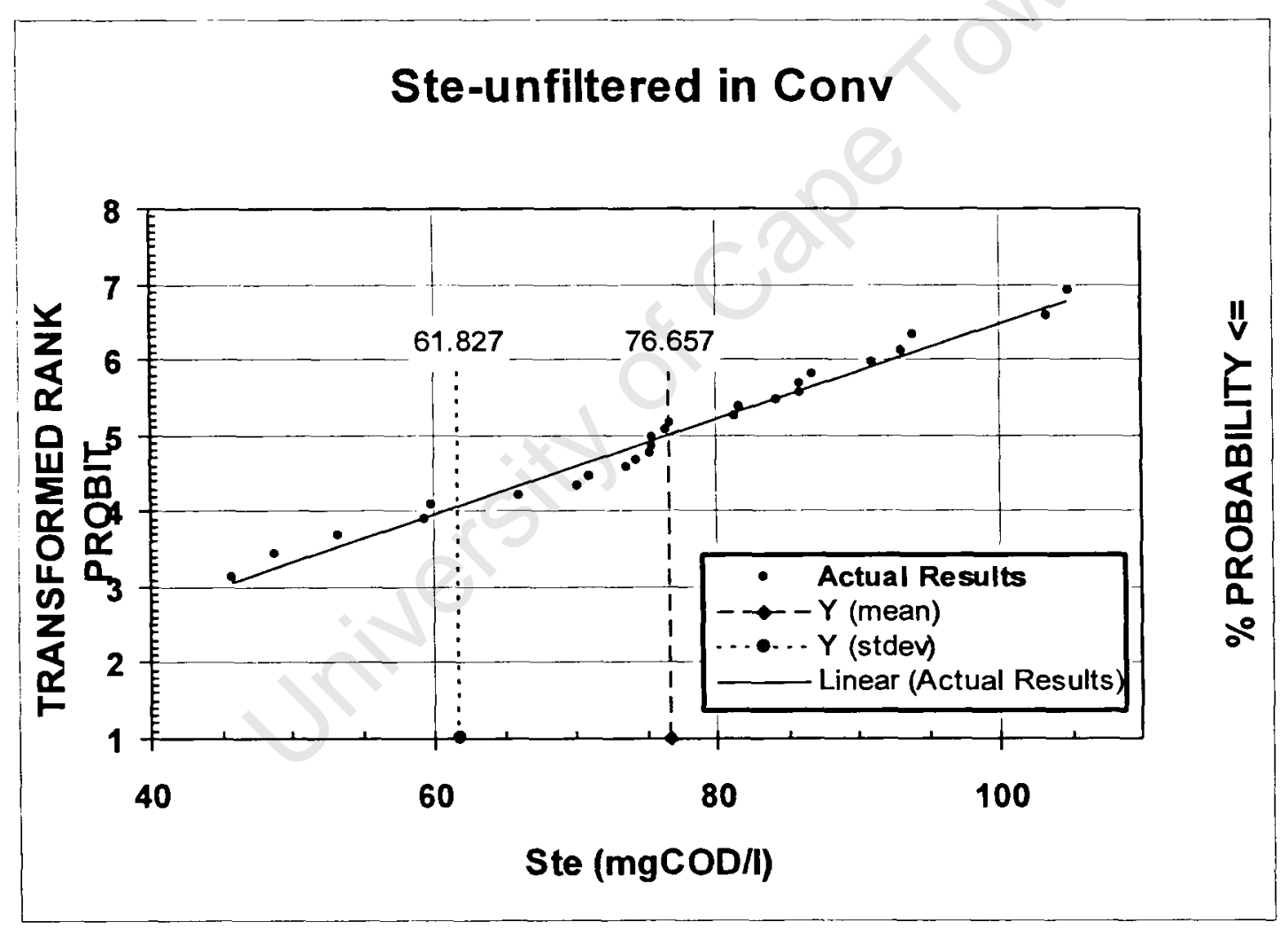

Figure 5.20: Statistical plot of the effluent unfiltered COD (Ste) in the conventional UCT system. 


\section{Ste filtered in Conv}

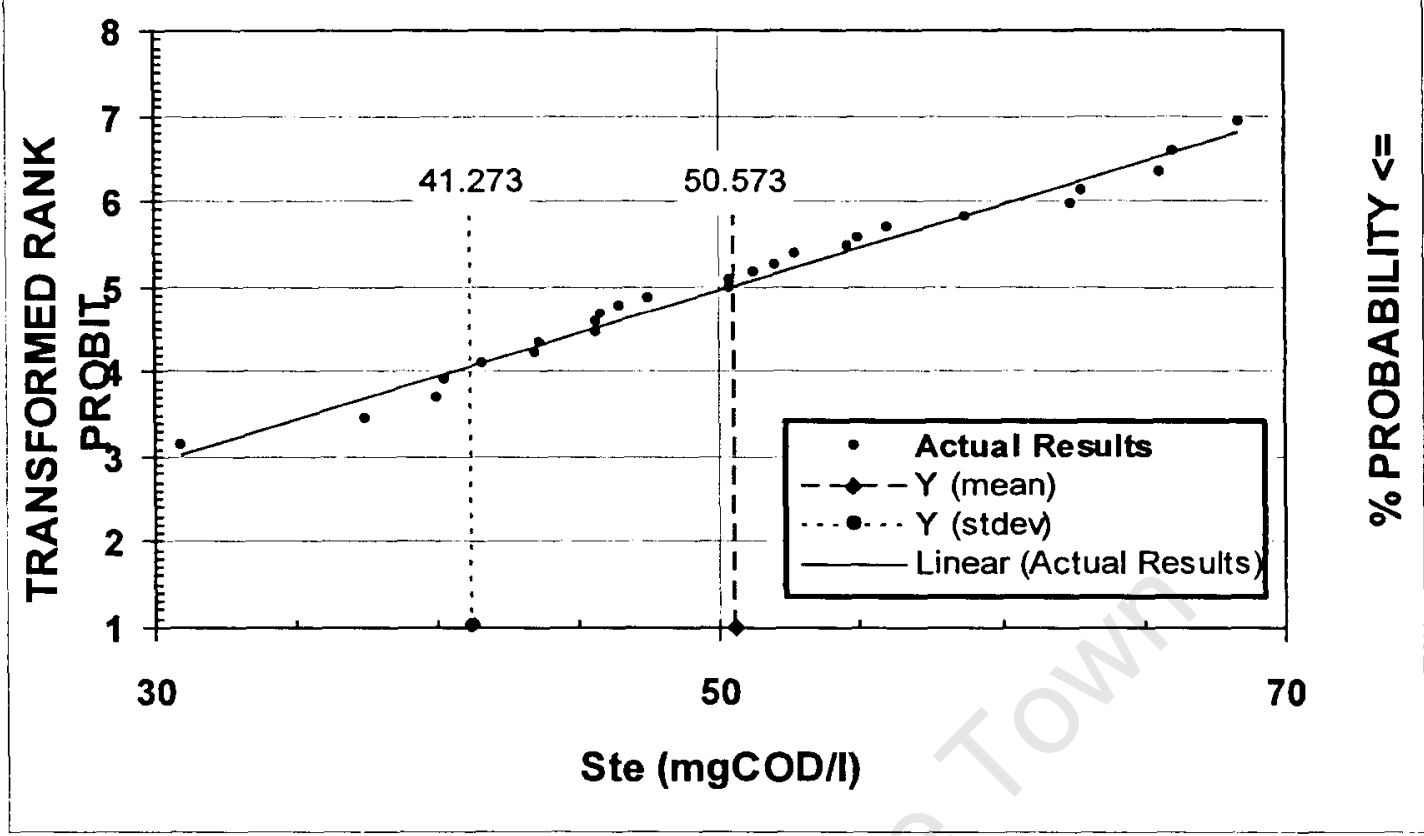

Figure 5.21: Statistical plot of the effluent filtered COD in the conventional UCT system.

- The conventional UCT system provided reasonably high COD removal efficiency. Despite fluctuations in the influent feed, from $785 \mathrm{mgCOD} / \ell$ (sewage batch 8) to $1147 \mathrm{mgCOD} / \ell$ (sewage batch 24), effluent COD removals remained within the range of $46-105 \mathrm{mgCOD} / \ell$ for the unfiltered effluent $\mathrm{COD}$ and $31-66 \mathrm{mgCOD} / \ell$ in the effluent filtered COD.

- Statistical plots of the effluent COD filtered and unfiltered concentrations are presented in Figs. 5.20 and 5.21. The mean average effluent COD concentrations are $76.7 \mathrm{mgCOD} / \ell(\mathrm{SSD}=14.8 \mathrm{mgCOD} / \ell)$ for the unfiltered effluent $\mathrm{COD}$ and $50.6 \mathrm{mgCOD} / \ell(\mathrm{SSD}=9.3$ ) for the filtered effluent COD. Thus, throughout the investigation an average of $26.1 \mathrm{mgCOD} / \ell$ was lost through the effluent as non-settleable solids. Using the $f_{C V}$ and VSS/TSS ratios calculated in Section 5.3 this amounts to approximately $21.5 \mathrm{mgTSS} / \ell$ lost in the effluent.

- The COD removal efficiencies of $0.45 \mu \mathrm{m}$ filtered and unfiltered samples in the system were $95.7 \%$ and $92.7 \%$ respectively.

- The difference between $0.45 \mu \mathrm{m}$ filtered and unfiltered effluent COD demonstrates the loss of solids with the effluent in conventional systems with SSTs. This solids loss, as noted by Ramphao et al. (2004) impacts directly on the quality of the effluent.

\subsubsection{Nitrogen Removal}

System nitrogen removal through the nitrification and denitrification mechanisms is examined in Fig. 5.22. 
TKN-removal performance of the Conv. System

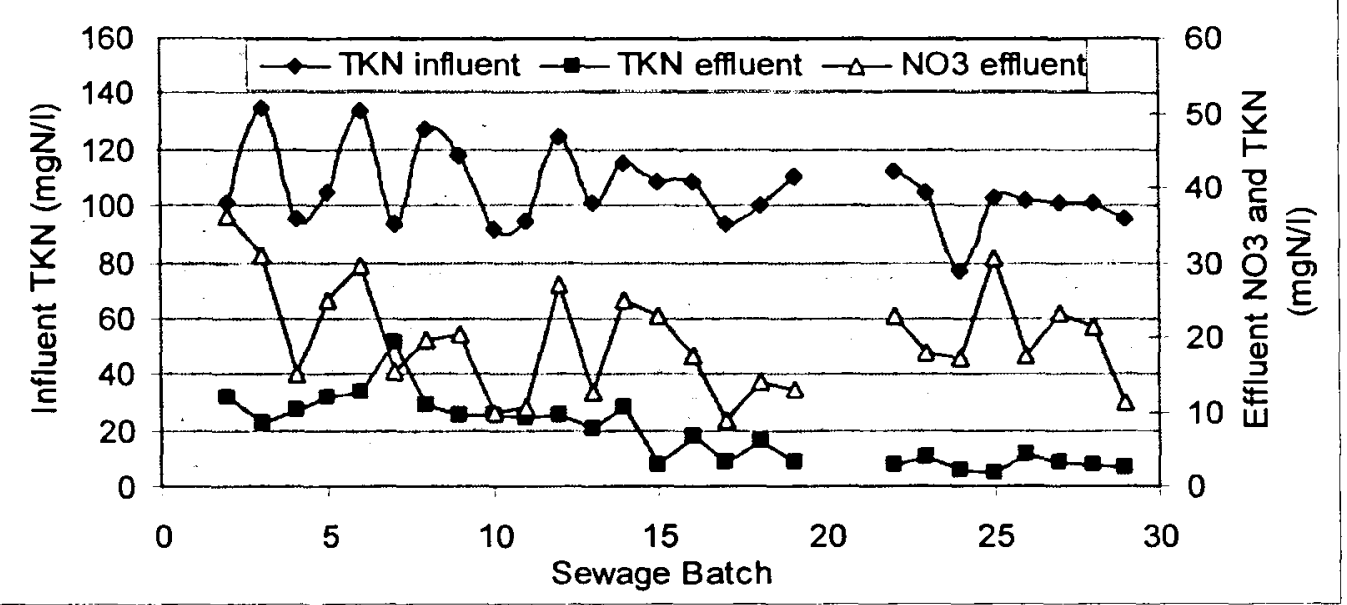

Figure 5.22: System influent $\left(N_{t i}\right)$ and effluent $\left(N_{t e}\right)$ TKN concentrations plotted against the effluent nitrate $\left(N_{n e}\right)$.

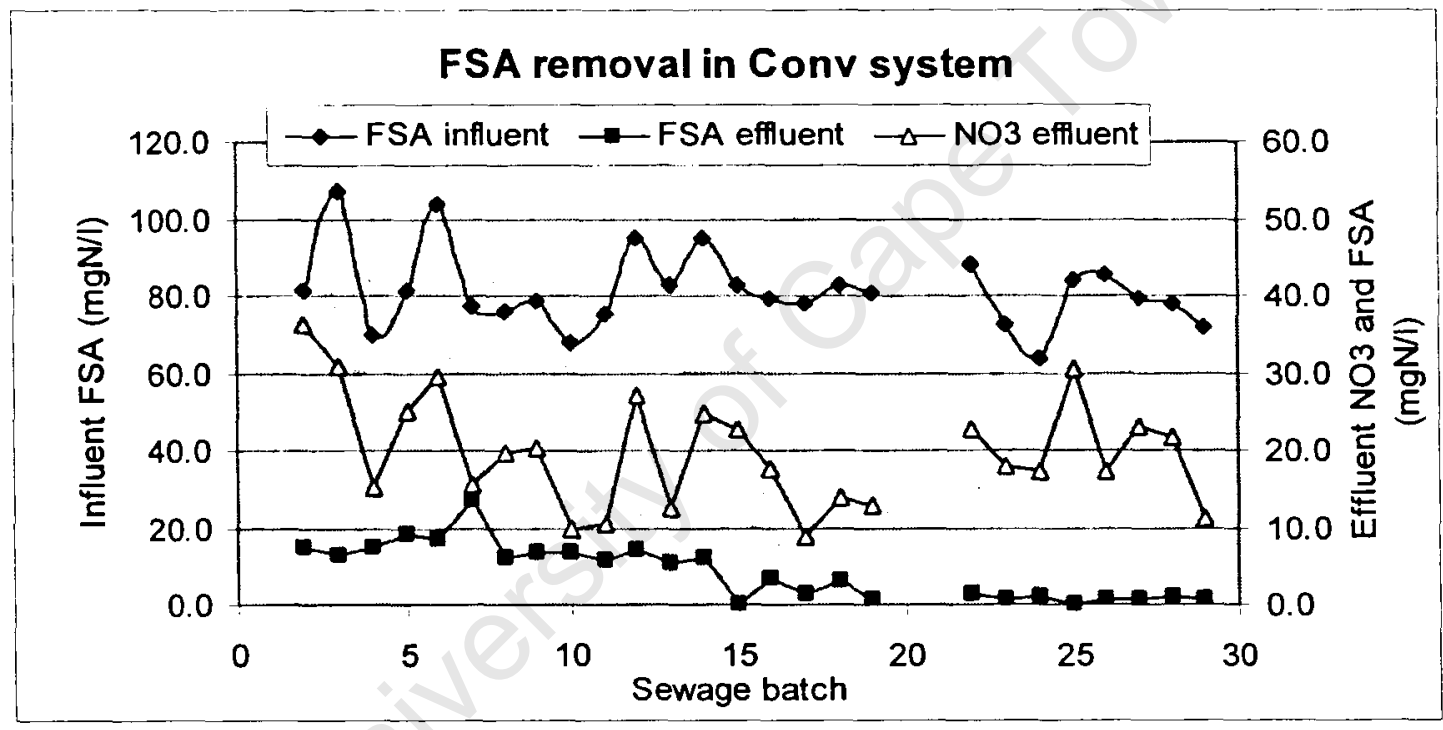

Figure 5.23: System influent $\left(N_{a i}\right)$ and effluent $\left(N_{a e}\right)$ TKN concentrations plotted against the effluent nitrate $\left(N_{n e}\right)$. 


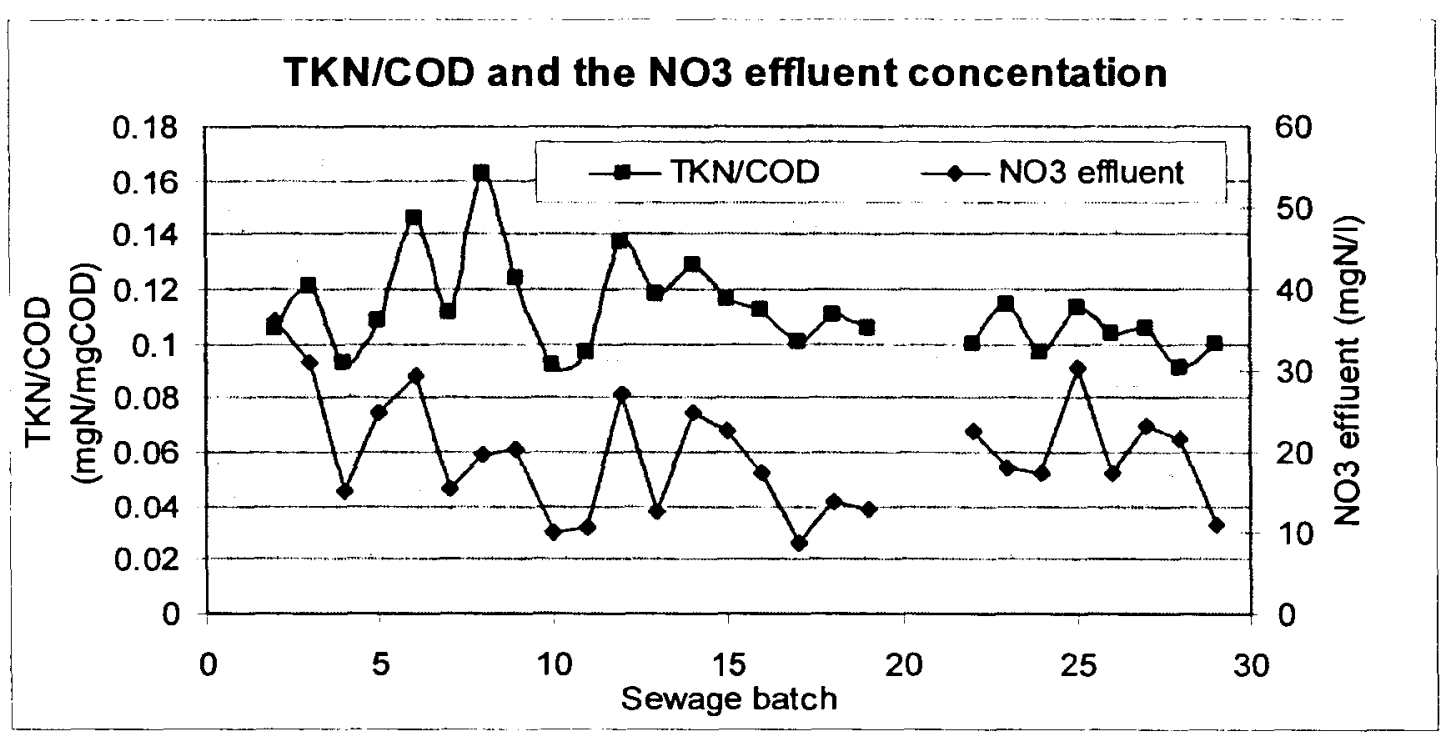

Figure 5.24: TKN/COD ratio on the influent feed and its influence on the effluent nitrate.

\section{Nitrification}

Variations in influent and effluent TKN and FSA concentrations (as well as effluent nitrate) are presented in Figs. 5.23 and 5.24.

- Approximately $80 \%$ of the influent TKN is FSA with the balance being organic nitrogen. Early tests on the influent confirmed that nitrate and nitrite concentrations in the influent were negligible. The high FSA fraction of the TKN is due to the dosing of ammonia to the feed in order to maintain a $\mathrm{TKN} / \mathrm{COD}$ ratio of $0.1 \mathrm{mgN} / \mathrm{mgCOD}$.

- For the first 12 sewage batches $20 \mathrm{mgN} / \ell$ ammonium chloride was added to the feed daily. However it was noticed that the TKN/COD ratio fluctuated dramatically ranging from 0.09 to $0.16 \mathrm{mgN} / \mathrm{mgCOD}$. The TKN/COD ratio was dependant on the collected wastewater characteristics and the dilution necessary to obtain an influent feed of $800 \mathrm{mCOD} / \ell$ to be augmented with RBCOD. Thereafter, from day 204 (sewage batch 13), an attempt was made to maintain the TKN/COD ratio at $0.1 \mathrm{mgN} / \mathrm{mgCOD}$ by controlled dosing of ammonium. This strategy significantly reduced the variation in influent TKN/COD ratio, Fig.5.24.

- Two distinct periods of testing are observed. From sewage batch 2-15 high effluent ammonia $\left(\mathrm{N}_{\mathrm{ae}}\right.$ ) was measured ranging from 2.4 (day 134) to 20.7 (day 117) with an average of $7.4 \mathrm{mgN} / \ell$. This could be attributed to poor testing for that period, or poor nitrification. Poor nitrification would be due to poor aeration which would effectively reduce the aerated sludge mass fraction; or to a reduced sludge age, close to the minimum sludge age for nitrification which would result in incomplete nitrification.

- From sewage batch 15 onwards there was a dramatic decrease in the measured FSA ranging from 0 to $3.9 \mathrm{mgN} / 1$ with a mean average of $1.3 \mathrm{mgN} / \ell$.

- This variation in FSA impacted directly onto the TKN measured. Hence for the purposes of evaluating conventional $\mathrm{N}$ removal performance only sewage batches 15-29 are used. 
- The filtered effluent TKN and FSA, and the unfiltered effluent TKN values were averaged for sewage batches 15 - 29. Statistical plots of these results are shown in Figs. 5.25-5.27. The mean average values for the unfiltered $N_{t e}$, filtered $\mathrm{N}_{\mathrm{te}}$ and filtered $\mathrm{N}_{\mathrm{ae}}$ were $3.45 \mathrm{mgN} / \ell(\mathrm{SSD}=1.39), 1.97 \mathrm{mgN} / \ell$ $(\mathrm{SDD}=1.07)$ and $1.15 \mathrm{mgN} / \ell(\mathrm{SDD}=1.01)$ respectively.

- Accepting that the difference between the filtered effluent TKN and FSA is the organic unbiodegradable soluble nitrogen $\left(\mathrm{N}_{\text {ouse }}\right)$, the average $\mathrm{N}_{\text {ouse }}$ over the investigation is calculated as $1.97-1.15=0.82 \mathrm{mgN} / \ell$. Expressed as a fraction of the influent $\mathrm{N}_{\mathrm{ti}}=100.9$ (for the period: batches $15-29$ ), $\mathrm{f}_{\text {Nouse }}=$ 0.009 . If it is accepted that on average $20 \mathrm{mgN} / \ell$ ammonia was dosed to the influent the resulting $\mathrm{f}_{\text {Nouse }}$ is 0.011 . As was noted in Chapter 4, Section 4.9.2.1, this is substantially lower than the value of $0.025-0.030$ conventionally accepted for typical South African waste waters.

- The difference between the filtered and unfiltered TKN is the unsettleable solids that are lost from the SST. In the conventional system this difference was on average $1.48 \mathrm{mgN} / \ell$. Adopting the measured TKN/VSS $=0.094$ for this system (Section 5.3) the sludge loss using TKN measurements was on average $15.7 \mathrm{mgVSS} / \ell$. This is reasonably close to that estimated from COD removal (Section 5.6.1).

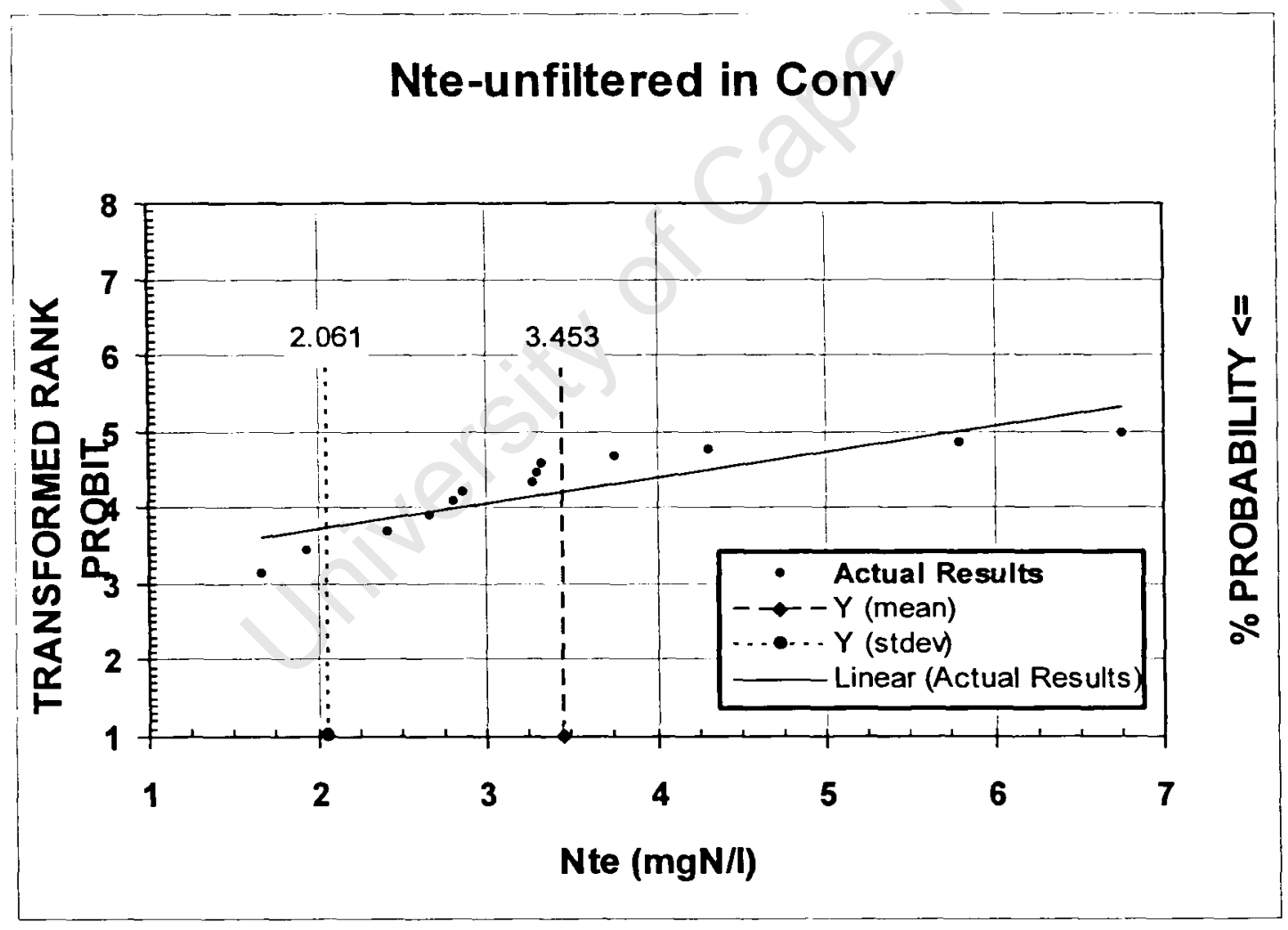

Figure 5.25: Unfiltered effluent TKN concentrations for sewage batches 15-29. 


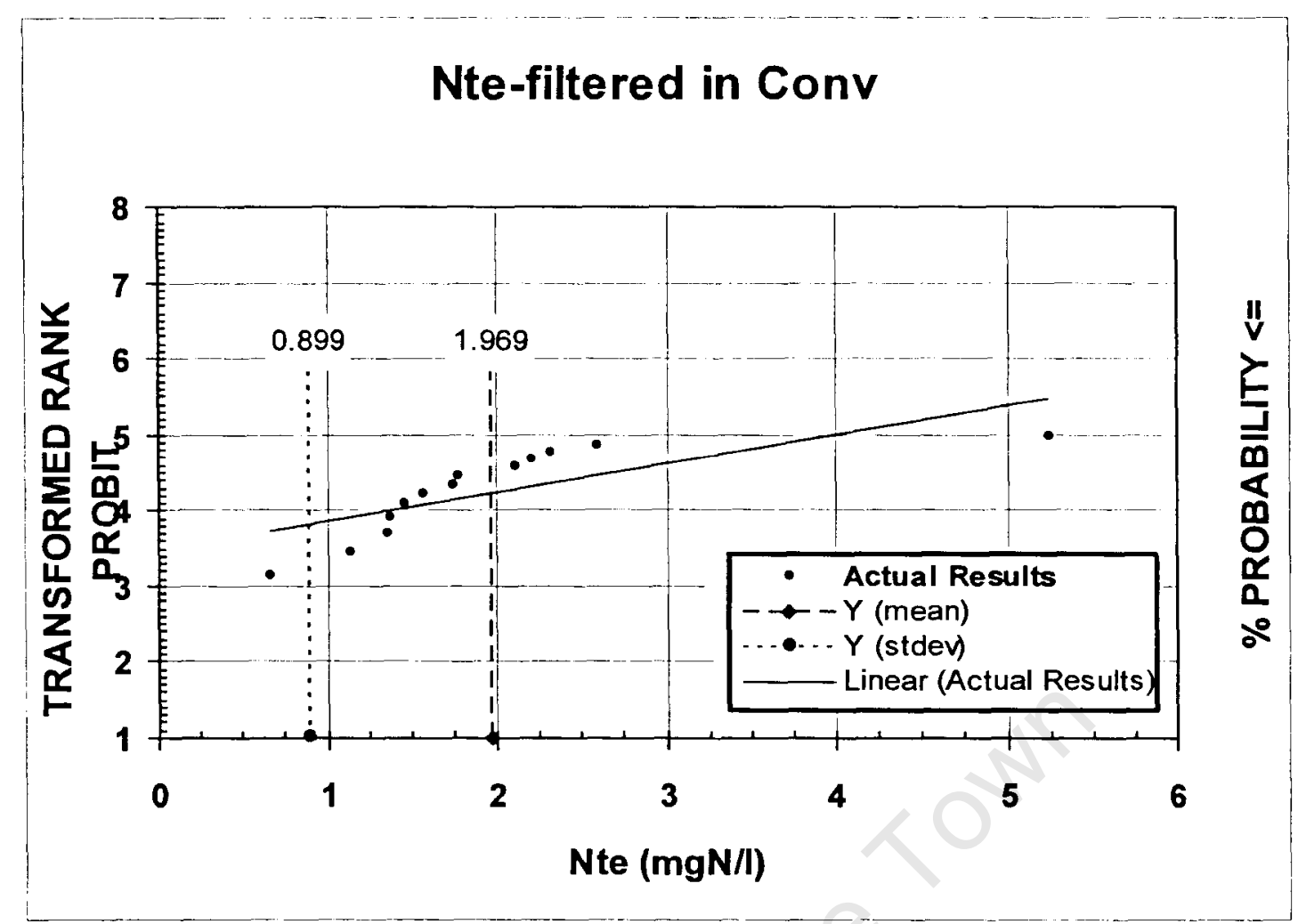

Figure 5.26: Filtered effluent TKN concentrations for sewage batches 15-29.

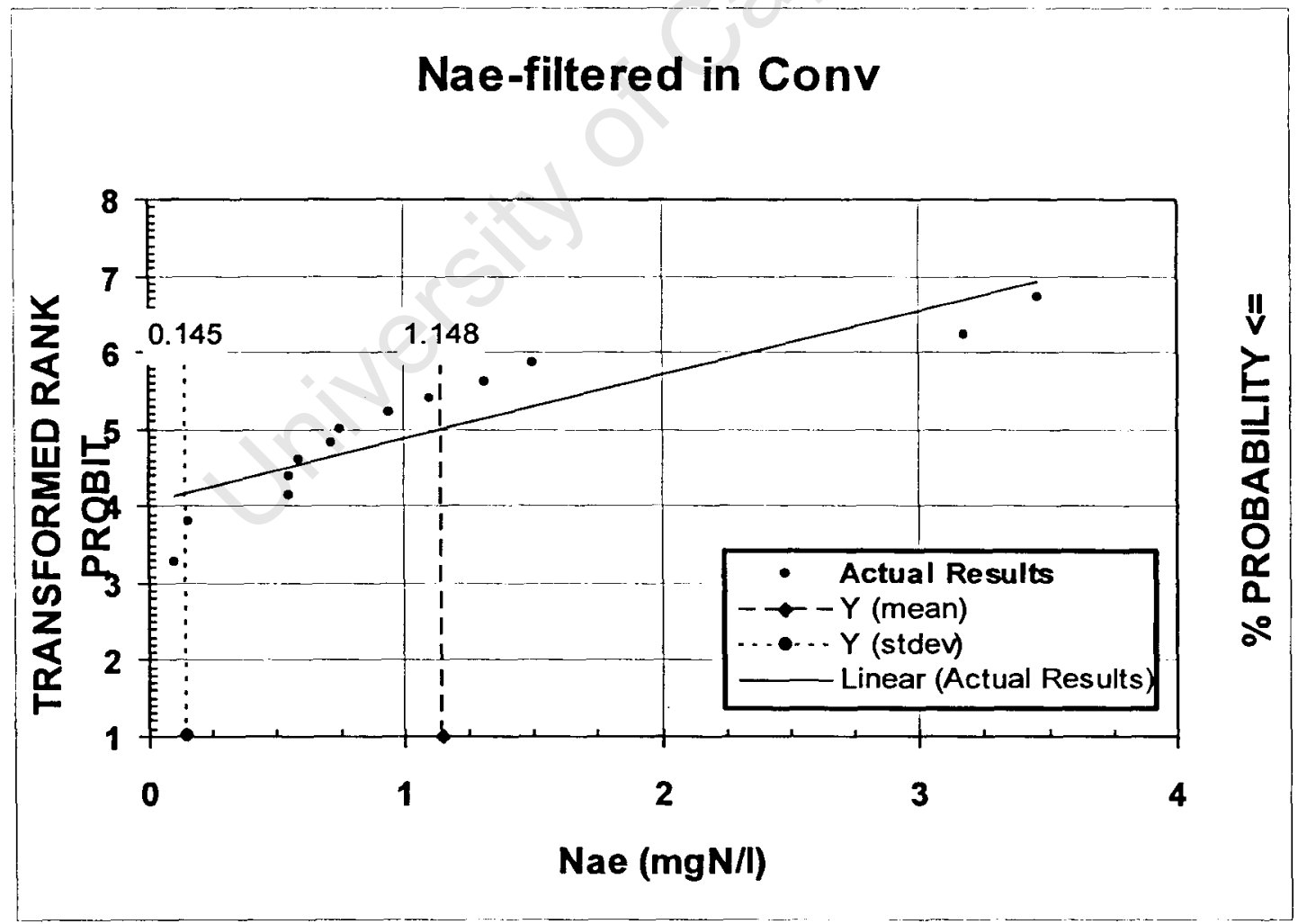

Figure 5.27: Filtered effluent FSA concentrations for sewage batches 15-29. 


\section{Denitrification}

Reactor and effluent $\mathrm{NO}_{3}$ concentrations are plotted in Figs. 5.27-5.30. Detailed results for all reactors and effluent are listed in Appendix B.

- $\mathrm{NO}_{2}$ concentrations across the reactors were typically negligible $(<1 \mathrm{mgN} / \ell)$ however on a few occasions across all the reactors significant $\mathrm{NO}_{2}$ concentrations were measured. For ease of assessment these $\mathrm{NO}_{2}$ concentrations have been added to the nitrate concentrations and the sum termed $\mathrm{NO}_{3}$.

- In general variations in the TKN/COD ratio were tracked by the effluent nitrate $\left(\mathrm{N}_{\mathrm{ne}}\right)$ concentrations as can be seen in Fig. 5.26. This is expected as in the conventional UCT-type configuration $\mathrm{N}_{\mathrm{ne}}$ is approximately:

$$
N_{n e}=\frac{N_{C}}{a+s+1} \quad \text { or } \quad N_{n e}>\frac{N_{C}}{a+s+1}
$$

Where:

$\mathrm{N}_{\mathrm{C}}=$ the nitrification capacity of the system which is directly dependant on the influent TKN/COD ratio (WRC, 1984).

$a$ and $s=$ the $a$-recycle and $s$-recycles respectively.

If $a$ and $s$ in Equation 5.3a are maintained constant as was the case here, and $\mathrm{N}_{\mathrm{C}}$ increases due to increased influent $T K N$, then the $\mathrm{N}_{\text {ne }}$ must increase. This relationship however accepts that all the $\mathrm{NO}_{3}$ recycled to the anoxic reactor is denitrified. If this is not true then Equation $5.3 \mathrm{~b}$ above applies, and $\mathrm{N}_{\text {ne }}$ increases disproportionately to an increase in $\mathrm{N}_{\mathrm{C}}$.

- The denitrification potential $\left(D_{P P}\right)$ as discussed in Chapter 4, Section 4.9.4.4, is the amount of $\mathrm{NO}_{3}$ an anoxic reactor can denitrify. It is dependant on the anoxic mass fraction, the sludge concentration and the influent COD. The nitrification load $\left(\mathrm{N}_{\mathrm{nL}}\right)$ is the amount of $\mathrm{NO}_{3}$ loaded on the anoxic reactor and is dependant on the recycles and nitrification capacity of the system. For a fixed underflow $s$-recycle the distribution of $\mathrm{NO}_{3}$ between the aerobic and anoxic reactors is thus dependant on the $a$-recycle. Ideally the $D_{\mathrm{PP}}$ should equal the $\mathrm{N}_{\mathrm{nL}}$ to denitrify the maximum amount of $\mathrm{NO}_{3}$, while still protecting BEPR in the anaerobic reactor. If $\mathrm{N}_{\mathrm{nL}}$ is greater than $\mathrm{D}_{\mathrm{PP}}$ then $\mathrm{NO}_{3}$ will be retumed to the aerobic reactor.

- The conventional system was designed such that $D_{P P}$ would equal $N_{n L}$, however in 22 out of the 26 measured sewage batches the anoxic $\mathrm{NO}_{3}$ was greater than $1 \mathrm{mgN} / 1$ indicating that the anoxic reactor was overloaded. This was however expected as the solids concentration throughout the system was substantially lower than the expected design solids for the duration of the investigation, and as noted above the $D_{P P}$ is dependant on the solids concentration. Thus the $D_{P P}$ was reduced substantially while the $\mathrm{N}_{\mathrm{nL}}$ remained the same.

- Clearly high concentrations of $\mathrm{NO}_{3}$ were recycled to the anaerobic reactor, which had detrimental effects on BEPR. This is examined in Section 5.6.3 (BEPR). 


\section{System Nitrogen Removal}

The system nitrogen removal is given by the difference between the influent nitrogen $\left(\mathrm{N}_{\mathrm{ti}}\right)$ and the sum of the effluent unfiltered TKN $\left(\mathrm{N}_{\mathrm{te}}\right)$ and $\mathrm{NO}_{3}$ concentrations $\left(\mathrm{N}_{\mathrm{ne}}\right)$. The influent $\left(\mathrm{N}_{\mathrm{ti}}\right)$ and summed effluent $\left(\mathrm{N}_{\mathrm{e}}\right)$ for the batch period is presented in Fig. 5.28 below.

The total system $\mathrm{N}$ removal ranged from 65 to $87 \%$. For the entire investigation period the system $\mathrm{N}$ removal mean was $74.7 \%(\mathrm{SSD}=7.5 \%)$, for the portion of the investigation from sewage batches $15-29$ the mean was $78.6 \%$ (SSD $=5.1 \%$ ) $\mathrm{mgN} / \ell$. Thus the higher $\mathrm{N}_{\mathrm{ae}}$ results in sewage batches 2-14 did influence total system $\mathrm{N}$-removal, and better control of the TKN/COD ratio reduced variation in the removals.

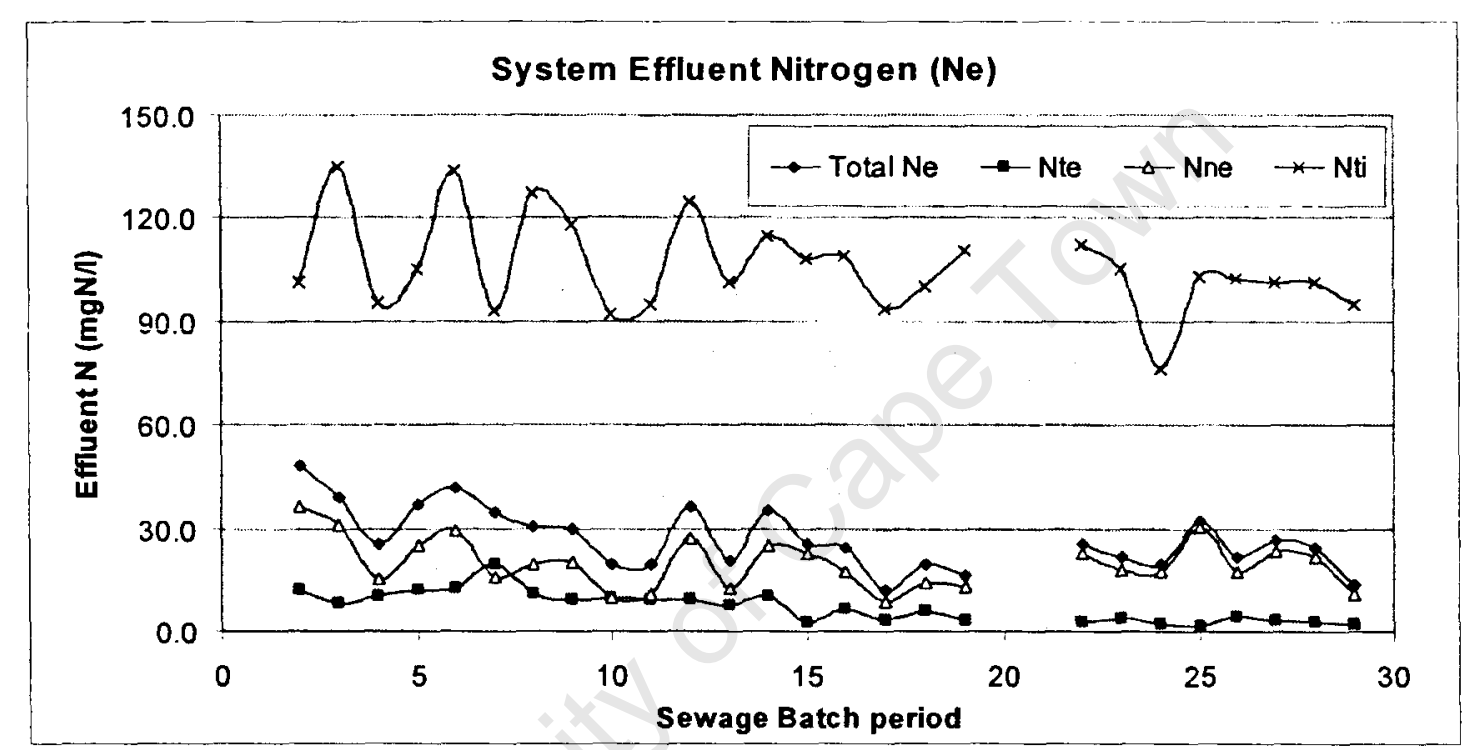

Figure 5.28: Total system N-removal, comparing the influent TKN (Nti) to the effluent TKN (Nte) and nitrate (Nne), which together give the effluent nitrogen (Ne).

\subsubsection{Biological Excess Phosphorus Removal (BEPR)}

Influent, effluent and reactor phosphorus concentrations are shown in Figs. 5.29-5.32. Following the procedures detailed in Chapter 4, Section 4.9.3, Table 5.3 lists the influent $\mathrm{P}, \mathrm{P}$ release/ $\mathrm{P}$ uptake across each reactor and the SST, and the overall $\mathrm{P}$ removal. 


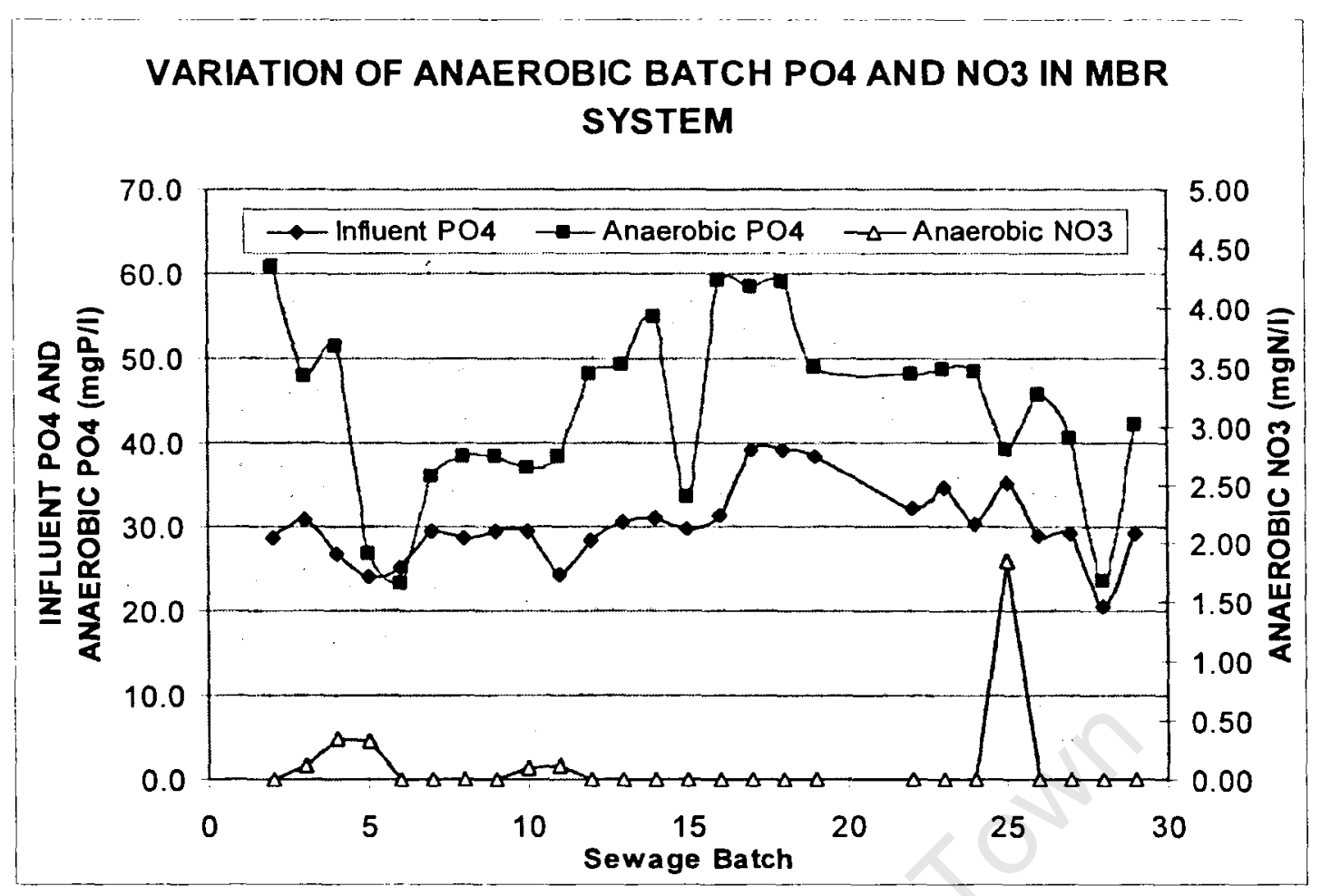

Figure 5.29: Time dependant variation in anaerobic total soluble phosphorus and anaerobic nitrate concentrations.

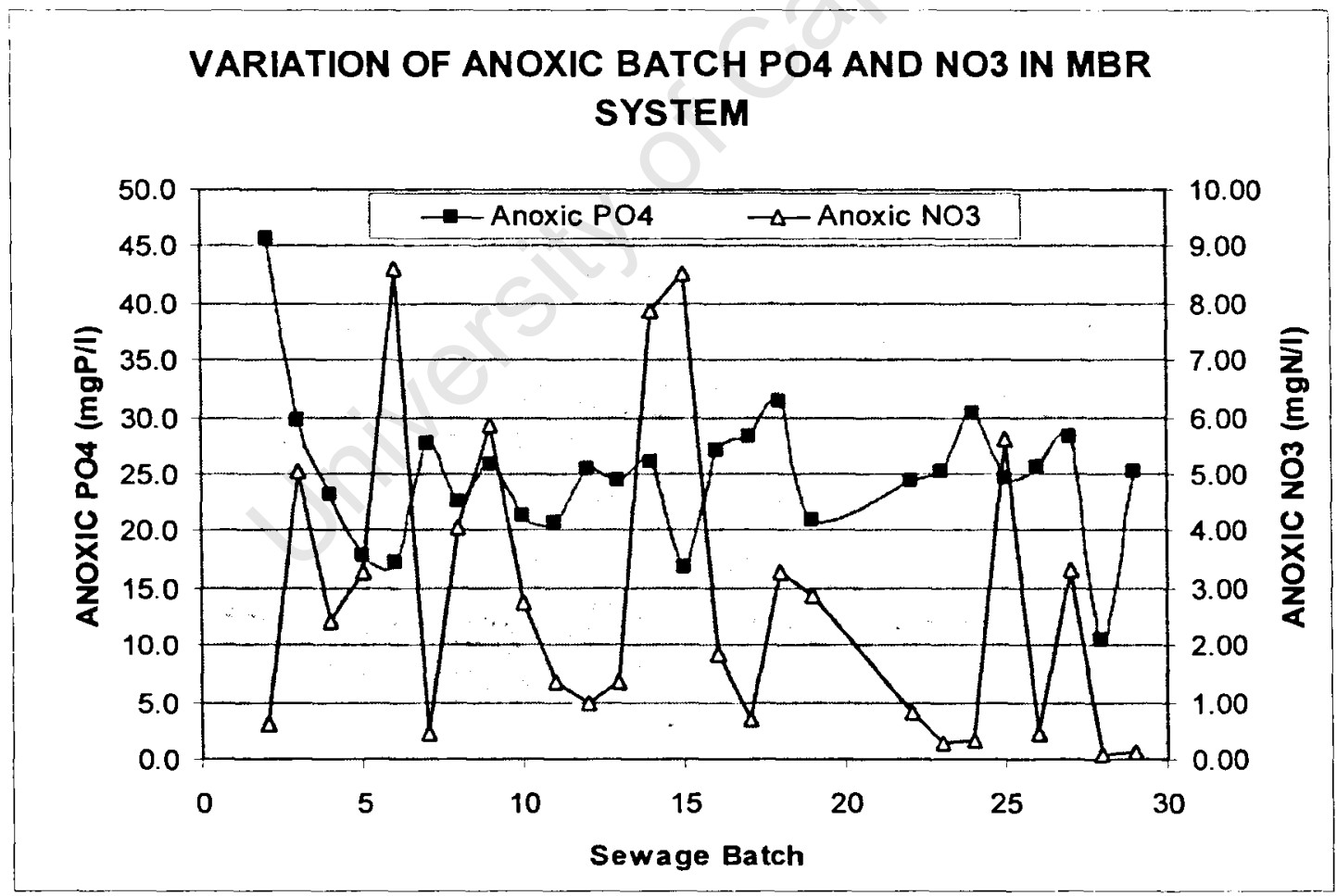

Figure 5.30: Time dependant variation in anoxic total soluble phosphorus and anoxic nitrate concentrations. 


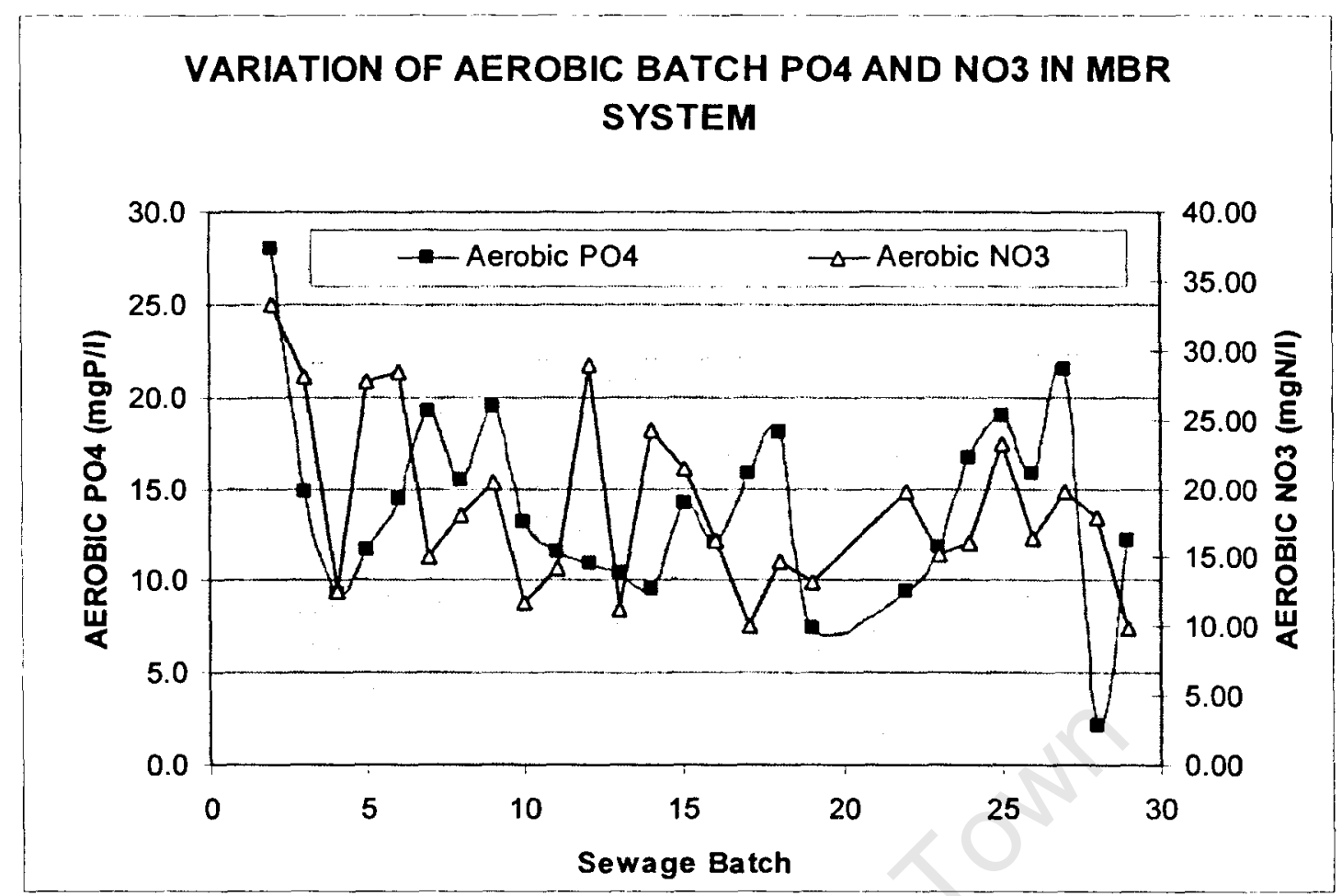

Figure 5.31: Time dependant variation in aerobic total soluble phosphorus and aerobic nitrate concentrations.

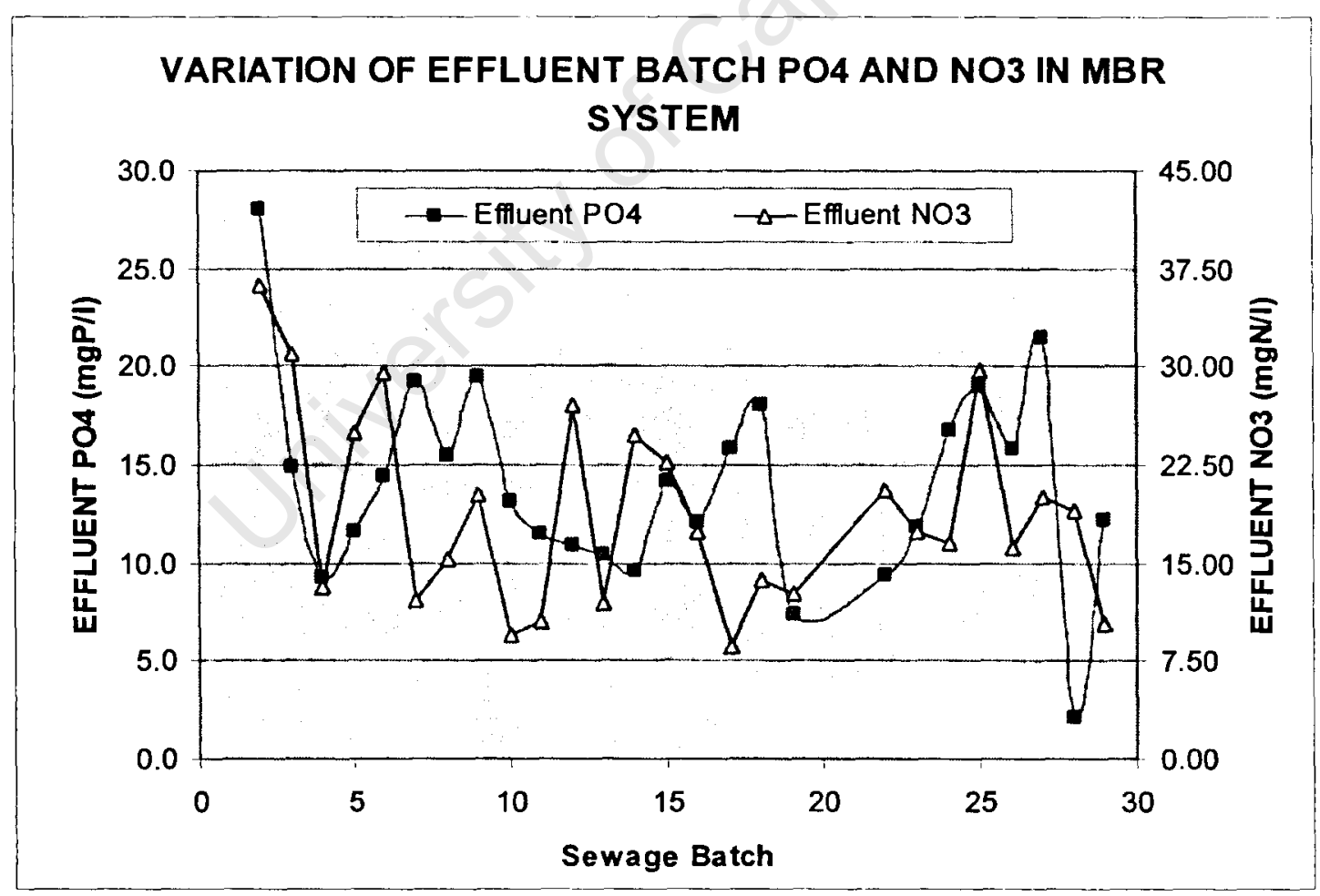

Figure 5.32: Time dependant variation in effluent total soluble phosphorus and effluent nitrate concentrations. 
Table 5.3: $\quad$ Sewage batch average $P$ release (-ve) and $P$ uptake ( $+v e)$ across the reactors and settling tank for the conventional UCT system.

\begin{tabular}{|c|c|c|c|c|c|c|}
\hline $\begin{array}{c}\text { Batch } \\
\text { Number }\end{array}$ & Influent & Anaerobic & Anoxic & Aerobic & SST & M Prem \\
\hline & mgP/linf & mgP/linf & mgP/linf & mgP/linf & mgP/linf & mgP/linf \\
\hline 2 & 28.60 & -47.28 & 0.00 & 68.48 & 1.63 & 0.58 \\
\hline 3 & 30.94 & -34.79 & 0.00 & 56.79 & 1.91 & 16.01 \\
\hline 4 & 26.66 & -53.11 & 15.74 & 54.30 & 0.76 & 17.32 \\
\hline 5 & 23.96 & -11.75 & 1.32 & 21.90 & 1.51 & 12.25 \\
\hline 6 & 25.19 & -3.75 & 4.37 & 9.45 & 1.16 & 10.67 \\
\hline 7 & 29.47 & -14.69 & 0.00 & 20.16 & 8.42 & 9.78 \\
\hline 8 & 28.67 & -25.70 & 12.10 & 26.01 & 1.43 & 13.15 \\
\hline 9 & 29.52 & -21.42 & 5.31 & 26.37 & -0.35 & 10.08 \\
\hline 10 & 29.35 & -23.43 & 8.64 & 30.28 & 1.27 & 16.15 \\
\hline 11 & 24.38 & -31.52 & 9.98 & 33.16 & 2.28 & 12.81 \\
\hline 12 & 28.31 & -42.40 & 8.46 & 47.95 & 6.18 & 17.22 \\
\hline 13 & 30.51 & -43.63 & 10.93 & 51.18 & 2.79 & 19.94 \\
\hline 14 & 30.98 & -52.53 & 10.47 & 62.21 & 2.23 & 21.31 \\
\hline 15 & 29.76 & -20.49 & 22.07 & 15.78 & -3.26 & 15.67 \\
\hline 16 & 31.36 & -60.04 & 33.06 & 39.04 & 12.95 & 18.78 \\
\hline 17 & 39.06 & -49.44 & 23.88 & 48.17 & 1.16 & 23.22 \\
\hline 18 & 39.10 & -47.51 & 16.70 & 50.96 & 1.62 & 20.99 \\
\hline 19 & 38.43 & -38.51 & 19.39 & 48.04 & 3.83 & 30.91 \\
\hline 22 & 32.04 & -39.75 & 5.22 & 55.64 & 2.83 & 22.57 \\
\hline 23 & 34.72 & -37.11 & 9.27 & 49.23 & 2.64 & 22.77 \\
\hline 24 & 30.16 & -36.47 & 0.00 & 58.18 & -2.23 & 13.50 \\
\hline 25 & 35.21 & -18.46 & 11.69 & 26.96 & -0.89 & 16.30 \\
\hline 26 & 28.89 & -37.03 & 11.83 & 39.27 & 0.34 & 13.05 \\
\hline 27 & 29.14 & -23.91 & 7.20 & 22.80 & 2.90 & 7.60 \\
\hline 28 & 20.66 & -15.86 & 3.13 & 30.06 & 2.30 & 18.51 \\
\hline 29 & 29.18 & -29.72 & 3.19 & 38.84 & 8.39 & 16.67 \\
\hline Average & 30.16 & -33.09 & 9.77 & 39.66 & 2.45 & 16.07 \\
\hline & & & & & & \\
\hline
\end{tabular}

- The influent $P$ values for sewage batches 2-14 of this investigation were measured independently of the influent to the MBR system, despite both feeds being the same. Hence there is a slight variation between the influent batch averages for the conventional system (Table 5.3) and the MBR system (Chapter 4, Table 4.10).

- The results in the table above show that overall $\mathrm{P}$ removal in the conventional UCT system was poor. The average removal was $16.1 \mathrm{mgP} / \ell$ which is substantially less than that measured in Phase 1 of $21.5 \mathrm{mgP} / \ell$ (Ramphao et $a l ., 2004$ ) or in the MBR system for the Phase 2 investigation $22.4 \mathrm{mgP} / \ell$, but is attributed to the substantially lower TSS concentration in the conventional UCT system due to suspected unaccounted for sludge loss.

- P release predominately occurred in the anaerobic reactor, only 4 out of 26 sewage batches showed net anoxic $P$ release. The $P$ release was generally very poor with an average $\mathrm{P}$ uptake of only $33.5 \mathrm{mgP} / \ell$. Ramphao et al. (2004) noted that $P$ releases of at least $100 \mathrm{mgP} / \ell$ should have occurred considering 
that $200 \mathrm{mgCOD} / \ell$ was readily biodegradable $\mathrm{COD}$ throughout the investigation $(0.5 \mathrm{mgPreleased} / \mathrm{mgCOD}$ is generally sequestered by PAOs).

- Anoxic nitrate concentrations due to overloading the anoxic reactor with nitrate were consistently high. This would have had an adverse effect on $P$ release as for every $1 \mathrm{mgN}-\mathrm{NO} 3 / \ell$ recycled to the anaerobic reactor $8.6 \mathrm{mgCOD} / \ell$ would be lost to the PAOs. However this alone can not justify how low $\mathrm{P}$ removal was.

- The majority of $\mathrm{P}$ uptake occurred in the aerobic reactor ranging from 9.45 $\mathrm{mgP} / \ell$ to $68.5 \mathrm{mgP} / \ell$ with an average of $39.7 \mathrm{mgP} / \ell$.

- Anoxic P uptake occurred in 22 out of 26 sewage batches and in one case, sewage batch 15 , it was dominant over aerobic $P$ uptake. The average anoxic $P$ uptake was $9.7 \mathrm{mgP} / \ell$.

- P uptake did occur in the SST, though it was negligible for all but four sewage batches, these were sewage batches $7,(8.4 \mathrm{mgP} / \ell)$; batch $12,(6.2 \mathrm{mgP} / \ell)$; batch $16,(13.0 \mathrm{mgP} / \ell)$ and batch $29,(8.4 \mathrm{mgP} / \ell)$.

- The total P uptake, on average $51.8 \mathrm{mgP} / \ell$, was also substantially lower than expected, but is relative to the low $P$ release in the anaerobic reactor.

Thus it is concluded that the low solids concentration, and hence low PAO population, combined with the recycling of $\mathrm{NO}_{3}$ to the anaerobic reactor are responsible for the poor $\mathrm{P}$ removal in the conventional UCT system.

\section{Anoxic P uptake}

Significant anoxic P uptake was observed in the conventional system as is illustrated in Figs. 5.33 and 5.34 below. This implies that the PAOs exhibited significant denitrification as has previously been described in Chapter 4, Section 4.9.3. Significantly reduced BEPR has been reported with anoxic $P$ uptake by denitrifying (D)PAOs (Ekama and Wentzel, 1999; Hu et al., 2002). This has been attributed to less efficient utilization of the influent RBCOD (Hu et al., 2002).

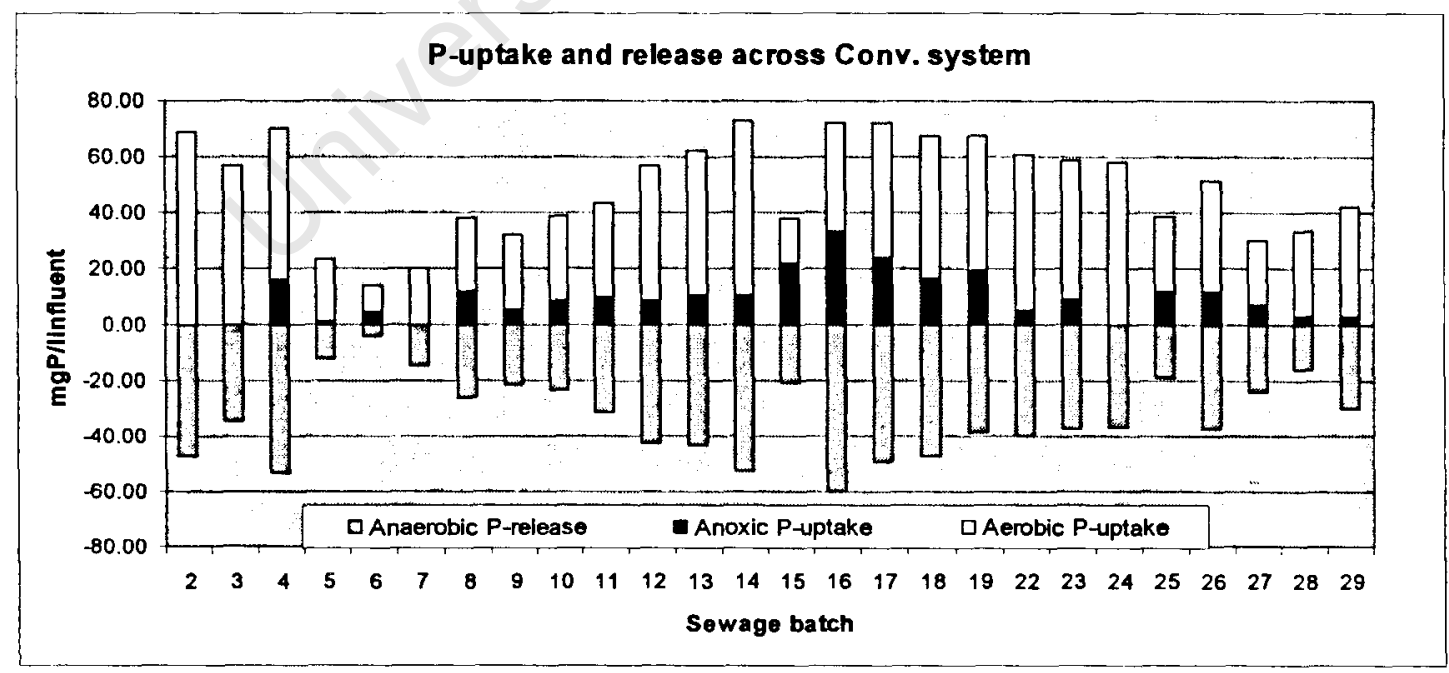

Figure 5.33: P-release/uptake by the system reactors for each batch period. 


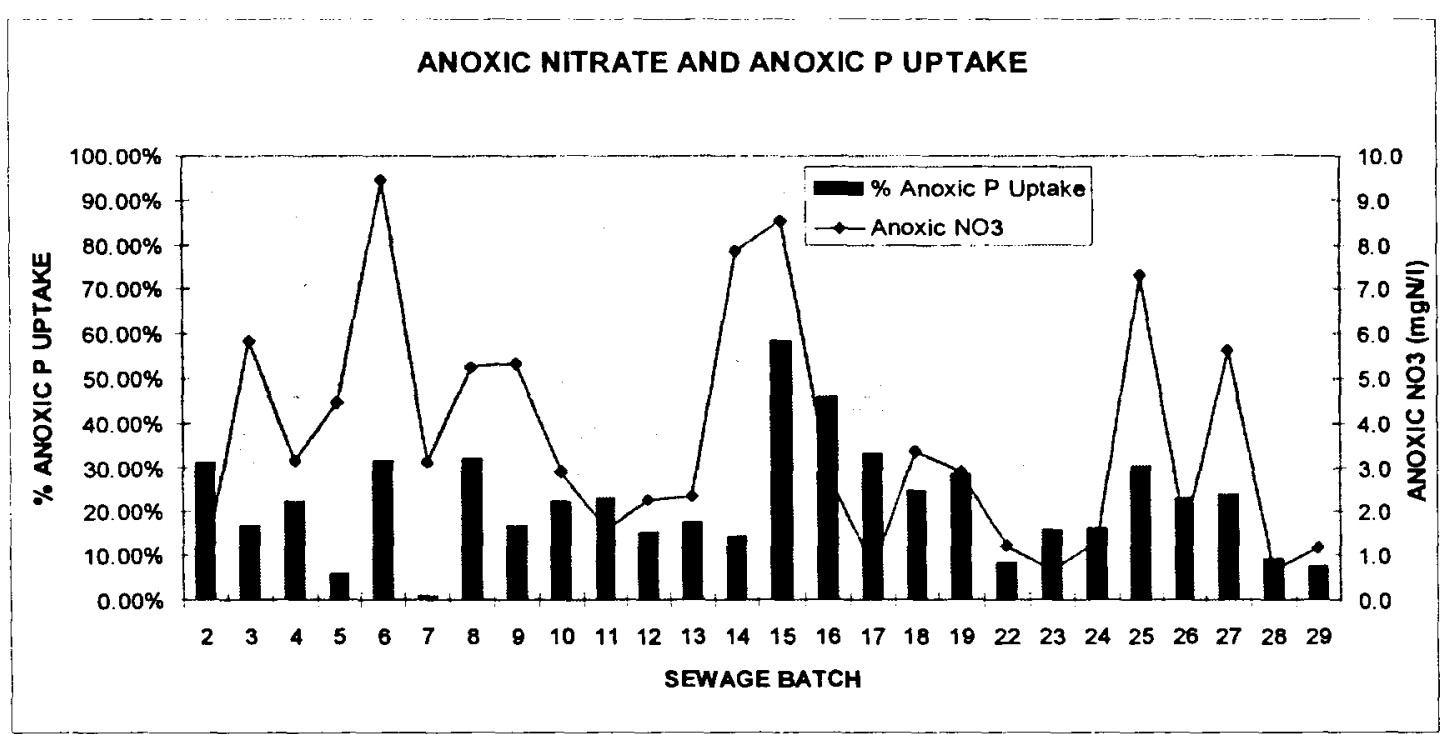

Figure 5.34: The influence of anoxic nitrate on anoxic $P$ uptake

- Anoxic $\mathrm{P}$ uptake as a percentage of total $\mathrm{P}$ uptake ranged from $0.7-58.3 \%$ with an average of $22.1 \%$ thus making it a significant factor in BEPR in the conventional UCT system.

- A link between anoxic $\mathrm{NO}_{3}$ and anoxic P-uptake is noted in Ramphao et al. (2004) citing Hu et al., (2002). This relationship would be illustrated in Fig. 5.34 however in this investigation no clear relationship can be inferred from Fig. 5.34.

- The influence of anoxic P uptake is illustrated in Fig. 5.35 and indicates that higher anoxic $\mathrm{P}$ uptake degrades BEPR. However it must be remembered that significant anoxic $\mathrm{P}$ uptake is reported throughout the investigation while very poor $\mathrm{P}$ removal also occurs throughout the investigation! Hence it is difficult to compare the influence of anoxic $\mathrm{P}$-uptake as at no point in the investigation did it not occur.

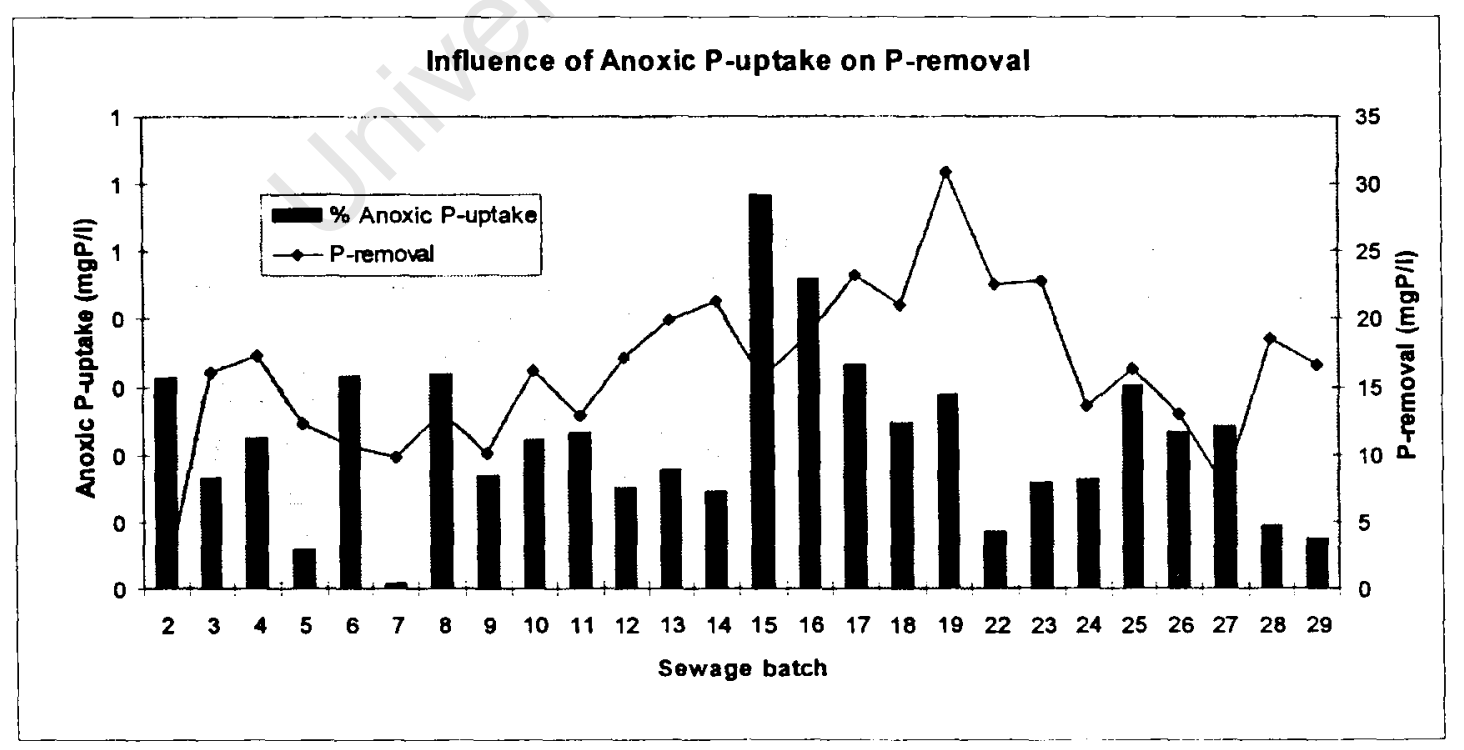

Figure 5.35: The influence of anoxic $P$ uptake on $P$ removal is not particularly clear in this investigation 


\subsection{CLOSURE}

Due to the uncertainties in the data gathered from the conventional UCT system, as illustrated by the poor mass balances and variance in solids concentrations, the data has been analysed cautiously in order to assess its performance and to allow as accurate a comparison with the MBR UCT system as possible. Little merit however could be found in conducting a theoretical evaluation of ND and BEPR performance in the conventional system, as was done with the MBR system. This however makes little difference to the outcomes of this investigation as the BNR performance of the MBR system is the focus of this project.

It is concluded that unaccounted for sludge losses resulted in the poor system mass balances. The consequent low solids concentrations resulted in low OUR readings and an overloading of the anoxic reactor with $\mathrm{NO}_{3}$ as the $\mathrm{D}_{\mathrm{PP}}$ decreased with decreasing solids concentration. As a consequence the system was not run optimally and nutrient removal was adversely affected.

In Chapter 6 the results from the two UCT systems are compared, as far as possible, to assess the performance of the MBR UCT system. 


\section{CHAPTER 6}

\section{COMPARISON OF SYSTEM PERFORMANCES}

\subsection{INTRODUCTION}

In Chapters 4 and 5 the performances of the MBR UCT and conventional UCT systems run in Phase 2 of this investigation were evaluated respectfully. In this chapter the performances of both systems are compared with each other and with those observed in Phase 1 of the investigation (Ramphao et al., 2004) in order to assess the advantages and disadvantages of membrane solid-liquid separation in BNR systems, and to evaluate what impact, if any, membranes have on the performance of BNR systems.

\subsection{SYSTEM OPERATIONAL PARAMETERS}

In order to compare the BNR performance of the two laboratory-scale systems their opcrational parameters were designed to be the same. In most respects (e.g. sludge age, influent concentrations, mass fractions, temperature) this was achieved. However some differences were apparent:

- The MBR UCT system was run at high MLSS concentrations. The high MLSS concentrations were necessary in order to maintain sufficient scour across the membranes to prevent fouling, and to observe the impact of concentration on BNR performance. In order to achieve the high MLSS concentrations required the COD loading per unit volume in the MBR UCT system was approximately 3.2 times that of the conventional UCT system, at $1801 \mathrm{mgCOD} / \ell$ reactor.d versus $571 \mathrm{mgCOD} / \ell$ reactor.d. Ramphao et al. (2004) noted the potential reactor volume savings that could be achieved by an MBR system, but pointed out that the volume savings were strongly influenced by the nature of the influent, particularly the PWWF/ADWF ratio, aerobic mass fraction and COD strength.

- As was observed in Phase 1 (Ramphao et al., 2004), the sludge distribution in the MBR UCT system differed from that in the conventional UCT system, and was linked to the a/as- and r-recycles. In a MBR UCT system the magnitude of the sludge distribution and zone mass fractions is linked to the inter-reactor recycles due to the solids liquid separation step occurring in the aerobic reactor thus concentrating missed liquor in the aerobic reactor as opposed to the SST in a conventional system (Ramphao et al., 2004). Additionally in the MBR UCT system the measured recycles were found to differ from the design recycles resulting in system mass fractions that differed from the design mass fractions: Average mass fractions for the MBR UCT system in Phase 2 were anaerobic:anoxic:aerobic $=0.139: 0.277: 0.584$ versus the original design values of $0.126: 0.279: 0.595$. In the conventional UCT system only the $r-$ recycle influenced the system mass fractions and was found to remain close to the design value, hence the system mass fractions were the same as those 
determined in design 0.126:0.279: 0.595. This resulted, as in Phase 1, in slight differences in the system mass fractions of the two systems.

\subsection{SYSTEM REMOVALS AND EFFLUENT QUALITY}

The average removals of both the MBR and conventional UCT systems for the entire Phase 2 investigation are summarised in Table 6.1.

Table 6.1: $\quad$ Summary of the influent and effluent qualities, and the resultant removals, of both UCT systems.

\begin{tabular}{|l|l|l|l|l|l|l|}
\hline \multicolumn{2}{|l|}{ Parameter } & \multirow{2}{*}{ Influent } & \multicolumn{2}{l|}{ MBR UCT } & Conv. UCT \\
\cline { 4 - 7 } & & & Effluent & Efficiency & Effluent & Efficiency \\
\hline COD & $\mathrm{mgCOD} / \mathrm{l}$ & 951.2 & 42.0 & $95.6 \%$ & $74.6^{1}\left(50.6^{2}\right)$ & $92.2 \%$ \\
\hline TKN & $\mathrm{mgN} / \mathrm{l}$ & 106.5 & 1.7 & $98.4 \%$ & $3.7^{1}\left(2.0^{2}\right)^{3}$ & $96.5 \%$ \\
\hline FSA & $\mathrm{mgN} / \mathrm{l}$ & 81.7 & 0.7 & $99.1 \%$ & 1.3 & $98.4 \%$ \\
\hline $\mathrm{NO} 3$ & $\mathrm{mgN}-\mathrm{NO} 3 / \mathrm{l}$ & 0 & 18.0 & - & 18.1 & - \\
\hline TN & $\mathrm{mgN} / \mathrm{l}$ & 106.5 & 19.7 & $81.5 \%$ & $21 .{ }^{*}$ & $79.5 \%$ \\
\hline TP & $\mathrm{mgP} / \mathrm{l}$ & 30.3 & 9.0 & $21.3 \mathrm{mgP} / \mathrm{linf}$ & 13.6 & $16.7 \mathrm{mgP} / \mathrm{linf}$ \\
\hline TSS & $\mathrm{mgTSS} / \mathrm{l}$ & $\mathrm{N} / \mathrm{A}$ & 0.0 & - & 21.5 & - \\
\hline e. coli & $\mathrm{CFU} / 100 \mathrm{ml}$ & $\mathrm{N} / \mathrm{A}$ & $<10$ & - & 2250 & - \\
\hline
\end{tabular}

${ }^{1}$ unfiltered sample; ${ }^{2} 0.45$ filtered sample; ${ }^{3}$ System averages for sewage batches $15-29$

$\mathrm{N} / \mathrm{A}=$ value not available

\subsubsection{COD Removals}

The COD removal efficiency of the MBR system (96\%) was superior to that of the conventional system ( $92 \%$ unfiltered, $95 \% 0.45 \mu \mathrm{m}$ filtered). These results were comparable to those observed in Phase 1 (Ramphao et al., 2004) of 96\% COD removal in the MBR UCT system and $93 \%$ unfiltered and $94 \% 0.45 \mu \mathrm{m}$ filtered in the conventional UCT system.

For the purposes of comparison three categories of MBR COD were measured: directly from the effluent line; as $0.45 \mu \mathrm{m}$ filtered effluent from the aerobic reactor; and as unfiltered COD taken as supernatant from the $800 \mathrm{ml}$ mark of the DSVI test (Chapter 4, Section 4.9.1). Similarly conventional COD was measured as $0.45 \mu \mathrm{m}$ filtered effluent, and as unfiltered COD taken from the $800 \mathrm{ml}$ mark of the DSVI test. The recorded batch average values are presented in Fig. 6.1.

The difference in filtered COD removals from both systems is attributed to the smaller pore size of the membranes which retain organics that would otherwise be considered soluble in a conventional system. However membrane specifications state that the nominal pore size of the Kubota ${ }^{\circledR}$ membranes used in this study were $0.4 \mu \mathrm{m}$, while the membranes used to filter the conventional system effluent are only marginally larger at $0.45 \mu \mathrm{m}$, thus the improved filterability of the membrane system is attributed rather to the development of a dynamic gel layer which reduces the effective pore size of the membranes. $0.45 \mu \mathrm{m}$ filtered samples differed substantially for both systems at 70.5 and $50.6 \mathrm{mgCOD} / \ell$ for the MBR and conventional systems respectively. The larger $\mathrm{MBR} 0.45 \mu \mathrm{m} \mathrm{COD}$ is attributed to the retention, and subsequent concentration of COD considered soluble in the conventional system but retained in the MBR system by the membranes. 
The MBR unfiltered "effluent" COD values were consistently higher than those in the conventional system which confirmed that the MBR system retains and accumulates unsettleable material which would flow out with the effluent in a conventional system. This was observed in the DSVI test in which the supernatant of the conventional system mixed liquor would become clear in time, whereas the MBR supernatant remained cloudy.

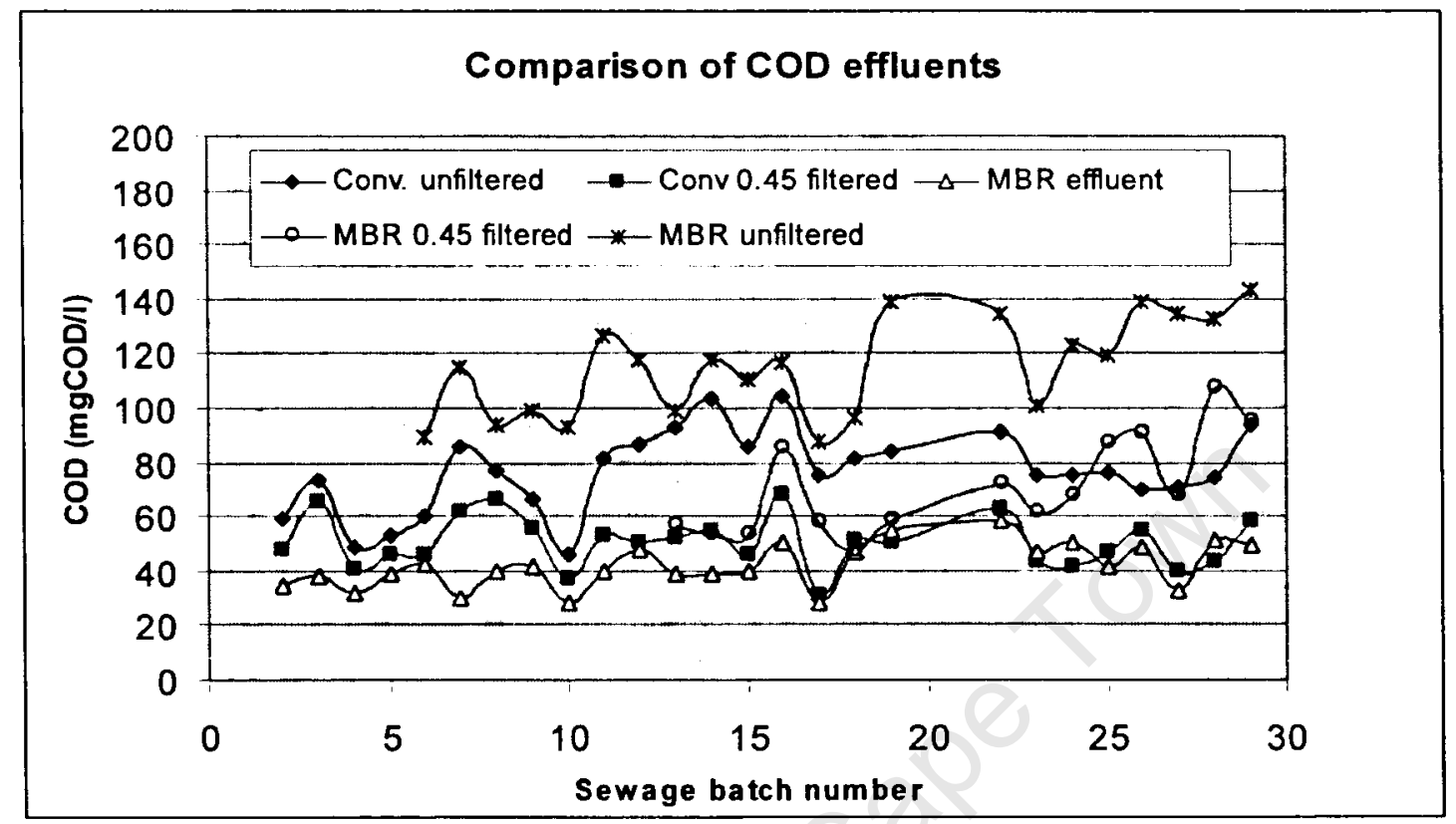

Figure 6.1: Conventional and MBR effluent filtered and unfiltered COD concentrations.

The difference between the filtered (50mgCOD/ $\ell$ ) and unfiltered $(75 \mathrm{mgCOD} / \ell)$ effluent COD measured in the conventional UCT system is attributed to the loss of non-settleable solids through the SST. Approximately $21.5 \mathrm{mgTSS} / \ell$ were lost as COD in the effluent.

After Ramphao et al. (2004), differences in the MBR UCT effluent COD and the conventional UCT effluent are accommodated in the steady state design models as differences in the soluble unbiodegradable COD fractions $\left(f_{\mathrm{S}, \text { us }}\right)$ which were 0.044 and 0.068 respectively.

\subsubsection{N Removals}

As was described in Chapter 5, Section 5.6.2.1, the system average TKN and FSA removals in the conventional UCT system were determined from sewage batches 15 29 only.

The TKN removal efficiency of the MBR system (98\%) was marginally better than that of the conventional system ( $97 \%$ unfiltered, $98 \% 0.45 \mu \mathrm{m}$ filtered). This is again attributed to the retention of solids by the membranes that would have been lost in the effluent of the conventional UCT system. FSA removal was also very similar for both systems $99 \%$ in the MBR system and $98 \%$ in the conventional system. Thus near complete nitrification was achieved in both systems. 
Effluent nitrate concentrations were virtually the same for both systems (18.0 and

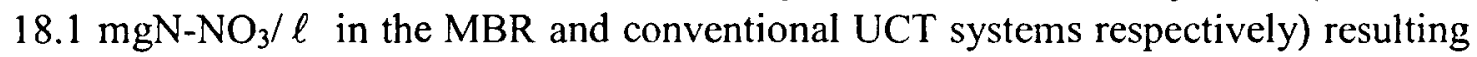
in similar total nitrogen (TN) removals, $(81.5 \%$ for the MBR system versus $79.5 \%$ for the conventional system). These results are higher than those achieved in Phase 1 (74 and $75 \%$ respectively), however the effluent nitrate concentrations were lower in Phase 2 than in Phase 1 ( 23 and $22 \mathrm{mgN}-\mathrm{NO}_{3} / \ell$, respectively).

A comparison of $\mathrm{N}$ removal behaviour of both systems is presented in Section 6.7.

\subsubsection{P removals}

In both systems TP was dosed in excess of the amount the system could remove in order to demonstrate BEPR. Thus $\mathrm{P}$ removal performance is quantified by the concentration of $P$ removed. Both systems exhibited $P$ removals substantially lower than those observed in Phase 1 however, this is discussed in Chapter 4, Section 4.9.3 and Chapter 5, Section 5.6.3. System average $\mathrm{P}$ removals of $21.3 \mathrm{mgP} / \ell$ and $16.7 \mathrm{mgP} / \ell$ were achieved indicating that total $\mathrm{P}$ removal would have been possible in both systems with influent $P$ concentrations of up to $\sim 20 \mathrm{mgP} / \ell$ and $\sim 16 \mathrm{mgP} / \ell$ respectively. Clearly however, the $P$ removal performance of the conventional UCT system was inferior to that of the MBR system. Reasons for this are discussed in Section 6.8 .

\subsubsection{Microbial Removals}

Periodic effluent samples were tested from both systems for the indicator microorganism e-coli using the membrane filtration method. Pathogen counts were consistently unobservable in the MBR UCT system whereas in the conventional UCT system pathogen counts ranged from 600 to $5600 \mathrm{cfu} / 100 \mathrm{ml}$ (Appendix E). The measured pathogen counts in the conventional system are still substantially lower than those measured on full scale plants (Sampson K, pers. com.). This reduction in pathogen count is attributed to the $4^{\circ} \mathrm{C}$ sewage storage conditions in the WRL which partially sterilise the wastewater.

Clearly from the removals described above the MBR UCT system produced an effluent that was equal, if not superior in quality to the conventional UCT system. Due to complete retention of solids, and pathogens, the membrane effluent is more viable for reuse purposes.

\subsection{MASS BALANCES}

$\mathrm{N}$ and COD mass balances were conducted on both the MBR and conventional UCT systems. The mass balances differed for both systems with the MBR system producing mass balances close to $100 \%$ (96.5 and $103.1 \%$ for the $\mathrm{N}$ and COD mass balances respectively), while the conventional UCT system mass balances were both low and close to $80 \%$ ( 79.6 and $79.4 \%$ respectively). Reasons for the low conventional UCT mass balances are presented in Chapter 5, Section 5.4. 


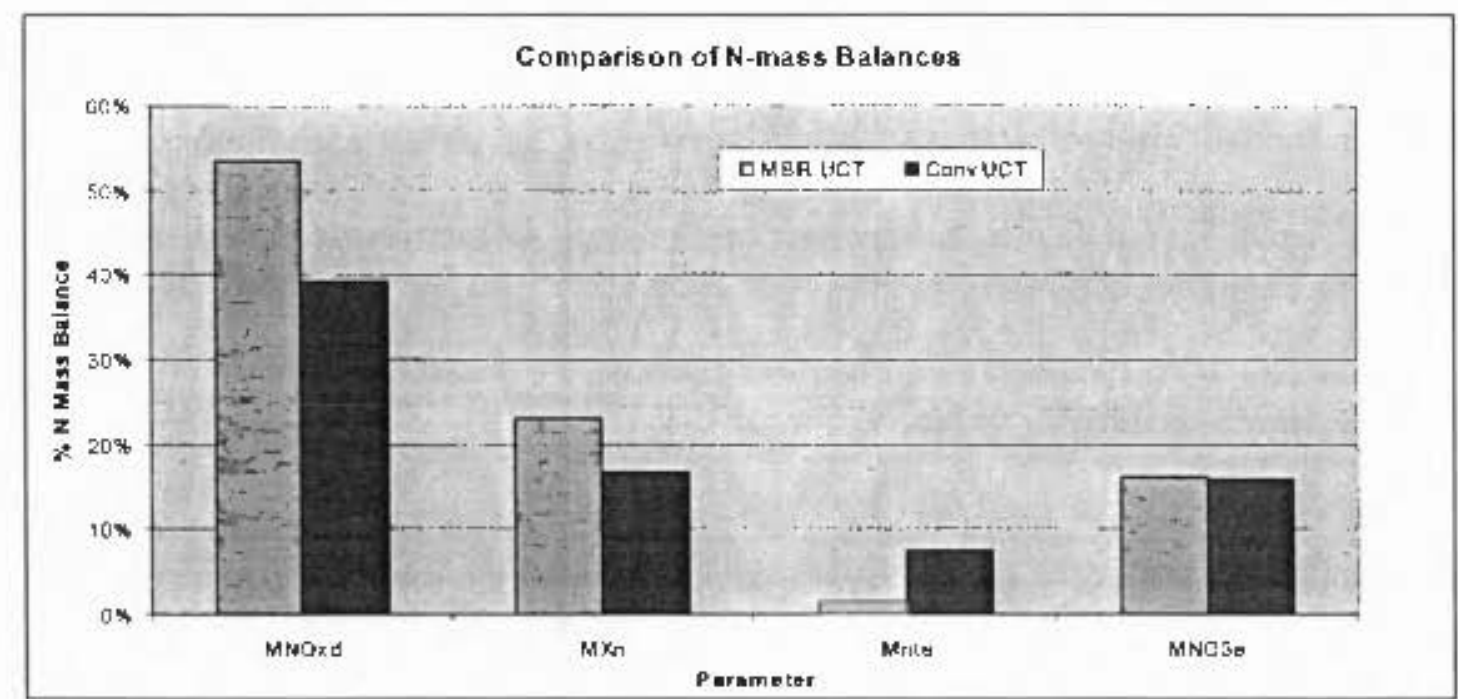

Figure 6.2: Comparison of $\%$ composition in $N$ mass balance: Nifrate/Nitrite denifrified $\left(M N O_{d}\right)$. N incorporated in waste mixed liquor $\left(M X_{n}\right)$. TKN in effuent $\left(M N_{i k}\right)$, nitrate in effluent ( $\left.M N O O_{3}\right)$.

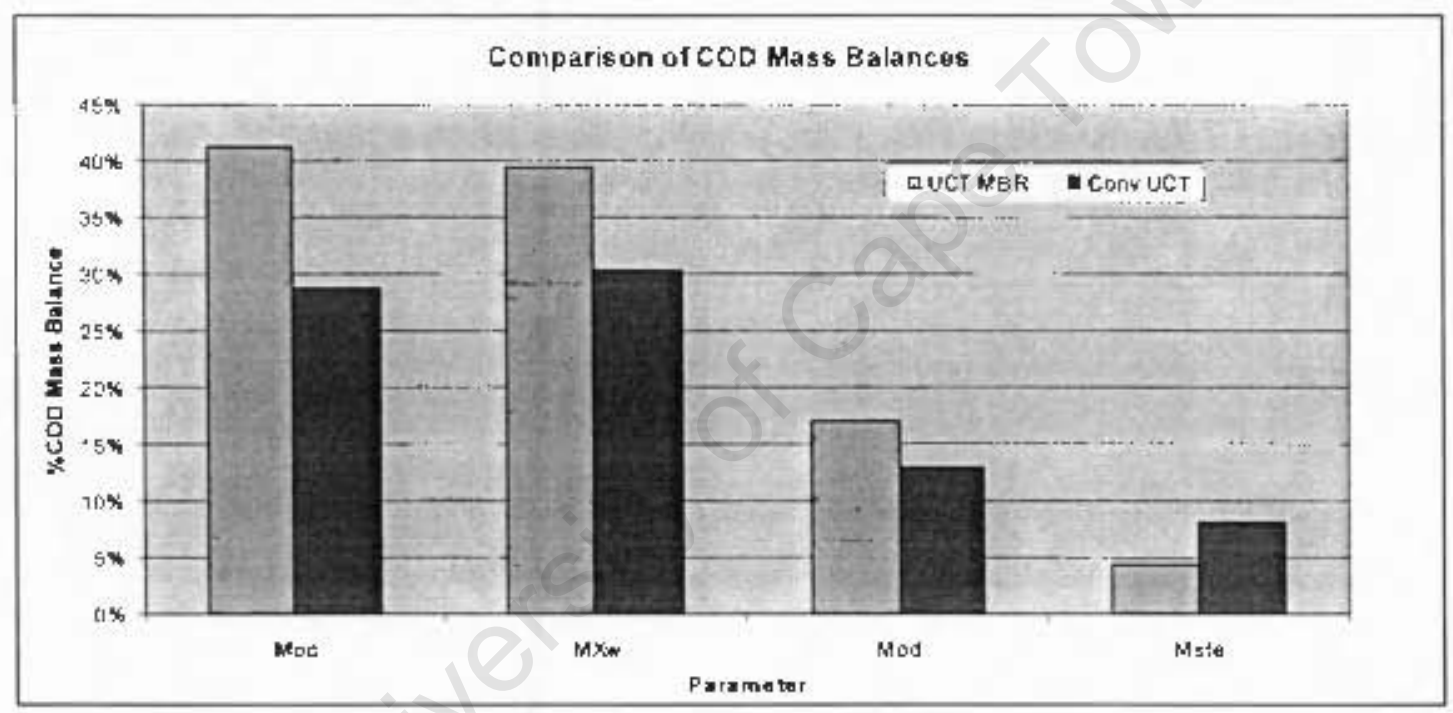

Figure 6.3: Comparison of \% composition in COD mass batance: Curbonaceous oxygen denand (MO). COD incorporated in waste mixed liquor $\left(M X_{w}\right)$, oxygen equivalent of nitrite/nitrate denitriffed $\left(M O_{d}\right), C O D$ in offuent (MS $)$.

Figs. 6.2 and 6.3 illustrate the percentile breakdown of the $N$ and COD mass balances respectively for both systems. The breakdown allows a comparison of the routes via which $\mathrm{N}$ and $\mathrm{COD}$ wete removed from the system.

- More nitrogen was removed from the MBR system through denitrification and sludge wasting than in the conventional system. This supports the observation of low sludge concentrations in the conventiona] UCT system, resulting in lower sludge mass removals and lower denitrification rates.

- N removed as nilrate was very similar for both systems.

- In Chaptet 5, Section 5.6.2, it is noted that the effluent TKN concentration was erratic and high up until sewage batch 15 after which it remained low and comparable to that of the MBR system. However lor the mass balance average 
all sewage batches were used thus producing a very high $\mathrm{N}_{\mathrm{t}}$, influenced by the high effluent TKN concentrations up to sewage batch 15 .

- In the COD mass balances the MBR removals are consistently higher for all parameters except eftluent $\mathrm{COD}$ as the membranes produced a low COD eftuent. This indicates that the mechanisns of $\mathrm{COD}$ removals in both systems are proportionally similar, however little more can be concluded from this comparison due to the low conventional COD mass balance.

\subsection{SLLDGE PRODUCTION}

In Phase 1 of the investigation sludge production in the two UCT systems differed significantly. A number of explanations were suggested by Ramphao ef al. (2004), however more data was required in order to validate the observations. Hence one of the objectives for Phase 2 of the investigation was to validale the observed discrepancy in sludge production in the two systems.

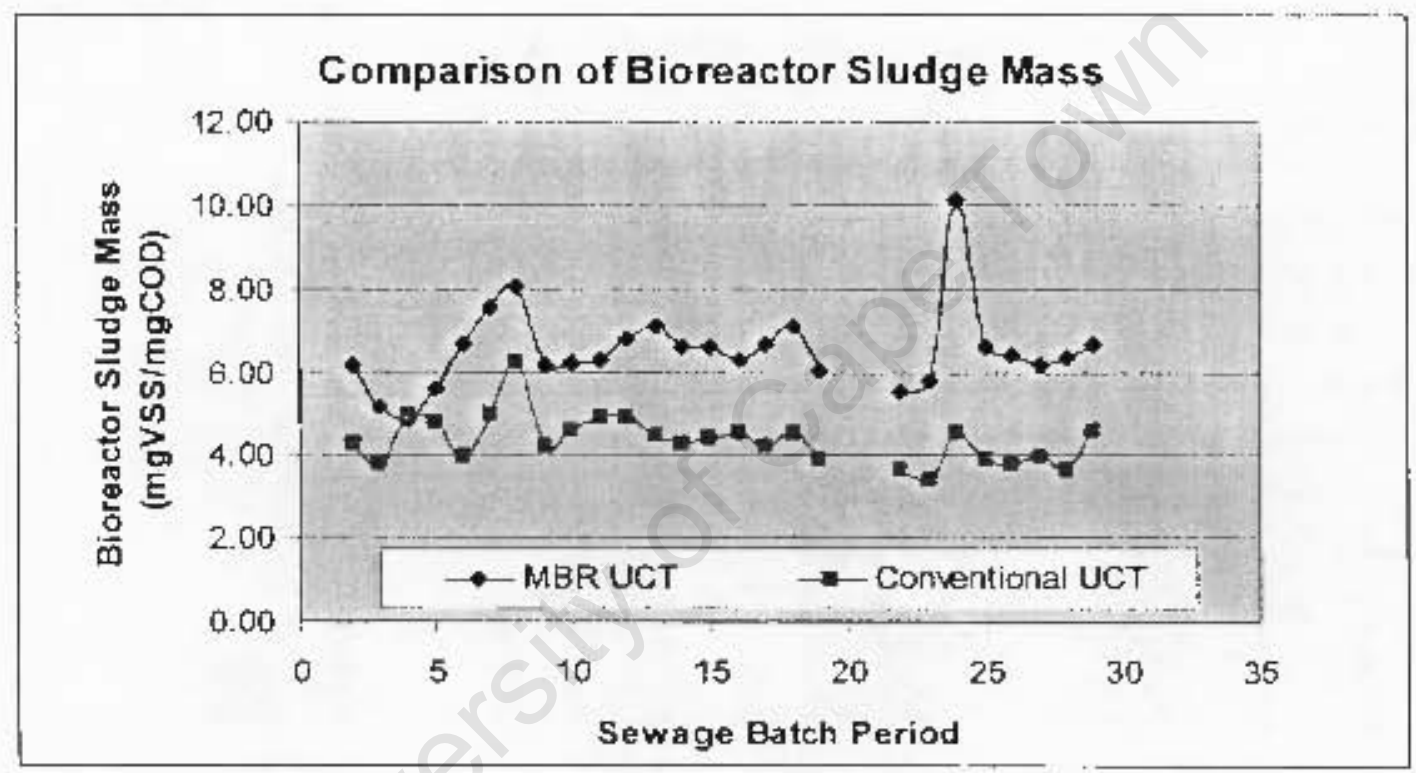

Figure 6.4: Comparison of bioreactor sladge mass per (OD load in MBR and conventional UCT systems.

The mass of sludge in the reactor systems per unit COD utilized in the influent daily feed is represented in Fig. 6.4.

- With the exception of sewage batch 4 the sludge production in the MRR system was consistently higher than that of the conventional UCT system by on average $50 \%$.

- The MBR system was not at steady state in sewage batch 4 due to substantial loss of mixed liquor through spillages on days $66-69$ and 74. Sirnilarly sewage butch 19 experienced substartial mixed liçuor losses through spills on days 294,298 and 305 , resulting in a drop in bioreactor sludge mass.

- The spike in both sysicms in sewage hatch 8 is altributed to particularly low influent $\mathrm{COD}$ concentrations $(785 \mathrm{mgCOD} / \mathrm{f}$ ) that characterised that sewage batch.

- Power failures in sewage batch 24 resulted in some sludge loss and retuced feeding due to the pursp not being able to make up backlogs in feeding. As a 
result the system was not fed on days 374 and 377 , and hence was not at stcady statc.

- Average sludge productions for the two systems were 0.311 and 0.205 mgVSSimgCOD for the MBR and conventional UCT systems respectively. In Phase 1 of the project Ramphao et al. (2004) reported simitar results, 0.32 and $0.22 \mathrm{mgVSS} / \mathrm{mgCO}$ ) respectively.

- The higher sludge produclion can be accommodated in the steady state design model by increasing the $f_{\text {s. up }}$ fraction. This was demonstrated by the high $f_{\text {s.up }}$ values observed is l'hase $1(0.224)$ (Ramplata et al, 2004) and Phase 2 $(0.200)$ in the MBR system.

As was noted by Ramphao et al. (2004) a number of factors contribute to the higher sludge production in the MBR system

- The retertion of solids by membranes in the MBR system resulted in approximately $17.2 \mathrm{mgTSS} / \ell$ accumulating in the MBR system that would have been lost through the SST in the conventional UCT system. This would have "increased" sludge production by $0.018 \mathrm{mgVSS} / \mathrm{mgCOD}$.

- In the MBR UeT system, organics that would be considered as soluble in the conventional system are retained. This is demonstrated by the difference in the $0.45 \mu \mathrm{m}$ tillered efiluent COD system averages from the MBR and conventional UCT systems of 8mgCOI) $\ell$. This would account for approximately 0.008 in the difference in the $f_{S}$,up values above.

Additionally Ramphao ef al. (2004) proposed two other explanations for the differcence in sludge production in the two systems.

- The higher P' removal in the MBR LCT system suggests a greater PAO population which would produce more sludge per unit influent COD than OlIOs due to their lower endogenous respiration rates (Wentzel et al, 1990).

- Particulate organics that are biodegradable in the conventional UCT systcm are no longer biodegadable in the MBR system due to factors such as high MLSS concentrations, or different floc morphology.

In the literature previous studies comparing conventional and MBR BNR syslems ruл under the same opcrating conditions have indicated that the sludge production of the two systems were very similar (Masse et al, 2006, Monti et al, 2006), however in both investigations the systems were rum at the same COD loading rate per unit reactor volume. Masse et at., (2006) included the sludge lost through the SST in sludge wasting calculations in order to corrpare sludge productions in the two systems.

Additionally sludge production in ND and VIJBEPR systems operated using the same wastewater source, for sludge ages in the region of 10 and 20 days, have produced sludge in comparable tnagnitudes to those abserved in the conventional system. Previous values are lisked in Table 6.2. Thus it would appear that there is an increase in sludge production in the MBR system linked to the increased MISS concentration in the MBR system and the setention by membranes of all solids. 
Table 6.2: Comparison of sidge productions in investigations using the same waste water.

\begin{tabular}{|c|c|c|c|c|c|}
\hline Author & $\begin{array}{l}\text { System } \\
\text { type }\end{array}$ & SRT & $f_{s u s}$ & $f_{\text {S.up }}$ & $\begin{array}{c}\text { Sludge } \\
\text { production }\end{array}$ \\
\hline units & - & days & - & - & $\begin{array}{l}\text { mgVSSt } \\
\text { mgCOD }\end{array}$ \\
\hline \multirow[t]{2}{*}{ Sneyders (1995) } & LCT & 10 & 0.05 & 0.045 & 0.26 \\
\hline & प̄CT & 10 & 0.06 & 0.062 & 0.27 \\
\hline Musvoto & MUCT & 20 & 0.073 & 0.32 & 0.31 \\
\hline Beeharry $(2001\}$ & MLE & 10 & 0.05 & 0.161 & 0.28 \\
\hline \multirow[b]{2}{*}{ Ubisi (1997) } & \multirow[b]{2}{*}{ MLE } & 12 & 0.095 & 0.12 & 0.22 \\
\hline & & 20 & 0.095 & 0.12 & 0.2 \\
\hline \multirow[b]{2}{*}{ Lee $(2002)$} & \multirow[b]{2}{*}{ MLE } & 10 & 0.043 & 0.165 & 0.32 \\
\hline & & 20 & 0.04 & 0.148 & 0,18 \\
\hline Crumje $(200)$ & MLE & 10 & 0.085 & 0.103 & 0.23 \\
\hline
\end{tabular}

\subsection{MIXED LIQUOR CHARACTFRISTICS}

In order to quantify the mixed liquor in both systems the VSS, TSS, COD and TKN concentrations of the mixed liquor were measured. Investigation average ratios between these parameters are listed in Table 6.3.

Table 6.3: Mixed liquor parameters

\begin{tabular}{|c|c|c|c|}
\hline Parameter & unit & MBR System & Conv. System \\
\hline VSS/TSS & mgVSS/mgTSS & 0.809 & 0.814 \\
\hline CODNSS & mgCODImgVSS & 1.402 & 1.496 \\
\hline TKNNSS & mgNimgVSS & 0.085 & 0.094 \\
\hline
\end{tabular}

- The VSSTSSS and TKNVVS average ratios were relatively close for both systems.

- Both systems exhibited high VSS/TSS ratios which are not characteristic of BFFR systems. In BEPR systems the development of a PAO population is encouraged. PAO's have internally a low VSS/TSS ratio due to the additional inorganic polyP in their cell mass.

- Although the CODVSS ratios differ substantially from each other in the two systems, they both fall very close to the expected and theoredieal $f_{\mathrm{CV}}$ values of 1.48 and 1.42 respectively (WRC., 1984).

- The CODVVS ratio indicates that the COD incorporated into the mixed liquor was lower in the MBR system than in the conventional system. However the comparison of mass balances showed a greater proportion of COr was removed from the MBR system via the mixed liquor wasted than in the conventional system. In order for this to occur proportionlally more mixed liquor would need to be wasted from the MBR systern than the converional syslem to achieve higher COD removals through wasting, particularly with lower COD incorporated in the mixed liquor. This is only possible, at the same sludge age, if the sludge production in the MBR system was substantially greater than that in the conventional system. 


\subsection{NITROGEN REMOVAL}

As described in Chapter 4, Section 4.9 .2 nitrogen is removed from BiVR systems either by the incorporation of nitrogen in mixed liquor and its subsequent removal through wasting, or through nitrification/denitrification.

- The intluent $\mathrm{N}$ incotporated in the mixed liquor was lower in the MBR UCI system than in the cornentional LCT system. This corresponds to the observation above of lower COD incorporated in the MLVSS.

- Regardless, N removal lhrough sludge wasting was higher in the MBR LCT system than the conventional UCT system, this is largely due to the higher sludge production in the MBR 5ystem, and consequent increased sludge mass wasted per unit influent $N$.

- The MBR LCT system additionalify displayed higher $N$ removals through denitrification. This was achieved despite similar mass fractions and that the conventional UCT system was frequnetsly fully loaded.

\subsection{BIOLOGICAL EXCESS PHOSPHORLS REMOVAL (BEPR) BEHAVIOUR}

In both systems TP was dosed in excess of the amount the system could remove in order to demonstrate BFPR. Thus $P$ removal performance is represented by $P$ removals. System average $P$ removals of $21.3 \mathrm{mgP} / \ell$ and $16.7 \mathrm{mgF} / t$ were achieved indicating that total $\mathrm{P}$ removal would have been possible in both systems with influent $P$ concentrations of tip to $-20 \mathrm{mg} P / \ell$ and $-16 \mathrm{mgP} / \mathrm{f}$ respectively. Clearly however, the P remenal perfommance of the conventional UCT system was inferior to that of the MBR system. Reasors for this are discussed below. A comparison of $P$ release. uptake and removal is presented in Fig. 6.4.

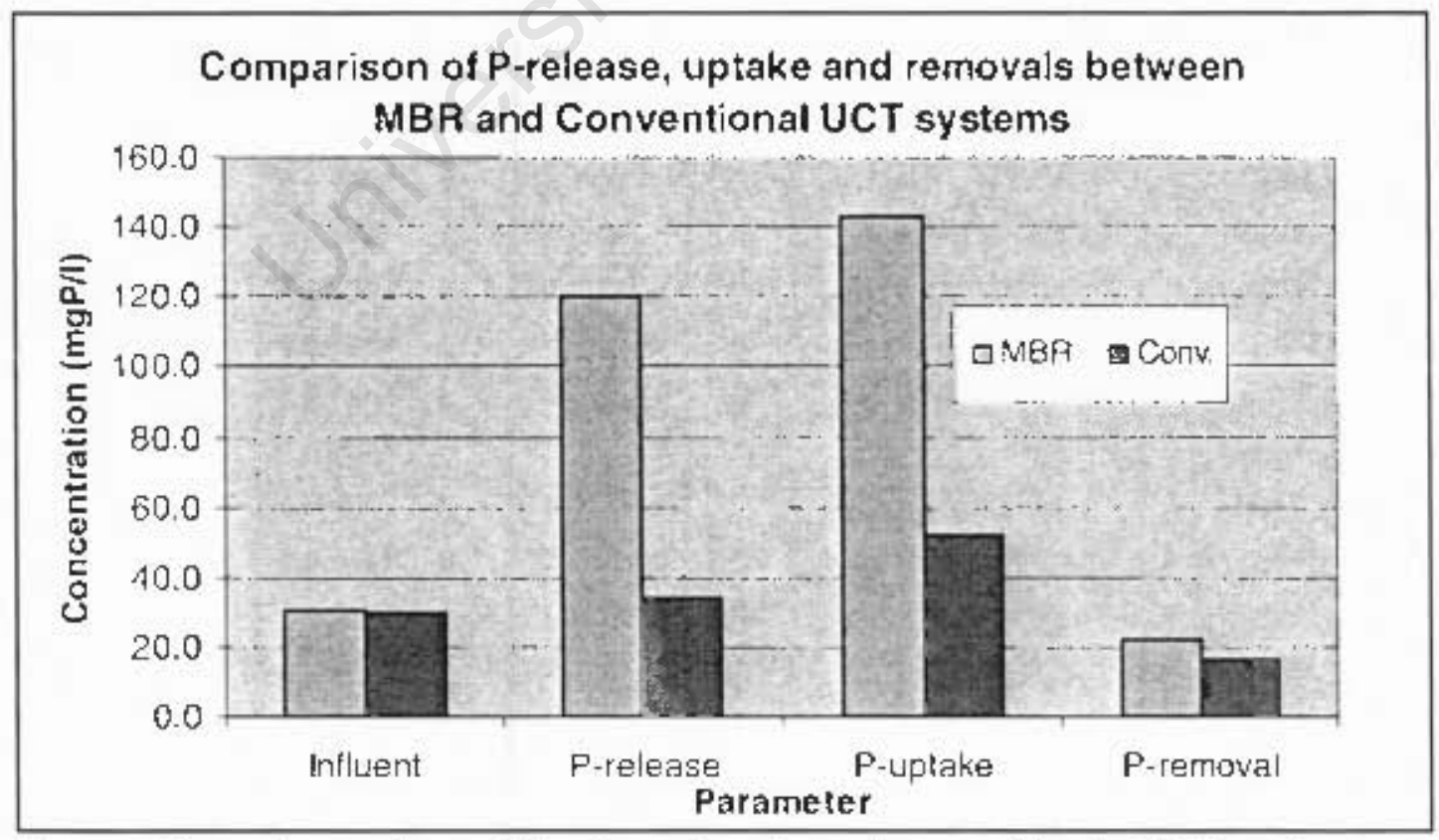

Figure 6.4: Comparison of P-retetastuptate and remonct in the MBR and Conventionad UCT systems. 
- P-removals differed significantly between the two systems, $22.4 \mathrm{mgP} / \mathrm{f}$ and 16.1 migP/ in the MBR and conventiond LCT systems renpectively.

- Denitrification was complete in the MBR system, but not in the conventional UCT system, evidenced by regular concentrations in the anoxic reactor $>1 \mathrm{mgN}-\mathrm{NO}_{3} f$. This loading of nitrate on the anacrobic reactor in the conventional [IC $[$ system would have reduced the RBCOD avaliable for $P A O S$ and conseguently reduce $P$ removil.

- the anoxic P uptake was more prevalent in the conventional system with $22.1 \%$ of $\mathrm{P}$ uptake taking place in the anoxic reactor, in contrast to only $8.5 \%$ anoxic $P$ uptake in the MBR system.

The above observations indicate that the conventional system was not being operated optimally (the anoxic reactor was overloitled), hence it is difficult to assess if the presence of membranes does indeed change the $\mathrm{P}$ removal efficiency of the MBR system.

\subsection{CIOOSLRE}

The behaviour and responses of the UCT ictivited sludge systems have been compared in this chapter. From the comparison it is clear that the MBR LCT system gave equal or superior quality response and performance jn terms of effluent quality and system removals. The MBR systen did however produce substantially more mixed liquer per unit COD load than the conventional system, which is in agreement with obserations from Pluase 1 (Ramphio, et al., 2004). The increased sludge production is explained in part by the retention, by the membranes, of additional COD and non-settleable solids which would otherwise now through SSTs, however this cannot explain the magnitude of difference in sludge production between the two systems. 


\section{CHAPTER 7}

\section{CONCLUSIONS AND RECOMMENDATIONS}

\subsection{INTRODUCTION}

Membranes for solids liquid separation in the activated sludge process are rapidly gaining acceptance internationally and have been implemented in many parts of the world. They have the inherent advantages that they produce a consistently solids free effluent, they reduce the footprint of wastewater treatment plants significantly by allowing AS systems to be run at high solids concentrations and obviating the need for SSTs, additionally experience has shown that membranes retain pathogens and in some cases virus' too which increases the reusability of the wastewater effluent. Stricter international water quality guidelines have stressed the importance of nutrient removal from wastewaters, spurring the development of methods for nutrient removal by chemical or biological means. In South Africa, and more recently internationally, the advantages of biological nutrient removal (BNR) have made it an attractive choice for nutrient removal. Little however is known of the effect of combining the attributes of membranes, namely higher concentration mixed liquors, the retention of all solids and the increased turbulence of aeration for membrane scour, on BNR systems. This investigation set out to add to the limited existing literature on BNR in MBR systems with the following specific objectives:

- Verifying the results obtained by Ramphao et al. (2004) with particular emphasis on explaining the phenomena of increased sludge production;

- Gaining a better understanding of the operating conditions and considerations of MBR BNR systems;

- Providing a parent system from which further testing into the kinetics of a MBR BNR system could be performed (Parco et al., 2006).

These objectives were met by running two activated sludge systems in UCT configurations. The first was a MBR UCT system with membranes in the aerobic reactor and the other a conventional UCT system with a SST with which to compare performance. All engineering parameters were monitored in order to assess the BNR performance of the systems. The results of the two systems were presented in Chapters 4 and 5 respectively, with a comparison and discussion of results in Chapter 6.

\subsection{CONCLUSIONS}

From this investigation, the following conclusions could be drawn:

- Membranes in a BNR system are a feasible nutrient removal solution with excellent organic and nutrient removal performance. The presence of membranes and consequently operating the system at high sludge concentrations did not adversely affect BNR performance, but produced an effluent of equal or superior quality to that produced by a conventional system 
using SSTs. In addition pathogen counts indicated that all pathogens were retained by the membranes. Thus the membrane effluent is safer and more viable for reuse purposes.

- Higher sludge productions of 0.311 and $0.320(\mathrm{mgVSS} / \mathrm{d}) /(\mathrm{mgCOD} / \mathrm{d})$ were observed in the MBR system in both Phase 1 (Ramphao et al., 2004) and Phase 2 of the investigation respectively. This higher sludge production is accommodated in steady state design theory by increasing the unbiodegradable particulate COD fraction $\left(\mathrm{f}_{\mathrm{S} \text {,up }}\right.$ ) fraction, in Phase 1 to 0.224 and in Phase 2 to 0.200 . The increased sludge production is justified in part by the retention of all solids. Similarly the unbiodegradable soluble COD fraction $\left(f_{\mathrm{S}, \text { us }}\right)$ must be decreased to account for the additional retention of "soluble" COD which is attributed to the finer membrane pore size.

- A theoretical evaluation of the BNR performance of the MBR system indicated that the current BNR theory was able to closely predict the system performance for COD removal and nitrification. However for denitrification the $\mathrm{D}_{\mathrm{PP}}$ was underpredicted requiring $\mathrm{K}_{2}$ 'T to be adjusted from 0.145 to 0.216 $\mathrm{mgN} / \mathrm{mgVSS} / \mathrm{d}$ at $20^{\circ} \mathrm{C}$ in order to match observed and predicted values. The BEPR predictions for aerobic $P$ uptake BEPR were close to those observed when the system PAO population reached a steady state (sewage batches $18-$ 25). $\mathrm{f}_{\mathrm{XBGP}}$ observed in this period $(0.376 \mathrm{mgP} / \mathrm{mgVSS})$ was close to that determined theoretically of $0.38 \mathrm{mgP} / \mathrm{mgVSS}$.

- Aeration testing was performed on the system, in order to determine alpha values for the high concentration sludge. Alpha values of $0.5-0.6$ for $\sim 15$ $000 \mathrm{mgTSS} / \ell$ and $0.2-0.3$ for $\sim 20000 \mathrm{mgTSS} / \ell$ were determined, which are higher than other values reported in the literature. These values are however specific to the laboratory system run in which factors such as reactor geometry and high aeration turbulence would have affected oxygen transfer in the system. Additionally the low sensitivity of the measuring apparatus resulted in substantial variance of results.

- Rheological testing on the sludge confirmed that there is a linear relationship between activated sludge viscosity and oxygen mass transfer.

- The sludge filterability through the membranes can be influenced by fine colloidal material, however observations indicated that the filterability would return to previous levels once colloids are removed from solution by assimilation into the mixed liquor.

\subsection{RECOMMENDATIONS FOR FURTHER STUDY}

Following from this investigation two recommendations are proposed:

- Accurate knowledge of the oxygen transferability in high concentration sludge is an important design consideration. However in this investigation the difficulty in measuring this parameter at a lab scale was realised. It is recommended that this parameter needs to be quantified on a full scale and be determined at a fully operational BNR MBR WWTP.

- The relationship between Alpha and viscosity of activated sludge needs to be investigated further in order to better understand the influence of high concentration sludges on oxygen mass transfer. This is an important parameter for design and thus accurate prediction of oxygen mass transfer co-efficients such as Alpha is essential. 


\section{REFERENCES}

Adam C., Gnirss R., Lesjean B., Buisson H. and Kraume M. (2002). Enhanced biological phosphorus removal in membrane bioreactors. Water Sci. Technol. 46(4/5), 281-86.

Ahn K.H., Song K.G., Cho E., Cho J., Yun H., Lee S. and Kim J. (2003). Enhanced biological phosphorus and nitrogen removal using a sequencing anoxic/anaerobic membrane bioreactor (SAM) process. Desalination 157, 345-352.

Baker D.R. et al (1975). Oxygen transfer at high solids concentrations Jour. Env. Eng. Div., ASCE, EE5, October, 759-774.

Barker P.S. and Dold P.L. (1996). Sludge production and oxygen demand in nutrient removal activated sludge systems. Water Sci. Technol. 34(5-6), 43-50.

Beeharry A.O., Wentzel M.C. and Ekama G.A. (2001). Evaluation of batch test for measurement of active biomass in activated sludge mixed liquor. Res. Rept. W112, Department of Civil Engineering, University of Cape Town, Rondebosch 7701, RSA.

Bratby J.R., Gaines B., Loyer M., Luiz F. and Parker D. (2002). Merits of alternative MBR systems. Proceedings of the Water Environment Federation 75th Annual Technical Exhibition \& Conference, Chicago, Illinois, USA, September 28 October 2.

Brindle K. and Stephenson T. (1996). The application of membrane biological reactor for treatment of wastewater. Biotechnol. and Bioeng. 49(6), 601-610.

Buisson H., Cote P., Paderie M. and Paillard H. (1998). The use of immersed membranes for upgrading wastewater treatment plants. Water Sci. Technol. 37(9), 89-95.

Churchouse S., Wildgoose D. (1999). Membrane bioreactors progress from the laboratory to full-scale use. Membrane Technology 111, 4-8.

Clayton J.A., Ekama G.A., Wentzel M.C. and Marais G.vR. (1989). Denitrification kinetics in biological nitrogen and phosphorus removal activated sludge systems, Res. Rept. W63, Department of Civil Engineering, University of Cape Town, Rondebosch, 7701, RSA.

Clayton J.A., Ekama G.A., Wentzel M.C., and Marais G.vR. (1991). Denitrification kinetics in biological $\mathrm{N}$ and $\mathrm{P}$ removal activated sludge systems treating municipal wastewaters. Water Sci. Technol. 23, 1025-1035.

Cornel P., Wagner M., and Krause S. (2003). Investigation of oxygen transfer rates in full scale membrane bioreactors. Water Sci. Technol. 47(11): 313-319. 
Cote P., Buisson H., Pound C. and Arakaki G. (1997). Immersed membrane activated sludge for the reuse of municipal wastewater. Desalination 113, 189-196.

Cronje G.L., Wentzel M.C. and Ekama G.A. (2000). Measurement of active heterotrophic organism concentration in nitrification-denitrification activated sludge systems. Res. Rept., W102, Department of Civil Engineering, University of Cape Town, Rondebosch, 7701, RSA.

Darton E.G. (1997). Scale inhibition techniques used in membrane systems. Desalination 113, 227-229.

Davies W.J., Le M.S. and Heath C.R. (1998). Intensified activated sludge process with submerged membrane microfiltration. Water Sci. Technol. 38(4-5), 421428.

de Haas D.W., Wentzel M.C. and Ekama G.A. (2001). The use of simultaneous chemical precipitation in modified activated sludge systems exhibiting biological excess phosphorus removal. Part 5: Experimental periods using a ferrous-ferric chloride blend. Water SA 27(2), 117-134.

Dold P.L., Wentzel M.C., Billing A.E., Ekama G.A. and Marais G.vR. (1991). Activated sludge system simulation programs, version 1.0. Water Research Commission, P O Box 824, Pretoria, 0001, RSA.

Ekama G.A. and Marais G.vR. (1978). The dynamic behaviour of the activated sludge process. Res. Rept. W27, Department of Civil Engineering, University of Cape Town, Rondebosch, 7700, RSA.

Ekama G.A. and Wentzel M.C. (1999). Denitrification kinetics in biological N and P removal activated sludge systems treating municipal wastewaters. Water Sci. Technol. 39(6), 69-77.

Ekama G.A. and Wentzel M.C. (2004). A predictive model for the reactor inorganic suspended solids concentration in activated sludge systems. Water Research 38(19), 4093-4106.

Ekama G.A., Dold P.L. and Marais G.vR. (1986). Procedures for determining influent COD fractions and the maximum specific growth rate of heterotrophs in activated sludge systems. Water Sci. Technol. 18(6), 91-114.

Fane A. and Chang S. (2002). Membrane bioreactors: design and operation options. Filtration and Separation 39(5), 26-29.

Fleischer E.J., Broderick T.A., Diagger G.T., Fonseca A.D., Holbrook R.D. and Murthy S.N. (2005). Evaluation of membrane bioreactor process capabilities to meet stringent effluent nutrient discharge requirements. Water Env. Res. $77(2), 162-178$. 
Gander M., Jefferson B. and Judd. S. (2000). Aerobic MBR's for domestic wastewater treatment: a review with cost considerations. Separation and Purification Technology 18(2), 119-130.

Gnirss R., Lesjean B., Adam C. and Buisson H. (2003). Cost effective and advanced phosphorus removal in membrane bioreactors for a decentralised wastewater technology. Water Sci. Technol. 47(12), 133-139.

Guadix A., Sorensen E., Papageorgiou L.G. and Gaudix E. M. (2004). Optimal design and operation of continuous ultrafiltration plants. Journal of Membrane Science 235(1-2), 131-138.

Gunder B., Krauth K. (1999). Replacement of secondary clarification by membrane separation - results with tubular, plate and hollow fibre modules. Water Sci. Technol. 40(4-5), 311-320.

Henze M., Grady C.P.L. (Jr.), Gujer W., Marais G.vR. and Matsuo T. (1987). Activated Sludge model no. 1. IAWPRC Scientific and Technical Report No. 1, IAWPRC, London.

Howell J.A. (2004). Future of membranes and membrane reactors in green technologies and for water reuse. Desalination 162(1), 1-11.

Hu Z.R., Wentzel M.C., and Ekama G.A. (2002). The significance of denitrifying polyphosphate accumulating organisms in biological nutrient removal activated sludge systems. Water Sci. Technol. 46(1-2), 129-138.

Ishiguro K., Imai. K. and Sawada S. (1994). Effects of biological treatment conditions on permeate flux of ultrafiltration membranes in membrane/activated sludge wastewater treatment system. Desalination 98, 119-126.

Ji L. and Zhou J. (2006). Influence of aeration on microbial polymers and membrane fouling in submerged membrane bioreactors. Journal of Membrane Science 276(1-2), 168-177.

Judd S. (2002). Submerged membrane bioreactors: Flat plate or hollow fibre? Filtration and Separation 39(5), 30-31.

Kennedy, S. Senior process engineer, Copa MBRTechnology. (2006). pers. com.

Kern-Jespersen J.P., and Henze M. (1993). Biological phosphorus uptake under anoxic and aerobic conditions. Water Research 27(4), 617-624.

Krampe J. and Krauth K. (2003). Oxygen transfer into activated sludge with high MLSS concentrations. Water Sci. Technol. 47(11), 297-303.

Kraume M., Bracklow U., Vocks M., and Drews A. (2005). Nutrients removal in MBR's for municipal wastewater treatment. Water Sci. Technol. 51(6/7), 391402. 
Kuba T., Smolders G., van Loosdrecht M.C.M. and Heijnen J.J. (1993). Biological phosphorus removal from wastewater by anaerobic-anoxic sequencing batch reactor. Water Sci. Technol. 27(5/6), 241-252.

Kuba T., van Loosdrecht M.C.M., Brandse F.A. and Heijnen J.J. (1997). Occurrence of denitrifying phosphorus removing bacteria in modified UCT-type wastewater treatment plants. Water Research 31(4), 777-786.

Lee B.J., Wentzel M.C. and Ekama G.A. (2002a). Batch tests for measurement of ordinary heterotrophic organism active biomass in activated sludge mixed liquor. MSc (Eng) Thesis, Department of Civil Engineering, University of Cape Town, Rondebosch 7701, RSA.

Lee Y, Cho J, Seo Y, Lee JW, Ahn K (2002b) Modelling of submerged membrane bioreactor process for wastewater treatment. Desalination 146, 451-457.

Lesjean B., Gnriss R., Buisson H., Keller S., Tazi-Pain A., and Luck F. (2005). Outcomes of a 2-year investigation on enhanced biological nutrients removal and trace organics elimination in membrane bioreactor (MBR). Water Sci. Technol. 52(10-11), 453-460.

Lesjean B., Gnirss R., Adam C., Kraume M. and Luck F. (2003). Enhanced biological phosphorus removal process implemented in membrane bioreactors to improve phosphorus recovery and recycling. Water Sci. Technol. 48(1), 87-94.

Lesjean B., Rosenberger S. and Schrotter J.C. (2004). Membrane aided biological wastewater treatment - an overview of applied systems. Membrane Technology 2004(8), 5-10.

Li H., Yang M., Zhang Y., Yu T. and Kamagata Y. (2006). Nitrification performance and microbial community dynamics in a submerged membrane bioreactor with complete sludge retention. Journal of Biotechnology 123(2006), 60-70.

Liebig T., Wagner M., Bjerrum L. and Denecke M. (2001). Nitrification performance and nitrifier community composition of a chemostat and a membrane assisted bioreactor for the nitrification of sludge reject water. Bioproc. Biosys. Eng. 24(2001), 203-210.

Maharaj S., Naidoo A. and Bux F. (2006). Comparison of the nitrifying population dynamics from an MBR and conventional activated sludge system. MSc Thesis, Department of Microbiology, Durban Institute of Technology, Durban.

Mahendraker V., Mavinic D.S., Rabinowitz B. and Hall B. K. (2005). The impact of influent ratios and biochemical reactions on oxygen transfer in an EBPR process - A theoretical explanation. Biotechnol ana Bioeng. 91(1), 22-42.

Manser J., Gujer W. and Siegrist H. (2005). Membrane bioreactor versus conventional activated sludge system: population dynamics of nitrifiers. Waier Sci. Technol. 52(10-11), 417-425. 
Marais G.vR. and Ekama G.A. (1976). The activated sludge process: Part I - Steady state behaviour. Water SA 2(4), 163-200.

Masse A., Sperandio M. and Cabassud C. (2006). Comparison of sludge characteristics and performance of a submerged membrane bioreactor and an activated sludge process at high solids retention time. Water Research 40(12), 2405-2415.

Mbewe A., Wentzel M.C., Ekama G.A. (1995). Characterization of carbonaceous materials in municipal wastewaters. Res. Rept. W84, Department of Civil Engineering, University of Cape Town, Rondebosch, 7701, RSA.

Mellin H.K.O., Lakay M.T., Wentzel M.C. and Ekama G.A. (1998). The effect of temperature on denitrification kinetics, biological excess phosphorus removal and bulking in nutrient activated sludge systems in tropical climates $\left(20^{\circ} \mathrm{C}\right.$ to $30^{\circ} \mathrm{C}$ ). Res. Rept. W91, Department of Civil Engineering, University of Cape Town, Rondebosch, 7701, RSA.

Monti A., Hall E.R., Dawson R.N., Husain H. and Kelly H.G. (2006). Comparative study of biological nutrient removal (BNR) processes with sedimentation and membrane-based separation. Biotechnology and Bioengineering 94(4), 740752.

Mouthon-Bello J. and Zhou H. (2005). Performance of a submerged membrane bioreactor system for biological nutrient removal. Water Env. Res. 78(5), 538547.

Muller A., Wentzel M.C. and Ekama G.A. (2003). Measurement of ordinary heterotroph organism anoxic yield in anoxic-aerobic activated sludge systems. MSc (Eng) Thesis, Department of Civil Engineering, University of Cape Town, Rondebosch 7701, RSA.

Musvoto E.V., Casey T.G., Ekama G.A, Wentzel M.C., Marais G.vR. (1992). The effect of large anoxic mass fraction and concentrations of nitrate and nitrite in the primary anoxic zone on low F/M filament bulking in nutrient removal activated sludge systems. MSc (Eng) Thesis, Department of Civil Engineering, University of Cape Town, Rondebosch, 7701, RSA.

Parco V., Wentzel M.C. and Ekama G.A. (2006). Kinetics of nitrogen removal in a membrane bioreactor biological nutrient removal activated sludge system. Water Institute of Southern Africa - WISA 2006 - Biennial Conference, 21-25 May 2006, Durban (South Africa).

Parco V., du Toit G.J.G., Wentzel M.C. and Ekama G.A. (2007). Biological nutrient removal in membrane bioreactors: Denitrification and Phosphorus removal kinetics. IWA $4^{\text {th }}$ International Membranes Conference, 15-1 $7^{\text {th }}$ May 2007 Harrogate (UK).

Ramphao M., Wentzel M.C., Merritt R., Ekama G.A., Young T., and Buckley C.A. (2005). Impact of membrane solid-liquid separation on design of biological 
nutrient removal activated sludge systems. Biotechnol. and Bioeng. 89(6), 630-646.

Ramphao M.C., Wentzel M.C., Lakay M.T., Mafungwa H. and Ekama G.A. (2004). The performance of biological nitrogen and phosphorus removal with ultrafiltration membranes for solid-liquid separation. MSc (Eng) thesis, Department of Civil Engineering, University of Cape Town, Rondebosch, 7701, RSA.

Randall E.W., Wilkinson A. and Ekama G.A. (1991). An instrument for the direct determination of oxygen utilization rate. Water $S A$ 17(1), 11-18.

Rosenberger S., Kruger U., Witzig R., Manz W., Szewzyk U. and Kraume M. (2002a). Performance of a bioreactor with submerged membranes for aerobic treatment of municipal wastewater. Water Research 36(2), 413-420.

Rosenberger S., Kubin K. and Kraume M. (2002b). Rheology of Activated Sludge in Membrane Bioreactors. Eng. Life. Sci. 2(9), 269-275.

Rosenberger S., Laabs C., Lesjean B., Gnirss R., Amy G., Jekel M. and Schrotter J.C. (2006). Impact of colloidal and soluble organic material on membrane performance in membrane bioreactors for municipal wastewater treatment Water Research 40(4), 710-720.

Sampson K. City Engineer, Cape Town (2006) pers. com.

Sneyders M.J., Ekama G.A. and Wentzel M.C. (1998). The effect of dosing unstabilized landfill leachate to nutrient removal activated sludge systems. Res. Rept. W95, Department of Civil Engineering, University of Cape Town, Rondebosch, 7701, RSA.

Sorm R., Bortone G., Saltarelli R., Jenicek P., Wanner J. and Tilche A. (1996). Phosphate uptake under anoxic conditions and fixed-film nitrification in nutrient removal activated sludge system. Water Research 30(7), 1573-1584.

Standard Methods (1985). Standard Methods for the examination of water and wastewater $\left(16^{\text {th }}\right.$ ed.) American Public Health Assoc., $101515^{\text {th }}$ Str. NW, Washington DC, 20005, USA.

Trussell R.S., Merlo R.P., Hermanowicz S.W. and Jenkins D. (2006). The effect of organic loading on process performance and membrane fouling in a submerged membrane bioreactor treating municipal wastewater. Water Research 40(14), 2675-2683.

Ubisi M.F., Wentzel M.C. and Ekama G.A. (1997). Organic and inorganic components of activated sludge mixed liquor. Res. Rept. W94, Department of Civil Engineering, University of Cape Town, 7701, RSA. 
Urbain V., Mobarry B., de Silva V., Stahl D.A., Rittmann B.E. and Manem J. (1998). Integration of performance, molecular biology and modelling to describe the activated sludge process. Water Sci. Technol. 37(4-5), 223-229.

Vocks M., Adam C., Lesjean B., Gnirss R. and Kraume M. (2005). Enhanced postdenitrification without addition of an external carbon source in membrane bioreactors. Water Research 39(14), 3360-3368.

Wagner M.R. and Pöpel H.J. (1998). Oxygen transfer and aeration efficiency influence of diffuser submergence, diffuser density, and blower type. Water Sci. Technol. 38(3), 1-6.

Warburton C.A., Lakay M.T., Casey T.G., Ekama G.A., Wentzel M.C. and Marais G.vR. (1991). The effect of sludge age and aerobic mass fraction on low F/M filament bulking in intermittent aeration nitrogen removal systems. Res. Rept. W65, Department of Civil Engineering, University of Cape Town, Rondebosch, 7701, RSA.

Wentzel M.C. and Ekama G.A. (1997). Principles in the design of single-sludge activated sludge systems for biological removal of carbon, nitrogen, and phosphorus. Water Env. Res. 69(7), 1222-1231.

Wentzel M., Ekama G.A. and Marais G.vR. (1992). Processes and modelling of nitrification denitrification biological excess phosphorus removal systems - a review. Water Sci. Technol. 25(6), 59-82.

Wentzel M.C., Ekama G.A., Dold P.L. and Marais G.vR. (1990). Biological excess phosphorous removal-steady state process design. Water $S A$ 16(1), 29-48.

Witzig R., Manz W., Rosenberger S., Krüger U., Kraume M. and Szewzyk U. (2002). Microbiological aspects of a bioreactor with submerged membranes for aerobic treatment of municipal wastewater. Water Research 36(2), 394-402.

WRC (1984). Theory, design and operation of nutrient removal activated sludge processes. Water Research Commision, P O Box 824, Pretoria, RSA.

Yamamoto K., Hiasa M., Mahmood T. and Matsuo T. (1989). Direct solid-liquid separation using hollow fibre membrane in an activated sludge aeration tank. Water Sci. Technol. 21, 43-54.

Yang W., Cicek N. and Ilg J. (2006). State-of-the-art of membrane bioreactors: Worldwide research and commercial applications in North America. Journal of Membrane science 270(1-2), 201-211. 


\section{APPENDIX A}

\section{MBR AND SQW DAILY MEASUREMENTS}

\section{TABLE OF CONTENTS}

A.1 Measured COD values for the MBR UCT and SQW systems.

A.2 Measured TKN, FSA and solids concentrations in the MBR UCT system.

A.3 Measured NO3, NO2 and soluble P in the MBR UCT system.

A.4 Miscellaneous measurements on the MBR UCT system.

A.5 Sewage Batch Averages for the MBR UCT System 


\section{A-1 COD MBR}

Appendix A-1

Measured COD values for the MBR UCT and SQW systems

\begin{tabular}{|c|c|c|c|c|c|c|c|c|c|c|c|c|c|}
\hline Day & Date & SB \# & Influent & Aerobic & Eff 0.1 & Eff 0.45 & $\begin{array}{l}\text { Eff } \\
\text { unfilt. }\end{array}$ & Tank & $\mid \begin{array}{l}\text { RB- } \\
\text { Mbewe* }\end{array}$ & SQW & $\begin{array}{l}\text { SBSi - } \\
\text { RBCOD }\end{array}$ & $\mathrm{fts}, \mathrm{s}$ & $f t s, T$ \\
\hline- & - & - & $(\mathrm{mgCOD} / \mathrm{l})$ & $(\mathrm{mgCOD} / 1)$ & $(\mathrm{mgCOD} / \mathrm{l})$ & $(\mathrm{mgCOD} / l)$ & $(\mathrm{mgCOD} / \mathrm{l})$ & $(\mathrm{mg} \operatorname{coD} / \mathrm{l})$ & $(\mathrm{mgCOD} / \mathrm{l})$ & $(\mathrm{mgCOD} / 1)$ & $(\mathrm{mgCOD} /)$ & - & - \\
\hline 21 & 3-Mar-05 & \multirow{15}{*}{. } & & & & & & & & & & & \\
\hline 22 & 4-Mar-05 & & & & & & & & & & & & \\
\hline 23 & 5-Mar-05 & & & & & & & & & & & & \\
\hline 24 & 6-Mar-05 & & & & & & & & & & & & \\
\hline 25 & 7-Mar-05 & & 1083.7 & 19514.4 & 18.7 & & & & & & & & \\
\hline 26 & 8-Mar-05 & & 1075.4 & 20760.0 & 41.5 & & & & & & & & \\
\hline 27 & 9-Mar-05 & & 1054.6 & 18351.8 & 62.3 & & & & & & & & \\
\hline 28 & 10-Mar-05 & & 1046.3 & & & & & & & & & & \\
\hline 29 & 11-Mar-05 & & 871.9 & 18517.9 & 29.1 & & & & & & & & \\
\hline 30 & 12-Mar-05 & & & & & & & & & & & & \\
\hline 31 & 13-Mar-05 & & & & & & & & & & & & \\
\hline 32 & 14-Mar-05 & & 988.2 & 134659 & 19.9 & & & & & & & & \\
\hline 33 & 15-Mar-05 & & 772.3 & 19043.5 & 31.9 & & & & & & & & \\
\hline 34 & 16-Mar-05 & & 950.8 & & & & & & & & & & \\
\hline 35 & 17-Mar-05 & & 860.5 & 17051.5 & 35.9 & & & & & & & & \\
\hline 36 & 18-Mar-05 & \multirow[b]{2}{*}{2} & 880.5 & & & & & & & $\infty$ & & & \\
\hline \multicolumn{2}{|c|}{ Batch Average } & & 958.4 & 18873.2 & 34.2 & & & & & $\square$ & & & \\
\hline 37 & 19-Mar-05 & \multirow{17}{*}{ 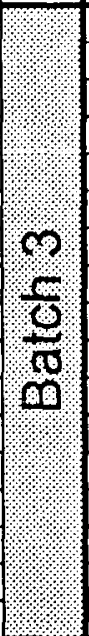 } & & & & & & & & - & & & \\
\hline 38 & 20-Mar-05 & & & & & & & & & 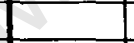 & & & \\
\hline 39 & 21-Mar-05 & & & 16095.4 & 21.9 & & & & & & & & \\
\hline 40 & 22-Mar-05 & & 7928 & & & & & & 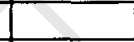 & 460.0 & 96.0 & 0.21 & 0.41 \\
\hline 41 & 23-Mar-05 & & 996.0 & 18953.0 & 64.0 & & & & & 460.0 & 104.5 & 0.23 & 0.38 \\
\hline 42 & 24-Mar-05 & & & & & & & & & & & & \\
\hline 43 & 25-Mar-05 & & 981.9 & 18009.0 & 36.4 & & & & & & & & \\
\hline 44 & 26-Mar-05 & & & & & & & & & & & & \\
\hline 45 & 27-Mar-05 & & 1038.0 & 21497.0 & 45.0 & & 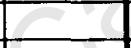 & L & & & & & \\
\hline 46 & 28-Mar-05 & & & & & & & & & & & & \\
\hline 47 & 29-Mar-05 & & 1156.0 & 20361.0 & 32.4 & $\mathrm{C}$ & & & & & & & \\
\hline 48 & 30-Mar-05 & & 1212.7 & & & & & & & & & & \\
\hline 49 & 31-Mar-05 & & 1249.2 & 20686.0 & 34.5 & & & & & 460.0 & & & 0.16 \\
\hline 50 & 1-Apr-05 & & 1249.2 & & & & & & & & & & \\
\hline 51 & 2-Apr-05 & & 1131.6 & 19793.3 & 32.4 & & & & & & & & \\
\hline 52 & 3-Apr-05 & & & & +1 & 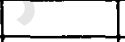 & & & & & & & \\
\hline 53 & 4-Apr-05 & & 1314.1 & 18414.2 & & & & & & & & & \\
\hline \multicolumn{2}{|c|}{ Batch Average } & 3 & 1147.7 & 19226.1 & 38.1 & & & & & 460.0 & 100.2 & 0.22 & 0.32 \\
\hline 54 & 5-Apr-05 & \multirow{22}{*}{ 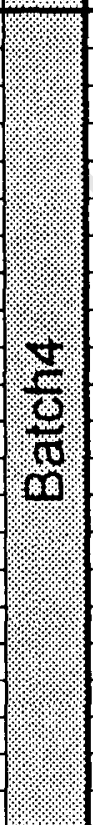 } & 1326.3 & 00 & & & & & & 460.0 & 94.2 & 0.20 & 0.32 \\
\hline 55 & 6-Apr-05 & & 1 & 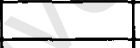 & & & & & & & & & \\
\hline 56 & 7-Apr-05 & & 2 & & & & & & & & & & \\
\hline 57 & 8-Apr-05 & & 880.0 & 19388.0 & 46.6 & & & & & & & & \\
\hline 58 & 9-Apr-05 & & 1 & & & & & & & & & & \\
\hline 59 & 10-Apr-05 & & 1155.9 & 20198.9 & 44.6 & & & & & & & & \\
\hline 60 & 11-Apr-05 & & 1302.0 & & & & & & & & & & \\
\hline 61 & 12-Apr-05 & & 1018.1 & 20523.4 & 38.5 & & & & & 460.0 & 63.9 & 0.14 & 0.31 \\
\hline 62 & 13-Apr-05 & & 904.5 & & & & & & & & & & \\
\hline 63 & 14-Apr-05 & & 1257.4 & 21496.8 & 14.2 & & & & & 460.0 & 54.8 & 0.12 & 0.26 \\
\hline 64 & 15-Apr-05 & & 1164.1 & & & & & & & & & & \\
\hline 65 & 16-Apr-05 & & 949.1 & 17765.3 & 24.3 & & & & & & & & \\
\hline 66 & 17-Apr-05 & & & & & & & & & & & & \\
\hline 67 & 18-Apr-05 & & 851.8 & 18819.8 & & & & & & & & & \\
\hline 68 & 19-Apr-05 & & 872.0 & 15007.2 & 38.5 & & & & & & & & \\
\hline 69 & 20-Apr-05 & & 1105.7 & & & & & & & & & & \\
\hline 70 & 21-Apr-05 & & 1326.0 & & & & & & & & & & \\
\hline 71 & 22-Apr-05 & & 726.2 & & & & & & & & & & \\
\hline 72 & 23-Apr-05 & & 790.9 & 141149 & 16.2 & & & & & & & & \\
\hline 73 & 24-Apr-05 & & & & & & & & & & & & \\
\hline 74 & 25-Apr-05 & & 681.4 & 102000 & 30.6 & & & & & & & & \\
\hline 75 & 26-Apr-05 & & 1056.7 & & & & & & & & & & \\
\hline Batcl & Average & 4 & 1021.6 & 18414.3 & 31.7 & & & & & 460.0 & 71.0 & 0.15 & 0.30 \\
\hline
\end{tabular}


A-1 COD MBR

\begin{tabular}{|c|c|c|c|c|c|c|c|c|c|c|c|c|c|}
\hline 76 & $27-A p r-05$ & \multirow{19}{*}{$\frac{\infty}{\infty}$} & & & & & & & & & & & \\
\hline 77 & 28-Apr-05 & & & & & & & & & & & & \\
\hline 78 & $29-A p r-05$ & & & 11995.2 & 40.8 & & & & & & & & \\
\hline 79 & 30-Apr-05 & & & & & & & & & & & & \\
\hline 80 & 1-May-05 & & 763.0 & & & & & & & & & & \\
\hline 81 & 2-May-05 & & & 12484.8 & 42.8 & & & & & 460.0 & 79.9 & 0.17 & \\
\hline 82 & 3-May-05 & & 1130.2 & & & & & & & & & & \\
\hline 83 & 4-May-05 & & 991.4 & & & & & & & & & & \\
\hline 84 & 5-May-05 & & 1224.0 & 14767.0 & 39.0 & & & & & 460.0 & 101.8 & 0.22 & 0.35 \\
\hline 85 & 6-May-05 & & 962.8 & 30110.4 & 34.7 & & & & & & & & \\
\hline 86 & 7-May-05 & & 6528 & & & & & & & 460.0 & 101.2 & 0.22 & 0.46 \\
\hline 87 & 8-May-05 & & 895.5 & & & & & & & & & & \\
\hline 88 & 9-May-05 & & 934.5 & 19747.2 & 34.7 & & & & & & & & \\
\hline 89 & 10-May-05 & & 942.7 & & & & & & & & & & \\
\hline 90 & 11-May-05 & & 1207.2 & 19747.2 & 44.9 & & & & & 460.0 & 58.7 & 0.13 & 0.27 \\
\hline 91 & 12-May-05 & & 981.3 & & & & & & & & & & \\
\hline 92 & 13-May-05 & & 881.3 & 20073.6 & 36.7 & & & & & 4600 & 60.2 & 0.13 & 0.33 \\
\hline 93 & 14-May-05 & & & & & & & & & & & & \\
\hline 94 & 15-May-05 & & & & & & & & & & & & \\
\hline Batc & Average & 5 & 992.2 & 18417.9 & 39.1 & & & & & 460.0 & 80.4 & 0.17 & 0.35 \\
\hline 95 & 16-May-05 & \multirow{16}{*}{$\begin{array}{l}0 \\
\frac{\sigma}{0} \\
\frac{\sigma}{\sigma} \\
m\end{array}$} & 947.0 & 17789.0 & 30.6 & & & & & & & & \\
\hline 96 & 17-May-05 & & & & & & & & & & & & \\
\hline 97 & 18-May-05 & & & & & & & & & & & & \\
\hline 98 & 19-May-05 & & 681.4 & & & & & & & & & & \\
\hline 99 & 20-May-05 & & 734.6 & 18468.0 & 61.6 & & & & & & 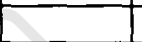 & & \\
\hline 100 & 21-May-05 & & 759.0 & & & & & & & & & & \\
\hline 101 & 22-May-05 & & 853.0 & & & & & & & 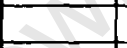 & & & \\
\hline 102 & 23-May-05 & & 907.2 & 20958.0 & & & & & & +2 & & & \\
\hline 103 & 24-May-05 & & 1153.9 & & & & & & & 460.0 & 69.0 & 0.15 & 0.30 \\
\hline 104 & 25-May-05 & & 1002.1 & 19726.0 & 4.30 & & & & & & & & \\
\hline 105 & 26-May-05 & & 969.2 & & & & & & & & & & \\
\hline 106 & 27-May-05 & & 1133.5 & 19479.5 & 43.2 & & 65.8 & & 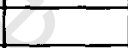 & & & & \\
\hline 107 & 28-May-05 & & 1072.6 & & & & & & & & & & \\
\hline 108 & 29-May-05 & & & & & & & $\square$ & & & & & \\
\hline 109 & 30-May-05 & & 741.3 & 21123.3 & 34.9 & & 113.0 & 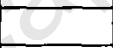 & & 460.0 & 50.1 & 0.11 & 0.35 \\
\hline 110 & 31-May-05 & & 907.0 & & & & & & & & & & \\
\hline Batc & Average & 6 & 912.4 & 19590.6 & 42.7 & & 89.4 & & & 460.0 & 59.6 & 0.13 & 0.32 \\
\hline 111 & 1-Jun-05 & \multirow{14}{*}{$\frac{1}{6}$} & & & & & $\therefore$ & & & & & & \\
\hline 112 & 2-Jun-05 & & 904.1 & & & & ${ }^{2}$ & & & & & & \\
\hline 113 & 3-Jun-05 & & 830.2 & 22931.6 & 41.1 & & 129.5 & & & & & & \\
\hline 114 & 4-Jun-05 & & & & 1 & & & & & & & & \\
\hline 115 & 5-Jun-05 & & 678.1 & & 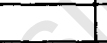 & & & & & & & & \\
\hline 116 & 6-Jun-05 & & 905.8 & 20383.6 & 20.5 & & 69.9 & & & & & & \\
\hline 117 & $7-$ Jun-05 & & 888.4 & & & & & & & 460.0 & 81.5 & 0.18 & 0.36 \\
\hline 118 & 8-Jun-05 & & 846.6 & 20159.0 & 23.9 & & 141.4 & & & & & & \\
\hline 119 & 9-Jun-05 & & 788.8 & $+\infty$ & & & & & & & & & \\
\hline 120 & 10-Jun-05 & & 996.2 & 18328.8 & 43.0 & & 76.0 & & & & & & \\
\hline 121 & 11-Jun-05 & & 813.7 & & & & & & & & & & \\
\hline 122 & 12-Jun-05 & & 669.8 & & & & & & & & & & \\
\hline 123 & 13-Jun-05 & & 742.0 & 17760.0 & 24.0 & & 158.0 & & & & & & \\
\hline 124 & 14-Jun-05 & & 952.0 & & & & & & & 460.0 & 57.7 & 0.13 & 0.31 \\
\hline \multicolumn{2}{|c|}{ Batch Average } & 3 & 834.6 & 19912.6 & 30.5 & & 115.0 & & & 460.0 & 69.6 & 0.15 & 0.34 \\
\hline 125 & 15-Jun-05 & \multirow{8}{*}{$\frac{\infty}{6}$} & & & & & & & & 460.0 & 51.2 & 0.11 & 0.37 \\
\hline 126 & 16-Jun-05 & & 660.0 & & & & & & & & & & \\
\hline 127 & 17-Jun-05 & & 764.0 & & & & & & & & & & \\
\hline 128 & 18-Jun-05 & & 652.0 & 18240.0 & 36.0 & & 170.0 & & & & & & \\
\hline 129 & 19-Jun-05 & & & & & & & & & & & & \\
\hline 130 & 20-Jun-05 & & 684.0 & 20240.0 & 42.0 & & 82.0 & & & & & & \\
\hline 131 & 21-Jun-05 & & 966.5 & & & & & & & & & & \\
\hline 132 & 22-Jun-05 & & 986.0 & 18493.2 & 41.1 & & 28.8 & & & & & & \\
\hline Batc & h Average & 8 & 785.4 & 18991.1 & 39.7 & & 93.6 & & & 460.0 & 51.2 & 0.11 & 0.37 \\
\hline 133 & 23-Jun-05 & \multirow[b]{6}{*}{$\sigma$} & & & & & & & & & & & \\
\hline 134 & 24-Jun-05 & & 995.0 & 192329 & 61.6 & & 69.9 & & & 460.0 & 84.9 & 0.18 & 0.35 \\
\hline 135 & 25-Jun-05 & & 1052.0 & & & & & & & & & & \\
\hline 136 & $26-J u n-05$ & & 5800 & & & & & & & & & & \\
\hline 137 & 27-Jun-05 & & 648.0 & 18880.0 & 30.0 & & 62.0 & & & & & & \\
\hline 138 & 28-Jun-05 & & 902.0 & & & & & & & & & & \\
\hline
\end{tabular}




\section{A-1 COD MBR}

\begin{tabular}{|c|c|c|c|c|c|c|c|c|c|c|c|c|c|}
\hline 139 & 29-Jun-05 & \multirow{9}{*}{$\frac{\Gamma}{\frac{\sigma}{U}}$} & 1004.0 & 17920.0 & 52.0 & & 64.0 & & & & & & \\
\hline 140 & 30 -Jun-05 & & 1010.0 & 18480.0 & 40.0 & & 76.0 & & & & & & \\
\hline 141 & $1-J u l-05$ & & 960.0 & 18720.0 & 40.0 & & 120.0 & & & & & & \\
\hline 142 & 2-Jul-05 & & 840.0 & & & & & & & & & & \\
\hline 143 & 3-Jul-05 & & 1016.0 & & & & & & & & & & \\
\hline 144 & 4-Jul-05 & & 1144.0 & 18560.0 & 28.0 & & 120.0 & & & 460.0 & 85.8 & 0.19 & 0.33 \\
\hline 145 & $\overline{5-J u l-05}$ & & 980.0 & 18320.0 & 38.0 & & 1740 & & & 460.0 & 68.6 & 0.15 & 0.32 \\
\hline 146 & 6-Jul-05 & & 1136.0 & 18720.0 & 46.0 & & 120.0 & & & 460.0 & 76.6 & 0.17 & 0.31 \\
\hline 147 & 7-Jul-05 & & 1056.0 & 18000.0 & 38.0 & & 84.0 & & & & & & \\
\hline \multicolumn{2}{|c|}{ Batch Average } & 9 & 980.2 & 18537.0 & 41.5 & & 98.9 & & & 460.0 & 79.0 & 0.17 & 0.33 \\
\hline 148 & 8-Jul-05 & \multirow{20}{*}{$\frac{8}{8}$} & 1072.0 & 19280.0 & 36.0 & & 90.0 & & & & & & \\
\hline 149 & 9-Jul-05 & & & & & & & & & & & & \\
\hline 150 & 10-Jul-05 & & & & & & & & & & & & \\
\hline 151 & 11-Jul-05 & & 891.4 & 18288.0 & 36.6 & & 85.3 & & & & & & \\
\hline 152 & $12-J u l-05$ & & 901.9 & 18532.0 & 24.4 & & 97.5 & & & & & & \\
\hline 153 & 13-Jul-05 & & 965.1 & & & & & & & 460.0 & 80.4 & 0.17 & 0.35 \\
\hline 154 & 14-Jul-05 & & 1079.8 & 18531.8 & 38.6 & & 121.9 & & & 460.0 & 80.4 & 0.17 & 0.33 \\
\hline 155 & 15-Jul-05 & & 11420 & 19263.0 & 20.3 & & 79.2 & & & & & & \\
\hline 156 & 16-Jul-05 & & & & & & & & & 460.0 & 77.6 & 0.17 & \\
\hline 157 & 17-Jul-05 & & & & & & & & & & & & \\
\hline 158 & 18-Jul-05 & & 1123.7 & 18369.3 & 36.6 & & 81.3 & & & & & & \\
\hline 159 & 19-Jul-05 & & 953.0 & 19588.5 & 34.5 & & 97.5 & & & & & & \\
\hline 160 & $20-J u l-05$ & & 1093.2 & 18856.9 & 20.3 & & 73.2 & & & & & & \\
\hline 161 & 21-Jul-05 & & 1060.8 & 174752 & 24.4 & & 111.8 & & & & & & \\
\hline 162 & 22-Jul-05 & & 991.6 & & & & & & & & & & \\
\hline 163 & 23-Jul-05 & & & & & & & & & 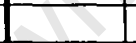 & & & \\
\hline 164 & 24-Jul-05 & & & & & & & & & + & & & \\
\hline 165 & 25-Jul-05 & & 832.3 & 19502.4 & 24.5 & & 79.6 & & e & & & & \\
\hline 166 & 26-Jul-05 & & 1079.2 & 19992.0 & 14.9 & & 1367 & & - & 460.0 & 60.9 & 0.13 & 0.29 \\
\hline 167 & $27-J u l-05$ & & 901.7 & 18849.6 & 32.6 & & 102.0 & & 8 & & & & \\
\hline \multicolumn{2}{|c|}{ Batch Average } & 10 & 1006.3 & 19004.9 & 28.6 & & 92.7 & & & 460.0 & 74.8 & 0.16 & 0.32 \\
\hline 168 & 28-Jul-05 & \multirow{21}{*}{$\begin{array}{l}\bar{\sigma} \\
\frac{\sigma}{\mathcal{O}} \\
\frac{\pi}{0} \\
\infty\end{array}$} & 824.2 & & & & & 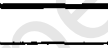 & & & & & \\
\hline 169 & 29-Jul-05 & & 1191.4 & 19176.0 & 34.7 & & 99.9 & - & & & & & \\
\hline 170 & 30-Ju\}-05 & & & & & & 1 & +2 & & & & & \\
\hline 171 & 31-Jut-05 & & & & & & & & & & & & \\
\hline 172 & 1-Aug-05 & & 838.9 & 20726.0 & 40.8 & 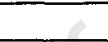 & 95.9 & & & 460.0 & 52.9 & 0.12 & 0.33 \\
\hline 173 & 2-Aug-05 & & 1087.3 & 19665.6 & 36.7 & 2 & 104.0 & & & 460.0 & 63.3 & 0.14 & 0.30 \\
\hline 174 & 3-Aug-05 & & 1122.0 & & & $\bar{C}$ & & & & 460.0 & 72.7 & 0.16 & 0.31 \\
\hline 175 & 4-Aug-05 & & 1014.0 & 18377.9 & 28.3 & 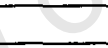 & 170.0 & & & & & & \\
\hline 176 & 5-Aug-05 & & 1129.4 & 17730.2 & 74.9 & & 121.4 & & & 460.0 & 73.4 & 0.16 & 0.31 \\
\hline 177 & 6-Aug-05 & & & & 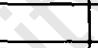 & & & & & & & & \\
\hline 178 & 7-Aug-05 & & & & 2 & & & & & & & & \\
\hline 179 & 8-Aug-05 & & 935.1 & 18701.8 & 42.5 & & 141.7 & & & 460.0 & 60.0 & 0.13 & 0.32 \\
\hline 180 & 9-Aug-05 & & 939.1 & 156253 & 32.4 & & 111.3 & & & & & & \\
\hline 181 & 10-Aug-05 & & 799.5 & 18944.6 & 36.4 & & 151.8 & & & & & & \\
\hline 182 & 11-Aug-05 & & & 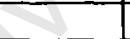 & & & & & & & & & \\
\hline 183 & 12-Aug-05 & & 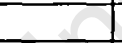 & -1 & & & & & & & & & \\
\hline 184 & 13-Aug-05 & & 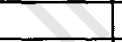 & & & & & & & & & & \\
\hline 185 & 14-Aug-05 & & 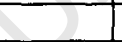 & & & & & & & & & & \\
\hline 186 & 15-Aug-05 & & 918.9 & 19349.4 & 46.6 & & 111.3 & & & & & & \\
\hline 187 & 16-Aug-05 & & 900.7 & 19673.3 & 38.5 & & 174.1 & & & 460.0 & 37.6 & 0.08 & 0.29 \\
\hline 188 & 17-Aug-05 & & 985.7 & 17649.3 & 28.3 & & 107.3 & & & 460.0 & 41.4 & 0.09 & 0.27 \\
\hline \multicolumn{2}{|c|}{ Batch Average } & 11 & 975.9 & 18999.4 & 40.0 & & 126.2 & & & 460.0 & 57.3 & 0.12 & 0.30 \\
\hline 189 & 18-Aug-05 & \multirow{14}{*}{$\frac{\infty}{\frac{\sigma}{\infty}}$} & 802.4 & & & & & & & & & & \\
\hline 190 & 19-Aug-05 & & 906.8 & & & & & & & & & & \\
\hline 191 & 20-Aug-05 & & & & & & & & & & & & \\
\hline 192 & 21-Aug-05 & & & & & & & & & & & & \\
\hline 193 & 22-Aug-05 & & 894.6 & 17973.1 & 52.6 & & 107.3 & & & & & & \\
\hline 194 & 23-Aug-05 & & 11352 & 18539.8 & 54.6 & & 101.2 & & & & & & \\
\hline 195 & 24-Aug-05 & & 959.6 & 17406.4 & 62.7 & & & & & 460.0 & 67.8 & 0.15 & 0.33 \\
\hline 196 & 25-Aug-05 & & 888.7 & 18944.6 & 40.5 & & & & & & & & \\
\hline 197 & 26-Aug-05 & & 830.2 & 18782.7 & 28.3 & & 137.6 & & & & & & \\
\hline 198 & 27-Aug-05 & & 866.3 & & & & & & & & & & \\
\hline 199 & 28-Aug-05 & & & & & & & & & & & & \\
\hline 200 & 29-Aug-05 & & 896.9 & 16434.9 & 40.5 & & 119.4 & & & & & & \\
\hline 201 & 30-Aug-05 & & 870.6 & & & & & & & & & & \\
\hline 202 & 31-Aug-05 & & 862.2 & 16839.7 & 526 & & 139.1 & & & & & & \\
\hline
\end{tabular}


A-1 COD MBR

\begin{tabular}{|c|c|c|c|c|c|c|c|c|c|c|c|c|c|}
\hline 203 & 1-Sep-05 & & \multirow[t]{2}{*}{987.5} & \multirow[t]{2}{*}{14977.6} & \multirow[t]{2}{*}{48.6} & & \multirow[t]{2}{*}{103.2} & & & \multirow[t]{2}{*}{460.0} & \multirow[t]{2}{*}{29.6} & 0.06 & 0.25 \\
\hline 204 & 2-Sep-05 & \multirow[b]{2}{*}{12} & & & & & & & & & & & \\
\hline \multicolumn{2}{|c|}{ Batch Average } & & 887.8 & 17487.4 & 47.6 & & 118.0 & & & 460.0 & 48.7 & 0.11 & 0.29 \\
\hline 205 & 3-Sep-05 & \multirow{14}{*}{$\frac{m}{\frac{8}{6}}$} & & & & & & & & & & & \\
\hline 206 & 4-Sep-05 & & & & & & & & & & & & \\
\hline 207 & 5-Sep-05 & & 829.5 & 15868.2 & 34.4 & & & & & 460.0 & 93.0 & 0.20 & 0.39 \\
\hline 208 & 6-Sep-05 & & 865.9 & 16093.4 & 40.6 & 48.8 & 101.6 & & 256.0 & 538.5 & 75.3 & 0.14 & 0.34 \\
\hline 209 & 7-Sep-05 & & 926.4 & & & & & & & & 70.2 & & \\
\hline 210 & 8-Sep-05 & & 707.1 & 16581.1 & 52.8 & 60.0 & 113.8 & 1081.0 & 268.2 & 550.7 & 77.8 & 0.14 & 0.38 \\
\hline 211 & 9-Sep-05 & & 934.7 & 19019.0 & 44.7 & 73.2 & 97.5 & 723.4 & 284.5 & 532.4 & 70.0 & 0.13 & 0.32 \\
\hline 212 & 10-Sep-05 & & 918.5 & & & & & & & & & & \\
\hline 213 & 11-Sep-05 & & & & & & & & & & & & \\
\hline 214 & 12-Sep-05 & & 910.3 & 16938.8 & 39.4 & 55.7 & 92.3 & 1005.4 & 222.3 & 425.5 & 72.3 & 0.17 & 0.35 \\
\hline 215 & 13-Sep-05: & & 827.9 & & & & & & & & & & \\
\hline 216 & 14-Sep-05 & & 735.6 & 17719.0 & 20.3 & 54.9 & 91.4 & 1003.8 & 213.4 & 404.4 & 48.2 & 0.12 & 0.36 \\
\hline 217 & 15-Sep-05 & & 837.2 & & & & & & & & & & \\
\hline 218 & 16-Sep-05 & & 918.5 & 16337.3 & 40.6 & 48.8 & 97.5 & & & 491.7 & & & \\
\hline Batch & Average & 13 & 855.6 & 16936.7 & 39.0 & 56.9 & 99.0 & 953.4 & 248.9 & 486.2 & 72.4 & 0.15 & 0.36 \\
\hline 219 & 17-Sep-05 & & 995.7 & & & & & & & & & & \\
\hline 220 & 18-Sep-05 & & & & & & & & & & & & \\
\hline 221 & 19-Sep-05 & & 866.9 & 18288.0 & 26.5 & & 185.5 & 1175.0 & 243.0 & 468.2 & 77.8 & 0.17 & 0.36 \\
\hline 222 & 20-Sep-05 & & 728.9 & & & & & & & & & & \\
\hline 223 & 21-Sep-05 & & 920.4 & 16581.1 & 50.8 & 68.5 & 134.7 & 12325 & 315.8 & 594.1 & 67.3 & 0.11 & 0.31 \\
\hline 224 & 22-Sep-05 & & 926.3 & & & & & & & & & & \\
\hline 225 & 23-Sep-05 & $\mathbf{4}$ & 939.2 & 18531.8 & 46.7 & 44.7 & 48.8 & 1365.5 & 256.0 & 654.3 & 71.7 & 0.11 & 0.30 \\
\hline 226 & 24-Sep-05 & - & 833.1 & & & & & & & & - & & \\
\hline 227 & 25-Sep-05 & $\frac{1}{5}$ & & & & & & & & 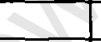 & & & \\
\hline 228 & 26-Sep-05 & + & 913.9 & 14467.8 & 36.6 & 38.6 & 111.8 & 1231.4 & 262.1 & 550.7 & & & \\
\hline 229 & 27-Sep-05 & $m$ & 924.7 & & & & & & 0 & & & & \\
\hline 230 & 28-Sep-05 & & 839.2 & 14711.7 & 37.5 & 59.6 & 139.1 & 1152.9 & 258.4 & 466.0 & 52.1 & 0.11 & 0.32 \\
\hline 231 & 29-Sep-05 & & 728.9 & & & & & & & & 27.1 & 0.06 & 0.32 \\
\hline 232 & 30-Sep-05 & & 11750 & & & & & 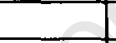 & - & & 30.3 & & 0.17 \\
\hline 233 & 1-Oct-05 & & & & & & & & & & & & \\
\hline 234 & $2-0 \mathrm{ct}-05$ & & 793.8 & & & & & $\overline{1}$ & & & & & \\
\hline 235 & $3-0 c t-05$ & & 897.6 & 18686.4 & 35.5 & & 88.7 & 0 & & & 60.1 & & 0.22 \\
\hline Batcl & Average & 14 & 869.9 & 16877.8 & 38.9 & 52.9 & 118.1 & 1231.5 & 267.1 & 546.7 & 55.2 & 0.11 & 0.29 \\
\hline 236 & 4-Oct-05 & & & & & & & & & & & & \\
\hline 237 & $5-0 c t-05$ & & & 18849.6 & 42.1 & 48.8 & 133.0 & 2607.7 & 368.1 & 634.2 & 69.6 & 0.11 & \\
\hline 238 & 6-Oct-05 & 5 & 1224.0 & & & & 2 & & & & & & \\
\hline 239 & $7-0 \mathrm{ct}-05$ & $\sigma$ & 1241.7 & 19665.6 & 28.8 & 46.6 & 122.0 & 2494.6 & 37.7 & 525.5 & 60.8 & 0.12 & 0.26 \\
\hline 240 & 8-Oct-05 & $\frac{1}{0}$ & 767.2 & & & & & & & & & & \\
\hline 241 & 9-Oct-05 & $\underset{\pi}{\pi}$ & & & $a_{1}$ & & & & & & & & \\
\hline 242 & $10-$ Oct-05 & $m$ & 860.9 & 17952.0 & 32.6 & 53.0 & 85.7 & 1362.7 & 359.0 & & & & \\
\hline 243 & 11-Oct-05 & & 701.8 & & & & & & & & & & \\
\hline 244 & $12-$ Oct-05 & & 807.8 & 20726.4 & 55.1 & 65.3 & 102.0 & 1256.6 & 273.4 & 673.2 & & & \\
\hline Batcl & Average & 15 & 933.9 & 19298.4 & 39.7 & 53.4 & 110.7 & 1930.4 & 259.5 & 611.0 & 65.2 & 0.11 & 0.26 \\
\hline 245 & 13-Oct-05 & & & 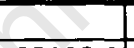 & & & & & & & & & \\
\hline 246 & 14-Oct-05 & & 979.2 & 20889.6 & 28.6 & 40.8 & 110.2 & 1468.8 & 297.8 & 971.0 & & & \\
\hline 247 & 15-Oct-05 & & 942.5 & & & & & & & & & & \\
\hline 248 & 16-Oct-05 & & & & & & & & & & & & \\
\hline 249 & 17-Oct-05 & & 950.6 & 17136.0 & 55.1 & 95.9 & 106.1 & 1240.3 & 291.7 & 883.3 & & & \\
\hline 250 & 18-Oct-05 & 1 & 995.5 & & & & & & & & & & \\
\hline 251 & 19-Oct-05 & 10 & 983.3 & 16809.6 & 30.6 & 79.6 & 75.5 & 1244.4 & 285.6 & 846.6 & 89.3 & 0.11 & 0.29 \\
\hline 252 & 20-Oct-05 & 8 & & & & & & & & & 93.0 & & \\
\hline 253 & $21-$ Oct-05 & 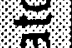 & 11832 & 17462.4 & 65.3 & 130.6 & 95.9 & 1334.2 & 291.7 & 814.0 & 84.6 & 0.10 & 0.26 \\
\hline 254 & 22-Oct-05 & 6 & & & & & & & & & & & \\
\hline 255 & 23-Oct-05 & & 938.4 & & & & & & & & & & \\
\hline 256 & 24-Oct-05 & & 930.2 & 19665.6 & 61.2 & 77.5 & 187.7 & 1268.9 & 328.4 & 846.6 & & & \\
\hline 257 & 25-Oct-05 & & 967.0 & & & & & & & & & & \\
\hline 258 & 26-Oct-05 & & 840.5 & 18931.2 & 61.2 & 83.6 & 124.4 & 1240.3 & 263.2 & 785.4 & 75.4 & 0.10 & 0.31 \\
\hline 259 & $27-0 c t-05$ & & & & & & & & & & & & \\
\hline Batcl & Average & 16 & 947.5 & 18482.4 & 50.3 & 84.7 & 116.6 & 1299.5 & 293.1 & 857.8 & 85.6 & 0.10 & 0.28 \\
\hline 260 & 28-Oct-05 & & 5998 & 17462.4 & 12.2 & 81.6 & 104.0 & 1224.0 & 202.0 & 911.9 & & & \\
\hline 261 & 29-Oct-05 & & 1011.8 & & & & & & & & & & \\
\hline 262 & 30-Oct-05 & & & & & & & & & & & & \\
\hline 263 & 31-Oct-05 & & 869.0 & 20563.2 & 46.9 & 87.7 & 104.0 & 1281.1 & 253.0 & & 68.8 & & 0.23 \\
\hline 264 & 1-Nov-05 & $N$ & 1289.3 & & & & & & & & 46.2 & & 0.16 \\
\hline
\end{tabular}




\begin{tabular}{|c|c|c|c|c|c|c|c|c|c|c|c|c|c|}
\hline 265 & 2-Nov-05 & \multirow{9}{*}{$\frac{\sigma}{\infty}$} & 995.5 & 20889.6 & 40.8 & 55.1 & 100.0 & 1146.5 & 255.0 & 779.3 & 95.2 & 0.12 & 0.30 \\
\hline 266 & 3-Nov-05 & & 9506 & & & & & & & & 52.8 & 0.07 & 0.27 \\
\hline 267 & 4-Nov-05 & & 869.0 & 21950.4 & 4.1 & 28.6 & 71.4 & 1179.1 & 246.8 & 728.3 & & & \\
\hline 268 & 5-Nov-05 & & 954.7 & & & & & & & & & & \\
\hline 269 & 6-Nov-05: & & & & & & & & & & & & \\
\hline 270 & 7-Nov-05 & & 865.0 & 18441.6 & 16.3 & 14.3 & 57.1 & 1150.6 & 7936 & 222.4 & & & \\
\hline 271 & 8-Nov-05 & & 983.3 & & & & & & & & & & \\
\hline 272 & $9-N o v-05$ & & 901.7 & 19584.0 & 46.9 & 79.6 & 89.8 & 1252.6 & 263.2 & 767.0 & & 0.09 & \\
\hline 273 & 10-Nov-05 & & 860.9 & & & & & & & & 70.2 & & 0.23 \\
\hline \multicolumn{2}{|c|}{ Batch Average } & 17 & 926.2 & 19815.2 & 27.9 & 57.8 & 87.7 & 1205.6 & 244.0 & 681.8 & 66.6 & 0.10 & $\overline{0.24}$ \\
\hline 274 & \multirow{2}{*}{$\begin{array}{l}11-\text { Nov-05 } \\
12-\text { Nov-05 }\end{array}$} & \multirow{18}{*}{$\frac{1}{4}$} & 897.6 & 25622.4 & 55.1 & 65.3 & 106.1 & 1183.2 & 297.8 & 577.3 & & & \\
\hline 275 & & & 8690 & & & & & & & & & & \\
\hline 276 & \multirow{2}{*}{\begin{tabular}{|l|}
$13-$ Nov-05 \\
$14-$ Nov-05
\end{tabular}} & & & & & & & & & & & & \\
\hline 277 & & & & & & & & & & & & & \\
\hline 278 & \multirow{2}{*}{$\frac{15-N o v-05}{16-N o v-05}$} & & 852.7 & 19584.0 & 32.6 & 40.8 & 89.8 & 1273.0 & & & 89.3 & & \\
\hline 279 & & & 954.7 & 20155.2 & 53.0 & 51.0 & 100.0 & 1379.0 & & & & & \\
\hline 280 & 17-Nov-05 & & & & & & & & & & & & \\
\hline 281 & \multirow{2}{*}{$\frac{18-N o v-05}{19-N o v-05}$} & & 1003.7 & 26356.8 & 49.0 & 51.0 & 87.7 & 1150.6 & 240.7 & 752.8 & 85.8 & 0.11 & 0.29 \\
\hline 282 & & & 889.4 & & & & & & & & & & \\
\hline 283 & $20-$ Nov-05 & & & & & & & & & & & & \\
\hline 284 & 21-Nov-05 & & 848.6 & & & & & & & & & & \\
\hline 285 & 22-Nov-05 & & 909.8 & 19992.0 & 61.2 & 46.9 & 71.4 & 1224.0 & 246.8 & 707.9 & 105.8 & 0.15 & 0.34 \\
\hline 286 & 23-Nov-05 & & 918.0 & 20318.4 & 34.7 & 34.7 & 114.2 & & & & & & \\
\hline 287 & 24-Nov-05 & & 958.8 & & & & & & & & 71.3 & & \\
\hline 288 & 25-Nov-05 & & 877.2 & 20073.6 & 44.9 & 26.5 & 91.8 & 1142.4 & 289.7 & 544.7 & 85.7 & 0.16 & 0.35 \\
\hline 289 & 26-Nov-05 & & & & & & & & & 2 & 112.2 & & \\
\hline 290 & 27-Nov-05 & & 905.8 & & & & & & & -2 & 103.7 & & \\
\hline 291 & 28-Nov-05 & & 999.6 & 20726.4 & 44.9 & 61.2 & 110.2 & & & & & & \\
\hline Batcl & Average & 18 & 914.2 & 21603.6 & 46.9 & 47.2 & 96.4 & 1225.4 & 268.8 & 645.7 & 93.4 & 0.14 & 0.33 \\
\hline 292 & 29-Nov-05 & & 1122.0 & & & & & & 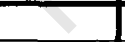 & & & & \\
\hline 293 & 30-Nov-05 & & 885.4 & 21134.4 & 40.8 & 63.2 & 161.2 & 1554.5 & 271.3 & 985.3 & & & \\
\hline 294 & 1-Dec-05 & & 1020.0 & & & & & $\square$ & & 826.2 & 106.4 & 0.13 & 0.30 \\
\hline 295 & 2-Dec-05 & & 1085.3 & 20726.4 & 85.7 & 73.4 & 130.6 & 1444.3 & 344.8 & 793.6 & & & \\
\hline 296 & 3-Dec-05 & & & & & & & -1 & & & & & \\
\hline 297 & 4-Dec-05 & & 975.1 & & & & & 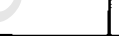 & & 818.0 & 125.6 & 0.15 & 0.33 \\
\hline 298 & 5-Dec-05 & & 1020.0 & 21950.4 & 20.4 & 61.2 & 128.5 & 1411.7 & 304.0 & 436.6 & & & \\
\hline 299 & 6-Dec-05 & & & & & $\gamma$ & & & & & & & \\
\hline 300 & 7-Dec-05 & 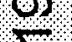 & 7670 & & & $\square$ & 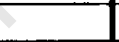 & & & 559.0 & & & \\
\hline 301 & 8-Dec-05 & 1 & 1060.8 & 23337.6 & 44.9 & 59.2 & 142.8 & 1419.8 & 285.6 & & & & \\
\hline 302 & 9-Dec-05 & 8 & 962.9 & 23011.2 & 34.7 & 36.7 & 138.7 & 1403.5 & 308.0 & 897.6 & & & \\
\hline 303 & 10-Dec-05 & $=$ & & & & & & & & & & & \\
\hline 304 & 11-Dec-05 & 10 & & & 0 & & & & & & 72.4 & 0.08 & \\
\hline 305 & 12-Dec-05 & & 1244.4 & & 0 & & & & & & 78.7 & 0.09 & 0.23 \\
\hline 306 & 13-Dec-05 & & 1211.8 & 23419.2 & 91.8 & 44.9 & 122.4 & 1685.0 & 322.3 & 883.3 & 85.0 & 0.10 & 0.25 \\
\hline 307 & 14-Dec-05 & & 1077.1 & +1 & & & & & & & 147.2 & 0.17 & 0.32 \\
\hline 308 & 15-Dec-05 & & 1183.2 & 18849.6 & 67.3 & 71.4 & 151.0 & & & & & & \\
\hline 309 & 16-Dec-05 & & 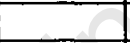 & 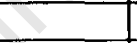 & & & & & & & & & \\
\hline 310 & 17-Dec-05 & & 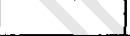 & & & & & & & & & & \\
\hline 311 & 18-Dec-05 & & & & & & & & & & & & \\
\hline 312 & 19-Dec-05 & & & & & & & & & & & & \\
\hline Batch & Average & 19 & 1070.7 & 21775.5 & 55.1 & 58.6 & 139.3 & 1486.5 & 306.0 & 774.9 & 102.5 & 0.12 & 0.29 \\
\hline 313 & 20-Dec-05 & & & & & & & & & & & & \\
\hline 314 & $21-\mathrm{Dec}-05$ & & & & & & & & & & & & \\
\hline 315 & 22-Dec-05 & & & & & & & & & & & & \\
\hline 316 & 23-Dec-05 & . & & & & & & & & & & & \\
\hline 317 & 24-Dec-05 & $8 \%$ & & & & & & & & & & & \\
\hline 318 & 25-Dec-05 & $1 \%$ & & & & & & & & & & & \\
\hline 319 & 26-Dec-05 & 8 & & & & & & & & & & & \\
\hline 320 & 27-Dec-05 & 8 & & & & & & & & & & & \\
\hline 321 & 28-Dec-05 & 10 & & & & & & & & & & & \\
\hline 322 & 29-Dec-05 & 88 & & & & & & & & & & & \\
\hline 323 & 30-Dec-05 & & & & & & & & & & & & \\
\hline 324 & 31-Dec-05 & & & & & & & & & & & & \\
\hline 325 & 1-Jan-06 & & & & & & & & & & & & \\
\hline 326 & 2-Jan-06 & & & & & & & & & & & & \\
\hline Batcl & Average & 20 & $n / a$ & $n / a$ & $\mathrm{n} / \mathrm{a}$ & $n / \mathbf{a}$ & $n / a$ & $n / a$ & $n / a$ & $n / a$ & $\mathrm{n} / \mathrm{a}$ & $n / a$ & $n / a$ \\
\hline 327 & 3-Jan-06 & & & & & & & & & & & & \\
\hline
\end{tabular}


A-1 COD MBR

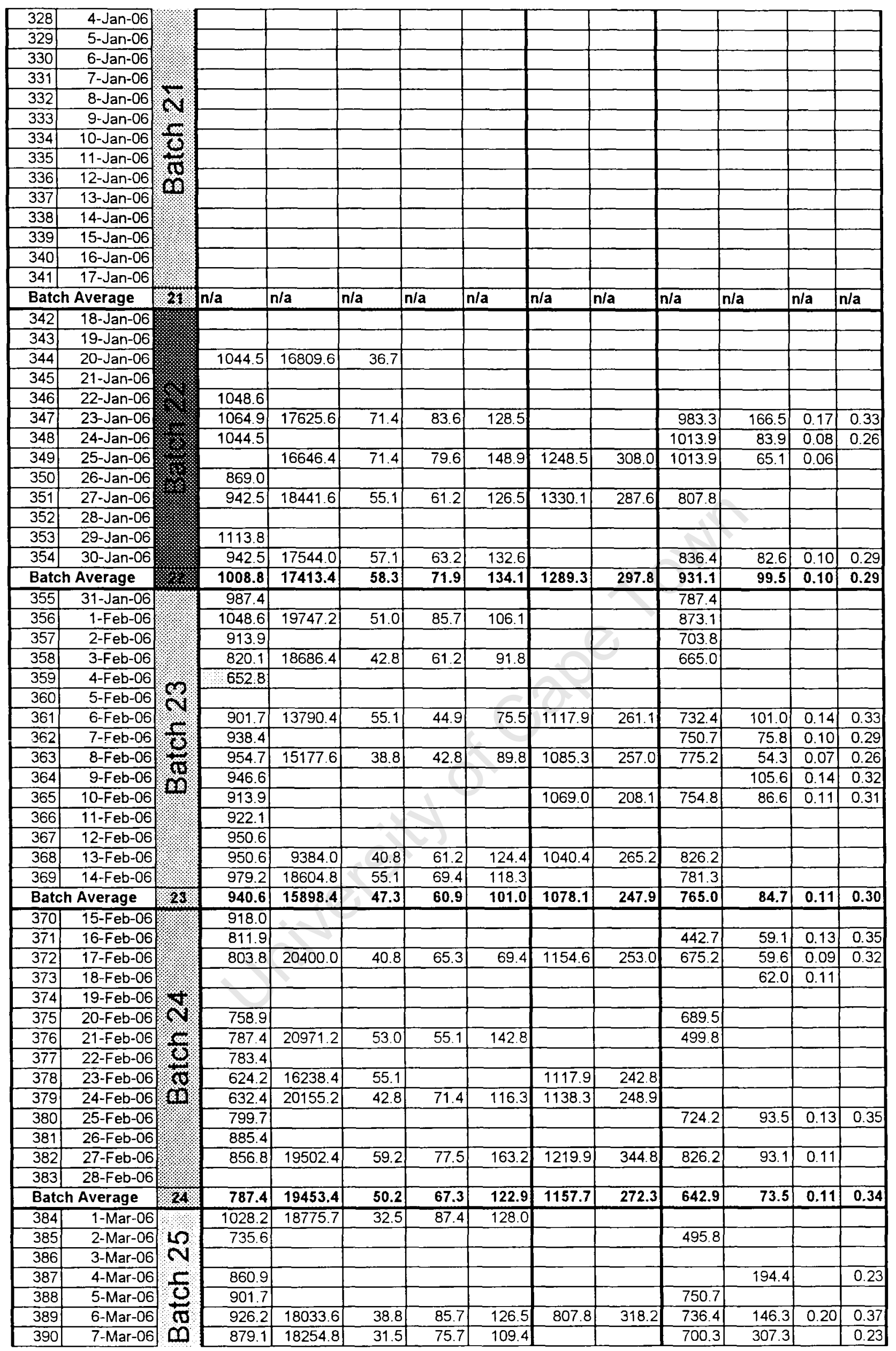


A-1 COD MBR

\begin{tabular}{|c|c|c|c|c|c|c|c|c|c|c|c|c|c|}
\hline 391 & 8-Mar-06 & & 1030.5 & 19012.0 & 65.2 & 96.7 & 113.6 & 820.2 & & 778.1 & 284.2 & & 0.19 \\
\hline \multicolumn{2}{|c|}{ Batch Average } & 25 & 908.9 & 18519.0 & 42.0 & 86.4 & 119.4 & 814.0 & 318.2 & 692.3 & 233.0 & 0.20 & 0.38 \\
\hline 392 & 9-Mar-06 & \multirow{15}{*}{$\begin{array}{c}\infty \\
\frac{\sigma}{\sigma} \\
\frac{\sigma}{\infty} \\
\infty\end{array}$} & 1179.1 & 19502.4 & 44.9 & 83.6 & & & & 958.8 & & & \\
\hline 393 & 10-Mar-06 & & & & & & & & & & 2274 & 0.19 & \\
\hline 394 & 11-Mar-06 & & 1195.4 & & & & & & & & & & \\
\hline 395 & 12-Mar-06 & & 897.6 & & & & & & & & & & \\
\hline 396 & 13-Mar-06 & & 958.8 & 19176.0 & 53.0 & 104.0 & 120.4 & & & & & & \\
\hline 397 & 14-Mar-06 & & 1007.8 & & & & & & & 781.3 & 148.9 & 0.19 & 0.35 \\
\hline 398 & 15-Mar-06 & & 910.3 & 19751.0 & 34.5 & 91.4 & 107.7 & 1475.2 & 278.4 & 737.6 & 124.2 & 0.17 & 0.35 \\
\hline 399 & 16-Mar-06 & & 1011.9 & & & & & & & 823.0 & 123.2 & 0.15 & 0.32 \\
\hline 400 & 17-Mar-06 & & 869.7 & 19588.5 & 54.9 & 89.4 & 132.1 & 1430.5 & 274.3 & 690.9 & 102.9 & 0.15 & 0.34 \\
\hline 401 & 18-Mar-06 & & 910.3 & & & & & & & 751.8 & 129.9 & 0.17 & 0.35 \\
\hline 402 & 19-Mar-06 & & 1081.0 & & & & & & & 747.8 & 148.9 & 0.20 & 0.35 \\
\hline 403 & 20-Mar-06 & & 1016.0 & 19100.8 & 61.0 & 89.4 & 166.6 & 1398.0 & 290.6 & 804.7 & 123.2 & 0.15 & 0.32 \\
\hline 404 & 21-Mar-06 & & & & & & & & & & 119.1 & 0.16 & \\
\hline 405 & 22-Mar-06 & & 886.0 & & & & & & & & & & \\
\hline 406 & 23-Mar-06 & & 934.7 & 19019.5 & 44.7 & 85.3 & 166.6 & 1341.1 & 280.4 & & 111.0 & 0.14 & 0.33 \\
\hline \multicolumn{2}{|c|}{ Batch Average } & 26 & 989.1 & 19356.4 & 48.8 & 90.5 & 138.7 & 1411.2 & 280.9 & 762.4 & 125.7 & 0.17 & 0.34 \\
\hline 407 & 24-Mar-06 & \multirow{15}{*}{$\begin{array}{l}N \\
N \\
\frac{\sigma}{U} \\
\frac{\sigma}{\pi} \\
\infty\end{array}$} & 1097.3 & 20238.7 & 38.6 & 93.5 & 158.5 & 1560.6 & 323.1 & 995.7 & & & \\
\hline 408 & 25-Mar-06 & & 938.8 & & & & & & & & & & \\
\hline 409 & 26-Mar-06 & & 930.7 & & & & & & & 749.8 & 132.6 & 0.18 & 0.35 \\
\hline 410 & 27-Mar-06 & & 1068.8 & 19425.9 & 14.2 & 0.0 & 144.3 & 1434.6 & 288.5 & 859.5 & 108.3 & 0.13 & 0.29 \\
\hline 411 & 28-Mar-06 & & 860.9 & & & & & & & 667.1 & 86.6 & 0.13 & 0.33 \\
\hline 412 & 29-Mar-06 & & 913.9 & 16809.6 & 12.2 & 49.0 & 114.2 & 1244.4 & 242.8 & 769.1 & 86.6 & 0.11 & 0.31 \\
\hline 413 & 30-Mar-06 & & 811.9 & & & & & & & 599.8 & & & 0.25 \\
\hline 414 & 31-Mar-06 & & 746.6 & 16646.4 & 26.5 & 69.4 & 126.5 & 1211.8 & 193.8 & & & & \\
\hline 415 & $1-A p r-0 E$ & & 799.7 & & & & & & -1 & 785.4 & 138.1 & 0.18 & 0.38 \\
\hline 416 & 2-Apr-06 & & 950.6 & & & & & & 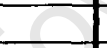 & 728.3 & 125.9 & 0.17 & 0.35 \\
\hline 417 & 3-Apr-06 & & 1028.2 & 20236.8 & 53.0 & 102.0 & 122.4 & 1346.4 & 259.1 & 860.9 & & & \\
\hline 418 & 4-Apr-06 & & 1293.4 & & & & & & & 897.6 & 151.6 & 0.17 & 0.30 \\
\hline 419 & 5-Apr-06 & & 1040.4 & 20073.6 & 51.0 & 89.8 & 138.7 & 1089.4 & 255.0 & 799.7 & & & \\
\hline 420 & 6-Apr-06 & & 1428:0 & & & & & & & & & & \\
\hline 421 & 7-Apr-06 & & 1089.4 & & & & & & & & & & \\
\hline \multicolumn{2}{|c|}{ Batch Average } & 27 & 969.3 & 18905.2 & 32.6 & 67.3 & 134.1 & 1314.5 & 260.4 & 792.1 & 118.5 & 0.15 & 0.32 \\
\hline 422 & 8-Apr-06 & \multirow{15}{*}{$\frac{1}{10}$} & 926.2 & & & & & & & & & & \\
\hline 423 & 9-Apr-06 & & 999.6 & & & & 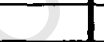 & & & & & & \\
\hline 424 & 10-Apr-06 & & 909.8 & 357408 & 53.0 & 104.0 & 102.0 & 1097.5 & 269.3 & & & & \\
\hline 425 & 11-Apr-06 & & & & & $\square$ & & & & 6528 & 71.7 & 0.11 & \\
\hline 426 & 12-Apr-06 & & 1069.0 & & & $\infty$ & & & & 787.4 & 117.8 & 0.15 & 0.31 \\
\hline 427 & 13-Apr-06 & & & 18931.2 & 30.6 & & 177.5 & 1093.4 & 230.5 & & 169.2 & & \\
\hline 428 & 14-Apr-06 & & 1024.1 & & 8 & & & & & 901.7 & 104.2 & 0.12 & 0.29 \\
\hline 429 & 15-Apr-06 & & 1028.2 & 18686.4 & 26.5 & 91.8 & 128.5 & 1007.8 & & 787.4 & 108.3 & 0.14 & 0.31 \\
\hline 430 & 16-Apr-06 & & 999.6 & 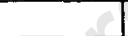 & \begin{tabular}{l|l}
2 & 1 \\
\end{tabular} & & & & & 767.0 & & & \\
\hline 431 & 17-Apr-06 & & 1166.9 & & & & & & & 860.9 & 141.9 & 0.16 & 0.31 \\
\hline 432 & 18-Apr-06 & & 1069.0 & 19257.6 & 73.4 & 126.5 & 134.6 & & & & 136.7 & & 0.19 \\
\hline 433 & 19-Apr-06 & & 1317.8 & +2 & & & & & & 901.7 & 106.9 & 0.12 & 0.25 \\
\hline 434 & 20-Apr-06 & & 1350.5 & & & & & & & 893.5 & & & \\
\hline 435 & 21-Apr-06 & & 1383.1 & 20726.4 & 73.4 & 106.1 & 120.4 & 1962.5 & 981.2 & 885.4 & & & \\
\hline 436 & 22-Apr-06 & & 1258.9 & & & & & & & 878.4 & 150.2 & & 0.16 \\
\hline \multicolumn{2}{|c|}{ Batch Average } & \multirow{14}{*}{$\frac{8}{6}$} & 1115.6 & 19400.4 & 51.4 & 107.1 & 132.6 & 1290.3 & 493.7 & 851.5 & 123.0 & 0.13 & 0.26 \\
\hline 437 & 23-Apr-06 & & 922.9 & & & & & & & 894.6 & & & \\
\hline 438 & 24-Apr-06 & & 1226.5 & 20806.7 & 52.6 & 103.2 & 153.8 & 1485.6 & 291.5 & 12468 & & & \\
\hline 439 & $25-\mathrm{Apr}-06$ & & 1028.2 & & & & & & & & 197.6 & 0.21 & 0.36 \\
\hline 440 & 26-Apr-06 & & 910.8 & 21373.4 & 30.4 & 81.0 & 115.4 & 1335.8 & 271.2 & 748.9 & 150.3 & 0.20 & 0.38 \\
\hline 441 & 27-Apr-06 & & 6324 & & & & & & & & & & \\
\hline 442 & 28-Apr-06 & & 999.6 & 21297.6 & 53.0 & 85.7 & 173.4 & 1354.6 & 306.0 & 799.7 & & & \\
\hline 443 & 29-Apr-06 & & 820.1 & & & & & & & 750.7 & & & \\
\hline 444 & 30-Apr-06 & & 926.2 & & & & & & & & 135.4 & 0.17 & 0.35 \\
\hline 445 & 1-May-06 & & 1040.4 & 20073.6 & 69.4 & 100.0 & 146.9 & 1342.3 & 253.0 & 889.4 & & & \\
\hline 446 & 2-May-06 & & 962.9 & & & & & & & & & & \\
\hline 447 & 3-May-06 & & 1105.7 & 18768.0 & 46.9 & 93.8 & 126.5 & 1256.6 & 244.8 & 881.3 & 94.7 & 0.11 & 0.27 \\
\hline 448 & 4-May-06 & & 967.0 & & & & & & & & 89.3 & & \\
\hline 449 & 5-May-06 & & 889.4 & 18360.0 & 44.9 & 106.1 & 144.8 & 1171.0 & 236.6 & 714.0 & 82.6 & 0.12 & 0.31 \\
\hline \multicolumn{2}{|c|}{ Batch Average } & 29 & 983.3 & 20113.2 & 49.5 & 95.0 & 143.5 & 1324.3 & 267.2 & 811.2 & 131.7 & 0.16 & 0.33 \\
\hline
\end{tabular}




\section{A-2 MBR TKN_FSA / TSS_VSS}

Appendix A-2

Measured TKN, FSA and solids concentrations in the MBR UCT system

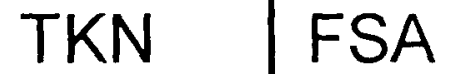

\begin{tabular}{|l|l|l|l|l|}
\hline Day & Date & SB \# & Infl. & ML \\
\hline
\end{tabular}

\section{TSS}

VSS

\begin{tabular}{|c|c|c|c|c|c|c|c|c|c|c|c|c|c|c|}
\hline & & & $(\mathrm{mgN} / \mathrm{l})$ & $(\mathrm{mgN} / \mathrm{l})$ & $(\mathrm{mgN} / \mathrm{l})$ & $(\mathrm{mgN} / \mathrm{l})$ & $(\mathrm{mgN} / \mathrm{l})$ & $(\mathrm{mgTSS} / \mathrm{l})$ & $(\mathrm{mgTSS} /)$ & $(m g T S S / l)$ & $(\mathrm{mgTSS} /)$ & $(m g \vee s S / l)$ & $\mid(m g \vee s s / 1)$ & $(m g V S S / 1)$ \\
\hline 19 & 1-Mar-05 & & & & & & & 6660 & 11514 & 19854 & & & 9738 & 16582 \\
\hline 20 & 2-Mar-05 & & & & & & & 8226 & 14256 & 17822 & & 6836 & 11658 & 14500 \\
\hline 21 & 3-Mar-05 & \multirow{16}{*}{$\frac{18}{48}$} & & & & & & 9374 & 15348 & 49526 & & 7554 & 12058 & 15144 \\
\hline 22 & 4-Mar-05 & & & & & & & 8018 & 14214 & 17276 & & 6626 & 11490 & 13850 \\
\hline 23 & 5-Mar-05 & & & & & & & & & & & & & \\
\hline 24 & 6-Mar-05 & & & & & & & & & & & & & \\
\hline 25 & 7-Mar-05 & & 104.4 & 1125.6 & 2.1 & 84.1 & 2.2 & 7768 & 12866 & 15066 & & 6496 & 10528 & 12268 \\
\hline 26 & 8 -Mar-05 & & 101.9 & 1192.8 & 1.8 & 88.2 & 0.6 & 8324 & 13916 & 17516 & & 6834 & 11206 & 14026 \\
\hline 27 & 9-Mar-05 & & 99.1 & 952.0 & 5.5 & 72.7 & 1.5 & 8020 & 13078 & 17186 & & 6664 & 10638 & 13840 \\
\hline 28 & 10-Mar-05 & & 112.0 & & & 86.0 & & & & & & & & \\
\hline 29 & 11-Mar-05 & & 89.3 & 1108.8 & 2.0 & 69.1 & 0.0 & 7010 & 12388 & 15754 & & 5756 & 10002 & 12594 \\
\hline 30 & 12-Mar-05 & & & & & & & & & & & & & \\
\hline 31 & 13-Mar-05 & & & & & & & & & & & & & \\
\hline 32 & 14-Mar-05 & & 94.5 & 940.8 & 3.8 & 75.7 & 1.3 & 7568 & 12132 & 15128 & & 6136 & 9658 & 12016 \\
\hline 33 & 15-Mar-05 & & 91.2 & $\overline{1212.4}$ & 2.2 & 69.7 & 0.9 & 8402 & 13890 & 17438 & & 6804 & 11108 & 13888 \\
\hline 34 & 16-Mar-05 & & 96.3 & 1092.0 & 4.3 & 80.7 & 1.6 & 8124 & 13272 & 17402 & & 6626 & 10688 & 13934 \\
\hline 35 & 17-Mar-05 & & 115.0 & 1092.0 & 4.3 & 94.6 & 1.6 & 6924 & 9580 & 15456 & & 5736 & 7850 & 12506 \\
\hline 36 & 18-Mar-05 & & 110.6 & & & 89.9 & & 6950 & 11644 & 14884 & & 5736 & 9430 & 11982 \\
\hline Batcl & h Average & 2 & 101.4 & 1089.6 & 3.3 & 81.1 & 1.2 & 7711 & 13275 & 16311 & & 6452 & 10681 & 13277 \\
\hline 37 & 19-Mar-05 & \multirow{17}{*}{$\frac{\infty}{\frac{c}{6}}$} & & & & & & & & & & & & \\
\hline 38 & 20-Mar-05 & & & & & & & & & & & & & \\
\hline 39 & 21-Mar-05 & & 140.0 & 1055.6 & 5.0 & 102.5 & 3.6 & 8800 & 12010 & 14144 & & 7380 & 9890 & 11686 \\
\hline 40 & 22-Mar-05 & & 89.0 & & & 65.7 & & 6636 & 12020 & 15212 & & 5474 & 9870 & 12442 \\
\hline 41 & 23-Mar-05 & & 123.1 & 1131.2 & 3.2 & 101.7 & 1.1 & 7382 & 12594 & 15292 & & & & \\
\hline 42 & 24-Mar-05 & & & & & & & 8222 & 12598 & 15540 & & 6896 & 10528 & 13002 \\
\hline 43 & 25-Mar-05 & & 141.4 & 1176.0 & 2.0 & 105.0 & 4.2 & 7658 & 12412 & 15306 & & 6524 & 10256 & 12566 \\
\hline 44 & 26-Маг-05 & & & & & & & & & & & & & \\
\hline 45 & 27-Mar-05 & & 114.8 & 1139.6 & 2.7 & 93.0 & 1.5 & 9460 & 13058 & 14362 & & 8054 & 10868 & 11996 \\
\hline 46 & 28-Mar-05 & & & & & & & & & & & & & \\
\hline 47 & 29-Mar-05 & & 111.2 & 1324.4 & 4.5 & 101.6 & 7.1 & 6482 & 15210 & 17650 & & 5678 & 12416 & 14376 \\
\hline 48 & $30-$ Mar-05 & & 151.8 & & & 134.7 & & & & & & & & \\
\hline 49 & 31-Mar-05 & & 134.7 & 1414.0 & 2.5 & 102.4 & 2.4 & 8034 & 14192 & 18604 & & 6792 & 11592 & 15208 \\
\hline 50 & 1-Apr-05 & & 150.1 & & $\theta$ & 109.9 & & & & & & & & \\
\hline 51 & 2-Aрг-05 & & 160.4 & 1274.0 & 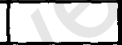 & 116.2 & 2.2 & 7760 & 13200 & 16770 & & 6396 & 10634 & 13400 \\
\hline 52 & 3-Apr-05 & & & & & & & & & & & & & \\
\hline 53 & 4-Apr-05 & & 166.8 & 1100.4 & 7.6 & 148.1 & 1.5 & 11860 & 14436 & 15570 & & 9698 & 11606 & 12454 \\
\hline Batcl & h Average & 3 & 139.4 & 1201.9 & 3.9 & 107.3 & 2.4 & 7826 & 13173 & 15845 & & 6649 & 10851 & 13014 \\
\hline 54 & 5-Арг-05 & \multirow{18}{*}{$\frac{9}{6}$} & 113.1 & 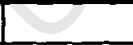 & & 83.9 & & 7036 & 13374 & 16450 & & 5956 & 10940 & 13374 \\
\hline 55 & 6-Apr-05 & & & & & & & & & & & & & \\
\hline 56 & 7-Apr-05 & & & & & & & & & & & & & \\
\hline 57 & 8-Apr-05 & & 97.4 & 1190.0 & & 64.7 & 1.1 & 7906 & 13178 & 15692 & & 6538 & 10578 & 12446 \\
\hline 58 & 9-Apr-05 & & & & & & & & & & & & & \\
\hline 59 & 10-Apr-05 & & 99.4 & 1293.6 & 1.7 & 68.6 & 1.8 & 6334 & 13040 & 16776 & & 5484 & 10694 & 13624 \\
\hline 60 & 11-Apr-05 & & 105.3 & & & 83.7 & & & & & & & & \\
\hline 61 & 12-Apr-05 & & 98.0 & 1313.2 & 5.0 & 75.4 & 1.3 & 8422 & 4562 & 18324 & & 6844 & 11596 & 14522 \\
\hline 62 & 13-Apr-05 & & 67.1 & & & 51.0 & & & & & & & & \\
\hline 63 & 14-Apr-05 & & 108.7 & 1341.2 & 3.2 & 80.0 & 2.2 & 8208 & 14136 & 19392 & & 6832 & 11462 & 15576 \\
\hline 64 & 15-Apr-05 & & 75.9 & & & 51.5 & & & & & & & & \\
\hline 65 & 16-Арг-05 & & 91.8 & 1156.4 & 1.6 & 60.5 & 2.2 & 7514 & 12710 & 16124 & & 6322 & 10250 & 12964 \\
\hline 66 & 17-Арr-05 & & & & & & & & & & & & & \\
\hline 67 & 18-Арг-05 & & 89.9 & & & 77.8 & & 5794 & 10516 & 14146 & & 4944 & 8574 & 11328 \\
\hline 68 & 19-Apr-05 & & 96.3 & 1148.0 & 2.1 & 62.7 & 0.0 & 6038 & 10764 & 13510 & & 5038 & 8694 & 10748 \\
\hline 69 & 20-Арг-05 & & 105.1 & & & 81.2 & & 5338 & 9706 & 12922 & & 4430 & 7806 & 10250 \\
\hline 70 & 21-Apr-05 & & 105.4 & & & 78.1 & & 6158 & 11316 & 14272 & & 5018 & 9076 & 11380 \\
\hline 71 & 22-Apr-05 & & 90.4 & & & 69.6 & & 4198 & 6642 & 7968 & & 3636 & 5564 & 6390 \\
\hline
\end{tabular}


A-2 MBR TKN FSA / TSS VSS

\begin{tabular}{|c|c|c|c|c|c|c|c|c|c|c|c|c|c|c|}
\hline 72 & 23-Apr-05 & & 86.5 & 686.0 & 4.1 & 59.9 & 1.0 & 4270 & 6866 & 8340 & & 3670 & 5640 & 6672 \\
\hline 73 & 24-Apr-05 & & & & & & & & & & & & & \\
\hline 74 & 25-Apr-05 & & 95.3 & 624.4 & 10.2 & 71.8 & 1.1 & 3090 & 6014 & 8744 & & 2730 & 5038 & 7110 \\
\hline 75 & 26-Apr-05 & & 94.2 & & & 71.8 & & & & & & & & \\
\hline \multicolumn{2}{|c|}{ Batch Average } & 4 & 97.0 & 1094.1 & 3.0 & 70.1 & 1.3 & 6177 & 10217 & 14051 & & 5188 & 8916 & 11260 \\
\hline \multicolumn{2}{|c|}{\begin{tabular}{|l|l|}
76 & $27-A p r-05$ \\
\end{tabular}} & \multirow[b]{8}{*}{4} & & & & & & & & & & & & \\
\hline \multicolumn{2}{|r|}{ 28-Apr-05 } & & & & & & & & & & & & & \\
\hline 78 & 29-Apr-05 & & 105.3 & 736.4 & 2.00 & 81.5 & 1.0 & 4686 & 7804 & 10350 & & 3972 & 6384 & 8282 \\
\hline 79 & 30-Apr-05 & & & & & & & & & & & & & \\
\hline 80 & 1-May-05 & & & & & & & & & & & & & \\
\hline 81 & 2-May-05 & & 12.0 & 856.8 & 2.59 & 126 & 0.8 & 7024 & 10490 & 12184 & & & & \\
\hline 82 & 3-May-05 & & & & & & & 3658 & 7534 & 16382 & & 3122 & 6204 & 13106 \\
\hline 83 & 4-May-05 & & 116.2 & 1500.8 & 2.80 & 88.3 & 1.7 & 2416 & 9092 & 16850 & & 1970 & 7340 & 12782 \\
\hline 84 & 5-May-05 & 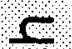 & 115.2 & & & 89.9 & & & & & & & & \\
\hline 85 & 6-May-05 & 3 & 93.1 & 1901.2 & 0.74 & 78.9 & 0.8 & & & & & & & \\
\hline 86 & 7-May-05 & (1) & 136.1 & & & & & & & & & & & \\
\hline 87 & 8-May-05 & 0 & 95.8 & & & & & & & & & & & \\
\hline 88 & 9-May-05 & & 119.4 & 1307.6 & 2.70 & 87.8 & 1.6 & 7048 & 12542 & 17218 & & 5840 & 10474 & 13788 \\
\hline 89 & 10-May-05 & & 99.1 & & & 84.3 & & 7770 & 12730 & 16156 & & 6516 & 10408 & 13148 \\
\hline 90 & 11-May-05 & & 108.2 & 1226.4 & 1.89 & 79.9 & 1.3 & 6740 & 12356 & 16728 & & 5642 & 9978 & 13406 \\
\hline 91 & 12-May-05 & & 123.8 & & & 103.5 & & 7636 & 13654 & 18604 & & 6282 & 10976 & 14888 \\
\hline 92 & 13-May-05 & & 113.1 & 1341.2 & 2.87 & 95.6 & 1.2 & 7112 & 13096 & 18412 & & 5938 & 10622 & 14820 \\
\hline 93 & 14-May-05 & & 125.7 & & & 96.0 & & & & & + & & & \\
\hline 94 & 15-May-05 & & & & & & & & & + & 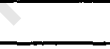 & & & \\
\hline \multicolumn{2}{|c|}{ Batch Average } & 5 & 112.6 & 1267.2 & 2.23 & 88.6 & 1.2 & 6010 & 11033 & 16567 & & 4910 & 9048 & 13705 \\
\hline 95 & 16-May-05 & \multirow{16}{*}{ 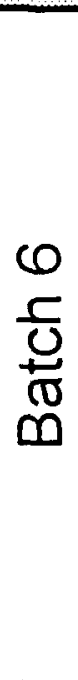 } & 119.6 & 1248.8 & 0.8 & 93.8 & 0.5 & 6948 & 11976 & 17042 & & 5806 & 9796 & 13784 \\
\hline 96 & 17-May-05 & & & & & & & 5536 & 12696 & 18386 & & 4438 & 10026 & 14576 \\
\hline 97 & 18-May-05 & & & & & & & & 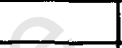 & & & & & \\
\hline 98 & 19-May-05 & & & & & & & 7592 & 12766 & 16940 & & 6170 & 10170 & 13450 \\
\hline 99 & 20-May-05 & & 113.0 & & 2.9 & 88.5 & 1.3 & 6974 & 12100 & 15856 & & 5640 & 9668 & 12678 \\
\hline 100 & 21-May-05 & & & & & & & 7092 & 12178 & 16056 & & 5926 & 10438 & 13268 \\
\hline 101 & 22-May-05 & & & & & & & & & & & & & \\
\hline 102 & 23-May-05 & & 148.1 & 1268.0 & 1.9 & 112.6 & 0.8 & 7808 & 13640 & 18318 & & 6502 & 11104 & 14832 \\
\hline 103 & 24-May-05 & & 146.6 & & & 117.0 & 2 & 10256 & 13372 & 14350 & & 8350 & 10770 & 11468 \\
\hline 104 & 25-May-05 & & 138.1 & 1151.0 & 0.7 & 109.2 & 0.9 & 7144 & 12866 & 17884 & & 5922 & 10442 & 14412 \\
\hline 105 & 26-May-05 & & 130.1 & & & 101.5 & & 6984 & 12678 & 17130 & & 5844 & 10334 & 13862 \\
\hline 106 & 27-May-05 & & 143.2 & 1184.4 & 1.8 & 100.3 & 1.1 & 7678 & 13362 & 17418 & & 6610 & 10932 & 14206 \\
\hline 107 & 28-May-05 & & & & & -1 & & & & & & & & \\
\hline 108 & 29-May-05 & & & & & & & & & & & & & \\
\hline 109 & 30-May-05 & & 132.9 & 1456.0 & 1.6 & 103.8 & 1.4 & 7604 & 15654 & 18724 & & 6194 & 12512 & 14860 \\
\hline 110 & 31-May-05 & & 130.8 & +2 & & 109.9 & & 8230 & 15822 & 18686 & & 6692 & 12630 & 14880 \\
\hline \multicolumn{2}{|c|}{ Batch Average } & 6 & 133.6 & 1261.6 & 1.6 & 104.1 & 1.0 & 7235 & 13026 & 17495 & & 5977 & 10563 & 14073 \\
\hline 111 & 1-Jun-05 & \multirow{14}{*}{$\frac{16}{6}$} & & & & & & 8534 & 15260 & 19886 & & 7016 & 12238 & 15888 \\
\hline 112 & 2-Jun-05 & & + & 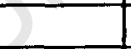 & & & & 7740 & 15348 & 20398 & & 6506 & 12470 & 16410 \\
\hline 113 & 3-Jun-05 & & 99.5 & 1397.2 & 2.3 & 79.8 & 0.9 & 7456 & 14464 & 20310 & & 6220 & 11624 & 16202 \\
\hline 114 & 4-Jun-05 & & & & & & & & & & & & & \\
\hline 115 & 5-Jun-05 & & & & & & & & & & & & & \\
\hline 116 & 6-Jun-05 & & 83.4 & 1288.0 & 1.1 & 83.8 & 0.2 & 8012 & 15670 & 20086 & & 6584 & 12518 & 15960 \\
\hline 117 & 7-Jun-05 & & 92.0 & & & 74.3 & & 7790 & 14822 & 17638 & & 6398 & 11942 & 14086 \\
\hline 118 & 8-Jun-05 & & 88.6 & 1184.4 & 1.2 & 71.8 & 0.0 & 7118 & 14294 & 17790 & & 5828 & 11382 & 14062 \\
\hline 119 & 9-Jun-05 & & 86.9 & & & 74.8 & & & & & & & & \\
\hline 120 & 10-Jun-05 & & 97.3 & 1159.2 & 2.1 & & 1.1 & 7122 & 14776 & 17408 & & 5888 & 11834 & 13808 \\
\hline 121 & 11-Jun-05 & & & & & & & 7140 & 12776 & 15562 & & 5852 & 9856 & 12456 \\
\hline 122 & 12-Jun-05 & & & & & & & 7134 & 12916 & 15578 & & 5872 & 10068 & 11830 \\
\hline 123 & 13-Jun-05 & & 95.5 & 1064.0 & 0.9 & 79.5 & 0.0 & 7390 & 14468 & 15926 & & 6022 & 11496 & 12656 \\
\hline 124 & 14-Jun-05 & & 101.8 & & & 78.5 & & & & & & & & \\
\hline \multicolumn{2}{|c|}{ Batch Average } & 18 & 93.1 & 1218.6 & 1.5 & 77.5 & 0.4 & 7434 & 14479 & 18058 & & 6219 & 11543 & 14336 \\
\hline 125 & 15-Jun-05 & \multirow{3}{*}{80} & & & & & & 7642 & 14634 & 17706 & & 6242 & 11660 & 13730 \\
\hline 126 & 16-Jun-05 & & & & & & & 10498 & 14564 & 15290 & & 8318 & 11410 & 11756 \\
\hline 127 & 17-Jun-05 & & & & & & & & & & & & & \\
\hline 128 & 18-Jun-05 & 18 & 129.1 & 1209.6 & 1.9 & 90.4 & 0.0 & 7594 & 15500 & 18470 & & 6252 & 12438 & 14688 \\
\hline 129 & 19-Jun-05 & 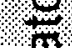 & 101.8 & & & & & & & & & & & \\
\hline
\end{tabular}


A-2 MBR TKN FSA / TSS VSS

\begin{tabular}{|c|c|c|c|c|c|c|c|c|c|c|c|c|c|c|}
\hline 130 & 20-Jun-05 & \multirow{3}{*}{9} & 123.1 & 1254.4 & 0.7 & 77.0 & 1.2 & 7896 & 15424 & 19078 & & 6500 & 12332 & 15122 \\
\hline 131 & 21-Jun-05 & & 137.3 & & & 52.6 & & 8530 & 14300 & 16704 & & 6904 & 11388 & 13166 \\
\hline 132 & 22-Jun-05 & & 145.4 & 1159.2 & 0.3 & 84.0 & 0.2 & 7580 & 14198 & 19356 & & 6184 & 11214 & 13248 \\
\hline \multicolumn{2}{|c|}{ Batch Average } & 8 & 127.3 & 1207.7 & 1.0 & 76.0 & 0.5 & 8290 & 14770 & 17767 & & 6733 & 11740 & 13618 \\
\hline 133 & 23-Jun-05 & \multirow{15}{*}{$\begin{array}{c}\sigma \\
\frac{5}{\sigma} \\
+\frac{0}{\infty}\end{array}$} & & & & & & 9200 & 14798 & 19356 & & 7486 & 11792 & 15290 \\
\hline 134 & 24-Jun-05 & & 133.3 & 9016 & 1.47 & 73.3 & 0.3 & 6958 & 12284 & 16258 & & 5746 & 9816 & 12730 \\
\hline 135 & 25-Jun-05 & & & & & & & & & & & & & \\
\hline 136 & 26-Jun-05 & & & & & & & & & & & & & \\
\hline 137 & 27-Jun-05 & & 103.7 & 1192.8 & 3.40 & 67.2 & 2.6 & 7158 & 13298 & 17490 & & 5870 & 10608 & 13806 \\
\hline 138 & 28-Jun-05 & & 115.5 & 1159.2 & 1.80 & 68.3 & 0.5 & 7576 & 13896 & 18298 & & 6174 & 11012 & 14348 \\
\hline 139 & 29-Jun-05 & & 121.5 & 1173.2 & 1.20 & 75.9 & 0.4 & 7458 & 13444 & 17460 & & 6028 & 10592 & 13614 \\
\hline 140 & 30-Jun-05 & & 117.6 & 1215.2 & 0.00 & 69.1 & 2.1 & 7652 & 13730 & 18280 & & 6192 & 10806 & 14236 \\
\hline 141 & 1-Jul-05 & & 112.4 & 1008.0 & 0.80 & 94.4 & 0.6 & 7152 & 13788 & 18634 & & 5790 & 10784 & 14448 \\
\hline 142 & 2-Jul-05 & & & & & & & & & & & & & \\
\hline 143 & 3-Jul-05 & & & & & & & & & & & & & \\
\hline 144 & 4-Jul-05 & & 121.9 & 1114.4 & 2.00 & 102.2 & 0.9 & 7176 & 13726 & 17996 & & 5808 & 10764 & 13894 \\
\hline 145 & 5-Jul-05 & & 124.1 & 1131.2 & 1.30 & 75.1 & 0.4 & 7466 & 13270 & 17364 & & 5866 & 10062 & 12912 \\
\hline 146 & 6-Jul-05 & & 119.4 & 1122.8 & 2.30 & 93.5 & 0.7 & 7370 & 13626 & 18074 & & 6062 & 10840 & 14174 \\
\hline 147 & 7-Jul-05 & & 108.4 & 1162.0 & 1.80 & 67.8 & 0.0 & 6896 & 13142 & 18328 & & 5632 & 10428 & 14492 \\
\hline \multicolumn{2}{|c|}{ Batch Average } & 9 & 117.8 & 1142.1 & 1.4 & 78.7 & 0.7 & 7286 & 13420 & 18128 & & 5917 & 10571 & 13995 \\
\hline 148 & 8-Jul-05 & \multirow{20}{*}{$\frac{1}{\frac{1}{6}}$} & 91.3 & 1187.2 & 1.7 & 71.4 & 0.4 & 6830 & 13078 & 18574 & & 5532 & 10256 & 14492 \\
\hline 149 & 9-Jul-05 & & & & & & & & & & & & & \\
\hline 150 & 10-Jul-05 & & & & & & & & & & & & & \\
\hline 151 & 11-Jul-05 & & 91.7 & 1229.2 & 1.8 & 58.8 & 0.4 & 7550 & 14802 & 17192 & 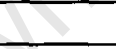 & 6272 & 11952 & 13790 \\
\hline 152 & 12-Jul-05 & & 91.6 & 1290.8 & 1.4 & 56.8 & 0.4 & 7450 & 13890 & 17348 & & 6212 & 11246 & 13870 \\
\hline 153 & 13-Jul-05 & & 93.3 & & & 73.6 & & 7492 & 13790 & 17052 & & 6272 & 11166 & 13650 \\
\hline 154 & 14-Jut-05 & & 1001 & 1117.2 & 2.5 & 73.2 & 0.9 & 7798 & 14576 & 17972 & & 6552 & 11918 & 14586 \\
\hline 155 & 15-Jul-05 & & 90.9 & 1226.4 & 0.8 & 73.9 & 0.4 & 6882 & 14406 & 18120 & & 5760 & 11696 & 14576 \\
\hline 156 & 16-Jul-05 & & & & & & & & 3 & & & & & \\
\hline 157 & 17-Jut-05 & & & & & & & & & & & & & \\
\hline 158 & 18-Jul-05 & & 99.7 & 1162.0 & 1.8 & 69.2 & 0.7 & 7428 & 14908 & 16610 & & 6230 & 12200 & 13450 \\
\hline 159 & 19-Jul-05 & & 92.0 & 1125.6 & 1.6 & 62.1 & 0.1 & 6990 & 13886 & 16980 & & 5976 & 11462 & 13930 \\
\hline 160 & 20-Jul-05 & & 87.5 & 1150.8 & 1.4 & 62.9 & 0.5 & 6986 & 13394 & 17082 & & 5928 & 11058 & 14028 \\
\hline 161 & 21-Jul-05 & & 95.1 & 1178.8 & 1.5 & 67.0 & 0.6 & 7324 & 14556 & 18674 & & 6352 & 12252 & 15596 \\
\hline 162 & 22-Jul-05 & & 87.9 & & & 70.1 & & & & & & & & \\
\hline 163 & 23-Jul-05 & & & & & & & 8096 & 14148 & 17590 & & 6750 & 11614 & 14322 \\
\hline 164 & 24-Jul-05 & & & & & +2 & & & & & & & & \\
\hline 165 & 25-Jul-05 & & 90.9 & 1240.4 & 1.4 & 72.9 & 12 & 7778 & 15484 & 18180 & & 6736 & 12972 & 15052 \\
\hline 166 & 26-Jul-05 & & 87.5 & 1243.2 & 1.4 & 69.6 & 0.1 & 7944 & 13912 & 17922 & & 6632 & 11396 & 14606 \\
\hline 167 & 27-Jul-05 & & 89.5 & 1204.0 & 2.1 & 64.6 & 0.2 & 7658 & 15818 & 17602 & & 6422 & 12958 & 14318 \\
\hline \multicolumn{2}{|c|}{ Batch Average } & \multirow{22}{*}{$\begin{array}{c}F \\
\frac{r}{0} \\
\frac{\sigma}{\sigma} \\
\infty\end{array}$} & 91.5 & 1196.3 & 1.5 & 67.6 & 0.4 & 7443 & 14332 & 17636 & & 6315 & 11838 & 14205 \\
\hline 168 & 28-Jul-05 & & & & & & & 7830 & 14388 & 19344 & & 6660 & 11828 & 15776 \\
\hline 169 & 29-Jul-05 & & 90.7 & 1232.0 & 1.1 & 591 & 0.7 & 7188 & 14482 & 17474 & & 6112 & 11930 & 14242 \\
\hline 170 & 30-Jul-05 & & & & & & & 6802 & 12308 & 16090 & & 5744 & 10078 & 13088 \\
\hline 171 & $31-J u l-05$ & & & 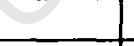 & & & & & & & & & & \\
\hline 172 & 1-Aug-05 & & 88.8 & 1285.2 & 0.3 & 75.4 & 0.5 & 8018 & 14040 & 19264 & & 6666 & 11470 & 15634 \\
\hline 173 & 2-Aug-05 & & 95.3 & 1302.0 & 0.4 & 73.2 & 0.7 & 6812 & 14244 & 17754 & & 5706 & 11538 & 14332 \\
\hline 174 & 3-Aug-05 & & 102.5 & & & 80.9 & & 6812 & 14244 & 17754 & & 5706 & 11538 & 14332 \\
\hline 175 & 4-Aug-05 & & 93.7 & 1120.0 & 1.1 & 70.4 & 0.2 & 6864 & 12932 & 17668 & & 5834 & 10650 & 14444 \\
\hline 176 & 5-Aug-05 & & 1088 & 1111.6 & 1.1 & 82.2 & & 7140 & 13054 & 15810 & & 6080 & 10720 & 12908 \\
\hline 177 & 6-Aug-05 & & & & & & & & & & & & & \\
\hline 178 & 7-Aug-05 & & & & & & & & & & & & & \\
\hline 179 & 8-Aug-05 & & 95.3 & 1128.4 & 0.6 & 75.7 & 0.2 & 6642 & 15644 & 16910 & & 5664 & 12768 & 13796 \\
\hline 180 & 9-Aug-05 & & 94.9 & 1058.4 & 1.3 & 82.0 & 0.2 & 6526 & 12296 & 16064 & & 5488 & 10006 & 13026 \\
\hline 181 & 10-Aug-05 & & 97.1 & 1226.4 & 1.2 & 73.9 & 0.2 & 7030 & 13036 & 17368 & & 5832 & 10560 & 14022 \\
\hline 182 & 11-Aug-05 & & & & & & & 8290 & 14294 & 15880 & & 6972 & 11698 & 12928 \\
\hline 183 & 12-Aug-05 & & & & & & & 7214 & 13238 & 15492 & & 6070 & 10800 & 12584 \\
\hline 184 & 13-Aug-05 & & & & & & & & & & & & & \\
\hline 185 & 14-Aug-05 & & & & & & & & & & & & & \\
\hline 186 & 15-Aug-05 & & 89.2 & 1120.0 & 0.7 & 74.9 & 0.0 & 7668 & 15722 & 16800 & & 6388 & 12814 & 13614 \\
\hline 187 & 16-Aug-05 & & 89.6 & 1128.4 & 0.8 & 78.0 & 0.4 & 7644 & 14266 & 17330 & & 6290 & 11508 & 14022 \\
\hline 188 & 17-Aug-05 & & 89.5 & 1106.0 & 1.1 & 73.7 & 0.0 & 7474 & 13468 & 17400 & & 6144 & 10894 & 13950 \\
\hline
\end{tabular}


A-2 MBR TKN FSA / TSS VSS

\begin{tabular}{|c|c|c|c|c|c|c|c|c|c|c|c|c|c|c|}
\hline \multicolumn{2}{|c|}{ Batch Average } & 11 & 93.3 & 1165.3 & 0.9 & 76.4 & 0.3 & 7178 & 13854 & 17150 & & 6026 & 11300 & 13795 \\
\hline 189 & 18-Aug-05 & \multirow{16}{*}{$\frac{\infty}{\frac{1}{9}}$} & 69.2 & & & 54.9 & & 7564 & 12710 & 16894 & & 6280 & 10324 & 13672 \\
\hline 190 & 19-Aug-05 & & & & & & & 7402 & 12730 & 16146 & & 6118 & 10274 & 12930 \\
\hline \multirow{2}{*}{\multicolumn{2}{|c|}{\begin{tabular}{|l|l|}
191 & 20 -Aug-05 \\
192 & $21-$ Aug-05 \\
\end{tabular}}} & & & & & & & & & & & & & \\
\hline & & & & & & & & & & & & & & \\
\hline 193 & 22-Aug-05 & & & & & & & 6760 & 13432 & 15800 & & 5674 & 10878 & 12666 \\
\hline \multirow{2}{*}{$\begin{array}{l}194 \\
195\end{array}$} & 23-Aug-05 & & 138.5 & & & 111.2 & & 8724 & 12646 & 15710 & & 7200 & 10254 & 12670 \\
\hline & 24-Aug-05 & & 144.3 & & & 107.4 & & 10660 & 14932 & 15938 & & 8684 & 12082 & 12744 \\
\hline \multirow{2}{*}{$\begin{array}{l}196 \\
197 \\
\end{array}$} & 25-Aug-05 & & 110.3 & 1190.0 & 0.6 & 86.8 & 1.0 & 8038 & 13800 & 17608 & & 6704 & 11270 & 14192 \\
\hline & 26-Aug-05 & & 110.0 & 1181.6 & 1.5 & 87.4 & & 7370 & 12824 & 17518 & & 6026 & 10224 & 13910 \\
\hline \multirow{2}{*}{$\begin{array}{l}198 \\
199\end{array}$} & 27-Aug-05 & & & & & & & 7378 & 13330 & 16752 & & 6146 & 10772 & 13450 \\
\hline & 28-Aug-05 & & & & & & & & & & & & & \\
\hline 200 & 29-Aug-05 & & 142.1 & 994.0 & 0.0 & 107.1 & 0.8 & 10778 & 14886 & 15280 & & 8722 & 11866 & 12128 \\
\hline \multirow{2}{*}{$\frac{201}{202}$} & 30-Aug-05 & & 128.1 & & & 98.3 & & 7786 & 13014 & 16718 & & 6374 & 10328 & 13268 \\
\hline & 31-Aug-05 & & 137.5 & 1089.2 & 1.5 & 97.4 & 0.4 & 6262 & 14054 & 14960 & & 5164 & 11142 & 13176 \\
\hline$\frac{202}{203}$ & $1-$ Sep-05 & & 142.9 & 996.8 & 1.8 & 101.4 & 0.4 & 7564 & 12582 & 15280 & & 6410 & 10098 & 12104 \\
\hline \multirow{2}{*}{$\frac{204}{\text { Batch }}$} & 2-Sep-05 & & & & & & & 7696 & 12194 & 15086 & & 6330 & 9654 & 13868 \\
\hline & h Average & 12 & 131.7 & 1090.3 & 1.1 & 99.6 & 0.6 & 7504 & 13318 & 16130 & & 6221 & 10705 & 13137 \\
\hline 205 & 3-Sep-05 & & & & & & & & & & & & & \\
\hline 206 & 4-Sep-05 & & & & & & & & & & & & & \\
\hline 207 & $5-$ Sep-05 & & 87.4 & 910.0 & 0.8 & 75.1 & 0.4 & 11644 & 14108 & 14912 & & 9302 & 11196 & 11780 \\
\hline 208 & 6-Sep-05 & & 99.5 & 870.8 & 0.5 & 69.4 & 0.4 & 7606 & 13556 & 18050 & & 6484 & 11032 & 14496 \\
\hline 209 & 7-Sep-05 & $\infty$ & 103.5 & & & 68.2 & & 7542 & 13792 & 18458 & & 6296 & 11118 & 14700 \\
\hline 210 & 8 -Sep-05 & r. & 111.6 & 1069.6 & 1.8 & 112.3 & 0.7 & 7274 & 13128 & 17302 & & 5968 & 10520 & 13692 \\
\hline 211 & 9-Sep-05 & 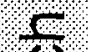 & 101.5 & 1142.4 & 1.4 & 82.1 & 0.0 & 9246 & 14814 & 17970 & & 7402 & 11654 & 14042 \\
\hline 212 & 10-Sep-05 & $\$$ & & & & & & & 2 & -1 & & & & \\
\hline 213 & 11-Sep-05 & 0 & & & & & & & & & & & & \\
\hline 214 & 12-Sep-05 & m & 101.9 & 1075.2 & 1.6 & 86.4 & 0.6 & 7160 & 12472 & 17118 & & 5796 & 9804 & 13352 \\
\hline 215 & 13-Sep-05 & & 101.5 & & & 78.0 & & 7002 & 12362 & 15252 & & 5836 & 9860 & 12056 \\
\hline 216 & 14-Sep-05 & & 99.3 & 1100.4 & 1.7 & 91.1 & 0.3 & 6756 & 12904 & 17080 & & 5572 & 10268 & 13422 \\
\hline 217 & 15-Sep-05 & & & & & & & 6622 & 14436 & 16782 & & 5368 & 11368 & 13114 \\
\hline 218 & 16-Sep-05 & & 105.7 & 1229.6 & 2.3 & 83.1 & 0.6 & 6756 & 13768 & 15878 & & 5550 & 10840 & 12302 \\
\hline Batc & h Average & 13 & 101.3 & 1056.9 & 1.4 & 79.2 & 0.4 & 7329 & 13534 & 16880 & & 6030 & 10766 & 13296 \\
\hline 219 & 17-Sep-05 & & & & & & & & & & & & & \\
\hline 220 & $18-S$ & & & & & & 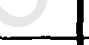 & & & & & & & \\
\hline 221 & 19-Sep-05 & & 113.1 & 1278.3 & 1.6 & 94.0 & 0.5 & & & & & & & \\
\hline 222 & 20-Sep-05 & & & & & & & 8084 & 12834 & 16836 & & 6508 & 10076 & 13150 \\
\hline 223 & 21-Sep-05 & & 114.8 & 1168.7 & 1.8 & 92.3 & 0.4 & 7496 & 12528 & 16008 & & 5992 & 9774 & 12448 \\
\hline 224 & $22-S e p-05$ & & 102.3 & & - & 85.1 & & 7088 & 12618 & 16158 & & 5750 & 9956 & 12662 \\
\hline 225 & 23-Sep-05 & 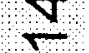 & 106.3 & 966.0 & 1.7 & 84.1 & 1.2 & 6840 & 11740 & 15774 & & 5522 & 9158 & 12204 \\
\hline 226 & 24-Sep-05 & & & $\pi$ & & & & & & & & & & \\
\hline 227 & 25-Sep-05 & 0 & & & & & & & & & & & & \\
\hline 228 & 26-Sep-05 & 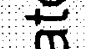 & 106.3 & 775.6 & 0.8 & 89.3 & 0.4 & 6844 & 11350 & 14286 & & 5508 & 8900 & 11068 \\
\hline 229 & 27-Sep-05 & $m$ & 122.9 & & & 104.9 & & 7588 & 12310 & 15584 & & 6284 & 9846 & 12322 \\
\hline 230 & 28-Sep-05 & & 109.6 & 856.8 & 1.1 & 94.2 & 0.0 & 9484 & 12556 & 13808 & & 7526 & 9784 & 10714 \\
\hline 231 & 29-Sep-05 & & 117.6 & & & 97.3 & & 8476 & 13958 & 17666 & & 6850 & 10974 & 13832 \\
\hline 232 & 30-Sep-05 & & 135.2 & & & 109.2 & & 8152 & 13842 & 17414 & & 6686 & 10926 & 13748 \\
\hline 233 & 1-Oct-05 & & & & & & & & & & & & & \\
\hline 234 & 2-Oct-05 & & & & & & & & & & & & & \\
\hline 235 & 3-Oct-05 & & \begin{tabular}{|l|}
118.7 \\
\end{tabular} & 1279.6 & 0.6 & 96.6 & 0.0 & 8326 & 13614 & 17084 & & 6628 & 10612 & 13246 \\
\hline Batc & ch Average & 14 & 112.4 & 1054.2 & 1.3 & 94.7 & 0.4 & 7838 & 12735 & 16062 & & 6325 & 10001 & 12539 \\
\hline 236 & 4-Oct-05 & & & & & & & 8716 & 13510 & 16628 & & 6994 & 10614 & 12966 \\
\hline 237 & 5-Oct-05 & & 106.1 & 1220.8 & 1.0 & 76.2 & 0.9 & 8768 & 13740 & 17294 & & 7056 & 10844 & 13564 \\
\hline 238 & 6-Oct-05 & & & & & & & & & & & & & \\
\hline 239 & 7-Oct-05 & & 109.5 & 1254.4 & 1.5 & 83.2 & 0.2 & 8432 & 13898 & 18212 & & 6808 & 11008 & 14316 \\
\hline 240 & 8-Oct-05 & $\frac{1}{0}$ & & & & & & & & & & & & \\
\hline 241 & 9-Oct-05 & & & & & & & & & & & & & \\
\hline 242 & 10-Oct-05 & $m$ & 120.4 & 1033.2 & 2.4 & 90.2 & 3.9 & 8402 & 13472 & 17104 & & 6866 & 10704 & 13488 \\
\hline 243 & 11-Oct-05 & & & & & & & 6943 & 11153 & 16947 & 12308 & 5697 & 8872 & 13373 \\
\hline 244 & $12-$ Oct-05 & & 98.3 & 1380.4 & 0.4 & 80.4 & 0.0 & 7032 & 13152 & 18884 & & 5856 & 10564 & 15032 \\
\hline Batc & ch Average & 15 & 108.6 & 1222.2 & 1.3 & 82.5 & 1.3 & 8049 & 13554 & 17512 & 12308 & 6546 & 10747 & 13790 \\
\hline 245 & 13-Oct-05 & & & & & & & 6942 & 13326 & 18108 & & 5728 & 10618 & 14310 \\
\hline
\end{tabular}


A-2 MBR TKN FSA / TSS VSS

\begin{tabular}{|c|c|c|c|c|c|c|c|c|c|c|c|c|c|c|}
\hline 246 & 14-Oct-05 & \multirow{14}{*}{$\frac{6}{6}$} & 94.4 & 1316.0 & 1.9 & 77.0 & 0.0 & 6958 & 12960 & 18024 & 13366 & 5798 & 10374 & 14316 \\
\hline 247 & $15-$-Oct-05 & & & & & & & & & & & & & \\
\hline 248 & 16-Oct-05 & & & & & & & & & & & & & \\
\hline 249 & 17-Oct-05 & & 105.3 & 1047.2 & 3.7 & 83.4 & 1.0 & 6966 & 12876 & 17208 & & 5790 & 10286 & 13534 \\
\hline 250 & 18 -Oct-05 & & & & & & & 6756 & 12496 & 16656 & & 5608 & 9956 & 13138 \\
\hline 251 & 19-Oct-05 & & 105.8 & 1027.6 & 3.4 & 84.0 & 0.2 & 8342 & 12938 & 15898 & & 8710 & 10158 & 12418 \\
\hline 252 & $20-$-ct-05 & & & & & & & 7880 & 12820 & 15852 & 12862 & 6442 & 10178 & 12474 \\
\hline 253 & $21-$ Oct-05 & & 137.8 & 1187.2 & 1.5 & 79.2 & 0.9 & 7088 & 12548 & 16256 & 12370 & 5770 & 9888 & 12702 \\
\hline 254 & 22-Oct-05 & & & & & & & & & & & & & \\
\hline 255 & 23-Oct-05 & & & & & & & & & & & & & \\
\hline 256 & 24-Oct-05 & & 108.9 & 1503.6 & 0.0 & 73.9 & 0.5 & 7148 & 13178 & 17180 & 13354 & 5820 & 10378 & 13382 \\
\hline 257 & $25-$ Oct-05 & & & & & & & 6980 & 12864 & 17508 & 12716 & 5654 & 10108 & 13648 \\
\hline 258 & 26-Oct-05 & & 101.1 & 1055.6 & 0.5 & 76.4 & 0.0 & 7406 & 13174 & 17956 & 13030 & 6020 & 10386 & 14012 \\
\hline 259 & 27-Oct-05 & & & & & & & 7448 & 13262 & 17620 & 13358 & 7082 & 10420 & 13744 \\
\hline Batc & h Average & 16 & 108.9 & 1189.5 & 1.8 & 79.0 & 0.4 & 7157 & 12949 & 17115 & 13008 & 5971 & 10250 & 13425 \\
\hline 260 & 28 -Oct-05 & \multirow{14}{*}{$\begin{array}{c}\frac{N}{\sigma} \\
\frac{\sigma}{0} \\
\frac{\sigma}{0}\end{array}$} & 96.6 & 1092.0 & 4.0 & 83.4 & 1.8 & 6784 & 12696 & 17398 & 13026 & 5568 & 10066 & 13562 \\
\hline 261 & 29-Oct-05 & & & & & & & & & & & & & \\
\hline 262 & 30-Oct-05 & & & & & & & & & & & & & \\
\hline 263 & 31-Oct-05 & & 92.4 & 1268.4 & 1.3 & 64.7 & 0.8 & 6996 & 13296 & 18124 & 13148 & 5710 & 10488 & 14188 \\
\hline 264 & 1-Nov-05 & & & & & & & 8262 & 14022 & 17904 & 13838 & 6698 & 11092 & 14036 \\
\hline 265 & 2-Nov-05 & & 97.4 & 1327.2 & 1.7 & 100.5 & 1.5 & 7176 & 13910 & 18346 & 13384 & 5878 & 11020 & 14426 \\
\hline 266 & 3-Nov-05 & & & & & & & & & & & & & \\
\hline 267 & 4-Nov-05 & & 84.8 & 1506.4 & 2.6 & 75.9 & 1.2 & 6844 & 13162 & 19954 & 13180 & 5786 & 10452 & 15620 \\
\hline 268 & 5-Nov-05 & & & & & & & & & & & & & \\
\hline 269 & 6-Nov-05 & & & & & & & & & & & & & \\
\hline 270 & 7-Nov-05 & & 98.8 & 1302.0 & 2.6 & 76.2 & 1.5 & 8510 & 14142 & 17618 & 14250 & 6918 & 11228 & 13892 \\
\hline 271 & 8-Nov-05 & & & & & & & 8442 & 14130 & 18548 & 12866 & 6950 & 11298 & 14740 \\
\hline 272 & 9-Nov-05 & & 90.4 & 1366.4 & 1.6 & 67.5 & 1.1 & 7650 & 13726 & 18290 & 13772 & 6238 & 10900 & 14412 \\
\hline 273 & 10-Nov-05 & & & & & & & 7240 & 13814 & 19242 & 11870 & 5980 & 11030 & 15244 \\
\hline Batc & h Average & 17 & 93.4 & 1310.4 & 2.3 & 78.0 & 1.3 & 7545 & 13655 & 18184 & 13433 & 6192 & 10842 & 14458 \\
\hline 274 & 11-Nov-05 & \multirow{18}{*}{$\frac{\infty}{\frac{\infty}{\infty}}$} & 101.9 & 1243.2 & 1.5 & 72.2 & 0.8 & 7436 & 13682 & 18880 & 13726 & 6096 & 10884 & 14914 \\
\hline 275 & 12-Nov-05 & & & & & & & & & & & & & \\
\hline 276 & 13-Nov-05 & & & & & & & & & & & & & \\
\hline 277 & 14-Nov-05 & & & & & & & 6926 & 12984 & 17950 & 12984 & 5702 & 10384 & 14292 \\
\hline 278 & $15-N o v-05$ & & 97.4 & 1386.0 & 1.9 & 81.8 & 0.8 & 8050 & 14132 & 18796 & 13946 & 6646 & 11326 & \\
\hline 279 & 16-Nov-05 & & 94.9 & 1405.6 & 35 & 97.2 & 1.2 & 10254 & 15050 & 16624 & 14728 & 8272 & 11946 & 13196 \\
\hline 280 & 17-Nov-05 & & & & & & & 7946 & 12972 & 17968 & 13924 & 6542 & 10300 & 14394 \\
\hline 281 & 18-Nov-05 & & 88.2 & 1330.0 & 1.8 & 79.0 & 1.1 & 7774 & 13150 & 18104 & 13614 & 6298 & 10224 & 13962 \\
\hline 282 & 19-Nov-05 & & & & & 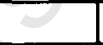 & & & & & & & & \\
\hline 283 & 20-Nov-05 & & & & - & & & & & & & & & \\
\hline 284 & 21-Nov-05 & & & & & & & 7898 & 13824 & 19242 & 13788 & 6330 & 10970 & 14766 \\
\hline 285 & $22-N o v-05$ & & 101.1 & 1341.2 & 2.7 & 81.5 & 1.3 & 13998 & 16214 & 17350 & 15726 & 11184 & 12908 & 13808 \\
\hline 286 & 23-Nov-05 & & 105.6 & 1472.8 & 1.8 & 84.0 & 1.1 & 7848 & 13988 & 19834 & 14166 & 6490 & 11250 & 15822 \\
\hline 287 & 24-Nov-05 & & & & & & & 7894 & 13598 & 16916 & 13262 & 6460 & 10904 & 13464 \\
\hline 288 & 25-Nov-05 & & 107.2 & 1204.0 & 1.6 & 80.6 & 1.2 & 8144 & 14232 & 18814 & 14244 & 6532 & 11036 & 14444 \\
\hline 289 & 26-Nov-05 & & & & & & & & & & & & & \\
\hline 290 & 27-Nov-05 & & & & & & & & & & & & & \\
\hline 291 & 28 -Nov-05 & & 104.2 & 1355.2 & 1.6 & 86.8 & 0.7 & 8056 & 14058 & 18716 & 14058 & 6564 & 11240 & 14792 \\
\hline \multicolumn{2}{|c|}{ Batch Average } & 18 & 100.1 & 1342.3 & 1.8 & 80.8 & 1.0 & 8021 & 13788 & 18266 & 14014 & 6926 & 11114 & 14350 \\
\hline 292 & 29-Nov-05 & \multirow{13}{*}{$\frac{3}{\frac{5}{6}}$} & & & & & & 8794 & 14742 & 19068 & 14840 & 7378 & 11788 & 15176 \\
\hline 293 & 30-Nov-05 & & 96.9 & 1456.0 & 2.4 & 73.6 & 1.3 & 8126 & 14722 & 18972 & 14652 & 6484 & 11526 & 14598 \\
\hline 294 & 1-Dec-05 & & & & & & & 7896 & 14118 & 18378 & 14038 & 6438 & 11236 & 14540 \\
\hline 295 & 2-Dec-05 & & 139.7 & 1327.2 & 1.9 & 103.0 & 1.2 & 7844 & 13568 & 18850 & 13936 & 6400 & 10760 & 14950 \\
\hline 296 & 3-Dec-05 & & & & & & & & & & & & & \\
\hline 297 & 4-Dec-05 & & & & & & & & & & & & & \\
\hline 298 & 5-Dec-05 & & 90.7 & 1125.6 & 0.3 & 70.6 & 0.0 & 5840 & 15052 & 19450 & 14824 & 4878 & 11982 & 15468 \\
\hline 299 & 6-Dec-05 & & & & & & & 6392 & 15840 & 20098 & 15200 & 5242 & 12646 & 15994 \\
\hline 300 & 7-Dec-05 & & & & & & & 5158 & 14486 & 19242 & 13598 & 4262 & 11078 & 14412 \\
\hline 301 & 8-Dec-05 & & 73.6 & 1271.2 & 2.0 & 70.8 & 0.0 & 5920 & 15292 & 19354 & 15064 & 4850 & 11768 & 14820 \\
\hline 302 & 9-Dec-05 & & 114.8 & 1276.8 & 1.9 & 82.9 & 0.0 & 5614 & 14890 & 20514 & 14752 & 4606 & 11814 & 16190 \\
\hline 303 & 10-Dec-05 & & & & & & & & & & & & & \\
\hline 304 & 11-Dec-05 & & & & & & & & & & & & & \\
\hline
\end{tabular}


A-2 MBR TKN FSA / TSS VSS

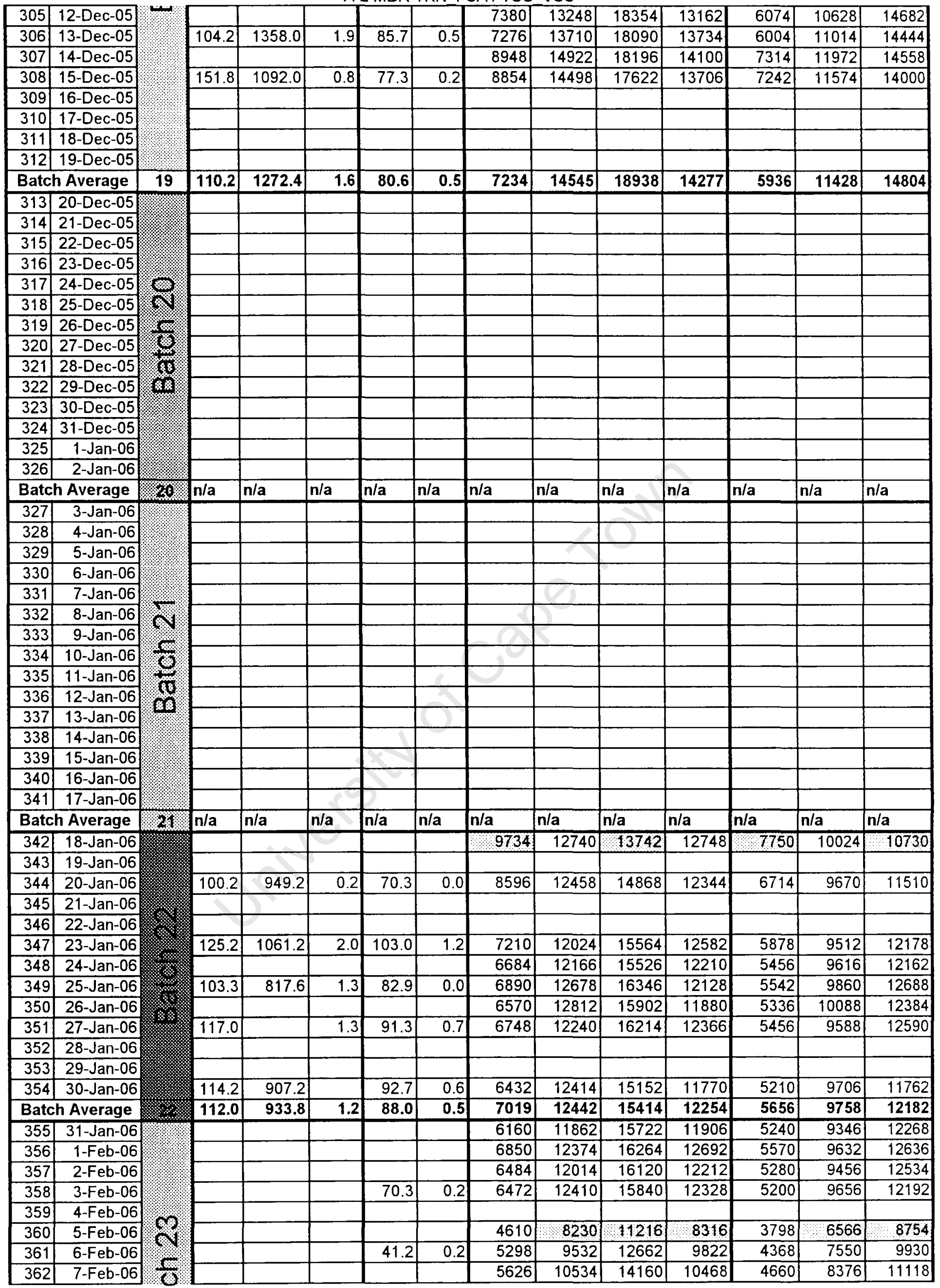


A-2 MBR TKN FSA / TSS VSS

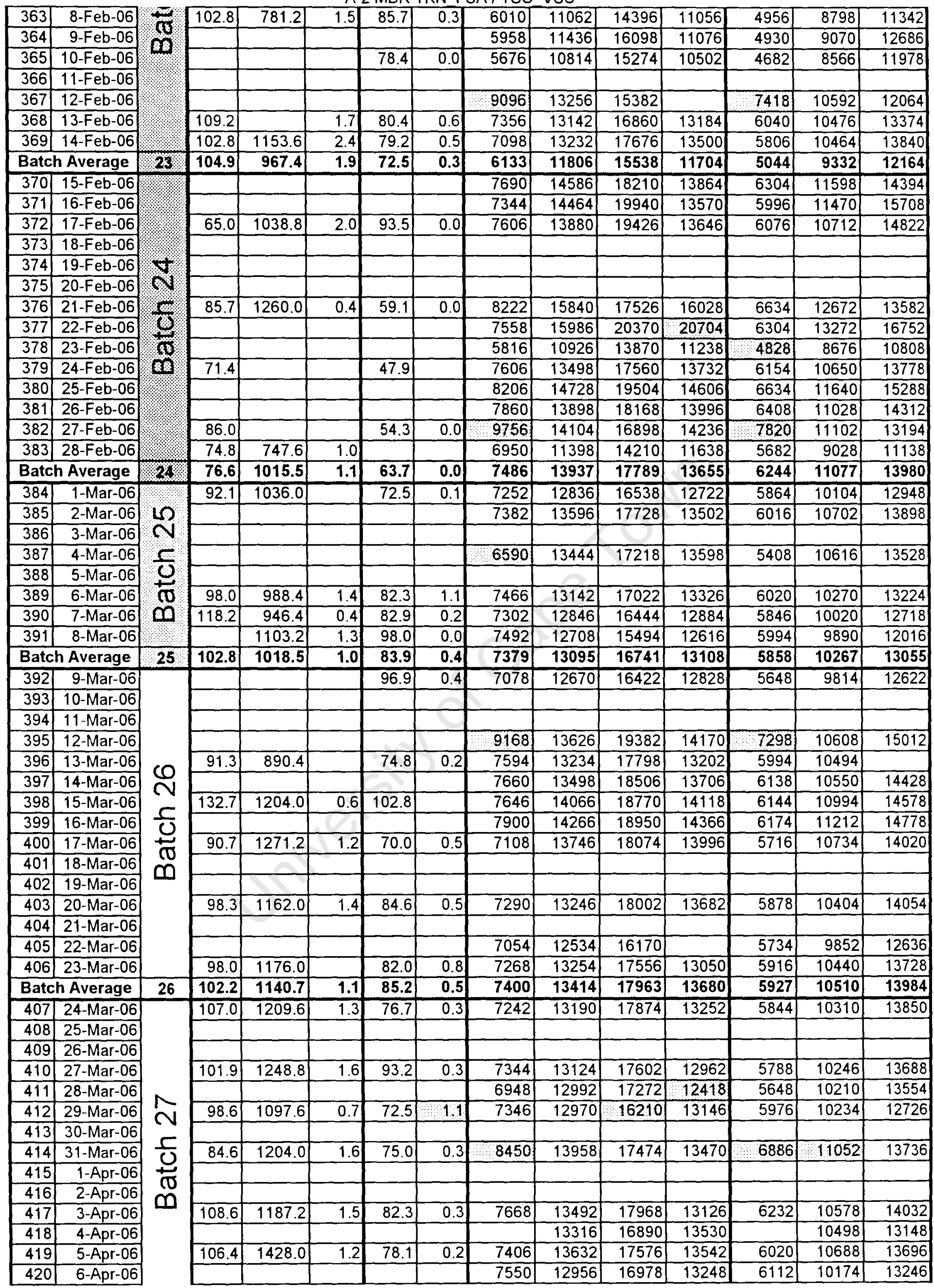


A-2 MBR TKN FSA / TSS VSS

\begin{tabular}{|c|c|c|c|c|c|c|c|c|c|c|c|c|c|c|}
\hline 421 & 7-Apr-06 & & & & & & & & & & & & & \\
\hline \multicolumn{2}{|c|}{ Batch Average } & 27 & 101.2 & \multirow[t]{2}{*}{1229.2} & 1.3 & \multirow[t]{2}{*}{79.7} & 0.3 & 7358 & \multirow[t]{2}{*}{13292} & \multirow[t]{2}{*}{17454} & \multirow[t]{2}{*}{13285} & \multirow[t]{2}{*}{5946} & \multirow[t]{2}{*}{10367} & \multirow[t]{2}{*}{13520} \\
\hline 422 & 8-Apr-06 & \multirow{15}{*}{$\frac{19}{8}$} & & & & & & & & & & & & \\
\hline 423 & 9-Apr-06 & & & & & & & 7564 & 13682 & 18248 & 17412 & 6072 & 10748 & 14360 \\
\hline 424 & 10-Apr-06 & & 80.6 & 905.8 & 1.1 & 58. & 0.3 & 7164 & 13006 & 17166 & 13232 & 5852 & 10288 & 13428 \\
\hline 425 & 11-Apr-06 & & & & & & & 7214 & 13168 & 16020 & 13426 & 5858 & 10428 & 13064 \\
\hline 426 & 12-Apr-06 & & & & & & & 7692 & 13806 & 18450 & 13796 & 6350 & 11078 & 14640 \\
\hline 427 & 13-Apr-06 & & 100.2 & 1184.4 & 1.3 & 83. & 0.6 & 6960 & 12734 & 16990 & 13070 & 5726 & 10176 & 13424 \\
\hline 428 & 14-Apr-06 & & 94.9 & & & & & 7454 & 13212 & 17130 & 13094 & 6176 & 10570 & 13634 \\
\hline 429 & 15-Apr-06 & & 100.0 & 1198.4 & 0.8 & 81. & 0.8 & 7474 & 12848 & 15474 & 13308 & 6130 & 10244 & 12258 \\
\hline 430 & 16-Apr-06 & & 98.0 & & & & & & & & & & & \\
\hline 431 & 17-Apr-06 & & 101.9 & & & & & & & & & & & \\
\hline 432 & 18-Apr-06 & & 117.0 & 1136.8 & 1.9 & 80. & 0.2 & 7074 & 11678 & 15828 & 11240 & 5880 & 9464 & 12702 \\
\hline 433 & 19-Арг-06 & & & & & & & 7276 & 12892 & 17290 & 12872 & 6124 & 10496 & 13936 \\
\hline 434 & 20-Apr-06 & & & & & & & 6902 & 12062 & 16226 & 12002 & 5872 & 9876 & 13208 \\
\hline 435 & 21-Apr-06 & & 118.2 & 1240.4 & 2.1 & 86. & 1.8 & 7352 & 13354 & 17898 & 13272 & 6174 & 10858 & 14462 \\
\hline 436 & 22-Apr-06 & & & & & & & & & & & & & \\
\hline \multicolumn{2}{|c|}{ Batch Average } & 棸6 & 101.4 & 1133.2 & 1.5 & 78. & 0.8 & 7284 & 13076 & 16975 & 12931 & 6019 & 10476 & 13556 \\
\hline 437 & 23-Apr-06 & \multirow{13}{*}{$\frac{6}{\frac{1}{6}}$} & & & & & & & & & & & & \\
\hline 438 & 24-Apr-06 & & 105.8 & 1204.0 & 1.2 & 77. & 0.1 & 6900 & 13166 & 17508 & 13048 & 5902 & 10914 & 14436 \\
\hline 439 & 25-Apr-06 & & & & & & & 7254 & 13733 & 18664 & 13992 & 6184 & 11418 & 15400 \\
\hline 440 & 26-Apr-06 & & 94.4 & 1316.0 & 1.6 & 76. & 0.0 & 6786 & 12874 & 17772 & 13098 & 5770 & 10636 & 14966 \\
\hline 441 & 27-Apr-06 & & & & & & & 7102 & 13362 & 17980 & 13652 & 6034 & 11014 & 14744 \\
\hline 442 & 28-Apr-06 & & 88.2 & 1377.6 & 1.7 & 69. & 0.0 & 6460 & 12500 & 17580 & 16190 & & & \\
\hline 443 & 29-Apr-06 & & & & & & & & & 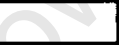 & & & & \\
\hline 444 & 30-Apr-06 & & & & & & & 7222 & 12820 & 18118 & 13012 & 5894 & 10210 & 14364 \\
\hline 445 & 1-May-06 & & & & & 79. & 2.0 & 7250 & 13042 & 17456 & 13412 & 5934 & 10416 & 13838 \\
\hline 446 & 2-May-06 & & & & & & & & 2 & & & & & \\
\hline 447 & 3-May-06 & & 96.9 & 1128.4 & 2.2 & 64. & 0.9 & 8300 & 14484 & 18520 & 14862 & 6766 & 11608 & 14734 \\
\hline 448 & 4-May-06 & & & & & & & & 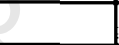 & & & & & \\
\hline 449 & 5-May-06 & & 89.6 & 1150.8 & 0.5 & 63. & 0.3 & 7482 & 12798 & 16778 & 12838 & & & \\
\hline \multicolumn{2}{|c|}{ Batch Average } & 89 & 95.0 & 1235.4 & 1. & 71. & 0.6 & 7057 & 13037 & 17820 & 13489 & 5953 & 10888 & 14640 \\
\hline
\end{tabular}




\section{A-3 MBR NOx/P}

Appendix A-3

Measured NO3, NO2 and soluble $\mathrm{P}$ in the MBR UCT system

\section{$\mathrm{NO} 3$ \\ $\mathrm{NO} 2$ \\ Ortho-P}

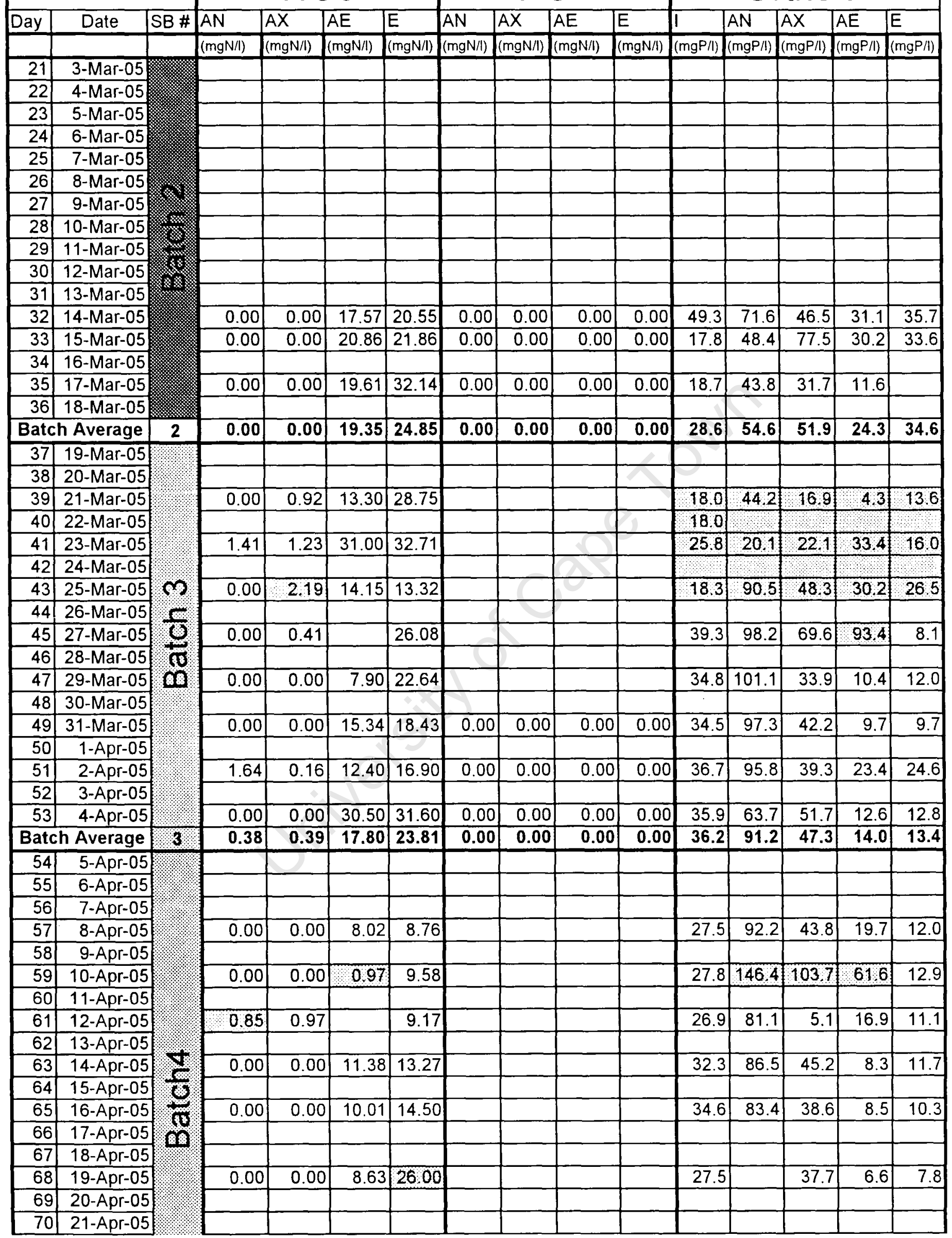


A-3 MBR NOx / P

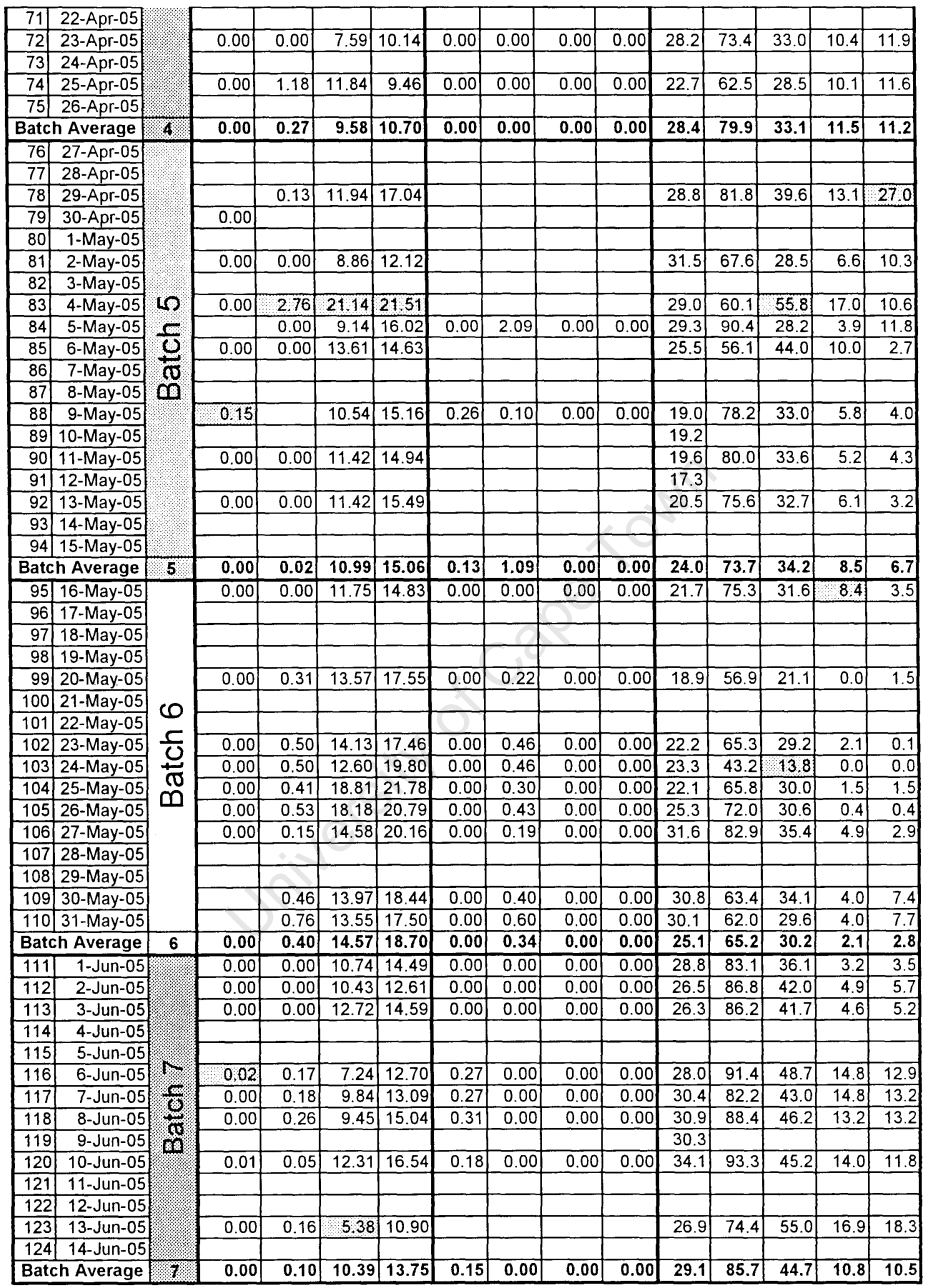




\begin{tabular}{|c|c|c|c|c|c|c|c|c|c|c|c|c|c|c|c|}
\hline 125 & 15-Jun-05 & \multirow[b]{3}{*}{$\infty$} & & & & & & & & & & & & & \\
\hline 126 & 16-Jun-05 & & & & & & & & & & & & & & \\
\hline 127 & 17-Jun-05 & & & & & & & & & & & & & & \\
\hline 128 & 18-Jun-05 & \multirow{2}{*}{$\frac{5}{9}$} & 0.00 & 0.15 & 9.97 & 16.09 & 0.00 & 0.00 & 0.00 & 0.00 & 25.8 & 87.8 & 42.8 & 15.8 & 15.3 \\
\hline 129 & 19-Jun-05 & & & & & & & & & & & & & & \\
\hline 130 & 20-Jun-05 & \multirow{3}{*}{ nos, } & 0.00 & 0.11 & 10.48 & 16.94 & 0.00 & 0.00 & 0.00 & 0.00 & 28.3 & 87.2 & 41.7 & 9.7 & 9.4 \\
\hline 131 & 21-Jun-05 & & 0.00 & 0.24 & 13.79 & 17.19 & 0.00 & 0.00 & 0.00 & 0.00 & 28.9 & 61.7 & 28.1 & 5.8 & 7.2 \\
\hline 132 & 22-Jun-05 & & 0.00 & 0.21 & 16.34 & 18.89 & & & & & 30.8 & 80.8 & 41.9 & 8.9 & 8.1 \\
\hline \multicolumn{2}{|c|}{ Batch Average } & 8 & 0.00 & 0.18 & 12.64 & 17.28 & 0.00 & 0.00 & 0.00 & 0.00 & 28.5 & 79.4 & 38.6 & 10.1 & 10.0 \\
\hline 133 & 23-Jun-05 & \multirow{15}{*}{ 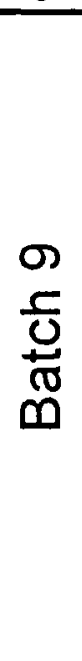 } & 0.00 & 0.15 & 14.73 & 18.89 & & & & & 33.6 & 73.6 & 36.1 & 3.6 & 9.4 \\
\hline 134 & 24-Jun-05 & & 0.00 & 0.00 & & 10.48 & & & & & 33.1 & 96.7 & 64.7 & 16.4 & 6.9 \\
\hline 135 & 25-Jun-05 & & & & & & & & & & & & & & \\
\hline 136 & 26-Jun-05 & & & & & & & & & & & & & & \\
\hline 137 & 27-Jun-05 & & 0.36 & 0.47 & 7.41 & 12.32 & & & & & 26.5 & 83.6 & 43.5 & & 8.9 \\
\hline 138 & 28-Jun-05 & & 0.11 & 0.11 & 7.55 & 13.42 & & & & & 26.5 & 76.6 & 38.7 & 10.5 & \\
\hline 139 & 29-Jun-05 & & 0.60 & 0.44 & 9.05 & 14.24 & & & & & 29.5 & 88.5 & 42.7 & 13.2 & 8.9 \\
\hline 140 & 30-Jun-05 & & 0.11 & 0.09 & 7.68 & 17.10 & & & & & 28.6 & 85.5 & 41.9 & 7.5 & 6.7 \\
\hline 141 & 1-Jul-05 & & 0.36 & 0.30 & 12.32 & 16.42 & & & & & 28.9 & 91.0 & 41.9 & 8.1 & 10.0 \\
\hline 142 & 2-Jul-05 & & & & & & & & & & & & & & \\
\hline 143 & 3-Jul-05 & & & & & & & & & & & & & & \\
\hline 144 & 4-Jul-05 & & 0.00 & 1.74 & 19.53 & 21.42 & 0.00 & 0.00 & 0.00 & 0.00 & 35.7 & 92.6 & 43.8 & 11.8 & 11.8 \\
\hline 145 & 5-Jul-05 & & 0.00 & 0.00 & 10.23 & 26.26 & 0.00 & 0.00 & 0.00 & 0.00 & 31.5 & 81.2 & 40.5 & 12.5 & 15.5 \\
\hline 146 & 6-Jul-05 & & 0.00 & 1.71 & 17.77 & 20.42 & 0.00 & 0.00 & 0.00 & 0.00 & 18.7 & 75.5 & 29.3 & 1.9 & 2.4 \\
\hline 147 & 7 -Jul-05 & & & & & & 0.00 & 0.00 & 0.00 & 0.00 & 12.5 & 76.6 & 33.2 & 2.2 & 1.6 \\
\hline \multicolumn{2}{|c|}{ Batch Average } & 9 & 0.10 & 0.50 & 11.81 & 17.10 & 0.00 & 0.00 & 0.00 & 0.00 & 29.3 & 84.5 & 39.8 & 9.5 & 9.0 \\
\hline 148 & 8-Jul-05 & \multirow{10}{*}{9} & 0.00 & 0.00 & 16.44 & 19.92 & 0.00 & 0.00 & 0.00 & 0.00 & 10.9 & 69.8 & 31.5 & 1.6 & 1.4 \\
\hline 149 & 9-Jul-05 & & 0.00 & 0.00 & 13.81 & 16.18 & & & & & & 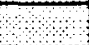 & 1 & & \\
\hline 150 & $10-\mathrm{Jul}-05$ & & & & & & & & & & & & & & \\
\hline 151 & 11-Jul-05 & & 0.00 & 0.00 & 11.59 & 13.10 & 0.00 & 0.00 & 0.00 & 0.00 & 9.6 & 74.2 & 36.7 & 1.3 & 1.0 \\
\hline 152 & 12-Jul-05 & & 0.00 & 0.00 & 11.15 & 14.93 & 0.00 & 0.00 & 0.00 & 0.00 & 10.1 & 73.3 & 31.9 & 1.8 & 1.0 \\
\hline 153 & 13-Jul-05 & & & & & & & & & & 13.2 & & & & \\
\hline 154 & 14-Jul-05 & & 0.00 & 0.00 & 12.23 & 14.93 & 0.00 & 0.00 & 0.00 & 0.00 & 127 & 68.2 & 27.4 & 3.0 & 7.3 \\
\hline 155 & 15-Jul-05 & & 0.00 & 0.00 & 11.48 & 16.45 & 0.00 & 0.00 & 0.00 & 0.00 & 15.2 & 76.7 & 325 & 3.0 & 1.8 \\
\hline 156 & $16-J u l-05$ & & & & & & & & & & $\%$ & & & & \\
\hline 157 & 17-Jul-05 & & & & & & & & & & & & & & \\
\hline 158 & 18-Jul-05 & \multirow{10}{*}{$\frac{6}{96}$} & 0.00 & 0.00 & 11.96 & 12.41 & 0.00 & 0.03 & 0.00 & 0.00 & 112 & 68.7 & 29.2 & 10 & $\$ .0$ \\
\hline 159 & 19-Jul-05 & & 0.00 & 0.00 & 12.23 & 13.63 & 0.00 & 0.03 & 0.00 & 0.00 & 122 & 63.4 & 25.6 & 1.5 & 0.5 \\
\hline 160 & $20-J u l-05$ & & 0.00 & 0.00 & 11.84 & 13.77 & 0.00 & 0.03 & 0.00 & 0.00 & & 61.4 & 25.6 & 1.5 & 0.2 \\
\hline 161 & 21-Jul-05 & & 0.00 & 0.00 & 12.28 & 13.87 & 0.00 & 0.05 & 0.00 & 0.00 & 11.7 & 71.8 & 26.3 & 1.5 & 0.5 \\
\hline 162 & 22-Jul-05 & & & $=0$ & & & & & & & 12.5 & & & & \\
\hline 163 & 23-Jul-05 & & & ${ }^{\circ}$ & 6.48 & 11.54 & 0.08 & 0.12 & 0.00 & 0.00 & 9.6 & 68.6 & 27.0 & 3.3 & 1.3 \\
\hline 164 & 24-Jul-05 & & & - & & & & & & & & & & & \\
\hline 165 & 25-Jul-05 & & & $\pi$ & 11.89 & 14.31 & 0.08 & 0.05 & 0.00 & 0.00 & 28.7 & 71.4 & 37.4 & 5.7 & 8.0 \\
\hline 166 & 26-Jul-05 & & & 0.17 & 10.80 & 13.43 & 0.04 & 0.05 & 0.00 & 0.00 & 30.2 & 71.9 & 33.9 & 9.3 & 9.3 \\
\hline 167 & $27-\mathrm{Jul}-05$ & & & & 12.89 & 14.49 & 0.04 & 0.05 & 0.00 & 0.00 & 29.2 & 96.2 & 37.7 & 8.8 & 9.3 \\
\hline \multicolumn{2}{|c|}{ Batch Average } & 10 & 0.00 & 0.00 & 12.01 & 14.08 & 0.02 & 0.03 & 0.00 & 0.00 & 29.3 & 79.8 & 36.3 & 8.0 & $8 . \overline{9}$ \\
\hline 168 & 28-Jul-05 & \multirow{12}{*}{$\begin{array}{l}\frac{\sigma}{\frac{\sigma}{0}} \\
\frac{\pi}{\sigma}\end{array}$} & 0.00 & 0.00 & 17.90 & 16.61 & 0.03 & 0.08 & 0.00 & 0.00 & & 80.7 & 46.0 & 11.8 & 11.0 \\
\hline 169 & 29-Jul-05 & & & & & & 0.06 & 0.06 & 0.00 & 0.00 & 27.8 & 73.6 & 36.9 & 8.5 & 11.8 \\
\hline 170 & 30-Jul-05 & & & & & & & & & & & & & & \\
\hline 171 & 31-Jul-05 & & & & & & & & & & & & & & \\
\hline 172 & 1-Aug-05 & & 0.00 & 0.00 & 11.93 & 14.69 & 0.04 & 0.04 & 0.00 & 0.00 & 23.3 & 51.7 & 32.4 & 3,0 & 7.1 \\
\hline 173 & 2-Aug-05 & & & & 13.17 & 14.45 & 0.04 & 0.07 & 0.00 & 0.00 & 22.0 & 78.2 & 38.5 & 8.5 & 8.7 \\
\hline 174 & 3-Aug-05 & & 0.00 & 0.00 & 12.95 & 14.37 & & 0.09 & 0.00 & 0.00 & 23.3 & 82.9 & 40.7 & 10.9 & 12.9 \\
\hline 175 & 4-Aug-05 & & 0.00 & 0.00 & 12.43 & 15.08 & & & 0.00 & 0.00 & 23.3 & 73.0 & 34.6 & 9.3 & 15.6 \\
\hline 176 & 5-Aug-05 & & & & & & & & & & 25.3 & 76.0 & 43.7 & 10.9 & 10.9 \\
\hline 177 & 6-Aug-05 & & & & & & & & & & & & & & \\
\hline 178 & 7-Aug-05 & & & & & & & & & & & & & & \\
\hline 179 & 8-Aug-05 & & 0.00 & 0.00 & 12.94 & 14.31 & & & 0.00 & 0.00 & & & & & \\
\hline
\end{tabular}




\begin{tabular}{|c|c|c|c|c|c|c|c|c|c|c|c|c|c|c|c|}
\hline 180 & 9-Aug-05 & $m$ & 0.00 & 0.00 & 11.88 & 12.01 & & & 0.00 & 0.00 & 23.3 & 71.3 & 31.6 & 7.8 & 8.4 \\
\hline 181 & 10-Aug-05 & & 0.00 & 0.00 & 16.76 & 17.03 & & & 0.00 & 0.00 & 22.8 & 70.3 & 31.0 & 8.4 & 8.6 \\
\hline 182 & 11-Aug-05 & & & & & & & & & & 20.6 & 60.7 & 28.1 & 8.1 & 6.8 \\
\hline 183 & 12-Aug-05 & & 0.00 & 0.00 & 18.20 & 18.67 & & & 0.00 & 0.00 & & & & & \\
\hline 184 & 13-Aug-05 & & & & & & & & & & & & & & \\
\hline 185 & 14-Aug-05 & & & & & & & & & & & & & & \\
\hline 186 & 15-Aug-05 & & 1.60 & 101 & 14.20 & 16.08 & & 0.00 & 0.00 & 0.00 & 31.0 & 63.9 & 38.0 & 6.5 & 7.8 \\
\hline 187 & 16-Aug-05 & & & & & & & & & & 20.6 & 60.1 & 32.1 & 7.3 & 6.8 \\
\hline 188 & 17-Aug-05 & & 0.00 & 0.00 & 17.07 & 20.39 & & 0.00 & 0.00 & & 24.4 & 56.9 & 26.0 & 6.0 & 6.5 \\
\hline Batcl & h Average & 11 & 0.00 & 0.00 & 14.49 & 15.33 & 0.04 & 0.05 & 0.00 & 0.00 & 24.0 & 69.2 & 35.4 & 8.7 & 8.9 \\
\hline 189 & 18-Aug-05 & \multirow{16}{*}{$\begin{array}{c}\frac{9}{5} \\
\frac{5}{\infty} \\
\frac{8}{\infty}\end{array}$} & 0.96 & 0.00 & 15.52 & 19.07 & 0.00 & 0.00 & 0.00 & 0.00 & & & & & \\
\hline 190 & 19-Aug-05 & & & & & & & & & & & & & & \\
\hline 191 & 20-Aug-05 & & & & & & & & & & & & & & \\
\hline 192 & 21-Aug-05 & & & & & & & & & & & & & & \\
\hline 193 & 22-Aug-05 & & 0.00 & 0.00 & 17.38 & 21.48 & & & 0.00 & 0.00 & 27.4 & 77.5 & 40.3 & 12.7 & 10.0 \\
\hline 194 & 23-Aug-05 & & 0.00 & 0.00 & 20.67 & 19.77 & & & 0.00 & 0.00 & 28.7 & 59.8 & 20.7 & 5.4 & 5.7 \\
\hline 195 & 24-Aug-05 & & 0.00 & 0.00 & 14.97 & 15.74 & & & 0.00 & 0.00 & 30.9 & 60.1 & 23.9 & 6.3 & 6.0 \\
\hline 196 & 25-Aug-05 & & 0.19 & 0.96 & 21.22 & 22.68 & 0.00 & 0.00 & 0.00 & 0.00 & 27.4 & 76.2 & 28.7 & 5.7 & 4.9 \\
\hline 197 & 26-Aug-05 & & 0.00 & 0.00 & 23.18 & 24.48 & & & 0.00 & 0.00 & 27.7 & 80.4 & 34.1 & 7.3 & 6.3 \\
\hline 198 & 27-Aug-05 & & & & & & & & & & & & & & \\
\hline 199 & 28-Aug-05 & & & & & & & & & & & & & & \\
\hline 200 & 29-Aug-05 & & 0.00 & 5.87 & 27.66 & 28.47 & & & 0.00 & 0.00 & 26.6 & 50.4 & 20.2 & 2.1 & 1.2 \\
\hline 201 & 30-Aug-05 & & 0.00 & 5.41 & 25.44 & 24.04 & & & & & + & 76.3 & 27.7 & 8.2 & 5.4 \\
\hline 202 & 31-Aug-05 & & & & & & & & & G & & 90.9 & 31.9 & 8.8 & 8.5 \\
\hline 203 & 1-Sep-05 & & 0.00 & 6.39 & 27.20 & 28.33 & & & 8 & C & 28.3 & 1241 & 57.6 & 24.1 & 23.6 \\
\hline 204 & 2-Sep-05 & & 0.00 & 6.03 & 22.54 & 26.32 & & & & $\nabla$ & 29.4 & 99.8 & 37.2 & 2.1 & 10.2 \\
\hline Batc & h Average & 12 & 0.02 & 2.47 & 21.58 & 23.04 & 0.00 & 0.00 & 0.00 & 0.00 & 28.3 & 74.6 & 29.4 & 6.5 & 6.5 \\
\hline 205 & 3-Sep-05 & \multirow{14}{*}{$\frac{8}{\frac{6}{6}}$} & & & & & & & & & & & & & \\
\hline 206 & 4-Sep-05 & & & & & & & 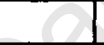 & & & & & & & \\
\hline 207 & 5-Sep-05 & & & & & & & & & & & & & & \\
\hline 208 & 6-Sep-05 & & 0.00 & 0.41 & 9.20 & 9.39 & e & D & & & 27.7 & 96.7 & 44.6 & 3.8 & 5.0 \\
\hline 209 & 7-Sep-05 & & & & & & & & & & & & & & \\
\hline 210 & 8-Sep-05 & & 0.00 & 0.36 & 12.72 & 12.79 & & & & & 29.9 & 99.9 & 45.9 & 7.3 & 8.5 \\
\hline 211 & 9-Sep-05 & & & & 9.50 & 11.72 & & & & & 30.3 & 81.7 & 25.1 & 1.2 & 0.6 \\
\hline 212 & 10-Sep-05 & & & & & & & & & & 31.2 & & & & \\
\hline 213 & 11-Sep-05 & & & & 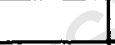 & _. & & & & & & & & & \\
\hline 214 & 12-Sep-05 & & 0.00 & 0.36 & 10.63 & 11.50 & & & & & 28.9 & 93.4 & 37.1 & 6.4 & 4.8 \\
\hline 215 & 13-Sep-05 & & & & 12.37 & 11.82 & & & & & 29.3 & 93.8 & 39.6 & 9.5 & 10.2 \\
\hline 216 & 14-Sep-05 & & 0.00 & 0.41 & 12.47 & 12.80 & & & & & & 95.3 & 39.9 & 6.1 & 4.2 \\
\hline 217 & 15-Sep-05 & & & 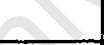 & 17.69 & 18.16 & & & & & 29.3 & 96.6 & 40.2 & 8.6 & 8.3 \\
\hline 218 & 16-Sep-05 & & 0.00 & 0.27 & 15.80 & 17.21 & & & & & 30.2 & 110.0 & 44.3 & 4.8 & 4.8 \\
\hline \multicolumn{2}{|c|}{ Batch Average } & 13 & 0.00 & 0.36 & 12.55 & 13.17 & 0.00 & 0.00 & 0.00 & 0.00 & 29.6 & 95.9 & 41.7 & 6.0 & 5.8 \\
\hline & \multirow{16}{*}{$\begin{array}{l}\frac{\sigma}{\sigma} \\
\frac{\sigma}{0} \\
\frac{0}{0}\end{array}$} & & & & & & & & & & & & & \\
\hline \multicolumn{2}{|c|}{\begin{tabular}{l|l}
220 & $18-S$ \\
\end{tabular}} & & & & & & & & & & & & & & \\
\hline \multicolumn{2}{|c|}{\begin{tabular}{l|l}
221 & $19-5$ \\
\end{tabular}} & & & & & & & & & & & & & & \\
\hline 222 & 20-Sep-05 & & 0.00 & 4.52 & 26.05 & \begin{tabular}{|l|}
25.04 \\
\end{tabular} & & & & & 45.9 & 74.3 & 31.9 & 6.1 & 2.0 \\
\hline 223 & 21-Sep-05 & & & & 19.65 & 20.41 & & & & & 29.1 & 88.3 & 36.0 & 9.8 & 10.4 \\
\hline 224 & 22-Sep-05 & & 0.00 & 1.07 & 19.60 & 20.87 & & 0.00 & 0.00 & 0.00 & 27.9 & 91.1 & 40.6 & 15.4 & 11.3 \\
\hline 225 & 23-Sep-05 & & 0.00 & 1.80 & 20.86 & 22.95 & & 0.00 & 0.00 & 0.00 & 29.7 & 97.6 & 40.9 & 9.2 & 9.2 \\
\hline 226 & 24-Sep-05 & & & & & & & & & & & & & & \\
\hline \multirow{2}{*}{$\frac{227}{228}$} & 25-Sep-05 & & & & & & & & & & & & & & \\
\hline & 26-Sep-05 & & 0.00 & 4.76 & 21.28 & 21.87 & & 0.00 & 0.00 & 0.00 & 33.2 & 79.6 & 31.6 & 8.2 & 8.9 \\
\hline 229 & 27-Sep-05 & & 0.00 & 5.45 & 24.25 & 24.71 & & 0.00 & 0.00 & 0.00 & 34.1 & 74.9 & 31.6 & 8.2 & 6.4 \\
\hline 230 & $28-\operatorname{sep}-05$ & & & & & & & & & & 34.1 & 69.0 & 22.9 & 5.4 & 3.2 \\
\hline 231 & 29-Sep-05 & & 0.00 & 0.46 & 8.60 & 9.21 & & 0.00 & 0.00 & 0.00 & & 71.4 & 30.1 & 4.7 & 6.3 \\
\hline 232 & 30-Sep-05 & & 0.00 & 0.58 & 18.07 & 20.03 & & 0.00 & 0.00 & 0.00 & 31.3 & 83.2 & 34.8 & 9.1 & 7.5 \\
\hline 233 & 1-Oct-05 & & & & & & & & & & & & & & \\
\hline 234 & 2-Oct-05 & & & & & & & & & & & & & & \\
\hline
\end{tabular}


A-3 MBR NOX / P

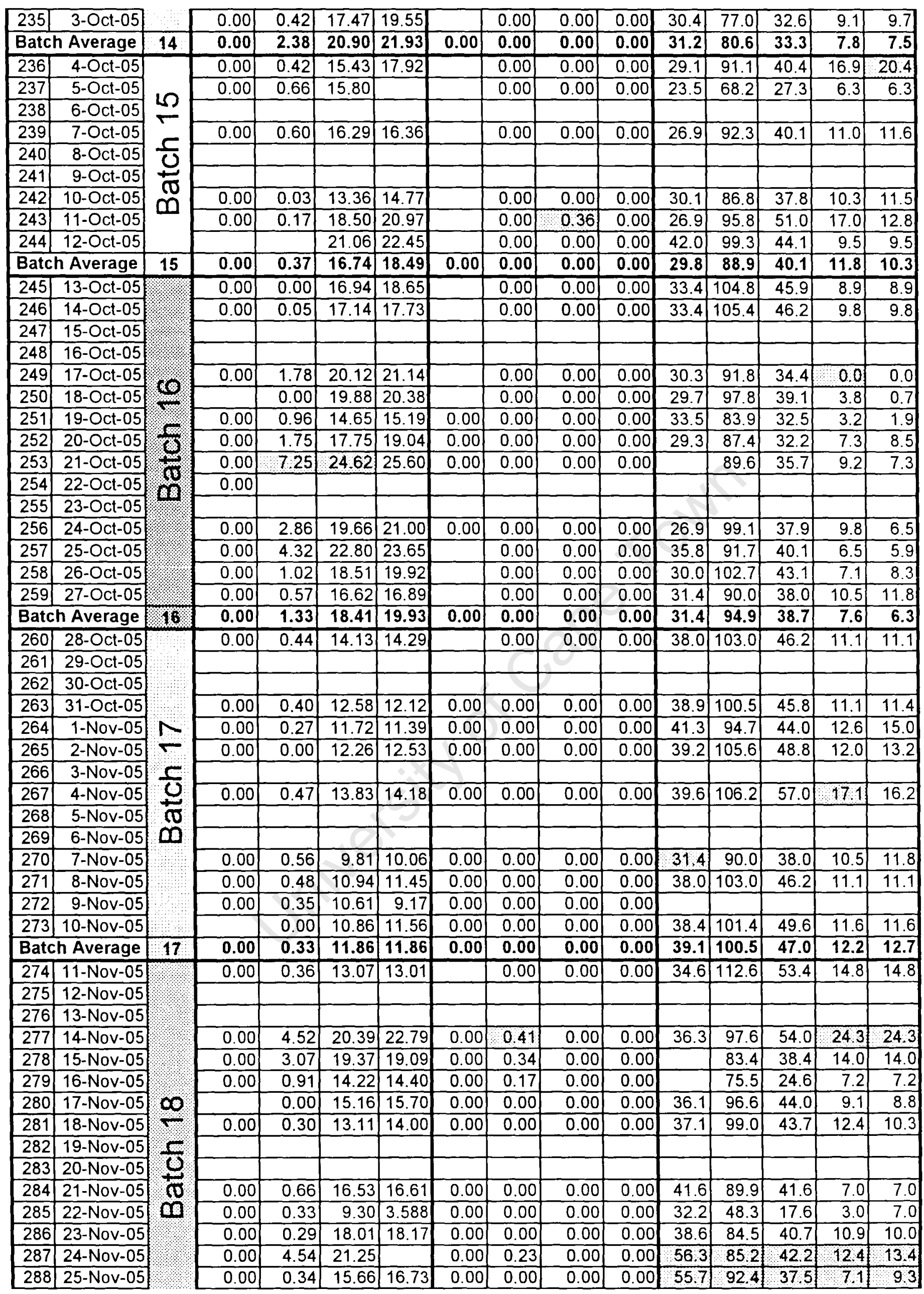




\section{A-3 MBR NOX / P}

\begin{tabular}{|c|c|c|c|c|c|c|c|c|c|c|c|c|c|c|c|}
\hline 289 & 26-Nov-05 & & & & & & & & & & & & & & \\
\hline 290 & $27-$ Nov-05 & & & & & & & & & & & & & & \\
\hline 291 & 28-Nov-05 & & 0.00 & 0.50 & 15.01 & 15.30 & 0.00 & 0.00 & 0.00 & 0.00 & 56,0 & 968 & 422 & 118 & 115 \\
\hline Batch & h Average & 18 & 0.00 & 1.32 & 15.92 & 15.40 & 0.00 & 0.07 & 0.00 & 0.00 & 36.6 & 87.5 & 39.8 & 9.8 & 9.9 \\
\hline 292 & 29-Nov-05 & \multirow{21}{*}{$\begin{array}{l}\frac{\$}{6} \\
\frac{5}{\infty} \\
0\end{array}$} & 0.00 & 0.42 & 12.71 & 13.34 & & 0.00 & 0.00 & 0.00 & 579 & 88.9 & 40.0 & 15.3 & 5.9 \\
\hline 293 & $30-N o v-05$ & & 0.00 & 0.23 & 13.78 & 14.06 & & 0.00 & 0.00 & 0.00 & 51.9 & 90.8 & 341 & 9.3 & 99 \\
\hline 294 & 1-Dec-05 & & 0.00 & 0.04 & 11.39 & 13.09 & & 0.00 & 0.00 & 0.00 & 29.3 & 81.3 & 38.5 & 10.8 & 10.2 \\
\hline 295 & 2-Dec-05 & & 0.00 & 0.01 & 12.91 & 13.16 & & 0.00 & 0.00 & 0.00 & 35.7 & 83.7 & 37.6 & 9.6 & 9.9 \\
\hline \multicolumn{15}{|c|}{\begin{tabular}{|l|l|}
296 & $3-D e c-05$ \\
\end{tabular}} & \\
\hline 297 & 4-Dec-05 & & & & & & & & & & & & & & \\
\hline 298 & 5-Dec-05 & & 0.00 & 0.18 & 14.07 & 13.70 & & 0.00 & 0.00 & 0.00 & 35.4 & 87.1 & 33.9 & 11.7 & 10.5 \\
\hline 299 & 6-Dec-05 & & 0.00 & 0.04 & 12.98 & 12.05 & & 0.00 & 0.00 & 0.00 & 41.9 & 86.8 & 28.6 & 7.1 & 6.5 \\
\hline 300 & 7-Dec-05 & & & & & & & & & & 44.4 & 114.1 & 41.6 & 5.8 & 6.4 \\
\hline 301 & 8-Dec-05 & & 0.00 & 0.23 & 12.05 & 12.68 & & 0.00 & 0.00 & 0.00 & 36.9 & 92.5 & 34.1 & 5.8 & 7.0 \\
\hline 302 & 9-Dec-05 & & 0.00 & 0.16 & 13.64 & 12.71 & & 0.00 & 0.00 & 0.00 & 33.7 & 88.7 & 30.6 & 6.4 & 3.0 \\
\hline 303 & 10-Dec-05 & & & & & & & & & & & & & & \\
\hline \multicolumn{15}{|c|}{304 11-Dec-05 } & \\
\hline \multicolumn{15}{|c|}{\begin{tabular}{l|l}
305 & $12-\mathrm{Dec}-05$ \\
\end{tabular}} & \\
\hline 306 & 13-Dec-05 & & 0.00 & 0.18 & 12.66 & 13.01 & & 0.00 & 0.00 & 0.00 & 33.4 & 79.0 & 31.9 & 1.7 & 1.4 \\
\hline 307 & 14-Dec-05 & & 0.00 & 0.19 & 11.56 & 11.90 & & 0.00 & 0.00 & 0.00 & 39.1 & 74.6 & 25.6 & 6.4 & 1.7 \\
\hline 308 & 15-Dec-05 & & 0.00 & 0.18 & 12.12 & 12.72 & & 0.00 & 0.00 & 0.00 & 41.0 & 82.7 & 31.5 & 3.6 & 1.4 \\
\hline 309 & 16-Dec-05 & & & & & & & & & & 34.4 & 89.1 & 37.8 & 8.9 & 8.5 \\
\hline 310 & 17-Dec-05 & & & & & & & & & & -1 & & & & \\
\hline 311 & 18-Dec-05 & & & & & & & & & & + & & & & \\
\hline 312 & 19-Dec-05 & & & & & & & & & 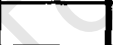 & & & & & \\
\hline \multicolumn{2}{|c|}{ Batch Average } & 19 & 0.00 & 0.14 & 12.72 & 12.95 & 0.00 & 0.00 & 0.00 & 0.00 & 36.8 & 84.5 & 33.8 & 7.1 & 6.0 \\
\hline \multicolumn{16}{|c|}{313 20-Dec-05 } \\
\hline \multicolumn{16}{|c|}{314 21-Dec-05 } \\
\hline \multicolumn{16}{|c|}{\begin{tabular}{l|l}
315 & $22-[$ \\
\end{tabular}} \\
\hline \multicolumn{16}{|c|}{\begin{tabular}{|c|c|}
316 & $23-\mathrm{Dec}-05$ \\
\end{tabular}} \\
\hline 317 & 24-Dec-05 & $\%$ & & & & & 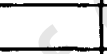 & + & & & & & & & \\
\hline \multicolumn{15}{|c|}{\begin{tabular}{|l|l|}
318 & $25-$ Dec-05
\end{tabular}} & \\
\hline \multicolumn{16}{|c|}{\begin{tabular}{|l|l|}
319 & $26-$ Dec-05 \\
\end{tabular}} \\
\hline \multicolumn{16}{|c|}{\begin{tabular}{l|l|}
320 & $27-D e c-05$ \\
\end{tabular}} \\
\hline 321 & 28-Dec-05 & 10 & & & & & & & & & & & & & \\
\hline 322 & 29-Dec-05 & $\%$ & & & & 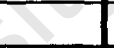 & & & & & & & & & \\
\hline 323 & 30-Dec-05 & & & & & 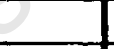 & & & & & & & & & \\
\hline 324 & 31-Dec-05 & & & & 80 & & & & & & & & & & \\
\hline 325 & 1-Jan-06 & & & & & & & & & & & & & & \\
\hline 326 & 2-Jan-06 & & & 2 & & & & & & & & & & & \\
\hline Batcl & h Average & 20 & $n / a$ & $n / a$ & $n / a$ & $n / a$ & $\mathrm{n} / \mathrm{a}$ & $n / a$ & $n / a$ & $n / a$ & $n / a$ & $n / a$ & $n / a$ & $n / a$ & $n / a$ \\
\hline 327 & 3-Jan-06 & & & D & & & & & & & & & & & \\
\hline 328 & 4-Jan-06 & & & & & & & & & & & & & & \\
\hline 329 & 5-Jan-06 & & & & & & & & & & & & & & \\
\hline 330 & 6-Jan-06 & & & & & & & & & & & & & & \\
\hline 331 & 7-Jan-06 & & & & & & & & & & & & & & \\
\hline 332 & 8-Jan-06 & $18 \%$ & & & & & & & & & & & & & \\
\hline 333 & 9-Jan-06 & 18 & & & & & & & & & & & & & \\
\hline 334 & 10-Jan-06 & 8 & & & & & & & & & & & & & \\
\hline 335 & 11-Jan-06 & $\frac{1}{60}$ & & & & & & & & & & & & & \\
\hline 336 & 12-Jan-06 & 18 & & & & & & & & & & & & & \\
\hline 337 & 13-Jan-06 & & & & & & & & & & & & & & \\
\hline 338 & 14-Jan-06 & & & & & & & & & & & & & & \\
\hline 339 & 15-Jan-06 & & & & & & & & & & & & & & \\
\hline 340 & 16-Jan-06 & & & & & & & & & & & & & & \\
\hline 341 & 17-Jan-06 & & & & & & & & & & & & & & \\
\hline Batc & h Average & 21 & $n / a$ & $n / a$ & $n / a$ & $n / a$ & $n / a$ & $n / a$ & $n / a$ & $n / a$ & $n / a$ & $n / a$ & $n / a$ & $n / a$ & $n / a$ \\
\hline 342 & 18-Jan-06 & & & & & & & & & & & & & & \\
\hline
\end{tabular}


A-3 MBR NOx / P

\begin{tabular}{|c|c|c|c|c|c|c|c|c|c|c|c|c|c|c|c|}
\hline \multicolumn{16}{|c|}{ 9-Jan-06 } \\
\hline 344 & 20-Jan-06 & \multirow{3}{*}{ (8: } & 0.03 & 0.00 & & 23.87 & 0.00 & 0.00 & 0.00 & 0.00 & 36.2 & 30.8 & & & 7.1 \\
\hline 345 & 21-Jan-06 & & & & & & & & & & & & & & \\
\hline 346 & 22-Jan-06 & & & & & & & & & & & & & & \\
\hline 347 & 23-Jan-06 & \multirow{5}{*}{. } & 0.00 & 0.99 & 25.12 & 24.50 & 0.00 & 0.66 & 0.00 & 0.00 & 32.4 & 29.8 & 8.5 & 8.9 & 4.3 \\
\hline 348 & 24-Jan-06 & & 0.00 & 0.00 & 17.17 & 17.17 & 0.00 & 0.00 & 0.00 & 0.00 & 32.0 & 94.4 & 37.1 & 6.6 & 5.3 \\
\hline 349 & 25-Jan-06 & & 0.00 & 0.00 & 19.89 & 17.17 & 0.00 & 0.09 & 0.00 & 0.00 & 32.4 & 96.0 & 34.3 & 5.0 & 3.7 \\
\hline 350 & 26-Jan-06 & & 0.00 & 0.00 & 19.68 & 18.21 & 0.00 & 0.00 & 0.00 & 0.00 & 30.1 & 95.1 & 34.3 & 6.5 & 5.3 \\
\hline 351 & 27-Jan-06 & & 0.00 & 0.00 & 19.05 & 19.05 & 0.00 & 0.00 & 0.00 & 0.00 & & 65.8 & 38.4 & 5.0 & 5.9 \\
\hline 352 & 28-Jan-06 & & & & & & & & & & & & & & \\
\hline 353 & 29-Jan-06 & & & & & & & & & & & & & & \\
\hline 354 & 30-Jan-06 & & 0.00 & 0.00 & 20.73 & 18.84 & 0.00 & 0.14 & 3.47 & 0.00 & 29.2 & 98.6 & 49.5 & 6.6 & 5.9 \\
\hline \multicolumn{2}{|c|}{ Batch Average } & 24 & 0.00 & 0.00 & 20.27 & 19.83 & 0.00 & 0.04 & 0.00 & 0.00 & 32.0 & 72.9 & 33.7 & 6.4 & 5.4 \\
\hline \multicolumn{2}{|c|}{$355 \quad 31-J a n-06 \mid$} & \multirow{7}{*}{9} & & & & & & & & & & & & & \\
\hline 356 & $1-F_{E}$ & & 0.00 & 0.02 & 6.43 & 21.68 & 0.05 & 0.13 & 2.71 & 3.39 & 32.4 & 95.7 & 42.1 & 10.6 & 7.1 \\
\hline 357 & 2-Feb-06 & & 0.00 & 0.57 & 9.06 & 12.26 & 0.05 & 0.10 & 0.00 & 4.19 & 33.9 & 106.1 & 47.2 & 9.9 & 10.6 \\
\hline 358 & 3-Feb-06 & & 0.00 & 0.70 & 12.64 & 11.13 & 0.05 & 0.11 & 0.00 & 0.00 & 33.6 & 104.9 & 46.8 & 11.2 & 11.5 \\
\hline 359 & 4-Feb-06 & & & & & & & & & & & & & & \\
\hline 360 & 5-Feb-06 & & & & & & & & & & & & & & \\
\hline 361 & $6-F e b-06$ & & 0.00 & 0.74 & 18.85 & 16.40 & 0.04 & 0.21 & 0.00 & 0.00 & 33.0 & 94.2 & 48.7 & 9.3 & 9.9 \\
\hline 362 & 7-Feb-06 & \multirow{6}{*}{$\frac{6}{68}}$. & 0.00 & 0.36 & 14.34 & 17.54 & 0.00 & 0.41 & 0.00 & 0.00 & 34.2 & 98.3 & 40.1 & 11.0 & 11.6 \\
\hline 363 & 8-Feb-06 & & 0.00 & 0.00 & 15.14 & 17.74 & 0.00 & 0.00 & 0.00 & 0.00 & 35.1 & 91.8 & 40.4 & 11.3 & 11.9 \\
\hline 364 & 9-Feb-06 & & 0.00 & 0.00 & 14.14 & 18.74 & 0.00 & 0.00 & 0.00 & 0.00 & 36.1 & 98.3 & 41.6 & 12.5 & 12.2 \\
\hline 365 & $10-F e b-06$ & & & & & & & & & & 37.6 & & & & \\
\hline 366 & 11-Feb-06 & & & & & & & & & 4 & 0 & & & & \\
\hline 367 & 12-Feb-06 & & & & & & & & & & & & & & \\
\hline 368 & 13-Fel & & 0.00 & 0.00 & 15.94 & 15.74 & 0.00 & 0.00 & 0.00 & 0.00 & 36.4 & 94.9 & 41.0 & 10.4 & 9.8 \\
\hline 369 & 14-Feb-06 & & 0.00 & 0.00 & 14.40 & 17.04 & 0.00 & 0.00 & 0.00 & 0.00 & 34.8 & 105.8 & 42.0 & 12.2 & 12.2 \\
\hline \multirow{2}{*}{\multicolumn{2}{|c|}{\begin{tabular}{|l|l|} 
Batch Average \\
370 & $15-$ Feb-06 \\
\end{tabular}}} & 23 & 0.00 & 0.27 & 13.44 & 16.48 & 0.02 & 0.07 & 0.00 & 0.42 & 34.7 & 98.9 & 43.3 & 10.9 & 11.2 \\
\hline \multirow{2}{*}{\begin{tabular}{l|l|}
370 & $15-F e b-06$ \\
371 & $16-F e b-06$
\end{tabular}} & & \multirow{6}{*}{8} & & & & & & & & & & & & & \\
\hline & 16-Feb-06 & & 0.00 & 0.00 & 10.88 & 15.72 & 0.00 & 0.18 & 0.00 & 0.00 & 30.1 & 96.5 & 36.3 & 10.1 & 5.9 \\
\hline 372 & 17-Feb-06 & & 0.00 & 0.00 & 13.96 & 15.50 & 0.00 & 0.00 & 0.00 & 0.00 & 32.5 & 105.8 & 46.2 & 6.8 & 7.4 \\
\hline 373 & 18-Feb-06 & & & & & & - & 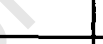 & & & & & & & \\
\hline 374 & 19-Feb-06 & & & & & & $=$ & & & & & & & & \\
\hline 375 & $20-F e b-06$ & & & & & & & & & & & & & & \\
\hline 376 & 21-Feb-06 & \multirow{8}{*}{$\frac{10}{10}$} & 0.00 & 0.00 & 12.20 & 14.40 & 0.00 & 0.00 & 0.00 & 0.00 & 27.2 & 92.3 & 44.3 & 10.0 & 8.8 \\
\hline 377 & $22-F e b-06$ & & 0.00 & 0.09 & 19.24 & 20.56 & 0.00 & 0.81 & 0.00 & 0.00 & 401 & 100.1 & 41.9 & 8.5 & 9.7 \\
\hline 378 & 23-Feb-06 & & 0.00 & 0.00 & 11.54 & 13.30 & 0.00 & 0.00 & 0.00 & 0.00 & 30.2 & 103.7 & 50.4 & 8.2 & 6.7 \\
\hline 379 & 24-Feb-06 & & 0.00 & 0.68 & 18.14 & 19.68 & 0.11 & 0.81 & 0.00 & 0.00 & 27.8 & 87.4 & 37.4 & 5.8 & 5.5 \\
\hline 380 & $25-F e b-06$ & & & & + & & & & & & & & & & \\
\hline 381 & 26-Feb-06 & & & & & & & & & & & & & & \\
\hline 382 & 27-Feb-06 & & 0.00 & 0.00 & 11.22 & 11.85 & 0.00 & 0.00 & 0.00 & 0.00 & 31.0 & 70.2 & 28.0 & 4.6 & 1.9 \\
\hline 383 & $28-F e b-06$ & & 0.00 & 0.00 & 16.47 & 16.89 & 0.00 & 0.19 & 0.00 & 0.00 & 32.5 & 98.7 & 43.6 & 11.2 & 12.4 \\
\hline \multicolumn{2}{|c|}{ Batch Average } & 24 & 0.00 & 0.01 & 14.21 & 15.99 & 0.00 & 0.25 & 0.00 & 0.00 & 30.2 & 97.8 & 41.0 & 8.1 & 7.3 \\
\hline 384 & 1-Mar-06 & & 0.00 & 1.02 & 20.67 & 21.72 & 0.00 & 1.60 & 0.00 & 0.00 & 34.6 & 77.7 & 30.4 & 7.3 & 5.5 \\
\hline 385 & 2-Mar-06 & 10 & & & & & & & & & & & & & \\
\hline 386 & 3-Mar-06 & $\mathrm{N}$ & & & & & & & & & & & & & \\
\hline 387 & 4-Mar-06 & 5 & & & & & & & & & & & & & \\
\hline 388 & 5-Mar-06 & 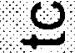 & & & & & & & & & & & & & \\
\hline 389 & 6-Mar-06 & $\pi$ & 0.00 & 0.91 & 27.39 & 29.49 & 0.00 & 0.44 & 0.00 & 0.00 & 40.0 & 104.0 & 44.8 & 13.3 & 10.6 \\
\hline 390 & 7-Mar-06 & 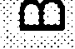 & 0.00 & 1.79 & 33.90 & 35.58 & 0.00 & 0.82 & 0.00 & 0.00 & 37.0 & 85.5 & 34.0 & 7.3 & 7.3 \\
\hline 391 & 8-Mar-06 & & 0.00 & 0.39 & 34.15 & 37.04 & 0.00 & 1.19 & 0.00 & 0.00 & 29.3 & 85.7 & 35.1 & 3.7 & 6.1 \\
\hline Batc & h Average & 25 & 0.00 & 1.03 & 29.03 & 30.96 & 0.00 & 1.01 & 0.00 & 0.00 & 35.2 & 88.2 & 36.0 & 7.9 & 7.4 \\
\hline 392 & 9-Mar-06 & & 0.00 & 0.45 & 32.82 & 34.37 & 0.00 & 1.30 & 0.00 & 0.00 & 32.0 & 89.4 & 33.2 & 5.8 & 5.2 \\
\hline 393 & 10-Mar-06 & & & & & & & & & & & & & & \\
\hline 394 & 11-Mar-06 & & & & & & & & & & & & & & \\
\hline 395 & 12-Mar-06 & & & & & & & & & & & & & & \\
\hline 396 & 13-Mar-06 & & 0.00 & 0.08 & 15.06 & 18.83 & 0.00 & 0.56 & 0.00 & 0.00 & 27.8 & 96.9 & 38.1 & & 6.4 \\
\hline
\end{tabular}


A-3 MBR NOx/P

\begin{tabular}{|c|c|c|c|c|c|c|c|c|c|c|c|c|c|c|c|}
\hline 397 & 14-Mar-06 & \multirow{10}{*}{$\frac{\frac{c}{U}}{\frac{0}{\pi}}$} & 0.00 & 0.00 & 15.28 & 16.84 & 0.00 & 0.02 & 0.00 & 0.00 & 26.0 & 90.6 & 38.4 & 7.3 & 4.0 \\
\hline 398 & 15-Mar-06 & & 0.00 & 0.00 & 12.00 & 15.42 & 0.04 & 0.05 & 0.00 & 0.00 & 25.4 & 104.7 & 41.2 & 9.8 & 10.4 \\
\hline 399 & 16-Mar-06 & & 0.00 & 0.00 & 14.56 & 21.20 & 0.04 & 0.05 & 0.00 & 0.00 & 32.4 & 103.8 & 45.2 & 7.4 & 8.6 \\
\hline 400 & 17-Mar-06 & & 0.00 & 0.00 & 14.99 & 16.92 & 0.04 & 0.14 & 0.00 & 0.00 & 25.4 & 93.4 & 41.8 & 8.9 & 9.8 \\
\hline 401 & 18-Mar-06 & & & & & & & & & & & & & & \\
\hline 402 & 19-Mar-06 & & & & & & & & & & & & & & \\
\hline 403 & 20-Mar-06 & & 0.00 & 0.00 & 19.49 & 22.48 & 0.06 & 0.77 & 0.00 & 0.00 & 34.5 & 111.4 & 43.1 & & 6.7 \\
\hline 404 & 21-Mar-06 & & 0.00 & 0.00 & 6.43 & 7.07 & 0.03 & 0.05 & 0.00 & 0.00 & 29.9 & 79.4 & 33.6 & 9.8 & 8.9 \\
\hline 405 & 22-Mar-06 & & & & & & & & & & & & & & \\
\hline 406 & 23-Mar-06 & & 0.00 & 0.00 & 13.70 & 17.27 & 0.05 & 0.00 & 0.00 & 0.00 & 27.0 & 92.8 & 38.2 & 8.3 & 7.2 \\
\hline Batch & h Average & 26 & 0.00 & 0.01 & 13.94 & 17.00 & 0.03 & 0.21 & 0.00 & 0.00 & 28.9 & 95.8 & 39.2 & 8.2 & 7.5 \\
\hline 407 & 24-Mar-06 & \multirow{15}{*}{$\frac{N}{\frac{N}{\sigma}}$} & 0.00 & 0.00 & 19.64 & 21.82 & 0.04 & 0.00 & 0.00 & 0.00 & 29.3 & 95.7 & 38.2 & 7.8 & 7.5 \\
\hline 408 & 25-Mar-06 & & & & & & & & & & & & & & \\
\hline 409 & 26-Mar-06 & & & & & & & & & & & & & & \\
\hline 410 & 27-Mar-06 & & 0.00 & 1.10 & 21.03 & 23.41 & 0.05 & 0.00 & 0.00 & 0.00 & 33.9 & 53.4 & 29.0 & 17.0 & 178 \\
\hline 4111 & 28-Mar-06 & & 0.00 & 6.50 & 14.69 & 17.07 & 0.00 & 0.00 & 0.00 & 0.00 & 29.6 & 98.6 & 37.6 & 7.5 & 8.0 \\
\hline 412 & 29-Mar-06 & & & & & & & & & & & & & & \\
\hline 413 & 30-Mar-06 & & 0.04 & 0.51 & 8.16 & 16.23 & 0.20 & 0.51 & 0.00 & 0.00 & 25.4 & 63.8 & 69.6 & 19.0 & 5.9 \\
\hline 414 & 31-Mar-06 & & 0.00 & 0.58 & 13.09 & 14.88 & 0.08 & 0.54 & 0.00 & 0.00 & 27.7 & 80.1 & 33.1 & 15.5 & 7.8 \\
\hline 415 & 1-Apr-06 & & 0.00 & 0.08 & 9.96 & 18.24 & 0.13 & 0.22 & 0.00 & 0.00 & 27.7 & 110.5 & 48.1 & 16.8 & 9.4 \\
\hline 416 & 2-Apr-06 & & 0.00 & 0.00 & 13.09 & 18.69 & 0.06 & 0.08 & 0.00 & 0.00 & 30.5 & 105.1 & 49.4 & 17.1 & 10.7 \\
\hline 417 & 3-Apr-06 & & 0.00 & 0.30 & 16.90 & 20.04 & 0.08 & 0.38 & 0.00 & 0.00 & 27.7 & 99.9 & 39.5 & 10.7 & 5.6 \\
\hline 418 & 4-Apr-06 & & 0.00 & 1.73 & 17.80 & 22.28 & 0.08 & 1.19 & 0.00 & 0.00 & 31.8 & 104.4 & 41.4 & 15.2 & 8.5 \\
\hline 419 & 5-Apr-06 & & 0.00 & 0.00 & 14.66 & 23.00 & 0.11 & 0.86 & 0.73 & 0.31 & 24.5 & 91.4 & 37.9 & 9.8 & 7.7 \\
\hline 420 & 6-Apr-06 & & 0.00 & 0.00 & 15.03 & 20.86 & 0.08 & 0.12 & 0.79 & 0.31 & 30.0 & 88.3 & 43.1 & 13.8 & 8.0 \\
\hline 421 & 7-Apr-06 & & 0.00 & 0.00 & 17.02 & 21.50 & 0.05 & 0.25 & 0.73 & 0.31 & 32.4 & 94.4 & 37.0 & 12.3 & 8.0 \\
\hline Batcl & h Average & 27 & 0.00 & 0.39 & 15.09 & 19.84 & 0.07 & 0.27 & 0.19 & 0.08 & 29.2 & 93.8 & 39.5 & 13.5 & 7.9 \\
\hline 422 & 8-Apr-06 & \multirow{15}{*}{9} & & & & & & & & & & & & & \\
\hline 423 & 9-Apr-06 & & & & & & & & & & & & & & \\
\hline 424 & 10-Apr-06 & & 0.00 & 0.00 & 16.10 & 19.36 & 0.06 & 0.09 & 0.79 & 0.31 & 28.2 & 91.4 & 38.8 & 5.6 & 1.9 \\
\hline 425 & 11-Apr-06 & & 0.00 & 0.00 & 17.60 & 21.29 & 0.04 & 0.08 & 0.79 & 0.31 & 14.4 & 92.6 & 36.4 & 2.2 & 0.4 \\
\hline 426 & 12-Apr-06 & & 0.00 & 0.00 & 17.13 & 24.09 & 0.11 & 0.08 & 0.00 & 0.00 & 17.7 & $115: 6$ & 39.2 & & \\
\hline 427 & 13-Apr-06 & & 0.00 & 1.38 & 20.61 & 25.95 & 0.10 & 1.16 & 0.00 & 0.00 & & 97.5 & 35.2 & & \\
\hline 428 & 14-Apr-06 & & 0.00 & 0.00 & 17.83 & 21.08 & 0.05 & 0.20 & 0.00 & 0.00 & 18.4 & 96.5 & 30.1 & & \\
\hline 429 & 15-Apr-06 & & 0.00 & 0.00 & 16.67 & 21.31 & 0.05 & 0.08 & 0.00 & 0.00 & 18.1 & 95.1 & 33.8 & 5.7 & \\
\hline 430 & 16-Apr-06 & & & & & D & & & & & & & & & \\
\hline 431 & 17-Apr-06 & & & & & & & & & & & & & & \\
\hline 432 & 18-Арг-06 & & 0.00 & 0.69 & 19.22 & 21.50 & 0.07 & 1.79 & 0.00 & 0.00 & 25.6 & 75.2 & 31.1 & 3.6 & 0.7 \\
\hline 433 & 19-Apr-06 & & 0.00 & 11.22 & 17.89 & 19.79 & 0.10 & 0.11 & 0.00 & 0.00 & 19.5 & 98.1 & 41.3 & 2.7 & 1.3 \\
\hline 434 & 20-Apr-06 & & 0.00 & 0.00 & 16.56 & 18.84 & 0.09 & 0.08 & 0.00 & 0.00 & 20.1 & 94.6 & 39.5 & 9.7 & 0.4 \\
\hline 435 & 21-Apr-06 & & 0.00 & 0.00 & 15.99 & 19.03 & 0.06 & 0.11 & 0.00 & 0.00 & 23.9 & 98.1 & 39.8 & 1.9 & 0.4 \\
\hline 436 & $22-A p r-06$ & & & & & & & & & & & & & & \\
\hline \multicolumn{2}{|c|}{ Batch Average } & 28 & 0.00 & 0.23 & 17.22 & 20.70 & 0.07 & 0.22 & 0.16 & 0.06 & 20.7 & 95.5 & 36.5 & 4.5 & 0.8 \\
\hline \multicolumn{2}{|c|}{\begin{tabular}{|l|l|}
437 & $23-A p r-06$ \\
\end{tabular}} & \multirow{13}{*}{$\frac{9}{18}$} & & & & & & & & & & & & & \\
\hline 438 & 24-Apr-06 & & & & & 12.89 & 0.11 & 0.05 & 0.64 & 0.07 & 9.1 & 81.6 & 32.4 & 1.4 & 1.7 \\
\hline 439 & 25-Apr-06 & & & & & 11.03 & 0.05 & 0.05 & 0.75 & 0.07 & 10.3 & 88.0 & 33.7 & 1.7 & 1.4 \\
\hline 440 & 26-Apr-06 & & 0.00 & 0.00 & 8.03 & 12.19 & 0.04 & 0.06 & 0.75 & 0.07 & 27.8 & 81.3 & 31.8 & 1.1 & 2.6 \\
\hline 441 & 27-Apr-06 & & 0.00 & 0.00 & 8.60 & 13.12 & 0.04 & 0.04 & 0.64 & 0.07 & 18.7 & 76.7 & 28.7 & 1.4 & 1.1 \\
\hline 442 & 28-Apr-06 & & 0.00 & 0.00 & 12.55 & 14.28 & 0.05 & 0.05 & 0.64 & 0.07 & 34.9 & 91.7 & 37.6 & 2.3 & 1.4 \\
\hline 443 & 29-Apr-06 & & & & & & 0.03 & 0.11 & 0.00 & 0.00 & 45.8 & 1170 & 62.2 & 8.0 & 7.1 \\
\hline 444 & 30-Apr-06 & & 0.00 & 0.04 & 10.45 & 13.87 & 0.05 & 0.35 & 0.00 & 0.00 & 36.3 & 84.1 & 35.7 & 7.7 & 8.6 \\
\hline 445 & 1-May-06 & & 0.00 & 0.19 & 8.63 & 14.10 & 0.04 & 0.57 & 0.00 & 0.00 & 35.1 & 85.6 & 36.9 & 6.5 & 6.2 \\
\hline 446 & 2-May-06 & & & & & & & & & & & & & & \\
\hline 447 & 3-May-06 & & 0.00 & 0.00 & 8.40 & 9.31 & 0.03 & 0.11 & 0.00 & 0.00 & 54.0 & 82.6 & 31.7 & 2.2 & 8.0 \\
\hline 448 & 4-May-06 & & & & & & & & & & & & & & \\
\hline 449 & 5-May-06 & & 0.00 & 2.28 & 13.87 & 15.01 & 0.03 & 1.50 & 0.00 & 0.00 & 37.8 & 85.6 & 36.6 & 8.0 & 8.6 \\
\hline Batc & h Average & 29 & 0.00 & 0.04 & 10.08 & 13.31 & 0.05 & 0.15 & 0.34 & 0.04 & 31.0 & 84.1 & 33.9 & 4.0 & 4.7 \\
\hline
\end{tabular}




\section{A-4 MBR Misc}

Appendix A-4

Miscellaneous mcasurements on the MBR UCT system

\begin{tabular}{|c|c|c|c|c|c|c|c|c|c|c|c|c|c|c|c|}
\hline Day & Date & SB\# & \multicolumn{2}{|c|}{ msrd. adj. } & \multicolumn{2}{|l|}{$\mathrm{Qi}$} & \multicolumn{2}{|c|}{ meas. revised } & as & $r$ & Head & $\overline{A N}$ & $A X$ & $\overline{A E}$ & $\overline{R E}$ \\
\hline- & - & - & $(\mathrm{mm} / \mathrm{g}$ & $S S / L)$ & (l) & & $\mathrm{mgO}$ & D/hr & - & & $(\mathrm{mm})$ & & (l) & & \\
\hline 19 & 1-Mar-05 & & & & 140 & 2.85 & & & & & & 19.0 & 21.0 & 34.0 & \\
\hline 20 & 2-Mar-05 & & & & 140 & 2.85 & & & & & & 19.0 & 21.0 & 34.0 & \\
\hline 21 & 3-Mar-05 & & & & 140 & 2.85 & & & & & & 19.0 & 21.0 & 34.0 & \\
\hline 22 & 4-Mar-05 & & & & 140 & 2.85 & & & & & & 19.0 & 21.0 & 34.0 & \\
\hline 23 & 5-Mar-05 & & & & 140 & & & & & & & 19.0 & 21.0 & 34.0 & \\
\hline 24 & 6-Mar-05 & & & & 140 & & & & & & & 19.0 & 21.0 & 34.0 & \\
\hline 25 & 7-Mar-05 & & & & 140 & 2.85 & & & & & & 18.0 & 20.0 & 34.0 & \\
\hline 26 & $8-$ Mar-05 & & & & 140 & 2.30 & & & & & 95.0 & 19.0 & 21.0 & 34.0 & \\
\hline 27 & 9-Mar-05 & 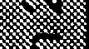 & 105.0 & 122.2 & 140 & 2.85 & & & & & 120.0 & 19.0 & 20.0 & 32.0 & \\
\hline 28 & 10-Mar-05 & 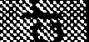 & & & 140 & 3.60 & & & & & 390 & 19.0 & 21.0 & 32.0 & \\
\hline 29 & 11-Mar-05 & 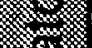 & & & 140 & 2.85 & & & & & & 19.0 & 21.0 & 34.0 & \\
\hline 30 & 12-Mar-05 & 1 & & & 140 & & & & & & & 19.0 & 21.0 & 34.0 & \\
\hline 31 & 13-Mar-05 & 8 & & & 140 & & & & & & & 19.0 & 21.0 & 34.0 & \\
\hline 32 & 14-Mar-05 & & 85.0 & 112.4 & 140 & 1.00 & & & & & 130.0 & 19.0 & 21.0 & 36.0 & \\
\hline 33 & 15-Mar-05 & & 90.0 & 103.2 & 140 & 4.80 & & & & & 130.0 & 19.0 & 21.0 & 34.0 & \\
\hline 34 & 16-Mar-05 & & & & 140 & 2.85 & & & & & 130.0 & 19.0 & 21.0 & 34.0 & \\
\hline 35 & 17-Mar-05 & & 90.0 & 116.5 & 140 & 2.85 & & & & & $\infty$ & 19.0 & 21.0 & 34.0 & \\
\hline 36 & 18-Mar-05 & & & & 140 & 2.85 & & & & & 130.0 & 19.0 & 21.0 & 34.0 & \\
\hline Batc & h Average & 2 & 92.5 & 113.4 & 140 & 2.88 & & & 4.00 & 1.00 & 122.5 & 18.9 & 20.9 & 33.9 & \\
\hline 37 & 19-Mar-05 & & & & 140 & & & & & & & 19.0 & 21.0 & 34.0 & \\
\hline 38 & 20-Mar-05 & & & & 140 & & & & & & E & 19.0 & 21.0 & 34.0 & \\
\hline 39 & 21-Mar-05 & & 750 & 106.1 & 140 & 2.85 & & & & $\square$ & & 19.0 & 21.0 & 34.0 & \\
\hline 40 & 22-Mar-05 & & & & 140 & 2.85 & & & & & 130.0 & 19.0 & 21.0 & 34.0 & \\
\hline 41 & 23-Mar-05 & & & & 140 & 2.85 & & & & & & 19.0 & 21.0 & 34.0 & \\
\hline 42 & 24-Mar-05 & & & & 140 & 2.85 & & & - & & 130.0 & 18.0 & 21.0 & 34.0 & \\
\hline 43 & 25-Mar-05 & $\infty$ & 75.0 & 98.0 & 140 & 2.85 & & & 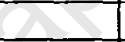 & & & 19.0 & 21.0 & 34.0 & \\
\hline 44 & 26-Mar-05 & 1 & & & 140 & & & & 0 & & & 19.0 & 21.0 & 34.0 & \\
\hline 45 & 27-Mar-05 & 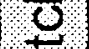 & & & 140 & 2.85 & & 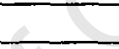 & & & & 19.0 & 21.0 & 34.0 & \\
\hline 46 & 28-Mar-05 & 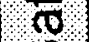 & & & 140 & & & 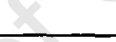 & & & & 19.0 & 21.0 & 34.0 & \\
\hline 47 & 29-Mar-05 & $\mathbf{a}$ & 90.0 & 102.0 & 140 & 2.85 & & 2 & & & 130.0 & 19.0 & 21.0 & 34.0 & \\
\hline 48 & 30-Mar-05 & & & & 140 & & & 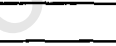 & & & & 19.0 & 21.0 & 34.0 & \\
\hline 49 & 31-Mar-05 & & 90.0 & 96.8 & 140 & 2.85 & & & & & & 19.0 & 21.0 & 34.0 & \\
\hline 50 & 1-Apr-05 & & & & 140 & & 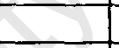 & & & & & 19.0 & 21.0 & 34.0 & \\
\hline 51 & 2-Apr-05 & & 85.0 & 101.4 & 140 & 2.85 & D & & & & & 19.0 & 21.0 & 34.0 & \\
\hline 52 & 3-Apr-05 & & & & 140 & & & & & & & 19.0 & 21.0 & 34.0 & \\
\hline 53 & 4-Apr-05 & & & & 140 & 2.85 & & & & & & 19.0 & 21.0 & 34.0 & \\
\hline Batc & h Average & 3 & 83.0 & 104.8 & 140 & 2.85 & & & 4.00 & 1.00 & 130.0 & 18.9 & 21.0 & 34.0 & \\
\hline 54 & 5-Apr-05 & & & & 140 & 3.35 & & & & & & 19.0 & 21.0 & 34.0 & \\
\hline 55 & 6-Apr-05 & & & & 140 & & & & & & & 19.0 & 21.0 & 34.0 & \\
\hline 56 & 7-Apr-05 & & & & 140 & & & & & & & 19.0 & 21.0 & 34.0 & \\
\hline 57 & 8-Apr-05 & & 80.0 & 102.0 & 140 & 2.85 & & & & & 19.0 & 19.0 & 21.0 & 34.0 & \\
\hline 58 & 9-Apr-05 & & & & 140 & & & & & & & 19.0 & 21.0 & 34.0 & \\
\hline 59 & 10-Aрг-05 & & & & 140 & 2.85 & & & & & & 19.0 & 21.0 & 34.0 & \\
\hline 60 & 11-Apr-05 & & & & 140 & & & & & & 30.0 & 19.0 & 21.0 & 34.0 & \\
\hline 61 & 12-Aрг-05 & & & & 140 & 2.85 & & & & & & 19.0 & 21.0 & 34.0 & \\
\hline 62 & 13-Apr-05 & & & & 140 & & & & & 0.95 & & 19.0 & 21.0 & 34.0 & \\
\hline 63 & 14-Apr-05 & 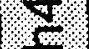 & 85.0 & 87.7 & 140 & 2.85 & & & & & 20.0 & 19.0 & 21.0 & 34.0 & \\
\hline 64 & 15-Apr-05 & $\$$ & & & 140 & & & & & 1.30 & & 19.0 & 21.0 & 34.0 & \\
\hline 65 & 16-Apr-05 & 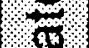 & & & 140 & 2.85 & & & & & & 19.0 & 21.0 & 34.0 & \\
\hline 66 & 17-Apr-05 & $m$ & & & 140 & & & & & & & 19.0 & 21.0 & 34.0 & \\
\hline 67 & 18-Apr-05 & & & & 140 & 2.85 & & & & & & 19.0 & 21.0 & 34.0 & \\
\hline 68 & 19-Apr-05 & & 55.0 & 81.4 & 140 & 2.85 & & & & 1.75 & 45.0 & 19.0 & 21.0 & 34.0 & \\
\hline 69 & 20-Apr-05 & & & & 140 & 2.85 & & & & & & 19.0 & 21.0 & 34.0 & \\
\hline 70 & 21-Apr-05 & & & & 140 & 2.85 & & & & & & 19.0 & 21.0 & 34.0 & \\
\hline 71 & 22-Apr-05 & & & & 140 & & & & 4.00 & 1.74 & & 19.0 & 21.0 & 34.0 & \\
\hline 72 & 23-Apr-05 & & 45.0 & 107.9 & 140 & & 36.1 & & & & & 19.0 & 21.0 & 34.0 & \\
\hline 73 & 24-Apr-05 & & & & 140 & & & & & & & 19.0 & 21.0 & 34.0 & \\
\hline 74 & 25-Apr-05 & & 40.0 & 91.5 & 140 & & 32.5 & & & & 35.0 & 19.0 & 21.0 & 34.0 & \\
\hline 75 & 26-Apr-05 & & & & 140 & & & & & & & 19.0 & 21.0 & 34.0 & \\
\hline
\end{tabular}


A-4 MBR Misc

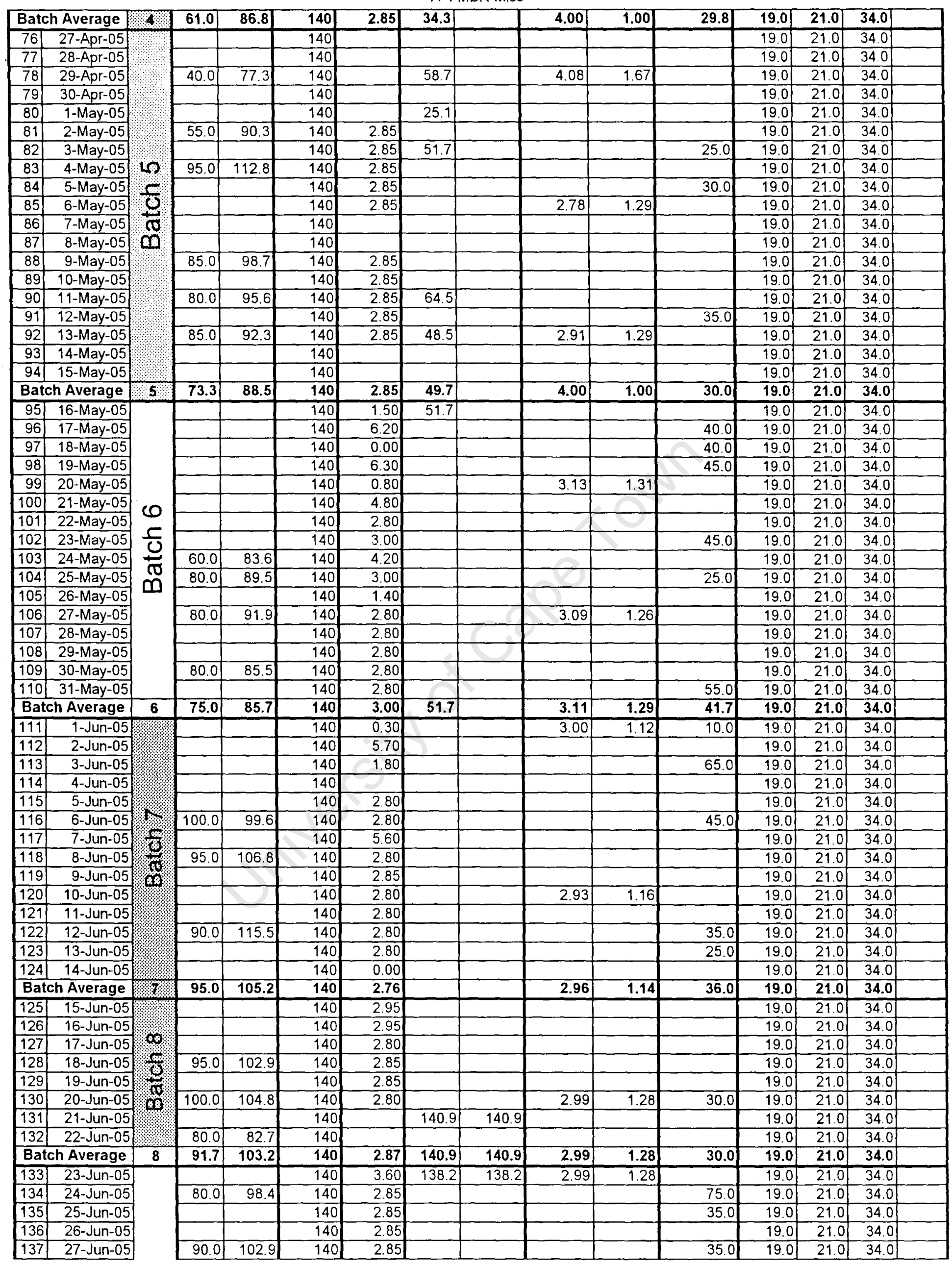




\section{A-4 MBR Misc}

\begin{tabular}{|c|c|c|c|c|c|c|c|c|c|c|c|c|c|c|c|}
\hline 138 & 28-Jun-05 & \multirow{10}{*}{$\begin{array}{l}\sigma \\
\frac{r}{U} \\
\frac{\mathbb{\pi}}{\sigma} \\
\infty\end{array}$} & 95.0 & 103.8 & 140 & 2.85 & & & & & & 19.0 & 21.0 & 34.0 & \\
\hline 139 & 29-Jun-05 & & 900 & 103.1 & 140 & 2.85 & & & & & & 19.0 & 21.0 & 34.0 & \\
\hline 140 & 30-Jun-05 & & 90.0 & 98.5 & 140 & 3.05 & 139.6 & 139.6 & & & 500 & 19.0 & 21.0 & 34.0 & \\
\hline 141 & 1-Jul-05 & & & & 140 & 2.85 & 116.2 & 116.2 & & & & 190 & 21.0 & 34.0 & \\
\hline 142 & 2-Jul-05 & & & & 140 & 2.85 & & & & & & 190 & 210 & 34.0 & \\
\hline 143 & 3-Jul-05 & & & & 140 & 2.85 & & & & & & 19.0 & 21.0 & 34.0 & \\
\hline 144 & 4-Jul-05 & & 85.0 & 94.5 & 140 & 2.85 & & & & & & 19.0 & 21.0 & 34.0 & \\
\hline 145 & 5-Jul-05 & & & & 140 & 2.85 & & & & & & 19.0 & 21.0 & 34.0 & \\
\hline 146 & 6-Jul-05 & & 85.0 & 94.1 & 140 & 2.95 & 132.1 & 132.1 & & & 40.0 & 19.0 & 21.0 & 34.0 & \\
\hline 147 & 7-Jul-05 & & 85.0 & 92.8 & 140 & 2.85 & & & 3.17 & 1.15 & & 19.0 & 21.0 & 34.0 & \\
\hline Batc & h Average & 9 & 87.5 & 96.5 & 140 & 2.92 & 131.5 & 131.5 & 3.08 & 1.22 & 47.0 & 19.0 & 21.0 & 34.0 & \\
\hline 148 & 8-Jul-05 & \multirow{20}{*}{$\frac{9}{9}$} & & & 140 & 2.85 & & & 3.17 & 1.15 & & 19.0 & 21.0 & 34.0 & \\
\hline 149 & $9-\mathrm{Jul}-05$ & & & & 140 & 2.85 & & & & & & 19.0 & 210 & 34.0 & \\
\hline 150 & 10-Jul-05 & & & & 140 & 2.85 & & & & & & 19.0 & 21.0 & 34.0 & \\
\hline 151 & 11-Jul-05 & & 70.0 & 81.4 & 140 & 2.90 & & & & & & 19.0 & 21.0 & 34.0 & \\
\hline 152 & 12-Jul-05 & & 80.0 & 92.2 & 140 & 2.85 & 123.2 & 123.2 & & & 35.0 & 19.0 & 21.0 & 340 & \\
\hline 153 & 13-Jul-05 & & & & 140 & 2.80 & 1200 & 1200 & & & & 19.0 & 21.0 & 340 & \\
\hline 154 & 14-Jul-05 & & 90.0 & 100.2 & 140 & 2.85 & 105.0 & 105.0 & & & & 19.0 & 21.0 & 34.0 & \\
\hline 155 & 15-Jul-05 & & & & 140 & 2.85 & & & & & & 19.0 & 21.0 & 34.0 & \\
\hline 156 & 16-Jut-05 & & & & 140 & 2.85 & & & & & 45.0 & 19.0 & 21.0 & 34.0 & \\
\hline 157 & 17-Jul-05 & & & & 140 & 2.85 & & & & & & 19.0 & 21.0 & 34.0 & \\
\hline 158 & 18-Jul-05 & & 80.0 & 96.3 & 140 & 2.90 & & & & & 45.0 & 19.0 & 21.0 & 34.0 & \\
\hline 159 & 19-Jul-05 & & 80.0 & 94.2 & 140 & 2.85 & 140.0 & 140.0 & & & & 19.0 & 21.0 & 34.0 & \\
\hline 160 & 20 -Jul-05 & & 80.0 & 93.7 & 140 & 3.00 & 126.0 & 126.0 & & & & 19.0 & 21.0 & 34.0 & \\
\hline 161 & $21-\mathrm{Jul}-05$ & & & & 140 & 2.85 & & & & & 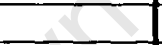 & 19.0 & 21.0 & 34.0 & \\
\hline 162 & 22-Jul-05 & & & & 140 & 2.85 & & & & & $\square$ & 19.0 & 21.0 & 34.0 & \\
\hline 163 & 23-Jul-05 & & & & 140 & 2.85 & 118.0 & 118.0 & & & 50.0 & 19.0 & 21.0 & 34.0 & \\
\hline 164 & 24-Jul-05 & & & & 140 & 2.85 & 116.0 & 116.0 & & 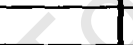 & & 19.0 & 21.0 & 34.0 & \\
\hline 165 & 25 -Jul-05 & & 110.0 & 121.0 & 140 & 2.85 & & & & & 70.0 & 19.0 & 21.0 & 34.0 & \\
\hline 166 & 26-Jul-05 & & 110.0 & 122.8 & 140 & 2.85 & & & & & 70.0 & 19.0 & 21.0 & 34.0 & \\
\hline 167 & $27-J u l-05$ & & 110.0 & 125.0 & 140 & 2.85 & & & & & & 19.0 & 21.0 & 34.0 & \\
\hline Batc & $\mathrm{h}$ Average & 籍 & 90.0 & 102.1 & 140 & 2.86 & 121.2 & 121.2 & 3.17 & 1.15 & 52.5 & 19.0 & 21.0 & 34.0 & \\
\hline 168 & 28-Jul-05 & \multirow{21}{*}{ 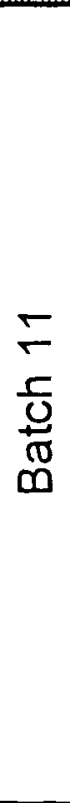 } & & & 140 & 2.85 & & & 3.17 & 1.15 & & 19.0 & 21.0 & 34.0 & \\
\hline 169 & 29-Jul-05 & & 110.0 & 125.9 & 140 & 290 & & & & & 70.0 & 19.0 & 21.0 & 34.0 & \\
\hline 170 & $30-J u l-05$ & & & & 140 & 2.85 & & & & & & 19.0 & 21.0 & 34.0 & \\
\hline 171 & 31-Jui-05 & & & & 140 & 2.85 & & & & & & 19.0 & 21.0 & 34.0 & \\
\hline 172 & 1-Aug-05 & & 110.0 & 114.2 & 140 & 3.00 & 111.0 & 111.0 & & & 45.0 & 19.0 & 21.0 & 34.0 & \\
\hline 173 & 2-Aug-05 & & 100.0 & 112.7 & 140 & 2.85 & 120.0 & 120.0 & & & & 19.0 & 21.0 & 34.0 & \\
\hline 174 & 3-Aug-05 & & & & 140 & 2.80 & & & & & 120.0 & 19.0 & 21.0 & 34.0 & \\
\hline 175 & 4-Aug-05 & & 100.0 & 113.2 & 140 & 2.85 & + & & & & & 19.0 & 21.0 & 34.0 & \\
\hline \begin{tabular}{|l|}
176 \\
\end{tabular} & 5-Aug-05 & & & & 140 & 2.85 & 2 & & & & 10.0 & 19.0 & 21.0 & 34.0 & \\
\hline 177 & 6-Aug-05 & & & & 140 & 2.85 & & & & & & 19.0 & 21.0 & 34.0 & \\
\hline \begin{tabular}{|l|}
178 \\
\end{tabular} & 7-Aug-05 & & & & 140 & 2.85 & & & & & & 19.0 & 21.0 & 34.0 & \\
\hline \begin{tabular}{|l|}
179 \\
\end{tabular} & 8-Aug-05 & & 90.0 & 106.4 & 140 & 2.90 & 102.0 & 102.0 & & & & 19.0 & 21.0 & 34.0 & \\
\hline 180 & 9-Aug-05 & & & & 140 & 2.85 & & & & & 70.0 & 19.0 & 21.0 & 34.0 & \\
\hline 181 & 10-Aug-05 & & 90.0 & 103.6 & 140 & 2.85 & 126.0 & 126.0 & 3.63 & 1.71 & 70.0 & 19.0 & 21.0 & 34.0 & \\
\hline 182 & 11-Aug-05 & & & & 140 & 2.85 & 111.0 & 1111.0 & & & 70.0 & 19.0 & 21.0 & 34.0 & \\
\hline 183 & 12-Aug-05 & & 85.0 & 109.7 & 140 & 2.85 & 112.0 & 131.1 & & & & 19.0 & 21.0 & 34.0 & \\
\hline 184 & 13-Aug-05 & & & & 140 & 2.85 & & & & & & 19.0 & 21.0 & 34.0 & \\
\hline 185 & 14-Aug-05 & & & & 140 & 2.85 & & & & & & 19.0 & 21.0 & 34.0 & \\
\hline 186 & 15-Aug-05 & & & & 140 & 2.85 & 107.0 & 114.3 & & & & 19.0 & 21.0 & 34.0 & \\
\hline 187 & 16-Aug-05 & & & & 140 & 2.70 & & & & & & 19.0 & 21.0 & 34.0 & \\
\hline 188 & 17-Aug-05 & & & & 140 & 2.85 & & & & & & 19.0 & 21.0 & 34.0 & \\
\hline Batc & Average & 11 & 97.9 & 114.1 & 140 & 2.85 & 112.7 & 116.5 & 3.40 & 1.43 & 65.0 & 19.0 & 21.0 & 34.0 & \\
\hline 189 & 18-Aug-05 & \multirow{13}{*}{$\begin{array}{l}\infty \\
\frac{c}{G} \\
\frac{c}{6} \\
m\end{array}$} & & & 140 & 2.85 & 114.0 & 151.5 & 3.38 & 1.56 & & 19.0 & 21.0 & 34.0 & \\
\hline 190 & 19-Aug-05 & & & & 140 & 2.85 & 119.0 & 150.9 & & & & 19.0 & 21.0 & 34.0 & \\
\hline 191 & 20-Aug-05 & & & & 140 & 2.85 & & & & & & 19.0 & 21.0 & 34.0 & \\
\hline 192 & 21-Aug-05 & & & & 140 & 2.85 & & & & & & 19.0 & 21.0 & 34.0 & \\
\hline 193 & 22-Aug-05 & & & & 140 & 2.85 & 96.0 & 119.3 & & & & 19.0 & 21.0 & 35.0 & \\
\hline 194 & 23-Aug-05 & & & & 140 & 2.85 & 99.0 & 105.7 & & & & 19.0 & 21.0 & 34.0 & \\
\hline 195 & 24-Aug-05 & & & & 140 & 2.85 & & & & & & & & & \\
\hline 196 & 25-Aug-05 & & 95.0 & 107.9 & 140 & 2.85 & 100.0 & 136.6 & & & & 19.0 & 21.0 & 34.0 & \\
\hline 197 & 26-Aug-05 & & & & 140 & 2.85 & 106.0 & 133.2 & & & & 19.0 & 21.0 & 34.0 & \\
\hline 198 & 27-Aug-05 & & & & 140 & 2.85 & & & & & & 21.0 & 23.0 & 36.0 & \\
\hline 199 & 28-Aug-05 & & & & 140 & 2.85 & & & & & & & & & \\
\hline 200 & 29-Aug-05 & & & & 140 & 2.85 & & & & & & 19.0 & 21.0 & 35.0 & \\
\hline 201 & 30-Aug-05 & & & & 140 & 2.35 & 108.0 & 115.0 & 3.24 & 1.31 & & 19.0 & 21.0 & 35.0 & \\
\hline
\end{tabular}


A-4 MBR Misc

\begin{tabular}{|c|c|c|c|c|c|c|c|c|c|c|c|c|c|c|c|}
\hline 202 & 31-Aug-05 & & & & 140 & 4.20 & 102.0 & 123.9 & & & 85.0 & & & & \\
\hline 203 & 1-Sep-05 & & & & 140 & 2.80 & 109.0 & 134.9 & & & 35.0 & 20.0 & 22.0 & 35.0 & \\
\hline 204 & 2-Sep-05 & & & & 140 & 2.85 & 117.0 & 141.7 & & & & & & & \\
\hline Batc & h Average & 12 & 95.0 & 117.8 & 140 & 2.90 & 107.0 & 131.3 & 3.31 & 1.44 & 60.0 & 19.3 & 21.3 & 34.5 & \\
\hline 205 & 3-Sep-05 & \multirow{14}{*}{$\frac{8}{\frac{8}{60}}$} & & & 140 & & & & & & & & & & \\
\hline 206 & 4-Sep-05 & & & & 140 & & & & & & & & & & \\
\hline 207 & 5-Sep-05 & & & & 140 & 2.35 & 102.0 & 135.8 & & & 85.0 & 19.0 & 21.0 & 35.0 & \\
\hline 208 & 6-Sep-05 & & & & 140 & 3.50 & & & & & 100.0 & 19.0 & 21.0 & 35.0 & \\
\hline 209 & 7-Sep-05 & & & & 140 & 2.85 & & & & & 75.0 & 20.0 & 22.0 & 340 & \\
\hline 210 & 8-Sep-05 & & 90.0 & 104.0 & 140 & 2.85 & & & 3.59 & 1.33 & 85.0 & 190 & 21.0 & 36.0 & \\
\hline 211 & 9-Sep-05 & & & & 140 & 3.35 & & & & & 95.0 & 19.0 & 21.0 & 35.0 & \\
\hline 212 & $10-$ Sep-05 & & & & 140 & 2.35 & & & & & & & & & \\
\hline 213 & 11-Sep-05 & & & & 140 & 285 & 102.0 & 140.0 & 3.83 & 1.56 & & 19.0 & 21.0 & 35.0 & \\
\hline 214 & 12-Sep-05 & & 900 & 105.2 & 140 & 2.85 & 110.0 & 151.0 & 3.37 & 1.23 & 110.0 & 19.0 & 22.0 & 35.0 & \\
\hline 215 & 13-Sep-05 & & & & 140 & 2.35 & 102.0 & 125.8 & & & 100.0 & 19.0 & 21.0 & 35.0 & \\
\hline 216 & 14-Sep-05 & & 90.0 & 105.4 & 140 & 3.00 & 111.0 & 146.9 & & & 95.0 & 19.0 & 21.0 & 35.0 & \\
\hline 217 & 15-Sep-05 & & & & 140 & 3.05 & & & 2.50 & 0.95 & 90.0 & 19.0 & 21.0 & 35.0 & \\
\hline 218 & 16-Sep-05 & & 80.0 & 100.8 & 140 & 3.15 & 113.0 & 130.3 & & & 80.0 & 19.0 & 23.0 & 36.0 & \\
\hline Batc & h Average & 13 & 87.5 & 103.7 & 140 & 2.88 & 106.7 & 138.3 & 3.32 & 1.27 & 91.5 & 19.1 & 21.4 & 35.1 & \\
\hline 219 & 17-Sep-05 & \multirow{17}{*}{$\begin{array}{l}\frac{J}{\sigma} \\
\frac{\sigma}{\mathcal{U}} \\
\frac{0}{0}\end{array}$} & & & 140 & 2.85 & & & & & & & & & \\
\hline 220 & 18-Sep-05 & & & & 140 & 2.85 & & & & & & & & & \\
\hline 221 & 19-Sep-05 & & 90.0 & & 140 & 2.85 & 104.0 & 136.4 & 3.12 & 1.24 & 45.0 & 19.0 & 21.0 & 35.0 & \\
\hline 222 & $20-\operatorname{Sep}-05$ & & 85.0 & 101.0 & 140 & 2.85 & 107.0 & 140.4 & & & 80.0 & 19.0 & 21.0 & 35.0 & \\
\hline 223 & $21-$ Sep-05 & & 800 & 100.0 & 140 & 2.85 & 112.0 & 143.1 & & & 83.0 & 19.0 & 21.0 & 35.0 & \\
\hline 224 & 22-Sep-05 & & 70.0 & 86.6 & 140 & 2.85 & 99.0 & 126.8 & & & 100.0 & 19.0 & 21.0 & 36.0 & \\
\hline 225 & 23-Sep-05 & & & & 140 & 2.50 & 109.0 & 146.5 & & & 83.0 & 19.0 & 21.0 & 35.0 & \\
\hline 226 & 24-Sep-05 & & & & 140 & 3.00 & & & 2.87 & 1.11 & & & & & \\
\hline 227 & 25 -Sep-05 & & & & 140 & 2.85 & & & & & & & & & \\
\hline 228 & $26-$ Sep-05 & & 700 & 98.0 & 140 & 2.85 & 96.0 & 120.8 & & & & 19.0 & 21.0 & 35.0 & \\
\hline 229 & 27-Sep-05 & & & & 140 & 2.85 & & & & & 95.0 & & & & \\
\hline 230 & $28-\operatorname{sep}-05$ & & & & 140 & 3.10 & 690 & 759 & 2.90 & 1.07 & & 19.0 & 21.0 & 35.0 & \\
\hline 231 & 29-Sep-05 & & & & 140 & 2.70 & & & & & & 19.0 & 21.0 & 35.0 & \\
\hline 232 & 30-Sep-05 & & & & 140 & 3.00 & & & & & & & & & \\
\hline 233 & 1-Oct-05 & & & & 140 & 2.85 & & $e^{2}$ & E & & & & & & \\
\hline 234 & 2-Oct-05 & & & & 140 & 2.85 & & & & & & & & & \\
\hline 235 & 3-Oct-05 & & 90.0 & 105.4 & 140 & 2.85 & & & & & 65.0 & & & & \\
\hline Batc & Average & 14 & 80.8 & 100.7 & 140 & 2.85 & 104.5 & 135.7 & 2.96 & 1.14 & 78.7 & 19.0 & 21.0 & 35.1 & \\
\hline 236 & 4-Oct-05 & \multirow{9}{*}{$\begin{array}{c}\frac{n}{\sigma} \\
\frac{c}{0} \\
\frac{\pi}{\omega}\end{array}$} & 90.1 & 108.3 & 140 & 2.85 & $\square$ & & & & 57.0 & 19.0 & 21.0 & 35.0 & 0.0 \\
\hline 237 & 5-Oct-05 & & 90. & 104.1 & 140 & 2.85 & & & & & 57.0 & 19.0 & 21.0 & 35.0 & 0.0 \\
\hline 238 & 6-Oct-05 & & & & 140 & 2.85 & & & & & 57.0 & & & & 0.0 \\
\hline 239 & 7-Oct-05 & & 110.0 & 120.8 & 140 & 2.85 & 69.0 & 90.4 & 3.15 & 1.25 & 56.0 & 19.0 & 21.0 & 35.0 & 00 \\
\hline 240 & 8-Oct-05 & & & & 140 & 2.85 & & & & & & & & & 0.0 \\
\hline 241 & 9-Oct-05 & & & & 140 & 2.85 & 82.0 & 107.4 & & & & & & & 0.0 \\
\hline 242 & 10-Oct-05 & & 90.1 & 105.2 & 140 & 285 & 104.0 & 132.0 & 2.97 & 1.14 & 100.0 & 19.0 & 21.0 & 35.0 & 0.0 \\
\hline 243 & 11-Oct-05 & & & & 140 & 2.85 & 104.0 & 143.2 & & & & 19.0 & 21.0 & 32.0 & 3.0 \\
\hline 244 & 12-Oct-05 & & 100. & 105.9 & 140 & 2.85 & 105.0 & 150.8 & & & 52.0 & 19.0 & 21.0 & 35.0 & 0.0 \\
\hline \multicolumn{2}{|c|}{ Batch Average } & 15 & 96.0 & 109.6 & 140 & 2.85 & 92.8 & 124.8 & 3.06 & 1.20 & 63.2 & 19.0 & 21.0 & 34.5 & 0.3 \\
\hline 245 & 13-Oct-05 & \multirow{15}{*}{$\frac{6}{10}$} & & & 140 & 2.85 & & & & & & & & & \\
\hline 246 & 14-Oct-05 & & 100. & 111.0 & 140 & 2.85 & 104.0 & 140.2 & 2.87 & 1.08 & 63.0 & 19.0 & 21.0 & 32.0 & 3.0 \\
\hline 247 & 15-Oct-05 & & & & 140 & 2.85 & & & & & & & & & \\
\hline 248 & 16 -Oct-05 & & & & 140 & 2.85 & & & & & & & & & \\
\hline 249 & \multirow{3}{*}{\begin{tabular}{|c|}
$17-$ Oct-05 \\
$18-$ Oct-05 \\
$19-$ Oct-05 \\
\end{tabular}} & & \multirow{2}{*}{100.0} & 116.2 & 140 & 2.85 & 105.0 & 129.4 & & & 50.0 & 19.0 & 21.0 & 32.0 & 3.0 \\
\hline 250 & & & & & 140 & 2.85 & & & & & 56.0 & 19.0 & 21.0 & 32.0 & 3.0 \\
\hline 251 & & & 80. & 100.6 & 140 & 2.85 & 111.0 & 136.8 & 2.75 & 1.13 & & 19.0 & 21.0 & 32.0 & 3.0 \\
\hline 252 & 20-Oct-05 & & & & 140 & 2.85 & 107.0 & 131.9 & & & 52.0 & 19.0 & 21.0 & 32.0 & 3.0 \\
\hline 253 & 21 -Oct-05 & & 70. & 86.1 & 140 & 2.85 & 117.0 & 1538 & & & 68.0 & 19.0 & 21.0 & 32.0 & 3.0 \\
\hline 254 & $22-$-ct-05 & & & & 140 & 2.85 & & & & & & 19.0 & 21.0 & 32.0 & 3.0 \\
\hline 255 & $23-$-ct-05 & & & & 140 & 2.85 & & & & & & & & & \\
\hline 256 & 24-Oct-05 & & 80. & 93.1 & 140 & 2.85 & 97.0 & 124.8 & & & & 19.0 & 21.0 & 32.0 & 3.0 \\
\hline 257 & $25-$-Oct-05 & & & & 140 & 2.85 & 97.0 & 133.6 & & & & 19.0 & 21.0 & 32.0 & 3.0 \\
\hline 258 & $26-$ - ct-05 & & 90. & 100.2 & 140 & 2.85 & 94.0 & 129.5 & & & 52.0 & 19.0 & 21.0 & 32.0 & 3.0 \\
\hline 259 & 27-Oct-05 & & & & 140 & 2.85 & 91.6 & 120.8 & 2.89 & 0.99 & & 19.0 & 21.0 & 32.0 & 3.0 \\
\hline Batc & ch Average & 16 & 86. & 101.3 & 140 & 2.85 & 102.6 & 130.9 & 2.84 & 1.07 & 56.8 & 19.0 & 21.0 & 32.0 & 3.0 \\
\hline 260 & 28 -Oct-05 & & 80 . & 92.0 & 140 & 2.90 & 84.9 & 113.4 & & & 52.0 & 19.0 & 21.0 & 32.0 & 3.0 \\
\hline 261 & $29-$ - ct-05 & & & & 140 & 2.85 & 78.4 & 104.7 & & & & 19.0 & 21.0 & 32.0 & 3.0 \\
\hline 262 & 30-Oct-05 & & & & 140 & 2.85 & & & & & & & & & \\
\hline 263 & 31-Oct-05 & & 80. & 88.3 & 140 & 2.85 & 82.8 & 114.1 & & & & 19.0 & 21.0 & 32.0 & 3.0 \\
\hline
\end{tabular}




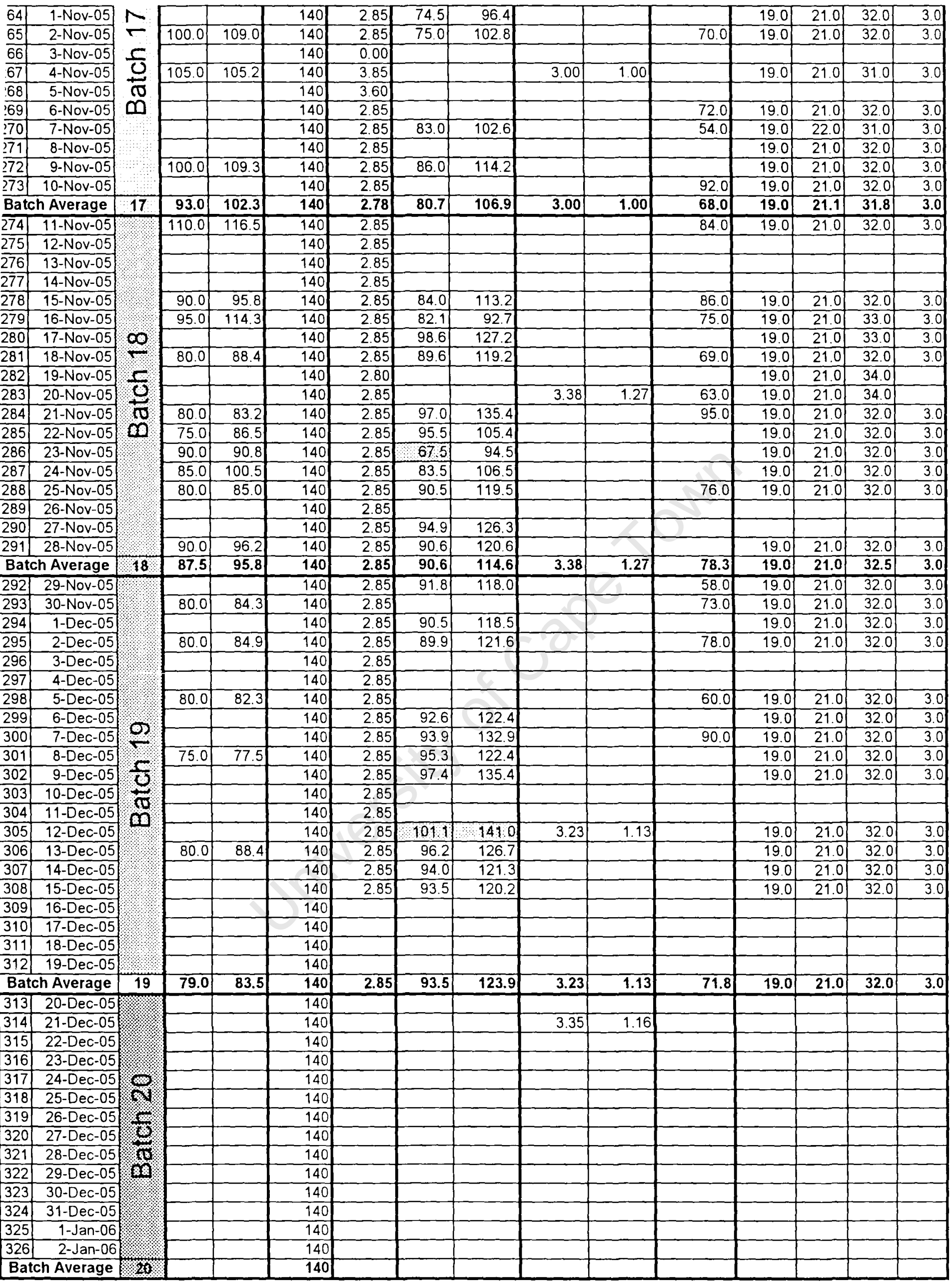


A-4 MBR Misc

\begin{tabular}{|c|c|c|c|c|c|c|c|c|c|c|c|c|c|c|c|}
\hline 90 & 7-Mar-06 & $\infty$ & 100.0 & 121.6 & 140 & 2.20 & 103.1 & 131.6 & 3.40 & 1.21 & 129.0 & 20.0 & 21.0 & 32.0 & 3.5 \\
\hline 91) & 8-Mar-06 & & 95.0 & 122.6 & 140 & 2.85 & 99.1 & 121.7 & & & 1303 & 19.0 & 21.0 & 32.0 & 3.5 \\
\hline 3atc & Average & 25 & 96.3 & 117.7 & 140 & 2.83 & 110.8 & 139.8 & 3.45 & 1.17 & 130.8 & 19.7 & 21.3 & 32.8 & 3.5 \\
\hline 92 & 9-Mar-06 & \multirow{15}{*}{$\begin{array}{c}0 \\
\frac{N}{U} \\
\frac{\sigma}{0} \\
\infty\end{array}$} & & & 140 & 2.95 & 116.5 & 149.1 & & & 126.9 & 19.0 & 21.0 & 33.0 & 3.5 \\
\hline 93 & 10-Mar-06 & & & & 140 & 2.85 & & & & & 1252 & & & & \\
\hline 94 & 11-Mar-06 & & & & 140 & 2.85 & & & & & & & & & \\
\hline 95 & 12-Mar-06 & & & & 140 & 2.00 & & & & & & 20.0 & 22.0 & 29.0 & 3.5 \\
\hline 96 & 13-Mar-06 & & 100.0 & 112.4 & 140 & 2.85 & 75.5 & 101.8 & & & & 21.0 & 23.0 & 34.0 & 3.5 \\
\hline 71 & 14-Mar-06 & & & 0.0 & 140 & 3.25 & 61.2 & 82.6 & & & & 20.0 & 22.0 & 35.0 & 3.5 \\
\hline 98 & 15-Mar-06 & & 95.0 & 101.2 & 140 & 2.45 & & & & & & 20.0 & 21.0 & 32.0 & 3.5 \\
\hline 99 & 16-Mar-06 & & & 0.0 & 140 & 3.05 & 84.0 & 110.8 & & & 146.9 & 20.0 & 22.0 & 33.0 & 3.5 \\
\hline 00 & 17-Mar-06 & & 95.0 & 105.1 & 140 & 2.65 & 82.3 & 106.3 & & & & 20.0 & 22.0 & 33.0 & 3.5 \\
\hline 01 & 18-Mar-06 & & & & 140 & 2.85 & & & & & & & & & \\
\hline & 19-Mar-06 & & & & 140 & 2.85 & 69.3 & 89.5 & & & 140.1 & & & & \\
\hline & 20-Mar-06 & & 90.0 & 100.0 & 140 & 2.85 & 88.6 & 116.6 & & & 147.1 & 19.0 & 21.0 & 33.0 & 3.5 \\
\hline & 21-Mar-06 & & & & 140 & 2.85 & 81.1 & 104.7 & 3.91 & 1.23 & 143.3 & & & & \\
\hline & $22-$ Mar-06 & & & & 140 & 2.85 & 87.0 & 114.5 & & & & 20.0 & 22.0 & 33.0 & 3.5 \\
\hline & 23-Mar-06 & & 95.0 & 108.2 & 140 & 2.85 & 99.2 & 133.5 & & & 154.8 & 19.0 & 21.0 & 34.0 & 3.5 \\
\hline Batc & h Average & 26 & 95.0 & 75.3 & 140 & 2.80 & 80.9 & 110.9 & 3.91 & 1.23 & 140.6 & 19.8 & 21.7 & 32.9 & 3.5 \\
\hline 07 & 24-Mar-06 & \multirow{15}{*}{$\begin{array}{l}\text { N } \\
\frac{\Omega}{U} \\
\frac{0}{\pi} \\
\infty\end{array}$} & 100.0 & 111.9 & 140 & 2.85 & 87.7 & 118.3 & & & 142.4 & 19.0 & 21.0 & 32.0 & 3.5 \\
\hline & 25-Mar-06 & & & & 140 & 2.85 & & & & & 148.3 & & & & \\
\hline & 26-Mar-06 & & & & 140 & 2.85 & & & & & & & & & \\
\hline & 27-Mar-06 & & 80.0 & 90.9 & 140 & 2.85 & 77.1 & 104.7 & & & & 19.0 & 21.0 & 32.0 & 3.5 \\
\hline & 28-Mar-06 & & & & 140 & 2.85 & 71.9 & 100.0 & & & 157.5 & 21.0 & 23.0 & 34.0 & 35 \\
\hline & 29-Mar-06 & & 85.0 & 104.9 & 140 & 2.85 & & & & & 154.1 & 19.0 & 21.0 & 32.0 & 3.5 \\
\hline & 30-Mar-06 & & & & 140 & 2.85 & & & & & 156.4 & & & & \\
\hline & 31-Mar-06 & & 100.0 & 114.5 & 140 & 2.85 & & & 3.52 & 1.12 & 157.7 & 19.0 & 21.0 & 32.0 & 3.5 \\
\hline & 1-Apr-06 & & & & 140 & 2.85 & & & & & & & & & \\
\hline & 2-Apr-06 & & & & 140 & 2.85 & & & & & & & & & \\
\hline & 3-Apr-06 & & 90.0 & 100.2 & 140 & 2.85 & 90.2 & 123.5 & & & 152.0 & 19.0 & 21.0 & 32.0 & 3.5 \\
\hline & 4-Apr-06 & & & & 140 & 2.25 & 85.9 & 107.2 & 3.52 & 1.12 & 164.0 & 19.0 & 21.0 & 32.0 & 3.5 \\
\hline & 5-Apr-06 & & 90.0 & 102.4 & 140 & 3.25 & 73.1 & 94.9 & & & 164.6 & 19.0 & 21.0 & 32.0 & 3.5 \\
\hline & 6-Apr-06 & & & & 140 & 2.85 & & & . & & & 19.0 & 21.0 & 32.0 & 3.5 \\
\hline & 7-Apr-06 & & & & 140 & 2.85 & & & - & & 173.9 & & & & \\
\hline Bats & ch Average & 27 & 90.8 & 104.1 & 140 & 2.84 & 81.0 & 108.1 & 3.52 & 1.12 & 157.1 & 19.2 & 21.2 & 32.2 & 3.5 \\
\hline & 8-Apr-06 & \multirow{15}{*}{$\frac{1}{4}$} & & & 140 & 2.85 & & & & & & & & & \\
\hline & 9-Apr-06 & & & & 140 & 2.85 & & - & & & & 19.0 & 21.0 & 32.0 & 3.5 \\
\hline & 10-Apr-06 & & 95.0 & 110.7 & 140 & 2.85 & & - & 3.22 & 1.10 & 179.9 & 19.0 & 21.0 & 320 & 3.5 \\
\hline & 11-Apr-06 & & & & 140 & 2.85 & 95.8 & 114.3 & & & & 20.0 & 22.0 & 320 & 3.5 \\
\hline & 12-Apr-06 & & & & 140 & 2.85 & 58.8 & 78.6 & & & 173.6 & 19.0 & 21.0 & 33.0 & 3.5 \\
\hline & 13-Apr-06 & & 90.0 & 105.9 & 140 & 2.85 & 63.3 & 82.3 & & & & 19.0 & 21.0 & 32.0 & 3.5 \\
\hline 28 & 14-Apr-06 & & & & 140 & 2.85 & 67.9 & 88.8 & & & 181.6 & 19.0 & 21.0 & 34.0 & 3.5 \\
\hline & 15-Apr-06 & & 110.0 & 142.2 & 140 & 2.85 & 63.7 & 74.1 & 3.54 & 1.22 & 185.9 & 19.0 & 21.0 & 32.0 & 3.5 \\
\hline & 16-Apr-06 & & & & 140 & 285 & 65.5 & 76.2 & & & 197.8 & & & & \\
\hline & 17-Apr-06 & & & & 140 & 2.85 & & & & & & & & & \\
\hline & 18-Apr-06 & & 100.0 & 126.4 & 140 & 5.90 & 73.6 & 103.6 & & & 195.1 & 19.0 & 21.0 & 38.0 & 3.5 \\
\hline & 19-Apr-06 & & & & 140 & 2.85 & 82.5 & 110.8 & 2.69 & 0.97 & 202.5 & 19.0 & 21.0 & 32.0 & 3.5 \\
\hline & 20-Apr-06 & & & & 0 & 0.00 & & & & & 194.8 & 19.0 & 21.0 & 32.0 & \\
\hline & 21-Apr-06 & & 100.0 & 111.7 & 140 & 2.90 & 81.5 & 109.9 & & & & 19.0 & 21.0 & 32.0 & 3.5 \\
\hline 36 & $22-A p r-06$ & & & & 0 & 2.85 & & & & & & & & & \\
\hline Bat & ch Average & 28 & 99.0 & 119.4 & 121 & 2.87 & 72.5 & 93.2 & 3.15 & 1.10 & 188.9 & 19.1 & 21.1 & 32.8 & 3.5 \\
\hline 13 & 23-Apr-06 & \multirow{13}{*}{$\frac{9}{9}$} & & & 140 & 285 & & & & & 166.4 & & & & \\
\hline & 24-Apr-06 & & 95.0 & 108.5 & 140 & 285 & 69.9 & 93.8 & & & 151.8 & 19.0 & 22.0 & 34.0 & 3.5 \\
\hline & 25-Apr-06 & & & & 140 & 2.85 & 66.3 & 88.4 & & & 150.3 & 20.0 & 23.0 & 33.0 & 3.5 \\
\hline 40 & 26-Apr-06 & & 100.0 & 112.5 & 140 & 2.85 & 88.5 & 120.1 & & & 142.2 & 19.0 & 21.0 & 33.0 & 35 \\
\hline & $27-A p r-06$ & & & & 140 & 2.85 & 88.6 & 116.7 & & & 136.3 & 19.0 & 21.0 & 33.0 & 3.5 \\
\hline 142 & 28-Apt-06 & & 110.0 & 125.1 & 140 & 2.85 & 93.7 & 101.7 & 3.21 & 1.20 & 138.2 & 19.0 & 23.0 & 34.0 & 3.5 \\
\hline 143 & 29-Apr-06 & & & & 140 & 2.85 & & & & & & 19.0 & 23.0 & 32.0 & 4.0 \\
\hline 144 & 30-Apr-06 & & & & 140 & 285 & 71.0 & 98.9 & & & 151.4 & 19.0 & 22.0 & 33.0 & 3.5 \\
\hline 445 & 1-May-06 & & 95.0 & 108.8 & 140 & 2.85 & 68.9 & 89.7 & & & 150.4 & 19.0 & 21.0 & 32.0 & 3.5 \\
\hline 146 & 2-May-06 & & & & 140 & 3.10 & 68.5 & 89.2 & & & 154.2 & & & & \\
\hline 447 & 3-May-06 & & 90.0 & 97.2 & 140 & 2.95 & 62.7 & 78.1 & & & 149.2 & 19.0 & 21.0 & 32.0 & 3.5 \\
\hline 448 & 4-May-06 & & & & 140 & 2.86 & & & & & 158.6 & & & & \\
\hline 449 & 5-May-06 & & 90.0 & 107.3 & 140 & 2.85 & & & & & 148.5 & 19.0 & 22.0 & 32.5 & 3.5 \\
\hline Bat & ch Average & 29 & 96.7 & 109.9 & 140 & 2.88 & 75.3 & 97.4 & 3.21 & 1.20 & 149.8 & 19.1 & 21.9 & 32.9 & 3.6 \\
\hline
\end{tabular}




\section{Appendix A-5}

Sewage Batch Averages for the MBR UCT System

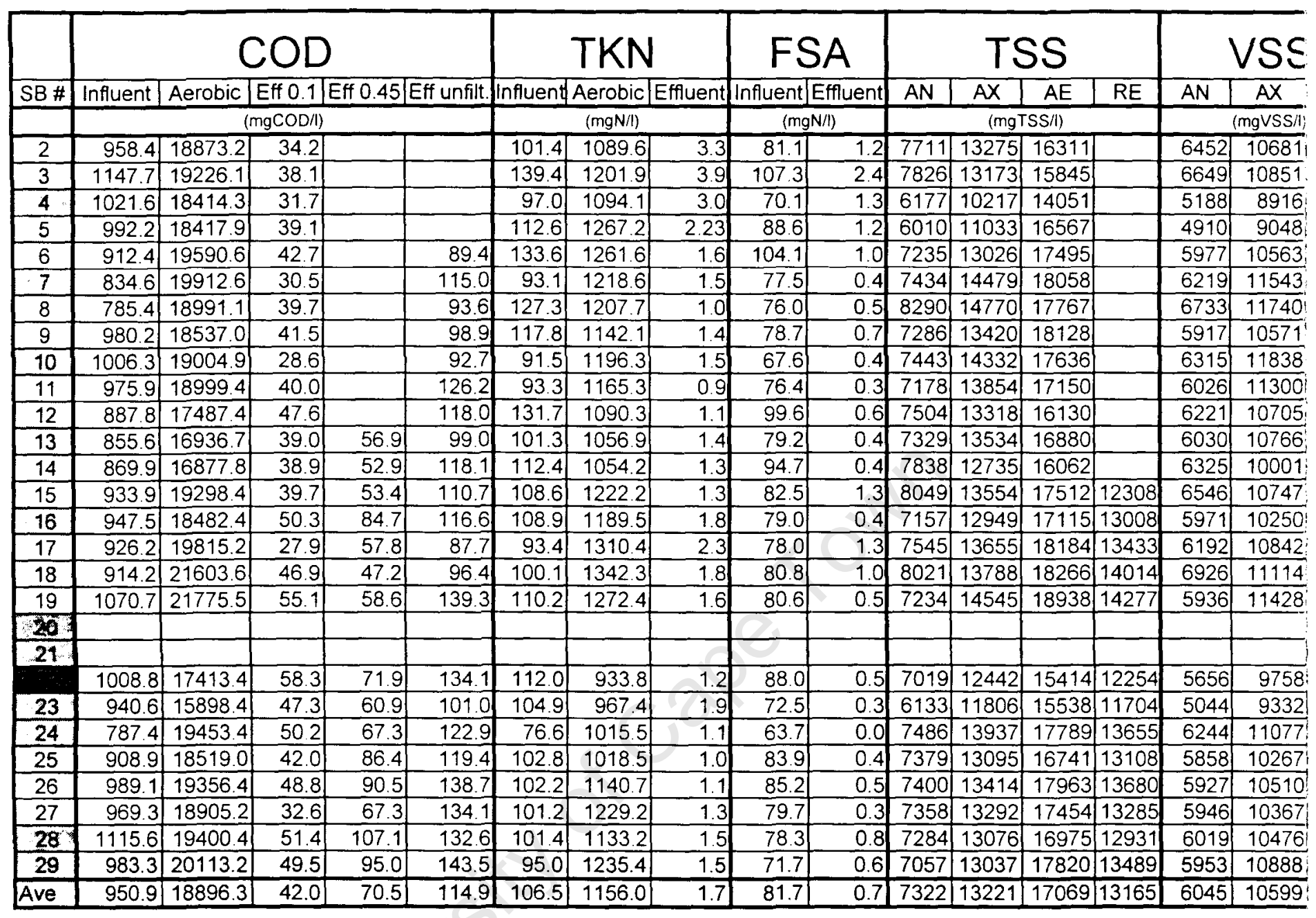




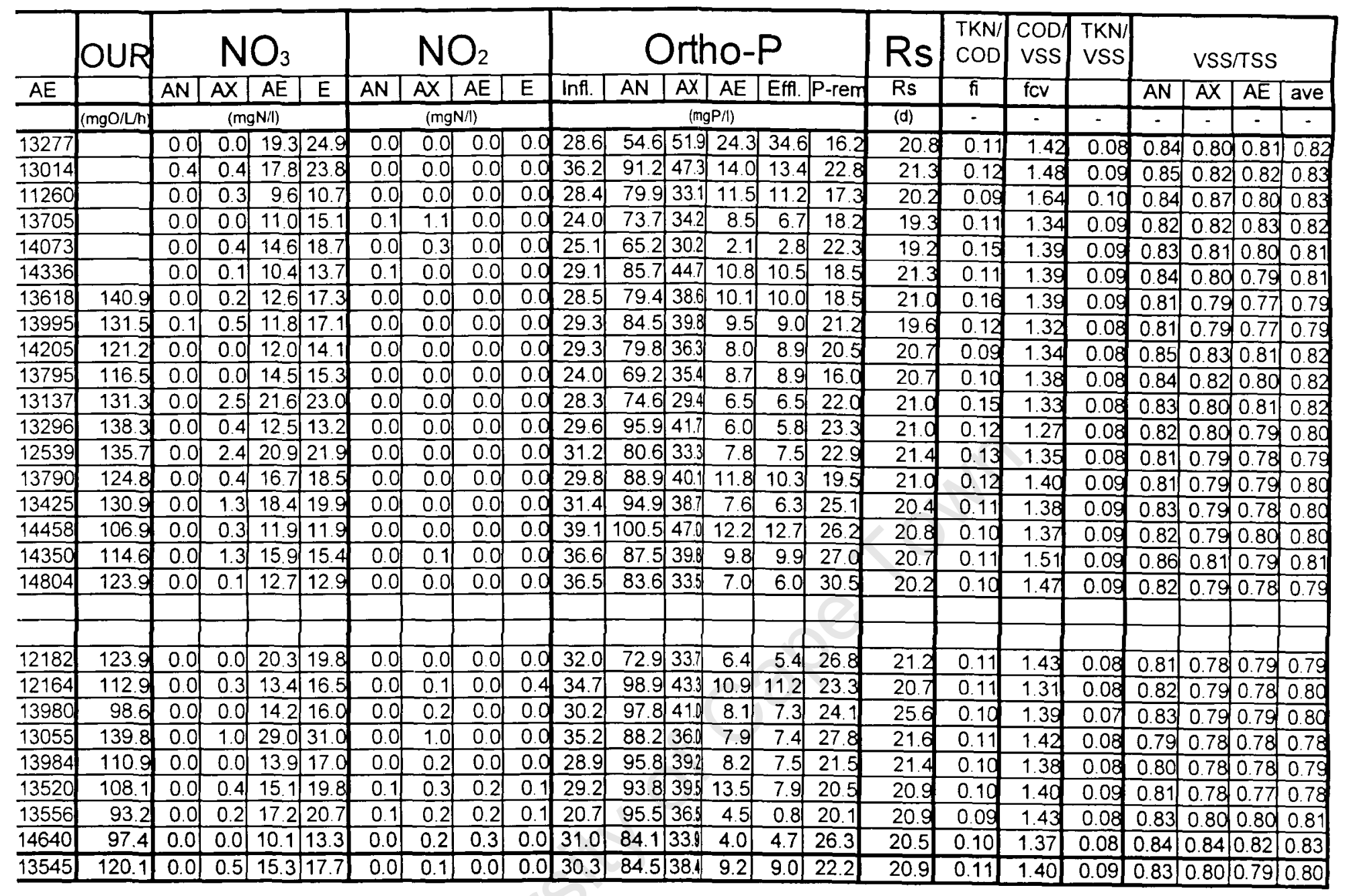




\section{APPENDIX B}

\section{CONVENTIONAL UCT DAILY MEASUREMENTS}

\section{TABLE OF CONTENTS}

B.1 Measured COD, TSS and VSS values for the conventional UCT system.

B.2 Measured TKN and FSA in the conventional UCT system.

B.3 Measured NO3, NO2 and soluble $\mathrm{P}$ in the conventional UCT system.

B.4 Miscellaneous measurements on the conventional UCT system.

B.5 Sewage Batch Averages for the conventional UCT system. 
B-1 Conv COD / TSS_vSS

Appendix B-1

Measured COD, TSS and VSS values for the conventional UCT system

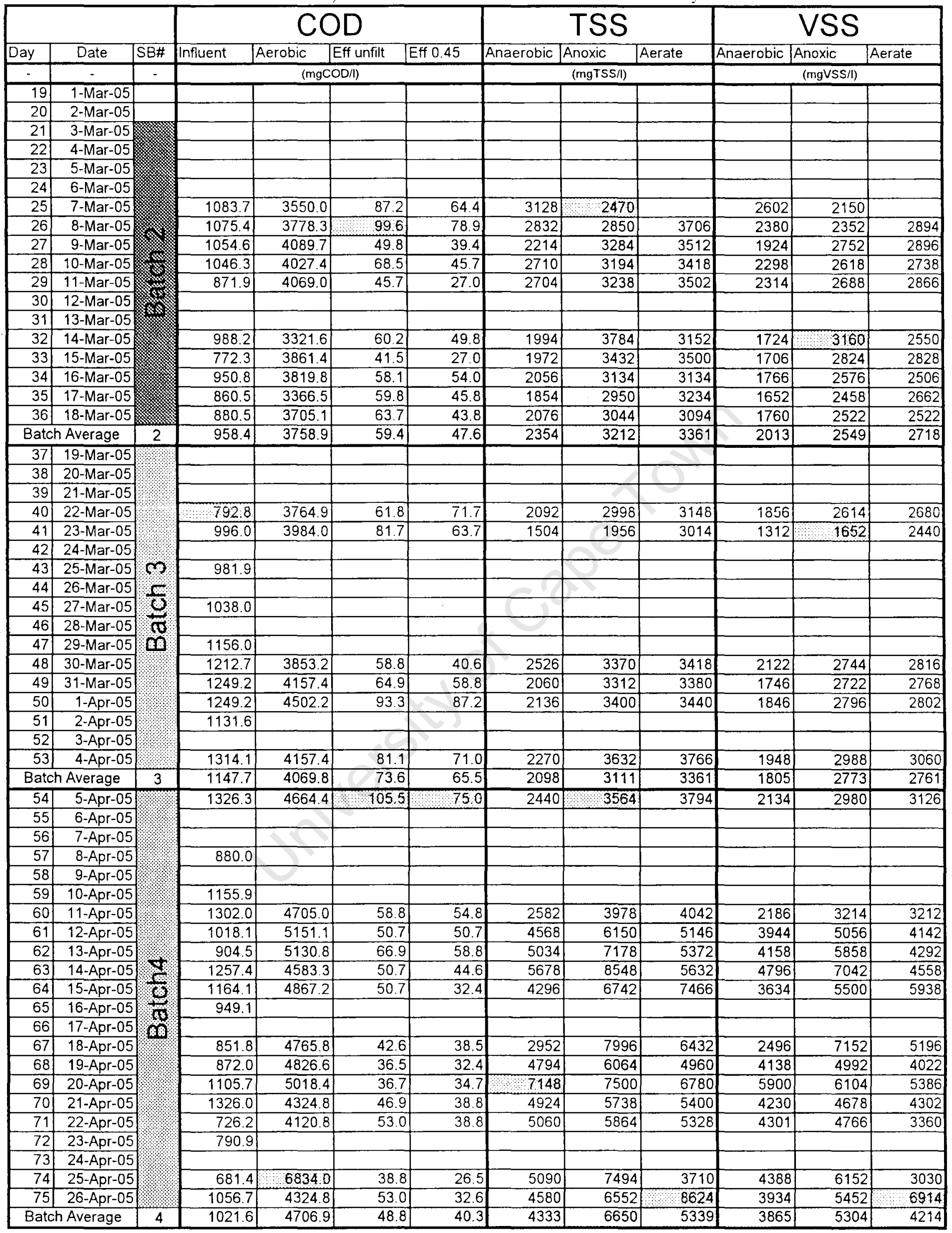


B-1 Conv COD / TSS_VSS

\begin{tabular}{|c|c|c|c|c|c|c|c|c|c|c|c|c|}
\hline 76 & 27-Apr-05 & & & & & & & & & & & \\
\hline 77 & 28-Apr-05 & & & & & & & & & & & \\
\hline 78 & 29-Apr-05 & & & & & & & & & & & \\
\hline 79 & 30-Apr-05 & & & & & & & & & & & \\
\hline 80 & 1-May-05 & & & & & & & & & & & \\
\hline 81 & 2-May-05 & & 763.0 & & & & & & & & & \\
\hline 82 & 3-May-05 & & & & & & & & & & & \\
\hline 83 & 4-May-05 & 10 & 1130.2 & 4834.8 & 151.0 & 1346 & 5392 & 7322 & 6514 & 4504 & 6094 & 5290 \\
\hline 84 & 5-May-05 & ᄃ & 991.4 & 3651.6 & 61.2 & 49.0 & 4770 & 6708 & 4130 & 4118 & 5690 & 3490 \\
\hline 85 & 6-May-05 & 0 & 12240 & 3774.0 & 53.0 & 38.8 & 4392 & 5122 & 7576 & 3788 & 4316 & 6136 \\
\hline 86 & 7-May-05 & $\pi$ & 962.8 & & & & & & & & & \\
\hline 87 & 8-May-05 & $m$ & 652.8 & & & & & & & & & \\
\hline 88 & 9-May-05 & & 895.5 & 4141.2 & 63.2 & 61.2 & 3916 & 6466 & 7920 & 3296 & 5390 & 6414 \\
\hline 89 & 10-May-05 & & 934.5 & 5201.9 & 48.8 & 48.8 & 3434 & 5326 & 5284 & 2966 & 4512 & 4356 \\
\hline 90 & 11-May-05 & & 942.7 & 5262.9 & 44.7 & 40.6 & 3834 & 6808 & 7108 & 3306 & 5682 & 5790 \\
\hline 91 & 12-May-05 & & 1207.2 & 4490.7 & 42.7 & 40.6 & 4740 & 3818 & 8142 & 4152 & 3170 & 4190 \\
\hline 92 & 13-May-05 & & 9813 & 4572.0 & 58.9 & 40.6 & 5688 & 6146 & 6056 & 4760 & 5112 & 4874 \\
\hline 93 & 14-May-05 & & 881.3 & & & & & & & & & \\
\hline 94 & 15-May-05 & & & & & & & & & & & \\
\hline Batch & h Average & 5 & 992.2 & 4491.1 & 53.2 & 45.7 & 4521 & 5965 & 6591 & 3861 & 4996 & 5068 \\
\hline 95 & 16-May-05 & & 947.0 & & & & & & & & & \\
\hline 96 & 17-May-05 & & & & & & & & & & & \\
\hline 97 & 18-May-05 & & & & & & & & & & & \\
\hline 98 & 19-May-05 & & 681.4 & & & & & & & & & \\
\hline 99 & 20-May-05 & & 734.6 & & & & & & & + & & \\
\hline 100 & 21-May-05 & & 759.0 & & & & & & & & & \\
\hline 101 & 22-May-05 & 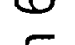 & 853.0 & & & & & & & & & \\
\hline 102 & 23-May-05 & $\frac{5}{0}$ & 907.2 & 3180.6 & 51.3 & 34.9 & 2930 & 3024 & 4310 & 2444 & 2508 & 3650 \\
\hline 103 & 24-May-05 & \pm & 1153.9 & 3057.5 & 61.6 & 49.2 & 2308 & 4430 & 4354 & 1984 & 3688 & 3706 \\
\hline 104 & 25-May-05 & $m$ & 1002.1 & 3098.5 & 69.8 & 59.5 & 2426 & 2354 & 1698 & 2066 & 2024 & 1512 \\
\hline 105 & 26-May-05 & & 969.2 & 3775.7 & 55.4 & 51.3 & 3524 & 6200 & 6952 & 3032 & 5304 & 5752 \\
\hline 106 & 27-May-05 & & 1133.5 & 3180.6 & 51.3 & 28.7 & 5252 & 6918 & 5106 & 4536 & 5834 & 4214 \\
\hline 107 & 28-May-05 & & 1072.6 & & & & & $\square$ & & & & \\
\hline 108 & 29-May-05 & & & & & & 0 & & & & & \\
\hline 109 & 30-May-05 & & 741.3 & 3406.3 & 51.3 & 45.1 & 1944 & 2376 & 2554 & 1672 & 2020 & 2156 \\
\hline 110 & 31-May-05 & & 907.0 & 3016.4 & 78.0 & 51.3 & 4378 & 7640 & 5466 & 3798 & 6600 & 4474 \\
\hline Batch & h Average & 6 & 912.4 & 3156.7 & 59.8 & 45.7 & 3252 & 4706 & 4349 & 2790 & 3997 & 3638 \\
\hline 111 & 1-Jun-05 & & & & & & \begin{tabular}{|l|} 
\\
\end{tabular} & & & & & \\
\hline 112 & 2-Jun-05 & & 904.1 & & & & & & & & & \\
\hline 113 & 3-Jun-05 & & 830.2 & 3764.9 & 73.7 & 59.8 & 4524 & 6920 & 4808 & 3890 & 5866 & 4000 \\
\hline 114 & 4-Jun-05 & & & & & & & & & & & \\
\hline 115 & 5-Jun-05 & & 678.1 & & & 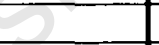 & & & & & & \\
\hline 116 & 6-Jun-05 & 18 & 905.8 & 3067.7 & 65.7 & 45.8 & 2894 & 7098 & 6858 & 2472 & 5978 & 5624 \\
\hline 117 & 7-Jun-05 & 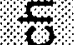 & 888.4 & 3824.6 & 67.7 & 67.7 & 3160 & 4370 & 5874 & 2704 & 3896 & 4666 \\
\hline 118 & 8-Jun-05 & (1). & 846.6 & 3565.7 & 89.6 & 55.8 & 2871.6 & 3016 & 2622 & 2477.6 & 2542 & 2184 \\
\hline 119 & 9-Jun-05 & $\mathrm{g}$ & 788.8 & 3207.1 & 61.8 & 59.8 & 2624 & 3040 & 3020 & 2298 & 2510 & 2530 \\
\hline 120 & 10-Jun-05 & & 996.2 & 3365.3 & 73.9 & 57.5 & 2288 & 3014 & 2778 & 2154 & 2730 & 2546 \\
\hline 121 & 11-Jun-05 & & 813.7 & 0 & & & 2680 & 2650 & 1842 & 2430 & 2370 & 1734 \\
\hline 122 & 12-Jun-05 & & 669.8 & 0 & & & 2718 & 2608 & 1868 & 2534 & 2462 & 1784 \\
\hline 123 & 13-Jun-05 & & 742.0 & 3652.6 & 121.1 & 90.3 & 2442 & 2794 & 2922 & 2194 & 2500 & 2596 \\
\hline 124 & \begin{tabular}{|l|}
$14-J u n-05$ \\
\end{tabular} & & 952.0 & 3693.6 & 133.4 & 110.8 & 1800 & 2562 & 2704 & & & \\
\hline Batch & h Average & 7 & 834.6 & 3517.7 & 85.9 & 62.4 & 2609 & 3807 & 3530 & 2408 & 3428 & 3074 \\
\hline 125 & 15-Jun-05 & & & & & & & & & & & \\
\hline 126 & 16-Jun-05 & & 660.0 & & & & & & & & & \\
\hline 127 & 17-Jun-05 & $\infty$ & 764.0 & & & & & & & & & \\
\hline 128 & 18-Jun-05 & $\frac{1}{6}$ & 652.0 & & & & & & & & & \\
\hline 129 & 19-Jun-05 & & & & & & & & & & & \\
\hline 130 & 20-Jun-05 & $\mathrm{co}$ & 684.0 & 4320.0 & 72.0 & 72.0 & 4114 & 4028 & 5102 & 3644 & 3426 & 4278 \\
\hline 131 & 21-Jun-05 & & 966.5 & 44000 & 88.0 & 64.0 & 3646 & 3218 & 3596 & 2658 & 2726 & 3002 \\
\hline 132 & 22-Jun-05 & & 986.0 & 5080.0 & 70.0 & 62.0 & 2322 & 3698 & 6022 & 1952 & 3142 & 5012 \\
\hline Batch & $\mathrm{h}$ Average & 8 & 785.4 & 4600.0 & 76.7 & 66.0 & 3361 & 3648 & 4907 & 2751 & 3098 & 4097 \\
\hline 133 & \begin{tabular}{|l} 
23-Jun-05 \\
\end{tabular} & & & & & & & & & & & \\
\hline 134 & 24-Jun-05 & & 995.0 & 3700.0 & 76.0 & 66.0 & 1824 & 3100 & 3058 & 1580 & 2664 & 2580 \\
\hline 135 & 25-Jun-05 & & 1052.0 & & & & & & & & & \\
\hline 136 & 26-Jun-05 & & 580,0 & & & & & & & & & \\
\hline 137 & 27-Jun-05 & & 648.0 & 3420.0 & 74.0 & 54.0 & 1822 & 2968 & 3064 & 1588 & 2534 & 2560 \\
\hline 138 & \begin{tabular}{|l|} 
28-Jun-05 \\
\end{tabular} & $\sigma$ & 902.0 & 3960.0 & 64.0 & 58.0 & 2100 & 4060 & 3004 & 1834 & 3444 & 2492 \\
\hline
\end{tabular}


B-1 Conv COD / TSS_VSS

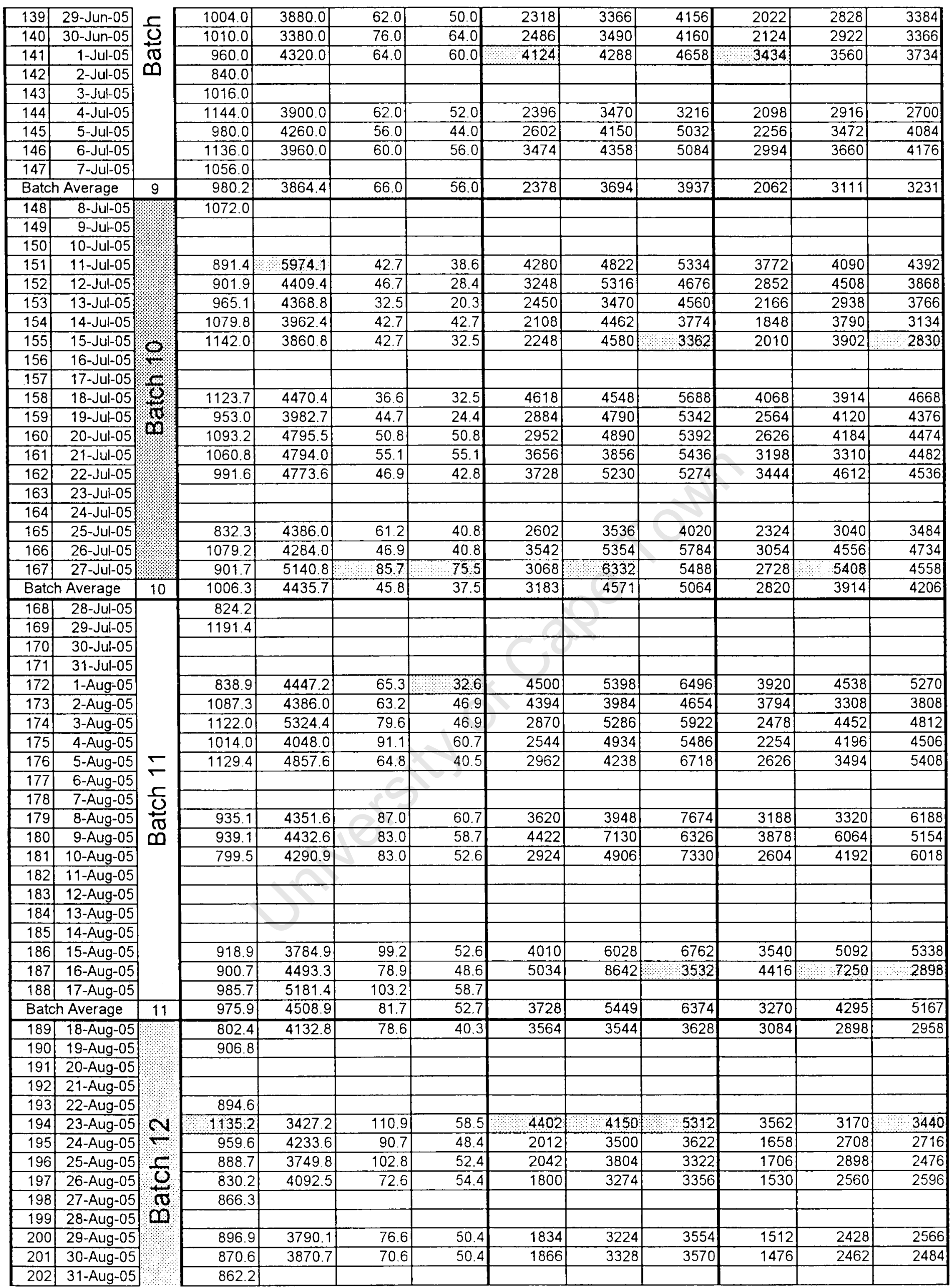


B-1 Conv COD / TSS VSS

\begin{tabular}{|c|c|c|c|c|c|c|c|c|c|c|c|c|}
\hline 203 & 1-Sep-05 & & 987.5 & 3689.3 & 90.7 & 48.4 & 1974 & 3228 & 3534 & 1700 & 2554 & 2730 \\
\hline 204 & 2-Sep-05 & & & & & & & & & & & \\
\hline Batch & Average & 12 & 887.8 & 3873.2 & 86.7 & 50.4 & 2156 & 3415 & 3512 & 2029 & 2710 & 2647 \\
\hline 205 & 3-Sep-05 & \multirow{14}{*}{ 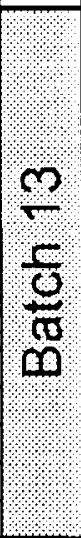 } & & & & & & & & & & \\
\hline 206 & 4-Sep-05 & & & & & & & & & & & \\
\hline 207 & 5-Sep-05 & & 829.5 & 3738.9 & 52.8 & 36.6 & 1854 & 3288 & 3308 & 1586 & 2606 & 2552 \\
\hline 208 & 6-Sep-05 & & 865.9 & 3413.8 & 52.8 & 42.7 & 1852 & 3404 & 3494 & 1562 & 2626 & 2622 \\
\hline 209 & $7-$ Sep-05 & & 926.4 & 3820.2 & 164.6 & 54.9 & 1756 & 2988 & 3222 & 1456 & 2290 & 2354 \\
\hline 210 & 8-Sep-05 & & 707.1 & 3718.6 & 111.8 & 65.0 & 2168 & 3348 & 3540 & 1804 & 2680 & 2796 \\
\hline 211 & 9-Sep-05 & & 934.7 & 3434.1 & 87.4 & 44.7 & 1970 & 2540 & 3126 & 1684 & 2058 & 2464 \\
\hline 212 & 10 -Sep-05 & & 918.5 & & & & & & & & & \\
\hline 213 & 11-Sep-05 & & & & & & & & & & & \\
\hline 214 & 12-Sep-05 & & 910.3 & 2783.8 & 93.5 & 65.0 & 2260 & 2492 & 2652 & 1840 & 2008 & 2118 \\
\hline 215 & 13-Sep-05 & & 827.9 & 2743.2 & 89.4 & 54.9 & 2408 & 2402 & 2544 & 1908 & 1896 & 2016 \\
\hline 216 & 14-Sep-05 & & 735.6 & & & & 2384 & 2192 & 2654 & 1872 & 1762 & 2070 \\
\hline 217 & 15-Sep-05 & & 837.2 & & & & & & & & & \\
\hline 218 & 16-Sep-05 & & 918.5 & & & & & & & & & \\
\hline Batch & Average & 13 & 8556 & 3378.9 & 93.2 & 52.0 & 2082 & 2832 & 3068 & 1714 & 2241 & 2374 \\
\hline 219 & 17-Sep-05 & \multirow{17}{*}{$\begin{array}{l}\mathbb{J} \\
\frac{\sigma}{\mathcal{O}}\end{array}$} & 995.7 & & & & & & & & & \\
\hline 220 & 18-Sep-05 & & & & & & & & & & & \\
\hline 221 & 19-Sep-05 & & 866.9 & 3185.3 & 84.7 & 38.3 & 1842 & 3122 & 3322 & 1546 & 2496 & 2608 \\
\hline 222 & 20-Sep-05 & & 7289 & & & & & & & & & \\
\hline 223 & 21-Sep-05 & & 920.4 & 3306.2 & 139.1 & 62.5 & 2116 & 2608 & 3132 & 1728 & 2072 & 2436 \\
\hline 224 & 22-Sep-05 & & 926.3 & 3407.0 & 98.8 & 84.7 & 1618 & 3134 & 3270 & 1358 & 2440 & 2516 \\
\hline 225 & 23-Sep-05 & & 939.2 & 3386.9 & 98.8 & 54.4 & 1720 & 3000 & 3030 & 1424 & 2360 & 2344 \\
\hline 226 & 24-Sep-05 & & 833.1 & & & & & & & & & \\
\hline 227 & 25-Sep-05 & & & & & & & & & & & \\
\hline 228 & 26-Sep-05 & & 913.9 & 3508.8 & 2713 & 61.2 & 1654 & 3054 & 3012 & 1420 & 2388 & 2338 \\
\hline 229 & $27-$ Sep-05 & & 924.7 & 2570.4 & 89.8 & 40.8 & 1498 & 2762 & 2956 & 1242 & 2156 & 2290 \\
\hline 230 & 28-Sep-05 & & 839.2 & 3325.2 & 93.8 & 59.2 & 1908 & 2620 & 3074 & 1650 & 2082 & 2380 \\
\hline 231 & $29-\operatorname{Sep}-05$ & & 728.9 & 3141.6 & 124.4 & 44.9 & 1726 & 2586 & 3034 & 1480 & 2108 & 2438 \\
\hline 232 & 30-Sep-05 & & 1175.0 & 3264.0 & 106.1 & 55.1 & 1728 & 3100 & 3236 & 1510 & 2482 & 2552 \\
\hline 233 & 1-Oct-05 & & & & & & & 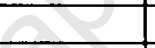 & & & & \\
\hline 234 & 2-Oct-05 & & 793.8 & & & & $\Rightarrow$ & & & & & \\
\hline 235 & 3-Oct-05 & & 897.6 & 3570.0 & 93.8 & 49.0 & 2368 & 3618 & 3366 & 1964 & 2882 & 2632 \\
\hline \multicolumn{2}{|c|}{ Batch Average } & 14 & 869.9 & 3343.9 & 103.3 & 55.0 & 1757 & 2887 & 3143 & 1484 & 2287 & 2453 \\
\hline 236 & 4-Oct-05 & \multirow{9}{*}{ 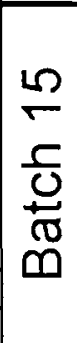 } & & & & & 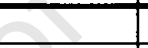 & & & & & \\
\hline 237 & 5-Oct-05 & & & & & & & & & & & \\
\hline 238 & 6-Oct-05 & & 1224.0 & & & & & & & & & \\
\hline 239 & 7-Oct-05 & & 1241.7 & 3621.0 & & 8 & 2508 & 3688 & 3260 & 2120 & 2942 & 2574 \\
\hline 240 & $8-$ Oct-05 & & 767.2 & 3774.0 & 80.8 & 40.8 & & & & & & \\
\hline 241 & 9-Oct-05 & & & & & 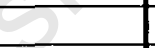 & & & & & & \\
\hline 242 & 10-Oct-05 & & 860.9 & 3468.0 & 91.0 & 51.0 & 2046 & 3310 & 3624 & 1654 & 2628 & 2838 \\
\hline 243 & 11-Oct-05 & & 701.8 & & 0 & & 1873 & 3044 & 3347 & 1568 & 2407 & 2603 \\
\hline 244 & 12-Oct-05 & & 807.8 & & 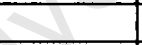 & & 2286 & 3210 & 2918 & 1884 & 2964 & 2260 \\
\hline \multicolumn{2}{|c|}{ Batch Average } & 15 & 933.9 & 3621.0 & 85.9 & 45.9 & 2178 & 3313 & 3287 & 1807 & 2735 & 2569 \\
\hline 245 & 13-Oct-05 & \multirow{15}{*}{$\frac{6}{\frac{6}{6}}$} & & & & & 1734 & 3078 & 3000 & 1462 & 2450 & 2364 \\
\hline 246 & 14-Oct-05 & & 979.2 & 3682.2 & 151.0 & 63.2 & 2180 & 3104 & 3202 & 1874 & 2488 & 2514 \\
\hline 247 & 15-Oct-05 & & 942.5 & & & & & & & & & \\
\hline 248 & 16-Oct-05 & & & & & & & & & & & \\
\hline 249 & 17-Oct-05 & & 950.6 & 3529.2 & 100.0 & 55.1 & 1894 & 3186 & 2946 & 1658 & 2646 & 2408 \\
\hline 250 & $18-$ Oct-05 & & 995.5 & & & & 2050 & 3070 & 3132 & 1788 & 2632 & 2612 \\
\hline 251 & 19-Oct-05 & & 983.3 & 36720 & 120.4 & 81.6 & 1714 & 2742 & 3222 & 1458 & 2156 & 2502 \\
\hline 252 & $20-0 c t-05$ & & & & & & 1816 & 3306 & 2738 & 1574 & 2740 & 2208 \\
\hline 253 & 21-Oct-05 & & 11832 & 4080.0 & 44.9 & 85.7 & 1984 & 3346 & 3102 & 1720 & 2708 & 2474 \\
\hline 254 & 22-Oct-05 & & & & & & & & & & & \\
\hline 255 & 23-Oct-05 & & 938.4 & & & & & & & & & \\
\hline 256 & 24-Oct-05 & & 930.2 & 3631.2 & 100.0 & 61.2 & 2330 & 3240 & 2946 & 1928 & 2602 & 2296 \\
\hline 257 & 25-Oct-05 & & 967.0 & & & & 2134 & 3196 & 3230 & 1774 & 2534 & 2546 \\
\hline 258 & 26-Oct-05 & & 8405 & 3447.6 & 112.2 & 63.2 & 1914 & 3190 & 3030 & 1600 & 2542 & 2382 \\
\hline 259 & 27-Oct-05 & & & & & & 2900 & 2898 & 2948 & 2304 & 2288 & 2332 \\
\hline \multicolumn{2}{|c|}{ Batch Average } & 16 & 947.5 & 3673.7 & 104.7 & 68.3 & 2059 & 3123 & 3076 & 1684 & 2563 & 2422 \\
\hline 260 & $28-O c t-05$ & \multirow{5}{*}{$\begin{array}{r} \\
\mathrm{N}\end{array}$} & 5998 & 3672.0 & 79.6 & 26.5 & 2534 & 3270 & 2928 & 2128 & 2608 & 2338 \\
\hline 261 & 29-Oct-05 & & & & & & & & & & & \\
\hline 262 & $30-0 c t-05$ & & 1011.8 & & & & & & & & & \\
\hline 263 & $31-$ Oct-05 & & 869.0 & 2488.8 & 104.0 & 26.5 & 1910 & 3034 & 3070 & 1604 & 2416 & 2424 \\
\hline 264 & 1-Nov-05 & & 1289.3 & & & & 1750 & 3178 & 3338 & 1468 & 2568 & 2626 \\
\hline
\end{tabular}


B-1 Conv COD / TSS_VSS

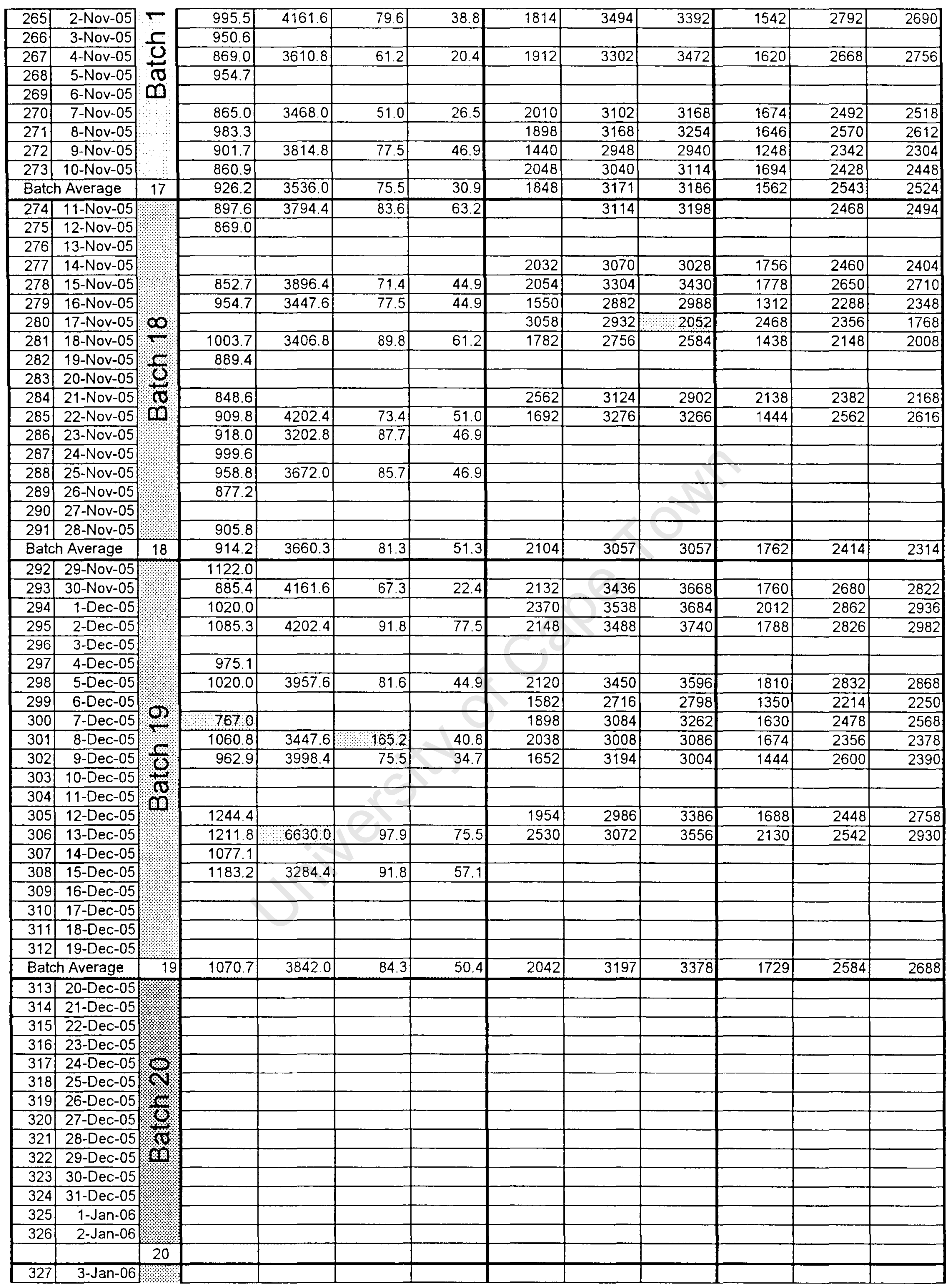


B-1 Conv COD / TSS_VSS

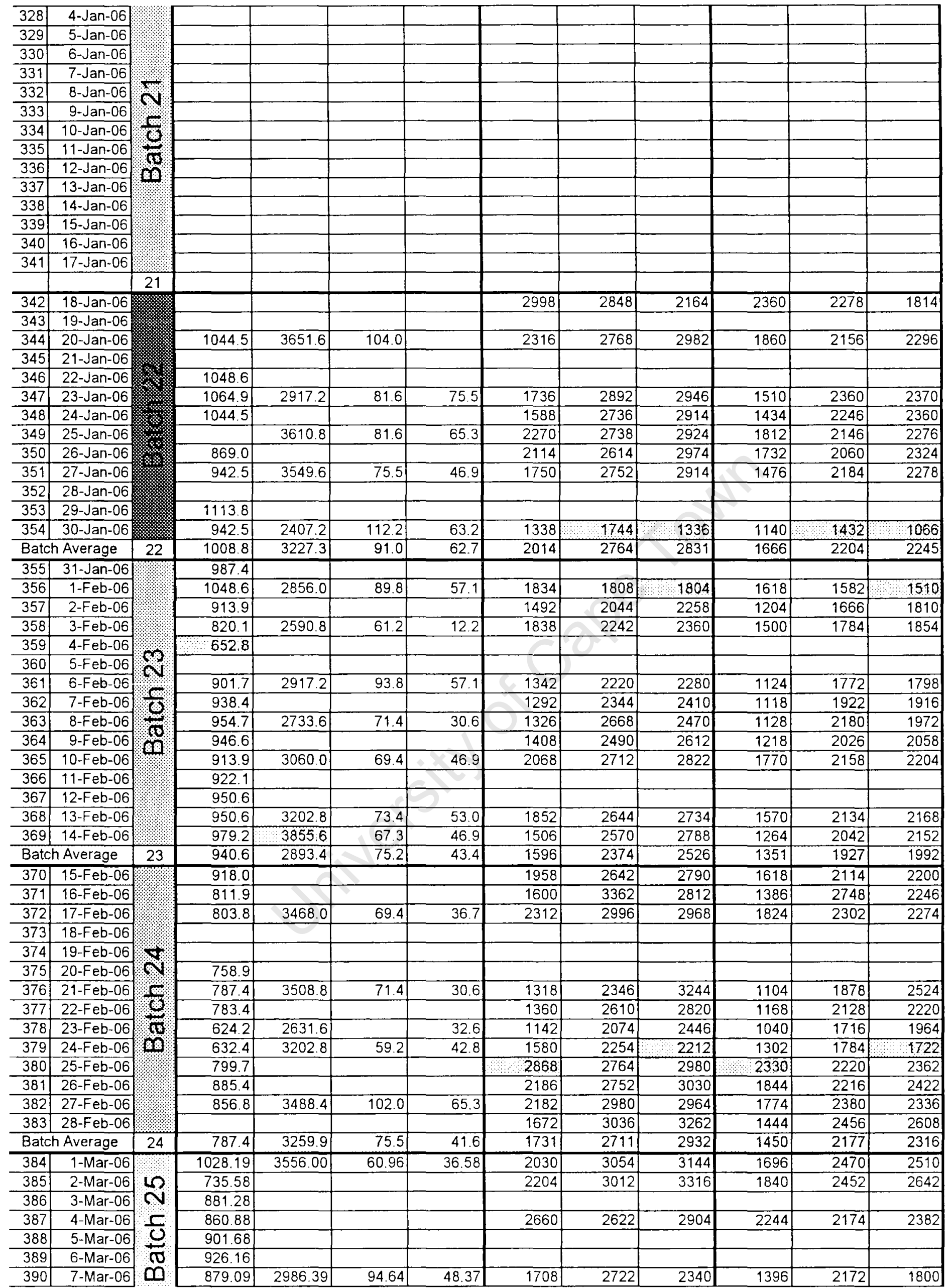


B-1 Conv COD / TSS_VSS

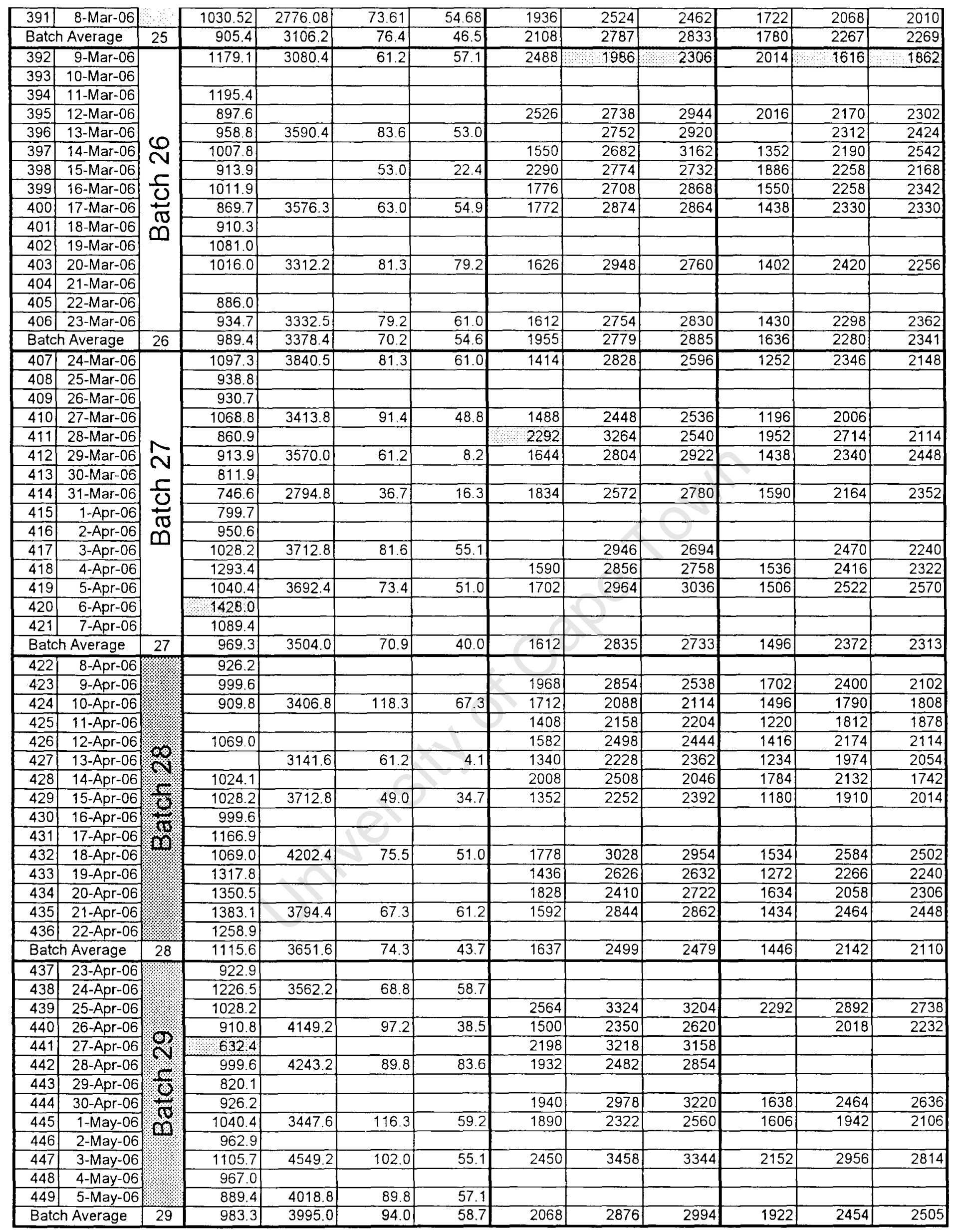


Appendix B-2

Measured TKN and FSA in the conventional UCT system

\begin{tabular}{|c|c|c|c|c|c|c|c|c|}
\hline \multirow[b]{2}{*}{ Day } & \multirow[b]{2}{*}{ Date } & \multirow[b]{2}{*}{ SB\# } & \multicolumn{4}{|c|}{ TKN } & \multicolumn{2}{|c|}{ FSA } \\
\hline & & & Influent & Aerobic & $\begin{array}{l}\text { Effluent } \\
\text { Unfilt. }\end{array}$ & $\begin{array}{l}\text { Effluent } \\
0.45 \text { Filt. }\end{array}$ & Influent & $\begin{array}{l}\text { Effluent } \\
0.45 \text { Filt. }\end{array}$ \\
\hline- & - & $=$ & \multicolumn{4}{|c|}{$(\mathrm{mgN} / \mathrm{l})$} & \multicolumn{2}{|c|}{$(\mathrm{mgN} / \mathrm{l})$} \\
\hline 19 & 1-Mar-05 & & & & & & & \\
\hline 20 & 2-Mar-05 & & & & & & & \\
\hline 21 & 3-Mar-05 & \multirow{16}{*}{ 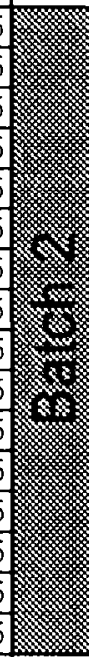 } & & & & & & \\
\hline 22 & 4-Mar-05 & & & & & & & \\
\hline 23 & 5-Mar-05 & & & & & & & \\
\hline 24 & 6-Mar-05 & & & & & & & \\
\hline 25 & 7-Mar-05 & & 104.4 & 245.7 & 12.6 & 8.0 & 84.1 & 6.6 \\
\hline 26 & 8-Mar-05 & & 101.9 & 235.9 & 15.4 & 12.2 & 88.2 & 9.0 \\
\hline 27 & 9-Mar-05 & & 99.1 & & 9.0 & 8.8 & 72.7 & 6.2 \\
\hline 28 & 10-Mar-05 & & 112.0 & 265.3 & 8.3 & 8.1 & 86.0 & 6.2 \\
\hline 29 & 11-Mar-05 & & 89.3 & 270.2 & 12.6 & 7.1 & 69.1 & 3.6 \\
\hline 30 & 12-Mar-05 & & & & & & & \\
\hline 31 & 13-Mar-05 & & & & & & & \\
\hline 32 & 14-Mar-05 & & 94.5 & 228.9 & 265 & 25.6 & 75.7 & $\overline{16.4}$ \\
\hline 33 & 15-Mar-05 & & 91.2 & 240.8 & 9.7 & 9.1 & 69.7 & 7.3 \\
\hline 34 & 16-Mar-05 & & 96.3 & 253.4 & 7.3 & 6.2 & 80.7 & 5.7 \\
\hline 35 & 17-Mar-05 & & 115.0 & 228.2 & 7.8 & 7.8 & 94.6 & 5.9 \\
\hline 36 & 18-Mar-05 & & 110.6 & 259.7 & 10.6 & 9.5 & 89.9 & 6.7 \\
\hline \multicolumn{2}{|c|}{ Batch Average } & 2 & 101.4 & 247.6 & 10.4 & 8.5 & 81.1 & 6.3 \\
\hline 37 & 19-Mar-05 & \multirow{17}{*}{ 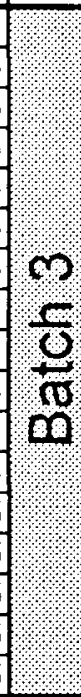 } & & & & & & \\
\hline 38 & 20-Mar-05 & & & & & & & \\
\hline 39 & 21-Mar-05 & & 140.0 & & & & 102.5 & \\
\hline 40 & $22-$ Mar-05 & & 890 & 272.3 & & & 65.7 & \\
\hline 41 & 23-Mar-05 & & 123.1 & 259.0 & & & 101.7 & \\
\hline 42 & 24-Mar-05 & & & & & 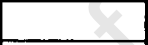 & & \\
\hline 43 & 25-Mar-05 & & 141.4 & & & $\bar{a}$ & 105.0 & \\
\hline 44 & 26-Mar-05 & & & & & 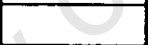 & & \\
\hline 45 & 27-Mar-05 & & 114.8 & & 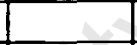 & & 93.0 & \\
\hline 46 & 28-Mar-05 & & & & & D & & \\
\hline 47 & 29-Mar-05 & & 111.2 & & $\theta$ & & 101.6 & \\
\hline 48 & 30-Mar-05 & & 151.8 & 276.5 & 8.1 & 7.4 & 134.7 & 6.6 \\
\hline 49 & 31-Mar-05 & & 134.7 & 278.6 & 8.3 & 7.8 & 102.4 & 6.4 \\
\hline 50 & 1-Apr-05 & & 150.1 & 279.3 & 8.0 & 7.6 & 109.9 & 6.9 \\
\hline 51 & 2-Apr-05 & & 160.4 & -2 & & & 116.2 & \\
\hline 52 & 3-Apr-05 & & +1 & 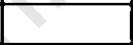 & & & & \\
\hline 53 & 4-Apr-05 & & 166.8 & 260.4 & 9.0 & 8.4 & 148.1 & 6.2 \\
\hline \multicolumn{2}{|c|}{ Batch Average } & 3 & 139.4 & 271.0 & 8.3 & 7.8 & 107.3 & 6.5 \\
\hline 54 & 5-Apr-05 & \multirow{16}{*}{ 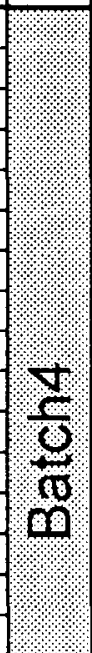 } & 113.1 & 287.0 & 12.0 & 11.1 & 83.9 & 7.0 \\
\hline 55 & 6-Apr-05 & & & & & & & \\
\hline 56 & 7-Apr-05 & & & & & & & \\
\hline 57 & 8-Apr-05 & & 97.4 & & & & 64.7 & \\
\hline 58 & 9-Apr-05 & & & & & & & \\
\hline 59 & 10-Apr-05 & & 99.4 & & & & 68.6 & \\
\hline 60 & 11-Apr-05 & & 105.3 & 313.6 & 8.8 & 8.8 & 83.7 & 8.4 \\
\hline 61 & 12-Apr-05 & & 98.0 & 289.8 & 9.5 & 10.5 & 75.4 & 7.3 \\
\hline 62 & 13-Apr-05 & & 671 & 302.4 & 10.8 & 11.5 & 51.0 & 8.8 \\
\hline 63 & 14-Apr-05 & & 108.7 & 296.1 & 9.8 & 7.1 & 80.0 & 7.3 \\
\hline 64 & 15-Apr-05 & & 75.9 & 308.0 & 13.7 & 9.9 & 51.5 & 8.3 \\
\hline 65 & 16-Apr-05 & & 91.8 & & & & 60.5 & \\
\hline 66 & 17-Apr-05 & & & & & & & \\
\hline 67 & 18 -Apr-05 & & 89.9 & 301.0 & 9.5 & 9.7 & 77.8 & 8.5 \\
\hline 68 & 19-Apr-05 & & 96.3 & 293.3 & 8.7 & 8.4 & 62.7 & 5.9 \\
\hline 69 & $20-A p r-05$ & & 105.1 & 285.6 & 11.1 & 9.0 & 81.2 & 7.0 \\
\hline
\end{tabular}


B-2 Conv TN

\begin{tabular}{|c|c|c|c|c|c|c|c|c|}
\hline 70 & 21-Apr-05 & & 105.4 & 266.0 & 8.7 & 8.1 & 78.1 & 7.0 \\
\hline 71 & 22-Apr-05 & & 90.4 & 277.2 & 10.2 & 7.3 & 69.6 & 7.6 \\
\hline 72 & 23-Apr-05 & & 86.5 & & & & 59.9 & \\
\hline 73 & 24-Apr-05 & & & & & & & \\
\hline 74 & 25-Apr-05 & & 95.3 & & 9.0 & 6.6 & 71.8 & 4.3 \\
\hline 75 & 26-Apr-05 & & 94.2 & 277.2 & 11.3 & 10.4 & 71.8 & 8.5 \\
\hline \multicolumn{2}{|c|}{ Batch Average } & 4 & 97.0 & 291.4 & 10.0 & 9.1 & 70.1 & 7.6 \\
\hline \multicolumn{2}{|c|}{\begin{tabular}{|l|r|}
76 & $27-A p r-05$ \\
\end{tabular}} & \multirow{19}{*}{$\begin{array}{l}\frac{1}{\sigma} \\
\frac{\infty}{\infty} \\
\infty\end{array}$} & & & & & & \\
\hline \multicolumn{2}{|c|}{\begin{tabular}{|l|l|}
77 & $28-A p r-05$ \\
\end{tabular}} & & & & & & & \\
\hline \multicolumn{2}{|c|}{\begin{tabular}{l|l|}
78 & 29-Apr-05 \\
\end{tabular}} & & 105.3 & & & & 81.5 & \\
\hline \multicolumn{2}{|c|}{\begin{tabular}{l|l|}
79 & $30-A p r-05$ \\
\end{tabular}} & & & & & & & \\
\hline 80 & 1-May-05 & & & & & & & \\
\hline 81 & 2-May-05 & & 120 & & & & 126 & \\
\hline \multirow{2}{*}{\begin{tabular}{|l|}
82 \\
83
\end{tabular}} & 3-May-05 & & & & & & & \\
\hline & 4-May-05 & & 116.2 & 244.3 & 10.4 & 7.7 & 88.3 & 6.6 \\
\hline$\frac{83}{84}$ & 5-May-05 & & 115.2 & 243.6 & 10.1 & 9.9 & 89.9 & 9.4 \\
\hline \multirow{2}{*}{\begin{tabular}{l|}
85 \\
86
\end{tabular}} & 6-May-05 & & 93.1 & 244.3 & 7.8 & 7.8 & 78.9 & 8.0 \\
\hline & 7-May-05 & & 136.1 & & & & & \\
\hline 87 & 8-May-05 & & 95.8 & & & & & \\
\hline 88 & 9-May-05 & & 119.4 & 283.5 & 211 & 196 & 87.8 & 167 \\
\hline \multirow{2}{*}{\begin{tabular}{|l|}
89 \\
90
\end{tabular}} & 10-May-05 & & 99.1 & 277.9 & 10.6 & 11.5 & 84.3 & 9.1 \\
\hline & 11-May-05 & & 108.2 & 335.3 & 11.6 & 9.9 & 79.9 & 8.0 \\
\hline 91 & 12-May-05 & & 123.8 & 308.0 & 13.7 & 11.8 & 103.5 & 7.4 \\
\hline 92 & 13-May-05 & & 113.1 & 300.3 & 9.1 & 7.4 & 95.6 & 7.1 \\
\hline 93 & 14-May-05 & & 125.7 & & & & 96.0 & 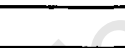 \\
\hline 94 & 15-May-05 & & & & & & & 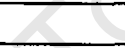 \\
\hline Batc & h Average & 5 & 112.6 & 279.7 & 10.5 & 9.4 & 88.6 & 7.9 \\
\hline 95 & 16-May-05 & & 119.6 & & & & 93.8 & \\
\hline 96 & 17-May-05 & & & & & & 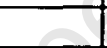 & 7 \\
\hline 97 & 18-May-05 & & & & & & & \\
\hline 98 & 19-May-05 & & & & & 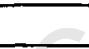 & 0 & \\
\hline 99 & 20-May-05 & & 113.0 & & & & 88.5 & \\
\hline 100 & 21-May-05 & $\mathrm{co}$ & & & & - & & \\
\hline 101 & 22-May-05 & & & & & & & \\
\hline 102 & 23-May-05 & $\frac{c}{0}$ & 148.1 & 264.6 & 9.5 & 8.0 & 112.6 & 8.0 \\
\hline 103 & 24-May-05 & 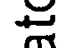 & 146.6 & 262.5 & 12.6 & 10.6 & 117.0 & 8.5 \\
\hline 104 & 25-May-05 & $m$ & 138.1 & 216.3 & 14.3 & 11.3 & 109.2 & 7.0 \\
\hline 105 & 26-May-05 & & 130.1 & 226.8 & & & 101.5 & \\
\hline 106 & 27-May-05 & & 143.2 & 223.3 & & & 100.3 & \\
\hline 107 & 28-May-05 & & & $a$ & & & & \\
\hline 108 & 29-May-05 & & & 0 & & & & \\
\hline 109 & 30-May-05 & & 132.9 & 2352 & 12.5 & 10.9 & 103.8 & 9.4 \\
\hline 110 & 31-May-05 & & 130.8 & 217.0 & 13.4 & 10.8 & 109.9 & 9.4 \\
\hline Batc & h Average & 6 & 133.6 & 235.1 & 12.5 & 10.3 & 104.1 & 8.5 \\
\hline 111 & 1-Jun-05 & & & & & & & \\
\hline 112 & 2-Jun-05 & & & & & & & \\
\hline 113 & 3-Jun-05 & & 99.5 & 252.0 & 10.4 & 9.5 & 79.8 & 8.5 \\
\hline 114 & 4-Jun-05 & & & & & & & \\
\hline 115 & 5-Jun-05 & & & & & & & \\
\hline 116 & 6-Jun-05 & ?. & 83.4 & 215.6 & 11.9 & 11.2 & 83.8 & 9.9 \\
\hline 117 & 7-Jun-05 & 13 & 92.0 & 260.4 & 21.8 & 21.0 & 74.3 & 20.7 \\
\hline 118 & 8-Jun-05 & $\frac{1}{\pi}$ & 88.6 & 229.6 & 27.7 & 24.2 & 71.8 & 18.8 \\
\hline 119 & 9-Jun-05 & 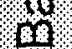 & 86.9 & 205.1 & 8.0 & 6.6 & 74.8 & 3.8 \\
\hline 120 & 10-Jun-05 & & 97.3 & & & & & \\
\hline 121 & 11-Jun-05 & & & & & & & \\
\hline 122 & 12-Jun-05 & & & & & & & \\
\hline 123 & 13-Jun-05 & & 95.5 & 219.8 & 39.3 & 35.3 & 79.5 & 26.2 \\
\hline 124 & 14-Jun-05 & & 101.8 & 212.1 & 16.0 & 10.5 & 78.5 & 7.0 \\
\hline Batc & Average & 7 & 93.1 & 227.8 & 19.3 & 16.9 & 77.5 & 13.6 \\
\hline 125 & 15-Jun-05 & & & & & & & \\
\hline 126 & 16-Jun-05 & & & & & & & \\
\hline
\end{tabular}


B-2 Conv TN

\begin{tabular}{|c|c|c|c|c|c|c|c|c|}
\hline 127 & 17-Jun-05 & 0 & & & & & & \\
\hline 128 & 18-Jun-05 & 1 & 129.1 & & & & 90.4 & \\
\hline 129 & 19-Jun-05 & $\frac{9}{\pi}$ & 101.8 & & & & & \\
\hline 130 & 20-Jun-05 & $\infty$ & 123.1 & 313.6 & 10.5 & 9.5 & 77.0 & 7.8 \\
\hline 131 & $21-J u n-05$ & & 137.3 & 261.8 & 11.2 & 8.5 & 52.6 & 5.7 \\
\hline 132 & 22-Jun-05 & & 145.4 & 291.9 & 10.8 & 9.7 & 84.0 & 4.9 \\
\hline Bato & Average & 8 & 127.3 & 289.1 & 10.8 & 9.2 & 76.0 & 6.2 \\
\hline 133 & 23-Jun-05 & \multirow{15}{*}{$\begin{array}{l}\sigma \\
\frac{\sigma}{U} \\
\frac{U}{\sigma} \\
\infty\end{array}$} & & & & & & \\
\hline 134 & 24-Jun-05 & & 133.3 & 249.9 & 6.4 & 5,0 & 73.3 & 2.9 \\
\hline 135 & 25-Jun-05 & & & & & & & \\
\hline 136 & 26-Jun-05 & & & & & & & \\
\hline 137 & 27-Jun-05 & & 103.7 & 236.6 & 9.9 & 10.5 & 67.2 & 7.8 \\
\hline 138 & 28-Jun-05 & & 115.5 & 259.0 & 9.1 & 8.4 & 68.3 & 6.9 \\
\hline 139 & 29-Jun-05 & & 121.5 & 244.3 & 9.9 & 10.2 & 75.9 & 6.2 \\
\hline 140 & 30-Jun-05 & & 117.6 & 228.9 & 8.8 & 8.4 & 69.1 & 6.7 \\
\hline 141 & 1-Jul-05 & & 112.4 & 250.6 & 10.1 & 9.2 & 94.4 & 7.4 \\
\hline 142 & 2-Jul-05 & & & & & & & \\
\hline 143 & 3-Jul-05 & & & & & & & \\
\hline 144 & 4-Jul-05 & & 121.9 & 265.3 & 11.5 & 11.3 & 102.2 & 8.4 \\
\hline 145 & 5-Jul-05 & & 124.1 & 291.2 & 10.1 & 9.5 & 75.1 & 8.0 \\
\hline 146 & 6-Jul-05 & & 119.4 & 277.2 & 8.7 & 8.7 & 93.5 & 6.2 \\
\hline 147 & 7-Jul-05 & & 108.4 & & & & 67.8 & \\
\hline \multicolumn{2}{|c|}{ Batch Average } & 9 & 117.8 & 255.9 & 9.8 & 9.5 & 78.7 & 7.2 \\
\hline 148 & 8-Jul-05 & \multirow{20}{*}{$\frac{8}{8}$} & 91.3 & & & & 71.4 & \\
\hline 149 & 9-Jul-05 & & & & & & & \\
\hline 150 & 10-Jul-05 & & & & & & & 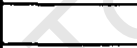 \\
\hline 151 & 11-Jul-05 & & 91.7 & 3717 & 10.1 & 98 & 58.8 & 8.7 \\
\hline 152 & 12-Jul-05 & & 91.6 & 256.9 & 10.4 & 10.4 & 56.8 & 6.9 \\
\hline 153 & 13-Jul-05 & & 93.3 & 299.6 & 6.6 & 7.0 & 73.6 & 5.6 \\
\hline 154 & 14-Jul-05 & & 100,1 & 313.6 & 9.4 & 8.1 & 73.2 & 5.2 \\
\hline 155 & 15-Jul-05 & & 90.9 & 264.6 & 9.5 & 8.4 & 73.9 & 7.0 \\
\hline 156 & 16-Jul-05 & & & & & & & \\
\hline 157 & 17-Jul-05 & & & & & 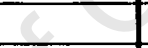 & & \\
\hline 158 & 18-Jul-05 & & 99.7 & 290.5 & 12.0 & 9.7 & 69.2 & 7.6 \\
\hline 159 & 19-Jul-05 & & 92.0 & 250.6 & 10.6 & 9.8 & 62.1 & 8.8 \\
\hline 160 & 20-Jul-05 & & 87.5 & 261.8 & 8.3 & 8.1 & 62.9 & 7.0 \\
\hline 161 & 21-Jul-05 & & 95.1 & 277.9 & 9.4 & 21 & 67.0 & 5.7 \\
\hline 162 & $22-J u \mid-05$ & & 87.9 & 291.9 & 10.2 & 9.8 & 70.1 & 6.9 \\
\hline 163 & 23-Jul-05 & & & 9 & & & & \\
\hline 164 & 24-Jul-05 & & & $a+1$ & & & & \\
\hline 165 & 25-Jul-05 & & 90.9 & 267.4 & 7.3 & 6.0 & 72.9 & 5.0 \\
\hline 166 & $26-J u l-05$ & & 87.5 & 280.0 & 8.7 & 7.7 & 69.6 & 7.8 \\
\hline 167 & $27-J u l-05$ & & 89.5 & 353.5 & 12.5 & 7.1 & 64.6 & 6.6 \\
\hline \multicolumn{2}{|c|}{ Batch Average } & 10 & 91.5 & 284.0 & 9.6 & 8.5 & 67.6 & 6.8 \\
\hline 168 & 28-Jul-05 & \multirow{17}{*}{$\begin{array}{l}\sigma \\
\frac{\sigma}{0} \\
\frac{\pi}{\pi} \\
\dot{\infty}\end{array}$} & & & & & & \\
\hline 169 & 29-Jul-05 & & 90.7 & & & & 59.1 & \\
\hline 170 & 30-Jul-05 & & & & & & & \\
\hline 171 & $31-J u l-05$ & & & & & & & \\
\hline 172 & 1-Aug-05 & & 88.8 & 268.8 & 12.2 & 8.4 & 75.4 & 6.6 \\
\hline 173 & 2-Aug-05 & & 95.3 & 289.8 & 9.7 & 9.8 & 73.2 & 7.3 \\
\hline 174 & 3-Aug-05 & & 102.5 & 323.4 & 9.4 & 9.1 & 80.9 & 7.8 \\
\hline 175 & 4-Aug-05 & & 93.7 & 242.2 & 9.7 & 8.1 & 70.4 & 5.9 \\
\hline 176 & 5-Aug-05 & & 108.8 & 298.9 & 10.8 & 9.7 & 82.2 & 6.6 \\
\hline 177 & 6-Aug-05 & & & & & & & \\
\hline 178 & 7-Aug-05 & & & & & & & \\
\hline 179 & 8-Aug-05 & & 95.3 & 230.3 & 10.5 & 6.4 & 75.7 & 24 \\
\hline 180 & 9-Aug-05 & & 94.9 & 277.2 & 7.4 & 5.0 & 82.0 & 3.6 \\
\hline 181 & 10-Aug-05 & & 97.1 & 252.7 & 6.3 & 7.3 & 73.9 & 5.7 \\
\hline 182 & 11-Aug-05 & & & & & & & \\
\hline 183 & 12-Aug-05 & & & & & & & \\
\hline 984 & 13-Aug-05 & & & & & & & \\
\hline
\end{tabular}


B-2 Conv TN

\begin{tabular}{|c|c|c|c|c|c|c|c|c|}
\hline 185 & 14-Aug-05 & & & & & & & \\
\hline 186 & 15-Aug-05 & & 892 & 224.0 & 7.8 & 7.7 & 74.9 & 5.7 \\
\hline 187 & 16-Aug-05 & & 89.6 & 269.5 & 7.7 & 7.3 & 78.0 & 5.5 \\
\hline 188 & 17-Aug-05 & \multirow[b]{2}{*}{11} & 89.5 & 282.1 & 7.3 & 6.0 & 73.7 & 5.6 \\
\hline \multicolumn{2}{|c|}{ Batch Average } & & 93.3 & 269.0 & 9.0 & 7.7 & 76.4 & 6.0 \\
\hline 189 & 18-Aug-05 & \multirow{16}{*}{$\frac{\omega}{\infty}$} & 692 & 261.1 & 7.3 & 7.1 & 549 & 5.3 \\
\hline 190 & 19-Aug-05 & & & & & & & \\
\hline 191 & 20-Aug-05 & & & & & & & \\
\hline 192 & 21-Aug-05 & & & & & & & \\
\hline 193 & 22-Aug-05 & & & & & & & \\
\hline 194 & 23-Aug-05 & & 138.5 & 231.7 & 151 & 14,4 & 111.2 & 11.3 \\
\hline 195 & 24-Aug-05 & & 144.3 & 293.3 & 11.1 & 9.8 & 107.4 & 9.9 \\
\hline 196 & 25-Aug-05 & & 110.3 & 262.5 & 8.3 & & 86.8 & 7.4 \\
\hline 197 & 26-Aug-05 & & 110.0 & 281.4 & 7.6 & 7.3 & 87.4 & 6.2 \\
\hline 198 & 27-Aug-05 & & & & & & & \\
\hline 199 & 28-Aug-05 & & & & & & & \\
\hline 200 & 29-Aug-05 & & 142.1 & 275.8 & 8.8 & 7.7 & 107.1 & 5.2 \\
\hline 201 & 30-Aug-05 & & 128.1 & 266.0 & 7.7 & 7.4 & 98.3 & 6.2 \\
\hline 202 & 31-Aug-05 & & 137.5 & & & & 97.4 & \\
\hline 203 & 1-Sep-05 & & 142.9 & 249.2 & 9.8 & 8.3 & 101.4 & 6.6 \\
\hline 204 & 2-Sep-05 & & & & & & & \\
\hline Batc & ch Average & 12 & 131.7 & 265.1 & 8.6 & 7.9 & 99.6 & 7.3 \\
\hline 205 & 3-Sep-05 & & & & & & & \\
\hline 206 & 4-Sep-05 & & & & & & & \\
\hline 207 & 5-Sep-05 & & 874 & 246.4 & 8.5 & 7.8 & 75.1 & 6.2 \\
\hline 208 & 6-Sep-05 & & 99.5 & 236.6 & 8.7 & 7.7 & 69.4 & 6.0 \\
\hline 209 & 7-Sep-05 & s & 103.5 & 237.3 & 7.3 & 6.9 & 68.2 & 5.5 \\
\hline 210 & 8-Sep-05 & 1 & 111.6 & 254.8 & 7.6 & 7.1 & 1123 & 5.2 \\
\hline 211 & 9-Sep-05 & 1 & 101.5 & 224.0 & 7.7 & 6.4 & 82.1 & 4.8 \\
\hline 212 & 10-Sep-05 & 1 & & & & & 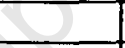 & \\
\hline 213 & 11-Sep-05 & 8 & & & & 0 & 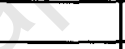 & \\
\hline 214 & 12-Sep-05 & $m$ & 101.9 & 223.3 & 7.3 & 6.7 & 86.4 & 5.7 \\
\hline 215 & 13-Sep-05 & & 101.5 & 180.6 & 9.0 & 8.4 & 78.0 & \\
\hline 216 & 14-Sep-05 & & 99.3 & 196.7 & 9.2 & 5.6 & 91.1 & \\
\hline 217 & 15-Sep-05 & & & & & & & \\
\hline 218 & 16-Sep-05 & & 105.7 & & & & 83.1 & \\
\hline Batc & h Average & 13 & 103.1 & 225.0 & 8.2 & 7.1 & 79.2 & 5.6 \\
\hline 219 & 17-Sep-05 & & & 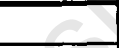 & & & & \\
\hline 220 & 18-Sep-05 & & & & & & & \\
\hline 221 & 19-Sep-05 & & 113.1 & 232.4 & 13.9 & 11.1 & 94.0 & 6.7 \\
\hline 222 & 20-Sep-05 & & & 2 & & & & \\
\hline 223 & 21-Sep-05 & & 114.8 & 229.6 & 13.0 & 9.8 & 92.3 & 7.6 \\
\hline 224 & 22-Sep-05 & & 102.3 & 2478 & 9.1 & 7.8 & 85.1 & 6.4 \\
\hline 225 & 23-Sep-05 & $\mathbf{S}$ & 106.3 & 231.7 & 7.8 & 7.0 & 84.1 & 5.2 \\
\hline 226 & 24-Sep-05 & - & & & & & & \\
\hline 227 & 25-Sep-05 & 0 & & & & & & \\
\hline 228 & 26-Sep-05 & 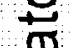 & 106.3 & 224.7 & 12.2 & 9.0 & 89.3 & 7.8 \\
\hline 229 & 27-Sep-05 & $m$ & 122.9 & 222.6 & 9.4 & 6.7 & 104.9 & 6.2 \\
\hline 230 & 28-Sep-05 & & 109.6 & 221.9 & 13.2 & 8.8 & 94.2 & 5.6 \\
\hline 231 & 29-Sep-05 & & 117.6 & 223.3 & 12.7 & 9.1 & 97.3 & 6.9 \\
\hline 232 & 30-Sep-05 & & 1352 & 236.6 & 4.8 & 7.8 & 109.2 & 4.3 \\
\hline 233 & 1-Oct-05 & & & & & & & \\
\hline 234 & 2-Oct-05 & & & & & & & \\
\hline 235 & 3-Oct-05 & & 118.7 & 230.3 & 9.5 & 8.3 & 96.6 & 5.7 \\
\hline Bat & h Average & 14 & 112.4 & 228.1 & 10.6 & 8.5 & 94.7 & 6.2 \\
\hline 236 & 4-Oct-05 & & & & & & 76.2 & \\
\hline 237 & 5-Oct-05 & & 106.1 & & & & & \\
\hline 238 & 6-Oct-05 & 5 & & & & & 83.2 & \\
\hline 239 & 7-Oct-05 & & 109.5 & & & & & \\
\hline 240 & 8-Oct-05 & $\frac{1}{0}$ & & & & & & 0.0 \\
\hline 241 & 9-Oct-05 & \pm & & & & & 90.2 & \\
\hline
\end{tabular}


B-2 Conv TN

\begin{tabular}{|c|c|c|c|c|c|c|c|c|}
\hline 242 & $10-0 c t-05$ & \multirow{3}{*}{$\dot{0}$} & 120.4 & 330.4 & 5.2 & & & 0.3 \\
\hline 243 & $11-0 c t-05$ & & & & & & 80.4 & \\
\hline 244 & 12-Oct-05 & & 98.3 & 212.8 & 2.8 & 1.8 & & \\
\hline \multicolumn{2}{|c|}{ Batch Average } & 15 & 108.6 & 271.6 & 4.0 & 1.8 & 82.5 & 0.2 \\
\hline \multicolumn{2}{|c|}{\begin{tabular}{|l|l|}
245 & $13-0 c t-05$ \\
\end{tabular}} & \multirow{7}{*}{$\stackrel{1}{1}$} & & & & & & \\
\hline 246 & 14-Oct-05 & & 94.4 & 242.9 & 2.2 & 1.8 & 77.0 & 1.4 \\
\hline 247 & $15-$ Oct-05 & & & & & & & \\
\hline 248 & 16-Oct-05 & & & & & & & \\
\hline 249 & $17-$ Oct-05 & & 105.3 & 252.0 & 4.3 & 2.7 & 83.4 & 2.2 \\
\hline 250 & 18-Oct-05 & & & & & & & \\
\hline 251 & 19-Oct-05 & & 105.8 & 280.0 & 160 & 10.3 & 84.0 & 135 \\
\hline 252 & 20-Oct-05 & \multirow{8}{*}{$\begin{array}{l}\frac{6}{6} \\
\frac{10}{6} \\
0\end{array}$} & & & & & & \\
\hline 253 & $21-$ Oct-05 & & 137.8 & 345.1 & 4.4 & 1.9 & 79.2 & 1.5 \\
\hline 254 & 22-Oct-05 & & & & & & & \\
\hline 255 & 23-Oct-05 & & & & & & & \\
\hline 256 & 24-Oct-05 & & 108.9 & 116.2 & 2.835 & 2.9 & 73.9 & 2.3 \\
\hline 257 & 25-Oct-05 & & & & & & & \\
\hline 258 & 26-Oct-05 & & 101.1 & 217.7 & 3.15 & 3.6 & 76.4 & 0.0 \\
\hline 259 & $27-$ Oct-05 & & & & & & & \\
\hline \multicolumn{2}{|c|}{ Batch Average } & \multirow{15}{*}{$\begin{array}{c}N \\
\sigma \\
+ \\
\sigma \\
\omega\end{array}$} & 108.9 & 242.3 & 3.4 & 2.6 & 79.0 & 1.5 \\
\hline 260 & 28-Oct-05 & & 96.6 & 138.6 & 3.8 & 2.1 & 83.4 & 1.5 \\
\hline 261 & 29-Oct-05 & & & & & & & \\
\hline 262 & $30-$ Oct-05 & & & & & & & \\
\hline 263 & 31-Oct-05 & & 92.4 & 218.4 & 2.2 & 2.0 & 64.7 & 1.7 \\
\hline 264 & $1-N o v-05$ & & & & & & & \\
\hline 265 & 2-Nov-05 & & 97.4 & 282.8 & 2.8 & 1.9 & 100.5 & 1.5 \\
\hline 266 & 3-Nov-05 & & & & & & & + \\
\hline 267 & 4-Nov-05 & & 84.8 & 272.3 & 2.9 & 2.7 & 75.9 & 1.2 \\
\hline 268 & 5-Nov-05 & & & & & & & 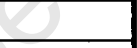 \\
\hline 269 & 6-Nov-05 & & & & & & 1 & \\
\hline 270 & 7-Nov-05 & & 98.8 & 280.0 & 6.4 & 2.2 & 762 & 2.0 \\
\hline 271 & $8-N o v-05$ & & & & & & 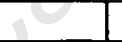 & \\
\hline 272 & 9-Nov-05 & & 90.4 & 105.7 & 1.8 & 3.0 & 67.5 & 1.2 \\
\hline 273 & 10-Nov-05 & & & & & & & \\
\hline \multicolumn{2}{|c|}{ Batch Average } & 17 & 93.4 & 216.3 & 3.3 & 2.3 & 78.0 & 1.5 \\
\hline 274 & 11-Nov-05 & \multirow{8}{*}{$\infty$} & 101.9 & 246.4 & 3.5 & 2.3 & 72.2 & 0.8 \\
\hline 275 & 12-Nov-05 & & & & & & & \\
\hline 276 & 13-Nov-05 & & & & 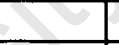 & & & \\
\hline 277 & 14-Nov-05 & & & 0 & & & & \\
\hline 278 & 15-Nov-05 & & 97.4 & 292.6 & 3.2 & 2.6 & 81.8 & 1.3 \\
\hline 279 & 16-Nov-05 & & 94.9 & 305.2 & 5.7 & 2.7 & 972 & 2.1 \\
\hline 280 & 17-Nov-05 & & 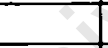 & + & & & & \\
\hline 281 & 18-Nov-05 & & 88.2 & 219.8 & 9.5 & 9.6 & 79.0 & 3.9 \\
\hline 282 & 19-Nov-05 & \multirow{10}{*}{$\begin{array}{c}\stackrel{\sigma}{\sigma} \\
\infty\end{array}$} & +4 & & & & & \\
\hline 283 & 20-Nov-05 & & 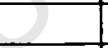 & & & & & \\
\hline 284 & 21-Nov-05 & & & & & & & \\
\hline 285 & 22-Nov-05 & & 101.1 & 307.3 & 4.3 & 2.7 & 81.5 & 1.8 \\
\hline 286 & 23-Nov-05 & & 105.6 & 263.2 & 11.4 & 11.6 & 84.0 & 93 \\
\hline 287 & 24-Nov-05 & & & & & & & \\
\hline 288 & 25-Nov-05 & & 107.2 & 240.8 & 3.0 & 5.3 & 80.6 & 3.0 \\
\hline 289 & 26-Nov-05 & & & & & & & \\
\hline 290 & $27-$ Nov-05 & & & & & & & \\
\hline 291 & 28-Nov-05 & & 104.2 & & & & 86.8 & \\
\hline \multicolumn{2}{|c|}{ Batch Average } & 18 & 100.1 & 267.9 & 5.8 & 5.2 & 80.8 & 2.2 \\
\hline \multicolumn{2}{|c|}{\begin{tabular}{l|l|}
292 & $29-N o v-05$ \\
\end{tabular}} & \multirow{7}{*}{$\sqrt{6}$} & & & & & & \\
\hline 293 & 30-Nov-05 & & 96.9 & 295.4 & 5.6 & 2.0 & 73.6 & 1.6 \\
\hline 294 & 1-Dec-05 & & & & & & & \\
\hline 295 & 2-Dec-05 & & 139.7 & 293.3 & 4.5 & 1.6 & 103.0 & 2.8 \\
\hline 296 & 3-Dec-05 & & & & & & & \\
\hline 297 & 4-Dec-05 & & & & & & & \\
\hline 298 & 5-Dec-05 & & 90.7 & 259.0 & 2.2 & 1.2 & 70.6 & 0.0 \\
\hline
\end{tabular}


B-2 Conv TN

\begin{tabular}{|c|c|c|c|c|c|c|c|c|}
\hline 299 & 6-Dec-05 & \multirow{2}{*}{0} & & & & & & \\
\hline 300 & 7-Dec-05 & & & & & & & \\
\hline 301 & 8-Dec-05 & \multirow{5}{*}{$\begin{array}{l}\frac{}{3} \\
\frac{3}{\infty} \\
\infty\end{array}$} & 73.6 & 221.9 & 3.7 & 1.3 & 70.8 & 0.4 \\
\hline 302 & 9-Dec-05 & & 114.8 & 235.9 & 2.2 & 1.6 & 82.9 & 0.0 \\
\hline 303 & 10-Dec-05 & & & & & & & \\
\hline 304 & 11-Dec-05 & & & & & & & \\
\hline 305 & 12-Dec-05 & & & & & & & \\
\hline 306 & 13-Dec-05 & \multirow{7}{*}{ r } & 104.2 & 249.2 & 0.0 & 0.0 & 85.7 & 0.0 \\
\hline 307 & 14-Dec-05 & & & & & & & \\
\hline 308 & 15-Dec-05 & & 151.8 & 179.2 & 4.9 & 1.8 & 77.3 & 0.3 \\
\hline 309 & 16-Dec-05 & & & & & & & \\
\hline 310 & 17-Dec-05 & & & & & & & \\
\hline 311 & 18-Dec-05 & & & & & & & \\
\hline 312 & 19-Dec-05 & & & & & & & \\
\hline \multicolumn{2}{|c|}{ Batch Average } & 19 & 110.2 & 247.7 & 3.3 & 1.4 & 80.6 & 0.7 \\
\hline \multicolumn{2}{|c|}{\begin{tabular}{|l|l|}
313 & $20-$ Dec-05 \\
\end{tabular}} & \multirow{6}{*}{9} & & & & & & \\
\hline \multicolumn{2}{|r|}{ 21-Dec-05 } & & & & & & & \\
\hline 315 & $22-D e c-05$ & & & & & & & \\
\hline 316 & 23-Dec-05 & & & & & & & \\
\hline 317 & 24-Dec-05 & & & & & & & \\
\hline 318 & 25-Dec-05 & & & & & & & \\
\hline 319 & 26-Dec-05 & \multirow{8}{*}{$\frac{8}{8}$} & & & & & & \\
\hline 320 & 27-Dec-05 & & & & & & & \\
\hline 321 & 28-Dec-05 & & & & & & & \\
\hline 322 & 29-Dec-05 & & & & & & & \\
\hline 323 & 30-Dec-05 & & & & & & & \\
\hline 324 & 31-Dec-05 & & & & & & & 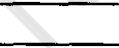 \\
\hline 325 & 1-Jan-06 & & & & & & & \\
\hline \multirow[t]{2}{*}{326} & 2-Jan-06 & & & & & & & \\
\hline & & 20 & & & & & 1 & \\
\hline 327 & 3-Jan-06 & \multirow{7}{*}{ \% } & & & & 1 & 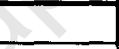 & \\
\hline 328 & 4-Jan-06 & & & & & & & \\
\hline 329 & 5-Jan-06 & & & & & -2 & & \\
\hline 330 & 6-Jan-06 & & & & & & & \\
\hline 331 & 7-Jan-06 & & & & & & & \\
\hline 332 & 8-Jan-06 & & & & & & & \\
\hline \multirow{2}{*}{$\begin{array}{l}333 \\
34 \\
\end{array}$} & 9-Jan-06 & & & - & + & & & \\
\hline & 10-Jan-06 & \multirow{8}{*}{$\frac{\mathrm{S}}{\mathrm{\infty}}$} & & 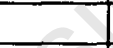 & 2 & & & \\
\hline 335 & 11-Jan-06 & & & 8 & & & & \\
\hline 336 & 12-Jan-06 & & & 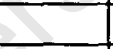 & & & & \\
\hline 337 & 13-Jan-06 & & & 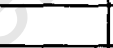 & & & & \\
\hline 338 & 14-Jan-06 & & & & & & & \\
\hline 339 & 15-Jan-06 & & & & & & & \\
\hline 340 & 16-Jan-06 & & & & & & & \\
\hline \multirow[t]{2}{*}{341} & 17-Jan-06 & & & & & & & \\
\hline & & 23 & & & & & & \\
\hline 342 & 18-Jan-06 & \multirow{6}{*}{ 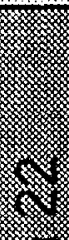 } & & & & & & \\
\hline 343 & 19-Jan-06 & & & & & & & \\
\hline 344 & 20-Jan-06 & & 100.2 & & & & 70.3 & \\
\hline 345 & 21-Jan-06 & & & & & & & \\
\hline 346 & 22-Jan-06 & & & & & & & \\
\hline 347 & $23-\operatorname{Jan}-06$ & & 125.2 & 216.3 & 3.8 & & 103.0 & 1.6 \\
\hline 348 & 24-Jan-06 & 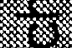 & & & & & & \\
\hline 349 & 25-Jan-06 & 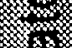 & 103.3 & 206.5 & 1.9 & 0.9 & 82.9 & 0.5 \\
\hline 350 & 26-Jan-06 & 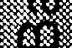 & & & & & & \\
\hline 351 & $27-J a n-06$ & & 117.0 & 191.8 & 2.9 & 2.2 & 91.3 & \\
\hline 352 & 28-Jan-06 & & & & & & & \\
\hline 353 & 29-Jan-06 & & & & & & & \\
\hline 354 & $30-\operatorname{Jan}-06$ & 准 & 114.2 & 152.6 & & & 92.7 & 1.8 \\
\hline Bat & h Average & 22 & 112.0 & 191.8 & 2.9 & 1.6 & 88.0 & 1.3 \\
\hline 355 & 31-Jan-06 & & & & & & & \\
\hline
\end{tabular}


B-2 Conv TN

\begin{tabular}{|c|c|c|c|c|c|c|c|c|}
\hline 356 & 1-Feb-06 & \multirow{6}{*}{ 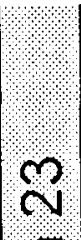 } & & & & & & \\
\hline 357 & 2-Feb-06 & & & & & & & \\
\hline 358 & 3-Feb-06 & & & & & & 70.3 & 0.2 \\
\hline 359 & 4-Feb-06 & & & & & & & \\
\hline 360 & 5-Feb-06 & & & & & & & \\
\hline 361 & $6-F e b-06$ & & & & & & 41.2 & 0.5 \\
\hline 362 & 7-Feb-06 & \multirow{8}{*}{ 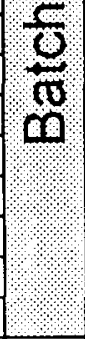 } & & & & & & \\
\hline 363 & 8 -Feb-06 & & 102.8 & 177.8 & 3.5 & 1.5 & 85.7 & 0.2 \\
\hline 364 & 9-Feb-06 & & & & & & & \\
\hline 365 & 10-Feb-06 & & & 156.8 & 4.0 & 1.7 & 78.4 & 0.6 \\
\hline 366 & 11-Feb-06 & & & & & & & \\
\hline 367 & 12-Feb-06 & & & & & & & \\
\hline 368 & 13-Feb-06 & & 109.2 & 169.4 & 3.3 & 2.8 & 80.4 & 1.1 \\
\hline 369 & 14-Feb-06 & & 1028 & 213.5 & 4.3 & 1.2 & 79.2 & 0.7 \\
\hline \multicolumn{2}{|c|}{ Batch Average } & 23 & 104.9 & 179.4 & 3.8 & 1.8 & 72.5 & 0.5 \\
\hline 370 & 15-Feb-06 & \multirow{7}{*}{8} & & & & & & \\
\hline 371 & 16-Feb-06 & & & & & & & \\
\hline 372 & 17-Feb-06 & & 65.0 & 210.0 & 3.0 & 1.8 & 93.5 & 0.4 \\
\hline 373 & 18-Feb-06 & & & & & & & \\
\hline 374 & 19-Feb-06 & & & & & & & \\
\hline 375 & $20-F e b-06$ & & & & & & & \\
\hline 376 & 21-Feb-06 & & 85.7 & 217.0 & 2.3 & 1.0 & 59.1 & 1.5 \\
\hline 377 & 22-Feb-06 & \multirow{7}{*}{$\begin{array}{l}\frac{3}{\infty} \\
0 \\
1\end{array}$} & & & & & & \\
\hline 378 & $23-F e b-06$ & & & & & & & \\
\hline 379 & $24-F e b-06$ & & 71.4 & & 1.3 & & 47.9 & \\
\hline 380 & 25-Feb-06 & & & & & & & \\
\hline 381 & 26-Feb-06 & & & & & & & \\
\hline 382 & $27-F e b-06$ & & 86.0 & 249.2 & & & 54.3 & $\infty$ \\
\hline 383 & 28-Feb-06 & & 74.8 & 210.0 & 1.2 & 0.6 & & 12 \\
\hline \multicolumn{2}{|c|}{ Batch Average } & 24 & 76.6 & 221.6 & 1.9 & 1.1 & 63.7 & 0.9 \\
\hline 384 & 1-Mar-06 & \multirow{4}{*}{ लि } & 92.1 & 200.2 & 1.5 & & 72.5 & 0.2 \\
\hline 385 & 2-Mar-06 & & & & & & 10 & \\
\hline 386 & 3-Mar-06 & & & & & & 3 & \\
\hline 387 & 4-Mar-06 & & & & & + & & \\
\hline 388 & 5-Mar-06 & \multirow{2}{*}{$\underset{\mathbb{O}}{\mathcal{O}}$} & & & & & & \\
\hline 389 & 6-Mar-06 & & 98.0 & & & & 82.3 & \\
\hline 390 & 7-Mar-06 & \multirow{2}{*}{$\infty$} & 118.2 & 147.7 & +2 & 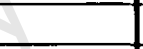 & 82.9 & \\
\hline 391 & 8-Mar-06 & & & 139.3 & $\begin{array}{r}.8 \\
\end{array}$ & 0.7 & 98.0 & 0.0 \\
\hline \multicolumn{2}{|c|}{ Batch Average } & 25 & 102.8 & 162.4 & 1.7 & 0.7 & 83.9 & 0.1 \\
\hline 392 & 9-Mar-06 & \multirow{15}{*}{$\begin{array}{c}c \\
\sim \\
\frac{\sigma}{\sigma} \\
\frac{\sigma}{\infty}\end{array}$} & & $\square$ & 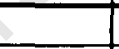 & & 96.9 & 0.4 \\
\hline 393 & 10-Mar-06 & & & 0 & & & & \\
\hline 394 & 11-Mar-06 & & & +2 & & & & \\
\hline 395 & 12-Mar-06 & & $\square$ & $\mathrm{L}$ & & & & \\
\hline 396 & 13-Mar-06 & & 91.3 & 275.8 & & 0.4 & 74.8 & \\
\hline 397 & 14-Mar-06 & & 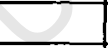 & & & & & \\
\hline 398 & 15-Mar-06 & & 132.7 & 209.3 & & 1.5 & 102.8 & 0.4 \\
\hline 399 & 16-Mar-06 & & & & & & & \\
\hline 400 & 17-Mar-06 & & 90.7 & 214.2 & 4.0 & 1.8 & 70.0 & 0.7 \\
\hline 401 & 18-Mar-06 & & & & & & & \\
\hline 402 & 19-Маг-06 & & & & & & & \\
\hline 403 & 20-Mar-06 & & 98.3 & 205.8 & 5.6 & 1.5 & 84.6 & 1.0 \\
\hline 404 & 21-Mar-06 & & & & & & & \\
\hline 405 & 22-Mar-06 & & & & & & & \\
\hline 406 & 23-Mar-06 & & 98.0 & 224.0 & 3.4 & 2.1 & 82.0 & 0.6 \\
\hline \multicolumn{2}{|c|}{ Batch Average } & 26 & 102.2 & 225.8 & 4.3 & 1.5 & 85.2 & 0.6 \\
\hline \begin{tabular}{ll|}
407 \\
\end{tabular} & 24-Mar-06 & & 107.0 & & 36 & & 76.7 & 0.7 \\
\hline 408 & 25-Mar-06 & & & & & 1.7 & & \\
\hline 409 & 26-Маг-06 & & & & & & & \\
\hline 410 & 27-Mar-06 & & 101.9 & 201.6 & & & 93.2 & 0.0 \\
\hline 411 & 28-Mar-06 & & & & & & & \\
\hline 412 & 29-Mar-06 & & 98.6 & 224.7 & 3.2 & & 72.5 & 0.6 \\
\hline
\end{tabular}


B-2 Conv TN

\begin{tabular}{|c|c|c|c|c|c|c|c|c|}
\hline 413 & 30-Mar-06 & \multirow{9}{*}{$\frac{\sigma}{\tilde{U}}$} & & & & 1.3 & & \\
\hline 414 & 31-Mar-06 & & 84.6 & 199.5 & 2.9 & & 75.0 & 0.2 \\
\hline 415 & 1-Apr-06 & & & & & 0.3 & & \\
\hline 416 & 2-Apr-06 & & & & & & & \\
\hline 417 & 3-Apr-06 & & 108.6 & 2058 & 3.1 & & 82.3 & 0.8 \\
\hline 418 & 4-Apr-06 & & & & & 2.5 & & \\
\hline 419 & 5-Apr-06 & & 106.4 & 253.4 & 3.6 & & 78.1 & 0.4 \\
\hline 420 & 6-Apr-06 & & & & & 1.2 & & \\
\hline 421 & 7-Apr-06 & & & & & & & \\
\hline \multicolumn{2}{|c|}{ Batch Average } & 27 & 101.2 & 217.0 & 3.3 & 1.4 & 79.7 & 0.5 \\
\hline \multicolumn{2}{|c|}{\begin{tabular}{l|l}
422 & $8-A p r-06$ \\
\end{tabular}} & \multirow{7}{*}{8} & & & & & & \\
\hline 423 & 9-Apr-06 & & & & & & & \\
\hline 424 & 10-Apr-06 & & 80.6 & 186.2 & & 3.4 & 58.8 & 2.2 \\
\hline 425 & 11-Apr-06 & & & & & & & \\
\hline 426 & 12-Apr-06 & & & & & & & \\
\hline 427 & 13-Apr-06 & & 100.2 & 189.7 & 4.3 & 2.2 & 83.7 & 0.9 \\
\hline 428 & 14-Apr-06 & & 94.9 & & & & & \\
\hline 429 & 15-Apr-06 & \multirow{8}{*}{$\frac{6}{6}$} & 100.0 & 204.4 & 1.4 & 1.4 & 81.8 & 1.2 \\
\hline 430 & 16-Apr-06 & & 98.0 & & & & & \\
\hline 431 & 17-Apr-06 & & 101.9 & & & & & \\
\hline 432 & 18-Apr-06 & & 117.0 & 250.6 & 2.4 & 1.8 & 80.4 & 0.4 \\
\hline 433 & 19-Apr-06 & & & & & & & \\
\hline 434 & 20-Apr-06 & & & & & & & \\
\hline 435 & 21-Apr-06 & & 118.2 & 238.7 & 2.5 & 2.3 & 86.8 & 0.9 \\
\hline 436 & $22-A p r-06$ & & & & & & & 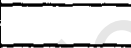 \\
\hline \multicolumn{2}{|c|}{ Batch Average } & 28 & 101.4 & 213.9 & 2.7 & 2.2 & 78.3 & 1.1 \\
\hline \multicolumn{2}{|c|}{$4 3 7 \longdiv { 2 3 - A p r - 0 6 }$} & \multirow{5}{*}{8} & & & & & & 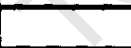 \\
\hline 438 & 24-Apr-06 & & 105.8 & 238.0 & 2.6 & 1.3 & 77.0 & 0.5 \\
\hline 439 & 25-Apr-06 & & & & & & & 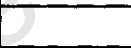 \\
\hline 440 & 26-Aрг-06 & & 94.4 & 222.6 & & 2.6 & 76.7 & 0.0 \\
\hline 441 & 27-Apr-06 & & & & & $\infty$ & + & \\
\hline 442 & 28-Apr-06 & \multirow{8}{*}{$\begin{array}{c}\frac{0}{5} \\
00 \\
08\end{array}$} & 88.2 & 236.6 & 2.4 & 1.8 & 69.7 & 0.4 \\
\hline 443 & 29-Apr-06 & & & & & - & & \\
\hline 444 & 30-Apr-06 & & & & & & & \\
\hline 445 & 1-May-06 & & & & & & 79.5 & 1.2 \\
\hline 446 & 2-May-06 & & & & & & & \\
\hline 447 & 3-May-06 & & 96.9 & 252.0 & + & 2.2 & 64.1 & 1.1 \\
\hline 448 & 4-May-06 & & & & & & & \\
\hline 449 & 5-May-06 & & 89.6 & 242.2 & 2.3 & 2.7 & 63.0 & 1.4 \\
\hline \multicolumn{2}{|c|}{ Batch Average } & 29 & 95.0 & 238.3 & 2.4 & 2.1 & 71.7 & 0.8 \\
\hline
\end{tabular}


Appendix B-3

Measured NO3, NO2 and soluble $\mathbf{P}$ in the conventional UCT system

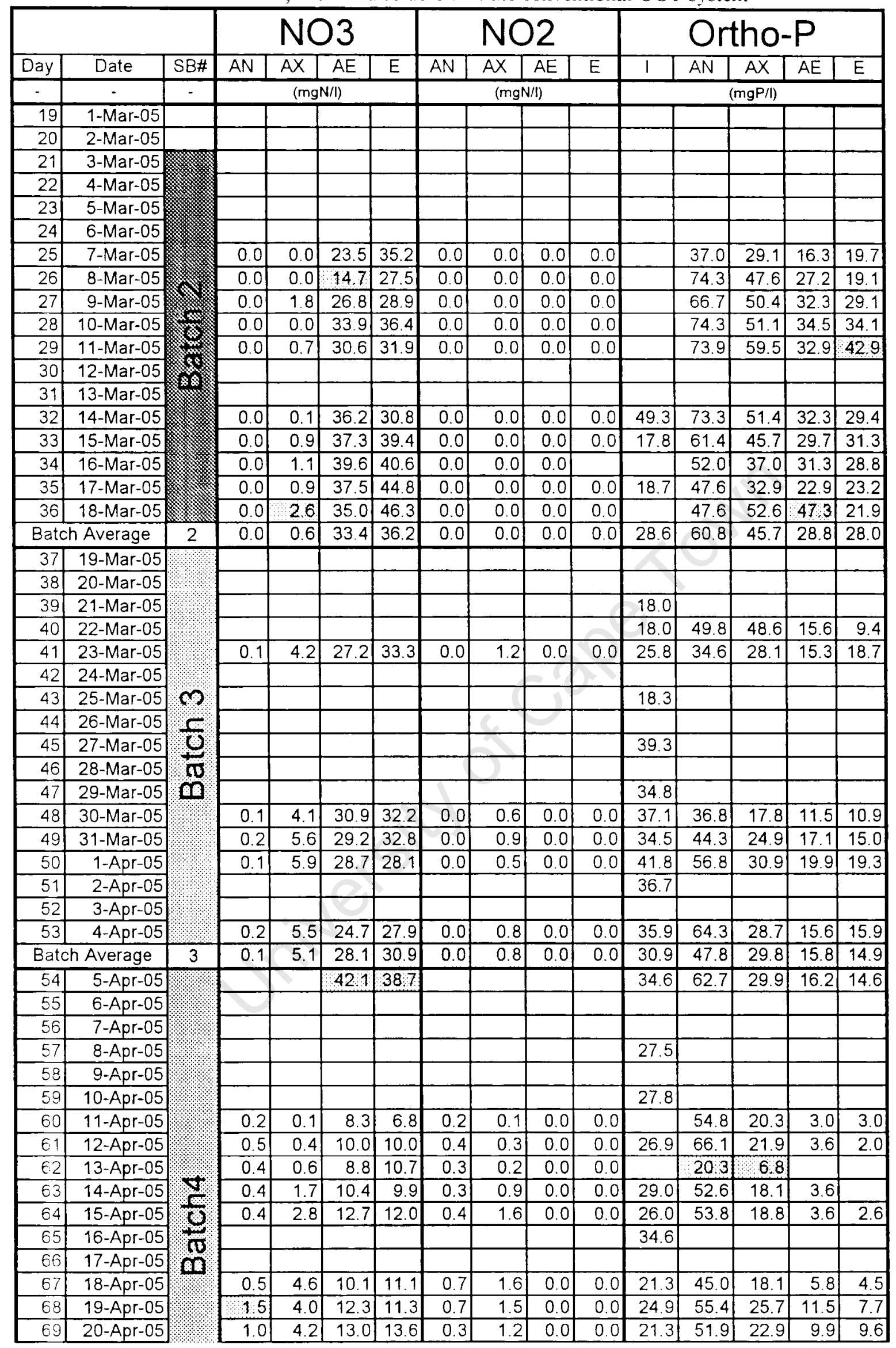




\begin{tabular}{|c|c|c|c|c|c|c|c|c|c|c|c|c|c|c|c|}
\hline 70$]$ & 21-Apr-05 & & 0.3 & 3.8 & 12.4 & 14.2 & 0.0 & 1.3 & 0.0 & 0.0 & 28.5 & 50.0 & 22.9 & 9.9 & 11.2 \\
\hline 71 & 22-Apr-05 & & 0.0 & 5.2 & 18.5 & 25.8 & 0.1 & 0.1 & 0.0 & 0.0 & 22.6 & 45.3 & 28.7 & 17.3 & 15.3 \\
\hline 72 & 23-Apr-05 & & & & & & & & & & 26.3 & 38.1 & 25.1 & 15.3 & 17.0 \\
\hline 73 & 24-Apr-05 & & & & & & & & & & 25.9 & 42.0 & 25.9 & 16.7 & 14.8 \\
\hline 74 & 25-Apr-05 & & 0.0 & 1.1 & 15.2 & 15.6 & 0.1 & 0.1 & 0.0 & 0.0 & 22.7 & & & & \\
\hline 75 & 26-Apr-05 & & 0.1 & 0.5 & 18.1 & 17.9 & 0.2 & 0.0 & 0.0 & 0.0 & & & & & \\
\hline \multicolumn{2}{|c|}{ Batch Average } & 4 & 0.4 & 2.4 & 12.5 & 13.2 & 0.3 & 0.7 & 0.0 & 0.0 & 26.7 & 51.5 & 23.2 & 9.7 & 9.3 \\
\hline \multicolumn{2}{|c|}{\begin{tabular}{l|l|}
76 & $27-A p r-05$ \\
\end{tabular}} & \multirow{19}{*}{$\begin{array}{l}5 \\
\frac{5}{0} \\
0 \\
0\end{array}$} & & & & & & & & & & & & & \\
\hline \multirow{2}{*}{\multicolumn{2}{|c|}{\begin{tabular}{|l|l|}
77 & $28-A p r-05$ \\
\end{tabular}}} & & & & & & & & & & & & & & \\
\hline 78 & & & & & & & & & & & 28.8 & & & & \\
\hline \multicolumn{2}{|c|}{\begin{tabular}{l|l|}
79 & $30-A p r-05$ \\
\end{tabular}} & & & & & & & & & & & & & & \\
\hline \multicolumn{2}{|c|}{\begin{tabular}{l|l|}
80 & $1-$ May-05 \\
\end{tabular}} & & & & & & & & & & & & & & \\
\hline \multirow{2}{*}{\multicolumn{2}{|c|}{\begin{tabular}{|l|l|}
81 & 2-May-05 \\
82 & 3-May-05 \\
\end{tabular}}} & & & & & & & & & & 31.5 & & & & \\
\hline & & & & & & & & & & & & & & & \\
\hline 83 & 4-May-05 & & 1.0 & 7.0 & 38.7 & 40.9 & 0.9 & 30 & 0.0 & 0.0 & 29.0 & 33.4 & 26.4 & 21.7 & 22.3 \\
\hline \multirow{2}{*}{\begin{tabular}{|l|}
84 \\
85
\end{tabular}} & 5-May-05 & & 0.7 & 4.7 & 32.8 & 34.2 & 0.7 & 1.5 & 0.0 & 0.0 & 29.3 & 32.0 & 25.3 & 20.6 & 21.4 \\
\hline & 6-May-05 & & 0.0 & 0.4 & 24.4 & 31.3 & 0.0 & 0.4 & 0.0 & 0.0 & 25.5 & 33.4 & 28.4 & 16.4 & 19.5 \\
\hline \multirow{2}{*}{\begin{tabular}{l|}
86 \\
87
\end{tabular}} & 7-May-05 & & & & & & & & & & & & & & \\
\hline & 8-May-05 & & & & & & & & & & & & & & \\
\hline$\frac{87}{88}$ & 9-May-05 & & 0.3 & 5.2 & 22.4 & 13.8 & 0.3 & 1.3 & 0.0 & 0.0 & 19.0 & 15.6 & 6.4 & & 5.9 \\
\hline 80 & 10-May-05 & & 00 & 0.8 & 17.3 & 10.3 & 0.1 & 0.4 & 0.0 & 0.0 & 19.2 & 30.3 & 16.4 & 6.2 & 7.0 \\
\hline 90 & 11-May-05 & & 0.3 & 1.6 & 24.0 & 18.5 & 0.3 & & 0.0 & 0.0 & 19.6 & 27.0 & 13.1 & 4.8 & 4.5 \\
\hline 91 & 12-May-05 & & 0.0 & 0.3 & 25.8 & 19.5 & 0.0 & 0.6 & 0.0 & 0.0 & 17.3 & 17.0 & 9.2 & 5.6 & 3.9 \\
\hline 92 & 13-May-05 & & 0.3 & 6.2 & 36.2 & 31.1 & 0.3 & 1.1 & 0.0 & 0.0 & 20.5 & 25.1 & 16.4 & 11.7 & 8.7 \\
\hline 93 & 14-May-05 & & & & & & & & & & & 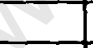 & & & \\
\hline 94 & 15-May-05 & & & & & & & & & & & & & & \\
\hline Batc & h Average & 5 & 0.3 & 3.3 & 27.7 & 25.0 & 0.3 & 0.9 & 0.0 & 0.0 & 24.0 & 26.7 & 17.7 & 12.4 & 11.7 \\
\hline 95 & 16-May-05 & & & & & & & & & & 21.7 & & & & \\
\hline 96 & 17-May-05 & & & & & & & & & & & & & & \\
\hline 97 & 18-May-05 & & & & & & & & & & & & & & \\
\hline 98 & 19-May-05 & & & & & & & & & & & & & & \\
\hline 99 & 20-May-05 & & & & & & & & - & & 18.9 & & & & \\
\hline 100 & 21-May-05 & & & & & & & & & & & & & & \\
\hline 101 & 22-May-05 & & & & & & 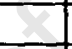 & & & & & & & & \\
\hline 102 & 23-May-05 & $\frac{5}{0}$ & 0.0 & 8.5 & 27.6 & 26.8 & 0.0 & 1.3 & 0.0 & 0.0 & 22.2 & 14.5 & 7.8 & 11.4 & 10.0 \\
\hline 103 & 24-May-05 & 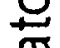 & 0.0 & 8.6 & 31.9 & 30.1 & 0.0 & 1.6 & 0.0 & 0.0 & 23.3 & 17.7 & 15.4 & 13.1 & 9.7 \\
\hline 104 & 25-May-05 & $m$ & 0.0 & 47 & 28.7 & 30.7 & 0.0 & 0.6 & 0.0 & 0.0 & 22.1 & 27.6 & 19.6 & 15.1 & 14.5 \\
\hline 105 & 26-May-05 & & 0.0 & 7.7 & 27.8 & 30.1 & 0.0 & 1.6 & 0.0 & 0.0 & 25.3 & 18.2 & 15.4 & 11.4 & 13.7 \\
\hline 106 & 27-May-05 & & 0.0 & 8.0 & 29.7 & 31.5 & 0.0 & 0.8 & 0.0 & 0.0 & 31.6 & 32.1 & 19.6 & 14.3 & $\overline{12.3}$ \\
\hline 107 & 28-May-05 & & & & & & & & & & & & & & \\
\hline 108 & 29-May-05 & & & & & & & & & & & & & & \\
\hline 109 & 30-May-05 & & 0.0 & 9.0 & 23.7 & 27.8 & 0.0 & 2.4 & 0.0 & 0.0 & 31.4 & 23.6 & 21.6 & 19.9 & 20.2 \\
\hline 110 & 31-May-05 & & 0.0 & 9.9 & 29.5 & 29.1 & & 1.5 & & & 30.1 & 28.1 & 21.6 & 20.2 & 20.8 \\
\hline Batc & h Average & 6 & 0.0 & 8.6 & 28.4 & 29.5 & 0.0 & 1.4 & 0.0 & 0.0 & 25.2 & 23.1 & 17.3 & 15.1 & 14.5 \\
\hline 111 & 1-Jun-05 & & & & & & & & & & 28.8 & & & & \\
\hline 112 & 2-Jun-05 & & & & & & & & & & 26.5 & & & & \\
\hline 113 & 3-Jun-05 & & 0.0 & 0.0 & 7.9 & 4.7 & 0.0 & 0.0 & 0.0 & 0.0 & 26.3 & 37.9 & 26.7 & 18.1 & 17.5 \\
\hline 114 & 4-Jun-05 & & & & & & & & & & & & & & \\
\hline 115 & 5-Jun-05 & & & & & & & & & & & & & & \\
\hline 116 & 6-Jun-05 & 18 & 0.0 & 0.0 & 11.9 & 14.2 & 0.0 & 0.0 & 0.0 & 0.0 & 28.4 & 37.3 & 26.7 & 18.4 & 19.8 \\
\hline 117 & 7-Jun-05 & 18 & 0.0 & 0.4 & & 4.9 & 0.0 & 0.0 & 0.0 & 0.0 & 30.4 & 41.2 & 48.2 & 53.5 & 30.1 \\
\hline 118 & 8-Jun-05 & $\frac{\pi}{\pi}$ & 0.0 & 0.0 & 18.5 & 12.7 & 0.0 & 0.0 & 0.0 & 0.0 & 30.9 & 37.3 & 20.3 & 17.0 & 24.2 \\
\hline 119 & 9-Jun-05 & 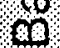 & 0.0 & 2.2 & 10.6 & 11.7 & 0.0 & 0.0 & 0.0 & 0.0 & 30.3 & 35.3 & 20.9 & 11.7 & 12.2 \\
\hline 120 & 10-Jun-05 & & & & & & 0.0 & 0.0 & 0.0 & 0.0 & 34.3 & 40.6 & 46.8 & 49.8 & 26.4 \\
\hline 121 & 11-Jun-05 & & & & & & & & & & & & & & \\
\hline 122 & 12-Jun-05 & & & & & & & & & & & & & & \\
\hline 123 & 13-Jun-05 & & 0.0 & 117 & 26.3 & 20.6 & 0.0 & 75 & 190 & 15.7 & 29.2 & 26.4 & 15.6 & 11.1 & 13.9 \\
\hline 124 & 14-Jun-05 & & 0.0 & 0.0 & 15.5 & 16.1 & 0.0 & 1.0 & 11.6 & 11.4 & 38.4 & 31.4 & 16.7 & 10.0 & 10.0 \\
\hline Batc & h Average & 7 & 0.0 & 0.4 & 15.1 & 12.1 & 0.0 & 0.1 & 1.7 & 3.4 & 29.5 & 35.9 & 27.7 & 23.7 & 19.3 \\
\hline 125 & 15-Jun-05 & & & & & & & & & & & & & & \\
\hline
\end{tabular}


B-3 Conv NOx / P

\begin{tabular}{|c|c|c|c|c|c|c|c|c|c|c|c|c|c|c|c|}
\hline 126 & 16-Jun-05 & \multirow[b]{2}{*}{$\infty$} & & & & & & & & & & & & & \\
\hline 127 & 17-Jun-05 & & & & & & & & & & & & & & \\
\hline 128 & 18-Jun-05 & \multirow{2}{*}{$\frac{1}{3}$} & & & & & & & & & 25.8 & & & & \\
\hline 129 & 19-Jun-05 & & & & & & & & & & & & & & \\
\hline 130 & 20-Jun-05 & \multirow{3}{*}{8} & 0.0 & 4.6 & 14.0 & 11.9 & 0.0 & 3.5 & 8.8 & 7.3 & 28.3 & 36.0 & 22.6 & 15.6 & 14.5 \\
\hline 131 & 21-Jun-05 & & 0.0 & 2.1 & 16.4 & 14.8 & 0.0 & 0.0 & 4.6 & 3.6 & 29.7 & 38.8 & 21.0 & 15.6 & 15.9 \\
\hline 132 & 22-Jun-05 & & 0.0 & 5.6 & 23.7 & 19.3 & 0.0 & 0.0 & 4.0 & 2.2 & 30.9 & 40.5 & 24.0 & 17.3 & 15.9 \\
\hline \multicolumn{2}{|c|}{ Batch Average } & 8 & 0.0 & 4.1 & 18.0 & 15.3 & 0.0 & 1.2 & 5.8 & 4.4 & 28.7 & 38.5 & 22.5 & 16.2 & 15.5 \\
\hline 133 & 23-Jun-05 & \multirow{15}{*}{$\begin{array}{l}0 \\
\frac{1}{0} \\
\frac{t}{0} \\
\infty\end{array}$} & & & & & & & & & 33.6 & & & & \\
\hline 134 & 24-Jun-05 & & 0.0 & 7.9 & & 25.1 & & & & & 31.3 & 36.3 & 23.7 & 20.4 & 18.7 \\
\hline 135 & 25-Jun-05 & & & & & & & & & & & & & & \\
\hline 136 & 26-Jun-05 & & & & & & & & & & & & & & \\
\hline 137 & $27-J u n-05$ & & 0.0 & 4.9 & & 20.7 & 0.0 & 0.0 & 0.0 & 0.0 & 27.5 & 37.7 & 25.4 & 20.7 & 20.1 \\
\hline 138 & 28-Jun-05 & & 0.0 & 4.4 & & 20.4 & 0.0 & 0.0 & 0.0 & 0.0 & 28.2 & 38.8 & 25.4 & 20.4 & 19.0 \\
\hline 139 & 29-Jun-05 & & 0.0 & 12 & & 19.6 & 0.0 & 0.0 & 0.0 & 0.0 & 31.2 & 43.9 & 25.1 & 19.6 & 20.1 \\
\hline 140 & 30-Jun-05 & & 0.0 & 6.7 & 22.5 & 21.4 & 0.0 & 0.0 & 0.0 & 0.0 & 30.1 & 37.7 & 26.8 & 20.7 & 21.2 \\
\hline 141 & $1-J u l-05$ & & 0.0 & 7.3 & 22.9 & 22.2 & 0.0 & 0.0 & 0.0 & 0.0 & 30.5 & 38.0 & 26.8 & 20.1 & 21.0 \\
\hline 142 & 2-Jul-05 & & & & & & & & & & & & & & \\
\hline 143 & 3-Jul-05 & & & & & & & & & & & & & & \\
\hline 144 & 4-Jul-05 & & 0.1 & 5.5 & 21.6 & 18.7 & & & & & 33.8 & 38.5 & 26.8 & 19.8 & 19.6 \\
\hline 145 & 5-Jul-05 & & 0.0 & 3.7 & 18.6 & 18.3 & & & & & 31.7 & 44.1 & 27.1 & 198 & 19.3 \\
\hline 146 & 6-Jul-05 & & 0.0 & 6.5 & 16.8 & 16.2 & & & & & 17.3 & 30.7 & 168 & 120 & 16.2 \\
\hline 147 & 7 -Jul-05 & & & & & & & & & & 125 & & 2 & & \\
\hline \multicolumn{2}{|c|}{ Batch Average } & 9 & 0.0 & 5.9 & 20.5 & 20.3 & 0.0 & 0.0 & 0.0 & 0.0 & 29.5 & 38.4 & 25.9 & 19.3 & 19.5 \\
\hline 148 & 8-Jul-05 & \multirow{20}{*}{$\frac{5}{6}$} & & & & & & & & & 10.9 & 2 & & & \\
\hline 149 & 9-Jul-05 & & & & & & & & & & & & & & \\
\hline 150 & $10-J u 1-05$ & & & & & & & & & & 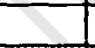 & & & & \\
\hline 151 & 11-Jul-05 & & & & 11.1 & 10.1 & 0.0 & 0.0 & 0.0 & 0.0 & 96 & 292 & 160 & 50 & 4,4 \\
\hline 152 & 12-Jul-05 & & 0.3 & 1.4 & 10.1 & 6.3 & 0.0 & 0.0 & 0.0 & 0.0 & 101 & 27.8 & 148 & 5.5 & 41 \\
\hline 153 & 13-Jul-05 & & & & & & 0.1 & 0.1 & 0.0 & 0.0 & 13.2 & 309 & 14,6 & 4.1 & 3.9 \\
\hline 154 & 14-Jul-05 & & & & 11.6 & & & & 0 & 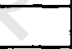 & 127 & 29.5 & 14,0 & 3.9 & 3.9 \\
\hline 155 & 15-Jul-05 & & & & 11.3 & \begin{tabular}{|l|}
11.2 \\
\end{tabular} & 0.0 & 0.0 & 0.0 & 0.0 & 15.2 & 351 & 129 & 4.4 & 4.1 \\
\hline 156 & $16-J u l-05$ & & & & & & & + & & & & & & & \\
\hline 157 & 17-Jul-05 & & & & & & & & & & & & & & \\
\hline 158 & 18-Jul-05 & & & & 10.8 & 9.7 & 0.0 & 0.0 & 0.0 & 0.0 & 112 & 337 & 15.1 & 4.4 & 39 \\
\hline 159 & 19-Jul-05 & & 0.0 & 1.8 & 11.4 & 6.3 & 0.1 & 0.0 & 0.0 & 0.5 & 122 & 295 & 140 & 39 & 53 \\
\hline 160 & 20-Jul-05 & & & & & & & & & & & 30.3 & 109 & 4.4 & 39 \\
\hline 161 & $21-J u l-05$ & & & & 10.4 & 6.7 & 8 & & 0.0 & 19 & 117 & 23.3 & 8,4 & 39 & 4.1 \\
\hline 162 & 22-Jul-05 & & & & 11.6 & 8.3 & & & 0.0 & 0.1 & 125 & 281 & 9.5 & 5.3 & 39 \\
\hline 163 & 23-Jul-05 & & & & -1 & & & & & & 9.6 & & & & \\
\hline 164 & 24-Jul-05 & & & $\Delta$ & 5 & & & & & & & & & & \\
\hline 165 & 25-Jul-05 & & & & 14.7 & 160 & 0.0 & 0.0 & 0.6 & 1.2 & 28.7 & 38.2 & 22.2 & 13.2 & 12.0 \\
\hline 166 & 26-Jul-05 & & 0.0 & 5.1 & 14.1 & 13.3 & 0.0 & 1.1 & 0.0 & 0.5 & 30.2 & 27.8 & 18.5 & 14.3 & 14.0 \\
\hline 167 & 27-Jul-05 & & $\square$ & & 12.1 & 6.7 & 0.0 & 0.0 & 0.0 & 0.1 & 29.2 & 45.0 & 23.0 & 14.0 & 13.4 \\
\hline \multicolumn{2}{|c|}{ Batch Average } & 10 & 0.1 & 2.8 & 11.7 & 9.5 & 0.0 & 0.1 & 0.1 & 0.2 & 29.3 & 37.0 & 21.2 & 13.8 & 13.2 \\
\hline 168 & 28-Jul-05 & \multirow{15}{*}{$\begin{array}{l}\frac{5}{5} \\
\frac{5}{0} \\
\frac{1}{\infty}\end{array}$} & & & & & & & & & & & & & \\
\hline 169 & 29-Jul-05 & & & & & & & & & & & & & & \\
\hline 170 & 30-Jul-05 & & & & & & & & & & 27.8 & & & & \\
\hline 171 & $31-J u l-05$ & & & & & & & & & & & & & & \\
\hline 172 & 1-Aug-05 & & & & 12.6 & 10.1 & & & 0.0 & 0.0 & 23.3 & 34.4 & 21.7 & 14.0 & 10.9 \\
\hline 173 & 2-Aug-05 & & 0.3 & 3.8 & 10.6 & 8.9 & 0.0 & 0.2 & 0.0 & 0.0 & 22.0 & 44.9 & 23.9 & 14.3 & 10.9 \\
\hline 174 & 3-Aug-05 & & & & 17.7 & 11.5 & & & 0.0 & 0.0 & 27.0 & 58.8 & 288 & 15.7 & 13.8 \\
\hline 175 & 4-Aug-05 & & & & 13.7 & 9.9 & & & 0.0 & 0.0 & 25.5 & 38.9 & 23.7 & 14.6 & 14.9 \\
\hline 176 & 5-Aug-05 & & & & 14.5 & 11.8 & & & 0.0 & 0.0 & 25.3 & 34.4 & 19.4 & 10.9 & 10.4 \\
\hline 177 & 6-Aug-05 & & & & & & & & & & & & & & \\
\hline 178 & 7-Aug-05 & & & & & & & & & & & & & & \\
\hline 179 & 8-Aug-05 & & & & 12.5 & 8.8 & & & 0.0 & 0.7 & 24.8 & 42.3 & 22.5 & 12.1 & 9.5 \\
\hline 180 & 9-Aug-05 & & 0.3 & 2.3 & 13.6 & 3.8 & 0.0 & 0.3 & 0.0 & 0.5 & 24.6 & 35.3 & 18.3 & 15.7 & 15.2 \\
\hline 181 & 10-Aug-05 & & & & 14.3 & 7.4 & & & 0.0 & 0.5 & 24.5 & 39.8 & 20.0 & 10.4 & 13.8 \\
\hline 182 & 11-Aug-05 & & & & & & & & & & 20.6 & & & & \\
\hline
\end{tabular}


B-3 Conv NOx / P

\begin{tabular}{|c|c|c|c|c|c|c|c|c|c|c|c|c|c|c|c|}
\hline 183 & 12-Aug-05 & & 0.0 & 00 & 14.2 & 8.4 & & & & & & & & & \\
\hline 184 & 13-Aug-05 & & & & & & & & & & & & & & \\
\hline 185 & 14-Aug-05 & & & & & & & & & & & & & & \\
\hline 186 & 15-Aug-05 & & 0.0 & 0.0 & 15.1 & 15.4 & & & & & 31.0 & 43.5 & 21.7 & 11.2 & 10.9 \\
\hline 187 & 16-Aug-05 & & 0.0 & 1.9 & 14.9 & 15.1 & & & & & 22.7 & 40.9 & 19.7 & 10.6 & 6.7 \\
\hline 188 & 17-Aug-05 & & 0.0 & 0.0 & 17.7 & 14.5 & & & & & 24.4 & 28.5 & 16.0 & 9.8 & 9.5 \\
\hline Batch & h Average & 11 & 0.1 & 1.3 & 14.3 & 10.5 & 0.0 & 0.3 & 0.0 & 0.2 & 24.4 & 38.3 & 20.7 & 12.7 & 11.5 \\
\hline 189 & 18-Aug-05 & \multirow{16}{*}{$\frac{\frac{N}{S}}{\frac{\Gamma}{\mathscr{O}}}$} & 0.0 & 0.0 & 20.0 & 18.8 & 0.0 & 0.0 & 0.0 & 0.0 & & 25.9 & 14.6 & 9.5 & 9.8 \\
\hline 190 & 19-Aug-05 & & & & & & & & & & & & & & \\
\hline 191 & 20-Aug-05 & & & & & & & & & & & & & & \\
\hline 192 & 21-Aug-05 & & & & & & & & & & & & & & \\
\hline 193 & 22-Aug-05 & & 0.0 & 0.0 & 0.0 & & 0.0 & 0.0 & 0.0 & 0.0 & 27.4 & & & & \\
\hline 194 & 23-Aug-05 & & 00 & 0.0 & 34.5 & 20.4 & 0.0 & 0.0 & 0.0 & 0.0 & 28.7 & 42.2 & 26.2 & 15.1 & 13.1 \\
\hline 195 & 24-Aug-05 & & 0.0 & 0.0 & 34.6 & 31.4 & 0.0 & 0.0 & 0.0 & 0.0 & 30.9 & 39.4 & 22.5 & 10.8 & 10.5 \\
\hline 196 & 25-Aug-05 & & 0.5 & 8.0 & 28.2 & 34.3 & 0.0 & 0.0 & 0.0 & 0.0 & 27.4 & 55.1 & 25.7 & 11.9 & 9.7 \\
\hline 197 & 26-Aug-05 & & 0.0 & 0.0 & 27.3 & 29.6 & 0.0 & 0.0 & 0.0 & 0.0 & 27.7 & 52.8 & 25.4 & 21.1 & $\overline{10.8}$ \\
\hline 198 & $27-A u g-05$ & & & & & & & & & & & & & & \\
\hline 199 & $28-A u g-05$ & & & & & & & & & & & & & & \\
\hline 200 & 29-Aug-05 & & 0.0 & 0.0 & 37.7 & 35.4 & 0.0 & 0.0 & 0.0 & 0.0 & 26.6 & 51.4 & 25.1 & 11.9 & 11.1 \\
\hline 201 & 30-Aug-05 & & 0.0 & 12.2 & 26.6 & 27.1 & 0.0 & 0.0 & 0.0 & 0.0 & & 49.7 & 25.7 & 12.2 & 11.1 \\
\hline 202 & 31-Aug-05 & & & & & & & & & & & 67.7 & 37.4 & 19.9 & 159 \\
\hline 203 & 1-Sep-05 & & 0.0 & 0.0 & 22.2 & 19.7 & & & & & 28.3 & & & & \\
\hline 204 & $2-$ Sep-05 & & & & & & & & & & 29.4 & & & & \\
\hline Batch & h Average & 12 & 0.0 & 1.0 & 28.9 & \begin{tabular}{|l|l|}
27.1 \\
\end{tabular} & 0.0 & 0.0 & 0.0 & 0.0 & 28.3 & 48.0 & 25.3 & \begin{tabular}{|l|}
14.1 \\
\end{tabular} & 10.9 \\
\hline 205 & 3-Sep-05 & \multirow{14}{*}{$\frac{9}{\frac{1}{6}}$} & & & & & & & & & & & & & \\
\hline 206 & 4-Sep-05 & & & & & & & & & 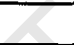 & 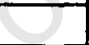 & & & & \\
\hline 207 & 5-Sep-05 & & 0.0 & 0.6 & 10.0 & 11.7 & 0.0 & 0.0 & 0.0 & 0.0 & 28.8 & 80.5 & 46.0 & \begin{tabular}{|l|}
17.2 \\
\end{tabular} & 11.3 \\
\hline 208 & $6-$ Sep-05 & & 0.0 & 0.0 & 9.1 & 10.1 & 0.0 & 0.0 & 0.0 & 0.0 & 28.3 & 58.6 & 26.9 & 12.2 & 9.7 \\
\hline 209 & 7 -Sep-05 & & 0.0 & 1.8 & 11.3 & 10.8 & 0.0 & 0.0 & 0.0 & 0.0 & 32.3 & 60.5 & 28.2 & \begin{tabular}{|l|}
14.1 \\
\end{tabular} & 10.6 \\
\hline 210 & 8 -Sep-05 & & & 0.0 & 13.3 & 14.0 & 0.0 & 0.0 & 0.0 & 0.0 & 30.5 & 62.3 & 28.2 & 13.8 & 11.6 \\
\hline 211 & 9-Sep-05 & & 0.0 & 1.9 & 11.4 & 11.7 & 0.0 & 0.0 & 0.0 & 0.0 & 30.6 & 57.0 & 24.4 & 10.3 & 10.9 \\
\hline 212 & $10-\operatorname{Sep}-05$ & & & & & & & & & & 31.2 & & & & \\
\hline 213 & 11-Sep-05 & & & & & & & 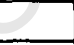 & & & & & & & \\
\hline 214 & 12-Sep-05 & & 0.0 & 9.3 & 167 & 181 & 0.0 & 0.0 & 0.0 & 0.0 & 32.2 & 26.6 & 15.0 & 9.7 & 10.0 \\
\hline 215 & 13-Sep-05 & & 0.0 & 0.0 & 11.1 & 13.8 & 0.0 & 0.0 & 0.0 & 0.0 & 31.7 & 16.0 & 10.3 & 8.7 & 9.1 \\
\hline 216 & 14-Sep-05 & & 0.0 & 5.2 & 12.1 & 11.1 & 0.0 & 0.0 & 0.0 & 0.0 & 30.7 & 32.6 & 16.0 & 9.4 & 10.6 \\
\hline 217 & 15-Sep-05 & & & & 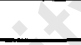 & 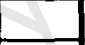 & & & & & 29.3 & & & & \\
\hline 218 & 16-Sep-05 & & & & & 2 & & & & & 30.2 & & & & \\
\hline \multicolumn{2}{|c|}{ Batch Average } & 13 & 0.0 & 1.3 & 11.2 & 11.9 & 0.0 & 0.0 & 0.0 & 0.0 & 30.5 & 49.3 & 24.4 & 11.9 & 10.5 \\
\hline \multicolumn{2}{|c|}{\begin{tabular}{l|l}
219 & $17-S e p-05$ \\
\end{tabular}} & \multirow{17}{*}{$\begin{array}{l}\frac{\sigma}{\sigma} \\
\frac{\sigma}{\mathcal{O}} \\
\frac{\pi}{\omega}\end{array}$} & & 0 & & & & & & & & & & & \\
\hline 220 & 18-Sep-05 & & & $O$ & & & & & & & & & & & \\
\hline 221 & 19-Sep-05 & & 0.0 & 8.7 & 24.6 & 24.6 & 0.0 & 0.0 & 0.0 & 0.0 & 29.5 & 42.3 & 19.9 & 7.1 & 8.7 \\
\hline 222 & $20-\operatorname{Sep}-05$ & & 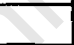 & & & & & & & & 459 & & & & \\
\hline 223 & 21-Sep-05 & & 0.0 & 3.9 & 19.7 & 19.0 & 0.0 & 0.0 & 0.0 & 0.0 & 29.9 & 41.0 & 24.0 & \begin{tabular}{|l|}
13.1 \\
\end{tabular} & 11.2 \\
\hline 224 & $22-\mathrm{Sep}-05$ & & 0.0 & & 16.2 & 16.5 & & & & & 29.0 & 54.8 & 25.0 & 8.7 & 6.1 \\
\hline 225 & 23-Sep-05 & & & 7.0 & 26.0 & 23.3 & & & & & 30.9 & 53.8 & 26.9 & 9.6 & 8.0 \\
\hline 226 & 24-Sep-05 & & & & & & & & & & & & & & \\
\hline 227 & $25-$ Sep-05 & & & & & & & & & & & & & & \\
\hline 228 & $26-\operatorname{sep}-05$ & & & 8.8 & 29.0 & 30.1 & & & & & 31.8 & 59.6 & 27.9 & 11.5 & 9.8 \\
\hline 229 & $27-$ Sep-05 & & & 11.6 & 31.7 & 31.5 & & & & & 30.0 & 53.2 & 28.2 & 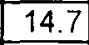 & 12.5 \\
\hline 230 & 28-Sep-05 & & & & & & & & & & 32.1 & 71.8 & 30.4 & 12.8 & 11.0 \\
\hline 231 & $29-S e p-05$ & & & & & & & & & & & 60.9 & 28.8 & 13.1 & 164 \\
\hline 232 & $30-S e p-05$ & & & 1.0 & 20.3 & 23.8 & & & & & 33.3 & 71.8 & 29.5 & 8.7 & 8.0 \\
\hline 233 & 1-Oct-05 & & & & & & & & & & & & & & \\
\hline 234 & 2 -Oct-05 & & & & & & & & & & & & & & \\
\hline 235 & 3-Oct-05 & & & 14.0 & 26.5 & 30.1 & & & & & 32.3 & 38.5 & 19.6 & 8.0 & 9.9 \\
\hline Batcl & h Average & 14 & 0.0 & 7.9 & 24.2 & 24.9 & 0.0 & 0.0 & 0.0 & 0.0 & 31.0 & 54.8 & 26.0 & \begin{tabular}{|l|}
10.7 \\
\end{tabular} & 9.6 \\
\hline 236 & 4-Oct-05 & & & & & & & & & & 29.1 & & & & \\
\hline 237 & 5-Oct-05 & & & & & & & & & & 23.5 & & & & \\
\hline 238 & 6-Oct-05 & (5) & & & & & & & & & & & & & \\
\hline
\end{tabular}


B-3 Conv NOx/P

\begin{tabular}{|c|c|c|c|c|c|c|c|c|c|c|c|c|c|c|c|}
\hline 239 & 7-Oct-05 & \multirow{6}{*}{$\frac{\sigma}{\frac{\sigma}{\sigma}}$} & 00 & 1.3 & 13.2 & 12.3 & 0.0 & 0.0 & 0.0 & 0.0 & 26.9 & & & & \\
\hline 240 & 8-Oct-05 & & & & & & & & & & & & & & \\
\hline 241 & 9-Oct-05 & & & & & & 0.0 & 0.0 & 0.0 & 0.0 & & & & & \\
\hline 242 & $10-$ Oct-05 & & 0.0 & 11.7 & 24.9 & 24.9 & 0.0 & 0.0 & 00 & 0.0 & 30.1 & 17.6 & 12.8 & 14.1 & 18.3 \\
\hline 243 & 11-Oct-05 & & 0.0 & 9.7 & 25.9 & 25.1 & & & & & 26.9 & 49.7 & 23.4 & 11.9 & 11.5 \\
\hline 244 & 12-Oct-05 & & 0.0 & 11.4 & 21.5 & 28.2 & & & & & 42.0 & 33.4 & 14.4 & 116 & 12.8 \\
\hline Batcl & h Average & 15 & 0.0 & 8.5 & 21.4 & 22.6 & 0.0 & 0.0 & 0.0 & 0.0 & 29.8 & 33.6 & 16.9 & 12.5 & 14.2 \\
\hline 245 & $13-$ Oct-05 & \multirow{15}{*}{$\frac{9}{\frac{9}{9}}$} & 0.0 & 9.7 & 27.0 & 26.9 & & & & & 33.4 & 54.8 & 28.8 & 17.7 & 19.3 \\
\hline 246 & 14-Oct-05 & & 0.0 & 4.6 & 22.1 & 25.6 & & & & & 33.4 & 63.1 & 30.6 & 17.4 & 14.4 \\
\hline 247 & 15-Oct-05 & & & & & & & & & & & & & & \\
\hline 248 & $16-$ Oct-05 & & & & & & 0.0 & 0.0 & 0.0 & 0.2 & & & & & \\
\hline 249 & 17-Oct-05 & & 0.0 & 0.0 & 15.5 & 12.1 & 0.0 & 0.0 & 0.2 & 0.2 & 30.3 & 59.6 & 29.4 & 13.0 & 13.0 \\
\hline 250 & 18-Oct-05 & & & & & & 0.0 & 0.0 & 0.0 & 0.0 & 29.7 & 66.9 & 79.5 & 61.2 & 42.9 \\
\hline 251 & 19-Oct-05 & & 0.0 & 1.2 & 10.2 & 10.0 & & & & & 33.5 & 56.5 & 30.9 & 24.6 & 9.2 \\
\hline 252 & $20-$ Oct-05 & & 0.0 & 0.4 & 16.5 & 14.1 & & & & & 29.3 & 58.0 & 29.3 & 11.7 & 9.8 \\
\hline 253 & $21-$ Oct-05 & & 0.0 & 0.2 & 15.8 & 15.7 & 0.0 & 0.0 & 0.0 & 0.2 & & 59.6 & 25.1 & 9.5 & 7.9 \\
\hline 254 & $22-$ Oct-05 & & & & & & 0.0 & 0.0 & 0.0 & 0.0 & & & & & \\
\hline 255 & 23-Oct-05 & & & & & & 0.0 & 0.0 & 0.2 & 0.2 & & & & & \\
\hline 256 & 24-Oct-05 & & 0.0 & 0.0 & 15.2 & 15.9 & 0.0 & 0.0 & 0.0 & 0.1 & 26.9 & 58.1 & 30.0 & 13.8 & 13.5 \\
\hline 257 & $25-$-Oct-05 & & 0.0 & 1.9 & 16.4 & 15.3 & & & & & 35.8 & 59.6 & 28.8 & 13.2 & 10.1 \\
\hline 258 & 26-Oct-05 & & 0.0 & 2.5 & 19.2 & 19.4 & & & & & 30.0 & 55.7 & 26.3 & 14.1 & 12.6 \\
\hline 259 & 27 -Oct-05 & & 0.0 & 5.7 & 15.9 & 19.1 & & & & & 31.4 & 17.2 & 10.8 & 10.5 & 11.1 \\
\hline Batc & Average & 16 & 0.0 & 1.8 & 16.3 & 17.4 & 0.0 & 0.0 & 0.0 & 0.1 & 31.4 & 59.2 & 27.0 & 18.8 & 12.1 \\
\hline 260 & $28-O c t-05$ & \multirow{14}{*}{$\frac{N}{\frac{N}{U}}$} & 0.0 & 1.8 & 6.8 & 4.7 & 0.0 & 0.0 & 0.0 & 0.0 & 38.0 & 51.3 & 19.3 & 13.9 & 14.8 \\
\hline 261 & $29-$-ct-05 & & & & & & & & & & & 2 & & & \\
\hline 262 & $30-$-Oct-05 & & & & & & & & & & & & & & \\
\hline 263 & $31-$ Oct-05 & & 0.0 & 0.4 & 6.9 & 5.0 & 0.0 & 0.0 & 0.0 & 0.0 & 38.9 & 54.6 & 292 & 19.3 & 14.7 \\
\hline 264 & 1-Nov-05 & & 0.0 & 0.3 & 11.6 & 10.9 & 0.0 & 0.0 & 0.0 & 0.0 & 41.3 & 63.0 & 45.8 & 16.2 & 16.2 \\
\hline 265 & 2-Nov-05 & & 0.0 & 0.0 & 12.0 & 10.8 & 0.0 & 0.0 & 0.0 & 0.0 & 39.2 & 59.7 & 28.9 & 16.2 & 16.5 \\
\hline 266 & 3-Nov-05 & & & & & & & & & & & & & & \\
\hline 267 & 4-Nov-05 & & 00 & 0.5 & 11.4 & 9.7 & 0.0 & 0.0 & 0.0 & 0.0 & 39.6 & 66.0 & 40.2 & 22.8 & 20.4 \\
\hline 268 & $5-$ Nov- 05 & & & & & & & & & & & & & & \\
\hline 269 & 6-Nov-05 & & & & & & & & 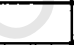 & & & & & & \\
\hline 270 & 7-Nov-05 & & 0.0 & 2.3 & 12.5 & 8.9 & 0.0 & 0.0 & 0.0 & 0.0 & 31,4 & 17.2 & 10.8 & 10.5 & 11.1 \\
\hline 271 & 8-Nov-05 & & 0.0 & 0.3 & 6.8 & & 0.0 & 0.0 & 0.0 & 0.0 & 38.0 & 51.3 & 19.3 & 13.9 & 14.8 \\
\hline 272 & 9-Nov-05 & & 0.0 & 0.8 & 11.3 & 8.9 & 0.0 & 0.0 & 0.0 & 0.0 & & & & & \\
\hline 273 & 10-Nov-05 & & 0.0 & 0.0 & 11.2 & 10.4 & 0.0 & 0.0 & 0.0 & 0.0 & 38.4 & 62.8 & 32.8 & 18.4 & 17.8 \\
\hline Batc & Average & 17 & 0.0 & 0.7 & 10.1 & 8.7 & 0.0 & 0.0 & 0.0 & 0.0 & 39.1 & 58.4 & 28.3 & 16.4 & 15.8 \\
\hline 274 & 11-Nov-05 & \multirow{18}{*}{$\frac{\infty}{\frac{c}{9}}$} & & 0.3 & 12.7 & 12.6 & 0.0 & 0.0 & 0.0 & 0.0 & 34.6 & 72.5 & 34.9 & 15.7 & 15.7 \\
\hline 275 & 12-Nov-05 & & & & 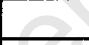 & & & & & & & & & & \\
\hline 276 & 13-Nov-05 & & & & & & & & & & & & & & \\
\hline 277 & 14-Nov-05 & & 0.0 & 5.4 & 15.4 & & 0.0 & 0.0 & 0.0 & 0.0 & 36.3 & 60.8 & 24.3 & 14.8 & \\
\hline 278 & 15-Nov-05 & & 0.0 & 7.5 & 20.3 & 17.8 & 0.0 & 0.0 & 0.0 & 0.0 & & 57.8 & 24.6 & 19.0 & 18.4 \\
\hline 279 & 16-Nov-05 & & 0.0 & 3.7 & 18.5 & 18.2 & 0.0 & 0.0 & 0.4 & 0.0 & & 56.1 & 34.0 & 22.2 & 16.9 \\
\hline 280 & 17-Nov-05 & & 0.0 & 0.0 & 15.9 & 17.3 & 0.0 & 0.0 & 19 & 0.5 & 36.1 & 55.9 & 32.2 & 20.6 & 17.3 \\
\hline 281 & $18-N o v-05$ & & 0.0 & 4.7 & 13.6 & 10.8 & 0.0 & 05 & 0.3 & 0.2 & 37.1 & 44.3 & & 16.7 & 22.2 \\
\hline 282 & 19-Nov-05 & & & & & & & & & & & & & & \\
\hline 283 & $20-$ Nov-05 & & & & & & & & & & & & & & \\
\hline 284 & 21-Nov-05 & & 0.0 & 0.6 & 14.3 & 13.1 & 0.0 & 0.0 & 0.0 & 0.0 & 41.6 & 70.5 & 35.5 & 21.9 & 18.5 \\
\hline 285 & 22-Nov-05 & & 0.0 & 0.3 & 14.7 & 13.4 & 0.0 & 0.0 & 0.0 & 0.0 & 32.2 & 57.1 & 29.8 & 14.6 & 17.6 \\
\hline 286 & $23-N o v-05$ & & 0.0 & 0.5 & 12.2 & 9.3 & 0.0 & 0.3 & 0.6 & 0.0 & 38.6 & 56.2 & 34.6 & 22.2 & 295 \\
\hline 287 & $24-N o v-05$ & & 0.0 & 9.7 & 16.0 & 12. & 0.0 & 0.3 & 0.0 & 0.0 & 56.3 & 25.9 & 33.1 & 21.2 & 17.8 \\
\hline 288 & $25-N o v-05$ & & & & & 12.7 & 0.0 & 0.0 & 0.0 & 0.2 & 557 & & & & \\
\hline 289 & $26-N o v-05$ & & & & & & & & & & & & & & \\
\hline 290 & $27-N o v-05$ & & & & & & & & & & & & & & \\
\hline 291 & 28-Nov-05 & & & & & & & & & & 56.0 & & & & \\
\hline \multicolumn{2}{|c|}{ Batch Average } & 18 & 0.0 & 3.3 & 14.8 & 13.8 & 0.0 & 0.1 & 0.1 & 0.0 & 39.1 & 59.0 & 31.4 & 18.9 & 18.0 \\
\hline 292 & \begin{tabular}{|l|}
$29-N o v-05$ \\
\end{tabular} & & & & & & & & & & 579 & & & & \\
\hline 293 & 30-Nov-05 & & 0.0 & 0.3 & 10.6 & 10.0 & 0.0 & 0.0 & 0.0 & 0.0 & 51.9 & 60.1 & 356 & 22.8 & 303 \\
\hline 294 & 1-Dec-05 & & 0.0 & 1.8 & 12.0 & 112 & 0.0 & 0.0 & 0.0 & 0.0 & 29.3 & 50.2 & 21.0 & 7.4 & 5.6 \\
\hline
\end{tabular}




\section{B-3 Conv NOX / P}

\begin{tabular}{|c|c|c|c|c|c|c|c|c|c|c|c|c|c|c|c|}
\hline 295 & 2-Dec-05 & \multirow{18}{*}{$\frac{\sigma}{\frac{\sigma}{\sigma}}$} & 0.0 & 2.5 & 14.5 & 12.7 & 0.0 & 0.2 & 0.0 & 0.0 & 35.7 & 52.6 & 21.6 & 8.3 & 6.2 \\
\hline 296 & 3-Dec-05 & & & & & & & & & & & & & & \\
\hline 297 & 4-Dec-05 & & & & & & & & & & & & & & \\
\hline 298 & 5-Dec-05 & & 0.0 & 6.4 & 18.9 & 18.6 & 0.0 & 0.4 & 0.0 & 0.0 & 35.4 & 45.0 & 24.0 & 14.2 & 10.8 \\
\hline 299 & 6-Dec-05 & & 0.0 & 5.6 & 16.9 & 18.9 & 0.0 & 0.0 & 0.0 & 0.0 & 41.9 & 50.5 & 28.6 & 17.0 & 14.8 \\
\hline 300 & 7-Dec-05 & & & & & & & & & & 44.4 & 51.0 & 19.0 & 6.4 & 2.7 \\
\hline 301 & 8-Dec-05 & & & 1.4 & & & 0.0 & $\overline{0.0}$ & 0.2 & 0.0 & 36.9 & 39.1 & 12.7 & 5.8 & 5.8 \\
\hline 302 & 9-Dec-05 & & 0.0 & 0.3 & 11.8 & 10.2 & 0.0 & 0.0 & 0.0 & 0.0 & 33.7 & 55.7 & 25.3 & 7.0 & 6.7 \\
\hline 303 & 10-Dec-05 & & & & & & & & & & & & & & \\
\hline 304 & 11-Dec-05 & & & & & & & & & & & & & & \\
\hline 305 & 12-Dec-05 & & & & & & & & & & & & & & \\
\hline 306 & 13-Dec-05 & & 0.0 & 3.8 & 16.1 & 10.7 & 0.0 & 0.0 & 0.5 & 0.0 & 33.4 & 41.3 & 21.8 & 4.2 & 10.8 \\
\hline 307 & 14-Dec-05 & & 0.0 & 6.1 & 5.8 & 11.0 & 0.0 & 0.0 & 0.0 & 0.0 & 39.1 & 43.8 & 11.4 & 9.9 & 5.2 \\
\hline 308 & 15-Dec-05 & & 0.0 & 0.3 & 12.4 & $\overline{11.2}$ & 0.0 & 0.0 & 0.0 & 0.0 & 41.0 & 903 & 23.7 & 13.3 & 5.2 \\
\hline 309 & 16-Dec-05 & & & & & & & & & & & & & & \\
\hline 310 & 17-Dec-05 & & & & & & & & & & & & & & \\
\hline 311 & 18-Dec-05 & & & & & & & & & & & & & & \\
\hline 312 & 19-Dec-05 & & & & & & & & & & & & & & \\
\hline Batcl & h Average & 19 & 0.0 & 2.8 & 13.2 & 12.7 & 0.0 & 0.0 & 0.0 & 0.0 & 38.4 & 48.9 & 20.9 & 9.4 & 7.4 \\
\hline 313 & 20 -Dec-05 & \multirow{14}{*}{$\frac{3}{9}$} & & & & & & & & & & & & & \\
\hline 314 & 21-Dec-05 & & & & & & & & & & & & & & \\
\hline 315 & 22 -Dec-05 & & & & & & & & & & & & & & \\
\hline 316 & 23-Dec-05 & & & & & & & & & & & & & & \\
\hline 317 & 24-Dec-05 & & & & & & & & & & & & & & \\
\hline 318 & 25-Dec-05 & & & & & & & & & & & E. & & & \\
\hline 319 & 26-Dec-05 & & & & & & & & & & & & & & \\
\hline 320 & 27-Dec-05 & & & & & & & & & T & & & & & \\
\hline 321 & $28-\mathrm{Dec}-05$ & & & & & & & & & & & & & & \\
\hline 322 & 29-Dec-05 & & & & & & & & & 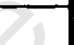 & & & & & \\
\hline 323 & 30-Dec-05 & & & & & & & & 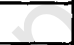 & & & & & & \\
\hline 324 & $31-\mathrm{Dec}-05$ & & & & & & & & & & & & & & \\
\hline 325 & 1-Jan-06 & & & & & & & & - & & & & & & \\
\hline 326 & $2-\operatorname{Jan}-06$ & & & & & & & & & & & & & & \\
\hline & & 26. & & & & & D & & & & & & & & \\
\hline 327 & 3-Jan-06 & \multirow{15}{*}{$\frac{\sqrt{0}}{\frac{6}{6}}}$. & & & & & 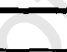 & & & & & & & & \\
\hline 328 & 4-Jan-06 & & & & & & C & & & & & & & & \\
\hline 329 & 5-Jan-06 & & & & & - & & & & & & & & & \\
\hline 330 & 6-Jan-06 & & & & & - & & & & & & & & & \\
\hline 331 & $7-\operatorname{Jan}-06$ & & & & C & 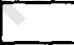 & & & & & & & & & \\
\hline 332 & 8-Jan-06 & & & & & & & & & & & & & & \\
\hline 333 & 9-Jan-06 & & & & & & & & & & & & & & \\
\hline 334 & 10-Jan-06 & & & & & & & & & & & & & & \\
\hline 335 & 11-Jan-06 & & & & & & & & & & & & & & \\
\hline 336 & 12-Jan-06 & & & & & & & & & & & & & & \\
\hline 337 & 13-Jan-06 & & & & & & & & & & & & & & \\
\hline 338 & 14-Jan-06 & & & & & & & & & & & & & & \\
\hline 339 & 15-Jan-06 & & & & & & & & & & & & & & \\
\hline 340 & 16-Jan-06 & & & & & & & & & & & & & & \\
\hline 341 & 17-Jan-06 & & & & & & & & & & & & & & \\
\hline & & 21 & & & & & & & & & & & & & \\
\hline 342 & 18-Jan-06 & \multirow{10}{*}{. } & & & & & & & & & & & & & \\
\hline 343 & 19-Jan-06 & & & & & & & & & & & & & & \\
\hline 344 & $20-\operatorname{Jan}-06$ & & 0.0 & 1.7 & 18.0 & 15.7 & 0.0 & 0.7 & 0.0 & 0.0 & 36.2 & 47.0 & 31.1 & 12.6 & 12.0 \\
\hline 345 & 21-Jan-06 & & & & & & & & & & & & & & \\
\hline 346 & $22-\operatorname{Jan}-06$ & & & & & & & & & & & & & & \\
\hline 347 & 23-Jan-06 & & 0.0 & 0.8 & 21.4 & 19.7 & 0.0 & 0.0 & 0.0 & 0.0 & 32.4 & 41.0 & 17.4 & 12.5 & 8.6 \\
\hline 348 & 24-Jan-06 & & 0.0 & 0.0 & 23.9 & 25.1 & 0.0 & 0.0 & 0.0 & 0.0 & 32.0 & 42.5 & 30.4 & 14.2 & 9.8 \\
\hline 349 & $25-\operatorname{Jan}-06$ & & 0.0 & 1.6 & 22.0 & 21.9 & 0.0 & 0.7 & 0.0 & 2.6 & 32.4 & 65.8 & 30.1 & 10.7 & 11.0 \\
\hline 350 & $26-\operatorname{Jan}-06$ & & 0.0 & 1.0 & 18.4 & 20.5 & 0.0 & 0.7 & 0.0 & 0.0 & 30.1 & 36.2 & 16.8 & 5.6 & 7.8 \\
\hline 351 & $27-J a n-06$ & & 0.0 & 0.5 & & & 0.0 & 0.0 & 0.0 & 0.0 & & 49.5 & 19.3 & 7.2 & 7.5 \\
\hline
\end{tabular}




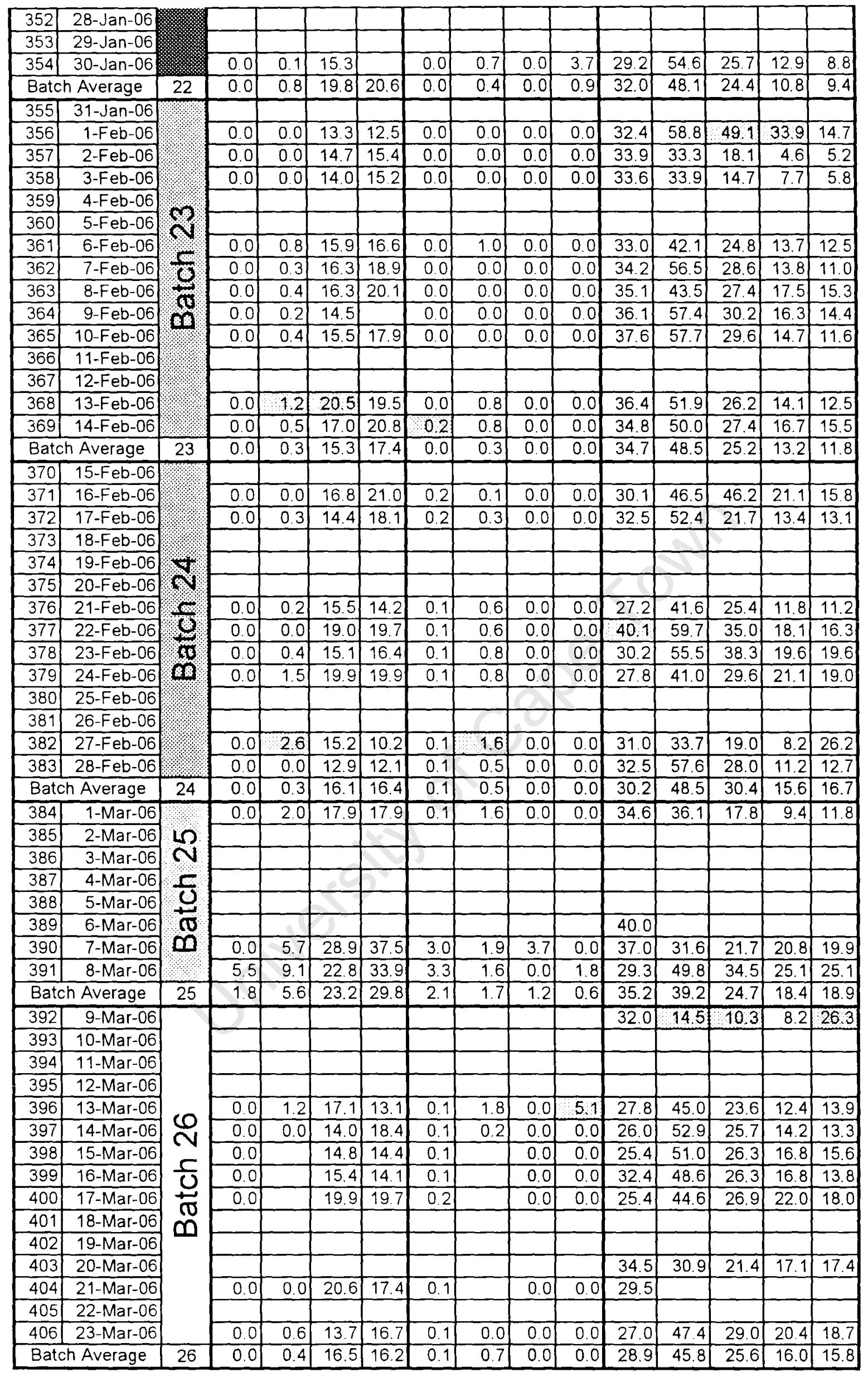




\begin{tabular}{|c|c|c|c|c|c|c|c|c|c|c|c|c|c|c|c|}
\hline 407 & 24-Mar-06 & \multirow{15}{*}{$\begin{array}{c}\frac{N}{U} \\
\frac{\sigma}{\pi} \\
\infty\end{array}$} & 0.0 & 2.0 & 17.7 & 15.7 & 0.1 & 1.8 & 0.0 & 0.0 & 29.3 & 45.4 & 28.7 & 22.4 & 20.4 \\
\hline 408 & 25-Mar-06 & & & & & & & & & & & & & & \\
\hline 409 & 26-Mar-06 & & & & & & & & & & & & & & \\
\hline 410 & 27-Mar-06 & & 0.0 & 2.6 & 21.2 & 18.7 & 0.1 & 2.7 & 0.0 & 0.0 & 33.9 & 29.9 & 24.7 & 23.3 & 22.4 \\
\hline 411 & 28-Mar-06 & & 0.0 & 5.1 & 18.3 & 11.4 & 0.1 & 27 & 1.5 & 3.1 & 29.6 & 35.1 & 25.3 & 21.8 & 28.4 \\
\hline 412 & 29-Mar-06 & & & & & & & & & & & & & & \\
\hline 413 & 30-Mar-06 & & 0.0 & 0.4 & 14.7 & 18.5 & 0.1 & 02 & 0.8 & 0.8 & 25.4 & 44.3 & 27.0 & 18.4 & 19.7 \\
\hline 414 & 31-Mar-06 & & 0.0 & 1.6 & 18.2 & 19.8 & 0.1 & 1.4 & 0.8 & 0.7 & 27.7 & 43.3 & 27.3 & 20.0 & 19.0 \\
\hline 415 & 1-Apr-06 & & 0.0 & 3.3 & 18.5 & 22.1 & 0.1 & 2.2 & 1.0 & 1.5 & 27.7 & 46.9 & 32.1 & 27.0 & 22.9 \\
\hline 416 & 2-Apr-06 & & 0.0 & 4.4 & 20.5 & 24.7 & 0.1 & 2.9 & 1.0 & 1.0 & 30.5 & 46.2 & 32.8 & 27.3 & 23.2 \\
\hline 417 & 3-Apr-06 & & 0.0 & 6.5 & 25.4 & 25.6 & 0.1 & 3.6 & 1.0 & 3.6 & 27.7 & 42.4 & 27.3 & 22.9 & 22.2 \\
\hline 418 & 4-Apr-06 & & 01 & 4.2 & 23.2 & 23.2 & 02 & 2.9 & 1.0 & 1.5 & 31.8 & 40.8 & 29.6 & 22.9 & 21.3 \\
\hline 419 & 5-Apr-06 & & & & & & & & & & 24.5 & 41.6 & 27.8 & & 21.1 \\
\hline 420 & 6-Apr-06 & & & & & & & & & & & & & & \\
\hline 421 & 7-Apr-06 & & & & & & & & & & 32.4 & 31.5 & 440 & 23.3 & 22.0 \\
\hline \multicolumn{2}{|c|}{ Batch Average } & 27 & 0.0 & 3.3 & 19.7 & 20.0 & 0.1 & 2.5 & 0.8 & 1.4 & 29.1 & 40.7 & 28.3 & 22.9 & 21.4 \\
\hline \multicolumn{2}{|c|}{\begin{tabular}{l|l|}
422 & $8-A p r-06$ \\
\end{tabular}} & \multirow{15}{*}{$\frac{8}{9}$} & & & & & & & & & & & & & \\
\hline \multicolumn{2}{|c|}{\begin{tabular}{|l|l|}
423 & $9-A p r-06$ \\
\end{tabular}} & & & & & & & & & & & & & & \\
\hline 424 & 10-Apr-06 & & 0.0 & 19 & 330 & 28.6 & 0.2 & 11 & 58 & 6.7 & 28.2 & 28.2 & 15.9 & 8.0 & 117 \\
\hline \multirow{2}{*}{\begin{tabular}{l|}
425 \\
426 \\
\end{tabular}} & 11-Apr-06 & & 0.0 & 0.7 & 24.7 & 325 & 0.1 & 0.6 & 0.7 & 3.3 & 14.4 & 22.7 & 10.4 & 5.9 & 7.4 \\
\hline & 12-Apr-06 & & 0.0 & 0.0 & 15.7 & 18.8 & 0.1 & 0.1 & 0.0 & 0.0 & 17.7 & 20.8 & 7.0 & 2.0 & 1.3 \\
\hline 427 & 13-Apr-06 & & 0.0 & 0.0 & 16.0 & 17.8 & 0.1 & 0.3 & 0.0 & 0.0 & & 20.1 & 6.3 & 1.3 & 0.3 \\
\hline \multirow{2}{*}{$\begin{array}{l}428 \\
429\end{array}$} & 14-Apr-06 & & & & & & & & & & 18.4 & & & & \\
\hline & 15-Apr-06 & & 0.0 & 0.0 & 16.9 & 17.4 & 0.1 & 0.3 & 0.0 & 0.0 & 18.1 & 22.4 & 10.0 & 2.0 & 0.3 \\
\hline \multirow{2}{*}{\begin{tabular}{|l|}
430 \\
431
\end{tabular}} & 16-Apr-06 & & & & & & & & & & 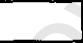 & +2 & & & \\
\hline & 17-Apr-06 & & & & & & & & & & & & & & \\
\hline $\begin{array}{ll}431 \\
132\end{array}$ & 18-Apr-06 & & 0.0 & 0.0 & 17.7 & 20.7 & 0.3 & 0.1 & 0.0 & 0.0 & 25.6 & 20.7 & 8.5 & 1.6 & 2.1 \\
\hline 433 & 19-Apr-06 & & 0.0 & 0.0 & 18.5 & 15.2 & 0.1 & 0.1 & 0.0 & 0.0 & 19.5 & 23.9 & 16.1 & 5.3 & 2.4 \\
\hline \multirow{2}{*}{\begin{tabular}{l|}
434 \\
435
\end{tabular}} & 20-Apr-06 & & 0.0 & 0.0 & 15.8 & 16.4 & 0.1 & 0.6 & 0.0 & 0.0 & 20.1 & 27.4 & 7.1 & 1.6 & 1.9 \\
\hline & 21-Apr-06 & & 0.0 & 0.0 & 17.9 & 16.8 & 0.0 & 0.1 & 0.0 & 0.0 & 23.9 & 25.6 & 13.2 & 1.9 & 1.0 \\
\hline 436 & 22-Apr-06 & & & & & & & & & & & & & & \\
\hline \multicolumn{2}{|c|}{ Batch Average } & 28 & 0.0 & 0.1 & 17.9 & 19.0 & 0.1 & 0.3 & 0.1 & 1.1 & 20.7 & 23.5 & 10.5 & 3.3 & 2.1 \\
\hline 437 & 23-Apr-06 & \multirow{10}{*}{$\frac{8}{4}$} & & & & & $\Leftrightarrow$ & 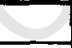 & & & & & & & \\
\hline 438 & 24-Apr-06 & & & & & 10.9 & - & & & & 9.1 & & & & 2.3 \\
\hline 439 & 25-Apr-06 & & 0.0 & 0.0 & 4.8 & 4.7 & 0.1 & 0.1 & & 0.9 & 10.3 & 38.3 & 16.5 & 2.9 & 1.7 \\
\hline 440 & 26-Apr-06 & & 0.0 & 0.0 & 7.9 & 7.4 & 0.0 & 0.1 & & 0.0 & 27.8 & 35.2 & 17.1 & 5.4 & 2.3 \\
\hline 441 & 27-Apr-06 & & & & & 12.2 & & & & 0.7 & 18.7 & & & & 3.2 \\
\hline 442 & 28-Apr-06 & & 0.0 & 0.2 & 12.7 & 14.3 & 0.1 & 1.0 & 1.2 & 0.1 & 34.9 & 49.0 & 25.1 & 13.7 & 7.8 \\
\hline 443 & 29-Apr-06 & & & & 0 & & & & & & 45.8 & & & & \\
\hline 444 & 30-Apr-06 & & 0.0 & 0.5 & 10.8 & 14.8 & 0.1 & 1.6 & 0.8 & 0.4 & 36.3 & 33.0 & 25.1 & 22.0 & 22.6 \\
\hline \multirow{2}{*}{$\frac{445}{446}$} & 1-May-06 & & 0.0 & 0.0 & 11.5 & 7.0 & 0.1 & 0.9 & 0.5 & 21 & 35.1 & 51.2 & 38.1 & 28.1 & 26.0 \\
\hline & 2-May-06 & & & 1 & & & & & & & & & & & \\
\hline \multirow{2}{*}{\begin{tabular}{ll|}
447 \\
448
\end{tabular}} & 3-May-06 & & 0.0 & 0.0 & 8.1 & 8.7 & 0.0 & 0.2 & 0.6 & 0.8 & 36.0 & 53.1 & 29.6 & 21.7 & 20.5 \\
\hline & 4-May-06 & & $\square$ & & & & & & & & & & & & \\
\hline$\frac{440}{449}$ & 5-May-06 & & 0.0 & 11 & 13.2 & 12.7 & 0.1 & 2.5 & 0.7 & 1.1 & 37.8 & 34.8 & 25.4 & 22.0 & 23.2 \\
\hline Batc & h Average & 29 & 0.0 & 0.1 & 9.9 & 10.3 & 0.1 & 0.9 & 0.7 & 0.6 & 29.2 & 42.1 & 25.2 & 16.5 & 12.2 \\
\hline
\end{tabular}


Appendix B-4

Miscellancous measurements on the conventional UCT system

\begin{tabular}{|c|c|c|c|c|c|c|c|c|c|c|c|c|c|}
\hline \multirow[t]{2}{*}{ Day } & Date & SB\# & \multicolumn{2}{|c|}{\begin{tabular}{|l|l|} 
msrd. & adj. \\
\end{tabular}} & Qi & Qw & $\mathrm{mgO} / \mathrm{l} / \mathrm{hr}$ & \multicolumn{3}{|r|}{ s } & AN & $A X$ & $\overline{A E}$ \\
\hline & $\cdot$ & - & \multicolumn{2}{|c|}{$(\mathrm{mm} / \mathrm{gTSS} / \mathrm{L})$} & \multicolumn{2}{|c|}{ (I) } & (mgO/L-h) & & . & & \multicolumn{3}{|c|}{ (1) } \\
\hline 21 & 3-Mar-05 & \multirow{16}{*}{$\frac{1}{8}$} & & & & & & & & & 6.2 & 5.6 & 13.2 \\
\hline 22 & 4-Mar-05 & & & & & & & & & & 6.2 & 5.6 & 13.2 \\
\hline 23 & 5-Mar-05 & & & & 15 & 1.1 & & 2.0 & 1.0 & 1.0 & 6.2 & 5.6 & 13.2 \\
\hline 24 & 6-Mar-05 & & & & 15 & 1.1 & & 2.0 & 1.0 & 1.0 & 6.2 & 5.6 & 13.2 \\
\hline 25 & 7-Mar-05 & & & & 15 & 1.1 & & 2.0 & 1.0 & 1.0 & 6.2 & 5.6 & 13.2 \\
\hline 26 & 8-Mar-05 & & & & 15 & 1.1 & & 2.0 & 1.0 & 1.0 & 6.2 & 5.6 & 13.2 \\
\hline 27 & 9-Mar-05 & & & & 15 & 1.1 & 40.1 & 2.0 & 1.0 & 1.0 & 6.2 & 5.6 & 13.2 \\
\hline 28 & 10-Mar-05 & & & & 15 & 1.1 & 30.8 & 2.0 & 1.0 & 1.0 & 6.2 & 5.6 & 13.2 \\
\hline 29 & 11-Mar-05 & & & & 15 & 1.1 & & 2.0 & 1.0 & 1.0 & 6.2 & 5.6 & 13.2 \\
\hline 30 & 12-Mar-05 & & & & 15 & 1.1 & & 2.0 & 1.0 & 1.0 & 6.2 & 5.6 & 13.2 \\
\hline 31 & 13-Mar-05 & & & & 15 & 1.1 & & 2.0 & 1.0 & 1.0 & 6.2 & 5.6 & 13.2 \\
\hline 32 & 14-Mar-05 & & & & 15 & 1.1 & 30.5 & 2.0 & 1.0 & 1.0 & 6.2 & 5.6 & 13.2 \\
\hline 33 & 15-Mar-05 & & & & 15 & 1.1 & 21.6 & 2.0 & 1.0 & 1.0 & 6.2 & 5.6 & 13.2 \\
\hline 34 & 16-Mar-05 & & & & 15 & 1.1 & 21.0 & 2.0 & 1.0 & 1.0 & 6.2 & 5.6 & 13.2 \\
\hline 35 & 17-Mar-05 & & & & 15 & 1.1 & 20.6 & 2.0 & 1.0 & 1.0 & 6.2 & 5.6 & 13.2 \\
\hline 36 & 18-Mar-05 & & & & 15 & 1.1 & 21.7 & 2.0 & 1.0 & 1.0 & 6.2 & 5.6 & 13.2 \\
\hline Batc & h Average & 2 & & & 15.0 & 1.1 & 26.6 & 2.0 & 1.0 & 1.0 & 6.20 & 5.60 & 13.20 \\
\hline 37 & 19-Mar-05 & & & & 15 & 1.1 & & 2.0 & 1.0 & 1.0 & 6.2 & 5.6 & 13.2 \\
\hline 38 & 20-Mar-05 & & & & 15 & 1.1 & & 2.0 & 1.0 & 1.0 & 6.2 & 5.6 & 13.2 \\
\hline 39 & 21-Mar-05 & & & & 15 & 1.1 & & 2.0 & 1.0 & 1.0 & 6.2 & 5.6 & 13.2 \\
\hline 40 & 22-Mar-05 & & & & 15 & 1.1 & 55.4 & 2.0 & 1.0 & 1.0 & 6.2 & 5.6 & 13.2 \\
\hline 41 & 23-Mar-05 & & & & 15 & 1.1 & & 2.0 & 1.0 & 1.0 & 6.2 & 5.6 & 13.2 \\
\hline 42 & 24-Mar-05 & & & & 15 & 1.1 & & 2.0 & 1.0 & 1.0 & 6.2 & 5.6 & 13.2 \\
\hline 43 & 25-Mar-05 & $\infty$ & & & 15 & 1.1 & & 2.0 & 1.0 & 1.0 & 6.2 & 56 & 13.2 \\
\hline 44 & 26-Mar-05 & 1 & & & 15 & 1.1 & & 2.0 & 1.0 & 1.0 & 6.2 & 5.6 & 13.2 \\
\hline 45 & 27-Mar-05 & 3 & & & 15 & 1.1 & & 2.0 & 1.0 & 1.0 & 6.2 & 5.6 & 13.2 \\
\hline 46 & 28-Mar-05 & 0 & & & 15 & 1.1 & & 2.0 & 1.0 & 1.0 & 6.2 & 5.6 & 13.2 \\
\hline 47 & 29-Mar-05 & $\infty$ & & & 15 & 1.1 & & 20 & 1.0 & 1.0 & 6.2 & 5.6 & 13.2 \\
\hline 48 & 30-Mar-05 & & & & 15 & 1.1 & 0 & 2.0 & 1.0 & 1.0 & 6.2 & 5.6 & 13.2 \\
\hline 49 & 31-Mar-05 & & & & 15 & 1.1 & 33.7 & 2.0 & 1.0 & 1.0 & 6.2 & 5.6 & 13.2 \\
\hline 50 & 1-Apr-05 & & & & 15 & 1.1 & 32.6 & 2.0 & 1.0 & 1.0 & 6.2 & 5.6 & 13.2 \\
\hline 51 & 2-Apr-05 & & & & 15 & 1.1 & 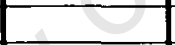 & 2.0 & 1.0 & 1.0 & 6.2 & 5.6 & 13.2 \\
\hline 52 & 3-Apr-05 & & & & 15 & 1.1 & & 2.0 & 1.0 & 1.0 & 6.2 & 5.6 & 13.2 \\
\hline 53 & 4-Apr-05 & & & & 15 & 1.1 & 41.5 & 2.0 & 1.0 & 1.0 & 6.2 & 5.6 & 13.2 \\
\hline Batc & h Average & 3 & & & 15.0 & 1.1 & 40.8 & 2.0 & 1.0 & 1.0 & 6.20 & 5.60 & 13.20 \\
\hline 54 & 5-Apr-05 & & 160.0 & 140.6 & 15 & 1.1 & 41.2 & 2.0 & 1.0 & 1.0 & 6.2 & 5.6 & 13.2 \\
\hline 55 & 6-Apr-05 & & & & 15 & 1.1 & & 2.0 & 1.0 & 1.0 & 6.2 & 5.6 & 13.2 \\
\hline 56 & 7-Apr-05 & & & & 15 & 1.1 & & 2.0 & 1.0 & 1.0 & 6.2 & 5.6 & 13.2 \\
\hline 57 & 8-Apr-05 & & & & 15 & 1.1 & & 2.0 & 1.0 & 1.0 & 6.2 & 5.6 & 13.2 \\
\hline 58 & 9-Apr-05 & & & & 15 & 1.1 & & 2.0 & 1.0 & 1.0 & 6.2 & 5.6 & 13.2 \\
\hline 59 & 10-Apr-05 & & & & 15 & 1.1 & & 2.0 & 1.0 & 1.0 & 6.2 & 5.6 & 13.2 \\
\hline 60 & 11-Apr-05 & & 150.0 & 123.7 & 15 & 1.1 & & 2.0 & 1.0 & 1.0 & 6.2 & 5.6 & 13.2 \\
\hline 61 & 12-Apr-05 & & 180.0 & 116.6 & 15 & 1.1 & 41.6 & 2.0 & 1.0 & 1.0 & 6.2 & 5.6 & 13.2 \\
\hline 62 & 13-Apr-05 & & 170.0 & 105.5 & 15 & 1.1 & 39.4 & 2.0 & 1.0 & 1.0 & 6.2 & 5.6 & 13.2 \\
\hline 63 & 14-Apr-05 & 8 & 165.0 & 97.7 & 15 & 1.1 & 39.4 & 2.0 & 1.0 & 1.0 & 6.2 & 5.6 & 13.2 \\
\hline 64 & 15-Apr-05 & $\frac{1}{4}$ & 160.0 & 71.4 & 15 & 1.1 & 48.0 & 2.0 & 1.0 & 1.0 & 6.2 & 5.6 & 13.2 \\
\hline 65 & 16-Apr-05 & $\frac{6}{6}$ & & & 15 & 1.1 & & 2.0 & 1.0 & 1.0 & 6.2 & 56 & $\overline{13.2}$ \\
\hline 66 & 17-Apr-05 & as & & & 15 & 1.1 & & 2.0 & 1.0 & 1.0 & 6.2 & 5.6 & 13.2 \\
\hline 67 & 18-Apr-05 & & 170.0 & 88.1 & 15 & 1.1 & 57.1 & 2.0 & 1.0 & 1.0 & 6.2 & 5.6 & 13.2 \\
\hline 68 & 19-Apr-05 & & 150.0 & 100.8 & 15 & 1.1 & 43.5 & 2.0 & 1.0 & 1.0 & 6.2 & 5.6 & 13.2 \\
\hline 69 & 20-Apr-05 & & 150.0 & 73.7 & 15 & 1.1 & 45.2 & 20 & 1.0 & 1.0 & 6.2 & 56 & 13.2 \\
\hline 70 & 21-Apr-05 & & 145.0 & 89.5 & 15 & 1.1 & 36.7 & 2.0 & 1.0 & 1.0 & 6.2 & 5.6 & 13.2 \\
\hline 71 & 22-Apr-05 & & 140.0 & 87.6 & 15 & 1.1 & 32.6 & 2.0 & 1.0 & 1.0 & 6.2 & 5.6 & 13.2 \\
\hline 72 & 23-Apr-05 & & & & 15 & 1.1 & & 2.0 & 1.0 & 1.0 & 6.2 & 5.6 & 13.2 \\
\hline 73 & 24-Apr-05 & & & & 15 & 1.1 & & 2.0 & 1.0 & 1.0 & 6.2 & 5.6 & 13.2 \\
\hline 74 & 25-Apr-05 & & 130.0 & 116.8 & 15 & 1.1 & 30.7 & 2.0 & 1.0 & 1.0 & 6.2 & 5.6 & 13.2 \\
\hline 75 & 26-Apr-05 & & 150.0 & 58.0 & 15 & 1.1 & & 2.0 & 1.0 & 1.0 & 6.2 & 5.6 & 13.2 \\
\hline Batc & h Average & 4 & & & 15.0 & 1.1 & 39.8 & 2.0 & 1.0 & 1.0 & 6.20 & 5.60 & 13.20 \\
\hline 76 & 27-Apr-05 & & & & 15 & 1.1 & & 2.0 & 1.0 & 1.0 & 6.2 & 5.6 & 13.2 \\
\hline 77 & 28-Apr-05 & & & & 15 & 1.1 & & 2.0 & 1.0 & 1.0 & 6.2 & 5.6 & 13.2 \\
\hline
\end{tabular}




\begin{tabular}{|c|c|c|c|c|c|c|c|c|c|c|c|c|c|}
\hline 78 & 29-Apr-05 & \multirow{17}{*}{$\frac{10}{\frac{\sigma}{\infty}}$} & & & 15 & 1.1 & & 2.0 & 1.0 & 1.0 & 6.2 & 5.6 & 13.2 \\
\hline 79 & 30-Apr-05 & & & & 15 & 1.1 & & 2.0 & 1.0 & 1.0 & 6.2 & 5.6 & 13.2 \\
\hline 80 & 1-May-05 & & & & 75 & $\overrightarrow{1.1}$ & & 2.0 & 1.0 & 1.0 & 6.2 & 5.6 & 13.2 \\
\hline 81 & 2-May-05 & & & & 15 & 1.1 & & 2.0 & 1.0 & 1.0 & 6.2 & 5.6 & 13.2 \\
\hline 82 & 3-May-05 & & & & 15 & 1.1 & & 2.0 & 1.0 & 1.0 & 6.2 & 5.6 & 13.2 \\
\hline 83 & 4-May-05 & & 140.0 & 71.6 & 15 & $\overrightarrow{1.1}$ & & 2.0 & 1.0 & 1.0 & 6.2 & 5.6 & 13.2 \\
\hline 84 & 5-May-05 & & 140.0 & 113.0 & 15 & 1.1 & & 2.0 & 1.0 & 1.0 & 6.2 & 5.6 & 13.2 \\
\hline 85 & 6-May-05 & & 135.0 & 59.4 & 15 & 1.1 & & 2.0 & 1.0 & 1.0 & 6.2 & 5.6 & 13.2 \\
\hline 86 & 7-May-05 & & & & 15 & 1.1 & & 20 & 1.0 & 1.0 & 6.2 & 5.6 & 13.2 \\
\hline 87 & 8-May-05 & & & & 15 & 1.1 & & 2.0 & 1.0 & 1.0 & 6.2 & 5.6 & 13.2 \\
\hline 88 & 9-May-05 & & 130.0 & 54.7 & 15 & 1.1 & 18.8 & 2.0 & 1.0 & 1.0 & 6.2 & 5.6 & 13.2 \\
\hline 89 & 10-May-05 & & 130.0 & 820 & 15 & 1.1 & & 2.0 & 1.0 & 1.0 & 6.2 & 5.6 & 132 \\
\hline 90 & 11-May-05 & & 130.0 & 61.0 & 15 & 1.1 & 24.3 & 2.0 & 1.0 & 1.0 & 6.2 & 5.6 & $\overline{13.2}$ \\
\hline 91 & 12-May-05 & & 130.0 & 53.2 & 15 & 1.1 & 20.5 & 2.0 & 1.0 & 1.0 & 6.2 & 5.6 & 13.2 \\
\hline 92 & 13-May-05 & & 135.0 & 74.3 & 15 & 1.1 & 20.4 & 2.0 & 1.0 & 1.0 & 6.2 & 5.6 & 13.2 \\
\hline 93 & 14-May-05 & & & & 15 & 1.1 & & 2.0 & 1.0 & 1.0 & 6.2 & 5.6 & 132 \\
\hline 94 & 15-May-05 & & & & 15 & 1.1 & & 2.0 & 1.0 & 1.0 & 6.2 & 5.6 & 13.2 \\
\hline Batc & h Average & 5 & & & 15.0 & 1.1 & 21.0 & 2.0 & 1.0 & 1.0 & 6.20 & 5.60 & 13.20 \\
\hline 95 & 16-May-05 & \multirow{16}{*}{$\begin{array}{c}0 \\
\frac{\sigma}{U} \\
\frac{1}{\sigma}\end{array}$} & & & 15 & 1.1 & & 2.0 & 1.0 & 1.0 & 6.2 & 5.6 & 13.2 \\
\hline 96 & 17-May-05 & & & & 15 & 1.1 & & 2.0 & 1.0 & 1.0 & 6.2 & 5.6 & 13.2 \\
\hline 97 & 18-May-05 & & & & 15 & 1.1 & & 2.0 & 1.0 & 1.0 & 6.2 & 5.6 & 13.2 \\
\hline 98 & 19-May-05 & & & & 15 & 1.1 & & 2.0 & 1.0 & 1.0 & 6.2 & 5.6 & 13.2 \\
\hline 99 & 20-May-05 & & & & 15 & 1.1 & & 2.0 & 1.0 & 1.0 & 6.2 & 5.6 & 13.2 \\
\hline 100 & 21-May-05 & & & & 15 & 1.1 & & 2.0 & 1.0 & 1.0 & 6.2 & 5.6 & 13.2 \\
\hline 101 & 22-May-05 & & & & 15 & 2.8 & & 2.0 & 1.0 & 1.0 & 6.2 & 5.6 & 13.2 \\
\hline 102 & 23-May-05 & & 110.0 & 85.1 & 15 & & & 2.0 & 1.0 & 1.0 & 6.2 & 5.6 & 13.2 \\
\hline 103 & 24-May-05 & & 100.0 & 76.6 & 15 & & 15.1 & 2.0 & 1.0 & 1.0 & 6.2 & 5.6 & 13.2 \\
\hline 104 & 25-May-05 & & 105.0 & 206.1 & 15 & & 15.3 & 2.0 & 1.0 & 1.0 & 6.2 & 5.6 & 13.2 \\
\hline 105 & 26-May-05 & & 110.0 & 52.7 & 15 & 1.1 & & 2.0 & 1.0 & 1.0 & 6.2 & 5.6 & 13.2 \\
\hline 106 & 27-May-05 & & 110.0 & 71.8 & 15 & 1.1 & 18.9 & 2.0 & 1.0 & 1.0 & 6.2 & 5.6 & 13.2 \\
\hline 107 & 28-May-05 & & & & 15 & & & 2.0 & 1.0 & 1.0 & 6.2 & 5.6 & 13.2 \\
\hline 108 & 29-May-05 & & & & 15 & 2.8 & & 2.0 & 1.0 & 1.0 & 6.2 & 5.6 & 13.2 \\
\hline 109 & 30-May-05 & & 100.0 & 130.5 & 15 & 0.5 & 25.0 & 2.0 & 1.0 & 1.0 & 6.2 & 5.6 & 13.2 \\
\hline 110 & 31-May-05 & & 90.0 & 54.9 & & 0.6 & $\Rightarrow$ & 0 & & & 6.2 & 5.6 & 13.2 \\
\hline Batc & h Average & 6 & & & 15.0 & 1.3 & 18.6 & 2.0 & 1.0 & 1.0 & 6.20 & 5.60 & 13.20 \\
\hline 111 & 1-Jun-05 & \multirow{14}{*}{$\frac{1}{6}$} & & & 15 & 1.1 & & 2.0 & 1.0 & 1.0 & 6.2 & 5.6 & 13.2 \\
\hline 112 & 2 -Jun-05 & & & & 15 & 1.1 & & 2.0 & 1.0 & 1.0 & 6.2 & 5.6 & 13.2 \\
\hline 113 & 3-Jun-05 & & 140.0 & 97.1 & 15 & 3.0 & 15.4 & 2.0 & 1.0 & 1.0 & 6.2 & 5.6 & 13.2 \\
\hline 114 & 4-Jun-05 & & & & 15 & & & 2.0 & 1.0 & 1.0 & 6.2 & 5.6 & 13.2 \\
\hline 115 & 5-Jun-05 & & & & 15 & 1.1 & & 2.0 & 1.0 & 1.0 & 6.2 & 5.6 & 13.2 \\
\hline 116 & 6-Jun-05 & & 100.0 & 48.6 & 15 & 1.1 & 21.1 & 2.0 & 1.0 & 1.0 & 6.2 & 5.6 & 13.2 \\
\hline 117 & 7 -Jun-05 & & 100.0 & 56.7 & 15 & 1.1 & 40.4 & 2.0 & 1.0 & 1.0 & 6.2 & 5.6 & 13.2 \\
\hline 118 & 8-Jun-05 & & 130.0 & 165.3 & 15 & 1.1 & 28.7 & 2.0 & 1.0 & 1.0 & 6.2 & 5.6 & 13.2 \\
\hline 119 & 9-Jun-05 & & 220.0 & 145.7 & 15 & 1.1 & 21.9 & 2.0 & 1.0 & 1.0 & 6.2 & 5.6 & 13.2 \\
\hline 120 & 10-Jun-05 & & 240.0 & 172.8 & 15 & 1.1 & 54.9 & 2.0 & 1.0 & 1.0 & 6.2 & 5.6 & 13.2 \\
\hline 121 & $11-$ Jun-05 & & & 此 & 15 & & & 2.0 & 1.0 & 1.0 & 6.2 & 5.6 & 13.2 \\
\hline 122 & 12-Jun-05 & & & (.). & 15 & & & 2.0 & 1.0 & 1.0 & 6.2 & 5.6 & 13.2 \\
\hline 123 & 13-Jun-05 & & 220.0 & 150.6 & 15 & 2.8 & 53.3 & 2.0 & 1.0 & 1.0 & 6.2 & 5.6 & 13.2 \\
\hline 124 & 14-Jun-05 & & - & & 15 & 1.1 & 24.5 & 2.0 & 1.0 & 1.0 & 6.2 & 56 & 13.2 \\
\hline Batc & h Average & 7 & & & 15.0 & 1.4 & 32.5 & 2.0 & 1.0 & 1.0 & 6.20 & 5.60 & 13.20 \\
\hline 125 & 15-Jun-05 & \multirow{8}{*}{$\frac{\infty}{4}$} & & & 15 & 1.1 & & 2.0 & 1.0 & 1.0 & 6.2 & 5.6 & 13.2 \\
\hline 126 & 16-Jun-05 & & & & 15 & 1.1 & & 2.0 & 1.0 & 1.0 & 6.2 & 5.6 & 13.2 \\
\hline 127 & 17-Jun-05 & & & & 15 & 2.8 & & 2.0 & 1.0 & 1.0 & 6.2 & 5.6 & 13.2 \\
\hline 128 & 18-Jun-05 & & & & 15 & 0.7 & & 2.0 & 1.0 & 1.0 & 6.2 & 5.6 & 13.2 \\
\hline 129 & 19-Jun-05 & & & & 15 & 1.1 & & 2.0 & 1.0 & 1.0 & 6.2 & 5.6 & 13.2 \\
\hline 130 & $20-$ Jun-05 & & 120.0 & 78.4 & 15 & 1.1 & 25.1 & 2.0 & 1.0 & 1.0 & 6.2 & 5.6 & 13.2 \\
\hline 131 & $21-J u n-05$ & & 125.0 & 115.9 & 15 & 1.1 & 23.5 & 2.0 & 1.0 & 1.0 & 6.2 & 5.6 & 13.2 \\
\hline 132 & \begin{tabular}{|l|}
$22-J u n-05$ \\
\end{tabular} & & 130.0 & 72.0 & 15 & & 28.3 & 2.0 & 1.0 & 1.0 & 6.2 & 5.6 & 13.2 \\
\hline Batc & Average & 8 & & & 15.0 & 1.3 & 25.6 & 2.0 & 1.0 & 1.0 & 6.20 & 5.60 & 13.20 \\
\hline 133 & $23-J u n-05$ & \multirow{8}{*}{$\begin{array}{l}\sigma \\
\frac{5}{0}\end{array}$} & & & 15 & 2.8 & & 2.0 & 1.0 & 1.0 & 6.2 & 56 & 13.2 \\
\hline 134 & $24-J u n-05$ & & 135.0 & 147.2 & 15 & 0.5 & 30.0 & 2.0 & 1.0 & 1.0 & 5.6 & 6.2 & 13.2 \\
\hline 135 & $25-J u n-05$ & & & & 15 & 1.1 & & 2.0 & 1.0 & 1.0 & 5.6 & 6.2 & 13.2 \\
\hline 136 & $26-J u n-05$ & & & & 15 & 1.1 & & 2.0 & 1.0 & 1.0 & 5.6 & 6.2 & 13.2 \\
\hline 137 & $27-J u n-05$ & & 130.0 & 141.4 & 15 & 1.1 & 28.5 & 2.0 & 1.0 & 1.0 & 5.6 & 6.2 & 13.2 \\
\hline 138 & 28 -Jun-05 & & 130.0 & 144.3 & 15 & 1.1 & 24.7 & 2.0 & 1.0 & 1.0 & 5.6 & 6.2 & 13.2 \\
\hline 139 & 29-Jun-05 & & 150.0 & 120.3 & 15 & 3.3 & 22.9 & 2.0 & 1.0 & 1.0 & 5.6 & 6.2 & 13.2 \\
\hline 140 & 30-Jun-05 & & 160.0 & 128.2 & 15 & & 31.9 & 2.0 & 1.0 & 1.0 & 5.6 & 6.2 & 13.2 \\
\hline
\end{tabular}




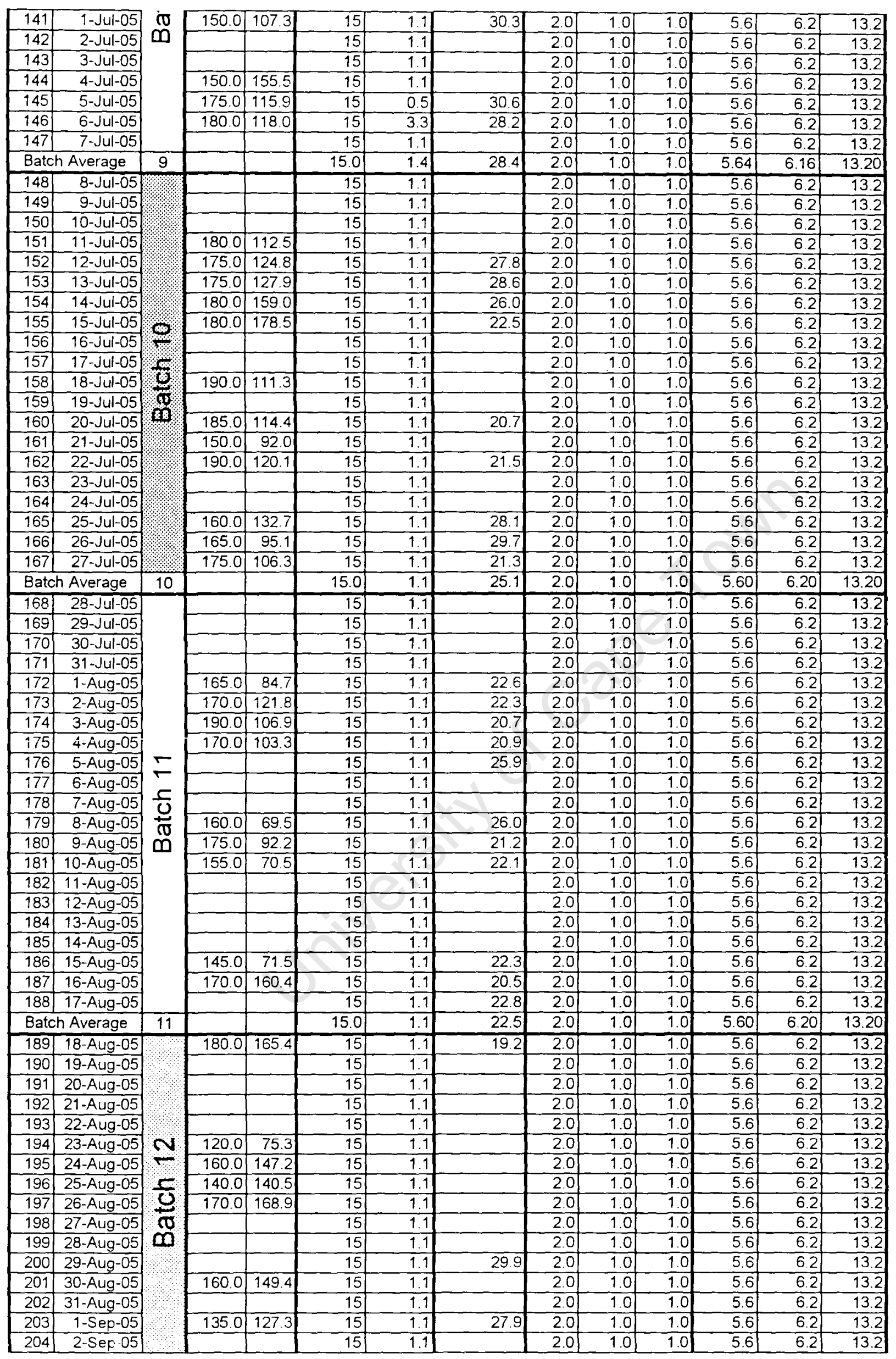




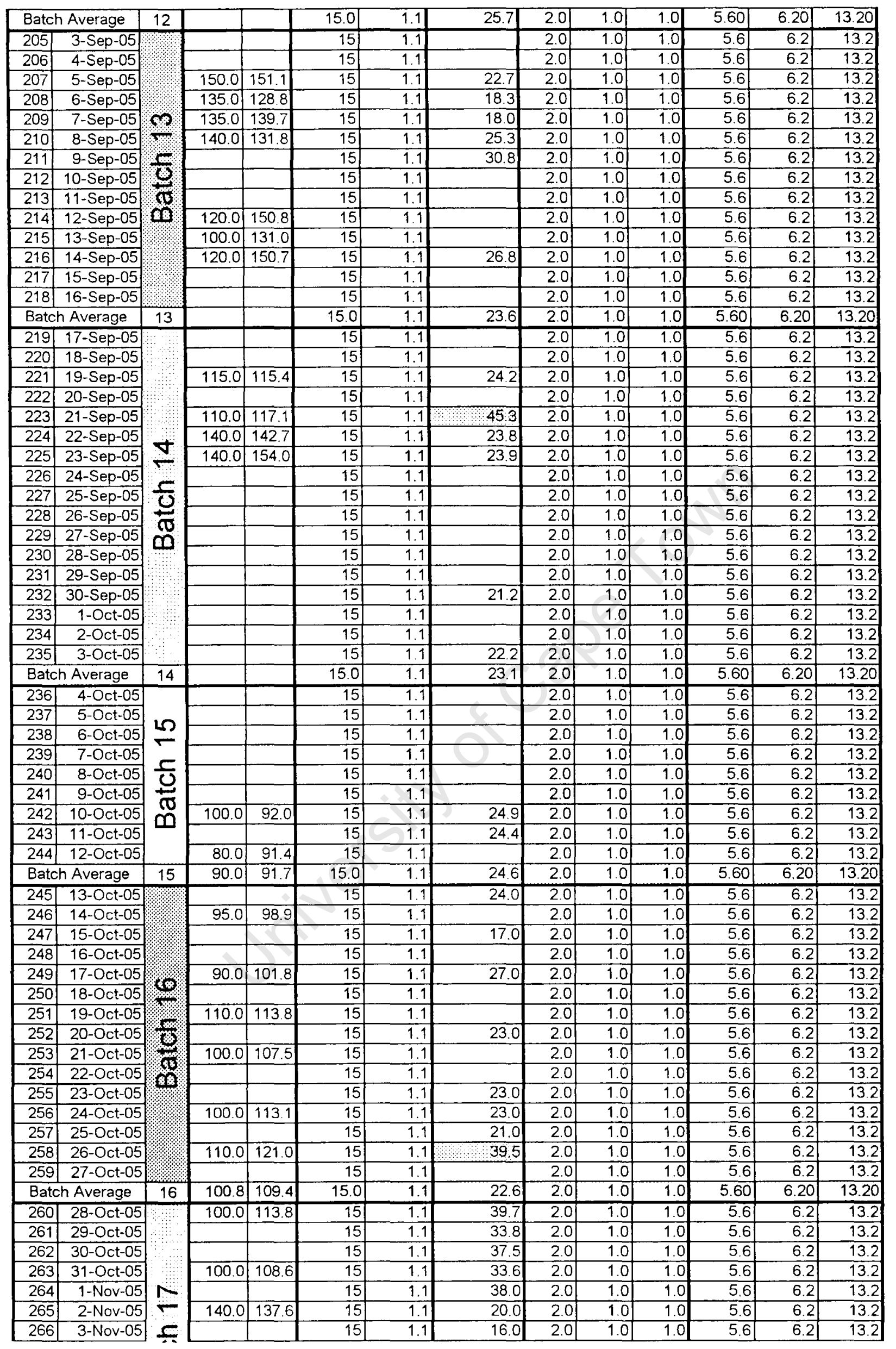




\begin{tabular}{|c|c|c|c|c|c|c|c|c|c|c|c|c|c|}
\hline 267 & 4-Nov-05 & \multirow{7}{*}{$\begin{array}{c}\frac{U}{\pi} \\
\frac{\pi}{0}\end{array}$} & 140.0 & 134.4 & 15 & 1.1 & & 2.0 & 1.0 & 10 & 5.6 & 6.2 & 13.2 \\
\hline 268 & 5-Nov-05 & & & & 15 & 1.1 & & 20 & 1.0 & 1.0 & 5.6 & 6.2 & 13.2 \\
\hline 269 & 6-Nov-05 & & & & 15 & 1.1 & & 2.0 & 1.0 & 1.0 & 5.6 & 62 & 13.2 \\
\hline 270 & $7-$ Nov-05 & & 130.0 & 136.8 & 15 & 1.1 & 14.0 & 2.0 & 1.0 & 1.0 & 5.6 & 6.2 & 13.2 \\
\hline 271 & $8-N o v-05$ & & & & 15 & 1.1 & & 2.0 & 1.0 & 1.0 & 5.6 & 6.2 & 13.2 \\
\hline 272 & 9-Nov-05 & & 130.0 & 147.4 & 15 & 1.1 & 23.0 & 2.0 & 1.0 & 1.0 & 5.6 & 6.2 & 13.2 \\
\hline 273 & 10-Nov-05 & & & & 15 & 1.1 & & 2.0 & 1.0 & 1.0 & 5.6 & 6.2 & 13.2 \\
\hline Batch & Average & 17 & 123.3 & 129.8 & 15.0 & 1.1 & 28.4 & 20 & 1.0 & 1.0 & 5.60 & 6.20 & 13.20 \\
\hline 274 & 11-Nov-05 & \multirow{18}{*}{$\frac{\infty}{\frac{\sigma}{\sigma}}$} & 145.0 & 151.1 & 15 & 1.1 & 24.0 & 2.0 & 1.0 & 1.0 & 5.6 & 6.2 & 13.2 \\
\hline 275 & 12-Nov-05 & & & & & & & & & & 5.6 & 6.2 & 13.2 \\
\hline 276 & 13-Nov-05 & & & & & & & & & & 5.6 & 6.2 & 13.2 \\
\hline 277 & 14-Nov-05 & & & & 15 & 1.1 & & 2.0 & 1.0 & 1.0 & 5.6 & 6.2 & 13.2 \\
\hline 278 & 15-Nov-05 & & 1200 & 1166 & 15 & 1.1 & 20.0 & 2.0 & 1.0 & 1.0 & 5.6 & 6.2 & 13.2 \\
\hline 279 & 16-Nov-05 & & 1300 & 145.0 & 15 & 1.1 & 25.0 & 2.0 & 1.0 & 1.0 & 5.6 & 6.2 & 132 \\
\hline 280 & 17-Nov-05 & & & & 15 & 1.1 & 38.0 & 2.0 & 1.0 & 10 & 5.6 & 6.2 & 13.2 \\
\hline 281 & 18-Nov-05 & & 110.0 & 141.9 & 15 & 1.1 & 24.0 & 2.0 & 1.0 & 1.0 & 5.6 & 6.2 & 13.2 \\
\hline 282 & 19-Nov-05 & & & & 15 & 1.1 & & 2.0 & 1.0 & 1.0 & 5.6 & 6.2 & 13.2 \\
\hline 283 & 20-Nov-05 & & & & & & & & & & 5.6 & 6.2 & 13.2 \\
\hline 284 & 21-Nov-05 & & 110.0 & 126.3 & 15 & 1.1 & 16.0 & 2.0 & 1.0 & 1.0 & 5.6 & 6.2 & 13.2 \\
\hline 285 & 22-Nov-05 & & 135.0 & 137.8 & 15 & 1.1 & 21.0 & 2.0 & 1.0 & 1.0 & 5.6 & 6.2 & 13.2 \\
\hline 286 & 23-Nov-05 & & 110.0 & & 15 & 1.1 & 18.4 & 2.0 & 1.0 & 1.0 & 5.6 & 6.2 & 13.2 \\
\hline 287 & 24-Nov-05 & & 130.0 & & & & 19.3 & & & & 5.6 & 6.2 & 13.2 \\
\hline 288 & 25-Nov-05 & & 130.0 & & & & 24.1 & & & & 5.6 & 6.2 & 13.2 \\
\hline 289 & $26-$ Nov-05 & & & & & & & & & & 5.6 & 6.2 & 13.2 \\
\hline 290 & 27-Nov-05 & & & & & & & & & & 5.6 & 6.2 & 13.2 \\
\hline 291 & 28-Nov-05 & & & & & & & & & & 5.6 & 6.2 & 13.2 \\
\hline Batc & h Average & 18 & 124.4 & 136.5 & 15.0 & 1.1 & 21.3 & 2.0 & 1.0 & 1.0 & 5.60 & 6.20 & 13.20 \\
\hline 292 & 29-Nov-05 & \multirow{21}{*}{$\frac{\sigma}{\sigma}$} & & & 15 & 1.1 & 20.4 & 2.0 & 1.0 & 1.0 & 5.6 & 6.2 & 13.2 \\
\hline 293 & $30-N o v-05$ & & 140.0 & 127.2 & 15 & 1.1 & 20.2 & 2.0 & 1.0 & 1.0 & 5.6 & 6.2 & 13.2 \\
\hline 294 & 1-Dec-05 & & & & 15 & 1.1 & 21.2 & 2.0 & 1.0 & 1.0 & 5.6 & 6.2 & 13.2 \\
\hline 295 & 2-Dec-05 & & 115.0 & 102.5 & 15 & 1.1 & 22.3 & 2.0 & 1.0 & 1.0 & 5.6 & 6.2 & 13.2 \\
\hline 296 & 3-Dec-05 & & & & 15 & 1.1 & & 2.0 & 1.0 & 1.0 & 5.6 & 6.2 & 13.2 \\
\hline 297 & 4-Dec-05 & & & & 15 & 1.1 & & 2.0 & 1.0 & 1.0 & 5.6 & 6.2 & 13.2 \\
\hline 298 & 5-Dec-05 & & 115.0 & 106.6 & 15 & 1.1 & 25.4 & 20 & 1.0 & 1.0 & 5.6 & 6.2 & 13.2 \\
\hline 299 & 6-Dec-05 & & & & 15 & 1.1 & 28.7 & 2.0 & 1.0 & 1.0 & 5.6 & 6.2 & 13.2 \\
\hline 300 & 7-Dec-05 & & & & 15 & 1.1 & 35.8 & 2.0 & 1.0 & 1.0 & 5.6 & 6.2 & 13.2 \\
\hline 301 & 8-Dec-05 & & 100.0 & 108.0 & 15 & 1.1 & 22.9 & 2.0 & 1.0 & 1.0 & 5.6 & 6.2 & 13.2 \\
\hline 302 & 9-Dec-05 & & & & 15 & 1.1 & 20.1 & 2.0 & 1.0 & 1.0 & 5.6 & 6.2 & 13.2 \\
\hline 303 & 10-Dec-05 & & & & 15 & 1.1 & 3 & 2.0 & 1.0 & 1.0 & 5.6 & 6.2 & 13.2 \\
\hline 304 & 11-Dec-05 & & & & 15 & 1.1 & 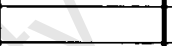 & 2.0 & 1.0 & 1.0 & 5.6 & 6.2 & 13.2 \\
\hline 305 & 12-Dec-05 & & & & 15 & 1.1 & 24.4 & 2.0 & 1.0 & 1.0 & 5.6 & 6.2 & 13.2 \\
\hline 306 & 13-Dec-05 & & 100.0 & 93.7 & 15 & 1.1 & 34.0 & 2.0 & 1.0 & 1.0 & 5.6 & 6.2 & 13.2 \\
\hline 307 & 14-Dec-05 & & & & 15 & 1.1 & 32.2 & 20 & 10 & 1.0 & 5.6 & 6.2 & 13.2 \\
\hline 308 & 15-Dec-05 & & & & 15 & 1.1 & 28.2 & 2.0 & 1.0 & 1.0 & 5.6 & 6.2 & 13.2 \\
\hline 309 & 16-Dec-05 & & & & + & 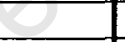 & & & & & 5.6 & 6.2 & 13.2 \\
\hline 310 & 17-Dec-05 & & & & + & & & & & & 5.6 & 6.2 & 13.2 \\
\hline 311 & 18-Dec-05 & & & & 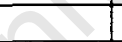 & & & & & & 5.6 & 6.2 & 13.2 \\
\hline 312 & 19-Dec-05 & & & & & & & & & & 5.6 & 6.2 & 13.2 \\
\hline \multicolumn{2}{|c|}{ Batch Average } & 19 & 114.0 & 107.6 & 15.0 & 1.1 & 25.8 & 2.0 & 1.0 & 1.0 & 5.60 & 6.20 & 13.20 \\
\hline \multicolumn{2}{|c|}{313,20 -Dec-05 } & \multirow{14}{*}{$\frac{6}{6} \frac{1}{6}$} & & & & & & & & & & & \\
\hline \multicolumn{2}{|c|}{\begin{tabular}{l|l}
314 & $21-$ Dec-05 \\
\end{tabular}} & & & & & & & & & & & & \\
\hline \multicolumn{13}{|c|}{\begin{tabular}{l|l|}
315 & $22-D e c-05$ \\
\end{tabular}} & \\
\hline \multicolumn{2}{|c|}{\begin{tabular}{l|l|}
316 & $23-\mathrm{Dec}-05$ \\
\end{tabular}} & & & & & & & & & & & & \\
\hline 317 & 24-Dec-05 & & & & & & & & & & & & \\
\hline 318 & 25-Dec-05 & & & & & & & & & & & & \\
\hline 319 & 26-Dec-05 & & & & & & & & & & & & \\
\hline 320 & 27-Dec-05 & & & & & & & & & & & & \\
\hline 321 & 28-Dec-05 & & & & & & & & & & & & \\
\hline 322 & 29-Dec-05 & & & & & & & & & & & & \\
\hline 323 & 30-Dec-05 & & & & & & & & & & & & \\
\hline 324 & 31-Dec-05 & & & & & & & & & & & & \\
\hline 325 & 1-Jan-06 & & & & & & & & & & & & \\
\hline 326 & 2-Jan-06 & & & & & & & & & & & & \\
\hline & & 20 & & & & & & & & & & & \\
\hline 327 & 3-Jan-06 & & & & & & & & & & & & \\
\hline 328 & 4-Jan-06 & & & & & & & & & & & & \\
\hline 329 & $5-\operatorname{Jan}-06$ & & & & & & & & & & & & \\
\hline
\end{tabular}




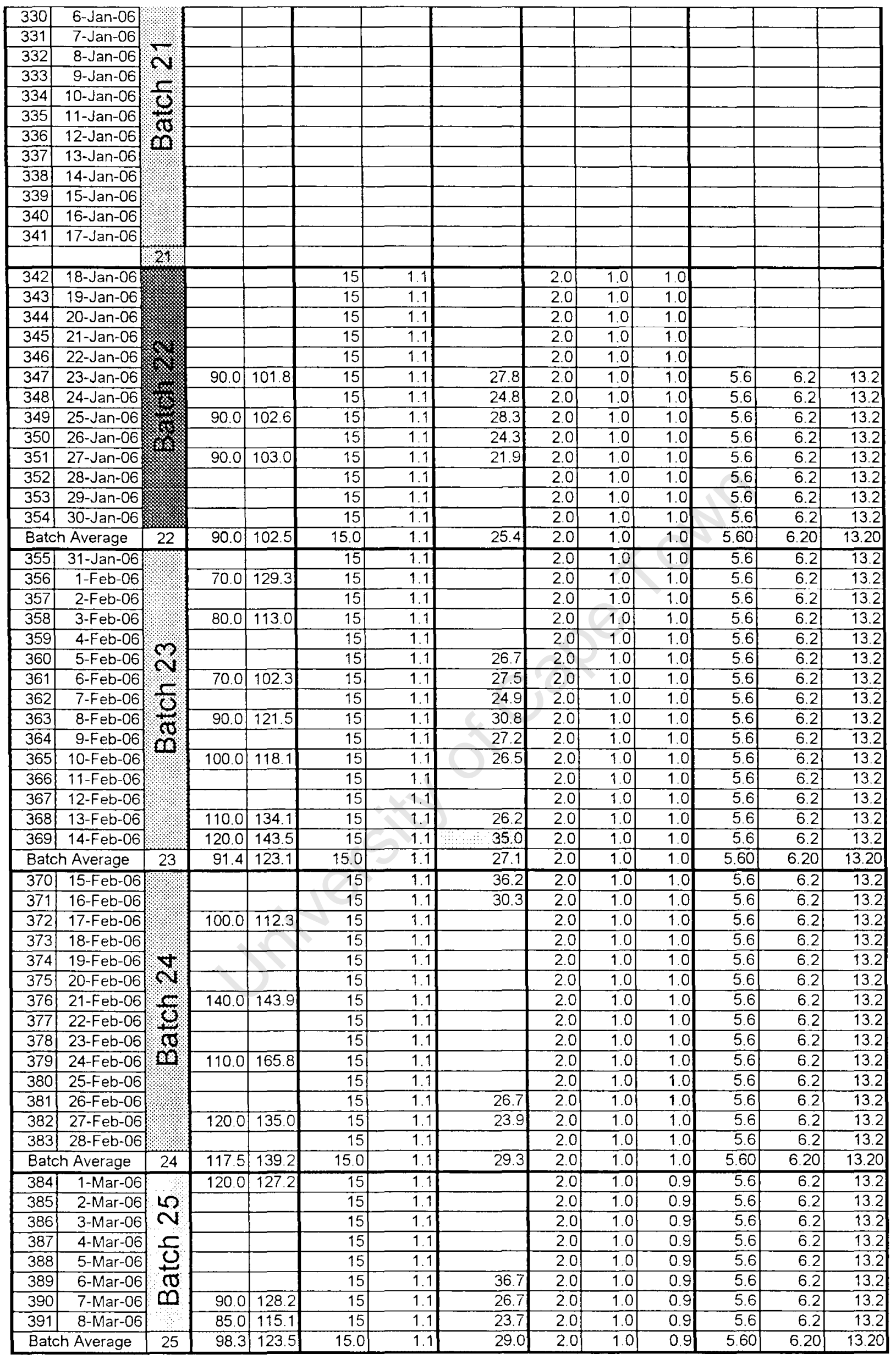




\begin{tabular}{|c|c|c|c|c|c|c|c|c|c|c|c|c|c|}
\hline 392 & 9-Mar-06 & \multirow{15}{*}{$\begin{array}{c}0 \\
\frac{N}{0} \\
\frac{\sigma}{\sigma} \\
\infty\end{array}$} & & & 15 & 1.1 & & 2.0 & 1.0 & 0.9 & 5.6 & 6.2 & 13.2 \\
\hline 393 & 10-Mar-06 & & & & 15 & 1.1 & & 2.0 & 1.0 & 0.9 & 5.6 & 62 & 13.2 \\
\hline 394 & 11-Mar-06 & & & & 15 & 1.1 & & 2.0 & 1.0 & 0.9 & 5.6 & 62 & 13.2 \\
\hline 395 & 12-Mar-06 & & & & 15 & 1.1 & 22.2 & 20 & 10 & 0.9 & 5.6 & 62 & 13.2 \\
\hline 396 & 13-Mar-06 & & 1200 & 137.0 & 15 & 1.1 & 23.4 & 2.0 & 1.0 & 0.9 & 5.6 & 62 & 13.2 \\
\hline 397 & 14-Mar-06 & & & & 15 & 1.1 & & 2.0 & 1.0 & 1.0 & 5.6 & 62 & 13.2 \\
\hline 398 & 15-Mar-06 & & 100.0 & 122.0 & 15 & 1.1 & 22.1 & 2.0 & 1.0 & 1.0 & 5.6 & 6.2 & 13.2 \\
\hline 399 & 16-Mar-06 & & & & 15 & 1.1 & 23.4 & 2.0 & 1.0 & 1.0 & 5.6 & 6.2 & 13.2 \\
\hline 400 & 17-Mar-06 & & 170.0 & 1979 & 15 & 1.1 & 24.7 & 2.0 & 1.0 & 1.0 & 56 & 6.2 & 13.2 \\
\hline 401 & 18-Mar-06 & & & & 15 & 1.1 & & 2.0 & 1.0 & 1.0 & 5.6 & 6.2 & 13.2 \\
\hline 402 & 19-Mar-06 & & & & 15 & 1.1 & 22.4 & 2.0 & 1.0 & 1.0 & 5.6 & 6.2 & 13.2 \\
\hline 403 & 20-Mar-06 & & 950 & 114.7 & 15 & 1.1 & 25.7 & 20 & 1.0 & 1.0 & 5.6 & 6.2 & 13.2 \\
\hline 404 & 21-Mar-06 & & & & 15 & 1.1 & & 20 & 1.0 & 10 & 5.6 & 6.2 & 13.2 \\
\hline 405 & 22-Mar-06 & & & & 15 & 1.1 & & 20 & 1.0 & 1.0 & 5.6 & 6.2 & 13.2 \\
\hline 406 & 23-Mar-06 & & 100.0 & 1178 & 15 & 1.1 & 22.3 & 2.0 & 1.0 & 1.0 & 5.6 & 6.2 & 13.2 \\
\hline \multicolumn{2}{|c|}{ Batch Average } & 26 & 117.0 & 137.9 & 150 & 1.1 & 23.3 & 2.0 & 1.0 & 1.0 & 5.60 & 6.20 & 13.20 \\
\hline 407 & 24-Mar-06 & \multirow{15}{*}{ 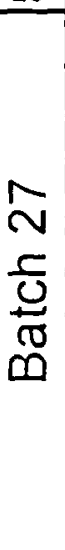 } & 110.0 & 141.2 & 15 & 1.1 & 21.7 & 2.0 & 1.0 & 1.0 & 56 & 6.2 & 13.2 \\
\hline 408 & 25-Mar-06 & & & & 15 & 1.1 & 21.3 & 2.0 & 1.0 & 1.0 & 5.6 & 6.2 & 13.2 \\
\hline 409 & 26-Mar-06 & & & & 15 & 1.1 & & 2.0 & 1.0 & 1.0 & 5.6 & 6.2 & 13.2 \\
\hline 410 & 27-Mar-06 & & 125.0 & 164.3 & 15 & 1.1 & & 2.0 & 1.0 & 1.0 & 5.6 & 6.2 & 13.2 \\
\hline 411 & 28-Mar-06 & & & & 15 & 1.1 & 28.5 & 2.0 & 1.0 & 1.0 & 5.6 & 6.2 & 13.2 \\
\hline 412 & 29-Mar-06 & & 140.0 & 159.7 & 15 & 1.1 & 24.0 & 2.0 & 1.0 & 1.0 & 5.6 & 6.2 & 13.2 \\
\hline 413 & 30-Mar-06 & & & & 15 & 1.1 & & 2.0 & 1.0 & 1.0 & 5.6 & 6.2 & 13.2 \\
\hline 414 & 31-Mar-06 & & 135.0 & 161.9 & 15 & 1.1 & 24.8 & 2.0 & 1.0 & 1.0 & 5.6 & 6.2 & 13.2 \\
\hline 415 & 1-Apr-06 & & & & 15 & 1.1 & 24.1 & 2.0 & 1.0 & 1.0 & 5.6 & 6.2 & 13.2 \\
\hline 416 & 2-Apr-06 & & & & 15 & 1.1 & 28.1 & 2.0 & 1.0 & 1.0 & 5.6 & 6.2 & 13.2 \\
\hline 417 & 3-Apr-06 & & 1100 & 136.1 & 15 & 1.1 & 36.6 & 2.0 & 1.0 & 1.0 & 5.6 & 6.2 & 13.2 \\
\hline 418 & 4-Apr-06 & & & & 15 & 1.1 & 34.0 & 2.0 & 1.0 & 1.0 & 5.6 & 6.2 & 13.2 \\
\hline 419 & $5-A p r-05$ & & 130.0 & $\overline{142.7}$ & & & & & & -1 & 5.6 & 6.2 & 13.2 \\
\hline 420 & 6-Apr-06 & & & & & & & & & & 5.6 & 6.2 & 13.2 \\
\hline 421 & $7-A p r-06$ & & & & & & & & & & 5.6 & 6.2 & 13.2 \\
\hline \multicolumn{2}{|c|}{ Batch Average } & 27 & 125.0 & 151.0 & 15.0 & 1.1 & 27.0 & 2.0 & 1.0 & 1.0 & 560 & 6.20 & 13.20 \\
\hline 422 & 8-Apr-06 & \multirow{15}{*}{$\frac{96}{6}$} & & & & 11 & & - & -1 & & 5.6 & 6.2 & 13.2 \\
\hline 423 & 9-Apr-06 & & & & & 1.1 & & - & -1 & & 5.6 & 6.2 & 13.2 \\
\hline 424 & 10-Apr-06 & & 140.0 & 220.8 & 15 & 0.0 & 24.1 & 2.0 & 1.0 & 1.0 & 5.6 & 6.2 & 13.2 \\
\hline 425 & 11-Apr-06 & & & & 15 & 0.0 & 18.0 & 2.0 & 1.0 & 1.0 & 5.6 & 6.2 & 13.2 \\
\hline 426 & 12 -Apr-06 & & & & 15 & 0.0 & 18.3 & 2.0 & 10 & 1.0 & 5.6 & 6.2 & 13.2 \\
\hline 427 & 13-Apr-06 & & 150.0 & 211.7 & 15 & 0.0 & 26.9 & 2.0 & 1.0 & 1.0 & 5.6 & 6.2 & 13.2 \\
\hline 428 & 14-Apr-06 & & & & 15 & 1.0 & -3 & 2.0 & 1.0 & 1.0 & 5.6 & 6.2 & 13.2 \\
\hline 429 & 15-Apr-06 & & 130.0 & 181.2 & 15 & 1.1 & 23.1 & 2.0 & 1.0 & 1.0 & 5.6 & 6.2 & 13.2 \\
\hline 430 & 16-Apr-06 & & & & 15 & 1.1 & & 2.0 & 10 & 1.0 & 5.6 & 6.2 & 13.2 \\
\hline 431 & 17-Apr-06 & & & & 15 & 1.1 & 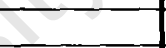 & 2.0 & 1.0 & 1.0 & $5 . \overline{6}$ & 6.2 & 13.2 \\
\hline 432 & 18-Apr-06 & & 145.0 & 163.6 & 15 & 1.1 & 23.6 & 2.0 & 1.0 & 1.0 & 5.6 & 6.2 & 13.2 \\
\hline 433 & 19-Apr-06 & & & & 15 & 1.1 & 24.6 & 2.0 & 1.0 & 1.0 & 5.6 & 6.2 & 13.2 \\
\hline 434 & 20-Apr-06 & & & & 15 & 1.1 & & 2.0 & 1.0 & 1.0 & 5.6 & 6.2 & 13.2 \\
\hline 435 & 21-Apr-06 & & 130.0 & 151.4 & 15 & 1.1 & 23.6 & 2.0 & 1.0 & 1.0 & 5.6 & 6.2 & 13.2 \\
\hline 436 & 22-Apr-06 & & & & & & 22.1 & & & & 5.6 & 6.2 & 13.2 \\
\hline \multicolumn{2}{|c|}{ Batch Average } & \multirow{14}{*}{$\frac{8}{8}$} & 139.0 & 185.7 & 15.0 & 0.8 & 22.7 & 2.0 & 1.0 & 1.0 & 5.60 & 6.20 & 13.20 \\
\hline 437 & 23-Apr-06 & & & & 15 & 1.1 & & 2.0 & 1.0 & 1.0 & 5.6 & 6.2 & 13.2 \\
\hline 438 & 24-Apr-06 & & 135.0 & & 15 & 1.1 & & 2.0 & 1.0 & 1.0 & 5.6 & 6.2 & 13.2 \\
\hline 439 & $25-A p r-06$ & & & & 15 & 1.1 & & 2.0 & 1.0 & 1.0 & 5.6 & 6.2 & 13.2 \\
\hline 440 & 26-Apr-06 & & 105.0 & 133.6 & 15 & 1.1 & & 2.0 & 1.0 & 1.0 & 5.6 & 6.2 & 13.2 \\
\hline 441 & $27-A p r-06$ & & & & 15 & 1.1 & 17.0 & 2.0 & 1.0 & 1.0 & 5.6 & 6.2 & 13.2 \\
\hline 442 & 28-Apr-06 & & 120.0 & 140.2 & 15 & 1.1 & 14.7 & 2.0 & 1.0 & 1.0 & 5.6 & 6.2 & 13.2 \\
\hline 443 & 29-Apr-06 & & & & 15 & 1.1 & & 2.0 & 1.0 & 1.0 & 5.6 & 62 & 13.2 \\
\hline 444 & 30-Apr-06 & & & & 15 & 1.1 & 18.6 & 2.0 & 1.0 & 1.0 & 5.6 & 6.2 & 13.2 \\
\hline 445 & 1-May-06 & & 1000 & 1302 & 15 & 1.1 & & 2.0 & 1.0 & 1.0 & 5.6 & 62 & 13.2 \\
\hline$\overline{446}$ & 2-May-06 & & & & 15 & 1.1 & & 2.0 & 1.0 & 1.0 & 5.6 & 6.2 & 13.2 \\
\hline 447 & 3-May-06 & & 110.0 & 109.6 & 15 & 1.1 & & 2.0 & 1.0 & 1.0 & 5.6 & 6.2 & 13.2 \\
\hline 448 & 4-May-06 & & & & 15 & 1.1 & & 2.0 & 1.0 & 1.0 & 5.6 & 6.2 & 13.2 \\
\hline 449 & 5-May-06 & & 135.0 & & 15 & 1.1 & 19.3 & 2.0 & 1.0 & 1.0 & 5.6 & 6.2 & 13.2 \\
\hline \multicolumn{2}{|c|}{ Batch Average } & 29 & 117.5 & 128.4 & 15.0 & $\overline{1.1}$ & 17.4 & 2.0 & 1.0 & 1.0 & 5.6 & 6.2 & 13.2 \\
\hline
\end{tabular}


Appendix B-5

Sewage Batch Averages for the Conventional UCT System

\begin{tabular}{|c|c|c|c|c|c|c|c|c|c|c|c|c|c|c|c|c|}
\hline \multirow{3}{*}{$\mathrm{SB} \#$} & \multicolumn{4}{|c|}{ COD } & \multicolumn{4}{|c|}{ TKN } & \multicolumn{2}{|c|}{ FSA } & \multicolumn{3}{|c|}{ TSS } & \multicolumn{3}{|c|}{ VSS } \\
\hline & Influent & Aerobic & ff unfilt & Eff 0.45 & Influent & Aerobic & JEff unfilt. & Eff 0.45 & influent & Eff 0.45 & AN & TAX & $\mathrm{AE}$ & AN & & TAE \\
\hline & \multicolumn{4}{|c|}{$(\mathrm{mgCOD} / 1)$} & \multicolumn{4}{|c|}{$(\mathrm{mgN} / 1)$} & \multicolumn{2}{|c|}{$(\mathrm{mgN} / 1)$} & \multicolumn{3}{|c|}{ (mgTSS $/)$} & \multicolumn{3}{|c|}{$\left(\mathrm{mgVSS} /{ }^{\prime}\right)$} \\
\hline 2 & 958.4 & 3758.9 & 59.4 & 47.6 & 101.4 & 247.6 & 10.4 & 8.5 & 81.1 & 6.3 & 2354 & 3212 & 3361 & 2013 & 2549 & 2718 \\
\hline 3 & 1147.7 & 4069.8 & 73.6 & 65.5 & 139.4 & 271.0 & 8.3 & 7.8 & 107.3 & 6.5 & 2098 & 3111 & 3361 & 1805 & 2773 & 2761 \\
\hline 4 & 1021.6 & 4706.9 & 48.8 & $\overline{40.3}$ & 97.0 & $\overline{291.4}$ & 10.0 & $\overline{9.1}$ & 70.1 & 7.6 & 4333 & 6650 & 5339 & 3865 & 5304 & $4 ?: 14$ \\
\hline 5 & 992.2 & 4491.1 & 53.2 & 45.7 & 112.6 & 279.7 & 10.5 & $\overline{9.4}$ & 88.6 & 7.9 & 4521 & 5965 & 6591 & 3861 & 4996 & 5068 \\
\hline 6 & 912.4 & 3156.7 & 598 & 45.7 & 133.6 & 235.1 & 12.5 & 10.3 & 104.1 & 8.5 & 3252 & 4706 & 4349 & 2790 & 3997 & 3638 \\
\hline 7 & & & 85.9 & 62.4 & & 227.8 & & 16.9 & 77.5 & 13.6 & 2609 & 3807 & 3530 & 2408 & 3428 & 3074 \\
\hline 8 & 785.4 & 4600.0 & 76.7 & 66.0 & 127.3 & 289.1 & 10.8 & 9.2 & 76.0 & 6.2 & 3361 & 3648 & 4907 & 2751 & 3098 & 4097 \\
\hline 9 & 980.2 & 3864.4 & 66.0 & 56.0 & 117.8 & 255.9 & 9.8 & 9.5 & 78.7 & 7.2 & 2378 & 3694 & 3937 & 2062 & 3111 & 3.31 \\
\hline 10 & 1006.3 & 443 & 45.8 & 37.5 & 91.5 & 284.0 & 9.6 & $\overline{8}$ & 67.6 & 6.8 & 3183 & 4571 & 5064 & 2820 & 3914 & $4: 06$ \\
\hline 11 & 975.9 & 4508.9 & 81.7 & 52.7 & 93.3 & 269.0 & 9.0 & 7.7 & 76.4 & 6.0 & 3728 & 5449 & 6374 & 3270 & 4295 & $5: 67$ \\
\hline 12 & 887.8 & 3873.2 & 86.7 & 50.4 & 131.7 & 265.1 & 8.6 & 7.9 & 99.6 & 7.3 & 2156 & 3415 & 3512 & 2029 & 2710 & $26 \overline{47}$ \\
\hline 13 & 855.6 & 3378.9 & 93.2 & 52.0 & 3.1 & 225.0 & 8.2 & 7.1 & 79.2 & 5.6 & 2082 & 2832 & 3068 & 1714 & 2241 & 2374 \\
\hline 14 & 869.9 & 3343.9 & 103.3 & 55.0 & & 228.1 & 10.6 & $\overline{8.5}$ & 94. & 6.2 & 1757 & 2887 & 3143 & 1484 & 2287 & 2.553 \\
\hline 15 & 933.9 & 3621.0 & 85.9 & 45.9 & & 271.6 & 4.0 & 1.8 & 82.5 & 0.2 & 2178 & 3313 & 3287 & 1807 & 2735 & 52669 \\
\hline 16 & 47.5 & 3673.7 & 104.7 & 68.3 & 108 & 242.3 & 3.4 & 2.6 & 79.0 & 1.5 & 2059 & 3123 & 3076 & 1684 & 2563 & $\sqrt{2}+22$ \\
\hline 17 & 926.2 & 3536.0 & 75.5 & 30.9 & 93.4 & 216.3 & 3 & & 78.0 & 1.5 & 1848 & 3171 & 3186 & 1562 & 2543 & 2524 \\
\hline 18 & 914.2 & 3660.3 & 81.3 & 51.3 & 00.1 & 267.9 & 5.8 & 5.2 & 80.8 & 2.2 & 2104 & 3057 & 3057 & 1762 & 2414 & 2.314 \\
\hline 19 & 1070.7 & 3842.0 & 84.3 & 50.4 & & 247.7 & 3.3 & 1.4 & 80.6 & 0.7 & 2042 & 3197 & 3378 & 1729 & 2584 & 2658 \\
\hline \multicolumn{17}{|l|}{20} \\
\hline 21 & & & & & & & & & & & & & & & & \\
\hline & 1008.8 & 3227.3 & 91.0 & 62.7 & 12.0 & 191.8 & 2.9 & 1.6 & 88.0 & 1.3 & 2014 & 2764 & 2831 & 1666 & 2204 & 2245 \\
\hline 23 & 940.6 & 2893.4 & & & 104.9 & 179.4 & 3.8 & 1.8 & 72.5 & 0.5 & 1596 & 2374 & 2526 & 1351 & 1927 & 1992 \\
\hline 24 & 787.4 & 3259.9 & 75.5 & 41.6 & 76.6 & $\overline{221.6}$ & 1.9 & 1.1 & 63.7 & 0.9 & 1731 & 2711 & 2932 & 1450 & 2177 & $\sqrt{2316}$ \\
\hline 25 & 905.4 & 3106.2 & 76.4 & 46.5 & 102.8 & 162.4 & 1.7 & 0.7 & 83.9 & 0.1 & 2108 & 2787 & 2833 & 1780 & 2267 & $2 \% 69$ \\
\hline 26 & 989.4 & 3378.4 & 70.2 & 54.6 & 102.2 & 225.8 & 4.3 & 1.5 & 85.2 & 0.6 & 1955 & 2779 & 2885 & 1636 & 2280 & 2341 \\
\hline 27 & & & 70.9 & & & 217.0 & & & 79.7 & 0.5 & 1612 & 2835 & 2733 & 1496 & 2372 & 2.313 \\
\hline 28 & 1115.6 & 3651.6 & 74.3 & 43.7 & 101.4 & 213.9 & 2.7 & 2.2 & 78.3 & 1.1 & 1637 & 2499 & 2479 & 1446 & 2142 & 2110 \\
\hline 29 & 983.3 & 3995.0 & 94.0 & 58.7 & & 238 & 2.4 & 2. & 71.7 & 0.8 & 2068 & 2876 & 2994 & 1922 & 2454 & $25 \overline{505}$ \\
\hline Ave & 951.2 & 3732.9 & 76.7 & 50.6 & 106.6 & 241.0 & 6.9 & 5.6 & 81.7 & 4.1 & 2414 & 3517 & 3644 & \begin{tabular}{|l|}
2083 \\
\end{tabular} & 2899 & 2933 \\
\hline
\end{tabular}




\begin{tabular}{|c|c|c|c|c|c|c|c|c|c|c|c|c|c|c|c|c|c|c|c|c|c|c|}
\hline DUR & \multicolumn{4}{|c|}{$\mathrm{NO}_{3}$} & \multicolumn{5}{|c|}{$\mathrm{NO}_{2}$} & \multicolumn{5}{|c|}{ Ortho-P } & \multirow{2}{*}{$\frac{R s}{R s}$} & \multirow{2}{*}{\begin{tabular}{|c|}
$\mathrm{TKN} /$ \\
$\mathrm{COD}$ \\
$\operatorname{lnf}$ \\
\end{tabular}} & \multirow{2}{*}{\begin{tabular}{|l|}
$C O D$ \\
VSS \\
$f C V$ \\
\end{tabular}} & \multirow{2}{*}{$\begin{array}{l}\text { TKN/ } \\
\text { VSS } \\
\end{array}$} & \multicolumn{4}{|c|}{ VSSITSS } \\
\hline & $\overline{A N}$ & $A X$ & TAE & $\mathrm{E}$ & AN & $\overline{A X}$ & $\mathrm{AE}$ & & & Infi. & AN & $A X$ & AEE & JEffil. & & & & & AN & $A \times \mid$ & AE & ave \\
\hline $0 /(-h)$ & & $1 \mathrm{mg}$ & gN/II & & & & $(m g N / /)$ & & & & & & & & & & & & & & & \\
\hline 26.6 & 0.0 & 0.6 & 33.4 & 36.2 & 0.0 & 0.0 & 0.0 & & 0.0 & 28.6 & 60.8 & 45.7 & 28.8 & 328.0 & 20.6 & 0.11 & 1.39 & 0.09 & 0.86 & 0.83 & 0.81 & $\widehat{0.78}$ \\
\hline 40.8 & 0.1 & 5.1 & 28.1 & 30.9 & 0.0 & 0.8 & 0.0 & & & 30.9 & 47.8 & 29.8 & 15.8 & $\widehat{149}$ & 20.1 & 0.12 & 1.48 & 0.10 & 0.86 & 0.83 & 0.82 & 10.8 \\
\hline & & 2.4 & 12.5 & 13.2 & 3 & 0.7 & 0.0 & & & & 51.5 & 23.2 & 9.7 & 9.3 & 22.7 & |09 & 1.16 & & & & & \\
\hline 21.0 & 0.3 & 3.3 & 27.7 & 25.0 & 0.3 & 0.9 & 0.0 & & 0.0 & 24.0 & 26.7 & 17.7 & 12.4 & 411.7 & 20.1 & 0.11 & 0.92 & 0.06 & 0.86 & 0.84 & 0.78 & 0.8 \\
\hline 18.6 & 0.0 & 8.6 & 28.4 & 29.5 & 0.0 & 1.4 & 0.0 & & & 25.2 & 23.1 & 17.3 & 15.1 & 114.5 & 18.5 & 0.14 & 1.06 & & 0.86 & 0.85 & 0.84 & 0.8 \\
\hline & 0.0 & 0.4 & 15.1 & & ती & 0.1 & & & & & 35.9 & 27.7 & 23.7 & 19.3 & 8.1 & & & & 0.89 & & & \\
\hline 25.6 & 0.0 & 4.1 & 18.0 & 15.3 & 0.0 & 1.2 & 5.8 & & 1.4 & & 38.5 & 22.5 & 16.2 & 215.5 & 21.4 & 0.17 & 1.21 & & 0.82 & & 0.04 & \\
\hline 28.4 & 0.0 & 5.9 & 20.5 & 20.3 & 0.0 & 0.0 & 0.0 & & & 29.5 & 38.4 & 25.9 & 19.3 & 39.5 & 24.1 & 0.12 & 1.23 & 0.0 & 0.86 & 0.84 & 0.82 & 10.8 \\
\hline 25.1 & 0.1 & 2.8 & 11.7 & 9.5 & 0.0 & 0.1 & 0.1 & & & 3 & 37.0 & 21.2 & 13.8 & 13.2 & 20.7 & 0.09 & 1.13 & 0.0 & 0.89 & 0.86 & 0.83 & 0.8 \\
\hline 22.5 & 0.1 & 1.3 & 14.3 & 10.5 & 0.0 & 0.3 & 0.0 & & & 4 & 38.3 & 20.7 & 12.7 & 11.5 & 20.8 & 0.10 & 0.94 & $\overline{0 .}$ & 0.88 & 0.84 & 0.8 & 0.8 \\
\hline 25.7 & 0.0 & 1.0 & 28.9 & 27.1 & 0.0 & 0.0 & 0.0 & & .0 & 3 & 48.0 & 25.3 & 14.1 & 10.9 & 20.6 & 0.14 & 1.43 & 0.10 & 0.83 & 0.77 & 0.74 & 0.77 \\
\hline 23.6 & 0.0 & 1.3 & 11.2 & 11.9 & 0.0 & 0.0 & 0.0 & & & & 49.3 & 24.4 & 11.9 & 10.5 & 20.4 & 0.1 & 1.40 & 0. & 0.84 & 0.79 & 0.77 & 0.79 \\
\hline 23.1 & 0.0 & 7.9 & 24.2 & 24.9 & 0.0 & 0 & & & & & 54.8 & 26.0 & 10.7 & 9.6 & 20.2 & 0. & 1.33 & 0.05 & 0.84 & 0.79 & 0.78 & 0.80 \\
\hline 24.6 & 0.0 & 8.5 & 21.4 & 22.6 & 0.0 & 0.0 & 0.0 & & 0.0 & 29.8 & 33.6 & 16.9 & 12.5 & 414.2 & 21.1 & 0.12 & $\overline{1.31}$ & 0.07 & 0.83 & 0.83 & 0.78 & 0.8 \\
\hline & 0.0 & 18 & 16.3 & 17.4 & 0.0 & 0.0 & 0.0 & & & & 59.2 & 27.0 & 18.8 & $\begin{array}{l}12.1 \\
\end{array}$ & 21.2 & 0.11 & 1.51 & 0. & 0.85 & 0.81 & & \\
\hline 28.4 & 0.0 & 0.7 & 10.1 & 8.7 & 0.0 & 0.0 & & & & 39.1 & 58.4 & 28.3 & 16.4 & & 20.7 & 0.11 & 1.41 & 0.1 & 0.85 & 0.80 & 0.79 & \\
\hline 21.3 & 0.0 & \begin{tabular}{l|}
3.3 \\
\end{tabular} & 14.8 & 13.8 & 0.0 & 0.1 & 0.1 & & 0.0 & 39.1 & 59.0 & 31.4 & 18.9 & $9 \longdiv { 1 8 . 0 }$ & 21.4 & 0.11 & 1.55 & 0.11 & 0.84 & 0.79 & 0.79 & 0.78 \\
\hline 25.8 & 0.0 & 2.8 & 13.2 & 12.7 & 0.0 & 0.0 & 0.0 & & 0.0 & 38.4 & 48.9 & 20.9 & 9.4 & 7.4 & 20.4 & 0.10 & 1.48 & 0.10 & 0.85 & 0.81 & 0.80 & $\longdiv { 0 . 8 1 }$ \\
\hline & & & & & & & & & & & & & & & & & & & & & & \\
\hline & & & & & & & & & & & & & & & & & & & & & & \\
\hline 25.4 & 0.0 & 0.8 & 19.8 & 20.6 & 0.0 & 0.4 & 0.0 & & 0.9 & 32.0 & 48.1 & 24.4 & 10.8 & $\begin{array}{l}39.4 \\
3\end{array}$ & 21.2 & 0.11 & 1.64 & 0.10 & 0.83 & 0.80 & 0.80 & 0.8 \\
\hline & 0.0 & 0.3 & 15.3 & 17.4 & 0.0 & 0.3 & 0.0 & & & 34.7 & 48.5 & 25.2 & 13.2 & 211.8 & 20.8 & 0.11 & 1.56 & 0. & 0.85 & 0.81 & 0.79 & \\
\hline 29.3 & 0.0 & 0.3 & 16.1 & 16.4 & 0.1 & 0.5 & 0.0 & & & & 48.5 & 30.4 & 15.6 & & 20.6 & 0.10 & 1.52 & 0.09 & 0.84 & & & \\
\hline 29.0 & 1.8 & 5.6 & 23.2 & 29.8 & 2.1 & 1.7 & 1.2 & & 0.6 & 35.2 & 39.2 & 24.7 & 18.4 & 18.9 & 21.4 & 0.11 & 1.49 & 0.08 & 0.84 & 0.81 & 0.80 & 0.8 \\
\hline 23.3 & 0.0 & 0.4 & 16.5 & 16.2 & 0.1 & 0.7 & 0.0 & & & 28.9 & 45.8 & 25.6 & 16.0 & $\overline{15.8}$ & 20. & 0.11 & 1.51 & 0.10 & 0.84 & 0.82 & 0.81 & \\
\hline 27.0 & 0.0 & 3.3 & 19.7 & 20.0 & 0.1 & 2.5 & & & & & 40.7 & 28.3 & 22.9 & $9 \longdiv { 2 1 . 4 }$ & 20.7 & 0.10 & 1.51 & 0.09 & 0.88 & & 0.84 & \\
\hline 22.7 & 0.0 & 0.1 & 17.9 & 19.0 & 0.1 & 0.3 & .1 & & & 20.7 & 23.5 & 10.5 & 3.3 & \begin{tabular}{l|l}
3 & 2.1 \\
\end{tabular} & 21.4 & 0.09 & 1.70 & 0.10 & 0.88 & 0.86 & 0.85 & 0.86 \\
\hline 17.4 & 0.0 & 0.1 & \begin{tabular}{|l|}
9.9 \\
\end{tabular} & 10.3 & 0.1 & \begin{tabular}{|c|}
0.9 \\
\end{tabular} & 0.7 & & & 29.2 & 42.1 & 25.2 & 16.5 & $\overline{172.2}$ & 20.8 & 0.09 & 1.70 & 0.09 & 0.87 & 0.85 & 0.84 & \\
\hline & 0.1 & 2.8 & 18.8 & 18.9 & 0.1 & 0.5 & 0.4 & & 0.5 & & 44.1 & 124.9 & 15.3 & 314.0 & 20 & 0.1 & 1.4 & 0.09 & 0.85 & 0.82 & 0.81 & 10.01 \\
\hline
\end{tabular}




\section{APPENDIX C}

\section{NITROGEN AND COD MASS BALANCES}

(After Musvoto 1992). In order to test the accuracy of the measured system response data, nitrogen and COD mass balances were performed on the system. These are discussed in detail below.

\section{C.1 NITROGEN MASS BALANCE}

The daily mass of nitrogen that enters the laboratory system in the form of influent TKN and dosed nitrate or nitrite should be accounted for as follows:

i) Nitrogen that is denitrified.

ii) Nitrogen in the waste sludge.

iii) Nitrogen in the effluent i.e. TKN plus nitrite and nitrate.

\section{i) Mass of nitrogen denitrified}

For the UCT configuration in the MBR and conventional systems (Figs. C.1 and C.2) this mass is obtained by a nitrate and nitrite mass balance around the anaerobic and anoxic sections of the system. Where significant amounts of nitrite are generated it is necessary to split the nitrite and nitrate in order to produce an accurate calculation particularly for the COD mass balance.

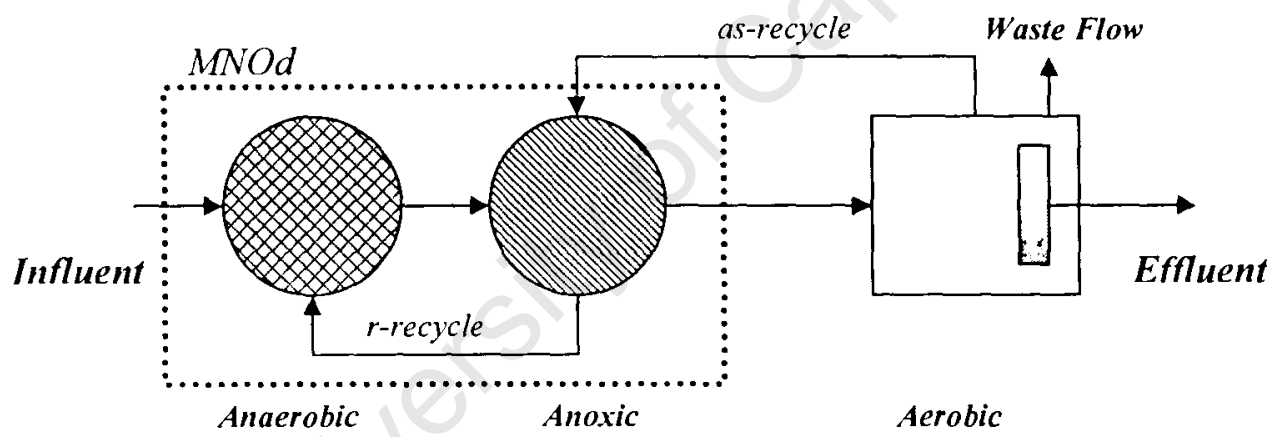

Figure C.1: Schematic layout of the MBR UCT system, the dotted line indicates the mass balance around the unaerated zones.

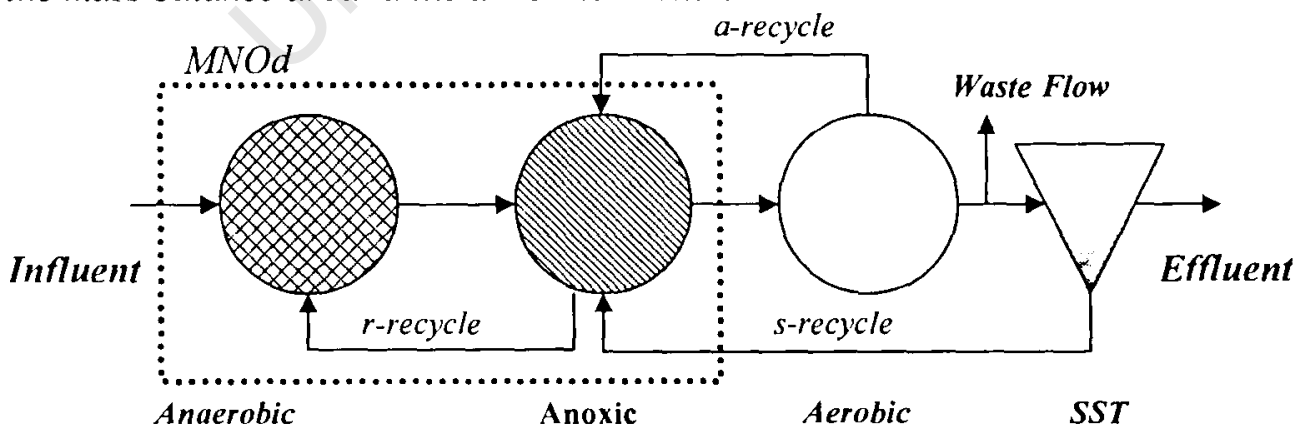

Figure C.2: Schematic layout of the conventional UCT system, the dotted line indicates the mass balance around the unaerated zones. 
Mass of nitrite and nitrate denitrified considering a UCT system is calculated using Equations (C.1) and (C.2) respectively.

$$
\begin{aligned}
& M N O_{2 d}=M_{N} O_{2 i n}-M N O_{2} m u
\end{aligned}
$$

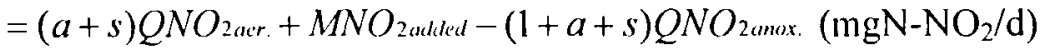

$$
\begin{aligned}
& M N O_{3} d=M N O_{3 m}-M N O_{3 m u t} \\
& =(a+s) Q N O_{3 \text { cerr. }}+M N O_{3 \text { ulded }}-(1+a+s) Q N O_{3 a m a x .}\left(\mathrm{mgN}-\mathrm{NO}_{3} / \mathrm{d}\right)
\end{aligned}
$$

Where:

$\mathrm{MNO} 2 \mathrm{~d}=$ mass of nitrite denitrified per day $\left(\mathrm{mgN}-\mathrm{NO}_{2} / \mathrm{d}\right)$

$\mathrm{MNO} 3 \mathrm{~d}=$ mass of nitrate denitrified per day $\left(\mathrm{mgN}-\mathrm{NO}_{3} / \mathrm{d}\right)$

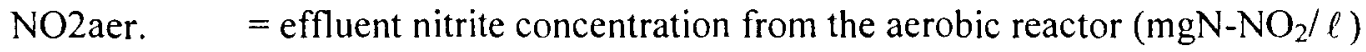

NO3aer. = effluent nitrite concentration from the aerobic reactor $\left(\mathrm{mgN}-\mathrm{NO}_{3} / \ell\right)$

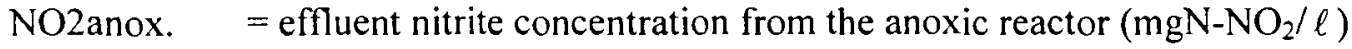

NO3anox. = effluent nitrite concentration from the anoxic reactor $\left(m g N-\mathrm{NO}_{3} / \ell\right)$

$\mathrm{Q}=$ daily influent flow rate $(\ell / \mathrm{d})$

$\mathrm{a}, \mathrm{s}, \mathrm{r} \quad=$ recycle ratios $(a+s$ is represented by $a s$ in the MBR system)

\section{ii) Mass of Nitrogen in the waste sludge}

The mass of $\mathrm{N}$ in the waste sludge is given by the product of the TKN/VSS $\left(f_{n}\right)$ ratio and the mass of VSS wasted per day.

$M X_{N}=f_{n} . M X_{\text {‘rasted }} \quad(\mathrm{mgN} / \mathrm{d})$

iii) Mass of Nitrogen in the effluent

This is the product of the daily flow rate and the sum of the effluent TKN, nitrate and nitrite concentrations.

$M N_{c}=\left(N_{t e}+N O_{2 e}+N O_{3 e}\right) \cdot Q \quad(\mathrm{mgN} / \mathrm{d})$

\section{iv) Nitrogen mass balance}

The $\% \mathrm{~N}$ mass balance is given by Equation (C.5)

$\% N_{-}$balance $=100\left(M N O+M N O_{3 d}+M X_{N}+M N_{e}\right) / M N_{i}$

Where $\mathrm{MN}_{\mathrm{i}}$ is the sum of the mass of TKN in the influent (given by the product of the influent TKN concentration and the daily flow rate) and the mass of nitrate and nitrite dosed, i.e.

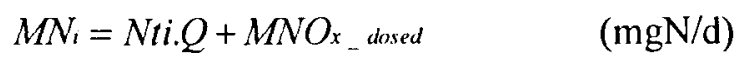




\section{C.2 COD MASS BALANCE}

The daily mass of COD $\left(\mathrm{MS}_{\mathrm{ti}}\right)$ that enters the system should be accounted by:

i) The mass of oxygen demand required per day for degradation of carbonaceous material in the aerobic reactor.

ii) The equivalent mass of oxygen demand per day by denitrification of nitrate and nitrite.

iii) COD mass in the waste sludge.

iv) COD mass in the effluent.

\section{i) Carbonaceous oxygen demand}

The total amount of oxygen utilized in the aerobic zone is made up of the nitrification demand and the carbonaceous oxygen demand. Since nitrification does not consume any of the influent COD, the oxygen demand due to nitrification must not be subtracted from the total measured oxygen demand. Stoichiometrically the oxygen requirements for nitrification of ammonia to nitrite and to nitrate is different, being slightly less in the former reaction (ie. $3.43 \mathrm{mgO} / \mathrm{mgN}$ and $4.57 \mathrm{mgO} / \mathrm{mgN}$ generated from ammonia). The oxygen demand for the nitrification of nitrite to nitrate is far less than these two being $1.14 \mathrm{mgO} / \mathrm{mgN}$. The calculation for the carbonaceous oxygen demand is as follows:

1) The mass of nitrate and nitrite generated by nitrification $\left(\mathrm{MNO}_{2 \mathrm{~g}}\right.$ and $\left.\mathrm{MNO}_{3 \mathrm{~g}}\right)$ is obtained by doing a nitrate and nitrite mass balance around the aerobic reactor of the system, as in Equations (C.7) and (C.8),

$$
\begin{array}{lr}
M N O_{2 g}=(1+a+s) Q N O_{2 a c r}-(1+a+s) Q N O_{2 a n o x .} & \left(m g N-N_{2} / \mathrm{d}\right) \\
M N O_{3 g}=(1+a+s) Q N O_{3 a e r .}-(1+a+s) Q N O_{3 a n o x .} & \left(m g N-\mathrm{NO}_{3} / \mathrm{d}\right)
\end{array}
$$

2) The nitrification oxygen demand is then given by Equation (C.9),

$$
M O_{n}=4.57 M N O_{3 g}+3.43 M N O_{2 g} \quad(\mathrm{mgCOD} / \mathrm{d})
$$

3) The carbonaceous oxygen demand $\left(\mathrm{MO}_{\mathrm{C}}\right)$ in the aerobic reactor is determined in Equation (C.10),

$$
M O_{c}=(O U R) \cdot V_{a} \cdot 24-M O_{n} \quad(\mathrm{mgCOD} / \mathrm{d})
$$

Where: OUR = oxygen utilization rate in the aerobic reactor $(\mathrm{mgO} / \ell / \mathrm{h})$

$\mathrm{V}_{\mathrm{a}} \quad=$ aerobic reactor volume $(\ell)$

ii) Equivalent oxygen demand for denitrification

During denitrification some influent biodegradable COD is oxidised with nitrate and nitrite. Stoichiometrically the equivalent amount of oxygen supplied during 
denitrification is different for nitrate and nitrite and therefore the equivalent oxygen demand per day for denitrification of nitrate and nitrite $\mathrm{MO}_{\mathrm{d}}$ is given by:

$$
M O_{d}=2.86 M N O_{3 d}+1.71 M N O_{2 d} \quad(\mathrm{mgCOD} / \mathrm{d})
$$

Where:

$2.86=$ equivalent mass of oxygen demand in denitrifying one $\mathrm{mgN}$ of

$1.71=$ equivalent mass of oxygen demand in denitrifying one $\mathrm{mgN}$ of nitrate to $\mathrm{N}_{2}\left(\mathrm{mgO} / \mathrm{mgN}^{\left.-\mathrm{NO}_{2}\right)}\right.$

$\mathrm{MNO}_{3 \mathrm{~d}}=$ mass of nitrate denitrified to nitrogen gas $\left(\mathrm{mgN}-\mathrm{NO}_{3} / \mathrm{d}\right)$

$\mathrm{MNO}_{2 \mathrm{~d}} \quad=$ mass of nitrate denitrified to nitrogen gas $\left(\mathrm{mgN}-\mathrm{NO}_{2} / \mathrm{d}\right)$

\section{iii) COD in waste sludge}

The amount of COD that passes out of the system via the waste sludge is given by Equation (C.12):

$$
M X_{s w w}=f\left(v \cdot M X_{v} \quad(\mathrm{mgCOD} / \mathrm{d})\right.
$$

Where:

$\mathrm{f}_{\mathrm{CV}} \quad=\mathrm{COD} / \mathrm{VSS}$ ratio $n$ activated sludge $(=1.48 \mathrm{mgCOD} / \mathrm{mgVSS})$

$\mathrm{MX}_{\mathrm{v}} \quad=$ mass of sludge wasted per day $(\mathrm{mgVSS} / \mathrm{d})$

\section{iv) COD in effluent}

This is given by the daily flow multiplied by the effluent COD concentration:

$$
M S_{t c}=Q . S_{t c}
$$

$(\mathrm{mgCOD} / \mathrm{d})$

\section{v) COD balance}

The percentage COD balance is then given by Equation (C.14),

$$
\% C O D_{-} \text {balance }=100\left(M O_{c}+M O_{d}+M X_{w w}+M S_{t c}\right) / M S_{u i}
$$




\section{APPENDIX D}

\section{CONSTRUCTION AND INTERPRETATION OF STATISTICAL PLOTS}

\section{D.1 INTRODUCTION}

(After Muller et al., 2003) Data from different tests could not be compared directly on a daily basis because of the variability in results from all the tests, due to variations in a multitude of factors that influence the data. Therefore a graphical approach was used to evaluate the data (Velz, 1950), to interpret the trends and compare the results between two test methods.

For a particular batch of wastewater, the data obtained from the different test methods were statistically analysed using a graphical procedure, to determine the mean, sample standard deviation (SSD), and standard deviation of the mean for the data set. This information could then be used to evaluate whether the difference between the means from two data sets is statistically significant at a selected confidence interval, or not.

\section{D.2 CONSTRUCTION OF STATISTICAL PLOT}

The experimental data is plotted using the procedure below:

- Arrange the data ( $\mathrm{n}$ in number) in order of ascending magnitude.

- Assign a serial number " $m$ " to each of the values $(1,2,3, \ldots n)$.

- Compute the $y$-axis plotting the position of each serial value, as the probability equal to or less than from the expression $[\mathrm{m} /(\mathrm{n}+1)]$. The $\mathrm{x}$-axis plotting position is the actual value for the data.

- The probability curve is linearized and plotted; for this investigation the transformed rank probability method (Scientific Tables, 1975) was used to linearize the probability curve, see Fig. DI.

\section{D.3 INTERPRETATION OF THE STATISTICAL PLOT}

The data plotted can give an indication of whether the data is normally distributed or not:

- If a straight line can be fitted to the plot it indicates that the data have a normal distribution.

- If a straight line cannot be fitted to the plot, the data are not normally distributed.

If the data are normally distributed it indicates that a multitude of factors have each had an independent small influence on the measurements; if the data are not normally distributed it indicates that one factor has had a dominating influence. 
From the above, provided a straight line can be fitted to the distribution (i.e. the data are normally distributed), is is possible to determine the mean and SSD graphically:

- The mean of the data plotted - this is determined as the $x$-value where the straight line of the distribution intercepts a vertical line extended from $y=5$.

- The SSD of the sample, which provides a measure of the variation of the data - this is the difference between the mean (i.e. the $x$-value that gives $y=5$, and the $x$ value that gives $y=4$, or $y=6$ ).

\section{D.4 TEST FOR STATISTICAL SIGNIFICANCE OF THE DIFFERENCES BETWEEN TWO MEAN VALUES}

Visual comparison of two data (or data sets) is a common method of appraisal, to determine whether they differ. However, observed differences or similarities may not be significant as these may arise solely by chance. Statistics defines the expected variations due to chance, to determine whether the observed differences between two data have arisen by chance alone or are significant. In the graphical method, by plotting of two or more series of data on the same probability plot, a quick visual appraisal of similarities and differences can be obtained. To test whether the visual differences in the two series of data are statistically significant, a mathematical significance test is performed as follows:

- Plot the two or more distributions to test for normality as described above.

- If normal, obtain the mean (M) and the sample standard deviation $(\sigma)$ of each series.

- Compute standard deviation of each mean:

$\operatorname{SD}($ mean $)=(\sigma / \sqrt{ } \mathrm{n})$

Where $n=$ number of data points.

- Compute the standard deviation of the difference between the two means:

$\mathrm{SD}($ difference $)=\sqrt{ }\left\{(\mathrm{SD} \text { mean } 1)^{2}-(\mathrm{SD} \text { mean } 2)^{2}\right\}$

- Compute the absolute value (i.e. positive) of the difference between the two means:

Mean $($ difference $)=\mid$ mean $1-$ mean2 $\mid$

- Decide upon a confidence interval for the test for significance, e.g. $95 \%$ certainty or $99 \%$ or any other desired level of confidence.

- Apply the test for statistical significance of the difference.

For example, if $95 \%$ is selected as the confidence level, subtract from the difference between the two means [ $\mathrm{SD}$ (difference)], i.e. [mean(difference) $-2 * \operatorname{SD}$ (difference)]. If a positive number is obtained it can be concluded that the difference between the two means is statistically significant at the selected level of confidence; if a negative value is obtained, then the difference between the two means was by chance alone, and it can be concluded that the apparent difference between the two means is NOT statistically different.

\section{D.5 EXAMPLE ILLUSTRATION}

An example plot is presented in Fig. D.1.

The mean of a set of values from an experiment is read off from the statistical graph as the value of $\mathrm{x}$ that gives $\mathrm{y}=5$, in this case $1.402 \mathrm{mgCOD} / \mathrm{mgVSS}$. The standard 
deviation of a set of values is calculated from the difference between the $x$-value that gives $y=5$, and the $x$-value that gives $y=4$ (or 6), as shown in Fig. D.1:

the $x$-value at $y=4=1.343$,

therefore the standard deviation $(\sigma)=1.402-1.343=0.059 \mathrm{mgCOD} / \mathrm{mgVSS}$.

The standard deviation of the mean is the standard deviation divided by the square root of the number of values in the data set. In this case:

Number of data in set $(n)=26$,

Therefore SD mean $=0.059 / \sqrt{ }(26)=0.116 \mathrm{mgCOD} / \mathrm{mgVSS}$.

\section{Batch COD to VSS ratio ( $\mathrm{fcv}$ ) in MBR}

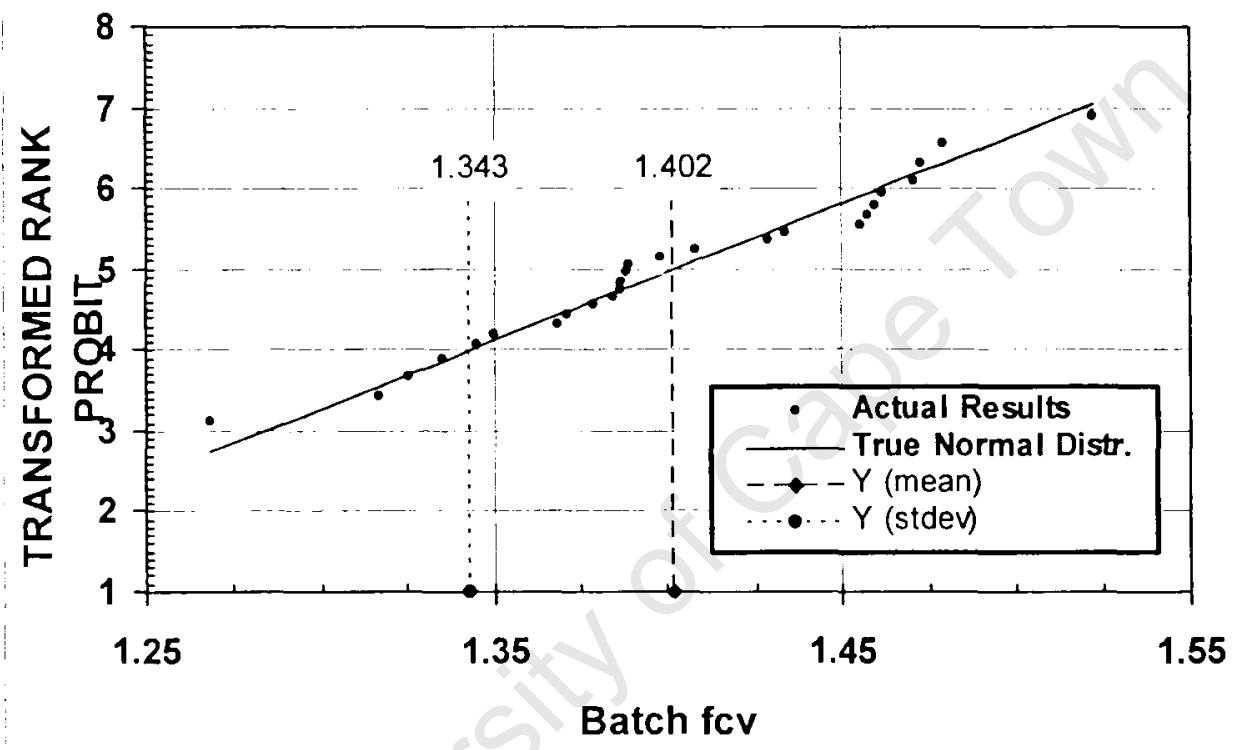

Figure D.1: Example of a linearized probability graph (after Fig 4.14, Chapter 4: Statistical plot of the sewage batch average COD to VSS ratio $\left(f_{C V}\right)$ in the MBR system) 


\section{APPENDIX E}

\section{CAPE METROPOLITAN SCIENTIFIC SERVICES PATHOGEN COUNTS}

\section{TABLE OF CONTENTS}

E.1 Scientific Services Laboratory Report 13-Nov-05

E.2 Scientific Services Laboratory Report 28-Mar-06 


\section{CITY OF CAPE TOWN}

WATER SERVICES

LABORATORY REPORT

File Ref: CB6/M2.1.2

Telephone: (021) 684 1028/5

TO: UCT Water Research Group

Attention: Geoff Du Toit

BACTERIOLOGICAL TESTING OF EFFLLENT WATER SAMPLES

\begin{tabular}{|lc|c|c|c|}
\hline Sampling Dale & Sample Code & & $\begin{array}{c}\text { Faecal Coliforms } \\
\text { per lo0 ml }\end{array}$ & E Coli per 100ml \\
\hline $2005-11-03$ & Conv UCT & & 6400 & 5700 \\
$2005-11-03$ & MBR UCT & & 10 & 10 \\
& & & & \\
\end{tabular}

KEY: E coli-Fscherichia coli

Ingrid Thomson for HEAD: WATER SERVICES 


\section{CITY OF CAPE TOWN}

WATER SERVICES

\section{LABORATORY REPORT}

File Ref: CB6/M2.1.2

TO: UCT Water Research Group

Attention: Gcoff Du Toit

BACTERIOLOGICAL TESTING OF EFFLUENT WATER SAMPLES

\begin{tabular}{|lccc|}
\hline Sampling Date & Sample Code & $\begin{array}{c}\text { Faecal Coliforms } \\
\text { per } 100 \mathrm{ml}\end{array}$ & E Coli per 100ml \\
\hline $2006-03-28$ & CONV UCT & 560 & 340 \\
$2006-03-28$ & MBR UCT & $<10$ & $<10$ \\
& & & \\
& & \\
\hline
\end{tabular}

KEY: Ecoli-Escherichia coli

\section{Ingrid Thomson}

for HEAD: WATER SERVICES 


\title{
APPENDIX F
}

\section{AERATION AND OXYGEN TRANSFER TESTING IN THE MBR SYSTEM}

\author{
TABLE OF CONTENTS \\ F.1 Steady-State Testing \\ F.2 Unsteady-State Testing \\ F.3 Viscosity and OTR Analysis \\ F.4 Rheology Testing on Sewage Sludge \\ F.5 Air Flow Rotameter Calibration
}


Steady State Testing without membranes

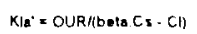

$\cos (20)=$

hota =

9.07

1.024

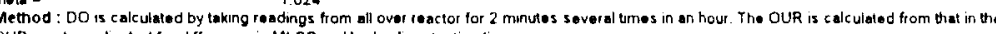

OUR resector, adiusted for difference in MLSS and hydradic retention time
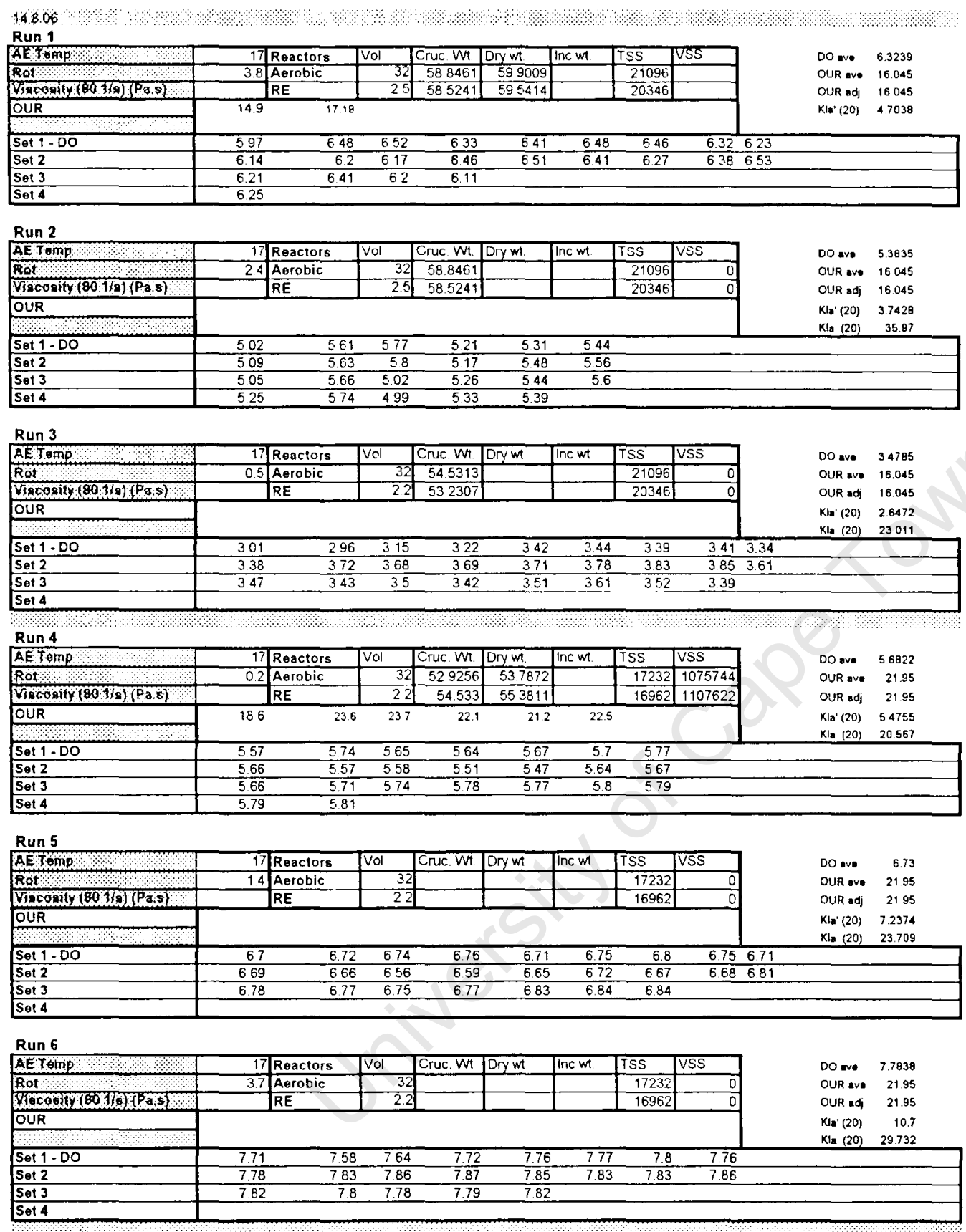

Run 7

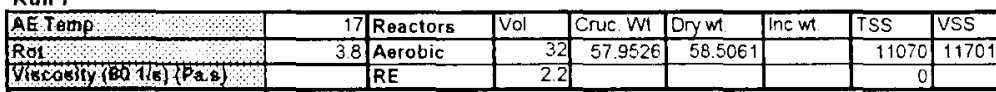

OUR

\begin{tabular}{|c|c|c|c|c|c|c|c|c|c|c|}
\hline OUR & 13.7 & 13.07 & 13.98 & 13.75 & 13.48 & 13.57 & & & $\begin{array}{l}\operatorname{Kl}_{12}(20) \\
K_{1} \mathbf{z}(20)\end{array}$ & $\begin{array}{l}15.043 \\
29.994\end{array}$ \\
\hline Sel 1-DO & 8.86 & 9.03 & 9.06 & 9.09 & 9.1 & 9.13 & 91 & 9.12 & & \\
\hline Set 2 & 9.13 & 9.11 & 9.1 & 903 & 9.02 & 9.06 & 9.03 & 8.97 & & \\
\hline Set 3 & 8.94 & 8.92 & 8.91 & 892 & 8.86 & 8.87 & & & & \\
\hline
\end{tabular}

Set 4

Run 8

\begin{tabular}{|c|c|c|c|c|c|c|c|c|c|c|}
\hline $\mathrm{Ral}$ & 1.4 & Aerobic & 32 & 57.9526 & \begin{tabular}{|l|}
58.5061 \\
\end{tabular} & & 11070 & 1170122 & \multirow{3}{*}{$\begin{array}{l}\text { OO ave } \\
\text { OUR ave } \\
\text { OUR adj } \\
\text { Kla' (20) }\end{array}$} & 13.592 \\
\hline 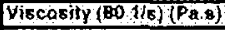 & & RE & 22 & & & & 0 & 0 & & 13.592 \\
\hline OUR & & & & & & & & & & 11745 \\
\hline Set 1-DO & 869 & 8.7 & 8.72 & 8.7 & $\overline{8.72}$ & 8 & 8.74 & 875 & & \\
\hline Set 2 & 878 & & 873 & 879 & 878 & 8.8 & 8.83 & & & \\
\hline Set 3 & & & & & & & & & & \\
\hline Set 4 & & & & & & & & & & \\
\hline
\end{tabular}

Do ave 90164

OUR ave 13.592

OUR odj 13592 $\begin{array}{rrrrrrr}9.03 & 9.06 & 9.09 & 9.1 & 9.13 & 91 & 912 \\ 911 & 91 & 903 & 902 & 9.06 & 903 & 897\end{array}$ $\begin{array}{lllll}8.92 & 8.91 & 8.92 & 8.86 & 8.87\end{array}$
Equatlon for Kla(20)
$y=09675 \times 2+14474 x+32601$ 
$\begin{array}{lr}\text { Cs(20): } & 907 \\ \text { Theta } & 1024\end{array}$

Testing without membranes

Rotameter readirig
Log (Cs-Cl gradient)
Kla (T)
Kla ( 20$)$
Temp
Vol

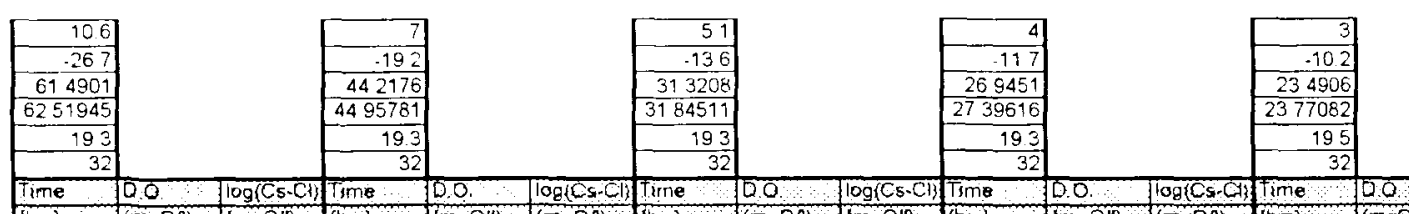

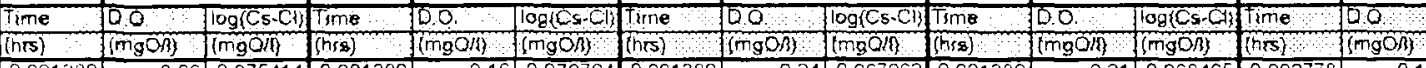

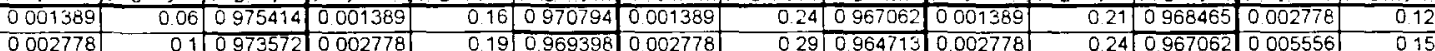
\begin{tabular}{|l|r|l|l|l|l|l|l|l|l|l|l|l|l|}
\hline 0002778 & 01 & 0973572 & 0002778 & 0.19 & 0.969398 & 0.002778 & 029 & 0.964713 & 0.002778 & 0.24 & 0.967062 & 0005556 & 015 \\
\hline 0.004167 & 013 & 0972185 & 0004167 & 0.23 & 0.96753 & 0.004167 & 0.35 & 0.961878 & 0.004167 & 027 & 0.965654 & 0.008333 & 0.16 \\
\hline
\end{tabular}

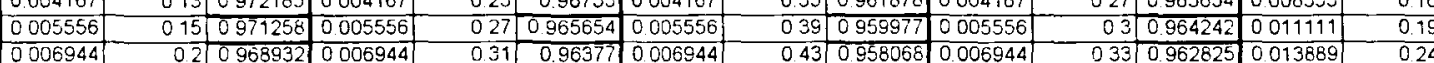
\begin{tabular}{|r|r|r|r|r|r|r|r|r|r|r|r|r|r|}
\hline 0006944 & 0.2 & 0968932 & 0006944 & 0.31 & 0.96377 & 0.006944 & 0.43 & 0958068 & 0.006944 & 033 & 0.962825 & 0.013889 & 0.24 \\
\hline 0008333 & 027 & 0965654 & 0008333 & 036 & 0961403 & 0008333 & 0.51 & 0954224 & 0008333 & 037 & 0960928 & 0.016667 & 0.31 \\
\hline
\end{tabular}

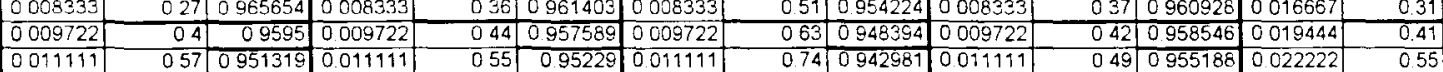

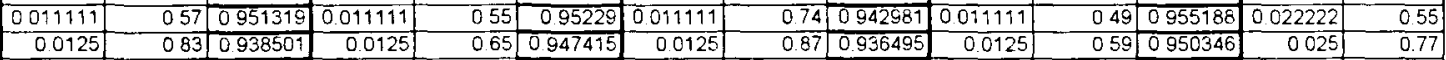

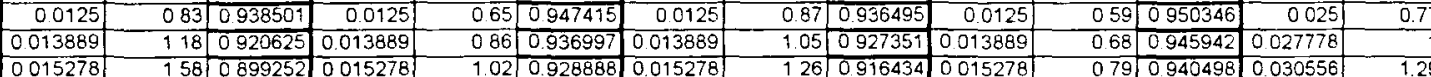

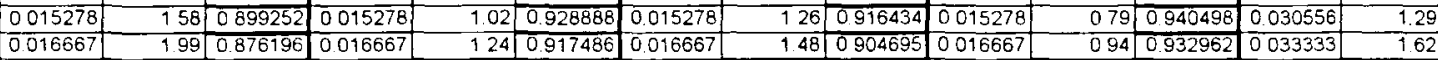
\begin{tabular}{|l|l|l|l|l|l|l|l|l|l|l|l|l|l|}
\hline 0018056 & 2.38 & 0853067 & 0018056 & 1.53 & 0.901982 & 0.018056 & 1.71 & 0892074 & 0.018056 & 1.07 & 0.926323 & 0.036111 & 1.99 \\
\hline
\end{tabular}

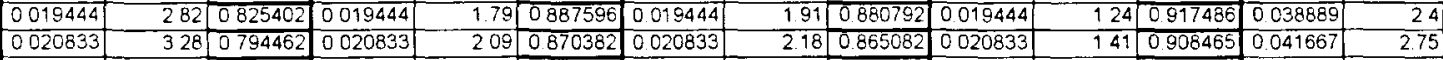

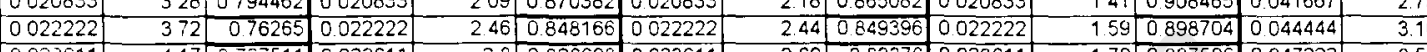

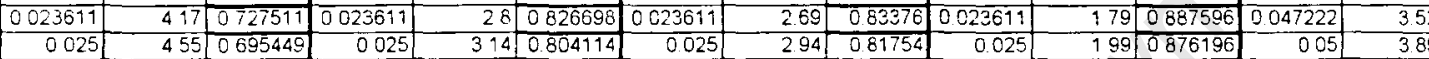

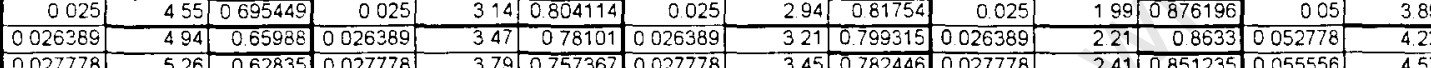

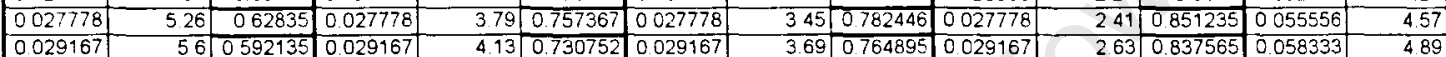
\begin{tabular}{|l|r|r|r|r|r|r|r|r|r|r|r|r|r|r|}
\hline 0030556 & 593 & 0553837 & 0030556 & 4.45 & 0.704118 & 0030556 & 394 & 0.745826 & 0.030556 & 2.85 & 082345 & 0061111 & 5.19 \\
\hline
\end{tabular}

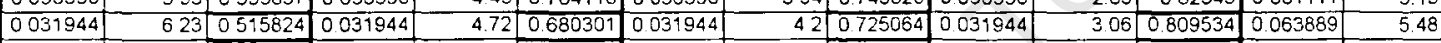

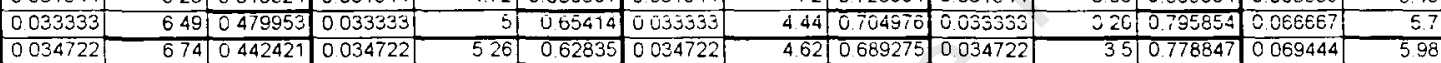

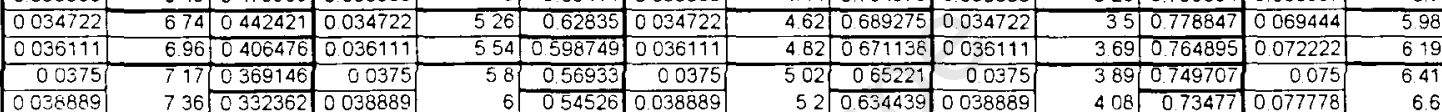
\begin{tabular}{ll|l|l|l|l|l|}
736 & 0332362 & 0038889 & 6 & 054526 & 0038889 \\
\hline 755 & 020212 & 0040278 & 051746 & 0040278 \\
\hline
\end{tabular}

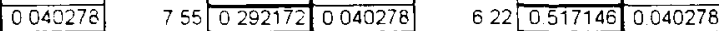

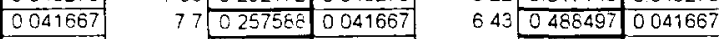

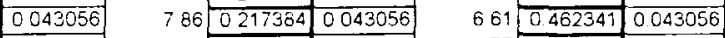

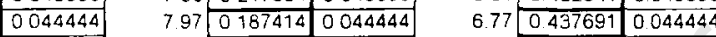

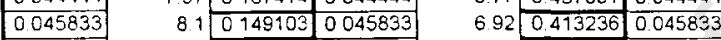
\begin{tabular}{|l|l|l|l|l|l|l|}
\hline 0.047222 & 82 & 0117146 & 0047222 & 705 & 0390868 & 0.047222 \\
\hline 0048
\end{tabular}

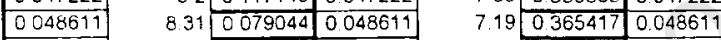

\begin{tabular}{r|r|r|}
7.39 & 0.365417 & 0.048611 \\
\hline & 0.344318 & 005 \\
\hline
\end{tabular} \begin{tabular}{r|r|r|}
7.3 & 0.344318 & 0.05 \\
\hline 745 & 0.313788 & 0.051389 \\
\hline
\end{tabular} \begin{tabular}{r|r|r|r|}
\hline 005 & 8.4 & 0045175 & 005 \\
\hline 0051389 & 845 & 0025151 & 0.051389 \\
\hline
\end{tabular} \begin{tabular}{|ll|l|l|}
\hline 0052778 & 853 & 000894 & 0052778 \\
\hline
\end{tabular}

\begin{tabular}{|ll|l|l|}
\hline 0054167 & 856 & .002245 & 0054167 \\
\hline
\end{tabular} \begin{tabular}{ll|l|l|l|l|l|l|l|l}
757 & 0287717 & 0052778 \\
\hline
\end{tabular} \begin{tabular}{ll|l|l|}
766 & 0267083 & 0054167 \\
\hline
\end{tabular} $7 7 5 \longdiv { 0 . 2 4 5 4 1 9 } 0 0 5 5 5 5 5$ \begin{tabular}{llll}
0055556 & 865 & 020569 & 055556 \\
\hline 0056944 & 87 & 005172 & 0056944 \\
\hline
\end{tabular}

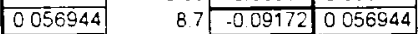
0056333 0.059722

0.061111 \begin{tabular}{|l|l|l|}
875 & .01194 & 0.058333 \\
\hline
\end{tabular} \begin{tabular}{ll|l|l|}
879 & 0.1429 & 0059722 \\
\hline
\end{tabular}

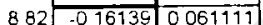
\begin{tabular}{l|l|l|}
887 & .0 .19408 & 0.0625 \\
\hline
\end{tabular} \begin{tabular}{l|l|r|}
88 & .0 .19408 & 0.0625 \\
\cline { 2 - 3 } & -020787 & 0063889 \\
\hline
\end{tabular} \begin{tabular}{|l|l|r|r|}
\hline 0063889 & 8.89 & -020787 & 0063889 \\
\hline 0065278 & 894 & -024441 & 0065278 \\
\hline
\end{tabular} \begin{tabular}{|l|l|l|l|}
\hline 0.065278 & 894 & -024441 & 0065278 \\
\cline { 3 - 4 } & & 0.065657 & 0.066667 \\
\hline
\end{tabular} 0066667

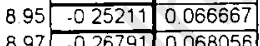
78502200090056944 \begin{tabular}{l|l|l|}
7.85 & 0.220009 & 0056944 \\
\cline { 2 - 3 } 794 & 0.195795 & 0058333 \\
\hline
\end{tabular} \begin{tabular}{ll|l}
8.01 & 0.175982 & 0.059722 \\
\hline
\end{tabular} 809 \begin{tabular}{ll|l|l|}
\hline & 0.152173 & 0.061111 \\
\hline
\end{tabular} \begin{tabular}{lll}
8.17 & 0.126982 & 0.0625 \\
\hline
\end{tabular} \begin{tabular}{|l|l|l|}
821 & 0.113817 & 0063889 \\
\cline { 2 - 3 }
\end{tabular} \begin{tabular}{l|l|l|l|}
827 & 0.093289 & 0.065278 \\
\hline
\end{tabular} \begin{tabular}{llll|}
8.32 & 0.075409 & 0.066667 \\
\hline
\end{tabular} 8370056761006805 \begin{tabular}{ll|l|l|}
842 & 0.037276 & 0.069444 \\
\hline
\end{tabular} \begin{tabular}{l|l|l|}
8.46 & 0.021033 & 0.070833 \\
\hline
\end{tabular} \begin{tabular}{|l|l|}
\hline Unsteady State 00 Aeration & 0.069444 \\
\hline 0070833 \\
\hline 0072222
\end{tabular} \begin{tabular}{ll|l|l|}
8.53 & -000894 & 0072222 \\
\hline
\end{tabular} \begin{tabular}{|r|r|r}
858 & -0.03169 & 0073611 \\
\hline 86 & 0.04114 & 0075 \\
\hline
\end{tabular} \begin{tabular}{l|l|l|}
52 & 0.634439 & 003888 \\
\cline { 2 - 4 } & 0612744 & 0040278 \\
\hline
\end{tabular} \begin{tabular}{|l|l|l|}
5458 & 0612744 & 0040278 \\
\cline { 2 - 3 } & 0594351 & 0041667 \\
\hline
\end{tabular} \begin{tabular}{l|l|l|}
576 & 0.573988 & 0043056 \\
\hline
\end{tabular} \begin{tabular}{|l|l|l|}
593 & 0553837 & 0044444 \\
\hline
\end{tabular} \begin{tabular}{|l|l|l|}
\hline 6.1 & 0.532706 & 0.045833 \\
\hline
\end{tabular} \begin{tabular}{l|l|l|}
6.24 & 0.514498 & 0.047222 \\
\hline
\end{tabular} 6.3804954920048611 \begin{tabular}{|l|r|r|}
6.38 & 0.495492 & 0.048611 \\
\hline 6510477067 & 005 \\
\hline
\end{tabular} \begin{tabular}{l|l|r|}
651 & 0.477067 & 0.05 \\
\hline 664 & 0457825 & 0051389 \\
\hline
\end{tabular} $6 7 6 \longdiv { 0 4 3 9 2 7 3 } 0 0 5 2 7 7 8$ 689044182390054167 699040133500055556 \begin{tabular}{lll}
711 & 0380143 & 0056944 \\
\hline
\end{tabular} \begin{tabular}{l|l|l|}
7.2 & 0353541 & 0058333 \\
\hline
\end{tabular} \begin{tabular}{l|l|l|}
729 & 0.346279 & 0.059722 \\
\hline 7
\end{tabular} \begin{tabular}{|l|r|r|}
738 & 0.328303 & 0.061111 \\
\hline
\end{tabular}

\begin{tabular}{|l|l|l|}
7.47 & 0.30955 & 00625 \\
\hline
\end{tabular} \begin{tabular}{l|l|l|}
7.55 & 0.292172 & 0.063889 \\
\hline
\end{tabular} \begin{tabular}{l|l|l|}
7.63 & 0.274071 & 0.065278 \\
\hline
\end{tabular} \begin{tabular}{l|l|l|}
77.7 & 0257588 & 0.066667 \\
\hline
\end{tabular} $\begin{array}{llll}777 & 0240455 & 0068056\end{array}$ \begin{tabular}{lll}
7.83 & 0.225212 & 0069444 \\
\hline
\end{tabular} \begin{tabular}{|l|l|l|}
792 & 0201294 & 0070833 \\
\hline
\end{tabular} \begin{tabular}{l|l|l|}
792 & 0201294 & 0.070833 \\
\cline { 2 - 3 } & 0.187414 & 0.072222 \\
\hline 8 & 17304 & 0.073611 \\
\hline
\end{tabular} \begin{tabular}{l|l|l|}
802 & 0173076 & 0.073611 \\
\hline
\end{tabular} $8 0 6 \longdiv { 0 1 6 1 2 5 5 } 0 0 7 5$ \begin{tabular}{ll|l|l|l|l|l|l|l|l|l|l|}
811 & 0146011 & 076389 \\
\hline
\end{tabular} \begin{tabular}{llll}
817 & 0.126982 & 0077778 \\
\hline 821 & 0.113817 & 0.079167
\end{tabular} \begin{tabular}{ll|l|l|l|l|l|l|l|l|l|l|}
821 & 0.113817 & 0.079167 \\
\hline
\end{tabular} \begin{tabular}{ll|l|l|}
8.26 & 0096779 & 0.080556 \\
\hline
\end{tabular} \begin{tabular}{ll|l|l|}
8.3 & 0.08265 & 0.081944 \\
\cline { 2 - 3 } & 0.056046 & 0.83333 \\
\hline
\end{tabular} \begin{tabular}{l|l|l|}
8.34 & 0068046 & 0083333 \\
\hline
\end{tabular} \begin{tabular}{l|l|l}
863 & .00557 & 00703
\end{tabular} \begin{tabular}{l|l|l}
8.67 & -0.07592 & 0077 \\
\hline 8.0 .0972 & 0.079
\end{tabular} \begin{tabular}{l|l|l|}
872 & -0.10258 & 0080556 \\
\hline
\end{tabular} \begin{tabular}{l|l|l|}
8.77 & -0.13099 & 0081944 \\
\hline
\end{tabular}

\begin{tabular}{llll}
721 & 0361656 & 0.141667 \\
\hline
\end{tabular}

\begin{tabular}{ll|l|l|}
727 & 0.350175 & 0144444 \\
\hline
\end{tabular}

\begin{tabular}{ll|l|l|}
737 & 0330337 & 0.147222 \\
\hline
\end{tabular}

\begin{tabular}{l|l|r|}
745 & 0.313788 & 0.15 \\
\hline
\end{tabular}

\begin{tabular}{ll|l|l|}
753 & 0.296582 & 0.152778 \\
\hline
\end{tabular}

\begin{tabular}{ll|l|l|}
759 & 0283216 & 0.155556 \\
\hline
\end{tabular}

\begin{tabular}{l|l|l|}
7.65 & 0.269425 & 0.158333 \\
\cline { 2 - 3 }
\end{tabular}

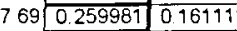

\begin{tabular}{ll|l|l|}
774 & 0.247881 & 0.163889 \\
\cline { 2 - 4 } & & 0.240455 & 0.166657 \\
\hline
\end{tabular}

\begin{tabular}{ll|l|l|}
777 & 0.240455 & 0.166667 \\
\hline
\end{tabular}
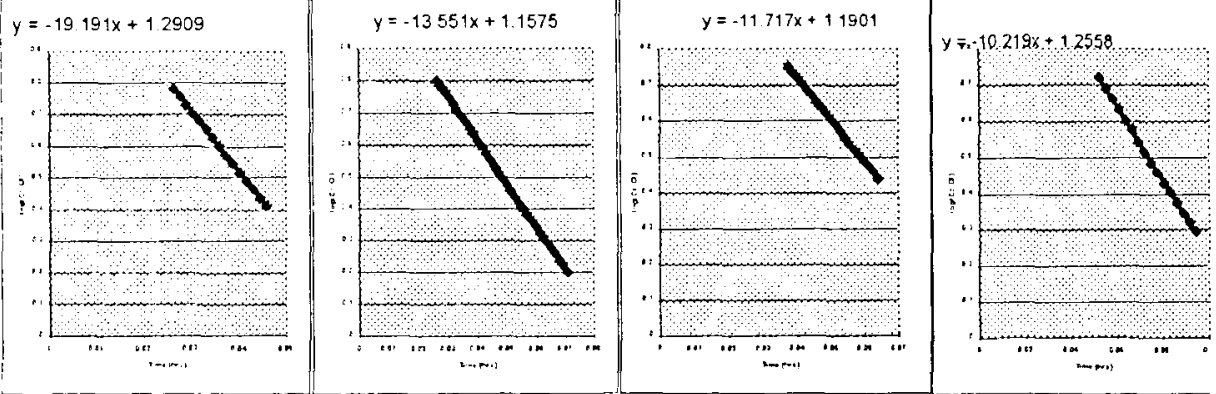
Testing with membranes

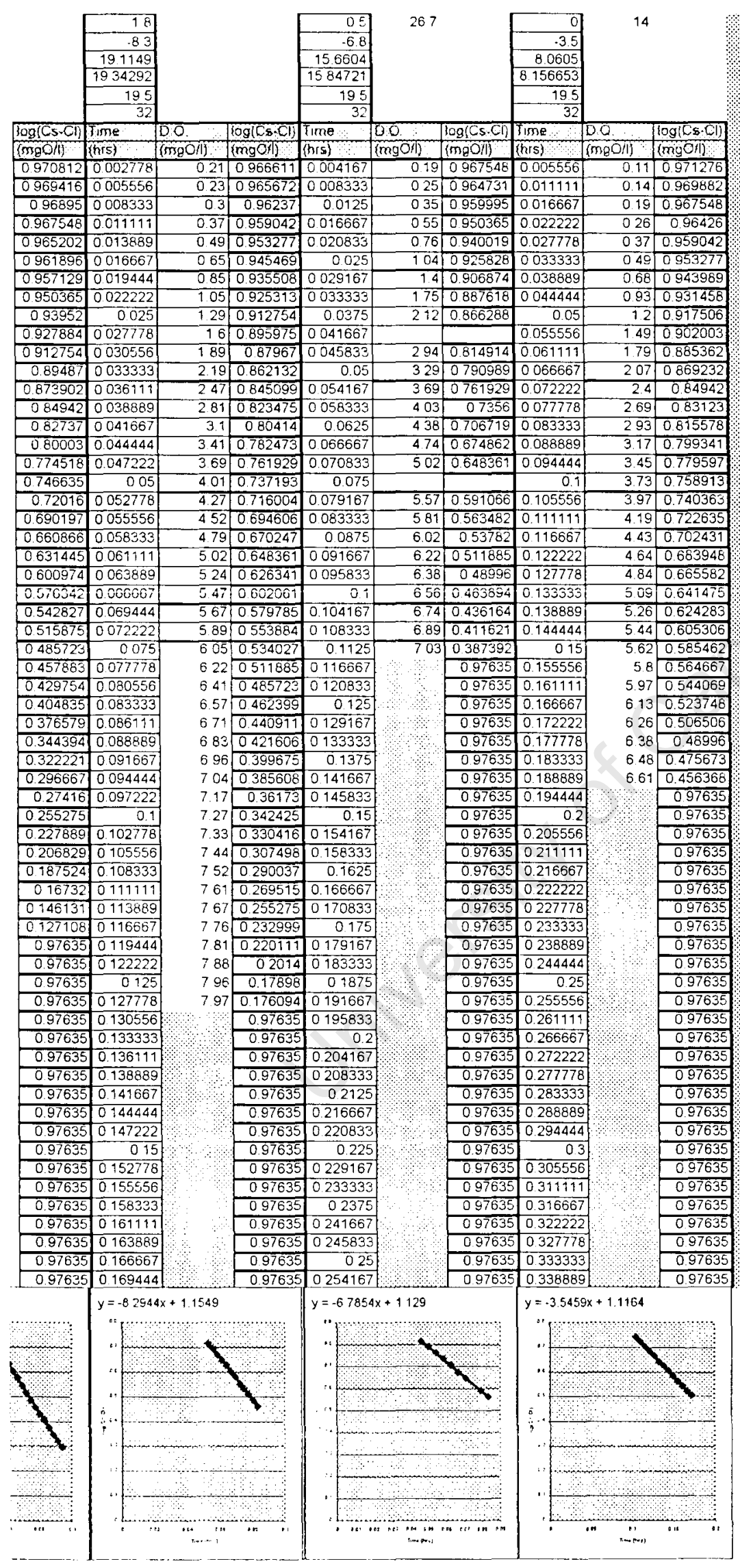

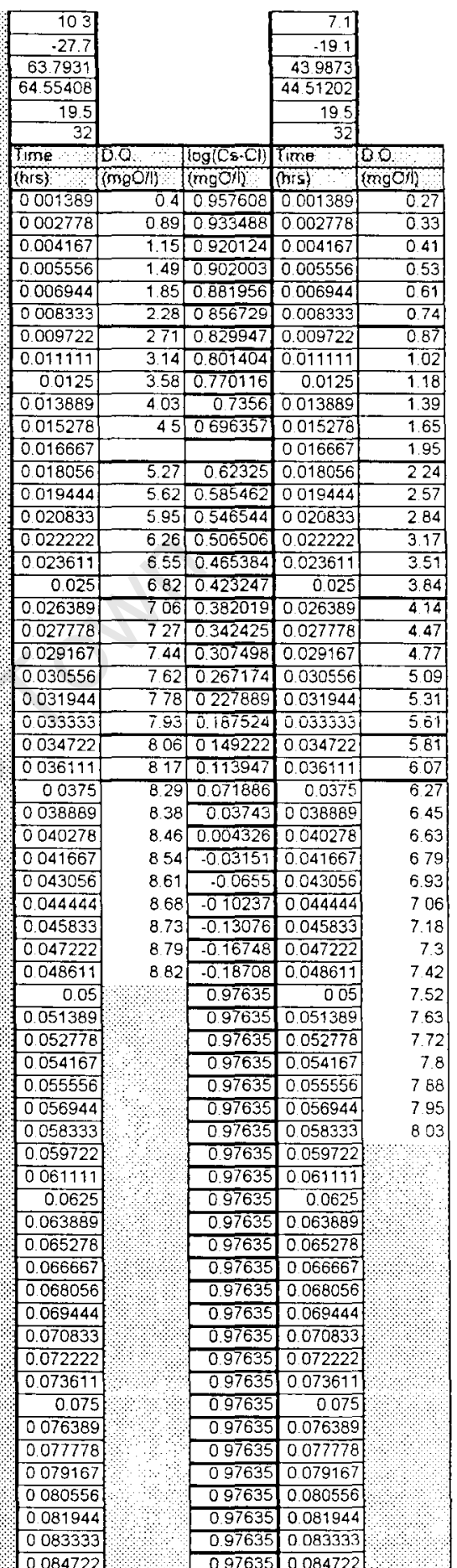

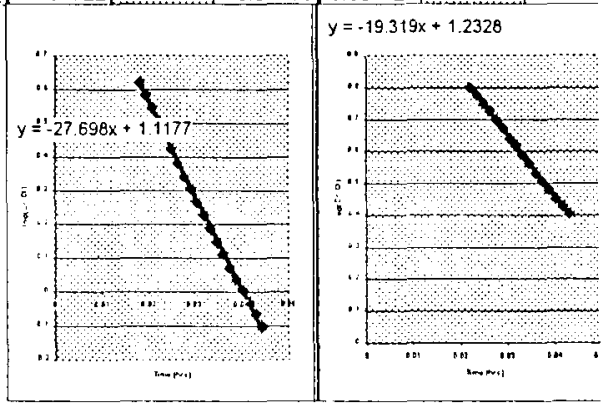




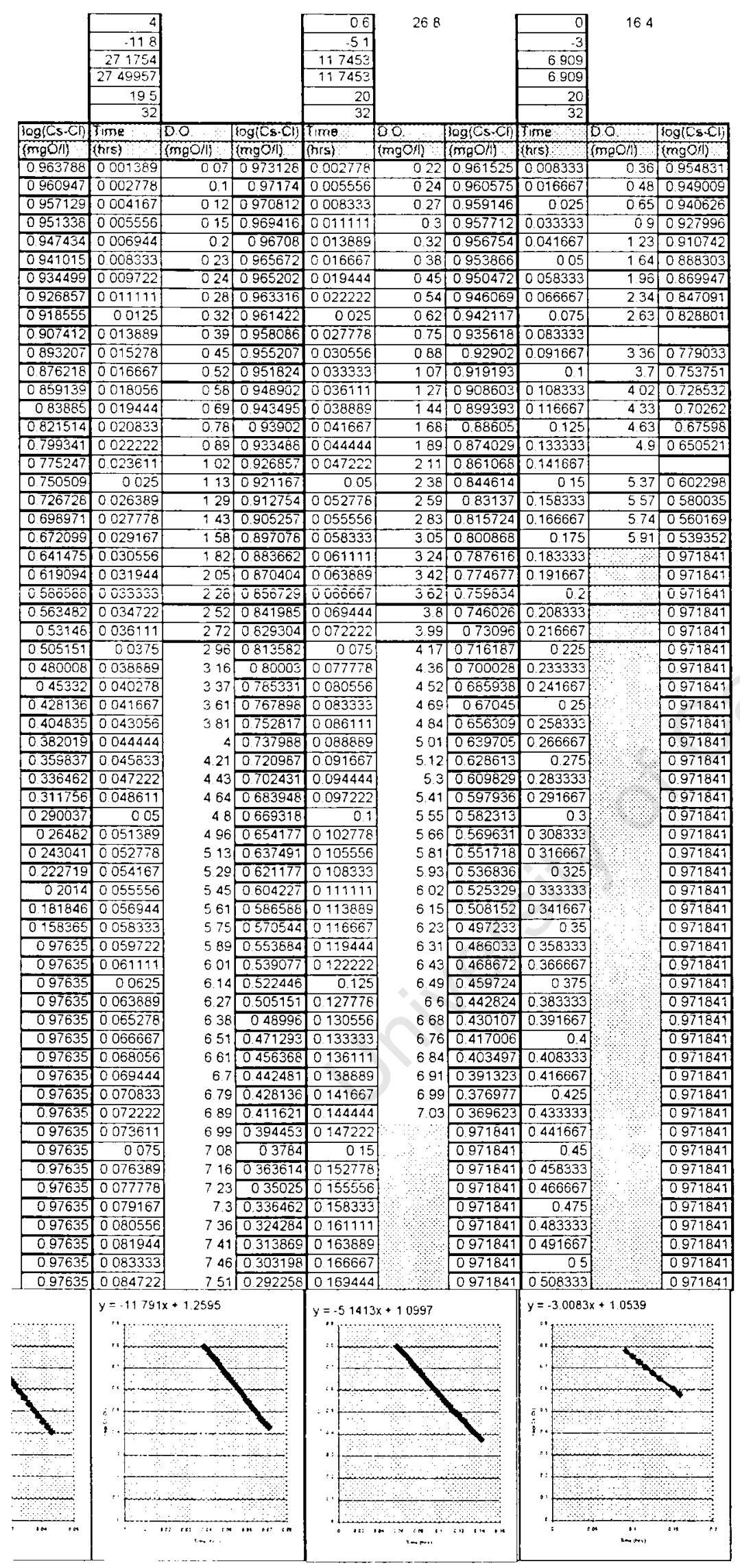


Appendix F-3: Summarized Rheology Results

\section{Rheology readings}

\begin{tabular}{|c|c|c|c|c|c|c|c|}
\hline Sample ID & \multicolumn{4}{|c|}{ Viscosity@shear rate } & MLSS & Ave Alpha & SOTE \\
\hline \# & $280.7 / \mathrm{s}$ & $148.7 / \mathrm{s}$ & $78.8 / \mathrm{s}$ & $57.4 / \mathrm{s}$ & mgTSS/l & & $\% / m$ \\
\hline A & 0.0291 & 0.04239 & 0.06547 & 0.0831 & 21096 & 0.186 & 0.203 \\
\hline$B$ & 0.0178 & 0.0247 & 0.0373 & 0.0468 & 17232 & 0.444 & 0.46 \\
\hline $\mathrm{C}$ & 0.0079 & 0.0096 & 0.0138 & 0.0166 & 11070 & 0.621 & 0.701 \\
\hline$D$ & 0.00537 & 0.0029 & 0.0015 & 0 & $\mathrm{n} / \mathrm{a}$ & $n / a$ & n/a \\
\hline
\end{tabular}

Plot A

Rheology of the MBR MLSS

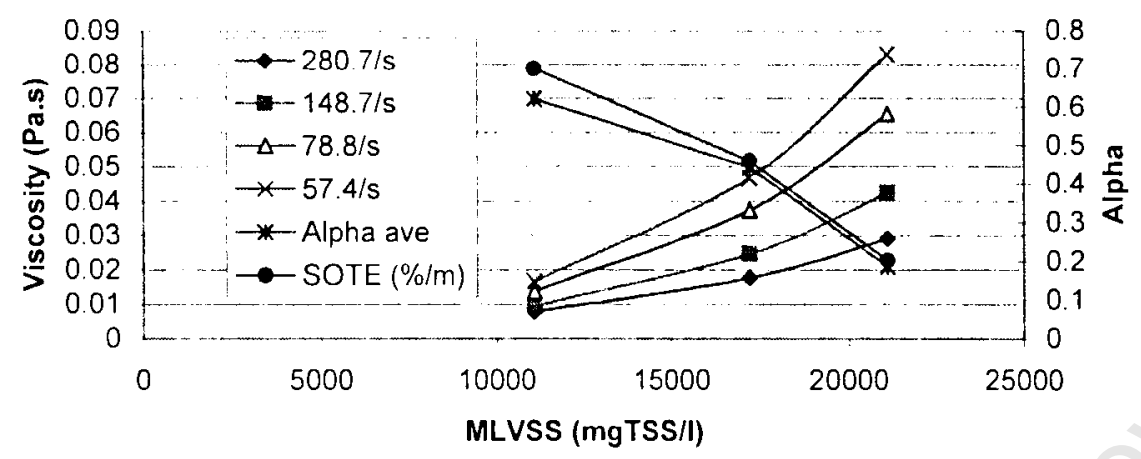

Plot B

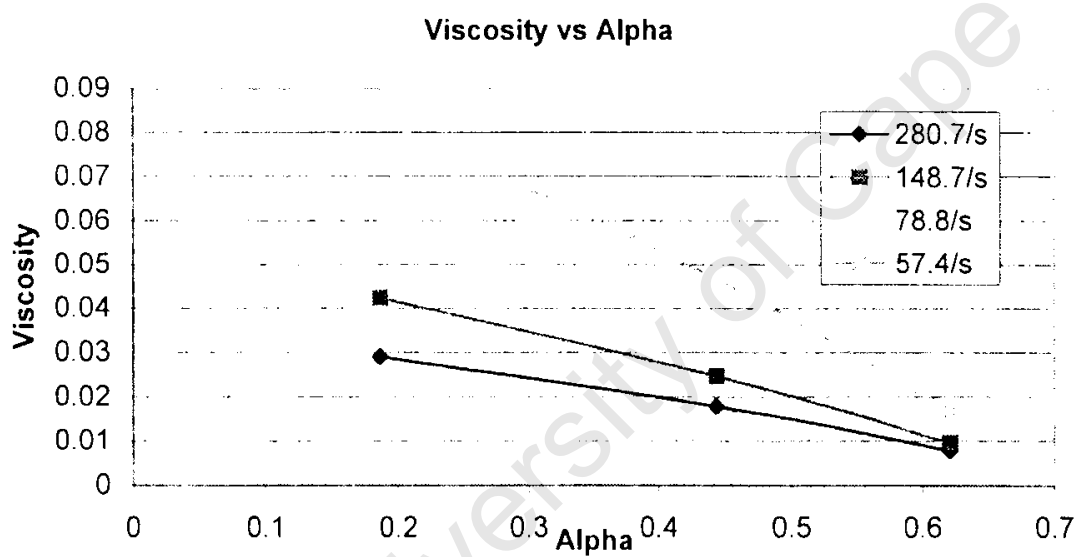

Plot C

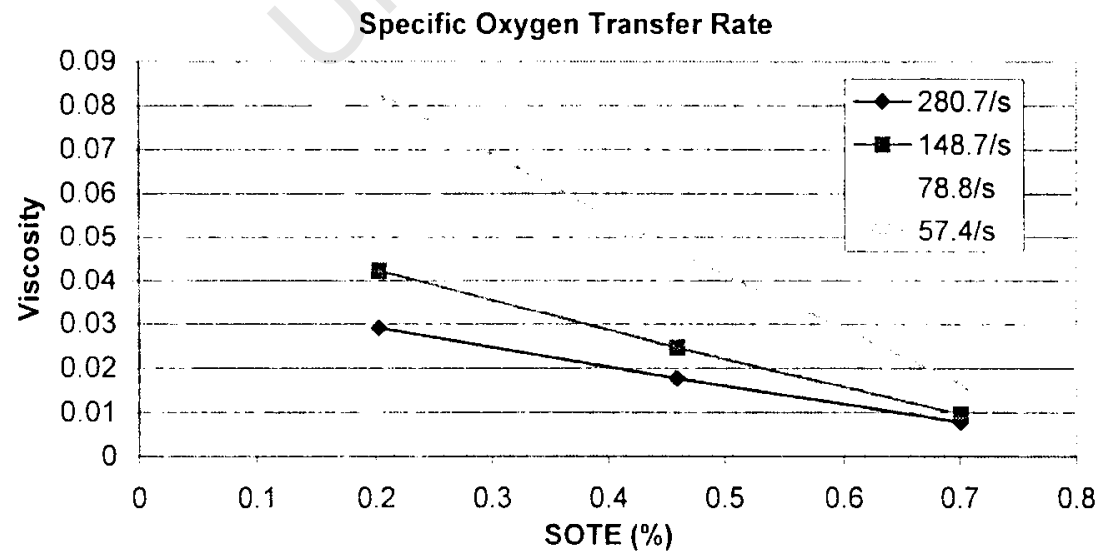




\begin{tabular}{|c|c|c|c|c|c|c|}
\hline \multicolumn{7}{|c|}{ Data Series Information } \\
\hline \multirow{2}{*}{\multicolumn{3}{|c|}{$\begin{array}{l}\text { Name: } \\
\text { Sample: }\end{array}$}} & \multicolumn{4}{|l|}{ Sample A 1} \\
\hline & & & \multirow{2}{*}{\multicolumn{4}{|c|}{ Sample A }} \\
\hline \multicolumn{3}{|c|}{ Operator: } & $\begin{array}{l}\text { Sample A } \\
\text { VGF \& GS }\end{array}$ & & & \\
\hline \multicolumn{3}{|c|}{ Remarks } & \\
\hline \multicolumn{3}{|c|}{ Number of intervals: } & \multicolumn{4}{|c|}{$\begin{array}{c}\text { Recerved } 15 \text { Aug } 2006 \\
1\end{array}$} \\
\hline \multirow{2}{*}{\multicolumn{3}{|c|}{ Application }} & \multicolumn{4}{|c|}{ US200/32 V2. $4321001629-33024$} \\
\hline & & & \multicolumn{4}{|c|}{ MC1+SN701754 } \\
\hline \multirow{2}{*}{\multicolumn{3}{|c|}{ Measurement Date. }} & \multicolumn{4}{|c|}{$8 / 15 / 2006$} \\
\hline & & Measurement Time & \multicolumn{4}{|l|}{$1638 \mathrm{PM}$} \\
\hline \multicolumn{3}{|c|}{ Measuring Systems: } & \multicolumn{4}{|c|}{$Z 2 \operatorname{DIN}(45 \mathrm{~mm})$} \\
\hline Calculating & Constants: & & & & & \\
\hline - Csr: & & & 12946486 & & & \\
\hline - Css & & & 39172248 & & & \\
\hline - Start Delas & $y$ Time [s] & & 2.924 & & & \\
\hline - Measurem & ent Type. & & 2 & & & \\
\hline Interval. & & & 1 & & & \\
\hline Number of $\square$ & Data Points & & 30 & & & \\
\hline Time Setting & & & 30 Meas. Pts & 50 & & \\
\hline Measuring $F$ & Profile: & & & & & \\
\hline Shear Rate & & & $d($ gamma $) / d t=$ & $=1 E+3 \ldots 0$ & 1 1/s Log & \\
\hline Meas Pts & $\begin{array}{l}\text { Shear Ratr } \\
1 / \mathrm{s}]\end{array}$ & $\begin{array}{l}\text { " Shear Stre } \\
{[\mathrm{Pa}]}\end{array}$ & $\begin{array}{l}\text { e Viscosity } \\
\text { [Pa:s] }\end{array}$ & $\begin{array}{l}\text { Speed } \\
{[1 / \mathrm{min}]}\end{array}$ & $\begin{array}{l}\text { Torque } \\
{[\mu \mathrm{Nm}]}\end{array}$ & $\begin{array}{l}\text { Statt } \\
\text { [] }\end{array}$ \\
\hline 1 & 1,000 & 23.08 & 0.02308 & 7726 & 5.892 & $32 M-M$ \\
\hline 2 & 727.9 & 15.51 & 0.0213 & 562.2 & 3.959 & $59 \mathrm{M}-$ \\
\hline 3 & 529.8 & 11.66 & 0.02201 & 4092 & 2.977 & $7 \mathrm{M}-$ \\
\hline 4 & 3856 & 9.627 & 0.02497 & 297.9 & 2.458 & $58 \mathrm{M}-$ \\
\hline 5 & 280.7 & 8179 & 0.02914 & 2168 & 2,088 & \\
\hline 6 & 204.3 & 7.124 & 0.03486 & 157.8 & 1,819 & $19 \mathrm{M}-$ \\
\hline 7 & 1487 & 6.304 & 0.04239 & 1149 & 1.609 & $9 \mathrm{M}$ - \\
\hline 8 & 108.3 & 5674 & 0.05241 & 83.61 & 1,448 & $48 \mathrm{M}-$ \\
\hline 9 & 788 & 6159: & $00054 \%$ & 8087 & 1,317 & $17 \mathrm{M}-$ \\
\hline 10 & 57.36 & 4764 & 0.08305 & 443 & 1.216 & $16 \mathrm{M}$ - \\
\hline 11 & 41.74 & 4.448 & 0.1066 & 32.24 & 1,135 & $35 \mathrm{M}-$ \\
\hline 12 & 3039 & 4188 & 0.1378 & 23.47 & 1,069 & $99 \mathrm{M}$. \\
\hline 13 & 22.12 & 3919 & 0.1772 & 17.08 & 1,000 & $20 \mathrm{M}$ \\
\hline 14 & 16.1 & 3.767 & 0.2339 & 12.44 & 961.5 & \\
\hline 15 & 11.72 & 3591 & 0.3065 & 905 & 9167 & \\
\hline 16 & 8534 & 3.477 & 0.4074 & 6592 & 887.6 & \\
\hline 17 & 6.214 & 3.366 & 0.5416 & 4.8 & 859.2 & \\
\hline 18 & 4515 & 3.261 & 0.7223 & 3488 & 8325 & \\
\hline 19 & 3291 & 3.141 & 0.9544 & 2.542 & 801.9 & \\
\hline 20 & 2.39 & 2.992 & 1.252 & 1846 & 763.8 & \\
\hline 21 & 1.748 & 2877 & 1.646 & 1.35 & 7344 & \\
\hline 22 & 1.271 & 2.739 & 2.155 & 0.9818 & 6991 & \\
\hline 23 & 0.9116 & 2519 & 2.764 & 0.7042 & 643.2 & \\
\hline 24 & 06689 & 2.448 & 366 & 0.5167 & 624.9 & \\
\hline 25 & 0.4909 & 2114 & 4.307 & 0.3792 & 539.8 & $8 \mathrm{M}$ \\
\hline 26 & 0.3834 & 1.966 & 5.128 & 0.2962 & 5019 & \\
\hline 27 & 0.383 & 1855 & 4.843 & 0.2958 & 473.5 & $5 \mathrm{M}$ \\
\hline 28 & 03938 & 1816 & 4.611 & 0.3042 & 4635 & $5 \mathrm{M}$. \\
\hline 29 & 0.3884 & 1618 & 4165 & 03 & & \\
\hline 30 & 03938 & 1554 & 3947 & 03042 & & \\
\hline
\end{tabular}

Name

Sample

Operator

Number of Intervals

Application

Device

Measurement Time.

Calculating Constants:

- Csr

- Start Delay Time [s]

- Measirement Type

Interval

Numbe- of Data Points:

Time Setting:

Measurng Profile

Shear Rate

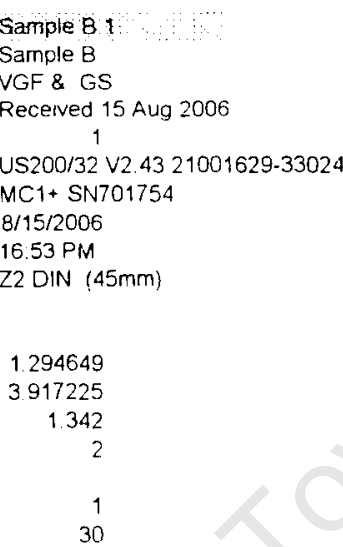

30 Meas Pts

Meas. Pt. Duration $5 \mathrm{~s}$

$\mathrm{d}($ gamma $) / \mathrm{dt}=1 \mathrm{E}+3 \ldots 0.11 / \mathrm{s} \mathrm{Log}$

Meas. Pts Shear Ratt Shear Stre Viscosity Speed Torque Status

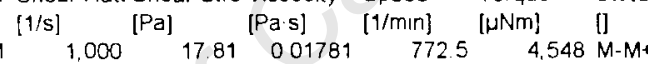
$\begin{array}{lllll}1.000 & 17.81 & 0001781 & 772.5 & 4.548 \mathrm{M}-\mathrm{M} \\ 728 & 11.96 & 0.01644 & 5622 & 3.054 \mathrm{M}-\end{array}$ $\begin{array}{rrrrr}128 & 11.96 & 0.01644 & 5622 & 3.054 \mathrm{M} \\ 529.8 & 8.075 & 0.01524 & 409.2 & 2.061 \mathrm{M} \text { - }\end{array}$ $\begin{array}{lllll}385.6 & 6.032 & 001564 & 297.9 & 1.540 \mathrm{M}\end{array}$ $\begin{array}{lllll}280.7 & 4.994 & 0.01779 & 216.8 & 1,275 \mathrm{M}\end{array}$ $\begin{array}{rrrrrr}6 & 204.3 & 4.278 & 0.02094 & 157.8 & 1,092 \mathrm{M}-\end{array}$ $\begin{array}{rlllll}148.7 & 3.671 & 0.02468 & 114.9 & 937 \mathrm{M}-\end{array}$ $\begin{array}{llllll}8 & 148.3 & 3.267 & 0.03018 & 8362 & 834 \mathrm{M}-\end{array}$

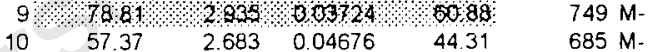
$\begin{array}{rrrrrr}10 & 57.37 & 2.683 & 0.04676 & 44.31 & 685 \mathrm{M} \text { - } \\ 11 & 41.75 & 2.49 & 0.05963 & 32.25 & 636 \mathrm{M} \text {. }\end{array}$ $\begin{array}{llllll}12 & 30.39 & 2.345 & 0.07715 & 23.48 & 599 \mathrm{M} \text {. } \\ 13 & 22.12 & 2.211 & 0.09995 & 17.09 & 565 \mathrm{M} \text {. }\end{array}$ $\begin{array}{rrrrrr}14 & 16.1 & 2.086 & 0.1296 & 12.43 & 532.6 \mathrm{M} \text { - } \\ 15 & 11.72 & 1.975 & 0.1685 & 9.055 & 50.3 \mathrm{M} \text {. }\end{array}$ $\begin{array}{llllll}15 & 11.72 & 1.975 & 0.1685 & 9.055 & 504.3 \mathrm{M} . \\ 16 & 8534 & 1.908 & 0.2236 & 6592 & 487.1 \mathrm{M} .\end{array}$ $\begin{array}{rrrrrr}16 & 8.534 & 1.908 & 0.2236 & 6.592 & 487.1 \mathrm{M} \text { - } \\ 17 & 6.214 & 1.832 & 0.2949 & 4.8 & 467.8 \mathrm{M} \text { - }\end{array}$ $\begin{array}{llllll}18 & 4.52 & 1.834 & 0.4058 & 3.492 & 468.2 \mathrm{M}-\end{array}$ $\begin{array}{llllll}19 & 3.291 & 1.712 & 0.5201 & 2.542 & 436.9 \mathrm{M}\end{array}$

$\begin{array}{llllll}20 & 1.39 & 1.665 & 0.6968 & 1.846 & 425.1 \mathrm{M} \\ 21 & 1742 & 1.563 & 0.8969 & 1346 & 399 \mathrm{M}\end{array}$

$\begin{array}{lllllll}22 & 1.268 & 1.555 & 1.227 & 0.3492 & 3969 \mathrm{M}\end{array}$ $\begin{array}{llllll}23 & 0.917 & 1.45 & 1.581 & 0.7083 & 370.2 \mathrm{M}-\end{array}$ $\begin{array}{llllll}24 & 0.6851 & 1.439 & 2.101 & 0.5292 & 367.5 \mathrm{M} \text { - } \\ 25 & 0.4963 & 1384 & 2.789 & 0.3833 & 353.4 \mathrm{M}-\end{array}$ $\begin{array}{llllll}25 & 0.4963 & 1.384 & 2.789 & 0.3833 & 353.4 \mathrm{M}- \\ 26 & 0.4046 & 1.528 & 3.778 & 0.3125 & 390.2 \mathrm{M}-\end{array}$ $\begin{array}{llllll}27 & 0.3884 & 1.281 & 3299 & 0.3 & 327.1 \mathrm{M}-\end{array}$ $\begin{array}{llllll}28 & 0.3884 & 1.181 & 3.042 & 0.3 & 301.6 \mathrm{M} \text { - }\end{array}$ $\begin{array}{rrrrrr}29 & 0.3825 & 1.03 & 2.693 & 0.2955 & 263 \mathrm{M}- \\ 30 & 0.3884 & 0.9426 & 2.427 & 03 & 240.6 \mathrm{M}-\end{array}$
Name

Sample:

Remarks

Number of Intervals

Application:

Measurement Date:

Measurement Time

Calculating Constants:

- Csr

- Start Delay Time [s]

- Measurement Type

Interval:

Number of Data Points:

TIme Setting

Measuring Profile:

Shear Rate

Sample C
VGF \& GS

Recelved 15 Aug 2006

US200/32 V2 4321001629.33024

$M C 1+$ SN701754

17:03 PM

Z2 DIN $(45 \mathrm{~mm})$

1294649

3.917225
4.005

?

30 Meas Pts

Meas Pt Duration $5 \mathrm{~s}$

$d(g a m m a) / d t=1 E+3 \ldots 011 / s \log$

Meas. Pts. Shear Ratt Shear Stre Viscosity Speed Torque Status

\begin{tabular}{|c|c|c|c|c|}
\hline$[1 / \mathrm{s}]$ & {$[\mathrm{Pa}]$} & [Pas] & {$[1 / \mathrm{min}]$} & {$[\mu \mathrm{Nm}]$} \\
\hline $1 \quad 1.000$ & 11.72 & 0.01172 & 7725 & 2,993 \\
\hline 727.9 & 7.566 & 0.01039 & 562.2 & 1.931 \\
\hline 529.8 & 5.004 & 0.009444 & 409.2 & 1,277 \\
\hline 3856 & 3.494 & 0.00906 & 297.9 & 892 \\
\hline 280.7 & 2.223 & 0.007918 & 2168 & 567.4 \\
\hline 204.3 & 1.736 & 0.008498 & 157.8 & 443.3 \\
\hline 148.7 & 1.43 & 0.009615 & 114.9 & 365 \\
\hline 1083 & 1.234 & 0.01139 & 83.62 & 314.9 \\
\hline 180 & 1001 & 001304 & 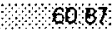 & 278.5 \\
\hline 57.37 & 0.9514 & 0.01658 & 44.32 & 242.9 \\
\hline 41.75 & 0.8882 & 0.02128 & 32.25 & 226.8 \\
\hline 304 & 0.8115 & 0.0267 & 23.48 & 207.2 \\
\hline 22.13 & 0.6832 & 0.03088 & 17.09 & 174.4 \\
\hline 16.11 & 0.5863 & 0.0364 & 12.44 & 149.7 \\
\hline 11.72 & 0.5564 & 0.04747 & 9.054 & 142 \\
\hline 8.539 & 0.592 & 0.06933 & 6.596 & 151.1 \\
\hline 6.214 & 0324 & 0.05214 & 48 & 82.71 \\
\hline 4.515 & 0.2894 & 0.06409 & 3.488 & 73.88 \\
\hline 3.285 & 0.4103 & 0.1249 & 2.537 & 104.8 \\
\hline 2.39 & 0.1668 & 0.0698 & 1.846 & 42.58 \\
\hline 1.742 & 0.2762 & 0.1585 & 1.346 & \\
\hline 1.265 & 0.7315 & 0.5781 & 0.9773 & 186.7 \\
\hline 0.917 & 0.6002 & 0.6544 & 0.7083 & 153 \\
\hline 0.6797 & 0.5657 & 0.8323 & 0.525 & 144 \\
\hline 0.4801 & 0.5624 & 1.172 & 0.3708 & 143.6 \\
\hline 0 3834 & 0.5629 & 1.468 & 02962 & 143.7 \\
\hline 0.383 & & 1.632 & 0.2958 & \\
\hline 0.3938 & 0.6405 & & 0.3042 & \\
\hline
\end{tabular}




\section{Rotameter Calibration}

Method: A five litre calibrated bucket is filled with water and held upside down under water. The rotameter is set at a certain value and for a set period of time flow is directed into the bucket, then removed. The bucket is lifted until the inner level and outer level are the same. The volume displaced divided by the time it took is the flow in litres/second.

\begin{tabular}{|c|c|c|c|c|c|c|c|c|}
\hline Rotameter Set & & 0.5 & 1 & 1.5 & 2 & 3 & 3.5 & 4 \\
\hline $5 \mathrm{~s}$ & 5 & 2000 & 2400 & 2550 & 2800 & 3250 & 3500 & 3750 \\
\hline & 5 & 2100 & 2400 & 2600 & 2900 & 3200 & 3500 & 3800 \\
\hline & 5 & 2200 & 2450 & 2600 & 2800 & 3200 & 3500 & 3900 \\
\hline ave flow $(\mathrm{l} / \mathrm{m})$ & & 25.2 & 29.0 & 31.0 & 34.0 & 38.6 & 42.0 & 45.8 \\
\hline $7 \mathrm{~s}$ & 7 & & & & & 4500 & 4900 & \\
\hline & 7 & & & & & 4600 & 4900 & \\
\hline & 7 & & & & & 4500 & 4900 & \\
\hline ave flow $(1 / \mathrm{m})$ & & & & & & 38.9 & 42.0 & \\
\hline $8 s$ & 8 & & & 4000 & 4550 & & & \\
\hline & 8 & & & 4000 & 4600 & & O & \\
\hline & 8 & & & 4000 & 4500 & & +2 & \\
\hline ave flow $(\mathrm{l} / \mathrm{m})$ & & & & 30.0 & 34.1 & & & \\
\hline $10 \mathrm{~s}$ & 10 & 4100 & 4750 & & & 8 & & \\
\hline & 10 & 4200 & 4700 & & & 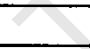 & & \\
\hline & 10 & 4200 & 4600 & & & & & \\
\hline & & 25.0 & 28.1 & & & & & \\
\hline ave flow $(1 / \mathrm{m})$ & & 25.1 & 28.6 & 30.5 & 34.1 & 38.7 & 42.0 & 45.8 \\
\hline ave flow $(\mathrm{m} 3 / \mathrm{h}$ & & 1.51 & 1.71 & 1.83 & 2.04 & 2.32 & 2.52 & 2.75 \\
\hline
\end{tabular}

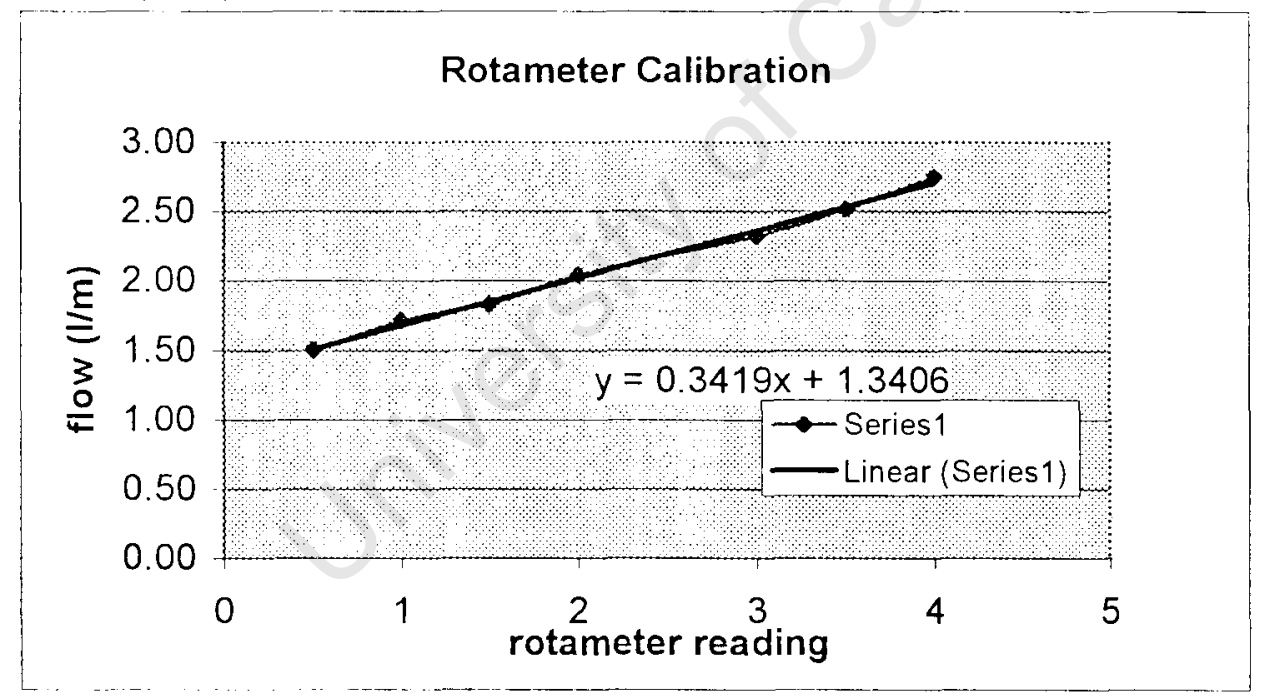




\section{APPENDIX G}

\section{DAY LONG SLUDGE AGE MBR DAILY MEASUREMENTS}

\section{TABLE OF CONTENTS}

G.1 Daily measurements for 4 Sewage Batch Periods investigated. 
40 Day Sludge Age Daily Measurements

\begin{tabular}{|c|c|c|c|c|c|c|c|c|c|c|c|c|c|c|c|c|c|c|c|c|c|c|c|c|c|c|c|c|c|c|c|c|c|c|}
\hline \multirow{2}{*}{ batch } & \multicolumn{3}{|c|}{$\mathrm{COD}$} & \multicolumn{3}{|c|}{ TKN } & \multicolumn{2}{|c|}{ FSA } & \multicolumn{3}{|c|}{ TSS } & \multicolumn{3}{|c|}{ vss } & Q & \multicolumn{2}{|c|}{ QW OUR } & \multicolumn{4}{|c|}{$\mathrm{NO} 3$} & \multicolumn{4}{|c|}{$\mathrm{NO} 2$} & \multicolumn{5}{|c|}{ P } & \multicolumn{2}{|c|}{ Recycles } & \multirow{2}{*}{\begin{tabular}{|l|}
$C O D$ \\
$\mathrm{VSS}$ \\
$\mathrm{fCV}$ \\
\end{tabular}} & \multirow{2}{*}{\begin{tabular}{|c|} 
TKN \\
VSS \\
TN \\
\end{tabular}} \\
\hline & $\operatorname{Inf}$ & Aer & Eff & $\operatorname{lnf}$ & Aer & Eff & $\operatorname{lnf}$ & Eff & AN & $A X$ & $A E$ & AN & $A X$ & $\mathrm{AE}$ & (I) & (1) & $\mathrm{mgO} / / \mathrm{hr}$ & AN & $A X$ & $A E$ & $E$ & AN & $A X$ & $A E$ & E & INF & AN & $A X$ & $\mathrm{AE}$ & EFF & $d$ & & & \\
\hline 19. Sep-06 & 9837 & 176493 & 243 & 107.5 & 1010.8 & 23 & 794 & 2.0 & 6568 & 17276 & \begin{tabular}{|c|}
16696 \\
\end{tabular} & 5676 & 15664 & 15630 & 80 & 15 & 64.9 & 0.0 & 00 & 57 & 10.7 & 0.0 & 0.0 & 0.8 & 05 & 319 & 684 & 881 & 29.4 & 135 & 300 & 1.20 & & \\
\hline 20-Sep-06 & 9800 & 123869 & 182 & 1158 & 9912 & 21 & 818 & 2.9 & 6238 & 13002 & 15366 & 5282 & |10528 & 12442 & 80 & 1.5 & 65.8 & 0.0 & 00 & 69 & 12.1 & 0.0 & 0.0 & 1.1 & 23 & 351 & 69.4 & 51.9 & 14.1 & 157 & 300 & 1.20 & 099 & 008 \\
\hline 21. Sep-06 & 1157.7 & 163539 & 283 & 105.1 & 949.2 & 20 & 82.5 & 15 & 6876 & 17940 & 14806 & 5972 & 14962 & 12128 & 80 & 15 & 612 & 00 & 00 & 0.0 & 3.6 & 00 & 0.0 & 25 & 15 & 416 & 784 & 46.3 & 10.4 & 141 & 3.00 & 1.20 & 1.35 & \\
\hline $22-\operatorname{Sep}-06$ & 11132 & 164349 & 324 & 101.5 & 963.2 & 22 & 745 & 12 & 7304 & 17722 & 16780 & 5980 & $\mid 14326$ & 15758 & 80 & 1.5 & 66.5 & 0.0 & 0.0 & 4.1 & 7.2 & 0.0 & 0.0 & 1.4 & 0.5 & 44.4 & 72.5 & 55.0 & 85 & 107 & 3.00 & 120 & 104 & $00 t$ \\
\hline $23.5 e p-06$ & 13035 & 182160 & 121 & 99.0 & 11900 & 08 & 710 & 1.9 & 4331 & 14172 & 16034 & 3741 & 11852 & 12672 & 80 & 1.5 & 869 & 0.0 & 00 & 0.0 & 11.3 & 0.0 & 0.1 & 2.6 & 04 & 475 & 80.9 & 347 & 11.6 & 12.3 & 300 & 1.20 & 1.44 & \\
\hline $24-\operatorname{sep}-06$ & 1218.4 & 182160 & 263 & 102.9 & 1092.0 & 0.6 & 78.7 & 2.5 & 6870 & 17798 & \begin{tabular}{|l|l|}
17944 \\
\end{tabular} & 5748 & 13274 & 12688 & 80 & 1.5 & 72.2 & 0.0 & 0.0 & 18 & 11.5 & 0.0 & 0.0 & 3.8 & 1.6 & 606 & 92.8 & 69.1 & 329 & 13.5 & 300 & 1.20 & 143 & 0.09 \\
\hline Average & 1126.1 & 16542.8 & 23.6 & 105.3 & 1032.7 & 1.7 & 78.0 & 2.0 & 6364 & 16318 & \begin{tabular}{|l|}
6271 \\
\end{tabular} & 5400 & 13434 & 13553 & 80 & 1.5 & 69.6 & 001 & \begin{tabular}{|l|}
0.0 \\
\end{tabular} & 3.1 & 9.4 & 0.0 & 0.0 & 2.1 & 1.1 & 53.5 & \begin{tabular}{|l|}
94.7 \\
\end{tabular} & 70.9 & 16.0 & 14.5 & 3.0 & 1.2 & 1.230 & 0.077 \\
\hline $26-5$ ep-06 & 1303.5 & 152205 & 263 & 99.8 & 1024.8 & 05 & 73.5 & 0.2 & 6138 & 14834 & 17702 & $\mid$ & 12298 & 14340 & 80 & 1.5 & 740 & 0.0 & 0.3 & 5.6 & 126 & 0.0 & 0.1 & 56 & 2.3 & 45.0 & 79.6 & 59.4 & 77.6 & 135 & 300 & 1.20 & \begin{tabular}{|l|}
1.06 \\
\end{tabular} & 0.07 \\
\hline $27-$ Sep-06 & 1181.4 & 187085 & 605 & 126.1 & 1044.4 & 20 & $8 \overline{64}$ & 03 & 6866 & 12230 & 18170 & 5776 & \begin{tabular}{|l|}
9886 \\
\end{tabular} & 14578 & 80 & 15 & 97.0 & 0.0 & 0.9 & 115 & 22.3 & 0.0 & 0.1 & 12.3 & 1.1 & 35.8 & 65.6 & 47.1 & 159 & 17.5 & 300 & 1.20 & 1.28 & 0.07 \\
\hline$\overline{2 \overline{-}-5 \mathrm{ep}-06} \%$ & 1201.5 & 188698 & 403 & 129.9 & 1075.2 & 19 & 1025 & 0.8 & 6620 & 14570 & 18486 & 5600 & 11838 & 15664 & 80 & 15 & 75.5 & 0.0 & \begin{tabular}{|l|}
00 \\
\end{tabular} & 0.0 & 23.1 & 0.0 & 0.1 & 1.3 & 0.2 & & & & & & 300 & 120 & 120 & \\
\hline $29-5$ ep-06 & 1153.2 & 199987 & 202 & 1309 & 1061.2 & 19 & 934 & 07 & 7748 & 13912 & 18988 & |6498 & 11486 & 15470 & 80 & 15 & 78.6 & 0.0 & 0.2 & 18.2 & 21.3 & 0.0 & 01 & 3.7 & 13 & 38 & 63.3 & 688 & 203 & 15.4 & 3.00 & 1.20 & 1.29 & \\
\hline $30-5 e p-06$ & 1229.8 & 19514.9 & 444 & 130.6 & 1248.8 & 1.8 & 1114 & 16 & 6956 & 14518 & 19468 & $\mid$ & 12024 & 15328 & 80 & 15 & 87.5 & 0.0 & 00 & 00 & 25.4 & 0.0 & 0.3 & 1.0 & 0.2 & 36.3 & 61.5 & 347 & 172 & 18.8 & 3.00 & 120 & 127 & 0.00 \\
\hline Average & 1213.9 & 18462.5 & 38.3 & 123.5 & 1090.9 & 1.6 & 93.4 & 0.7 & 6866 & 14013 & 18563 & 5806 & 11506 & 15076 & 80 & 1.5 & 82.5 & 0.0 & 0.3 & 7.1 & 20.9 & 0.0 & 0.1 & 4.8 & 1.0 & 37.8 & 89.8 & 100.6 & 67.4 & 20.8 & 3.0 & 1.2 & 1.221 & 0.072 \\
\hline 4 -0ct-06 & 10960 & 164000 & 140 & 127.1 & 11340 & 21 & 1028 & 0.7 & 7192 & 12870 & 15150 & (5966 & 10392 & 11592 & 80 & 1.5 & 92.7 & 0.0 & 0.2 & 131 & 28.7 & 0.0 & 0.2 & 4.8 & 0.2 & 372 & 70.1 & 62.8 & 30.2 & 18.1 & 300 & 1.20 & 1.41 & \begin{tabular}{|l|}
0.10 \\
\end{tabular} \\
\hline $5-0 \mathrm{ct}-06$ & 11080 & 208800 & 140 & 123.1 & 1103.2 & 2.2 & 1030 & 04 & 7182 & 11656 & 15776 & 6048 & \begin{tabular}{|l|}
9516 \\
\end{tabular} & 12688 & 80 & 1.5 & 858 & 0.0 & 0.0 & 0.0 & 17.9 & 0.0 & 0.2 & 1.7 & 09 & 533 & 850 & 49 & 23.4 & 25.2 & 300 & 1.20 & 1.64 & 0.0 \\
\hline 6 -oct-06 & 1088.0 & 163200 & 260 & 119.8 & 1187.2 & 15 & 994 & 0.2 & 6730 & 13072 & 14282 & 5708 & 10604 & 11266 & 80 & 15 & 88.1 & 0.0 & 1.9 & 20.8 & 19.2 & 0.0 & 0.1 & & 02 & 527 & 998 & 566 & 286 & 195 & 300 & 1.20 & 1.45 & 0.1 \\
\hline $7-0 \mathrm{ct}-06 \mathrm{Q}$ & 10280 & 168000 & 320 & 111.3 & 1005.2 & 2.2 & 102.9 & 0.2 & 7464 & 12056 & 15132 & 6253 & 10056 & 12948 & 80 & 15 & 86.8 & 0.0 & 0.0 & 6.1 & 17.9 & 0.0 & 0.1 & & 0.3 & 41.6 & 82.6 & 41.6 & 20.7 & 180 & 300 & 1.20 & 130 & 00 \\
\hline $8.0 c t-06 \approx$ & & & & & 0.0 & 0.0 & & 00 & & & & & & & & & & & & & & & & & & 45.8 & 83.8 & 103.5 & 159 & 18.6 & & & & \\
\hline $\mathrm{S}-\mathrm{OCt}-06$ & 1012.0 & 180000 & 40.0 & 125.0 & 1195.6 & 04 & 1056 & 0.1 & 6404 & 13054 & $\overline{14660}$ & 5302 & $10596 \mid$ & $\overline{11680}$ & 80 & 1.5 & 79.8 & 0.0 & 1.7 & 282 & 34.1 & 0.0 & 0.1 & & 0.1 & 36.4 & 47.9 & 304 & 13.2 & 150 & 300 & 1.20 & 154 & 01 \\
\hline $10.0 \mathrm{ct}-06$ & 7024.0 & 180800 & 38.0 & 1148 & 1262.8 & 13 & 93.2 & $|05|$ & 6200 & 11910 & 15622 & 5212 & \begin{tabular}{|l|}
9772 \\
\end{tabular} & 12598 & 80 & 1.5 & 88.1 & 0.0 & \begin{tabular}{|l|}
1.7 \\
\end{tabular} & 26.4 & 30.3 & 0.0 & 0.1 & 13 & 0.1 & & & & & & 300 & 1.20 & 1.43 & 0.1 \\
\hline Average & 1059.3 & 17746.7 & 27.3 & 120.2 & 1148.0 & 1.6 & 101.2 & 0.4 & 6862 & 12436 & 15104 & 5748 & 10156 & 12129 & 80 & 1.5 & 86.9 & 0.0 & \begin{tabular}{|l|}
0.9 \\
\end{tabular} & 15.8 & 24.7 & 0.0 & 0.1 & 2.6 & 0.3 & 44.5 & 78.2 & 57.3 & 22.0 & 19.1 & 3.0 & 1.2 & 1.462 & 0.095 \\
\hline 15-0ct-06 姼 & 11894 & 181440 & 32.3 & 132.3 & 1111.6 & 2.9 & 1022 & 0.4 & 5810 & 12318 & 17220 & 5044 & 10088 & 13668 & 80 & 1.5 & 993 & 0.0 & 0.0 & 155 & 23.7 & 0.0 & 0.1 & 2.3 & 12 & & & & & & 3.0 & 1.2 & 1.33 & 0.0 \\
\hline $16-0 \mathrm{ct}-06$ & 1181.4 & 176602 & 50.4 & 123.9 & 1190.0 & 22 & 1064 & 1.8 & 6232 & 12490 & 15714 & 5268 & 10124 & 12438 & 80 & 1.5 & 82.1 & 0.0 & 0.0 & 160 & 201 & 0.0 & 0.0 & 1.5 & 0.8 & & & & & & 3.0 & 1.2 & 1.42 & 0.1 \\
\hline 17 -OCt-06 & 999.9 & 199987 & 42.3 & 132.7 & 1131.2 & 3.1 & 98.8 & \begin{tabular}{|l|}
1.3 \\
\end{tabular} & 5790 & 13912 & 17312 & \begin{tabular}{|l|}
4872 \\
\end{tabular} & 11486 & 13892 & 80 & 1.5 & 860 & 0.0 & \begin{tabular}{|l|}
0.1 \\
\end{tabular} & 23.0 & 26.3 & 0.0 & 0.1 & 2.0 & 0.7 & & & & & & 3.0 & 1.2 & 144 & \\
\hline $18.0 \mathrm{ct}-06$ & 923.3 & 19031.0 & 28.2 & 108.2 & 1290.8 & 2.9 & 89.9 & \begin{tabular}{|l|}
0.8 \\
\end{tabular} & 5046 & 12280 & 15630 & \begin{tabular}{|l|l|}
4376 \\
\end{tabular} & 10298 & 12554 & 80 & 0.0 & 85.5 & 0.0 & \begin{tabular}{|l|}
0.0 \\
\end{tabular} & 18.4 & 27.5 & 0.0 & 0.0 & 1.6 & 0.9 & & & & & & 30 & 1.2 & 1.51 & 0.1 \\
\hline $20-0 c t-06$ & 840.3 & 20442.4 & 50.5 & 114.4 & 1066.8 & 20 & 90.4 & 0.6 & 6378 & 12826 & 19006 & 5240 & 10252 & 14432 & 80 & 1.5 & 108.7 & 0.0 & 0.0 & 17.5 & 22.3 & 0.0 & 0.1 & 3.1 & 1.7 & & & & & & 30 & 1.2 & 1.41 & 00 \\
\hline $21-0 \mathrm{ct}-06$ & 812.0 & 16887.2 & 44.4 & 129.8 & 910.0 & 0.3 & 91.4 & & 5360 & 9862 & 16230 & 4460 & 7996 & 12832 & 80 & 1.5 & 113.4 & 0.0 & 0.0 & 17.2 & 340 & 0.0 & 0.1 & 1.7 & 0.8 & & & & & & 30 & 1.2 & & \\
\hline $2 \overline{22.0 c t-06}$ & 8242 & 177760 & 48.5 & 115.6 & 985.6 & 2.0 & 97.4 & 06 & 4248 & 9680 & 16420 & 3670 & 7950 & 13024 & 80 & 1.5 & 117.9 & 0.0 & 0.2 & 196 & 37.6 & 0.0 & 0.1 & 5.1 & 0.8 & & & & & & 30 & 1.2 & 1.36 & $\overline{0.0}$ \\
\hline Average & 967.2 & 18562.8 & 42.4 & 122.4 & 1098.0 & $\frac{2.2}{2.2}$ & 96.7 & 0.8 & 5552 & 11910 & 16790 & 4704 & 9742 & 13263 & 80 & 1.3 & 99.0 & 0.0 & \begin{tabular}{|l|}
0.0 \\
\end{tabular} & 18.2 & 27.4 & 0.0 & 0.1 & 2.5 & 1.0 & & & & & & 3.0 & 1.2 & 1.40 & 0.08 \\
\hline
\end{tabular}

